\title{
A FINITE-ELEMENT SIMULATION MODEL FOR SATURATED- UNSATURATED, FLUID-DENSITY-DEPENDENT GROUND- WATER FLOW WITH ENERGY TRANSPORT OR CHEMICALLY- REACTIVE SINGLE-SPECIES SOLUTE TRANSPORT
}

By Clifford I. Voss 


\section{UNITED STATES DEPARTMENT OF THE INTERIOR WILLIAM P. CLARK. Secretary}

GEOLOGICAL SURVEY

Dallas L. Peck. Director

For additional information write to:

Copies of this report can be purchased from:

Chief Hydrologist

U.S. Geological Survey

U.S. Geological Survey Open-File Services Section

431 National Center

Reston, Virginia 22092 Western Distribution Branch Box 25425. Federal Center Denver, Colorado 80225 
11. A Finite-Element Simulation Model for Saturated-Unsaturated, Fluid-Density-Dependent Ground-Water Flow with Energy Transport or Chemically-Reactive Single Species Solute Transport. (UNCLASSIFIED)

19.

SUTRA flow simulation may be employed for areal and cross-sectional modeling of saturated ground-water flow systems, and for cross-sectional modeling of unsaturated zone flow. Solute transport simulation using SUTRA may be employed to model natural or man-induced chemical species transport including processes of solute sorption, production and decay, and may be applied to analyze ground-water contaminant transport problems and aquifer restoration designs. In addition, solute transport simulation with SUTRA may be used for modeling of variable density leachate movement, and for cross-sectional modeling of saltwater intrusion in aquifers at near-well or regional scales, with either dispersed or relatively sharp transition zones between fresh water and salt water. SUTRA energy transport simulation may be employed to model thermal regimes in aquifers, subsurface heat conduction, aquifer thermal energy storage systems, geothermal reservoirs, thermal pollution of aquifers, and natural hydrogeological convection systems.

Mesh construction is quite flexible for arbitrary geometries employing quadrilateral finite elements in Cartesian or radial-cylindrical coordinate systems. The mesh may be coarsened employing 'pinch nodes' in areas where transport is unimportant. Permeabilities may be anisotropic and may vary both in direction and magnitude throughout the system as may most other aquifer and fluid properties. Boundary conditions, sources and sinks may be time-dependent. A number of input data checks are made in order to verify the input data set. An option is available for storing the intermediate results and restarting simulation at the intermediate time. An option to plot results produces output which may be contoured directly on the printer paper. Options are also available to print fluid velocities in the system, and to make temporal observations at points in the system.

Both the mathematical basis for SUTRA and the program structure are highly general, and are modularized to allow for straightforward addition of new methods or processes to the simulation. The FORTRAN-77 coding stressed clarity and modularity rather than efficiency, providing easy access for eventual modifications.

18.

DESCRIPTORS: Two Dimensional Flow Decay Adsorption

IDENTIFIERS: Thermal Pollution Water Pollution Leaching

SUTRA (Saturated-Unsaturated Transport) 


\section{REPORT DOCUMENTATION PAGE}

13. REPORT SECURITY CLASSIFICATION

UNCLASSIFIED

20. SECURITY CLASSIFICATION AUTHORITY

3. DISTRIBUTION/AVAILABILITY OF REPORT

Approved for public release; distribution unlimited.

2b. DECLASSIFICATION/DOWNGRADING SCHEDULE

5. MONITORING ORGANIZATION REPORT NUMBER(S)

ESL-TR-85-10

Water-Resources Investigations

Report 84-4369

6. NAME OF PERFORMING ORGANIZATION

U.S. Geological Survey

6b. OFFICE SYMBOL (If applicable)

7. NAME OF MONITORING ORGANIZATION

HQ AFESC/RDVW

6c. ADDRESS (City, State and ZIP Code)

431 National Center

7b. ADDRESS (City, State and ZIP Code)

Tyndall AFB, Florida 32403

Reston, Virginia 22092

8. NAME OF FUNDING/SPONSORING ORGANIZATION JOIntly funded and sponsored by $6 \& 7$ above. 8c. ADDRESS (City, State and ZIP Code) 8b. OFFICE SYMBOL (If applicable)
9. PROCUREMENT INSTRUMENT IDENTIFICATION NUMBER

MIPR-N-83-18
10. SOURCE OF FUNDING NOS.

\section{PROGRAM} ELEMENT NO.

$63723 \mathrm{~F}$ PROJECT
NO.

2103
TASK NO.

90
WORK UNIT NO.

11. TITLE (Include Security Classification)

Voss, Clifford I.

12. PERSONAL AUTHOR(S)

13b. TIME COVERED FROM 821229 TO 850130
14. DATE OF REPORT (Yr., Mo., Day) 841230
15. PAGE COUNT 409

Final

16. SUPPLEMENTARY NOTATION

17. FIE 12

\begin{tabular}{|c|c|c|}
\multicolumn{3}{|c}{ COSATI CODES } \\
\hline GROUP & SUB. GR. \\
\hline 08 & \\
\hline 01 & \\
\hline
\end{tabular}

\begin{tabular}{|c|c|c|}
\hline Ground Water & Transport & Energy \\
\hline Mathematical Models & Flow & Fluid Flow \\
\hline Computer Programs & solutes & Radial Flow \\
\hline
\end{tabular}

19. ABSTRACT (Continue on reverse if necessary and identify by block number)

SUTRA (Saturated-Unsaturated Transport) is a computer program which simulates fluid movement and the transport of either energy or dissolved substances in a subsurface environment. The model employs a two-dimensional hybrid finite-element and integratedfinite-difference method to approximate the governing equations that describe the two interdependent processes that are simulated by SUTRA:

1. fluid density-dependent saturated or unsaturated ground-water flow, and either 2a. transport of a solute in the ground water, in which the solute may be subject to: equilibrium adsorption on the porous matrix, and both first-order and zero-order production or decay, or,

2b. transport of thermal energy in the ground water and solid matrix of the aquifer. SUTRA provides, as the primary calculated result, fluid pressures and either solute concentrations or temperatures, as they vary with time, everywhere in the simulated subsurface system. SUTRA may also be used to simulate simpler subsets of the above process 20. DISTRIBUTION/AVAILABILITY OF ABSTRACT

UnClassified/UNLIMITEO $\otimes$ Same As RPt. $\square$ otic users $\square$ 21. ABSTRACT SECURITY CLASSIFICATION UNCLASSIFIED 22. NAME OF RESPONSIBLE INDIVIOUAL

1Lt Edward Heyse 22b. TELEPHONE NUMBER

Tnclude Area Code

(904) 283-4628 22c. OFFICE SYMBOL

HQ AFESC/RDVW 
This report describes a complex computer model for analysis of fluid flow and solute or energy transport in subsurface systems. The user is cautioned that while the model will accurately reproduce the physics of flow and transport when used with proper discretization, it will give meaningful results only for well-posed problems based on sufficient supporting data.

The user is requested to kindly notify the originating office of any errors found in this report or in the computer program. Updates will occasionally be made to both the report and the computer program to include corrections of errors, addition of processes which may be simulated, and changes in numerical algorithms. Users who wish to be added to the mailing list for updates may send a request to the originating office at the following address:

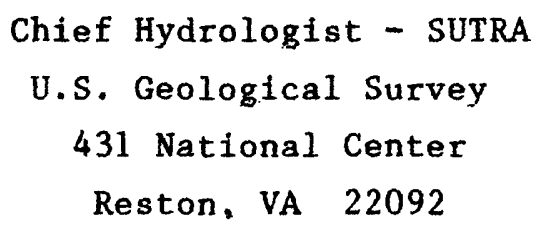

Copies of the computer program on tape are available at cost of processing from:

$$
\begin{aligned}
& \text { U.S. Geological Survey } \\
& \text { WATSTORE Program office } \\
& 437 \text { National Center } \\
& \text { Reston, VA } 22092 \\
& \text { Telephone: 703-860-6871 }
\end{aligned}
$$

This report has been reviewed by the Public Affairs Office (AFESC/PA) and is releasable to the National Technical Information Service (NTIS). At NTIS, it will be available to the general public, including foreign nationals.

$$
\text { (p.vii follows) }
$$


ABSTRACT

SUTRA ( Saturated-Unsaturated Transport) is a computer program which simulates fluid movement and the transport of either energy or dissolved substances in a subsurface environment. The model employs a two-dimensional hybrid fintte-element and integrated-finite-difference method to approximate the governing equations that describe the two interdependent processes that are simulated:

1) fluid density-dependent saturated or unsaturated ground-water flow, and either

2a) transport of a solute in the ground water, in which the solute may be subject to: equilibrium adsorption on the porous matrix, and both first-order and zero-order production or decay, or,

2b) transport of thermal energy in the ground water and solid matrix of the aquifer.

SUTRA provides, as the primary calculated result, fluid pressures and either solute concentrations or temperatures, as they vary with time, everywhere in the simulated subsurface system. SUTRA may also be used to simulate simpler subsets of the above process.

SUTRA flow simulation may be employed for areal and cross-sectional modeling of saturated ground-water flow systems, and for cross-sectional modeling of unsaturated zone flow. Solute transport simulation using SUTRA may be employed to model natural or man-Induced chemical species transport including processes of solute sorption, production and decay, and may be applied 
to analyze ground-water contaminant transport problems and aquifer restoration designs. In addition, solute transport simulation with SUTRA may be used for modeling of variable density leachate movement, and for cross-sectional modeling of salt-water intrusion in aquifers at near-well or regional scales, with either dispersed or relatively sharp transition zones between fresh water and salt water. SUTRA energy transport simulation may be employed to model thermal regimes in aquifers, subsurface heat conduction, aquifer thermal energy storage systems, geothermal reservoirs, thermal pollution of aquifers, and natural hydrogeologic convection systems.

Mesh construction is quite flexible for arbitrary geometries employing quadrilateral finite elements in Cartesian or radial-cylindrical coordinate systems. The mesh may be coarsened employing 'pinch nodes' in areas where transport is unimportant. Permeabilities may be anisotropic and may vary both in direction and magnitude throughout the system as may most other aquifer and fluid properties. Boundary conditions, sources and sinks may be time-dependent. A number of input data checks are made in order to verify the input data set. An option is available for storing intermediate results and restarting simulation at the intermediate time. An option to plot results produces output which may be contoured directly on the printer paper. Options are also available to print fluid velocities in the system, to print fluid mass and solute mass or energy budgets for the system, and to make temporal observations at points in the system.

Both the mathematical basis for SUTRA and the program structure are highly general, and are modularized to allow for straightforward addition of new methods or processes to the simulation. The FORTRAN-77 coding stresses clarity and modularity rather than efficiency, providing easy access for eventual modifications. 


\section{ACKNOWLEDGMENTS}

The SUTRA computer code and this report were prepared under a joint research project of the U.S. Geological Survey, Department of the Interior (USGS-MIPR-N-83-18) and the Engineering and Services Laboratory, U.S. Air Force Engineering and Services Center (AFESC-JON:2103-9025) entitled, "Groundwater model development for enhanced characterization of contaminant fate and transport." 
TABLE OF CONTENTS

Page

PREFACE

v

ABSTRACT -

ACKNOWLEDGMENTS-

TABLE OF CONTENTS-

LIST OF FIGURES-

\section{INTRODUCTION}

Chapter 1

Introduction- 3

1.1 Purpose and Scope-1

1.2 The Model- 4

1.3 SUTRA Processes- 6

1.4 Some SUTRA Applications- 7

1.5 SUTRA Numerical Methods- 8

1.6 SUTRA as a Tool of Analysis- 11 
SUTRA FUNDAMENTALS

Chapter 2

Physical-Mathematical Basis of SUTRA Simulation-

2.1 Physical Properties of Solid Matrix and Fluid-_ 16

Fluid physical properties- 16

Properties of fluid within the solid matrix- 19

2.2 Description of Saturated-Unsaturated Ground-water Flow------- 25

Fluid flow and flow properties- 25

Fluid mass balance- 33

2.3 Description of Energy Transport in Ground Water-

Subsurface energy transport mechanisms- 35

Solid matrix-fluid energy balance- 36

2.4 Description of Solute Transport in Ground Water- 38

Subsurface solute transport mechanisms- 38

Solute and adsorbate mass balances- 39

Adsorption and production/decay processes- 43

2.5 Description of Dispersion- 47

Pseudo-transport mechanism-_- 47

Isotropic-media dispersion model- 48

Anisotropic-media dispersion model- 50

Guidelines for applying dispersion model- 54

2.6 Unified Description of Energy and Solute Transport-- 56

Unified energy-solute balance- 56

Fluid-mass-conservative energy-solute balance-_- 58

Chapter 3

Fundamentals of Numerical Algorithms 63

3.1 Spatial Discretization by Finite Elements- 65

3.2 Representation of Coefficients in Space- 68

Elementwise discretization- 71

Nodewise discretization- 73

Cellwise discretization- 74

3.3 Integration of Governing Equation in Space- 75

Approximate governing equation and weighted residuals method--- 75

Cellwise integration of time-derivative term- 77

Elementwise integration of flux term and origin of

boundary fluxes- 79

Cellwise integration of source term- 83 
3.4 Time Discretization of Governing Equation

Time steps- 85

Resolution of non-linearities- 86

3.5 Boundary Conditions and Solution of Discretized Equation -- - - 87

Matrix equation and solution sequence- 87

Specification of boundary conditions 90

\section{DETAILS OF SUTRA METHODOLOGY}

Chapter 4

Numerical Methods- 95

4.1 Basis and Weighting Functions

4.2 Coordinate Transformations

4.3 Gaussian Integration-

4.4 Numerical Approximation of SUTRA Fluid Mass BalanceSpatial integration-- 106

Temporal discretization and iteration-

Boundary conditions, fluid sources and sinks-

4.5 Numerical Approximation of SUTRA Unified Solute Mass

and Energy Balance-

Spatial integration-

Temporal discretization and iteration-_-

Boundary conditions, energy or solute mass sources and sinks---123

4.6 Consistent Evaluation of Fluid Velocity-125

4.7 Temporal Evaluation of Adsorbate Mass Balance-_-

Chapter 5

Other Methods and Algorithms

5.1 Rotation of Permeability Tensor-

5.2 Radial Coordinates

5.3 Pinch Nodes-135

5.4 Solution Sequencing- 140

5.5 Velocity Calculation for Output_ 
5.6 Budget Calculations- 143

5.7 Program Structure and Subroutine Descriptions-

\section{SUTRA SIMULATION EXAMPLES}

Chapter 6

Simulation Examples-

6.1 Pressure Solution for Radial Flow to a Well

(Theis Analytical Solution)

6.2 Radial Flow with Solute Transport

(Analytical Solutions)

6.3 Radial Flow with Energy Transport

(Analytical Solution) - 186

6.4 Areal Constant-Density Solute Transport

(Example at Rocky Mountain Arsenal)

6.5 Density-Dependent Flow and Solute Transport

(Henry (1964) Solution for Sea-Water Intrusion)-_- 196

6.6 Density-Dependent Radial Flow and Energy Transport

(Aquifer Thermal Energy Storage Example)

6.7 Constant-Density Unsaturated Flow and Solute Transport

(Example from Warrick, Biggar, and Nielsen (1971))-_-_-_-_-_09

\section{SUTRA SIMULATION SETUP}

Chapter 7

Simulation Setup-

7.1 SUTRA Data Requirements-221

7.2 Discretization Rules-of-Thumb- 229 
7.3 Program Dimensions-235

7.4 Input and Output Files-237

7. 5 User-Supplied Programming-

Subroutine UNSAT-

Subroutine BCTIME-_- 239

7.6 Modes and Options-

Simulation modes-C-

Output options- 43

7.7 SUTRA Input Data List-

UNIT 5- UNIT $55-0-247$

UNIT 55-

REFERENCES-

APPENDICES

Appendix A: Nomenclature-1

Appendix B: SUTRA Program Listing (Model version V1284-2D)--_---_---301

Appendix C: Data File Listing for Radial Energy Transport Example----381

Appendix D: Output Listing for Radial Energy Transport Example-------391 
Figure 2.1

Saturation-capillary pressure relationship (schematic).

Figure 2.2

Definition of anisotropic permeability and effective

permeability, $\mathrm{k}$

Figure 2.3

Relative permeability-saturation relationship (schematic).

Figure 2.4

Definition of flow-direction-dependent longitudinal

dispersivity, $\alpha_{L}(\theta)$.

Figure 3.1

Two-dimensional finite-element mesh and quadrilateral

element.

Figure 3.2

Elementwise discretization of coefficient $k(x, y)$

Figure 3.3

Nodewise discretization of coefficient $h(x, y)$

Figure 3.4

Cells, elements and nodes for a two-dimensional

finite-element mesh composed of quadrilateral

elements. - 72

Figure 3.5

Schematic representation of specified head (or

pressure) boundary condition.

Figure 4.1

Quadrilateral finite element in local coordinate

system $(\xi, \eta)$

Figure 4.2

Perspectives of basis function $\Omega_{i}(\xi, n)$ at node 1.

Figure 4.3

Finite element in local coordinate system with Gauss

points.

Figure 5.1

Finite-element mesh in radial coordinates.

Figure 5.2

Finite-element mesh with pinch nodes. 
Figure 5.3

Detail of mesh with a pinch node.

Figure 5.4

Finite element in local coordinates $(\xi, n)$ with

pinch nodes.

Figure 5.5

Finite element in global coordinates $(x, y)$ with element

centroid.

Figure 5.6

Schematic of SUTRA output

Figure 5.7

SUTRA logic flow

Figure 6.1

Radial finite-element mesh for Theis solution.

Figure 6.2

Match of Theis analytical solution (solid line)

with SUTRA solution $(+)$.

Figure 6.3

Radial finite-element mesh for constant-density

solute and energy transport examples.

Figure 6.4

Match of analytical solutions for radial solute transport of Hoopes and Harleman (1967) (dashed),

Gelhar and Collins (1971), (solid), and SUTRA

solution (dash-dot). Number of elapsed time

steps is $n$.

F1gure 6.5

Match of analytical solution for radial energy

transport (modified from Gelhar and Collins (1971)

solid line) with SUTRA solution (dashed line).

Number of elapsed time steps is $n$.

Figure 6.6

Idealized representation for example at Rocky

Mountain Arsenal, and finite-element mesh.

Figure 6.7

Nearly steady-state conservative solute plume as simulated for the Rocky Mountain Arsenal example by SUTRA. 
Figure 6.8

Nearly steady-state solute plume (with solute half-life $\sim 20$. years) as simulated for the

Rocky Mountain Arsenal example by SUTRA.

Figure 6.9

Boundary conditions and finite-element mesh

for Henry (1964) solution.

Figure 6.10

Match of isochlors along bottom of aquifer

for numerical results of Huyakorn and Taylor

(1976) and SUTRA.

Figure 6.11

Match of isochlor contours for Henry analytical

solution (for 0.50 isochlor) (long dashes), INTERA

code solution (short dashes), SUTRA solution (solid

line).

Figure 6.12

Match of 0.50 isochlor contours for Henry problem

with simulated results for $D_{m}=6.6 \times 10^{-9}\left[\mathrm{~m}^{2} / \mathrm{s}\right]$

of Pinder and Cooper (1970), (short dashes), Segol,

et al (1975) (dotted line), Frind (1982) (long and

short dashes), Desai and Contractor (1977) (long dashes).

SUTRA results at isochlors $(0.25,0.50,0.75)$ (solid line).

Henry (1964) solution for $D_{m}=18.8571 \times 10^{-9}\left[\mathrm{~m}^{2} / \mathrm{s}\right]$,

( 0.50 isochlor, dash-dot)

Figure 6.13

Radial two-dimensional finite-element mesh for

aquifer thermal energy storage example.

Figure 6.14

SUTRA results after 30 days of hot water injection.

Figure 6.15

SUTRA results after 90 days of hot water injection.-10

Figure 6.16

SUTRA results after 30 days of pumping, (120 days

total elapsed time).:- 110

Figure 6.17

SUTRA results after 60 days of pumping, (150 days

total elapsed time.) 
Figure 6.18

SUTRA results after 90 days of pumping, (180 days

total elapsed time.)

Figure 6.19

Propagation of moisture front for unsaturated flow and solute transport example. Results of Van Genuchten

(1982) and SUTRA shown in same solid line.

Figure 6.20

Propagation of solute slug for unsaturated flow and solute

transport example. Results of Van Genuchten (1982) and

SUTRA shown in same solid line.

Figure 7.1

Minimization of band width by careful numbering of nodes.-

Figure 7.2

Allocation of sources and boundary fluxes

in equal-sized elements. 


\section{INTRODUCTION}

1

(p. 3 follows) 


\section{Chapter 1}

\section{Introduction}

\subsection{Purpose and Scope}

SUTRA ( Saturated-Unsaturated Transport) is a computer program which simulates fluid movement and transport of either energy or dissolved substances in a subsurface environment. The model employs a two-dimensional hybrid finiteelement and integrated-finite-difference method to approximate the governing equations that describe the two interdependent processes that are simulated:

1) fluid density-dependent saturated or unsaturated ground-water flow, and either

2a) transport of a solute in the ground water, in which the solute may be subject to: equilibrium adsorption on the porous matrix, and both first-order and zero-order production or decay, or,

2b) transport of thermal energy in the ground water and solid matrix of the aquifer.

SUTRA provides, as the primary calculated result, fluid pressures and either solute concentrations or temperatures, as they vary with time, everywhere in the simulated subsurface system. SUTRA may also be used to simulate simpler subsets of the above processes. 
This report describes the physical-mathematical basis and the numerical methodology of the SUTRA computer code. The report may be divided into three levels which may be read depending on the reader's interest. The overview of simulation with SUTRA and methods may be obtained from Chapter 1 - Introduction. The basis, at a fundamental level, for a reader who will carry out simulations with SUTRA may be obtained by additional reading of: Chapter 2 - PhysicalMathematical Basis of SUTRA Simulation, which gives a complete and detailed description of processes which SUTRA simulates and also describes each physical parameter required by SUTRA input data, Chapter 3 - Fundamentals of Numerical Algorithms, which gives an introduction to the numerical aspects of simulation with SUTRA, Chapter 6 - Simulation Examples, and Chapter 7 - Simulation Setup which includes the SUTRA Input Data List. Finally, for complete details of SUTRA methodology, the following additional sections may be read: Chapter 4 - Numerical Methods, and Chapter 5 - Other Methods and Algorithms. Chapter 4 provides the detail upon which program modifications may be based, while portions of Chapter 5 are valuable background for certain simulation applications.

\subsection{The Model}

SUTRA is based on a general physical, mathematical and numerical structure implemented in the computer code in a modular design. This allows straightforward modifications and additions to the code. Eventual modifications may be, for example, the addition of non-equilibrium sorption (such as two-site models), equilibrium chemical reactions or chemical kinetics, or addition of over- and underburden heat loss functions, a well-bore model, or confining bed leakage. 
The SUTRA model stresses general applicability, numerical robustness and accuracy, and clarity in coding. Computational efficiency is somewhat diminished to preserve these qualities. The modular structure of SUTRA, however allows implementation of any eventual changes which may improve efficiency. Such modifications may be in the configuration of the matrix equations, in the solution procedure for these equations, or in the finite-element integration routines. Furthermore, the general nature and flexibility of the input data allows easy adaptability to user-friendly and graphic input-output programming. The modular structure would also ease major changes such as modifications for multi-layer (quasi-three-dimensional) simulations, or for simultaneous energy and solute transport simulations.

SUTRA is primarily intended for two-dimensional simulation of flow, and either solute or energy transport in saturated variable-density systems. While unsaturated flow and transport processes are included to allow simulation of some unsaturated problems, SUTRA numerical algorithms are not specialized for the non-linearities of unsaturated flow as would be required of a model simulating only unsaturated flow. Lacking these special methods, SUTRA requires fine spatial and temporal discretization for unsaturated flow, and is therefore not an economical tool for extensive unsaturated flow modeling. The general unsaturated capability is implemented in SUTRA because it fits simply in the structure of other non-linear coefficients involved in density-dependent flow and transport simulation without requiring special algorithms. The unsaturated flow capability is thus provided as a convenience to the user for occasional analyses rather than as the primary application of this tool. 
Simulation using SUTRA is in two space dimensions, although a threedimensional quality is provided in that the thickness of the two-dimensional region in the third direction may vary from point to point. Simulation may be done in either the areal plane or in a cross-sectional view. The spatial coordinate system may be either Cartesian $(x, y)$ or radial-cylindrical $(r, z)$. Areal simulation is usually physically unrealistic for variable-density fluid problems. Ground-water flow is simulated through numerical solution of a fluid mass balance equation. The ground-water system may be either saturated, or partly or completely unsaturated. Fluid density may be constant, or vary as a function of solute concentrations or fluid temperature.

SUTRA tracks the transport of either solute mass or energy in the flowing ground water through a unified equation which represents the transport of either solute or energy. Solute transport is simulated through numerical solution of a solute mass balance equation where solute concentration may affect fluid density. The single solute species may be transported conservatively, or it may undergo equilibrium sorption (through linear, Freundlich or Langmuir isotherms). In addition, the solute may be produced or decay through first- or zero-order processes.

Energy transport is simulated though numerical solution of an energy balance equation. The solid grains of the aquifer matrix and fluid are locally assumed to have equal temperature, and fluid density and viscosity may be affected by the temperature. 
Almost all aquifer material, flow, and transport parameters may vary in value throughout the simulated region. Sources and boundary conditions of fluid, solute and energy may be specified to vary with time or may be constant. SUTRA dispersion processes include diffusion and two types of fluid velocity-dependent dispersion. The standard dispersion model for isotropic media assumes direction-independent values of longitudinal and transverse dispersivity. A velocity-dependent dispersion process for anisotropic media is also provided and is introduced in the SUTRA documentation. This process assumes that longitudinal dispersivity varies depending on the angle between the flow direction and the principal axis of aquifer permeability when permeability is anisotropic.

\subsection{Some SUTRA Applications}

SUTRA may be employed in one- or two-dimensional analyses. Flow and transport simulation may be either steady-state which requires only a single solution step, or transient which requires a series of time steps in the numerical solution. Single-step steady-state solutions are usually not appropriate for non-linear problems with variable density, saturation, viscosity and nonlinear sorption.

SUTRA flow simulation may be employed for areal and cross-sectional modeling of saturated ground-water flow systems, and unsaturated zone flow. Some aquifer tests may be analyzed with flow simulation. SUTRA solute transport simulation may be employed to model natural or man-induced chemical species transport including processes of solute sorption, production and decay. Such simulation may be used to analyze ground-water contaminant transport problems and aquifer restoration designs. SUTRA solute transport simulation may 
also be used for modeling of variable density leachate movement, and for crosssectional modeling of salt-water intrusion in aquifers at both near-well or regional scales with either dispersed or relatively sharp transition zones between fresh water and salt water. SUTRA energy transport simulation may be employed to model thermal regimes in aquifers, subsurface heat conduction, aquifer thermal energy storage systems, geothermal reservoirs, thermal pollution of aquifers, and natural hydrogeologic convection systems.

\subsection{SUTRA Numerical Methods}

SUTRA simulation is based on a hybridization of finite-element and integrated-finite-difference methods employed in the framework of a method of weighted residuals. The method is robust and accurate when employed with proper spatial and temporal discretization. Standard finite-element approximations are employed only for terms in the balance equations which describe fluxes of fluid mass, solute mass and energy. All other non-flux terms are approximated with a finite-element mesh version of the integrated-finitedifference methods. The hybrid method is the simplest and most economical approach which preserves the mathematical elegance and geometric flexibility of finite-element simulation, while taking advantage of finite-difference efficiency.

SUTRA employs a new method for calculation of fluid velocities. Fluid velocities, when calculated with standard finite-element methods for systems with variable fluid density, may display spurious numerically generated components within each element. These errors are due to fundamental numerical inconsistencies in spatial and temporal approximations for the pressure gradient 
and density-gravity terms which are involved in velocity calculation. Spurious velocities can significantly add to the dispersion of solute or energy. This false dispersion makes accurate simulation of all but systems with very low vertical concentration or temperature gradients impossible, even with fine vertical spatial discretization. Velocities as calculated in SUTRA, however, are based on a new, consistent, spatial and temporal discretization, as introduced in this report. The consistently-evaluated velocities allow stable and accurate transport simulation (even at steady state) for systems with large vertical gradients of concentration or temperature. An example of such a system that SUTRA successfully simulates is a cross-sectional regional model of a coastal aquifer wherein the transition zone between horizontally flowing fresh water and deep stagnant salt water is relatively narrow.

The time discretization used in SUTRA is based on a backwards finitedifference approximation for the time derivatives in the balance equations. Some non-linear coefficients are evaluated at the new time level of solution by projection, while others are evaluated at the previous time level for noniterative solutions. All coefficients are evaluated at the new time level for iterative solutions.

The finite-element method allows the simulation of irregular regions with irregular internal discretization. This is made possible through use of quadrilateral elements with four corner nodes. Coefficients and properties of the system may vary in value throughout the mesh. Manual construction and data preparation for an irregular mesh requires considerable labor, and it may be worthwhile for the user to develop or obtain interactive software for this purpose in the event that irregular mesh construction is often required. 
'Pinch nodes' may be introduced in the finite-element mesh to allow for quick changes in mesh size from a fine mesh in the region where transport is of primary interest, to the external region, where only a coarse mesh is needed to define the flow system. Pinch nodes, although simplifying mesh design and reducing the number of nodes required in a particular mesh, also increases the matrix equation band width. Because SUTRA employs a band solver, the increased band width due to the use of pinch nodes may offset the gain in computational efficiency due to fewer nodes. Substitution of a non-band-width-dependent solver would guarantee the advantage that pinch nodes can provide. However, mesh designs employing pinch nodes may be experimented with, using the present solver.

SUTRA includes an optional numerical method based on asymmetric finite element weighting functions which results in 'upstream weighting' of advective transport and unsaturated fluid flux terms. Although upstream weighting has typically been employed to achieve stable, non-oscillatory solutions to transport problems and unsaturated flow problems, the method is not recommended for general use as it merely changes the physical system being simulated by increasing the magnitude of the dispersion process. A practical use of the method is, however, to provide a simulation of the sharpest concentration or temperature variations possible with a given mesh. This is obtained by specifying a simulation with absolutely no physical diffusion or dispersion, and with $50 \%$ upstream weighting. The results may be interpreted as the solution with the minimum amount of dispersion possible for a stable result in the particular mesh in use. In general simulation analyses of transport, upstream weighting is discouraged. The non-upstream methods are also provided by SUTRA, and are based 
on symmetric weighting functions. These methods are robust and accurate when the finite-element mesh is properly designed for a particular simulation, and are those which should be used for most transport simulations.

\subsection{SUTRA as a Tool of Analysis}

SUTRA will provide clear, accurate answers only to well-posed, welldefined, and well-discretized simulation problems. In less-well-defined systems, SUTRA simulation can help visualize a conceptual model of the flow and transport regime, and can aid in deciding between various conceptual models. In such less-well-defined systems, simulation can help answer questions such as: Is the (inaccessible) aquifer boundary which is (probably) ten kilometers offshore either leaky or impermeable? How leaky? Does thịs boundary affect the primary analysis of onshore water supply?

SUTRA is not useful for making exact predictions of future responses of the typical hydrologic systems which are not well defined. Rather, SUTRA is useful for hypothesis testing and for helping to understand the physics of such a system. On the other hand, developing an understanding of a system based on simulation analysis can help make a set of worthwhile predictions which are predicated on uncertainty of both the physical model design and model parameter values. In particular, transport simulation which relies on large amounts of dispersion must be considered an uncertain basis for prediction, because of the highly idealized description inherent in the SUTRA dispersion process.

A simulation-based prediction made with certainty is often inappropriate, and an "if-then" prediction is more realistic. A reasonable type of result of SUTRA simulation analysis may thus be: "Based on the uncertainty in location 
and type of boundary condition $A$, and uncertainty in the distribution of values for parameters $B$ and $C$, the following predictions are made. The extreme, but reasonable combination of $A, B$, and $C$ results in prediction $X$; the opposite reasonable extreme combination of $A, B$, and $C$ results in prediction $Y$; the combination of best estimates of $A, B$, and $C$, results in prediction $Z$, and is considered most likely."

In some cases, the available real data on a system may be so poor that a simulation using SUTRA is so ambiguously defined that no prediction at all can be made. In this instance, the simulation may be used to point out the need for particular types of data collection. The model could be used to advantage in visualizing possible regimes of system behavior rather than to determine which is accurate. 
SUTRA FUNDAMENTALS

13

(p.15 follows) 


\section{Chapter 2}

\section{Physical-Mathematical Basis of SUTRA Simulation}

The physical mechanisms which drive thermal energy transport and solute transport in the subsurface environment are described by nearly identical mathematical expressions. SUTRA takes advantage of this similarity, and with a simple program structure provides for simulation of either energy or solute transport. In fact, SUTRA simulation combines two physical models, one to simulate the flow of ground water, and the second to simulate the movement of either thermal energy or a single solute in the ground water.

The primary variable upon which the flow model is based is fluid pressure, $\mathrm{p}\left[\mathrm{M} /\left(\mathrm{L} \cdot \mathrm{s}^{2}\right)\right]=\mathrm{p}(\mathrm{x}, \mathrm{y}, \mathrm{t})$. Pressure may vary spatially in the ground-water system, as well as with time. Pressure is expressed as a combination of fluid mass units, $[M]$, length units, [L], and time units in seconds, [s]. Fluid density may vary depending on the local value of fluid temperature or solute concentration. Variation in fluid density, aside from fluid pressure differences, may itself drive flows. The effects of gravity acting on fluids with different density must therefore be accounted for in the flow field.

The flow of ground water, in turn, is a fundamental mechanism upon which the physical models of energy transport and solute transport are based. The primary variable characterizing the thermal energy distribution in a groundwater system is fluid temperature, $T\left[{ }^{\circ} \mathrm{C}\right]=T(x, y, t)$, in degrees Celcius, which may vary spatially and with time. The primary variable characterizing the state of solute distribution in a ground-water system is solute mass fraction, $C\left[M_{S} / M\right]=C(x, y, t)$, which may also vary spatially and with time. The units are a ratio of solute mass, $\left[M_{S}\right]$ to fluid mass, $[M]$. The term 'solute mass fraction' 
may be used interchangeably with 'solute concentration', and no difference should be implied. Note that 'solute volumetric concentration', $c\left[M_{s} / L_{f}{ }^{3}\right]$, (mass of solute, $M_{s}$, per volume of fluid, $L_{f}{ }^{3}$, is not the primary variable characterizing solute transport referred to either in this report or in output from the SUTRA model. Note that the measure of solute mass $\left[M_{s}\right]$ may be in units such as $[\mathrm{mg}],[\mathrm{kg}],[$ moles], or $[\mathrm{lbm}]$, and may differ from the measure, $[M]$, of fluid mass.

SUTRA allows only the transport of either thermal energy or a single solute to be modeled in a given simulation. Thus, when simulating energy transport, a constant value of solute concentration is assumed in the ground water. When simulating solute transport, a constant ground-water temperature is assumed. SUTRA simulation is carried out in two space dimensions with parameters varying in these two directions. However, the region of space to be simulated may be defined as three dimensional, when the assumption is made that all SUTRA parameters and coefficients have a constant value in the third space direction. A SUTRA simulation may be carried out over a region defined over two space coordinates $(x, y)$ in which the thickness of the region measured in the third coordinate direction $(z)$ varies depending on $(x, y)$ position.

2.1 Physical Properties of Solid Matrix and Fluid

\section{Fluid physical properties}

The ground-water fluid density and viscosity may vary depending on pressure, temperature and solute concentration. These fundamental variables are defined as follows: 


$$
\begin{array}{lll}
\mathrm{p}(\mathrm{x}, \mathrm{y}, \mathrm{t}) & \left|\mathrm{M} /\left(\mathrm{L} \cdot \mathrm{s}^{2}\right)\right| & \text { fluid pressure } \\
\mathrm{T}(\mathrm{x}, \mathrm{y}, \mathrm{t}) & \left|{ }^{\circ} \mathrm{C}\right| & \text { fluid temperature (degrees Celcius) } \\
\mathrm{C}(\mathrm{x}, \mathrm{y}, \mathrm{t}) & \left|\mathrm{M}_{\mathrm{S}} / \mathrm{M}\right| & \text { fluid solute mass fraction } \\
& & \text { (or solute concentration) (mass } \\
& \text { solute per mass total fluid) }
\end{array}
$$

As a point of reference, the 'solute volumetric concentration' is defined in terms of fluid density, 0 :

$$
\begin{aligned}
& c(x, y, t) \quad\left|M_{s} / L_{f}^{3}\right| \quad \text { solute volumetric concentration } \\
& \text { (mass solute per volume total fluid) } \\
& p(x, y, t) \quad\left|M / L_{f}^{3}\right| \quad \text { fluid density } \\
& c=D C \\
& p=p_{W}+C
\end{aligned}
$$

Total fluid density is the sum of pure water density, $\rho_{W}$, and $c$. Note again that 'solute concentration' refers to solute mass fraction, $C$, and not $c$. Fluid density, while a weak function of pressure is primarily dependent upon fluid solute concentration and temperature. The approximate density models employed by SUTRA are first order Taylor expansions about a base (reference) density other density models may be substituted through minor modifications to the program. For energy transport:

$$
\begin{aligned}
& D=\rho(T) \simeq \rho_{0}+\frac{\partial \rho}{\partial T}\left(T-T_{0}\right) \\
& 0_{0} \quad\left|\mathrm{M} / \mathrm{L}_{f}^{3}\right| \quad \text { base fluid density at } \mathrm{T}=\mathrm{T}_{\mathrm{o}} \\
& \mathrm{T}_{\mathrm{O}} \quad \mathrm{I}^{\circ} \mathrm{Cl} \quad \text { base fluid temperature }
\end{aligned}
$$

where $p_{0}$ is the base fluid density at a base (reference) temperature of $T_{0}$, and $\partial p / \partial T$ is a constant value of density change with temperature. For the 
range $20^{\circ} \mathrm{C}$ to $60^{\circ} \mathrm{C}, \partial \rho / \partial \mathrm{T}$ is approximately $-.375\left[\mathrm{~kg} /\left(\mathrm{m}^{3}{ }^{\circ} \mathrm{C}\right)\right]$; however, this factor varies and should be carefully chosen for the temperature range of interest.

For solute transport:

$$
\begin{array}{ll} 
& \rho=\rho(C)=\rho_{0}+\frac{\partial \rho}{\partial C}\left(C-C_{0}\right) \\
\rho_{0} & {\left[M / L_{f}^{3}\right]} \\
C_{0} & {\left[M_{S} / M\right]}
\end{array}
$$

where $p_{0}$ is the base fluid density at base concentration, $C_{0}$. (Usually, $\mathrm{C}_{\mathrm{O}}=0$, and the base density is that of pure water.) The factor $\partial \rho / \partial \mathrm{C}$ is a constant value of density change with concentration. For example, for mixtures of fresh and sea water at $20^{\circ} \mathrm{C}$, when $\mathrm{C}$ is the mass fraction of total dissolved solids, $C_{0}=0$, and $\rho_{0}=998.2\left[\mathrm{~kg} / \mathrm{m}^{3}\right]$, then the factor, $\partial \rho / \partial C$, is approximately $700 .\left[\mathrm{kg} / \mathrm{m}^{3}\right]$.

Fluid viscosity, $\mu\left[M / L_{f} \cdot s\right]$, is a weak function of pressure and of concentration, (for all except very high concentrations), and depends primarily on fluid temperature. For energy transport the viscosity of pure water is given in $\mathrm{m}-\mathrm{k}-\mathrm{s}$ units by:

$$
\mu(T) \simeq\left(239.4 \times 10^{-7}\right) 10^{\left(\frac{248.37}{T+133.15}\right)}[\mathrm{kg} /(\mathrm{m} \cdot \mathrm{s})]
$$

(The units may be converted to those desired via a scale factor in the program input data.)

For solute transport, viscosity is taken to be constant. For example, at $20^{\circ} \mathrm{C}$ in $\mathrm{m}-\mathrm{k}-\mathrm{s}$ units:

$$
\left.\mu(\mathrm{C})\right|_{\mathrm{T}}=20^{\circ} \mathrm{C}=1.0 \times 10^{-3}[\mathrm{~kg} /(\mathrm{m} \cdot \mathrm{s})]
$$




\section{Properties of fluid within the solid matrix}

The total volume of a porous medium is composed of a matrix of solid grains typically of solid earth materials, and of void space which includes the entire remaining volume which the solid does not fill. The volume of void space may be fully or partly filled with gas or liquid, and is commonly referred to as the pore volume. Porosity is defined as a volume of voids in the soil matrix per total volume of voids plus matrix:
$\varepsilon(x, y, t)$
porosity

(volume of voids per total volume)

where [1] indicates a dimensionless quantity.

It should be noted that SUTRA employs only one type of porosity, $\varepsilon$. In some instances there may be need to distinguish between a porosity for pores which take part in fluid flow, and pores which contain stagnant fluid. (Modifications may be made by the user to include this process.)

The fraction of total volume filled by the fluid is $\varepsilon S_{w}$ where:

$$
S_{w}(x, y, t)
$$

water saturation (saturation)

(volume of water per volume of voids)

When $S_{w}=1$, the void space is completely filled with fluid and is said to be saturated. When $S_{W}<1$, the void space is only partly water filled and is referred to as being unsaturated.

When $S_{w}<1$, water adheres to the surface of solid grains by surface tension effects, and the fluid pressure is less than atmospheric. Fluid pressure, p, is measured with respect to background or atmospheric pressure. The negative pressure is defined as capillary pressure, which exists only for $p<0$ : 


$$
\begin{array}{ccc}
p_{c}(x, y, t) & {\left[M /\left(L \cdot \mathrm{s}^{2}\right)\right]} & \text { capillary pressure } \\
p_{c}=-p & \text { when } p<0 \\
p_{c}=0 & \text { when } p \geq 0
\end{array}
$$

In a saturated porous medium, as fluid (gauge) pressure drops below zero, air may not directly enter the void space, but may enter suddenly when a critical

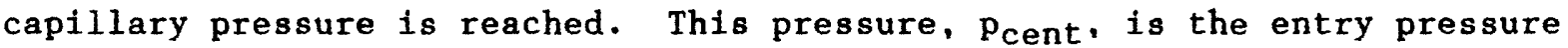
(or bubble pressure):

$$
\text { Pcent } \quad\left[M /\left(L \cdot \mathrm{s}^{2}\right)\right] \quad \text { entry capillary pressure }
$$

Typical values for $p_{\text {cent }}$ range from about $1 . \times 10^{3}\left[\mathrm{~kg} /\left(\mathrm{m} \cdot \mathrm{s}^{2}\right)\right]$ for coarse sand to approximately $5 . \times 10^{3}\left[\mathrm{~kg} /\left(\mathrm{m} \cdot \mathrm{s}^{2}\right)\right]$ for fine silty sand.

The relationship between fluid saturation and capillary pressure in a given medium is typically determined by laboratory experiment, and except for the portion near bubble pressure, tends to have an exponential character (Figure 2.1). Different functional relationships exists for different materials as measured in the laboratory. Also a number of general functions with parameters to be fitted to laboratory data are available. Because of the variety of possible functions, no particular function is set by SUTRA; any desired function may be specified for simulation of unsaturated flow. For example, a general function with three fitting parameters is (Van Genuchten, 1980):

$$
s_{w}=s_{\text {wres }}+\left(1-s_{\text {wres }}\right)\left[\frac{1}{1+\left(a_{c}\right)^{n}}\right]\left(\frac{n-1}{n}\right)
$$




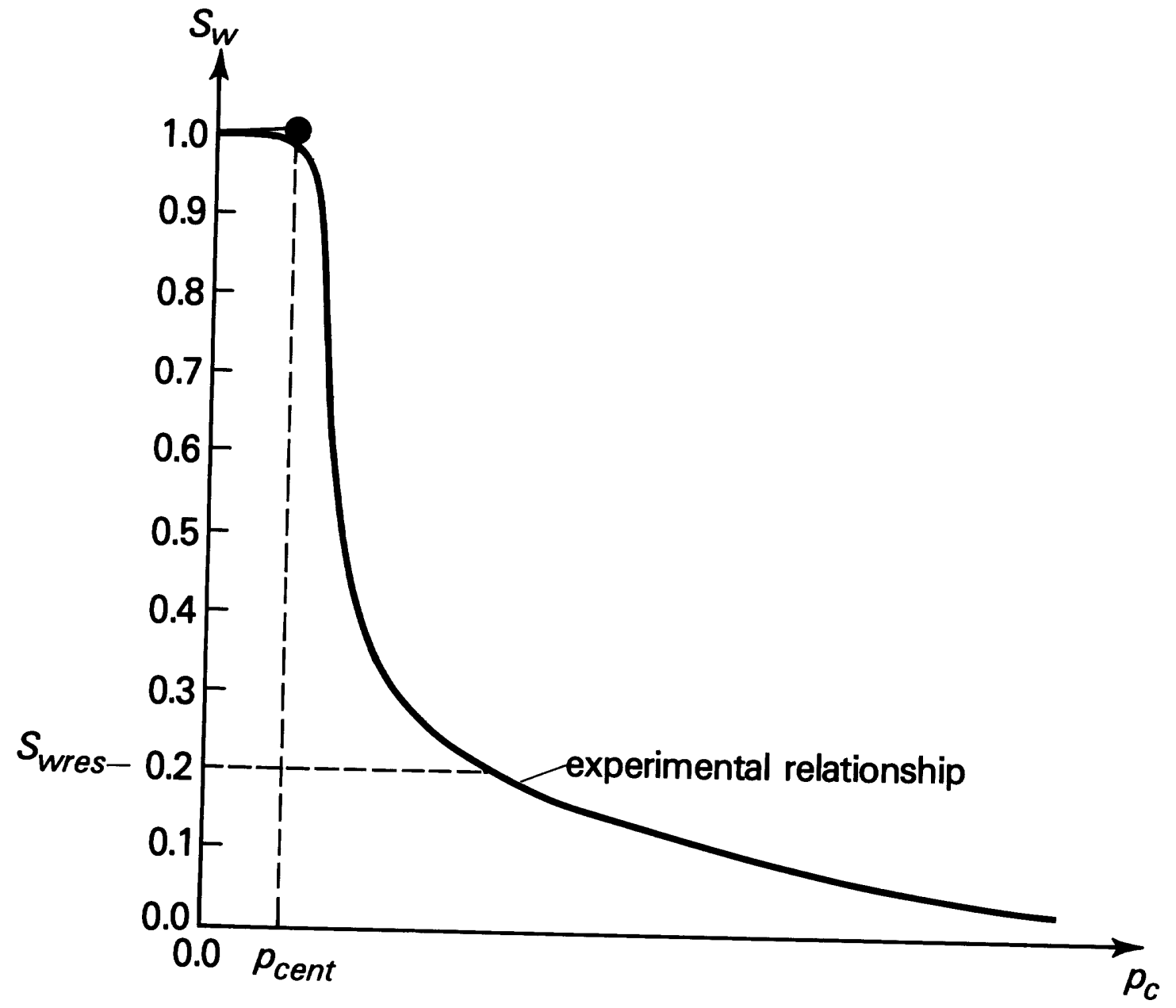

Figure 2.1

Saturation-capillary pressure relationship (schematic). 
where $S_{\text {wres }}$ is a residual saturation below which saturation is not expected to fall (because the fluid becomes immobile), and both a and $n$ are parameters. The values of these parameters depend upon a number of factors and must be carefully chosen for a particular material.

The total mass of fluid contained in a total volume, VOL, of solid matrix plus pore space is $\left(\varepsilon S_{w} \rho\right)$ VOL. The actual amount of total fluid mass contained depends solely on fluid pressure, $p$, and solute concentration, $C$, or fluid temperature, $T$. A change in total fluid mass in a volume, assuming VOL is constant, is expressed as follows:

$$
\operatorname{VOL} \cdot d\left(\varepsilon S_{w} p\right)=\operatorname{VOL} \cdot\left[\frac{\partial\left(\varepsilon S_{w} p\right)}{\partial p} d p+\frac{\partial\left(\varepsilon S_{w} p\right)}{\partial U} d U\right]
$$

where U represents either $C$ or $T$. Saturation, $S_{w}$, is entirely dependent on fluid pressure, and porosity, $\varepsilon$, does not depend on concentration or temperature:

$$
V O L \cdot d\left(\varepsilon S_{w} \rho\right)=V O L \cdot\left[\left(S_{w} \frac{\partial(\varepsilon \rho)}{\partial p}+\varepsilon \rho \frac{\partial S_{w}}{\partial p}\right) d p+\varepsilon S_{w} \frac{\partial \rho}{\partial U} d U\right]
$$

The factor, $\partial S_{w} / \partial p$, is obtained by differentiation of the chosen saturationcapillary pressure relationship. For the example function given as (2.8),

$$
\frac{d S_{w}}{d p}=\frac{a(n-1)\left(1-S_{w r e s}\right)\left(a p_{c}\right)^{(n-1)}}{\left(1+\left(a p_{c}\right)^{n}\right)\left(\frac{2 n-1}{n}\right)}
$$

The factor, $\partial \rho / \partial U$, is a constant value defined by the assumed density models, given by equations (2.3) and (2.4).

Aquifer storativity under fully saturated conditions is related to the factor, $\partial(\varepsilon \rho) / \partial p$, by definition, as follows (Bear, 1979): 


$$
\frac{\partial(\varepsilon \rho)}{\partial p} \equiv \rho S_{o p}
$$

where:

$$
\begin{aligned}
& \mathrm{S}_{\mathrm{op}} \equiv \frac{1}{\mathrm{VOL}}\left(\frac{\Delta \mathrm{VOL}}{\Delta \mathrm{w}}\right) \\
& \mathrm{S}_{\mathrm{op}}(\mathrm{x}, \mathrm{y}) \quad\left[\mathrm{M} /\left(\mathrm{L} \cdot \mathrm{s}^{2}\right)\right]^{-1}
\end{aligned}
$$

The specific pressure storativity, $S_{o p}$, is the volume of water released from saturated pore storage due to a unit drop in fluid pressure per total solid matrix plus pore volume. Note that the common specific storativity, $S_{O}\left[L^{-1}\right]$, which when multiplied by confined aquifer thickness gives the well known storage coefficient, $S[1]$, is related to $S_{o p}$ as, $S_{0}=\rho|g| S_{o p}$, where $|g|\left[L / s^{2}\right]$ is the magnitude of the gravitational acceleration vector. The common specific storativity, $S_{o}$, is analagous to specific pressure storativity, $S_{o p}$, used in SUTRA, except that $S_{0}$ expresses the volume of water released from pore storage due to a unit drop in piezometric head.

SUTRA employs an expanded form of the specific pressure storativity based on fluid and bulk porous matrix compressibilities. The relationship is obtained as follows by expanding equation (2.12)

$$
\rho S_{o p}=\rho \frac{\partial \varepsilon}{\partial p}+\varepsilon \frac{\partial \rho}{\partial p}
$$

The coefficient of compressibility of water is defined by

$$
\begin{aligned}
& B \equiv \frac{1}{\rho} \frac{\partial \rho}{\partial p} \\
& \beta \quad\left[M /\left(L \cdot s^{2}\right)\right]^{-1} \quad \text { fluid compressibility }
\end{aligned}
$$


which allows the last term of (2.14) to be replaced by $\varepsilon \rho \beta$. For pure water at $20^{\circ} \mathrm{C}, \beta \sim 4.47 \times 10^{-10}\left[\mathrm{~kg} /\left(\mathrm{m} \cdot \mathrm{s}^{2}\right)\right]^{-1}$ As the volume of solid grains $\mathrm{VOL}, \mathrm{s}$, in a volume, VOL, of porous solid matrix plus void space is $\mathrm{VOL}_{s}=(1-\varepsilon) \cdot \mathrm{VOL}$, the factor, $\partial \varepsilon / \partial p$, may be expressed as:

$$
\frac{\partial \varepsilon}{\partial p}=\frac{(1-\varepsilon)}{\text { VOL }} \frac{\partial(\text { VOL })}{\partial p}
$$

which assumes that individual solid grains are relatively incompressible. The total stress at any point in the solid matrix-fluid system is the sum of effective (intergranular) stress, $\sigma^{\prime}\left[M /\left(L \cdot s^{2}\right)\right]$, and fluid pore pressure, $p$. In systems where the total stress remains nearly constant, $d \sigma^{\prime}=-d p$, and any drop in fluid pressure increases intergranular stress by a like amount. This consideration allows (2.16) to be expressed in terms of bulk porous matrix compressibility, as: $\partial \varepsilon / \partial p=(1-\varepsilon) \alpha$, where

$$
\alpha \equiv-\frac{1}{\mathrm{VOL}} \frac{\partial(\mathrm{VOL})}{\partial \sigma^{\prime}}
$$

$$
\begin{array}{lll}
\alpha & {\left[M /\left(L \cdot s^{2}\right)\right]^{-1}} & \text { porous matrix compressibility } \\
\sigma^{\prime} & {\left[M /\left(L \cdot s^{2}\right)\right]} & \text { intergranular stress }
\end{array}
$$

Factor $\alpha$ ranges from $\alpha \sim 10^{-10}\left[\mathrm{~kg} /\left(\mathrm{m} \cdot \mathrm{s}^{2}\right)\right]^{-1}$ for sound bedrock to about $\alpha \sim 10^{-7}\left[\mathrm{~kg} /\left(\mathrm{m} \cdot \mathrm{s}^{2}\right)\right]^{-1}$ for clay. Thus equation (2.14) may be rewritten as $\rho S_{o p}=\rho(1-\varepsilon) \alpha+\varepsilon \rho B$, and, in effect, the specific pressure storativity, $S_{\text {op }}$, is expanded as:

$$
S_{\text {op }}=(1-\varepsilon) \alpha+\varepsilon \beta
$$

A more thorough discussion of storativity is presented by Bear (1979). 


\section{Fluid flow and flow properties}

Fluid movement in porous media where fluid density varies spatially may be driven by either differences in fluid pressure or by unstable variations in fluid density. Pressure-driven flows, for example, are directed from regions of higher than hydrostatic fluid pressure toward regions of lower than hydrostatic pressure. Density-driven flows occur when gravity forces act on denser regions of fluid causing them to flow downward relative to fluid regions which are less dense. A stable density configuration drives no flow, and is one in which fluid density remains constant or increases with depth.

The mechanisms of pressure and density driving forces for flow are expressed for SUTRA simulation by a general form of Darcy's law which is commonly used to describe flow in porous media:

$$
\underline{v}=-\left(\frac{\mathrm{kk}_{r}}{\varepsilon S_{w} \mu}\right) \cdot(\underline{\nabla p}-p g)
$$

where:

$$
\begin{array}{lll}
\mathbf{v}(\mathrm{x}, \mathrm{y}, \mathrm{t}) & {[\mathrm{L} / \mathrm{s}]} & \begin{array}{l}
\text { average fluid velocity } \\
\mathbf{k}(\mathrm{x}, \mathrm{y})
\end{array} \\
\mathbf{k}_{\mathrm{r}}(\mathrm{x}, \mathrm{y}, \mathrm{t}) & {[1]} & \begin{array}{l}
\text { solid matrix permeability } \\
(2 \mathrm{~L} 2 \text { tensor of values })
\end{array} \\
\mathbf{g} & {\left[\mathrm{L} / \mathrm{s}^{2}\right]} & \begin{array}{l}
\text { relative permeability to fluid flow } \\
\text { (assumed to be independent of direction.) }
\end{array} \\
& & \begin{array}{l}
\text { gravitational acceleration (gravity vector) } \\
(1 \mathrm{x} 2 \text { vector of values) }
\end{array}
\end{array}
$$

The gravity vector is defined in relation to the direction in which vertical elevation is measured: 
where $|g|$ is the magnitude of the gravitational acceleration vector. For example, if the $y$-space-coordinate is oriented directly upwards, then $\underline{\nabla}$ (ELEVATION) is a vector of values (for $x$ and $y$ directions, respectively): $(0,1)$, and $g=(0,-|g|)$. If for example, ELEVATION increases in the $x-y$ plane at a $60^{\circ}$ angle to the $x$-axis, then $\underline{\nabla}($ ELEVATION $)=\left((1 / 2),\left(3^{\frac{1}{2}} / 2\right)\right)$ and $g=\left(-(1 / 2)|g|,-\left(3^{\frac{1}{2}} / 2\right)|g|\right)$. The average fluid velocity, $\underline{v}$, is the velocity of fluid with respect to the stationary solid matrix. The so-called Darcy velocity, $q$, for the sake of reference, is $q=\varepsilon S_{w}$. This value is always less than the true average fluid velocity, $\underline{v}$, and thus, not being a true indicator of the speed of water movement, 'Darcy velocity', $q$, is not a useful concept in simulation of subsurface transport. The velocity is referred to as an 'average', because true velocities in a porous medium vary from point to point due to variations in the permeability and porosity of the medium at a spatial scale smaller than that at which measurements were made.

Fluid velocity, even for a given pressure and density distribution, may take on different values depending on how mobile the fluid is within the solid matrix. Fluid mobility depends on the combination of permeability, $k$, relative permeability, $k_{r}$, and viscosity, $\mu$, that occurs in equation (2.19a). Permeability is a measure of the ease of fluid movement through interconnected voids in the solid matrix when all voids are completely saturated. Relative permeability expresses what fraction of the total permeability remains when the voids are only partly fluid-filled and only part of the total interconnected void space is, in fact, connected by continuous fluid channels. Viscosity directly expresses ease of fluid flow; a less viscous fluid flows more readily under a driving force. 
As a point of reference, in order to relate the general form of Darcy's law, (2.19a), back to a better-known form dependent on hydraulic head, the dependence of flow on density and saturation must be ignored. When the solid matrix is fully saturated, $S_{w}=1$, the relative permeability to flow is unity $k_{r}=1$. When, in addition, fluid density is constant, the right side of (2.19a) expanded by (2.19b) may be multiplied and divided by $\underline{p|g|:}$

$$
\underline{v}=\frac{-k \rho|g|}{\varepsilon \mu} \cdot\left[\underline{\underline{\underline{g}} \rho}\left(\frac{p}{\rho|g|}\right)+\underline{\nabla}(\text { ELEVATION })\right]
$$

The hydraulic conductivity, $K(x, y, t)[L / s]$, may be identified in this equation as, $\underset{=}{K}=(\underline{k} \rho|g|) / \mu$; pressure head, $h_{p}(x, y, t)[L]$, is $h_{p}=p /(\rho|g|)$. Hydraulic head, $h(x, y, t)[L]$, is $h=h_{p}+$ ELEVATION. Thus, for constant density, saturated flow:

$$
\underline{v}=-\left(\frac{\mathrm{K}}{\varepsilon}\right) \cdot \underline{\nabla} \mathrm{h}
$$

which is Darcy's law written in terms of the hydraulic head. Even in this basic form of Darcy's law, flow may depend on solute concentration and temperature. The hydraulic conductivity, through viscosity, is highly dependent on temperature, and measurably, but considerably less on concentration. In cases where density or viscosity are not constant, therefore, hydraulic conductivity, $K$, is not a fundamental parameter describing ease of flow through the solid matrix. Permeability, $k$, is in most situations, essentially independent of pressure, temperature and concentration and therefore is the appropriate fundamental parameter describing ease of flow in the SUTRA model. 
Permeability, $k$, describes ease of fluid flow in a saturated solid matrix. When permeability to flow in a particular small volume of solid matrix differs depending upon in which direction the flow occurs, the permeability is said to be anisotropic. Direction-independent permeability is called isotropic. It is commonly assumed that permeability is the same for flow forward or backward along a particular line in space. When permeability is anisotropic, there is always one particular direction, $x_{p}$, along which permeability has an absolute maximum value, $k_{\max }\left[L^{2}\right]$. Somewhere in the plane perpendicular to the 'maximum direction' there is a direction, $x_{m}$, in which permeability has the absolute minimum value, $k_{m i n}\left[L^{2}\right]$, which exists for the particular volume of solid matrix. Thus, in two dimensions, there are two principal orthogonal directions of anisotropic permeability. Both principal directions, $x_{p}$ and $x_{m}$, are assumed to be within the $(x, y)$ plane of the two-dimensional model.

The permeability tensor, $k$, of Darcy's law, equation (2.19) has four components in two dimensions. These tensorial components have values which depend on effective permeabilities in the $x$ and $y$ coordinate directions which are not necessarily exactly aligned with the principal directions of permeability. The fact that maximum and minimum principal permeability values may change in both value and direction from place to place in the modeled region makes the calculation of the permeability tensor, which is aligned in $x$ and $y$, complex. The required coordinate rotations are carried out automatically by SUTRA according to the method described in section 5.1, "Rotation of Permeability Tensor".

An anisotropic permeability field in two dimensions is completely described by the values $k_{\max }$ and $k_{\min }$, and the angle orienting the principal directions, $x_{p}$ and $x_{m}$, to the $x$ and $y$ directions through the permeability ellipse shown in Figure 2.2. The semi-major and semi-minor axes of the ellipse are defined as 


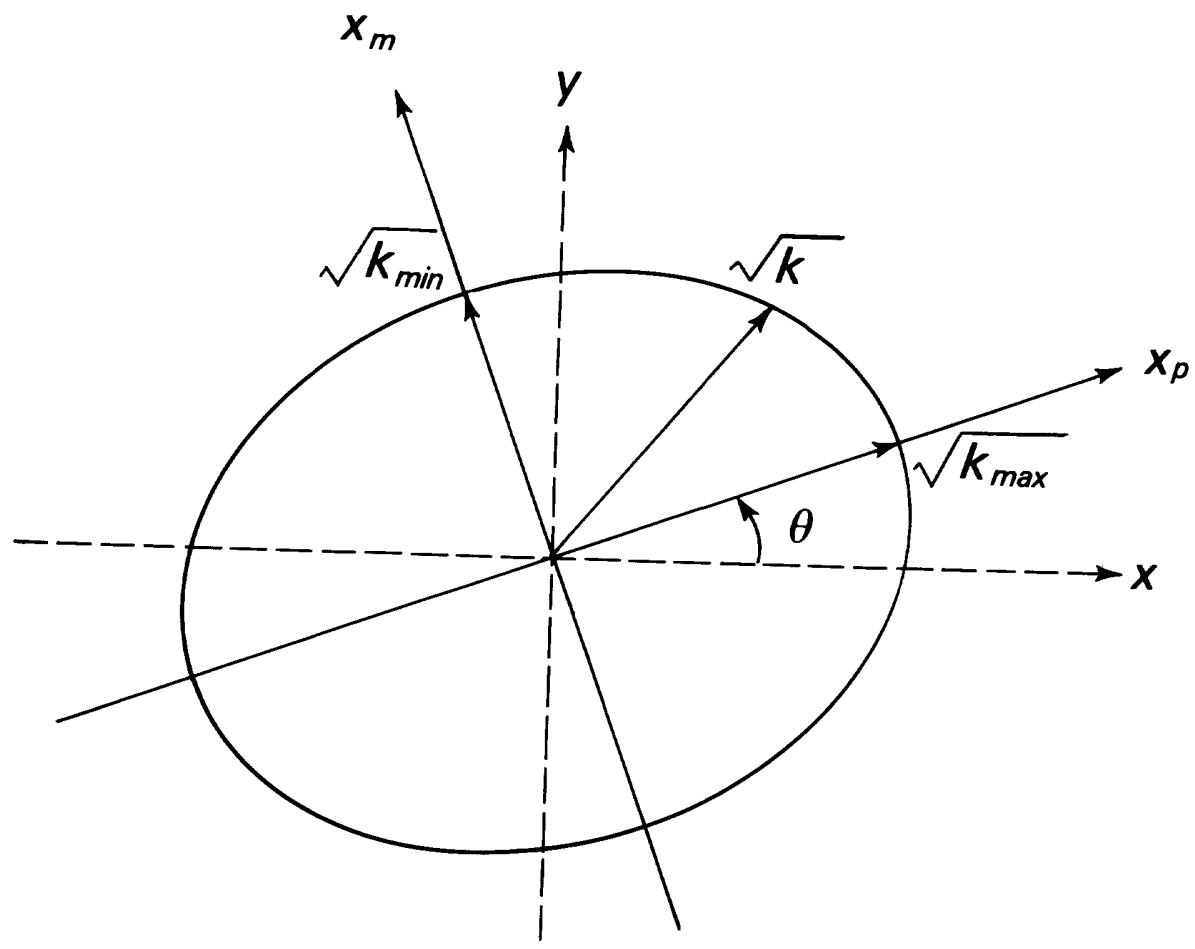

Figure 2.2 Definition of anisotropic permeability and effective
permeability, $k$. 
$\mathrm{k}_{\max }^{\frac{1}{2}}$, and $\mathrm{k}_{\min }^{\frac{1}{2}}$, respectively, and the length of any radius is $\mathrm{k}^{\frac{1}{2}}$, where $\mathrm{k}$ is the effective permeability for flow along that direction. Only, $k_{\max }, k_{m i n}$, and $\theta$, the angle between the $x$-axis and the maximum direction $x_{p}$ need be specified to define the permeability, $k$, in any direction, where:

$$
\begin{array}{lll}
k_{\max }(x, y) & {\left[L^{2}\right]} & \text { absolute maximum value of permeability } \\
k_{\min }(x, y) & {\left[L^{2}\right]} & \text { absolute minimum value of permeability } \\
\theta(x, y) & {\left[{ }^{\circ}\right]} & \begin{array}{l}
\text { angle from }+x \text {-coordinate axis to di- } \\
\text { rection of maximum permeability, } x_{p}
\end{array}
\end{array}
$$

In the case of isotropic permeability, $k_{\max }=k_{\min }$, and $\theta$ is arbitrary.

The discussion of isotropic and anisotropic permeability, $k$, applies as well to flow in an unsaturated solid matrix, $S_{w}<1$, although unsaturated flow has additional unique properties which require definition. When fluid capillary pressure, $p_{c}$, is less than entry capillary pressure, $p_{\text {cent }}$, the void space is saturated $S_{w}=1$, and local porous medium flow properties are not pressuredependent but depend only on void space geometry and connectivity. When $p_{c}>p_{\text {cent }}$, then air or another gas has entered the matrix and the void space is only partly fluid filled, $s_{w}<1$. In this case, the ease with which fluid can pass through the solid matrix depends on the remaining cross-section of well-connected fluid channels through the matrix, as well as on surface tension forces at fluid-gas, and fluid-solid interfaces. When saturation is so small such that no interconnected fluid channels exist and residual fluid is scattered about and tightly bound in the smallest void spaces by surface tension, flow ceases entirely. The relative permeability to flow, $k_{r}$, which is a measure of this behavior, varies from a value of zero or near-zero at the residual fluid 
saturation, $S_{\text {wres }}$, to a value of one at saturation, $S_{w}=1$. A relative permeability-saturation relationship (Figure 2.3) is typically determined for a particular solid matrix material in the laboratory as is the relationship, $S_{w}\left(p_{c}\right)$. Relative permeability is assumed in SUTRA to be independent of direction in the porous media.

SUTRA allows any desired function to be specified which gives the relative permeability in terms of saturation or pressure. A general function, for example, based on the saturation-capillary pressure relationship given as an example in (2.8) is (Van Genuchten, 1980):

$$
k_{r}=s_{w}^{* \frac{1}{2}}\left\{1-\left[1-s_{w}^{*}\left(\frac{n}{n-1}\right)\right]\left(\frac{n-1}{n}\right)\right\}^{2}
$$

where the a dimensionless saturation, $s_{w}^{*}$, is given by:

$$
s_{w}^{*}=\frac{S_{w}-S_{w r e s}}{1-S_{\text {wres }}}
$$

Flow in the gaseous phase that fills the remaining void space not containing fluid when $S_{w}<1$ is assumed not to contribute significantly to total solute or energy transport which is due primarily to fluid flow and other transport processes through both fluid and solid matrix. Furthermore it is assumed that pressure differences within the gas do not drive significant fluid flow. These assumptions are justified in most common situations when gas pressure is approximately constant throughout the solid matrix system. Should gas pressure vary appreciably in a field system, simulation with SUTRA, which is by definition a single phase flow and transport model, must be critically evaluated against the possible necessity of employing a multiphase fluid flow and transport model. 


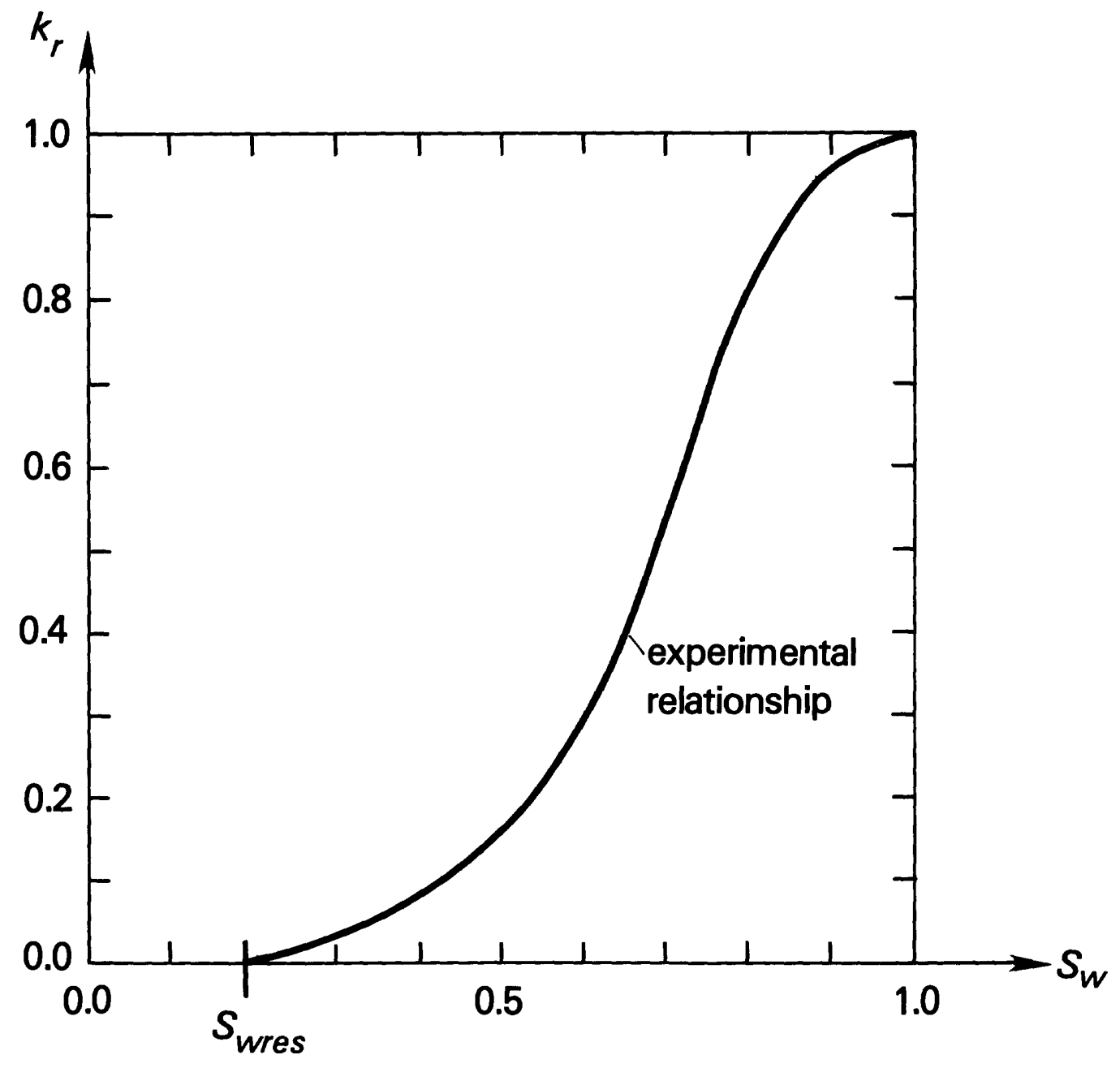

Figure 2.3

Relative permeability-saturation relationship (schematic). 


\section{Fluid mass balance}

The "so-called" flow simulation provided by SUTRA is in actuality a calculation of how the amount of fluid mass contained within the vold spaces of the solid matrix changes with time. In a particular volume of solid matrix and void space, the total fluid mass $\left(\varepsilon S_{w} p\right) \cdot V O L$, may change with time due to: ambient ground-water inflows or outflows, infection or withdrawal wells, changes in fluid density caused by changing temperature or concentration, or changes in saturation. SUTRA flow simulation is, in fact, a fluid mass balance which keeps track of the fluid mass contained at every point in the simulated ground-water system as it changes with time due to flows, wells, and saturation or density changes.

The fluid mass balance is expressed as the sum of pure water and pure solute mass balances for a solid matrix in which there is negligible net movement:

$$
\frac{\partial\left(\varepsilon S_{w} p\right)}{\partial t}=-\underline{\nabla} \cdot\left(\varepsilon S_{w} p \underline{v}\right)+Q_{p}+T
$$

where:

$$
\begin{array}{lll}
Q_{p}(x, y, t) & {\left[M /\left(L^{3} \cdot s\right)\right]} & \begin{array}{l}
\text { fluld mass source (Including pure } \\
\text { water mass plus solute mass dissolved } \\
\text { In source water) }
\end{array} \\
T(x, y, t) & {\left[M /\left(L^{3} \cdot s\right)\right]} & \begin{array}{l}
\text { solute mass source (e.g., dissolution } \\
\text { of solid matrix or desorption) }
\end{array}
\end{array}
$$

The term on the left may be recognized as the total change in fluid mass contained in the vold space with time. The term involving $\nabla$ represents contributions to local fluid mass change due to excess of fluid inflows over outflows at a point. The fluid mass source term, $Q_{p}$, accounts for external additions of fluid including pure water mass plus the mass of any solute dissolved in the source fluid. The pure solute mass source term, $T$, may account for external additions 
of pure solute mass not associated with a fluid source. In most cases, this contribution to the total mass is small compared to the total pure water mass contributed by fluid sources, $Q_{p}$. Pure solute sources, $T$, are therefore neglected in the fluid mass balance, but may be readily included in SUTRA for special situations. Note that solute mass sources are not neglected in the solute mass balance, which is discussed in section 2.4 .

While (2.22) is the most fundamental form of the fluid mass balance, it is necessary to express each mechanism represented by a term of the equation, in terms of the primary variables, $p, C$, and $T$. As SUTRA allows variation in only one of $\mathrm{C}$ or $\mathrm{T}$ at a time, the letter $\mathrm{U}$ is employed to represent either of these quantities. The development from equation (2.9) to (2.18) allows the time derivative in (2.22) to be expanded:

$$
\frac{\partial\left(\varepsilon S_{w} \rho\right)}{\partial t}=\left(S_{w} \rho S_{o p}+\varepsilon \rho \frac{\partial S_{w}}{\partial p}\right) \frac{\partial p}{\partial t}+\left(\varepsilon S_{w} \frac{\partial \rho}{\partial U}\right) \frac{\partial U}{\partial t}
$$

While the concepts upon which specific pressure storativity, $S_{\text {op' }}$ is based, do not exactly hold for unsaturated media, the error introduced by summing the storativity term with the term involving $\left(\partial S_{w} / \partial p\right)$ is insignificant as $\left(\partial S_{w} / \partial p\right) \gg S_{o p}$

The exact form of the fluid mass balance as implemented in SUTRA is obtained from (2.22) by neglecting $T$, substituting (2.23) and employing Darcy's law, $(2.19)$, for $\underline{v}$ :

$$
\begin{gathered}
\left(S_{w} \rho S_{o p}+\varepsilon \rho \frac{\partial S_{w}}{\partial p}\right) \frac{\partial p}{\partial t}+\left(\varepsilon S_{w} \frac{\partial \rho}{\partial U}\right) \frac{\partial U}{\partial t}-\underline{\nabla} \cdot\left[\left(\frac{\underline{\underline{z}} k_{r} \rho}{\mu}\right) \cdot(\underline{\nabla p}-\rho g)\right] \\
=Q_{p}
\end{gathered}
$$




\subsection{Description of Energy Transport in Ground Water}

\section{Subsurface energy transport mechanisms}

Energy is transported in the water-solid matrix system by flow of ground water, and by thermal conduction from higher to lower temperatures through both the fluid and solid. The actual flow velocities of the ground water from point to point in the three-dimensional space of an aquifer may vary considerably about an average two-dimensional velocity uniform in the $z$-direction, $\underline{v}(x, y, t)$, calculated from Darcy's law (2.22). As the true, not-average, velocity field is usually too complex to measure in real systems, an additional transport mechanism approximating the effects of mixing of different temperature ground waters moving both faster and slower than average velocity, $\underline{v}$, is hypothesized. This mechanism, called energy dispersion, is employed in SUTRA as the best currently available, though approximate description, of the mixing process. In the simple dispersion model employed, dispersion, in effect, adds to the thermal conductivity value of the fluid-solid medium in particular directions dependent upon the direction of fluid flow. In other words, mixing due to the existence of nonuniform, nonaverage velocities in three dimensions about the average-uniform flow, $\underline{v}$, is conceptualized in two dimensions as a diffusion-like process with anisotropic diffusivities.

The model has, in fact, been shown to describe transport well in purely homogeneous porous media with uniform one-dimensional flows. In heterogeneous field situations with non-uniform flow in, for example, irregular bedding or fractures, the model holds only at the pre-determined scale at which dispersivities are calibrated and it must be considered as a currently necessary approximation, and be carefully applied when extrapolating to other scales of transport. 


\section{Solid matrix-fluid energy balance}

The simulation of energy transport provided by SUTRA is actually a calculation of the time rate of change of the amount of energy stored in the solid matrix and fluid. In any particular volume of solid matrix plus fluid, the amount of energy contained is $\left[\varepsilon S_{w} \rho_{w}+(1-\varepsilon) \rho_{s} e_{s}\right] \cdot V O L$, where

$\begin{array}{lll}e_{w} & {[E / M]} & \text { energy per unit mass water } \\ e_{s} & \text { energy per unit mass solid matrix } \\ \rho_{S} & {\left[E / M_{G}\right]} & \text { density of solid grain in solid matrix }\end{array}$

and where $[E]$ are energy units $\left[M \cdot L^{2} / s^{2}\right]$.

The stored energy in a volume may change with time due to: ambient water with a different temperature flowing in, well water of a different temperature injected, changes in the total mass of water in the block, thermal conduction (energy diffusion) Into or out of the volume, and energy dispersion in or out.

This balance of changes in stored energy with various energy fluxes is expressed as follows:

$$
\begin{aligned}
& \frac{\partial\left[\varepsilon S_{w} p e_{w}+(1-\varepsilon) p_{s} e_{s}\right]}{\partial t}=-\underline{\nabla} \cdot\left(\varepsilon S_{w} p e_{w} v\right)+\underline{\nabla} \cdot[\lambda \underset{I}{I} \cdot \underline{\nabla} T] \\
& +\underline{\nabla} \cdot\left[\varepsilon S_{w} \rho c_{w} D \cdot \underline{\nabla T}\right]+Q_{p} c_{w} T^{*}+\varepsilon S_{w} p \gamma_{0}^{w}+(1-\varepsilon)_{p} \gamma_{0}^{s} \\
& \lambda(x, y, t) \quad\left[E /\left(s \cdot L \cdot{ }^{\circ} \mathrm{C}\right)\right] \\
& \text { I } \\
& \mathrm{c}_{\mathrm{w}} \quad\left[\mathrm{E} /\left(\mathrm{M} \cdot{ }^{\circ} \mathrm{C}\right)\right]
\end{aligned}
$$




$$
\begin{array}{lll}
\stackrel{D}{D}(\mathrm{x}, \mathrm{y}, \mathrm{t}) & {\left[\mathrm{L}^{2} / \mathrm{s}\right]} & \text { dispersion tensor }(2 \mathrm{X} 2) \\
\mathrm{T}^{*}(\mathrm{x}, \mathrm{y}, \mathrm{t}) & {\left[{ }^{\circ} \mathrm{C}\right]} & \text { temperature of source fluid } \\
\gamma_{0}^{\mathrm{w}}(\mathrm{x}, \mathrm{y}, \mathrm{t}) & {[\mathrm{E} / \mathrm{M} \cdot \mathrm{s}]} & \text { energy source in fluid } \\
\gamma_{\mathrm{o}}^{\mathrm{s}}(\mathrm{x}, \mathrm{y}, \mathrm{t}) & {\left[\mathrm{E} / \mathrm{M}_{\mathrm{G}} \cdot \mathrm{s}\right]} & \text { energy source in solid grains }
\end{array}
$$

The time derivative expresses the total change in energy stored in both the solid matrix and fluid per unit total volume. The term involving $\mathbf{v}$ expresses contributions to locally stored energy from average-uniform flowing fluid (average energy advection). The term involving bulk thermal conductivity, $\lambda$, expresses heat conduction contributions to local stored energy and the term involving the dispersivity tensor, $\underset{\equiv}{ }$, approximately expresses the contribution of irregular flows and mixing which are not accounted for by average energy advection. The term involving $Q$ accounts for the energy added by a fluid source with temperature, $\mathrm{T}^{*}$. The last terms account for energy production in the fluid and solid, respectively, due to endothermic reactions, for example.

While more complex models are available and may be implemented if desired, SUTRA employs a volumetric average approximation for bulk thermal conductivity, $\lambda$ :

$$
\begin{aligned}
& \lambda \equiv \varepsilon S_{w} \lambda_{w}+(1-\varepsilon) \lambda_{s} \\
& \lambda_{w} \quad\left[\mathrm{E} /\left(\mathrm{s} \cdot \mathrm{L} \cdot{ }^{\circ} \mathrm{C}\right)\right] \text { fluid thermal conductivity } \\
& \left(\lambda_{w} \sim 0.6\left[\mathrm{~J} /\left(\mathrm{s} \cdot \mathrm{m} \cdot{ }^{\circ} \mathrm{C}\right)\right] \text { at } 20^{\circ} \mathrm{C}\right) \\
& \lambda_{\mathrm{s}} \quad\left[\mathrm{E} /\left(\mathrm{s} \cdot \mathrm{L} \cdot{ }^{\circ} \mathrm{C}\right)\right] \text { solid thermal conductivity } \\
& \left(\lambda \sim 3.5\left[\mathrm{~J} /\left(\mathrm{s} \cdot \mathrm{m} \cdot{ }^{\circ} \mathrm{C}\right)\right] \text { at } 20^{\circ} \mathrm{C}\right. \\
& \text { for sandstone) }
\end{aligned}
$$

The specific energy content (per unit mass) of the fluid and the solid matrix depends on temperature as follows: 


$$
\begin{aligned}
& e_{w}=c_{w} T \\
& e_{s}=c_{s} T
\end{aligned}
$$

$$
c_{s} \quad\left[E /\left(M_{G} \cdot{ }^{\circ} \mathrm{C}\right)\right]
$$

solid grain specific heat $\left(\mathrm{c} \sim 8.4 \times 10^{2}\left[\mathrm{~J} /\left(\mathrm{kg} \cdot{ }^{\circ} \mathrm{C}\right)\right]\right.$ for sandstone at $20^{\circ} \mathrm{C}$ )

An expanded form of the solid matrix-fluid energy balance is obtained by substitution of $(2.27 \mathrm{a}, \mathrm{b})$ and $(2.26)$ into $(2.25)$. This yields:

$$
\begin{aligned}
& \frac{\partial}{\partial t}\left[\varepsilon S_{w} \rho c_{w}+(1-\varepsilon) \rho_{s} c_{s}\right] T+\underline{\nabla} \cdot\left(\varepsilon S_{w} \rho c_{w} \underline{T}\right) \\
& \left.-\underline{\nabla} \cdot\left\{\left[S_{w} \lambda_{w}+(1-\varepsilon) \lambda_{s}\right] I+\varepsilon S_{w} \rho c_{w} \underline{D}\right\} \cdot \nabla T\right] \\
& =Q_{p} c_{w} T^{*}+\varepsilon S_{w} \rho \gamma_{o}^{w}+(1-\varepsilon) \rho_{s} \gamma_{o}^{s}
\end{aligned}
$$

\subsection{Description of Solute Transport in Ground Water}

\section{Subsurface solute transport mechanisms}

Solute mass is transported through the porous medium by flow of ground water (solute advection) and by molecular or ionic diffusion, which while small on a field scale, carries solute mass from areas of high to low concentrations. The actual flow velocities of the ground water from point to point in threedimensional space of an aquifer may vary considerably about an average, uniform two-dimensional velocity, $\underline{v}$, which is calculated from Darcy's law (2.22). As the true, not-average, velocity field is usually too complex to measure in real systems, an additional transport mechanism approximating the effects of mixing of waters with different concentrations moving both faster and slower than the average velocity, $\underline{v}(x, y, t)$, is hypothesized. This mechanism, called solute dispersion, is employed in SUTRA as the best currently available, though approximate, description of the mixing process. In the simple dispersion model 
employed, dispersion, in effect, significantly adds to the molecular diffusivity value of the fluid in particular directions dependent upoon the direction of fluid flow. In other words, mixing due to the existence of non-uniform, non-average velocities in three dimensions about the average flow, $\underline{v}$, is conceptualized in two dimensions, as a diffusion-like process with anisotropic diffusivities.

The model has, in fact, been shown to describe transport well in purely homogeneous porous media with uniform one-dimensional flows. In heterogeneous field situations with non-uniform flows in, for example, irregular bedding or fractures, the model holds only at the pre-determined scale at which dispersivities are calibrated and it must be considered as a currently necessary approximation, and be carefully applied when extrapolating to other scales of transport.

\section{Solute and adsorbate mass balances}

SUTRA rolute transport simulation accounts for a single species mass stored in fluid solution as solute and species mass stored as adsorbate on the surfaces of solid matrix grains. Solute concentration, $C$, and adsorbate concentration, $C_{s}(x, y, t)\left[M / M_{G}\right]$, (where $[M]$ denotes units of solute mass, and $\left[M_{G}\right]$ denotes units of solid grain mass), are related through equilibrium adsorption isotherms. The species mass stored in solution in a particular volume of solid matrix may change with time due to ambient water with a different concentration flowing in, well water injected with a different concentration, changes in the total fluid mass in the block, solute diffusion or dispersion in or out of the volume, transfer of dissolved species to adsorbed species (or reverse), or a chemical or biological reaction causing solute production or decay. The species mass stored as 
adsorbate on the surface of solid grains in a particular block of solid matrix may change with time due to a gain of adsorbed species by transfer of solute from the fluid (or reverse), or a chemical or biological reaction causing adsorbate production or decay.

The separate balances for a single species stored in solution (solute) and on the solid grains (adsorbate), are expressed, respectively, as follows:

$$
\begin{gathered}
\frac{\partial\left(\varepsilon S_{w} \rho C\right)}{\partial t}=-f-\underline{\nabla} \cdot\left(\varepsilon S_{w} \rho \underline{v} C\right)+\underline{\nabla} \cdot\left[\varepsilon S_{w} \rho\left(D_{m} I+D\right) \cdot \underline{I} C\right] \\
+\varepsilon S_{w} \rho \Gamma_{w}+Q_{p} C^{*} \\
\frac{\partial\left[(1-\varepsilon) \rho_{S} C_{s}\right]}{\partial t}=+f+(1-\varepsilon) \rho_{s} \Gamma_{s}
\end{gathered}
$$$$
f(x, y, t) \quad\left[M_{s} /\left(L^{3} \cdot s\right)\right]
$$

volumetric adsorbate source (gain of absorbed species by transfer from fluid per unit total volume)

$D_{m} \quad\left[L^{2} / s\right]$

apparent molecular diffusivity of solute in solution in a porous medium inclyding tortuosity effects, $\left(D_{m} \sim 1 . \times 10^{-9}\left[\mathrm{~m}^{2} / \mathrm{s}\right]\right.$

identity tensor (ones on diagonal, zero elsewhere) $(2 \times 2)$

$$
\begin{array}{ll}
D(x, y, t) & {\left[L^{2} / s\right]} \\
\Gamma_{w}(x, y, t) & {\left[M_{s} / M \cdot s\right]}
\end{array}
$$

dispersion tensor

solute mass source in fluid (per unit fluid mass) due to production reactions 


$$
\begin{aligned}
& C^{*}(x, y, t) \quad\left[M_{s} / M\right] \\
& C_{s}(x, y, t) \quad\left[M_{s} / M_{G}\right] \\
& p_{s} \quad\left[M_{G} / L G\right] \\
& \Gamma_{8}(x, y, t) \quad\left[M_{s} / M_{G} \cdot 8\right]
\end{aligned}
$$

where $\left[\mathrm{L}_{G}{ }^{3}\right]$ is the volume of $8011 \mathrm{~d}$ grains.

Equation (2.29) is the solute mass balance in terms of the dissolved mass fraction (solute concentration), C. The time derivative expresses the total changes in solute mass with time in a volume due to the mechanisms represented by terms. on the right side of the equation. The term involving $f(x, y, t)$ represents the loss of solute mass from solution which becomes fixed on the solid grain surfaces as adsorbate. The adsorbate source, $f$, may, in general, depend on solute concentration, $C$, adsorbate concentration, $C_{s}$, and the rate of change of these concentrations, depending on either an equilibrium adsorption isotherm or on non-equilibrium adsorption processes. SUTRA algorithms are structured to directly accept non-equilibrium sorption models as an addition to the code. However, the current version of SUTRA assumes equilibrium sorption as shown in the following section, "Adsorption and production/decay processes."

The term involving fluid velocity, $\underline{v}$, represents average advection of solute mass into or out of the local volume. The term involving molecular diffusivity of solute, $D_{m}$, and dispersivity, $D$, expresses the contribution of solute diffusion and dispersion to the local changes in solute mass. The diffusion contribution is based on a true physical process often negligible at the field 
scale. The dispersion contribution is an approximation of the effect of solute advection and mixing in irregular flows which are not accounted for by solute advected by the average velocity. The solute mass source term involving $\Gamma_{w}(x, y, t)$. the solute mass production rate per unit mass of fluid, expresses the contribution to dissolved species mass of chemical, biological or radioactive reactions in the fluid. The last term accounts for dissolved species mass added by a fluid source with concentration, $C^{*}$.

Equation (2.30) is the balance of mass which has been adsorbed by solid grain surfaces in terms of species concentration on the solid (specific adsorbate concentation), $\mathrm{C}_{\mathrm{s}}$. The change in total adsorbate mass is expressed by the time derivative term. It may increase due to species leaving solution as expressed by adsorbate source term, $f$. The adsorbed mass may also change due to a production of adsorbate mass (per unit solid matrix mass), $\Gamma_{s}$ by radioactive or chemical processes within the adsorbate. Note that mass becomes immobile once adsorbed, and is affected only by possible desorption or chemical and biological processes.

The total mass of a species in a volume is given by the sum of solute mass and adsorbate mass. A balance of the total mass of a species is obtained by addition of (2.30) and (2.29). The general form of the total species mass balance used in SUTRA is this:

$$
\begin{aligned}
& \frac{\partial\left(\varepsilon S_{w} \rho C\right)}{\partial t}+\frac{\partial\left[(1-\varepsilon) \rho_{S} C_{s}\right]}{\partial t}=-\underline{\nabla} \cdot\left(\varepsilon S_{w} \rho \underline{v} C\right) \\
& +\underline{\nabla} \cdot\left[\varepsilon S_{w} \rho\left(D_{m} \underline{I}+\underline{D}\right) \cdot \nabla C\right]+\varepsilon S_{w} \rho \Gamma_{w}+(1-\varepsilon) \rho_{s} \Gamma_{s}+Q_{p} C^{*}
\end{aligned}
$$


Equation (2.31) is the basis for SUTRA solute transport simulation. In cases of solute transport where adsorption does not occur $\left(C_{s}=0\right)$, the adsorbate source term, $f$, simply has the value zero $(f=0)$, and the terms that stem from equation (2.30) are ignored. Further discussion of solute and adsorbate mass balances may be found in Bear (1979).

\section{Adsorption and production/decay processes}

The volumetric adsorbate source, $f$, of (2.29) and (2.30) may be expressed in the terms of a specific sorption rate, $f_{s}$, as:

$$
\begin{gathered}
f=(1-\varepsilon) \rho_{s} f_{s} \\
f_{s}(x, y, t) \quad\left[\begin{array}{l}
\text { specific solute mass adsorption rate } \\
\text { (per unit mass solid matrix) }
\end{array}\right.
\end{gathered}
$$

A particular non-equilibrium (kinetic) model of sorption is obtained by defining the functional dependence of the sorption rate, $f_{s}$, on other parameters of the system. For example, for a linear reversible non-equilibrium sorption model, the expression is: $f_{s}=m_{1}\left(c-m_{2} c_{s}\right)$, where $m_{1}$ and $m_{2}$ are sorption parameters. This particular model and a number of other non-equilibrium sorption models are accommodated by a general expression for $f_{s}$, as follows:

$$
f_{s}=k_{1} \frac{\partial C}{\partial t}+k_{2} C+k_{3}
$$

where:

$$
k_{1}=k_{1}\left(C, C_{s}\right), k_{2}=k_{2}\left(C, C_{s}\right), k_{3}=k_{3}\left(C, C_{s}\right)
$$

$$
\begin{array}{lll}
\kappa_{1}\left(C, C_{s}\right) & {\left[M / M_{G}\right]} & \text { first general sorption coefficient } \\
\kappa_{2}\left(C, C_{s}\right) & {\left[M / M_{G} \cdot s\right]} & \text { second general sorption coefficient } \\
\kappa_{3}\left(C, C_{s}\right) & {\left[M_{s} / M_{G} \cdot s\right]} & \text { third general sorption coefficient }
\end{array}
$$


Through a suitable definition of the general coefficients, $r_{i}\left(C, C_{s}\right)$, a number of non-equilibrium sorption models may be obtained. For example, the linear reversible non-equilibrium model mentioned above requires the definitions: $r_{1} \equiv 0, k_{2} \equiv m_{1}$, and $k_{3} \equiv-m_{1} m_{2} c_{s}$. The general coefficients $k_{1}, k_{2}$, and $k_{3}$ are included in the SUTRA code to provide generality for possible inclusion of such non-equilibrium (kinetic) sorption models.

The equilibrium sorption models are based on definition of the general coefficients through the following relation:

$$
\frac{\partial C}{\partial t}=r_{1} \frac{\partial C}{\partial t}
$$

Only general sorption coefficient, $k_{1}$, need be defined based on various equilibrium sorption isotherms as shown in the following. The other coefficients are set to zero, $k_{2}=\kappa_{3}=0$.

The linear equilibrium sorption model is based on the linear sorption isotherm assuming constant fluid density:

$$
\begin{aligned}
& C_{s}=\left(x_{1} p_{0}\right) C \\
& \frac{\partial C_{s}}{\partial t}=\left(X_{1} p_{0}\right) \frac{\partial C}{\partial t}
\end{aligned}
$$

where:

$$
\left.x_{1} \quad \mid L_{f}^{3} / M_{G}\right\rfloor \quad \text { linear distribution coefficient }
$$

and $\rho_{0}$ is the fluid base density

For linear sorption, general coefficient, ${ }^{\kappa_{1}}$, takes on the definition:

$$
r_{1}=x_{1} p_{0}
$$


The Freundich equilibrium sorption model is based on the following isotherm which assumes a constant fluid density, $\rho_{0}$ :

$$
\begin{aligned}
& c_{s}=x_{1}\left(\rho_{0} c\right)\left(\frac{1}{x_{2}}\right) \\
& \frac{\partial C_{s}}{\partial t}=\left(\frac{x_{1}}{x_{2}}\right)\left(\rho_{0} c\right)^{\left(\frac{1-x_{2}}{x_{2}}\right)} \rho_{0} \frac{\partial C}{\partial t}
\end{aligned}
$$

where:

$$
\begin{array}{lll}
x_{1} & {\left[L_{f}^{3} / M G\right]} & \text { a Freundlich distribution coefficient } \\
x_{2} & {[1]} & \text { Freundlich coefficient }
\end{array}
$$

when $x_{2}=1$, the Freundlich isotherm is equivalent to the linear isotherm. For Freundlich sorption, then, the general coefficient, $k_{1}$, takes the definition:

$$
x_{1}=\left(\frac{x_{1}}{x_{2}}\right) \rho_{0}^{\left(\frac{1}{x_{2}}\right)} c^{\left(\frac{1-x_{2}}{x_{2}}\right)}
$$

The Langmuir equilibrium sorption model is based on the following isotherm which assumes a constant fluid density, $\rho_{0}$ :

$$
\begin{aligned}
& c_{s}=\frac{x_{1}\left(p_{0} c\right)}{1+x_{2}\left(\rho_{0} C\right)} \\
& \frac{\partial C_{s}}{\partial t}=\frac{x_{1} \rho_{o}}{\left(1+x_{2} \rho_{0} C\right)^{2}} \frac{\partial C}{\partial t}
\end{aligned}
$$


where:

$$
\begin{array}{lll}
x_{1} & {\left[\mathrm{~L}_{\mathrm{f}}^{3} / \mathrm{M}_{\mathrm{G}}\right]} & \text { a Langmuir distribution coefficient } \\
\mathrm{x}_{2} & {\left[\mathrm{~L}_{\mathrm{f}}^{3} / \mathrm{M}_{\mathrm{s}}\right]} & \text { Langmuir coefficient }
\end{array}
$$

For very low solute concentrations, C, Langmuir sorption becomes linear sorption with linear distribution coefficient $x_{1}$. For very high solute concentrations, $C$, the concentration of adsorbate mass, $C_{s}$, approaches an upper limit equal to $\left(x_{1} / x_{2}\right)$. The general SUTRA coefficient, $\kappa_{1}$, is defined for Langmuir sorption as:

$$
\kappa_{1}=\frac{x_{1} \rho_{0}}{\left(1+x_{2} \rho_{0} c\right)^{2}}
$$

The production terms for solute, $\Gamma_{w}$, and adsorbate, $\Gamma_{s}$, allow for first-order mass production (or decay) such as linear BOD (biochemical oxygen demand) or radioactive decay, biological or chemical production, and zero-order mass production (or decay).

$$
\begin{aligned}
& \Gamma_{w}=\gamma_{1}^{w} \mathrm{c}+\gamma_{0}^{w} \\
& \Gamma_{s}=\gamma_{1}^{s} c_{s}+\gamma_{0}^{s}
\end{aligned}
$$

where:

$$
\begin{array}{lll}
r_{1}^{w} & {\left[s^{-1}\right]} & \text { first order mass production rate of solute } \\
r_{0}^{w} & {\left[\left(M_{s} / M\right) / s\right]} & \text { zero-order solute mass production rate } \\
r_{1}^{s} & \cdot\left[s^{-1}\right] & \begin{array}{l}
\text { first-order mass production rate of } \\
\text { adsorbate }
\end{array} \\
r_{0}^{s} & {\left[\left(M_{s} / M_{G}\right) / s\right]} & \begin{array}{l}
\text { zero-order adsorbate mass production } \\
\text { rate }
\end{array}
\end{array}
$$




\subsection{Description of Dispersion}

\section{Pseudo-transport mechanism}

Dispersion is a pseudo-transport process representing mixing of fluids which actually travel through the solid matrix at velocities different from the average velocity in two space dimensions, $\underline{v}$, calculated from Darcy's $l 3 w,(2.19)$. Dispersion is a pseudo-flux in that it only represents deviations from an average advective flux of energy or solute mass and as such does not represent a true mechanism of transport. Should it be possible to represent the true, complex, non-homogeneous velocity field in, for example, in the layers of an irregularly bedded field system, then the dispersion prucess need not be invoked to describe the transport, as the local variations in advection would provide the true picture of the transport taking place. However, as available data almost never allows for such a detailed velocity description, an approximate description, which helps to account for observed temperatures or concentrations different from that expected based on the average fluid advection, must be employed.

Current research trends are to develop dispersion models for various hydrogeological conditions, and SUTRA may be updated to include these new results as they become available. Currently, SUTRA dispersion is based on a new generalization for anisotropic media of the standard description for dispersion in isotropic homogeneous porous media. The standard description is, in fact, the only model available today for practical simulation. Because any inconsistencies which may arise in applying this dispersion model to particular 
field situation often would not be apparent due to the poor quality or small amount of measured data, the user is warned to exercise good judgement in interpreting results when large amounts of so-called dispersion are required to explain the field measurements.

In any case, the user is advised to consult up-to-date literature on field-scale dispersion, before employing this transport model.

\section{Isotropic-media dispersion model}

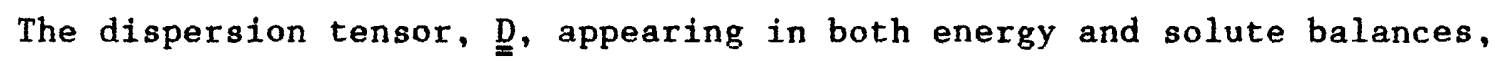
(2.28) and (2.31), is usually expressed for flow in systems with isotropic permeability and isotropic spatial distribution of inhomogeneities in aquifer materials as:

$$
D=\left[\begin{array}{ll}
D_{x x} & D_{x y} \\
D_{y x} & D_{y y}
\end{array}\right]
$$

where, $\mathrm{D}$ is, in fact, symmetric and the diagonal elements are:

$$
\begin{aligned}
& D_{x x}=\left(\frac{1}{v}\right)\left(d_{L} v_{x}^{2}+d_{T} v_{y}^{2}\right) \\
& D_{y y}=\left(\frac{1}{v}\right)\left(d_{T} v_{x}^{2}+d_{L} v_{y}^{2}\right)
\end{aligned}
$$

and the off-diagonal elements are:

$$
\begin{gathered}
D_{i j}=\left(\frac{1}{v 2}\right)\left(d_{L}-d_{T}\right)\left(v_{i} v_{j}\right) \\
i \neq j, \quad i=x, y \\
j=x, y
\end{gathered}
$$




$$
\begin{array}{lll}
v(x, y, t) & {[L / s]} & \text { magnitude of velocity } \underline{v} \\
v_{x}(x, y, t) & {[L / s]} & \text { magnitude of } x \text {-component of } \underline{v} \\
v_{y}(x, y, t) & {[L / s]} & \text { magnitude of } y \text {-component of } \underline{v} \\
d_{L}(x, y, t) & {\left[L^{2} / s\right]} & \text { longitudinal dispersion coefficient } \\
d_{T}(x, y, t) & {\left[L^{2} / s\right]} & \text { transverse dispersion coefficient }
\end{array}
$$

The terms $d_{L}$ and $d_{T}\left[L^{2} / s\right]$ are called longitudinal and transverse dispersion coefficients, respectively. These terms are analogous to typical diffusion coefficients. What is special, is that these are directional in nature. The term, $d_{L}$, acts as a diffusion coefficient which causes dispersion forward and backward along the local direction of fluid flow, and is called the longitudinal dispersion coefficient. The term, $d_{T}$, acts as a diffusion coefficient causing dispersion evenly in the directions perpendicular to the local flow direction, and is called the transverse dispersion coefficient. Thus, if $d_{L}$ and $d_{T}$ were of equal value, a circular disk of tracer released (in the $x-y$ plane) in ground water flowing, on the average uniformly and unidirectionally, would disperse in a perfectly symmetric circular manner as it moved downstream. However, if $d_{L}>d_{T}$ then the tracer would disperse in an elliptical manner with the long axis oriented in the flow direction, as it moved downstream.

The size of the dispersion coefficients are, in this model, for dispersion in isotropic permeability systems, dependent upon the absolute local magnitude of average velocity in a flowing system (Bear, 1979):

$$
\begin{array}{r}
d_{L}=\alpha_{L} v \\
d_{T}=\alpha_{T} v \\
\alpha_{L}(x, y)
\end{array}
$$

longitudinal dispersivity of solid matrix

transverse dispersivity of solid matrix 
When the isotropic-media dispersion model is applied to a particular field situation where aquifer inhomogeneities are much smaller than the field transport scale, then dispersivities $\alpha_{\mathrm{L}}$ and $\alpha_{\mathrm{T}}$ may be considered to be fundamental transport properties of the system just as, for example, permeability is a fundamental property for flow through porous media. In cases where inhomogeneities are large or scales of transport vary, dispersivities may possibly not be representive of a fundamental system property. In this case, dispersion effects must be interpreted with care, because dispersivity values are the only means avatlable to represent the dispersive characteristics of a given system to be simulated.

\section{Anisotropic-media dispersion model}

In a system with anisotropic permeability or anisotropic spatial distribution of inhomogeneities in aquifer materials, dispersivities may not have the same values for flows in all directions. In a case such as a layered aquifer, longitudinal dispersivity would clearly not have the same value for flows parallel to layers and perpendicular to layers. The fsotropic-media dispersion model, described in the previous section, does not account for such variability as $\alpha_{L}$ is isotropic (direction-independent). Transverse dispersivity would also tend to be dependent on the flow-direction, but because it typically is only a small fraction of longitudinal dispersivity, especially in anisotropic media (Gelhar and Axness, 1983), its variability is ignored here. This does not imply that transverse dispersion is an unimportant process, but the approximation is made because accurate simulation of low transverse dispersion is already limited, due to the requirement of a fine mesh for accurate representation of the process. The effect of any direction-dependence of transverse dispersivity would be obscured by the numerical discretization errors in a typical mesh. 
An ad-hoc model of flow-direction-dependent longitudinal dispersion is postulated. In this model, longitudinal dispersivity is assumed to have two principal directions (in two space dimensions) aligned with principal directions of permeability, $x_{p}$ and $x_{m}$. The principal values of longitudinal dispersivity, are $\alpha_{\text {Lmax }}$ and $\alpha_{\text {Lmin }}$ in these principal directions (see Figure 2.4). Note that the subscripts, Lmax and Lmin, refer only to the maximum and minimum permeability directions, and are not intended to imply the relation in magnitude of $\alpha_{\text {Lmax }}$ and $\alpha_{\text {Lmin' }}$, the principal values of longitudinal dispersivity.

If $F_{s}$ is the dispersive flux of solute (or energy) along a stream line of fluid flow, then

$$
F_{s}=-\alpha_{L} \frac{\partial U}{\partial s}
$$

where:

$$
\alpha_{L}(x, y, t) \quad[L] \quad \begin{aligned}
& \text { longitudinal dispersivity along a } \\
& \text { streamline }
\end{aligned}
$$

and $U$ represents either concentration or temperature, and $s$ is distance measured along a streamline. The dispersive flux components in the principal permeability directions $x_{p}$ and $x_{m}$ are:

$$
\begin{aligned}
& F_{p}=-\alpha_{L \max } \frac{\partial U}{\partial x_{p}}=F_{s} \cos \theta_{k v} \\
& F_{m}=-\alpha_{L \min } \frac{\partial U}{\partial x_{m}}=F_{s} \sin \theta_{k v}
\end{aligned}
$$

where:

$$
\begin{aligned}
& \alpha_{\operatorname{Lmax}}(x, y) \\
& \alpha_{\operatorname{Lmin}}(x, y) \\
& \theta_{k v}(x, y, t)
\end{aligned}
$$

Longltudinal dispersivity in the maximum permeability direction, $x_{p}$.

Longitudinal dispersivity in the minimum permeability direction, $x_{m}$.

Angle from maximum permeability direction, $x_{p}$, to local flow direction, ( $\left.\underline{v} /|\underline{v}|\right)$ 

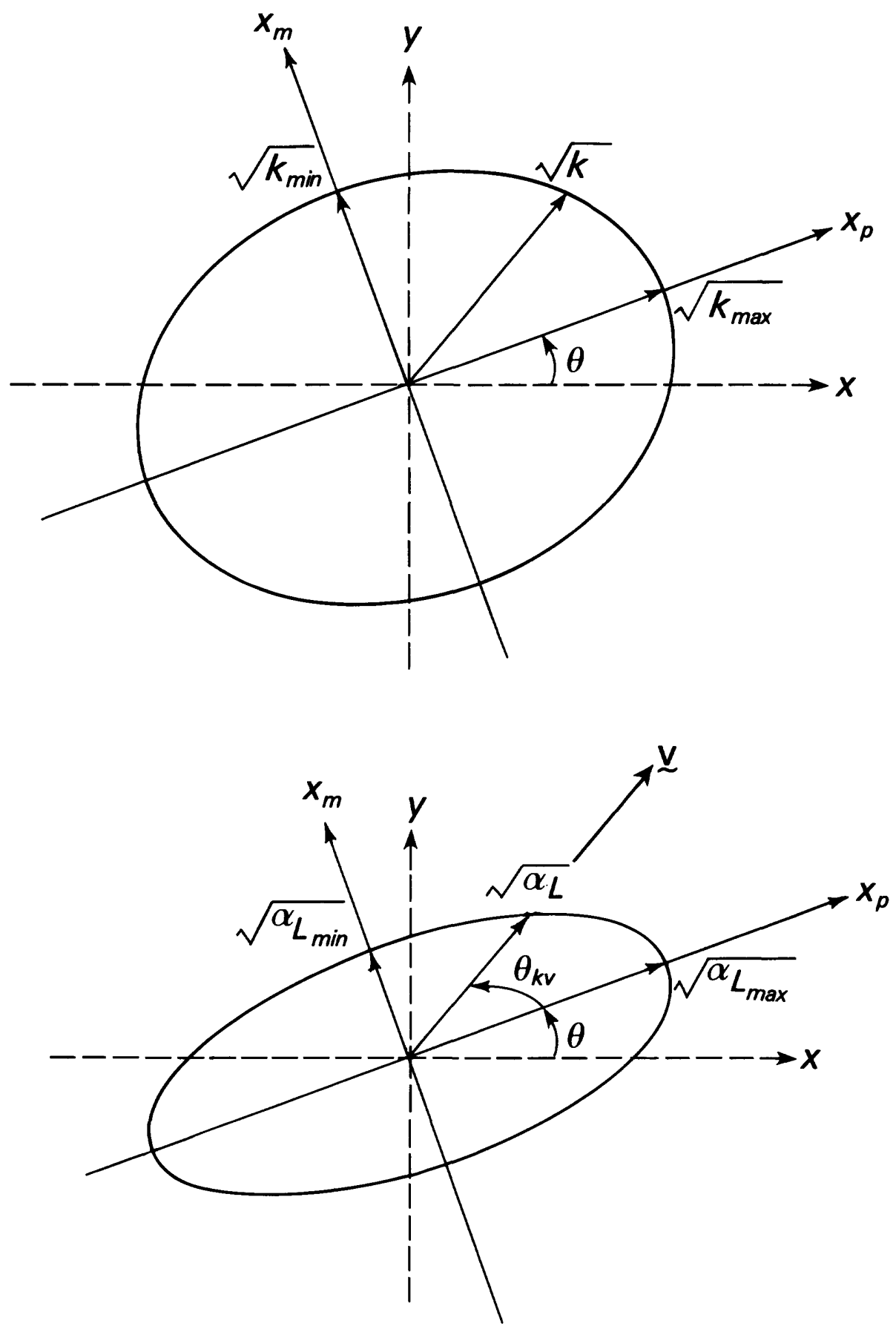

Figure 2.4

Definition of flow-direction-dependent longitudinal dispersivity, $\alpha_{L}(\theta)$. 
Because $U$ varies with $x$ and $y, U=U(x, y, t)$ :

$$
\begin{aligned}
& \frac{\partial U}{\partial s}=\frac{\partial U}{\partial x_{p}} \frac{\partial x_{p}}{\partial s}+\frac{\partial U}{\partial x_{m}} \frac{\partial x_{m}}{\partial s} \\
& \frac{\partial U}{\partial s}=\frac{\partial U}{\partial x_{p}} \cos \theta_{k v}+\frac{\partial U}{\partial x_{m}} \sin \theta_{k v}
\end{aligned}
$$

and:

$$
\begin{aligned}
& F_{s}=-\alpha_{L}\left(\cos \theta_{k v} \frac{\partial U}{\partial x_{p}}+\sin \theta_{k v} \frac{\partial U}{\partial x_{m}}\right) \\
& F_{s}=\alpha_{L}\left[\cos ^{2} \theta_{k v}\left(\frac{F_{s}}{\alpha_{L \text { max }}}\right)+\sin ^{2} \theta_{k v}\left(\frac{F_{s}}{\alpha_{L m i n}}\right)\right]
\end{aligned}
$$

This defines an ellipse as:

$$
\left(\frac{1}{\alpha_{L}}\right)=\left(\frac{\cos ^{2} \theta_{k v}}{\alpha_{L \max }}\right)+\left(\frac{\sin ^{2} \theta_{k v}}{\alpha_{L \min }}\right)
$$

with semi-major axis $\left(\alpha_{\operatorname{Lmax}}\right)^{1 / 2}$ and semi-minor axis $\left(\alpha_{L \min }\right)^{1 / 2}$. The length of a radius is $\left(\alpha_{L}\right)^{1 / 2}$, as shown in Figure 2.4 . This ellipse is analagous in concept to that which gives effective permeability in any direction in an anisotropic medium.

The value of effective longitudinal dispersivity as dependent on the flow direction is :

$$
\alpha_{L} \quad=\frac{\alpha_{L \max }{ }^{\alpha}{ }_{L \min }}{\left(\alpha_{L \min } \cos ^{2} \theta_{k v}+\alpha_{L \max } \sin ^{2} \theta_{k v}\right)}
$$

which is used by SUTRA to compute $\alpha_{L}$ for the anisotropic-media dispersion model. Note that if $\alpha_{L \max }=\alpha_{L \text { min }}$, then the isotropic dispersion-media model is obtained. 
This form of longitudinal dispersivity dependence on direction of flow relative to the principal permeability directions is similar to that obtained for a transversely isotropic medium in a stochastic analysis of macro-dispersion by Gelhar and Axness (1983).

\section{Guidelines for applying dispersion model}

Some informal guidelines may be given concerning values of dispersivities when other data are not available. Longitudinal dispersivities may be considered to be on the order of the same size as either the largest hydrogeologic or flow inhomogeneities along the transport reach or the distance between inhomogeneities, whichever is the greater value. For transport in pure homogeneous sand, longitudinal dispersivity is on the order of grain size. This is the type of situation where the isotropic-media dispersion model well describes observed transport behavior. In the case of a sandy aquifer containing well-distributed inclusions of less-permeable material, the longitudinal dispersivity required to correct an average advective transport which has passed by many of the inclusions would be of the order on the larger of either inclusion size or distance between inclusions.

Should the dispersivity, estimated on the basis of the size in homogeneities or distance between them, be greater than about one tenth of the longest transport reach, then the meaningful use of a constant-dispersivity dispersion model must be questioned. In such a case, the ideal action to take would be to more explicitly define the field distribution of velocity by taking into account the actual geometry of inhomogeneities. This would correctly account for most of the transport taking place as advective in nature, with much smaller con- 
tributions of the approximate dispersive process. Given a better-defined velocity field, and in the absence of other data, dispersivity should then be chosen based on the largest postulated inhomogeneities met along a given average stream tube. The size and distribution of inhomogeneities not explicitly taken into account by the average flow field may be postulated based on the best available knowledge of local geology.

Transverse dispersivity, $\alpha_{\mathrm{T}}$, is typically even less well known for field problems than longitudinal dispersivity. Values of $\alpha_{T}$ used in simulation are typically between one tenth and one third of $\alpha_{L}$. In systems with anisotropic permeability, $\alpha_{T}$ may be less than one hundredth of $\alpha_{L}$ for flows along the maximum permeability direction (Gelhar and Axness, 1983). Should simulated transport in a particular situation be sensitive to the value of transverse dispersivity, further data collection is necessary and the transport model must be interpreted with great care.

The ad-hoc model for longitudinal dispersion in anisotropic media presented in the previous section allows for simulation experiments with two principal longitudinal dispersivities which may be of special interest in systems with well-defined anisotropy values. Depending on the particular geometry of layers or inhomogeneities causing the permeability anisotropy, the longitudinal disper-

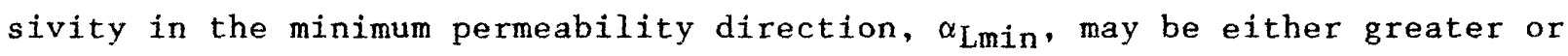
smaller than that in the maximum permeability direction $\alpha_{\text {Lmax }}$. However, use of the anisotropic-media dispersion model is advised only when clearly required by field data, and the additional longitudinal dispersion parameter is not intended for general application without evaluation of its applicability in a particular case. 


\section{Unifled energy-solute balance}

The saturated-unsaturated ground-water energy balance (2.28) is simply an accounting of energy fluxes, sources and sinks which keeps track of how the energy per unit volume of solid matrix plus fluid, $\left[\varepsilon S_{w} p c_{w}+(1-\varepsilon) p_{s} c_{s}\right] T$, changes with time at each point in space. The saturated-unsaturated groundwater balance of solute plus adsorbate mass, (2.31), is similarly an accounting of solute and adsorbate fluxes, sources and sinks, which keeps track of how the species mass (solute plus adsorbate mass) per unit volume of solid matrix plus fluid, $\left(\varepsilon S_{w} \rho C+(1-\varepsilon) \rho_{s} C_{s}\right)$, changes with time at each point in space. Both balances, therefore, track a particular quantity per unit volume of solid matrix plus fluid.

The fluxes of energy and solute mass in solution, moreover, are caused by simflar mechanisms. Both quantities undergo advection based on average flow velocity, $\underline{v}$. Both quantities undergo dispersion. Both quantities undergo diffusion; the diffusive solute mass flux is caused by molecular or ionic diffusion within the fluid, while the diffusive energy flux occurs by thermal conduction through both fluid and solid. Fluid sources and sinks give rise to similar sources and sinks of energy and solute mass. Energy and species mass may both be produced by zero-order processes, wherein energy may be produced by an endothermic reaction and solute may be produced, for example, by a biological process. The linear adsorption process affecting solutes is similar to the storage of energy in solid portion of an aquifer. Only the non-linear sorption processes and first-order production of solute and adsorbate, have no readily apparent analogy in terms of energy. 
Thus, the balances of energy per unit volume, (2.28), and total species mass per unit volume, (2.31), may be expressed in a single unified balance in terms of a variable, $U(x, y, t)$, which may either represent $T(x, y, t)$ or $C(x, y, t)$, as follows:

$$
\begin{gathered}
\frac{\partial}{\partial t}\left(\varepsilon S_{w} \rho c_{w} U\right)+\frac{\partial}{\partial t}\left[(1-\varepsilon) \rho_{s} U_{s}\right]+\underline{\nabla} \cdot\left(\varepsilon S_{w} \rho c_{w} v U\right) \\
-\underline{\nabla} \cdot\left\{\rho c_{w}\left[\varepsilon S_{w}\left(\sigma_{w} I+D\right)+(1-\varepsilon) \sigma_{s} I\right] \cdot \underline{V} U\right\} \\
=Q_{p} c_{w} U^{*}+\varepsilon S_{w} \rho \Gamma_{w}+(1-\varepsilon) \rho_{s} \Gamma_{s}
\end{gathered}
$$

where:

\section{for energy transport}

$$
\begin{aligned}
U \equiv T, U_{s} \equiv c_{s} T, U^{*} T^{*}, \sigma_{w}=\frac{\lambda_{w}}{\rho c_{w}}, \sigma_{s} \equiv \frac{\lambda_{s}}{\rho c_{w}} \\
\Gamma_{w} \equiv \gamma_{0}^{w}, \Gamma_{s} \equiv \gamma_{0}^{s}
\end{aligned}
$$

\section{for solute transport}

$U \equiv C, U_{s} \equiv C_{s}, U^{*} \equiv C^{*}, \sigma_{w} \equiv D_{m}, \sigma_{s} \equiv 0, c_{w} \equiv 1$

where $C_{s}$ is defined by $(2.34 a),(2.35 a)$ or $(2.36 a)$, depending on the isotherm.

By simple redefinition according to (2.47a) or $(2.47 b)$, equation (2.41) directly becomes the energy or species mass balance. This redefinition is automatically carried out by SUTRA as a result of whether the user specifies energy or solute transport simulation. 


\section{Fluid-mass-conservative energy-solute balance}

A further consideration is required before obtaining the form of the unified energy/solute balance as implemented in SUTRA. The amount of energy or solute per unit combined matrix-fluid volume may change either due to a change in the total fluid mass in the volume even when concentration and temperature remain constant (see relation $(2.10)$ ). Such a change in fluid mass may be caused by changes in fluid saturation, or by pressure changes affecting compressive storage.

The energy and solute balances as well as their unifled form, (2.47), track both types of contributions to changes in total stored energy or solute mass. However, the fluid saturation and pressure change contribution to energy and solute balances are already implicitly accounted for by the fluid mass balance.

The fluid mass balance contribution to solute and energy balances is expressed by the product of the fluid mass balance, equation (2.22) (which tracks changes in fluid mass per unit volume), with $c_{w} U$ (which represents either energy or solute mass per unit fluid mass). Note that $c_{w}=1$ for solute transport. This product tracks energy or solute mass changes per unit volume due to fluid mass changes per unit volume:

$$
\left(c_{w} U\right) \frac{\partial\left(\varepsilon S_{w} \rho\right)}{\partial t}+\left(c_{w} U\right) \underline{\nabla} \cdot\left(\varepsilon S_{w} \rho \underline{v}\right)=\left(c_{w} U\right) Q_{p}
$$

where the solute mass source, $T$, is neglected. Comparison of (2.48) with (2.47) will reveal that the terms on the left of (2.48) also appear in the unified balance equation. 
Before substituting (2.48) for the duplicate terms in (2.47), the search for redundant terms may be extended to a balance of species mass or energy stored in the solid matrix rather than in the fluid. A simple mass balance for the solid matrix is:

$$
\begin{array}{cc} 
& \frac{\partial}{\partial t}\left[(1-\varepsilon) \rho_{s}\right]+\nabla \cdot\left[(1-\varepsilon) \rho_{s} \underline{v}_{s}\right]=0 \\
\underline{v}_{s} & {[L / s] \quad \text { net solid matrix velocity }}
\end{array}
$$

Due to the assumption that the net solid matrix velocity, $\underline{v}_{s}$, is negligable. the associated term of (2.49) is dropped. The contribution of this simple solid matrix mass balance to the unified solute-energy balance may again be obtained by taking the product of (2.49) with $\mathrm{U}_{\mathrm{s}}$ :

$$
\left(U_{s}\right) \frac{\partial}{\partial t}\left[(1-\varepsilon) p_{s}\right]=0
$$

A comparison reveals that this term also appears in (2.47).

The redundant information in the unified energy-solute balance which keeps track of both solid matrix and fluid mass balance contributions may be directly removed from (2.47) by subtracting (2.48) and (2.50). The result is:

$$
\begin{gathered}
\varepsilon S_{w} \rho c_{w} \frac{\partial U}{\partial t}+(1-\varepsilon) \rho \frac{\partial U}{s}+\varepsilon t+S_{w} \rho c_{w} v \cdot \nabla U \\
-\underline{\nabla} \cdot\left\{\rho c_{w}\left[\varepsilon S_{w}\left(\sigma_{w=}+D\right)+(1-\varepsilon) \sigma_{s} \frac{I}{s}\right] \cdot \nabla \underline{U}\right\} \\
=Q_{p} c_{w}\left(U^{*}-U\right)+\varepsilon S_{w} \rho \Gamma_{w}+(1-\varepsilon) \rho_{s} \Gamma_{s}
\end{gathered}
$$


where:

\section{for energy transport}

$$
\begin{aligned}
U \equiv T, U_{s} \equiv c_{s} T U^{*} T^{*}, \sigma_{w} \equiv \frac{\lambda_{w}}{\rho c_{w}}, \sigma_{s} \equiv \frac{\lambda_{s}}{\rho c_{w}} \\
\Gamma_{w} \equiv \gamma_{0}^{w}, \Gamma_{s} \equiv \gamma_{0}^{s}
\end{aligned}
$$

\section{for solute transport}

$U \equiv C, U_{s} \equiv C_{s}, U^{*} \equiv C^{*}, \sigma_{w} \equiv D_{m}, \sigma_{s} \equiv 0 \quad c_{w} \equiv 1$

where $C_{s}$ is defined by $(2.34 a),(2.35 a)$ or (2.36a), depending on isotherm.

It is assumed in equation (2.51) that $c_{w}$ and $c_{s}$ are not time-dependent.

For numerical simulation, this equation may be termed a 'fluid-massconservative' form of the energy or species mass balance. When approximated numerically, the unified balance in the original form, (2.47), would contain approximation errors in both the fluid mass balance contributions (based on pressure and saturation changes) and the temperature or concentration change contribution. However, in the revised form, equation (2.51), the complete fluid mass balance contribution has already been analytically accounted for before any numerical approximation takes place. Thus, the total approximation error for the unified balance, (2.51), is significantly less as it is due to the temperature or concentration change contribution only.

The unified energy-species mass balance is brought to its final form by noticing that the form of the term, $\partial U_{s} / \partial t$, for energy transport, is the same as that for solute transport when using the equilibrium sorption relation (2.33), and that the form of the energy production of terms is simflar to that of relations $(2.37 \mathrm{a})$ and $(2.37 \mathrm{~b})$ for the mass production process: 


$$
\begin{aligned}
& \left\lfloor\varepsilon S_{w} \rho c_{w}+(1-\varepsilon) \rho_{S} c_{s}\right\rfloor \frac{\partial U}{\partial t}+\varepsilon S_{w} \rho c_{w} \underline{v} \cdot \underline{\nabla U} \\
& -\underline{\nabla} \cdot\left\{\rho c_{w}\left\lfloor\varepsilon S_{w}\left(\sigma_{w} \underline{I}+\underline{D}\right)+(1-\varepsilon) \sigma_{S} I\right\rfloor \cdot \underline{=}\right\} \\
& =Q_{p} c_{w}\left(U^{*}-U\right)+\varepsilon S_{w} \rho \gamma_{1}^{W} U+(1-\varepsilon) \rho_{s} \gamma_{1}^{s} U_{s}+\varepsilon S_{w} \rho \gamma_{0}^{W}+(1-\varepsilon) \rho_{s} \gamma_{0}^{s}
\end{aligned}
$$

where:

\section{for energy transport}

$$
U \equiv T, U^{*} \equiv T^{*}, \quad \sigma_{w} \equiv \frac{\lambda_{w}}{\rho C_{w}}, \sigma_{s} \equiv \frac{\lambda_{s}}{\rho c_{w}}, \gamma_{1}^{w} \equiv \gamma_{1}^{s} \equiv 0
$$

\section{for solute transport}

$\mathrm{U} \equiv \mathrm{C}, \mathrm{U}_{\mathrm{s}} \equiv \mathrm{C}_{\mathrm{s}}, \mathrm{U}^{*} \equiv \mathrm{C}^{*}, \sigma_{\mathrm{w}} \equiv \mathrm{D}_{\mathrm{m}}, \sigma_{\mathrm{s}} \equiv 0, \mathrm{c}_{\mathrm{s}} \equiv \mathrm{r}_{1}, \mathrm{c}_{\mathrm{w}} \equiv 1$

where $C_{s}$ is defined by $\left.(2.34 a), 2.35 a\right)$ or $(2.36 a)$, and $r_{1}$ is defined by $(2.34 c),(2.35 c)$ or $(2.36 c)$, depending on the isotherm.

The fluid-mass-conservative form of the unified energy-species mass balance, (2.52), is exactly that which is implemented in SuTRA. 


\section{Chapter 3}

\section{Fundamentals of Numerical Algorithms}

SUTRA methodology is complex because: (1) density-dependent flow and transport requires two interconnected simulation models, (2) fluid properties are dependent on local values of temperature or concentration, (3) geometry of a field area and distributions of hydrogeologic parameters may be complex, and (4) hydrologic stresses on the system may be distributed in space and change with time. Furthermore, a tremendous amount of data must be evaluated by SUTRA with precision. This requires great computational effort, and considerable numerical intricacy is required to minimize this effort. The mathematically elegant finite-element and integrated-finite-difference hybrid method employed by SUTRA allows great numerical flexibility in describing processes and characteristics of flow and transport in hydrologic field systems. Unlike simulation models based purely on the method of finite differences, however, the numerical aspects of which allow straight-forward interpretation at an intuitive level, some finite-element aspects of SUTRA methodology require interpretation at a less physical level and from a more mathematical point of view.

The following description of SUTRA numerical methods uses a simplified, constant-density water-table aquifer case as an illustrative example. While precise mathematically, this example is not used to demonstrate an actual application of SUTRA, as SUTRA does not, in fact, simulate a moving water table. The example is only used as a device through which to explain the theory and use of the primary numerical methods employed in SUTRA and the water table is invoked to allow discussion of a simple non-linearity. The basic methods, which are only demonstrated here, are applied in detail in 
Chapter 4, "Numerical Methods," to the SUTRA fluid mass balance and unified energy-species mass balance.

The water-table aquifer fluid mass balance equation is useful for demonstration of basic numerical methods employed on SUTRA governing equations, because it displays some of the salient aspects of the SUTRA equations: a time derivative, a non-linear term Involving space-derivatives, and a source term. The simplified fluid mass balance equation is as follows:

$$
S_{0} \frac{\partial h}{\partial t}-\underline{\nabla} \cdot(K \underline{\nabla} h)=Q^{*}
$$

where

$$
Q^{*}=\left(Q_{p} / 0\right)
$$

and

$$
\begin{aligned}
& S_{0}(x, y) \quad\left[L^{-1}\right\rfloor \quad \text { specific storativity } \\
& h(x, y, t) \quad \text { [L] hydraulic head (sum of pressure } \\
& \mathrm{k}(\mathrm{x}, \mathrm{y}) \quad[\mathrm{L} / \mathrm{s}] \quad \text { hydraulic conductivity (assumed for } \\
& Q^{*}(x, y) \quad\left[s^{-1}\right\rfloor \quad \text { volumetric fluid source (volume fluid } \\
& \text { injected per time / volume aquifer) } \\
& \text { (assumed constant for this example) } \\
& Q_{p}(x, y) \quad\left[M /\left(L^{3} \cdot s\right)\right\rfloor \quad \text { fluid mass source (mass fluid injected } \\
& \text { per time / volume aquifer) (assumed con- } \\
& \text { stant for this example) } \\
& \text { D }\left[M / L^{3}\right\rfloor \text { fluid density (assumed constant for } \\
& \text { this example) }
\end{aligned}
$$

This equation, (3.1), is obtained from the SUTRA fluid mass balance, (2.24), by assuming saturated conditions, constant concentration and temperature, constant fluid density, and using the definition of hydraulic conductivity, $\mathrm{K} \equiv(\mathrm{k} \rho|\mathrm{g}|) / \mu$, where $|\mathrm{g}|$ is the acceleration of gravity, and of hydraulic 
head, $h \equiv h_{p}+$ ELEVATION, where pressure head, $h_{p}=p /(p|g|)$. For clarity, hydraulic conductivity 18 assumed to be isotropic in this example. While (3.1) may be considered a fully three-dimensional mass balance equation, it is assumed that flow takes place only areally in a water-table aquifer with a fixed impermeable base (at z-position, $\operatorname{BASE}(x, y)$ ), and a moveable free surface (at z-position, $h(x, y, t))$. The $z$-direction is oriented vertically upward and the fluid is assumed to be in vertical hydrostatic equilibrium at any $(x, y)$ position (no vertical flow). Aquifer thickness, $B(x, y, t)[L]$, is measured as the distance along $z$ from the free surface to the aquifer base, and may change with time. Aquifer transmissivity, $\pi(x, y, t)$, is given by:

$$
\begin{array}{lll}
T \equiv \mathrm{KB} \equiv \mathrm{K}(\mathrm{h}-\mathrm{BASE}) & \\
\pi(\mathrm{x}, \mathrm{y}, \mathrm{t}) & {\left[\mathrm{L}^{2} / \mathrm{s}\right]} & \text { aquifer transmissivity } \\
\mathrm{B}(\mathrm{x}, \mathrm{y}, \mathrm{t}) & {[\mathrm{L}]} & \text { aquifer thickness } \\
\mathrm{BASE}(\mathrm{x}, \mathrm{y}) & {[\mathrm{L}]} & \text { elevation of aquifer base }
\end{array}
$$

The above assumption, in effect, makes (3.1) a two-dimensional mass balance equation which is applied to a finite thickness aquifer. The two-dimensional form of (3.1) describing an areal fluid mass balance for water-table aquifers in terms of a head-dependent transmissivity arises during the basic numerical analysis of (3.1) in section 3.3, "Integration of Governing Equation in Space."

\subsection{Spatial Discretization by Finite Elements}

Although SUTRA is a two-dimensional model, the region of space in which flow and transport 18 to be simulated may be defined in three space dimensions. The three-dimensional bounded volume of an aquifer which is to be simulated by 
SUTRA is completely divided up into a single layer of contiguous blocks. These blocks are called 'finite elements.' The subdivision is not done simply in a manner which creates one block (element) for each portion of the aquifer system which has unique hydrogeological characteristics. Each hydrogeologic unit is in fact divided into many elements giving the subdivided aquifer region the appearance of a fine net or mesh. Thus, subdivision of the aquifer region to be simulated into blocks is referred to as 'creating the finite-element mesh (or finite-element net).

The basic building block of a finite-element mesh is a finite element. The type of element employed by SUTRA for two-dimensional simulation is a quadrilateral which has a finite thickness in the third space dimension. This type of a quadrilateral element and a typical two-dimensional mesh is shown in Figure 3.1.

All twelve edges of the two-dimensional quadrilateral element are perfectly straight. Four of these edges are parallel to the z-coordinate direction. The $x-y$ plane (which contains the two coordinate directions of interest) bisects each of the edges parallel to $z$, so that the top and bottom surfaces of the element are mirror images of each other reflected about the central $x-y$ plane in the element. The mid-point of each $z$-edge (the point where the $x-y$ plane intersects) is referred to as a nodal point (or node). Thus, the element has a three-dimensional shape, but always has only exactly four nodes, each of which in fact, represents the entire $z$-edge on which it is located. The nodes mark the fact that, in this type of element, some aquifer parameters may be assigned a different value at each z-edge of the element. The lack of nodes outside of the $\mathrm{x}-\mathrm{y}$ plane is what makes this element two-dimensional; while some aquifer parameters may vary in value from node to node (i.e. from z-edge to z-edge), no parameters may be assigned varying values in the $z$-direction. 


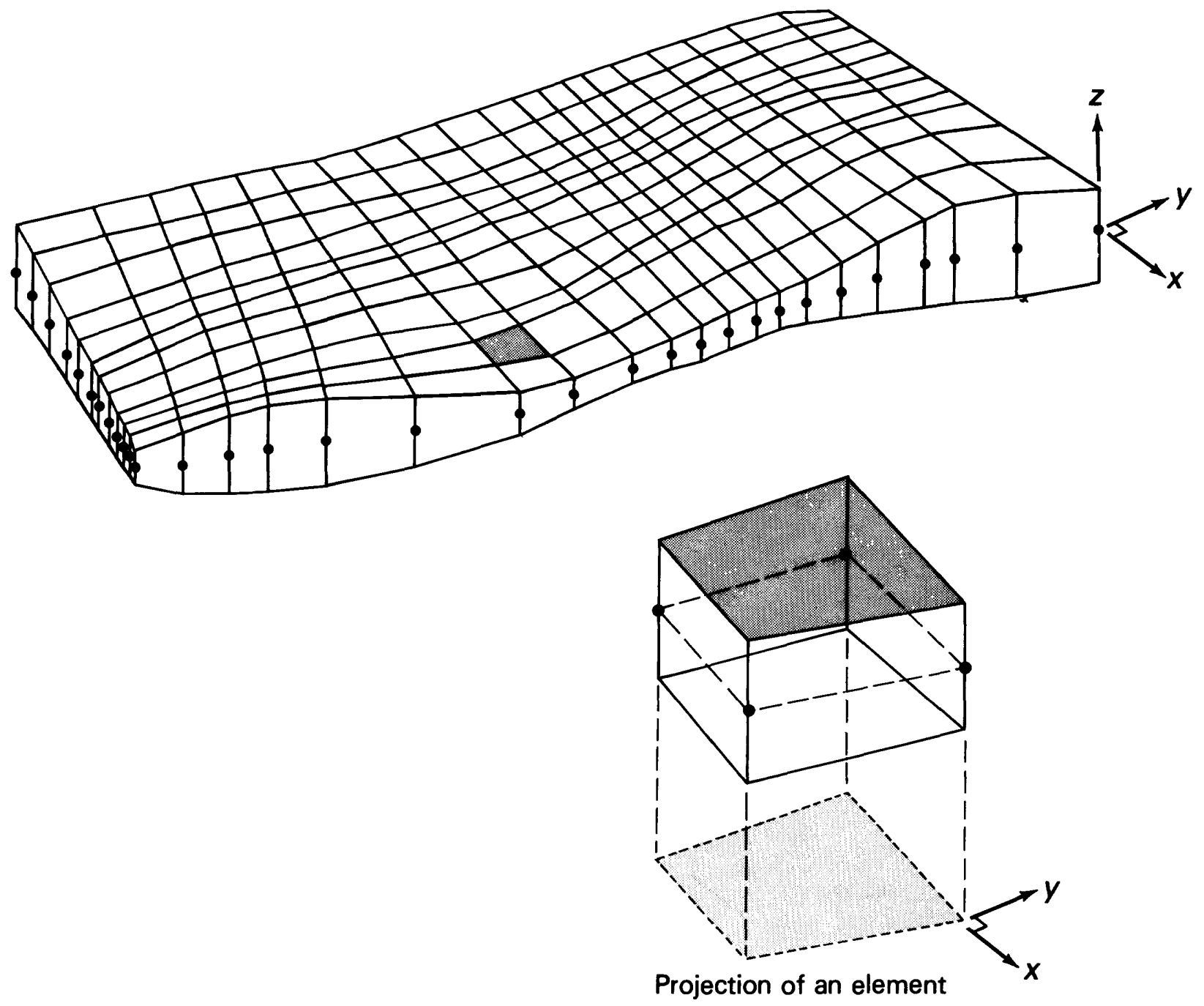

Figure 3.1

Two-dimensional finite-element mesh and quadrilateral element. 
Within a two-dimensional finite-element mesh there is only a single layer of elements, the nodes of which lie in the $x-y$ plane. Nodal points are always shared by the elements adjoining the node. Only nodes at external corners of the mesh are not contained in more than one element. The top and bottom surfaces are at every $(x, y)$ point equidistant from the $x-y$ plane, but the thickness of the mesh, measured in the $z$-direction, may vary smoothly from point to point. When projected on the $x-y$ plane, as in Figure 3.1, a finite-element mesh composed of the type of elements used by SUTRA appears as a mesh of contiguous quadrilaterals with nodes at the corners. Hence, the term, 'quadrilateral element'.

\subsection{Representation of Coefficients in Space}

Aquifer parameters and coefficients which vary from point to point in an aquifer such as specific storativity, $S_{o}$, and hydraulic conductivity, $K$, are represented in an approximate way in SUTRA. Parameters are either assigned a particular constant value in each element of a finite-element mesh (elementwise), or are assigned a particular value at each node in the mesh in two possible ways (nodewise or cellwise).

In the water-table aquifer, for a simple example, a regular two-dimensional mesh is used. The steplike appearance of elementwise assignment of $K$ values over this simple mesh is shown in Figure 3.2. Nodewise assignment for head over this mesh results in a continuous surface of $h$ values as shown in Figure 3.3 , with linear change in value between adjoining nodes along (profected) element edges. Cellwise assignment is employed for spectfic storativity, $S_{0}$, and the time derivative, $\frac{\partial h}{\partial t}$. This results in a steplike appearance of the assigned values 


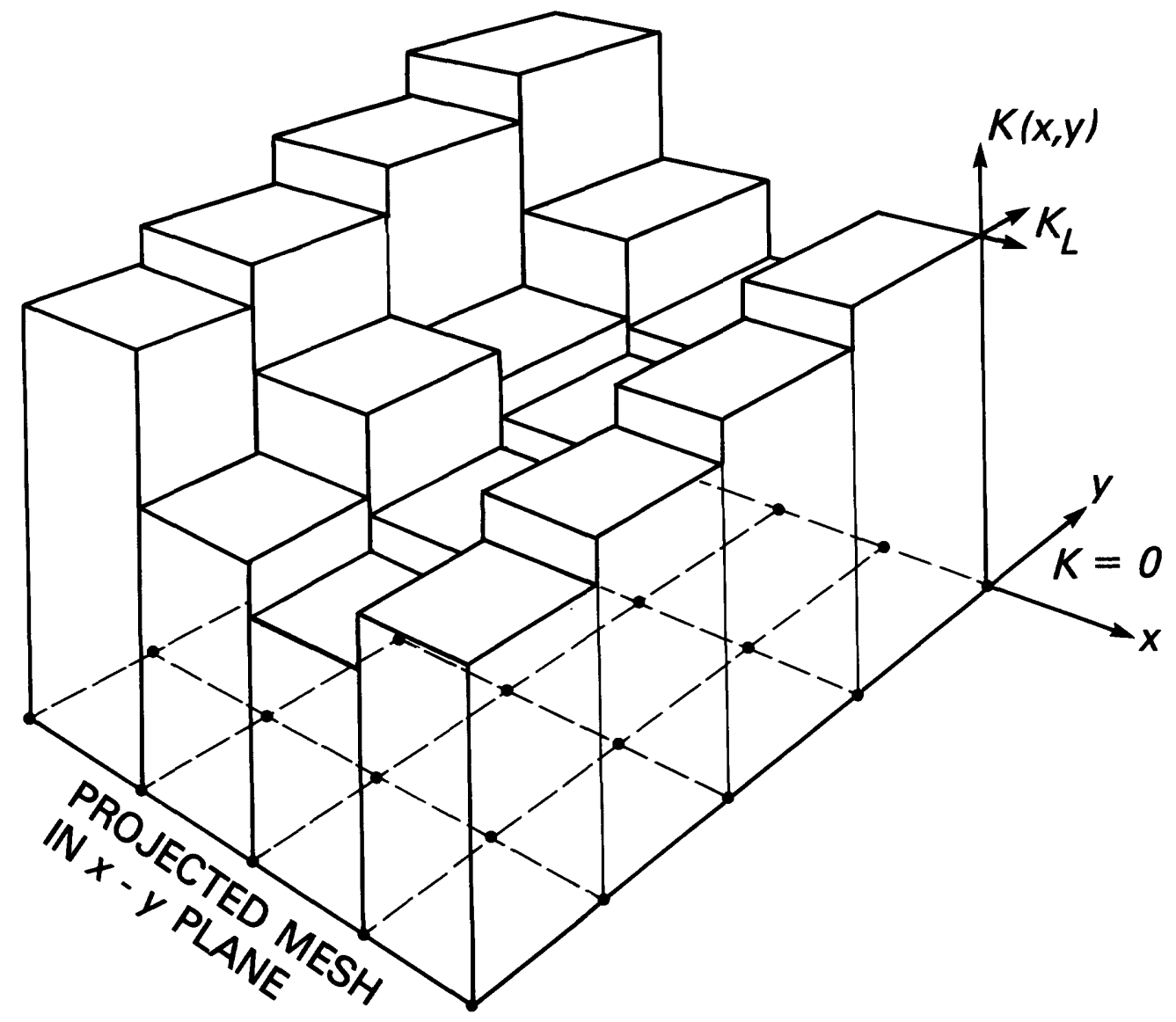

Figure 3.2

Elementwise discretization of coefficient $K(x, y)$. 


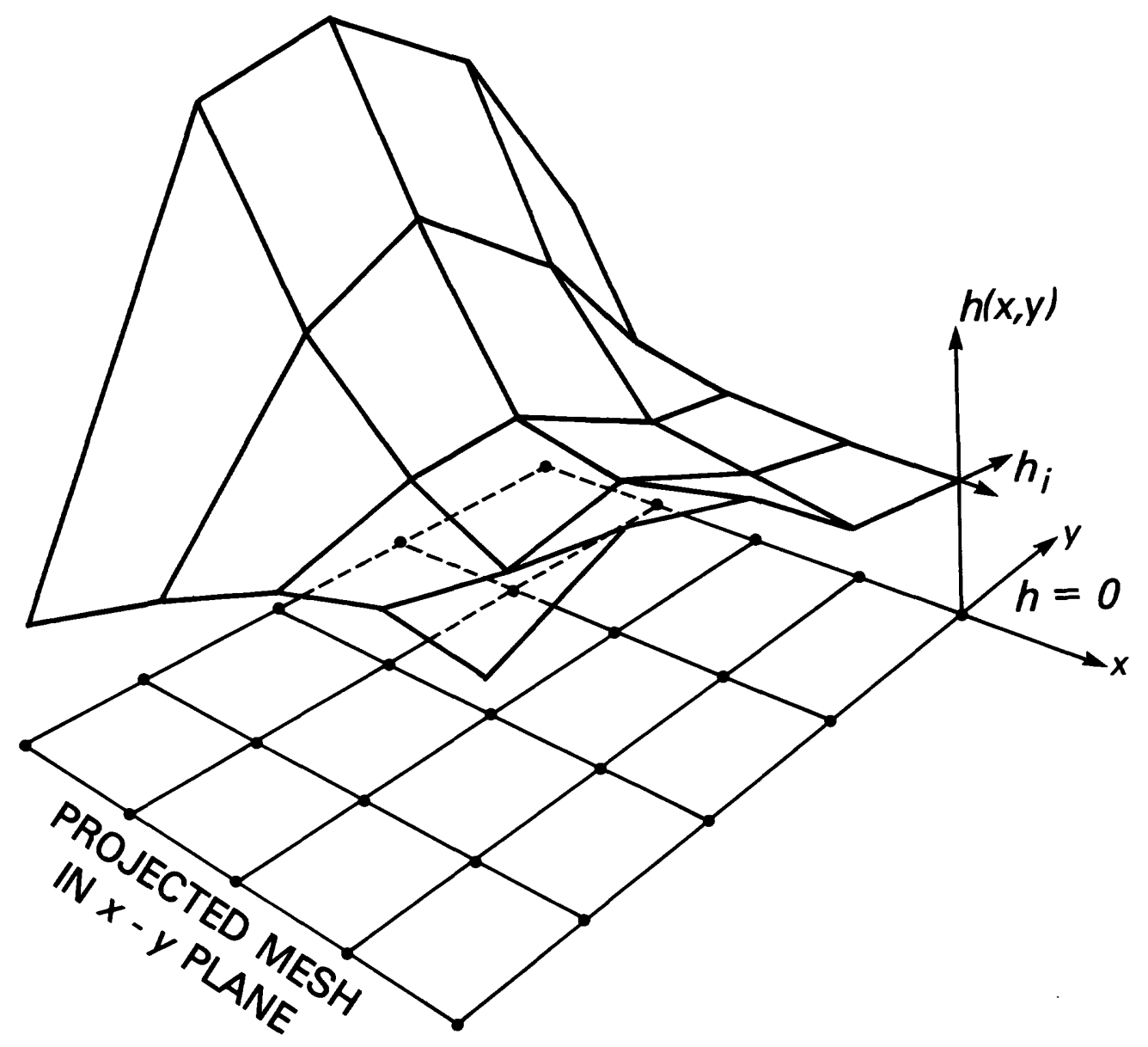

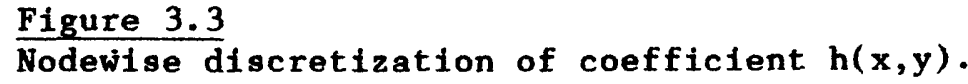


over the mesh similar to that of elementwise assignment in Figure 3.2 , but each cell is centered on a node, not on an element. Cell boundaries are half way between opposite sides of an element and are shown for the regular mesh in Figure 3.4. Thus the spatial distributions of parameters, $K, h$ and $S$, are discretized (i.e., assigned discrete values) in three different ways: $K$, elementwise, $h$, nodewise, and $s_{0}$, cellwise.

Because the internal program logic depends on the type of discretization, SUTRA expects certain particular parameters or equation terms to be discretized elementwise, nodewise, or cellwise. The primary dependent variables of the SUTRA code $p$, and $T$ or $C$, (in this example case, only hydraulic head, $h$ ), are expressed nodewise when used in terms which calculate fluxes of fluid mass, solute mass or energy.

\section{Elementwise discretization}

The equation which gives the values, over the finite element mesh, of an elementwise parameter, may be expressed for the hydraulic conductivity of the present example as:

$$
K(x, y) \approx \sum_{L=1}^{N E} K_{L}(x, y)
$$

where the elements have been numbered from one to $N E$ (total number of elements in the mesh), and $K_{L}(x, y)[L / s]$ has the value of hydraulic conductivity of element $L$ for $(x, y)$ coordinates within the element, and a value of zero outside the element. Thus $\mathrm{K}_{\mathrm{L}}(\mathrm{x}, \mathrm{y})$ is the flat-topped 'box' standing on an element $\mathrm{L}$, in Figure 3.2, and $\mathrm{K}(\mathrm{x}, \mathrm{y})$ is represented in a discrete approximate way by the sum of all the 'boxes'. Note that $K_{L}(x, y)$ has the same value in the $z$-direction from the top to the bottom of each two-dimensional element. 


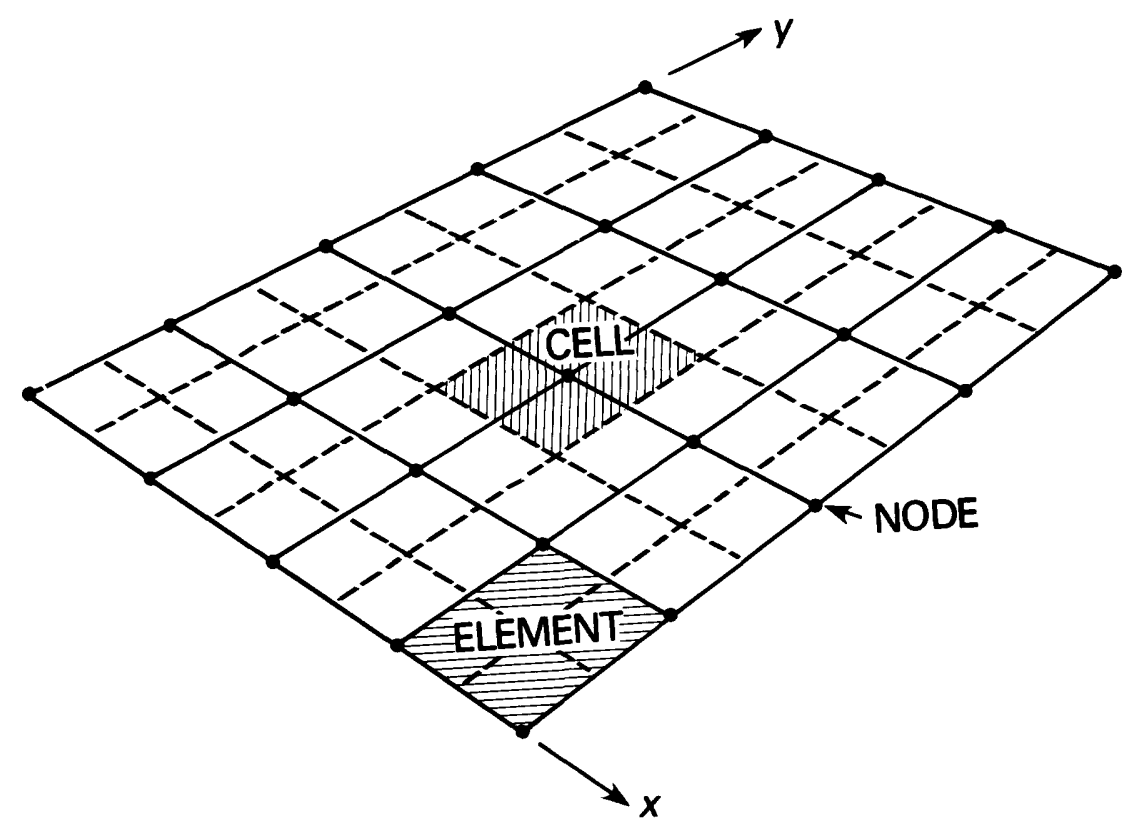

Figure 3.4

Cells, elements and nodes for a two-dimensional

finite-element mesh composed of quadrilateral

elements. 


\section{Nodewise discretization}

The equation which gives the values, over the finite-element mesh, of a nodewise value, may be expressed for the two-dimensional mesh as:

$$
h(x, y, t) \approx \sum_{j=1}^{N N} h_{j}(t) \phi_{j}(x, y)
$$

where the nodes have been numbered from one to NN (total number of nodes in the mesh). There are NN coefficients, $h_{j}(t)$, each of which is assigned the value of head at the coordinates $\left(x_{j}, y_{j}\right)$ of node number, $f$. These nodal head values may change with time to represent transient responses of the system. The function, $\phi_{j}(x, y)$, is known as the 'basis function'. It is the basis functions which spread values of head between the nodes when head is defined only at the nodal points by values of $h$. There is one basis function $\phi_{j}(x, y)$ defined for each node, $j$, of the NN nodes in the mesh. Suffice it to say, at this point, that at the node $f$, to which it belongs, the basis $\phi_{j}(x, y)$, has a value of one. At all other nodes 1 , $i \neq j$, in the mesh, it has a value of zero. It drops linearly in value from one to zero along each projected element edge to which the node $f$ is connected. This means that even when all the NN products of $h_{j}$ and $\phi_{j}(x, y)$ are summed (as in relation (3.4)), if the sum is evaluated at the coordinates $\left(x_{j}, y_{j}\right)$ of node $j$, then $h(x, y)$ exactly takes on the assigned value, $h_{j}$. This is because the basis function belonging to node $j$ has a value of one at node $j$, and all other basis functions belonging to other nodes, $1, i \neq j$, have a value zero at node $j$ dropping them from the summation in (3.4). Basis functions are described mathematically in section 4.1, "Basis and Weighting Functions." 


\section{Cellwise discretization}

The equation which gives the values, over the finite-element mesh, of a cellwise parameter may be expressed for the specific storativity of the present example as:

$$
s_{0}(x, y) \approx \sum_{i=1}^{N N} s_{i}(x, y)
$$

where $S_{i}(x, y)$ has the value of specific storativity of the cell centered on node $i$ for $(x, y)$ coordinates within the cell, and a value of zero outside the cell. Thus, $S_{i}(x, y)$ is a flat topped 'box' standing on a cell 1 in Figure 3.4 , and $S_{0}(x, y)$ is represented in a discrete approximate way by the sum of all the 'boxes'. Note $S_{1}(x, y)$ has the same value in the $z$-direction from the top to bottom of each two-dimensional element.

Reviewing the example problem, $K$ is assigned elementwise and both $S_{0}$ and $\frac{\partial h}{\partial t}$ are assigned cellwise. Hydrualic head, $h(x, y, t)$, and element thickness, $B(x, y, t)$, measured in the $z$-direction, are both discretized nodewise, with the nodewise expansion for thickness:

$$
B(x, y) \approx \sum_{i-1}^{N N} B_{i}(t) \phi_{i}(x, y)
$$

The values $B_{i}(t)$ are the $N N$ particular values which element thickness has at the nodes, and these values may change with time in the present water-table example. Relation (3.6) should call to mind a vision of discretized values of thickness represented by a surface similar to that of Figure 3.3 . The head surface of Figure 3.3 may stretch or shrink to move up or down as the head values at nodes, $h_{i}(t)$, change with time due to stresses on the aquifer system. The 
nodewise discretized surface may be viewed as the water table, and the element thickness as the thickness of the water-table aquifer.

\subsection{Integration of Governing Equation in Space}

\section{Approximate governing equation and weighted residuals method}

The governing equation for the water-table example may be written in operator form as:

$$
O(h)=S_{0} \frac{\partial h}{\partial t}-\underline{\nabla} \cdot(K \underline{\nabla})-Q^{*}=0
$$

Certain variables in this equation are approximated through elementwise and nodewise discretization. Particular terms of the equation are approximated through cellwise discretization. The result is that neither the derivatives, nor the variables are described exactly. Relation (3.7) no Ionger exactly equals zero:

$$
\widehat{O(h)}=R(x, y, t)
$$

\section{A}

where $O(h)$ is the result of approximating the terms of the equation and the variables, and $R(x, y, t)$ is the residual value of the approximated equation. When simulating a system with a numerical model based on approximation of the governing equation, $O(h)$, the residual, $R$, must be kept small everywhere in the simulated region and for the entire time of simulation in order to accurately reproduce the physical behavior predicted by the exact governing equation, (3.7).

In order to achieve a minimum error, a method of weighted residuals is applied to (3.8). The purpose of the method of weighted residuals is to minimize the error of approximation in particular sub-regions of the spatial domain 
to be simulated. This is done by forcing a weighted average of the residual to be zero over the sub-regions. This idea is the most abstract of those required to understand SUTRA methodology. The Galerkin method of weighted residuals chooses to use the 'basis function', $\phi_{i}(x, y)$, mentioned in the previous section, as the weighting function for calculation of the average residual:

$$
\begin{aligned}
\hat{O}_{V}(\mathrm{~h}) \phi_{i}(x, y) d V & =\int_{V} R(x, y, t) \phi_{i}(x, y) d V=0
\end{aligned}
$$

where $\mathrm{V}$ is the volume of the region to be modeled. The model volume is completely filled by a single layer of quadrilateral finite elements. Relation (3.9) is actually NN relations, one for each of $\mathrm{NN}$ nodes in the finite element mesh as indicated by the notation, $i=\overline{1, N N}$.

In each relation, the integral sums the residual weighted by the basis function over a volume of space. Each integrated weighted residual is forced to zero over the region of space in which $\phi_{1}(x, y)$ is non-zero. This region includes only elements which contain node $i$, because of the manner in wich the basis function is defined, as described earlier. Thus, over each of these NN sub-regions of a mesh, the sum of positive and negative residuals after weighting is forced to zero by relation (3.9). This, in effect, minimizes the average error in approximating the governing equation over each sub-region.

After stating that the integral of weighted residuals must be zero for each sub-region of the mesh as in (3.9), the derivation of the numerical methods becomes primarily a job of algebraic manipulation. The process is $\wedge$ begun by substitution of the governing equation for $O(h)$ in (3.9): 


$$
\begin{gathered}
\int_{V}\left(s_{0} \frac{\partial h}{\partial t}\right) \phi_{i}(x, y) d V-\int_{V}(\underline{\nabla} \cdot \hat{(K \nabla h)}) \phi_{i}(x, y) d V \\
\\
-\int_{V}\left(\hat{Q^{*}}\right) \phi_{i}(x, y) d V=0 \\
i=\overline{1, N N}
\end{gathered}
$$

The terms in large parentheses topped by a carat are the approximate discrete forms of the respective terms in (3.7). These are expanded in the manipulations that follow. Relation (3.10) is discussed term by term in the following paragraphs.

\section{Cellwise integration of time-dertvative term}

The first term involving the volume integral of the time derivative may be written in terms of the three space dimensions, $x, y$, and $z$. Although the governing equation and parameters vary only in two space dimensions, they apply to the complete three-dimensional region to be modeled.

$$
\begin{aligned}
\int_{V}\left(s_{0} \frac{\partial h}{\partial t}\right) \phi_{1}(x, y) d V & =\iint_{z} \int_{y}\left(s_{0} \hat{\frac{\partial h}{\partial t}}\right) \phi_{i}(x, y) d z d y d x \\
& =\iint_{y}\left(s \hat{\frac{\partial h}{\partial t}}\right) \phi_{i}(x, y)\left[\int_{z} d z\right] d x d y
\end{aligned}
$$

The rearrangement in the final term of (3.11) is possible because no parameter depends on $z$. In fact, referring to (3.2), the aquifer thickness, $B(x, y, t)$, 
may be defined as:

$$
B(x, y, t)=\int_{z(t)} d z=h(x, y, t)-\operatorname{BASE}(x, y)
$$

The final term of (3.11) is then:

$$
\iint_{y}\left(s_{0} \hat{\frac{\partial h}{\partial t}}\right) \phi_{i}(x, y) B(x, y, t) d x d y
$$

Now cellwise discretization is chosen for $S_{0}$ and for $\frac{\partial h}{\partial t}$, making these terms take on a constant value for the region of each cell $i$. The region of cell 1 is the same region over which $S_{1}(x, y)$ is non-zero. Then, for any cell 1 , term (3.13) becomes:

$$
s_{i} \frac{\partial h}{\partial t} i \int_{y} \int_{x} \phi_{i}(x, y) B(x, y, t) d x d y
$$

where $s_{1}$ and $\frac{\partial h}{\partial t} 1$ are the values taken by $s_{0}$ and $\frac{\partial h}{\partial t}$ in cell 1 .

It can be shown that the volume of cell $i$, denoted by $v_{i}(t)$, is, in fact, the integral in (3.14):

$$
v_{i}(t)=\iint_{y} \phi_{i}(x, y) B(x, y, t) d x d y
$$

For a particular finite-element mesh, the volume $v_{1}(t)$ of each cell is determined by numericai integration of (3.15). Numerical integration by Gaussian quadrature is discussed in section (4.3), "Gaussian Integration." 
Given the value of the specific storativity of each cell, $S_{i}$, the time derivative of head in each cell, $\frac{\partial h}{\partial t} i$, and given the volume of each cell, $v_{i}(t)$, determined numerically, the first term of the weighted residual statement takes on its discrete approximation in space:

$$
\int_{V}\left(s_{0} \frac{\partial h}{\partial t}\right) \phi_{i}(x, y) d V=s_{i} \frac{\partial h}{\partial t} i v_{i}(t)
$$

Elementwise integration of flux term and origin of boundary fluxes

Manipulation of the second integral in (3.10) begins with the application of Green's theorem which is an expanded form of the divergence theorem. This converts the integral into two terms, one of which is evaluated only at the surface of the region to be simulated. Green's theorem is:

$$
\int_{V}(\underline{\nabla} \cdot \underline{W}) A d V=\int_{\Gamma}(\underline{W} \cdot \underline{n}) A d \Gamma-\int_{V}(\underline{W} \cdot \underline{\nabla}) d V
$$

where $A$ is a scalar and $\underline{W}$ is a vector quantity. The boundary of volume $V$ is denoted by $\Gamma$ including both edges and upper and lower surfaces of the aquifer, and $\underline{n}$ is a unit outward normal vector to the boundary. Application of (3.17) to the second term in (3.10) results in:

$$
\begin{aligned}
& -\int_{V}[\underline{\nabla} \cdot(\hat{\mathrm{K} \nabla h})] \phi_{i}(\mathrm{x}, \mathrm{y}) \mathrm{dV}=-\int[(\hat{\mathrm{K} \nabla \mathrm{h}}) \cdot \underline{\mathrm{n}}] \phi_{i} \mathrm{~d} \Gamma \\
& +\int_{V}(\hat{\underline{\nabla}} \hat{h}) \cdot \underline{\nabla} \phi_{i} d V
\end{aligned}
$$


The first term on the right of (3.18) contains a fluid flux given by Darcy's law:

$$
\varepsilon \mathrm{v}_{\text {OUT }}=-\underline{K h} \cdot \underline{\mathbf{n}}
$$

where vOUT is the outward velocity at the boundary normal to the bounding surface. Thus the integral gives the total flow out across the bounding surface, Q QUVT 1 , in the vicinity of a node 1 on the surface:

$$
Q_{\text {OUT }_{1}}=\int_{\Gamma}\left(\varepsilon v_{\text {OUT }} \phi_{1}\right) d \Gamma
$$

An inflow would have a negative value of QouT $_{1}$, and the relation between an inflow, $Q_{I N_{1}}$, and outflow is: $Q_{I N_{1}}=-Q_{O U T_{1}}$. Thus, the first Integral on the right of (3.18) represents flows across boundaries of the water-table aquifer model.

The second integral on the right of (3.18) may be expressed in three spatial coordinates.

$$
\begin{aligned}
& \int_{V}\left(\hat{K} \underline{\nabla_{h}}\right) \cdot \underline{\nabla}_{1} d V=\iint_{x} \int_{y}\left(\hat{K} \underline{\nabla h}_{z}\right) \cdot \underline{\nabla}_{1} d z d y d x \\
& =\iint_{x}(\hat{\underline{\nabla} \underline{h}}) \cdot \underline{\nabla}_{1}\left[\int_{z} d z\right] d y d x=\iint_{x}(\hat{\underline{\nabla} \underline{h}}) \cdot \underline{\nabla}_{i} B(x, y, t) d y d x
\end{aligned}
$$

No term varies in the $z$-direction, allowing the use of (3.12) which defines aquifer thickness B. Notice that the transmissivity as given by (3.2), $T=\mathrm{KB}$ appears in the form of the integral just obtained. 
Now the approximation for the term $\widehat{K} \underline{\mathrm{V} h}$ is substituted into the integral. Hydraulic head, $h(x, y, t)$, is approximated in a nodewise manner as given by relation (3.4). The integral of (3.2) becomes:

$$
\begin{aligned}
& \iint_{x}(\hat{K} \underline{\nabla} \hat{h}) \cdot \nabla \phi_{i} B d y d x=\iint_{x}\left[\hat{K} \underline{\nabla} \sum_{j=1}^{N N} h_{j}(t) \phi_{j}(x, y)\right] \cdot \underline{\nabla}_{i} B d y d x \\
= & \sum_{j=1}^{N N} h_{j}(t) \iint_{x} \hat{k}\left(\underline{\nabla}_{j} \cdot \underline{\nabla}_{i}\right) B d y d x=\sum_{j=1}^{N N} h_{j}(t) I_{i j}(t)
\end{aligned}
$$

where $\hat{K}$ is the elementwise approximation for $K(x, y)$. The summation and $h_{j}(t)$ may be factored out of the integral because $h_{j}$ is a value of head at a node and does not vary with $x$ and $y$ location. The integral is represented by $I_{i j}(t)$ and depends on time because aquifer thickness, $B$, is time-dependent. For each node $i$, there are apparently $j=N N$ integrals which need to be evaluated. In fact, due to the way in which basis functions are defined, there are only a few which are non-zero, because $\left(\underline{\nabla} \phi_{j} \cdot \underline{\nabla} \phi_{i}\right)$ is non-zero only when nodes $i$ and $j$ are in the same finite element. When nodes $i$ and $j$ are in different elements, then $\nabla_{\phi} j$ is zero in the element containing node 1 .

The integrals are evaluated numerically by Gaussian integration. This is accomplished by first breaking up the integral over the whole volume to be simulated, into a sum of integrals, one each over every finite element in the mesh: 


$$
I_{i j}(t)=\iint_{x} \hat{K}\left(\underline{\nabla}_{j} \cdot \underline{\nabla}_{i}\right) B d y d x=\sum_{L=1}^{N E} \int_{x_{L} y_{L}} \hat{K}\left(\underline{\nabla}_{\phi_{j}} \cdot \underline{\nabla}_{i}\right) B d y d x
$$

There are NE elements in the mesh, $L$ is the element number, and $x_{L}$ and $y_{L}$ are the $x$ and $y$ spatial domains of element $L$. Thus, for a given $L$, the integral over $x_{L}$ and $y_{L}$ is integrated only over the area of element $L$.

Now the discrete elementwise approximation for hydraulic conductivity, as given by (3.3) allows one term for element $L$ in the summation of (3.23) to be written as:

$$
K_{L} \int_{x_{L} y_{L}}\left(\nabla_{\phi_{j}} \cdot \nabla_{\phi_{i}}\right) B d y d x
$$

Here, the thickness $B$ is specified to vary nodewise. The formula for B in this example is obtained by substituting the nodewise expression for head, (3.4), into the definition of $B$, relation (3.2).

The integral over one element, as given by term (3.24), must be evaluated numerically. In order to do this, the coordinates of the element $L$, which has an arbitrary quadrilateral shape as suggested in Figure 3.3 , is transformed to a new coordinate system in which the element is a two-by-two square. Then, Gaussian integration is carried out to evaluate the integral. For a given combination of nodes $i$ and $j$, this transformation and numerical integration $i s$ carried out for all elements in the mesh in which both nodes $i$ and $f$ appear. (There are $16 i-j$ combinations evaluated in each quadrilateral element.) The elementwise pieces of the Integral for each $1-j$ combination are then summed according to (3.23) in order to obtain the value of the integral over the whole 
region. The summation is called the 'assembly' process. This element transformation, integration of the 16 integrals arising in each element, and summation, makes up a large part of the computational effort of a finite-element model and also requires the most complex algorithm in a finite-element model. It is in this way that the second term of (3.10) is evaluated. More information on finiteelement integration and assembly may be found in numerical methods texts such as Wang and Anderson (1982), Pinder and Gray (1977), or Huyakorn and Pinder (1983). The details of this method as applied in SUTRA are given in Chapter 4, "Numerical Methods."

\section{Cellwise Integration of source term}

The last term of (3.10) deals with sources of fluid to the aquifer such as injection wells. The volume integral may, as before, may be written in $x, y$, and $z$ coordinates:

$$
\begin{aligned}
& -\int_{V} Q^{*}(x, y) \phi_{i}(x, y) d V=-\iint_{x} \int_{y}^{*} \phi_{i} d z d y d x \\
& =-\iint_{x} Q^{*} \phi_{i} B(x, y, t) d y d x
\end{aligned}
$$

where thickness $B$ is introduced because $Q^{*}$ and $\phi_{1}$ do not vary with $z$. It Is assumed that all fluid entering the aquifer within the region of cell 1 , which surrounds node $i$, enters at node $i$. If $Q_{i}^{*}\left[L^{3} / s\right]$ is defined as the volume of fluid entering cell $i$ per unit time, then $Q^{*}\left[s^{-1}\right]$, which 1 s the volume of fluid entering the aquifer per unit volume aquifer per unit time, is given as: 


$$
Q^{*}(x, y)=\sum_{i=1}^{N N}\left(\frac{Q^{*}}{v_{i}}\right)
$$

This is a cellwise discretization for the source term, $Q^{*}$. For cell 1 :

$$
-\iint_{x} Q^{*} \phi_{1} B d y d x=-\left(\frac{Q_{1}^{*}}{V_{1}}\right) \int_{x} \int_{y} \phi_{1} B d y d x=-Q_{1}^{*}
$$

Thus all recharges within cell $i$ due to areal infiltration, well injection or other types are allocated to the source at node $i$.

This completes the spatial integration of the governing equation for the example problem.

\subsection{Time Discretization of Governing Equation}

When the integrated terms of the governing equation are substituted in (3.10) the following results:

$$
\begin{gathered}
S_{i} v_{i}(t) \frac{d h}{d t} i+\sum_{j=1}^{N N} I_{i j}(t) h_{j}(t)=Q_{I N}+Q_{i}^{*} \\
i=\overline{1, N N}
\end{gathered}
$$

These are NN integrated weighted residual approximations of the governing differential equation, one at each node 1 in the mesh. Because of the summation term in (3.28), the integrated approximate equation for a node, 1 , may involve the values of head, $h_{j}(t)$, at all other nodes in the mesh. The other terms in (3.28) involve only values at node $i$ itself, at which the entire relation is evaluated. 
All the parameters in (3.28) are no longer functions of the space coordinates. Each parameter takes on a particular value at each node in the mesh. Some of these values vary with time and a particular time for evaluation of these values needs to be specified. Also, the time derivative requires discretization.

\section{Time steps}

Time is broken up into a series of discrete steps, or time steps. The length of a time step, $\Delta t$, is the difference in time between two discrete times, at the beginning and end of a time step:

$$
\Delta t_{n+1}=t^{n+1}-t^{n}
$$

where $\Delta t_{n+1}$ is the length of the $(n+1)^{\text {th }}$ time step, $t^{n}$ is the actual time at the beginning of the $(n+1)^{\text {th }}$ time step and $t^{n+1}$ is the actual time at the end of this time step. The time steps are chosen to discretize the time domain before a simulation just as a mesh (or 'spatial steps') is chosen to discretize space. The time step length may vary from step to step.

The entire spatially integrated governing equation, (3.28), is evaluated at the end of each time step, $t=t^{n+1}$. The time derivative of head in (2.28) is approximated, using a finite-difference approximation, as the change in head over a time step, divided by the time step length:

$$
\frac{d h}{d t} i \simeq \frac{h_{i}\left(t^{n}+\Delta t_{n+1}\right)-h_{i}\left(t^{n}\right)}{\Delta t_{n+1}}
$$

In order to simplify the notation, the head at the end of the time step, 
$h_{i}\left(t^{n}+\Delta t_{n+1}\right)$ is denoted $h_{i}^{n+1}$, and the head at the beginning of the time step $h_{i}\left(t^{n}\right)$ is denoted $h_{i}^{n}$. Thus,

$$
\frac{d h}{d t} i=\frac{h_{i}^{n+1}-h_{i}^{n}}{\Delta t}
$$

The parameters that depend on time in (3.28), $v_{i}(t), I_{i j}(t)$ and $h_{j}(t)$, are also evaluated at the time, $t^{n+1}$, at the end of a time step:

$$
\begin{aligned}
& \left.h_{j}(t)\right|_{t^{n+1}}=h_{j}^{n+1} \\
& \left.v_{i}(t)\right|_{t^{n+1}}=v_{i}^{n+1} \\
& \left.I_{i j}(t)\right|_{t^{n+1}}=I_{i j}^{n+1}
\end{aligned}
$$

The sources, $Q_{I N_{i}}$, and $Q_{i}^{*}$, are assumed constant in time for present example.

\section{Resolution of non-linearities}

The variability in time of cell volume, $v_{i}$, and the integral, $I_{i j}$, depends on the changlng thickness of the aquifer with time, $B(x, y, t)$. The aquifer thickness at node 1 at the end of a time step, $B_{1}{ }^{n+1}$, is not known until the head at the end of the time step is known giving the water-table elevation. This typifies a non-linear problem wherein the problem requires values of coefficients in order to be solved, but the values of these coefficients depend on the, as yet unobtained solution. This circular problem is avolded in this example by using estimates of the coefficient values in the solution. An estimate of the head at the end of the next time step is obtained by a linear projection:

$$
h_{i}^{p r o j}=h_{i}^{n}+\left(\frac{\Delta t}{\Delta t_{n}}\right)\left(h_{i}^{n}-h_{i}^{n-1}\right)
$$


where $h_{i}^{\text {proj }}$ is the projected or estimated head at the end of the, as yet unsolved time step, which would have an exact value, $h_{1}^{n+1}$. Actually, in addition to projection, SUTRA also employs a simple iterative process to resolve nonlinearities. This is described in sections 4.4 and 4.5 under the sub-heading "Temporal discretization and iteration."

A projected thickness may then be determined from (3.33) as:

$$
B_{i}^{n+1} \simeq B_{i}^{p r o j}=h_{i}^{p r o j}-\text { BASE }_{i}
$$

where $B_{i}^{n+1}$ is the value of thickness needed to evaluate $V_{i}^{n+1}$ and $I_{i j}^{n+1}, B_{i}^{p r o j}$ is the estimated value of $B_{i}^{n+1}$, and $\operatorname{BASE}_{i}$ is the value of $\operatorname{BASE}(x, y)$ at node $i$.

Now the spatially integrated equation, (3.28), may be written discretely in time:

$$
\begin{aligned}
S_{i} v_{i}^{n+1}\left(\frac{h_{i}^{n+1}-h_{i}^{n}}{\Delta t_{n+1}}\right)+\sum_{j=1}^{N N} I_{i j}^{n+1} h_{j}^{n+1}=Q_{I N}+Q_{i}^{*} \\
i=\overline{1, N N}
\end{aligned}
$$

where $V_{i}^{n+1}$ and $I_{i j}^{n+1}$ are evaluated based on projected thickness, $B_{i}^{p r o j}$.

\subsection{Boundary Conditions and Solution of Discretized Equation}

\section{Matrix equation and solution sequence}

The NN relations given by (3.35) may be rearranged and rewritten in matrix form: 
$\left(\frac{1}{\Delta t_{n+1}}\right)\left[\begin{array}{ccccc}s_{1} v_{1}^{n+1} & 0 & 0 & \cdots & 0 \\ 0 & s_{2} v_{2}^{n+1} & 0 & \cdots & 0 \\ 0 & 0 & s_{3} v_{3}^{n+1} & 0 \\ \vdots & \vdots & \vdots & \ddots & \vdots \\ 0 & 0 & 0 & \cdot s_{N N} v_{N N}^{n+1}\end{array}\right]\left\{\begin{array}{c}h_{1}^{n+1} \\ h_{2}^{n+1} \\ h_{3}^{n+1} \\ \vdots \\ h_{N N}^{n+1}\end{array}\right\}$

$+\left[\begin{array}{ccccccccc}I_{11}^{n+1} & I_{12}^{n+1} & I_{13}^{n+1} & I_{14}^{n+1} & \cdot & \cdot & \cdot & \cdot & I_{1, N N}^{n+1} \\ I_{21}^{n+1} & I_{22}^{n+1} & I_{23}^{n+1} & \cdot & \cdot & \cdot & \cdot & \cdot & \cdot \\ I_{31}^{n+1} & I_{32}^{n+1} & I_{33}^{n+1} & \cdot & \cdot & \cdot & \cdot & \cdot & \cdot \\ I_{41}^{n+1} & \cdot & \cdot & \cdot & \cdot & \cdot & \cdot & \cdot & \cdot \\ \cdot & \cdot & \cdot & \cdot & \cdot & \cdot & \cdot & \cdot & \cdot \\ \cdot & \cdot & \cdot & \cdot & \cdot & \cdot & \cdot & \cdot & \cdot \\ I_{N N}^{n+1} & \cdot & \cdot & \cdot & \cdot & \cdot & \cdot & \cdot & I_{N N, N N}^{n+1}\end{array}\right]\left\{\begin{array}{l}h_{1}^{n+1} \\ h_{2}^{n+1} \\ h_{3}^{n+1} \\ \cdot \\ h_{N N}^{n+1}\end{array}\right)$

$=\left(\frac{1}{\Delta t_{n+1}}\right)\left\{\begin{array}{ccc}s_{1} & v_{1}^{n+1} & h_{1}^{n} \\ s_{2} & v_{2}^{n+1} & h_{2}^{n} \\ s_{3} & v_{3}^{n+1} & h_{3}^{n} \\ \vdots & & \\ \vdots & & \\ s_{N N} & v_{N N}^{n+1} & h_{N N}^{n}\end{array}\right\}+\left\{\begin{array}{c}Q_{I_{1}} \\ Q_{I N_{2}} \\ Q_{I_{3}} \\ \vdots \\ Q_{I N_{N N}}\end{array}\right\}+\left\{\begin{array}{c}Q_{1}^{*} \\ Q_{2}^{*} \\ Q_{3}^{*} \\ \vdots \\ \vdots \\ Q_{N N}^{*}\end{array}\right\}$

By adding the two matrices on the left side, and the vectors on the right side, a matrix equation is obtained which may be solved for the model heads at the new time level, $t^{n+1}$, on each time step: 


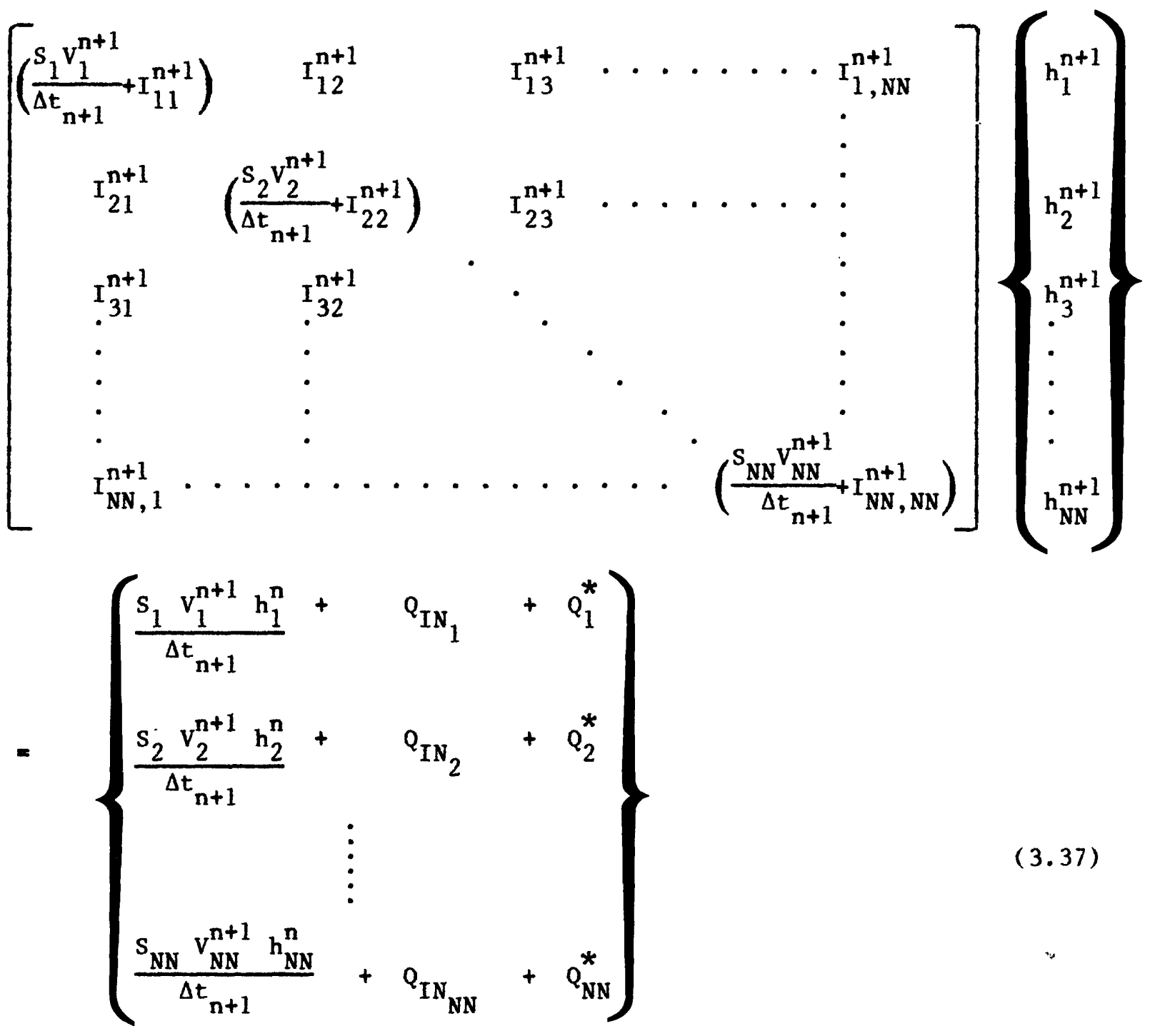

The solution progresses through time as follows: On a given time step, the nodal heads at the beginning of the step are known values and are placed in $h_{j}^{n}$ on the right hand side vector of (3.37). The thickness-dependent values are determined based on the projection of $B$ in (3.34) using projected head of (3.33). The integrals and volumes are evaluated and the matrix and vector completed. The nodal heads at the end of the current time step are solved for by Gaussian elimination for the (banded) matrix on the left of (3.37). The new heads are then placed on the right side of (3.37) into $h^{n}$, and a new time step is begun. 
Before solving the matrix equation as described above, information about boundary conditions must be included. In the case of solving for heads, the boundary conditions take the form of either specified fluid fluxes across boundaries which are directly entered in the terms, $Q_{1 N_{i}}$, or of particular head values specified at nodal locations. At a point of fixed head in an aquifer, a particular value of fluid inflow or outflow occurs at that point in order to keep the head constant when the aquifer is stressed. It is this flux of fluid which is added to the model aquifer in order to obtain fixed heads at nodes.

Consider the closed system of Figure 3.5 in which head at node $i, h_{1}$, is to have a specified value, $h_{B C}$, for all time. A well is removing water from the system at an internal node. A core of porous medium with conductance $\nu$ is connected to node 1 . The head outside the core is held at the specified value, $h_{B C}$. The head at node $1, h_{i}$, is calculated by the model. A flow of $Q_{B C}$ $\left[\mathrm{L}^{3} / \mathrm{s}\right]$ enters through the core at node $i$ in order to balance the rate of fluid removal at the well. The resulting head at node 1 depends on the conductance value $v$ of the core. If $v$ is very small, then a large head drop is required across the core in order to supply fluid at the rate the pumping well requires. This results in $h_{i}$ having quite a different value from $h_{B C}$. If, however, $v$ is very large, then the value of head at node 1 , is very close to $h_{B C}$, as only a minute head drop across the core supplies the fluid required by the well. Therefore, by applying flux to a node through a highly conductive core, the outside of which is held at a specified head value, the node responds with a head value nearly equal to that spectfied. An advantage of specifying head this way 


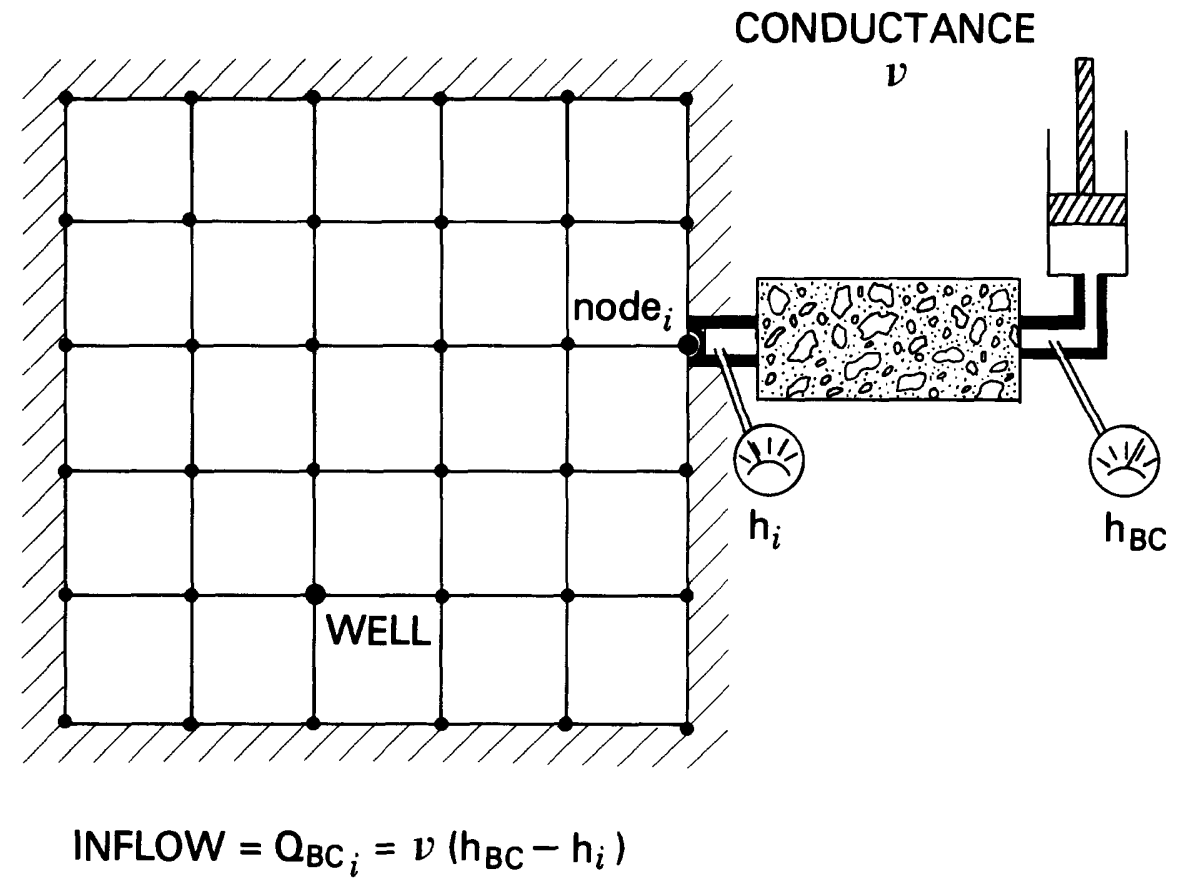

Figure 3.5

Schematic representation of specified head (or pressure) boundary condition. 
is that when head at a node in the mesh is fixed, a calculation of the flux entering the mesh at this node is obtained at the same time.

This flux is defined as follows:

$$
Q_{B C}=v\left(h_{B C}-h_{i}^{n+1}\right)
$$

where $Q_{B C}$ is the inflow at node $i$ resulting from the specified head boundary condition, $v$ is the conductance of the 'core', and $h_{B C}$ is the specified value of head at node $i$ on the boundary.

The matrix equation ( 3.37 ) may be written in short form as:

$$
\begin{gathered}
\sum_{j=1}^{N N} M_{i j}^{n+1} h_{j}^{n+1}=\left(\frac{S_{i} v_{i}^{n+1}}{\Delta t_{n+1}}\right) h_{i}^{n}+Q_{i}^{*}+Q_{I N_{1}}+Q_{B C_{i}} \\
i=\overline{1, N N}
\end{gathered}
$$

wherein an additional flux $Q_{B C}$ has been added to account for specified head nodes. At such a node, say node $A$, the equation is:

$$
\sum_{j=1}^{N N} M_{A j}^{n+1} h_{j}^{n+1}=\left(\frac{S_{A} v_{A}^{n+1}}{\Delta t_{n+1}}\right) h_{A}^{n}+Q_{A}^{*}+Q_{I N}+v\left(h_{B C_{A}}-h_{A}^{n+1}\right)
$$

where $v$ is very large, then the last term dominates the equation and $(3.40)$ becomes:

$$
\mathrm{h}_{\mathrm{A}}^{\mathrm{n}+1} \simeq \mathrm{h}_{\mathrm{BC}}
$$

Thus the specified head is set at node $A$, but as $h_{A}^{n+1}$ and $h_{B C}$ are slightly different, a flux may be determined from (3.38). 
DETAILS OF

SUTRA

METHODOLOGY

93

(p. 95 follows) 


\section{Chapter 4}

\section{Numerical Methods}

In this section, the numerical methods upon which SUTRA is based are presented in detail. The purpose of this presentation is to provide a complete reference for the computer code.

\subsection{Basis and Weighting Functions}

Basis functions, weighting functions and their derivatives are all described in local element geometry. In a local coordinate system, every element takes the shape of a two by two square. The local coordinates, $\xi$ and $\eta$, are shown along with a generic local finite element in Figure 4.1. The origin of the local coordinate system is at the center of the element. Local node one always has local coordinates $(\xi, \eta)=(-1,-1)$. The other nodes are numbered counterclockwise from the first node as shown in Figure 4.1.

The following one-dimensional basis functions are defined over the region of the element:

$$
\begin{aligned}
& \Xi_{-}(\xi)=\frac{1}{2}(1-\xi) \\
& \Xi_{+}(\xi)=\frac{1}{2}(1+\xi) \\
& H_{-}(n)=\frac{1}{2}(1-n) \\
& H_{+}(n)=\frac{1}{2}(1+n)
\end{aligned}
$$

These linear one-dimensional basis functions are continuous in $\xi$ and $\eta$ and have efther a value of zero or one depending on whether $\xi$ or $\eta$ have a value of +1 or -1 . The one-dimensional functions are combined to create the bilinear basis functions used in SUTRA: 


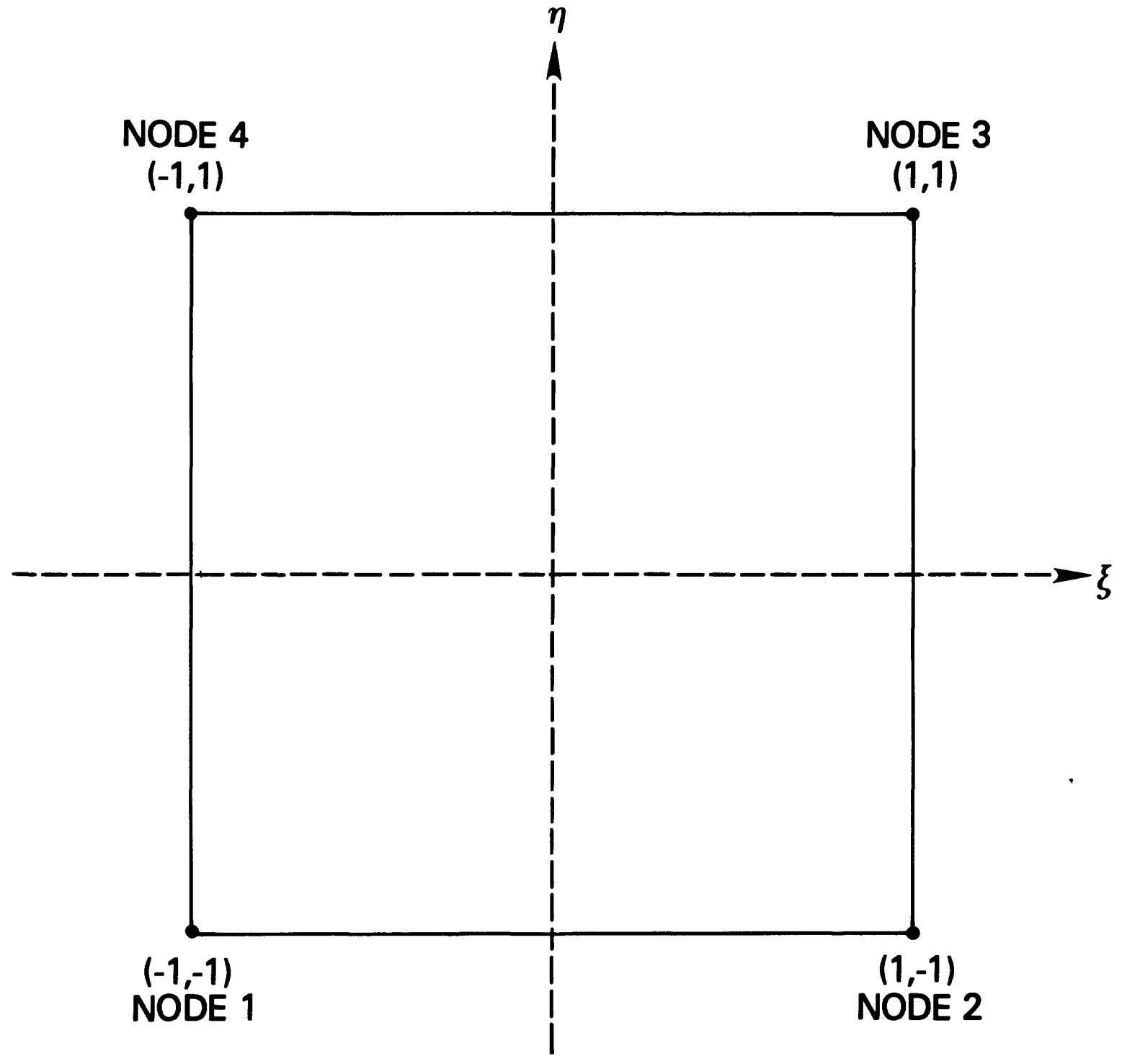

F1gure 4.1

Quadrilateral finite element in local coordinate system $(\xi, n)$. 


$$
\begin{aligned}
& \Omega_{1}(\xi, n)=\Xi_{-} H_{-} \\
& \Omega_{2}(\xi, n)=\Xi_{+} H_{-} \\
& \Omega_{3}(\xi, n)=E_{+} H_{+} \\
& \Omega_{4}(\xi, n)=E_{-} H_{+}
\end{aligned}
$$

The two-dimensional bi-linear basis functions, when defined in the local element coordinate system are denoted as $\Omega_{1}(\xi, n), i=1,2,3,4$. There is one basis function defined for each node.

The basis function $\Omega_{i}$, defined for node $i$, has a value of one at the node and $a$ value of zero at the other nodes. The surface representing $\Omega_{1}(\xi, n)$ over an element is curved due to the product of $\xi$ and $n$ in equations (4.5) through (4.8). A trajectory in the surface parallel to an element side, however, is a perfectly straight line as shown in Figure 4.2. This is born out in the derivatives of the bi-linear basis functions which depend on only one space coordinate:

$$
\begin{array}{llll}
\frac{\partial \Omega}{\partial \xi} 1 & =-\frac{1}{2} H_{-} & \frac{\partial \Omega}{\partial n} 1 & =-\frac{1}{2} \Xi_{-} \\
\frac{\partial \Omega}{\partial \xi} 2 & =+\frac{1}{2} H_{-} & \frac{\partial \Omega}{\partial n} 2=-\frac{1}{2} \Xi_{+} \\
\frac{\partial \Omega}{\partial \xi} 3=+\frac{1}{2} H_{+} & \frac{\partial \Omega}{\partial n} 3=+\frac{1}{2} \Xi_{+} \\
\frac{\partial \Omega}{\partial \xi} 4=-\frac{1}{2} H_{+} & \frac{\partial \Omega}{\partial n} 4=+\frac{1}{2} \Xi_{-}
\end{array}
$$

Asymmetric welghting functions are defined for use in a Galerkin-Petrov method (one version of which is described in Huyakorn and Pinder, 1983). These are not applied for nodewise discretization of parameters, but rather for weighting in the volume integrals of the governing equation. They may be used 

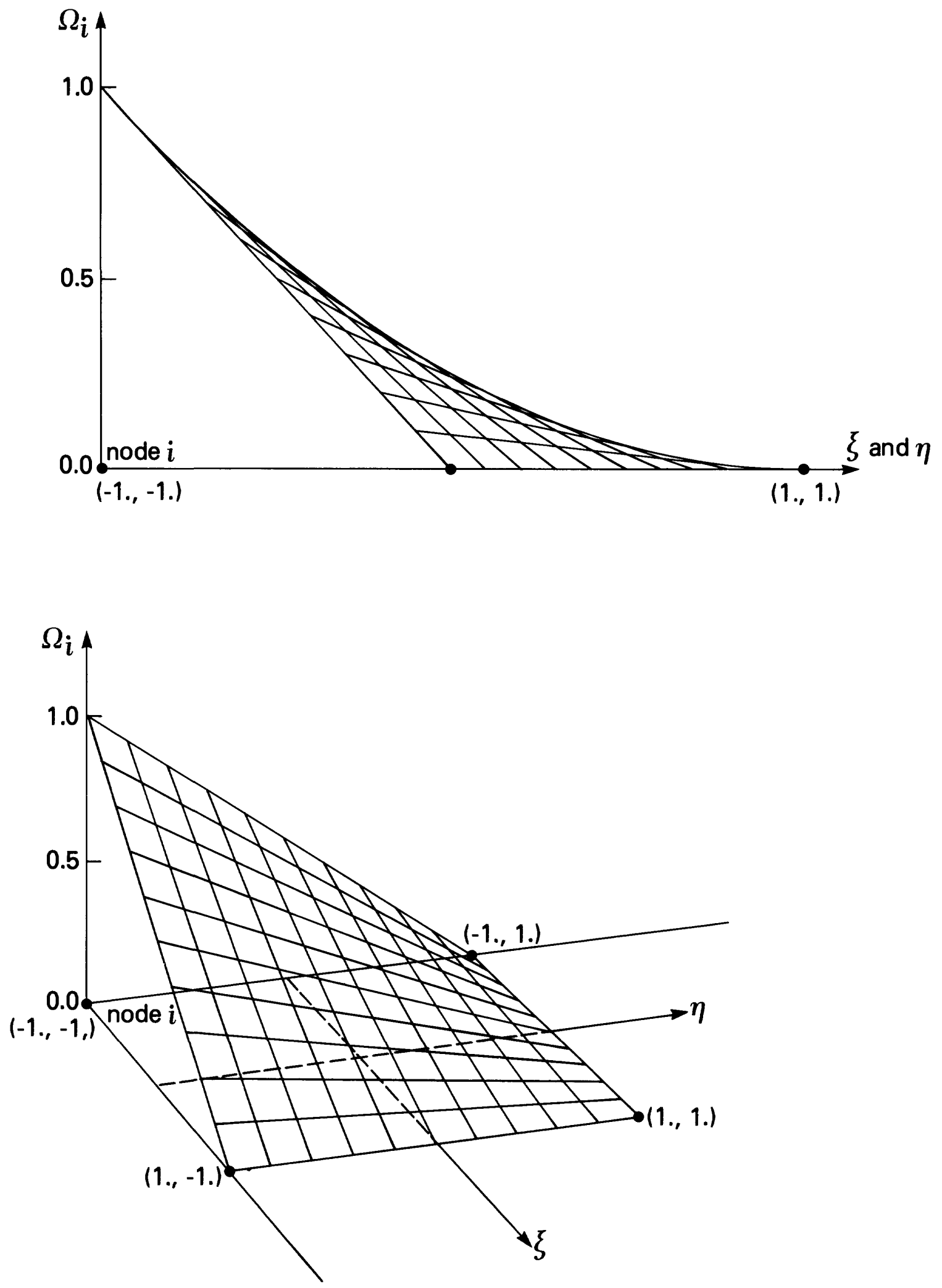

Figure 4.2

Perspectives of basis function $\Omega_{1}(\xi, n)$ at node 1 . 
to give an 'upstream weighting' to the advective flux term in the transport equations or to provide 'upstream weighting' to the fluid flux term in the fluid mass balance when the medium is unsaturated. The asymmetric functions are defined as follows:

$$
\begin{aligned}
& \Theta_{1}(\xi, \eta)=\left(\Xi_{-}-\Xi^{*}\right)\left(H_{-}-H^{*}\right) \\
& \Theta_{2}(\xi, n)=\left(\Xi_{+}+\Xi^{*}\right)\left(H_{-}-H^{*}\right) \\
& \theta_{3}(\xi, n)=\left(E_{+}+\Xi^{*}\right)\left(H_{+}+H^{*}\right) \\
& \Theta_{4}(\xi, n)=\left(\Xi_{-}-\Xi^{*}\right)\left(H_{+}+H^{*}\right)
\end{aligned}
$$

where:

$$
\begin{aligned}
& \Xi^{*}=3 a_{\xi} \Xi_{-} \Xi_{+} \\
& H^{*}=3 a_{\eta} \Xi_{-} \Xi_{+}
\end{aligned}
$$

The spatial derivatives are:

$$
\begin{array}{ll}
\frac{\partial \theta}{\partial \xi} 1=-\frac{1}{2}\left(1-3 a_{\xi} \xi\right)\left(H_{-}-H^{*}\right) & \frac{\partial \theta}{\partial \eta}=-\frac{1}{2}\left(1-3 a_{\eta} \eta\right)\left(\Xi_{-}-\Xi^{*}\right) \\
\frac{\partial \theta}{\partial \varepsilon} 2=+\frac{1}{2}\left(1-3 a_{\xi} \xi\right)\left(H_{-}-H^{*}\right) & \frac{\partial \theta}{\partial \eta} 2=-\frac{1}{2}\left(1-3 a_{\eta} \eta\right)\left(\Xi_{+}+\Xi^{*}\right) \\
\frac{\partial \theta}{\partial \xi} 3=+\frac{1}{2}\left(1-3 a_{\xi} \xi\right)\left(H_{+}+H^{*}\right) & \frac{\partial \Theta}{\partial \eta} 3=+\frac{1}{2}\left(1-3 a_{\eta} \eta\right)\left(\Xi_{+}+\Xi^{*}\right) \\
\frac{\partial \Theta}{\partial \xi} 4=-\frac{1}{2}\left(1-3 a_{\xi^{\xi}}\right)\left(H_{+}+H^{*}\right) & \frac{\partial \Theta}{\partial \eta} 4=+\frac{1}{2}\left(1-3 a_{\eta} \eta\right)\left(\Xi_{-}-\Xi^{*}\right)
\end{array}
$$

99 
The parameters $a_{\xi}$ and $a_{\eta}$ determine the amount of asymmetry (or upstream weight) in each coordinate direction. When these parameters have a value of zero, then the basis functions and their derivatives, equivalent to (4.5) through (4.12) are exactly obtained from (4.13) through (4.22). The values of ${ }^{a}{ }_{\xi}$ and $a_{\eta}$ depend on location in the element:

$$
\begin{aligned}
& { }_{a_{\xi}}(\xi, \eta)=\text { (UP) }\left(\frac{v_{\xi}}{\left|\underline{v}_{\text {local }}\right|}\right) \\
& a_{n}(\xi, \eta)=\text { (UP) }\left(\frac{v_{\eta}}{\left|\underline{v}_{l o c a l}\right|}\right)
\end{aligned}
$$

where UP is the fractional strength of upstream weighting desired (chosen by the model user), $v_{\xi}(\xi, n)$ and $v_{\eta}(\xi, n)$ are the components of fluid velocity given in terms of local element coordinates, and $\left|\underline{v}_{\text {local }}(\xi, n)\right|$ is the magnitude of fluid velocity given in terms of local coordinates. Each velocity component may vary in value throughout the element. A description of the calculation of fluid velocity is given in section 4.6, "Consistent Evaluation of Fluid Velocity."

Note that the basis functions, weighting functions and their derivatives are calculated by the SUTRA subroutine 'BASIS2'.

\subsection{Coordinate Transformations}

During calculations for the finite-element mesh and during integral evaluations, transformations are required between the global $(x, y)$ coordinate system in which an element may have an arbitrary size and quadrilateral shape, and the local $(\xi, \eta)$ coordinate system in which each element is a two by two square. Transformations are required in both directions. The transformation 
involves a linear remapping in each coordinate direction and employs the basis functions to provide mapping. The Jacobian matrix [J] is calculated separately for each element that requires transformation and may vary from point to point in an element.

$$
[\mathrm{J}]=\left[\begin{array}{cccc}
\frac{\partial \Omega}{\partial \xi} 1 & \frac{\partial \Omega}{\partial \xi} 2 & \frac{\partial \Omega}{\partial \xi} 3 & \frac{\partial \Omega}{\partial \xi} 4 \\
\frac{\partial \Omega}{\partial \eta} 1 & \frac{\partial \Omega}{\partial \eta} 2 & \frac{\partial \Omega}{\partial \eta} 3 & \frac{\partial \Omega}{\partial \eta} 4
\end{array}\right]\left[\begin{array}{cc}
\mathrm{x}_{1} & \mathrm{y}_{1} \\
\mathrm{x}_{2} & \mathrm{y}_{2} \\
\mathrm{x}_{3} & \mathrm{y}_{3} \\
\mathrm{x}_{4} & \mathrm{y}_{4}
\end{array}\right]
$$

The numbered subscripts refer to the local element numbering of Figure 4.1 .

The Jacobian matrix is used to transform derivatives of basis functions from the global to the local coordinate systems and the reverse:

$$
\begin{aligned}
& \left\{\begin{array}{c}
\frac{\partial \Omega}{\partial \xi} j \\
\frac{\partial \Omega}{\partial \eta} j
\end{array}\right\}=\left[\begin{array}{cc}
\frac{\partial x}{\partial \xi} & \frac{\partial y}{\partial \xi} \\
\frac{\partial x}{\partial \eta} & \frac{\partial y}{\partial \eta}
\end{array}\right]\left\{\begin{array}{l}
\frac{\partial \phi}{\partial x} \\
\frac{\partial \phi}{\partial y} j
\end{array}\right\} \\
& \left\{\begin{array}{l}
\frac{\partial \phi}{\partial x} j \\
\frac{\partial \phi}{\partial y}
\end{array}\right\}=\left[J^{-1}\right] \quad\left\{\begin{array}{l}
\frac{\partial \Omega}{\partial \xi} j \\
\frac{\partial \Omega}{\partial \eta} j
\end{array}\right\}
\end{aligned}
$$

where:

$$
[J]=\left[\begin{array}{ll}
\frac{\partial x}{\partial \xi} & \frac{\partial y}{\partial \xi} \\
\frac{\partial x}{\partial \eta} & \frac{\partial y}{\partial \eta}
\end{array}\right]
$$

The subscript $j$ refers to any one of the four nodes in an element and $\phi_{j}$ refers to the global basis function as defined for the $j^{\text {th }}$ node in an element. The same transformations apply to derivatives of the asymmetric weighting functions which are denoted $w_{j}$ in global coordinates. In $(4.27),\left[\mathrm{J}^{-1}\right]$ is the inverse Jacobian matrix defined as: 


$$
\left[J^{-1}\right]=\left(\frac{1}{\operatorname{det} J}\right)\left[\begin{array}{cc}
J_{22} & -J_{12} \\
-J_{21} & J_{11}
\end{array}\right]
$$

where $\operatorname{det} \mathrm{J}$ is the determinant of the Jacobian given by:

$$
\operatorname{det} \mathrm{J}=\mathrm{J}_{11} \mathrm{~J}_{22}-\mathrm{J}_{12} \mathrm{~J}_{21}
$$

The determinant may vary bi-linearly over an element.

Differential elements of area, dA, are transformed between local and global coordinate systems as:

$$
d A=d x d y=(\operatorname{det} J) d \xi d \eta
$$

Note that the Jacobian matrix, determinant of the Jacobian, and the derivatives of the basis functions in local and global coordinates are calculated in SUTRA subroutine, 'BASIS2'.

\subsection{Gaussian Integration}

Gaussian integration is a method by which exact integration of polynomials may be carried out through a simple summation of point values of the integrand. The method is:

$$
\int_{\tau=-1}^{\tau=+1} f(\tau) d \tau=\sum_{\mathrm{KG}=1}^{N P} G_{\mathrm{KG}} f\left(\tau_{\mathrm{KG}}\right)
$$

where $f(\tau)$ is the function to be integrated between $\tau=-1$ and $\tau=+1$. KG is the Gauss point number, NP is the total number of Gauss points, $G_{K G}$ is a constant, and $\tau_{K G}$ is the location of the KGth Gauss point. An exact integration is guaranteed by the sum in (4.32) If $(2 n-1)$ Gauss points are used for a polynomial $f(\tau)$ of order $n$. For evaluation of integrals which arise in the SUTRA methodology, only two Gauss points are used in a given coordinate direction as the integrals 
encountered are usually of order three or less. In this case, the constants, $G_{K G}$ have a value of one and $(4.32)$ simplifies to:

$$
\int_{\tau=-1}^{\tau=+1} f(\tau) d \tau=\sum_{K G=1}^{2} f\left(\tau_{K G}\right)
$$

The values of $\tau_{K G}$ for Gauss points one and two, are minus and plus 0.577350269189626 , (or $\pm 3^{-\frac{1}{2}}$ respectively).

The need to define a two by two element in local coordinates is apparent here. Gaussian integration is done over a range of two from -1 to +1 . In order to integrate a term of the differential governing equation over an arbitrary quadrilateral element in the mesh, the limits of the integral must first be transformed to values of -1 and +1 , that is, to local coordinates. When integrating a double integral, both integrals must be transformed to have limits of -1 and +1 , and two Gauss points are needed in each coordinate direction. These are defined as shown in Figure 4.3.

An example, evaluating the integral of (3.24) follows: The integral to evaluate is:

$$
A_{i j}=\int_{x_{L}} \int_{y_{L}}\left(\underline{\nabla} \phi_{j} \cdot \underline{\nabla} \phi_{i}\right) B_{i} d y d x
$$

where $x_{L}$ and $y_{L}$ indicate that the integral is over the area of an element $L$ in global coordinates. First, the $(x, y)$ integral is converted to an integral in local coordinates $(\xi, n)$ through use of the Jacobian:

$$
A_{i j}=\int_{\xi=-1}^{+1} \int_{\eta=-1}^{+1}\left(\nabla_{j} \cdot \underline{\nabla}_{i}\right) B_{i}(\operatorname{det} J) d \eta d \xi
$$




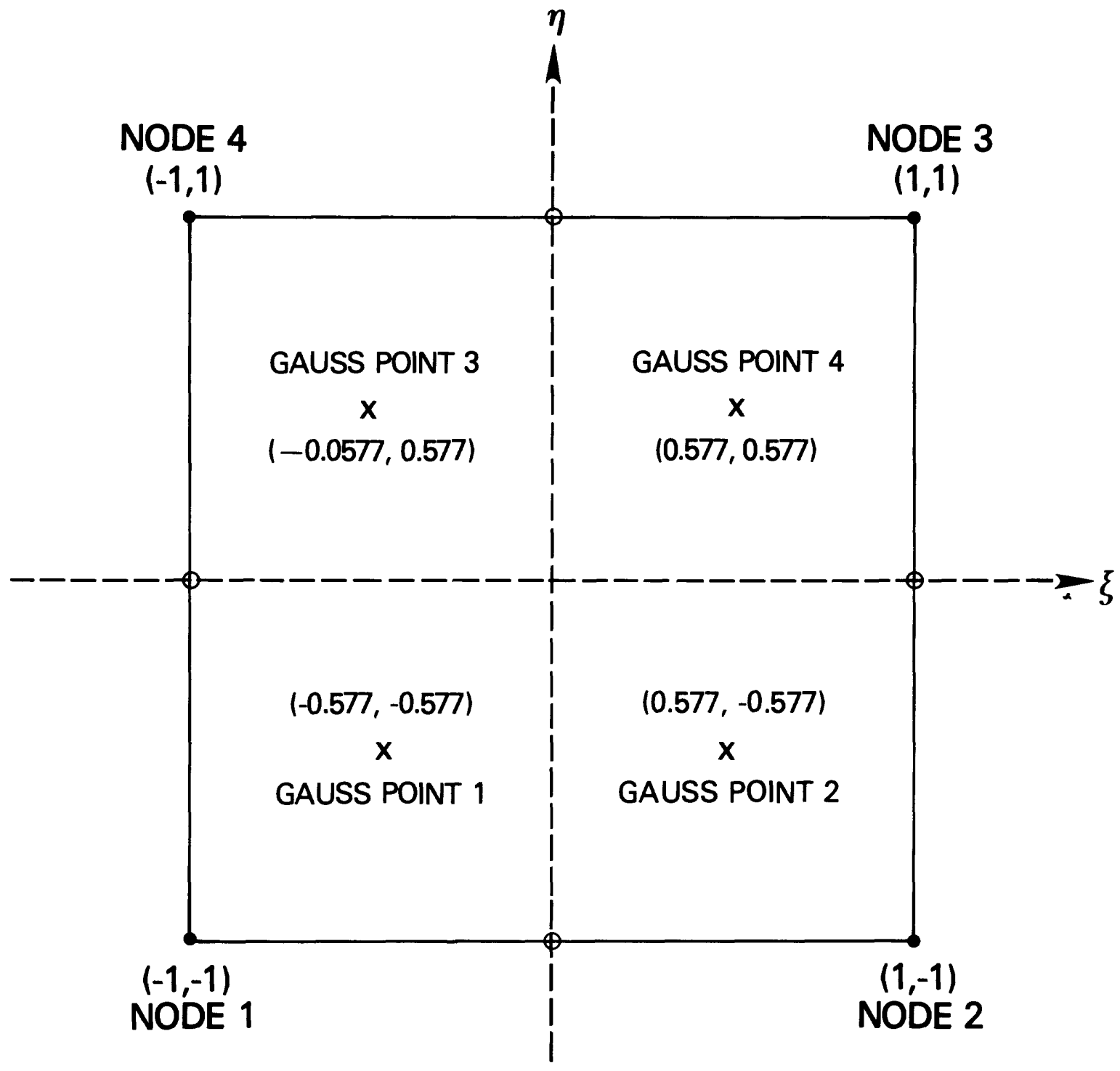

Figure 4.3

Finite element in local coordinate system with Gauss points. 
The values of $\underline{\nabla} \phi$ are in global coordinates and are obtained by transformation of derivatives calculated in local coordinates.

Gaussian integration is applied independently to each integral:

$$
A_{i j}=\sum_{k_{\xi}=1}^{2} \sum_{k_{n}=1}^{2}\left[\left(\nabla_{j} \cdot \underline{\nabla}_{i}\right) B_{i}(\operatorname{det} J)\right]\left(\xi_{K_{\xi}}, n_{K_{n}}\right)
$$

or equivalently as a single summation:

$$
A_{i j}=\sum_{K G=1}^{4}\left[\left(\nabla_{j} \cdot \underline{\nabla}_{i}\right) B_{i}(\operatorname{det} J)\right]\left(\xi_{K G}, n_{K G}\right)
$$

where $K_{\xi}$ and $K_{\eta}$ refer to Gauss point locations in the $\xi$ and $\eta$ directions, and where $\xi_{K G}$ and $n_{K G}$ refer to the four Gauss points arising in (4.36) as depicted In Figure 4.3. Thus, in order to evaluate the integral (4.34) over a given element, only four values of the integral need to be summed as given in (4.37), with one value determined at each of the four Gauss points.

In the case where an element is a non-rectangular quadrilateral with variable thickness $B$, the polynomial to be integrated in (4.35) is of fourth order as each of the terms may vary linearly in the same direction. Otherwise it is always of third order or less, and two-point Gauss integration provides exact results.

Note that the summation indication by (4.37) over the Gauss points is carried out by SUTRA subroutine 'ELEMEN' for each element in the mesh and for each integral which requires evaluation. 


\subsection{Numerical Approximation of SUTRA Fluid Mass Balance}

The governing equation representing the SUTRA fluid mass balance $(2.24)$, is modified by the addition of a point source term which is used to insert points at which pressure is specified. This is done as described in text referring to relation $(3.38)$.

$$
\begin{aligned}
& o_{p}(p, U)=\left(S_{w} \rho S_{o p}+\varepsilon \rho \frac{\partial S}{\partial p}\right) \frac{\partial p}{\partial t}+\left(\varepsilon S_{w} \frac{\partial \rho}{\partial U}\right) \frac{\partial U}{\partial t} \\
& -\underline{\nabla} \cdot\left[\left(\frac{k k_{r^{\rho}}}{\mu}\right) \cdot(\underline{\nabla}-\rho g)\right]-Q_{p} \\
& -v_{p}\left(p_{B C}-p\right)=0
\end{aligned}
$$

The last term is the source term arising from a specified pressure condition, wherein $\nu_{p}$ is a 'conductance' and $p_{B C}(t)$ is the externally specified pressure boundary condition value. When $\nu_{p}$ is set to a sufficiently large value, the last term becomes much larger than the others in (4.38), and $p \approx p_{B C}$, which is the desired boundary condition. Relation (4.38) is numerically approximated in the following sections.

\section{Spatial integration}

When the equation for $o_{p}(p, U)$ is approximated through nodewise, elementwise and cellwise discretizations, it no longer exactly equals zero. The approximate $\Lambda$

equation, $o_{p}(p, U)$, equals a spatially varying residual, $R_{p}(x, y, t)$, as shown in (3.8). A weighted residual formulation may be written as: 


$$
\int_{V} o_{p} \widehat{(p, U)} w_{i}(x, y) d V=0 \quad i=\overline{1, N N}
$$

where $W_{i}(x, y)$ is the weighting function in global coordinates chosen to be either the basis function, $\phi_{i}(x, y)$ or the asymmetric weighting function, $w_{i}(x, y)$, depending on the term of the equation. Relation (4.38) is approximated discretely and substituted for $o_{p}(p, u)$ in (4.39). The resulting set of integral terms is evaluated, one term at a time in the following paragraphs.

The first term is an integral of the pressure derivative:

$$
\int_{V}\left[\left(S_{w} \rho S_{o p}+\varepsilon \rho \frac{\partial S}{\partial p}\right) \frac{\partial p}{\partial t}\right] \phi_{i}(x, y) d V
$$

where the term in brackets is discretized cellwise, with one value of the term for each of the NN cells in the mesh, and the weighting function is chosen to be the basis function (written in global coordinates) The carat ( $)$ ) or large carat ( $\hat{N}$ ) over a term indicates that it has been approximated in one of the three ways. Because the cellwise-approximated term is constant for a node $i$, it is removed from the integral leaving only the basis function to be integrated. The volume integral of $\phi_{i}(x, y)$ gives the volume $v_{i}$ of cell $i$ according to relation (3.15). The term (4.40) becomes:

$$
\left(S_{w} \rho S_{o p}+\varepsilon \rho \frac{\partial S}{\partial p}\right)_{i} \frac{\partial p}{\partial t} v_{i}
$$

The second term of the expanded form of (4.39) is also a time derivative which is approximated cellwise:

$$
\int_{V}\left[\left(\varepsilon S_{w} \frac{\partial \rho}{\partial U}\right) \frac{\partial U}{\partial t}\right] \phi_{i}(x, y) d V=\left(\varepsilon S_{w} \frac{\partial \rho}{\partial U}\right)_{i} \frac{\partial U}{\partial t} i v_{i}
$$


The third term of expanded relation (4.39) involving the divergence of fluid flux is weighted with the asymmetric function. The asymmetry is intended for use only in unsaturated flow problems to maintain solution stability when the mesh has not been designed fine enough to represent sharp saturation fronts. In general, the usual symmetric function is used for weighting this flux term even for unsaturated flow, but the term is developed with the asymmetric function in order to provide generality. Green's Theorem (3.17) is applied yielding:

$$
\begin{aligned}
& -\int_{V}\left\{\underline{\nabla} \cdot\left[\left(\frac{k k_{r} \rho}{\mu}\right) \cdot(\underline{\nabla}-\rho g)\right]\right\} \omega_{i}(x, y) d V \\
& =-\int_{\Gamma}\left[\left(\frac{k k_{r} \rho}{\mu}\right) \cdot(\underline{\nabla}-\rho g)\right] \cdot \underline{n} \omega_{i}(x, y) d \Gamma \\
& +\int_{V}\left[\left(\frac{k k_{r} \rho}{\mu}\right) \cdot(\underline{\nabla}-\rho g)\right] \cdot \underline{\nabla}_{i} d V
\end{aligned}
$$

wherein the terms with carats are approximated discretely as described below, $\underline{n}$ is the unit outward normal to the three-dimensional surface bounding the region to be simulated, and $\Gamma$ is the surface of the region. The asymmetric weighting function in global (rather than local) coordinates is denoted, $\omega_{1}(x, y)$. The first term on the right of (4.43) is exactly the fluid mass flux (see Darcy's law, relation (2.19)) out across the region's boundary at node $i$, $q_{\text {our }}^{(t)}$ in units of $[M / s]$ :

$q_{\text {OUT }}(t)=\int_{\Gamma}(\hat{\varepsilon \rho \underline{v}} \cdot \underline{n}) \omega_{i} d \Gamma=\int_{\Gamma}\left[\left(\frac{\underline{\underline{k}} k_{r} \rho}{\mu}\right) \cdot(\underline{\nabla p}-\rho \underline{g})\right] \cdot \underline{n} \omega_{i} d \Gamma$ 
This term is used to specify fluid flows across boundaries in SUTRA. Note that an inflow, $q_{I N_{i}}(t)$ is $q_{I N_{i}}=-q_{O U T_{i}}$.

The second term on the right of (4.43) is approximated using a combination of elementwise and nodewise discretizations. The approximation of ( $\underline{p}-p g$ ) requires particular attention and is discussed in section 4.6 , "Consistent Evaluation of Fluid Velocity." The permeability tensor appearing in (4.43) in general has nine components, however, $(\underline{\nabla p}-\rho g)$ is always zero in the third spatial direction due to the assumption of a two-dimensional model. Thus only four components of the permeability tensor are required:

$$
\hat{k}^{L}=\left[\begin{array}{cc}
k_{x x}^{L} & k_{x y}^{L} \\
k_{y x}^{L} & k_{y y}^{L}
\end{array}\right]
$$

wherein $\underline{k}$ and is discretized elementwise as indicated by $\hat{k}^{L}$. The pressure is discretized nodewise:

$$
p(x, y, t) \simeq \sum_{i=1}^{N N} p_{i}(t) \phi_{i}(x, y)
$$

Relative permeability, $k_{r}$, depends on saturation which, in turn, depends on pressure. Relative permeabilities are evaluated at each Gauss point during numerical integration depending on the saturation (and pressure) at the Gauss point. Viscosity is evaluated at each Gauss point for energy transport as a function of nodewise discretized temperature, and is constant for solute transport. 
Density, $\rho$, when it appears in the permeability term, is also evaluated at each Gauss point depending on the nodewise discretized value of $U$ at the Gauss point. The density appearing in product with the gravity term is expressly not evaluated in this usual manner. A particular discretization is used which maintains consistency with the $\underline{\nabla p}$ term as described in section 4.6 , "Consistent Evaluation of Fluid Velocity". This consistently-evaluated $\rho g$ term is denoted $\hat{\mathrm{pg}}$, (see relation $(4.103))$.

The second term on the right of (4.43) is thus approximated as:

$$
\begin{aligned}
& \sum_{j=1}^{N N} p_{j}(t) \int_{x} \int_{y}\left\{\left[\left(\hat{k}^{L}\right)\left(\frac{k r}{\mu}\right)\right] \cdot \underline{\nabla}_{j}\right\} \cdot \underline{\nabla}_{i} B(x, y) d y d x \\
& -\int_{x} \int_{y}\left\{\left[\left(\hat{k}^{L}\right)\left(\frac{k r \rho}{\mu}\right)\right] \cdot[(\hat{\rho g})] \cdot \underline{\nabla}_{i} B(x, y) d y d x\right.
\end{aligned}
$$

where $\hat{\mathrm{k}}^{\mathrm{L}}$ indicates an elementwise discretized permeability tensor, $\left(\frac{\mathrm{k}_{\mathrm{r}} \mathrm{\rho}}{\mu}\right)$ indicates the value of the term based on nodewise discretized values of $p$ and $U$, and $(\hat{\rho g})$ indicates a discretization of $(\rho g)$ consistent with the discretization of $\underline{p}$. The thickness of the mesh, $B(x, y)$, is evaluated at each Gauss point depending on a nodewise discretization:

$$
B(x, y) \simeq \sum_{i=1}^{N N} B_{i} \phi_{i}(x, y)
$$

where $B_{i}$ is the mesh thickness at node $i$. Note that mesh thickness is fixed and may not vary in time as was allowed for illustrative purposes in Chapter 3, "Fundamentals of Numerical Algorithms."

The last two terms of (4.38) are approximated cellwise with a basis function for weighting. 
$-\int_{V} \hat{Q}_{p} \phi_{i}(x, y) d V-\int\left[\nu_{p}\left(p_{B C}-p\right)\right] \phi_{i}(x, y) d V=-Q_{i}-\nu_{i}\left(p_{B C}-p_{i}\right)$

The cellwise discretizations which are employed in the abcve evaluations are:

$$
\begin{aligned}
& \hat{Q}_{p}=\sum_{i=1}^{N N}\left(\frac{Q_{i}}{v_{i}}\right) \\
& {\left[\begin{array}{c}
\Lambda \\
Q_{P B C}
\end{array}\right]=\left[\nu_{p}\left(p_{B C}-p\right)\right]=\sum_{i=1}^{N N}\left[\left(\frac{v_{i}}{v_{i}}\right)\left(p_{B C}-p_{i}\right)\right]}
\end{aligned}
$$

where $V_{i}$ is the volume of cell $i, Q_{1}(t)[M / s]$ is the total mass source to cell $i$, $Q_{\mathrm{PBC}}\left[\mathrm{M} / \mathrm{L}^{3} \cdot \mathrm{s}\right]$ is the fluid mass source rate due to the specified pressure, and $\nu_{i}[\mathrm{~L} \cdot \mathrm{s}]$ is the pressure-based conductance for the specified pressure source in cell $i$. The conductance is set to zero for nodes at which pressure is not specified, and to a high value at nodes where prossure is specified.

By combining and rearranging the evaluations of approximate terms of (4.39), the following weighted residual relation is obtained:

$$
\begin{gathered}
A F_{i} \frac{d p_{i}}{d t}+C F_{i} \frac{d U}{d t} i+\sum_{j=1}^{N N} p_{j}(t) B F_{i j}+v_{i} p_{i}=Q_{i}+v_{i} p_{B C}+q_{I N}+D F_{i} \\
i=\overline{1, N N}
\end{gathered}
$$

where:

$$
\begin{aligned}
& A F_{i}=\left(S_{w} \rho S_{o p}+\varepsilon \rho \frac{\partial S}{\partial p} w\right)_{i} V_{i} \\
& C F_{i}=\left(\varepsilon S_{w} \frac{\partial \rho}{\partial U}\right)_{i} v_{i} \\
& B F_{i j}=\int_{x} \int_{y}\left\{\left[\left(\hat{\underline{k}}^{L}\right)\left(\frac{\hat{k}_{r}^{\rho}}{\mu}\right)\right] \cdot \underline{\nabla}_{j}\right\} \cdot \underline{\nabla}_{i} B d y d x
\end{aligned}
$$

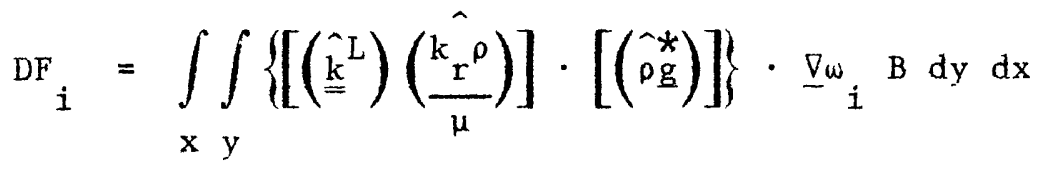


The only integrals requiring Gaussian integration are $\mathrm{BF}_{1 f}$ and $\mathrm{DF}_{1}$. Note that these are evaluated in SUTRA subroutine ELEMEN in an element by element manner. The other terms except for those involving $v_{1}$ are evaluated cellwise (one for each node). Note that this is done by subroutine NODALB, and the specified pressure terms are evaluated by subroutine BCB.

\section{Temporal discretization and iteration}

The time derivatives in the spatially discretized and integrated equation are approximated by finite differences. The pressure term is approximated as:

$$
\frac{d p_{1}}{d t} \simeq \frac{p_{1}^{n+1}-p_{1}^{n}}{\Delta t}
$$

where

$$
\begin{aligned}
& p_{1}^{n}=p_{1}\left(t^{n}\right) \\
& p_{1}^{n+1}=p_{1}\left(t^{n}+\Delta t t_{n+1}\right)=p_{1}\left(t^{n+1}\right)
\end{aligned}
$$

and

$$
\Delta t_{n+1}=t^{n+1}-t^{n}
$$

The new or current time step, $\Delta t_{n+1}$, begins at time $t^{n}$ and ends at $t i m e t^{n+1}$. The previous time step for which a solution has already been obtained at time $t^{n}$ is denoted $\Delta t_{n}$.

The term in (4.52) involving the time derivative of concentration or temperature, $\frac{d U}{d t}$, makes only a very small contribution to the fluid mass balance. For solution over the present time step, $\Delta t_{n+l}$, this derivative is evaluated using information from the previous time step, as these values are already known: 


$$
\frac{d U}{d t} i \simeq\left(\frac{d U}{d t} i\right)^{n}=\frac{U_{1}^{n}-U_{i}^{n-1}}{\Delta t}
$$

This approximation gives a simple method of accounting for this small contribution to the fluld mass balance.

All other terms in (4.51) are evaluated at the new time level $t^{n+1}$ for solution of the present time step, $\Delta t_{n+1}$, except for the density in the consistently discretized $\left(\begin{array}{l}\hat{*} \\ \rho \\ \mathrm{g}\end{array}\right)$ term. The density is evaluated based on $\mathrm{U}\left(\mathrm{t}^{\mathrm{n}}\right)$, the value of $U$ at the beginning of the present time step. Because coefficients depend on the, as yet, unknown values of $p$ and $U$ at the end of the time step, one or more iterations may be used to solve this non-linear problem. On the first iteration, and when only one iteration per time step is used, coefficents are based on a projected value of $p$ and $U$.

$$
\begin{aligned}
& p_{i}^{p r o j}=p_{i}^{n}+\left(\frac{\Delta t_{n+1}}{\Delta t_{n}}\right)\left(p_{i}^{n}-p_{i}^{n-1}\right) \\
& u_{i}^{p r o j}=U_{i}^{n}+\left(\frac{\Delta t_{n+1}}{\Delta t_{n}}\right)\left(U_{i}^{n}-U_{i}^{n-1}\right)
\end{aligned}
$$

These profections estimate the $p$ and $U$ values at a node $i, p_{i}^{p r o j}$ and $U_{i}^{p r o j}$, at the end of the present time step, $\Delta t_{n+1}$, based on linear extrapolation of the two previous values of $p$ and $U$. All $p$ and $U$ dependent coefficients (except $\hat{p} \hat{g}^{*}$ ) In (4.52) through (4.56) are estimated at time level $t^{\mathrm{n}+1}$. These coefficient values are based on the most recent values of $p$ and $U$, be they projections or solutions to the previous iteration. Iterations end when the maximum change in $p$ and $U$ at any node in the mesh falls below user-specified criteria of absolute change in $\mathrm{p}$ and $\mathrm{U}$. 
The weighted residual relations (4.52) may thus be written in a form which allows for solution of pressures at nodes, $p_{i}^{n+1}$, at the end of the present time step:

$$
\begin{gathered}
\left(\frac{A F_{i}^{n+1}}{\Delta t_{n+1}}\right) p_{i}^{n+1}+\sum_{j=1}^{N N} p_{i}^{n+1} B F_{i j}^{n+1}+v_{i} p_{i}^{n+1}=Q_{i}^{n+1} \\
+v_{i} p_{B C_{i}}^{n+1}+q_{I N}^{n+1}+D_{i}^{(n+1) *}+\left(\frac{A F_{i}^{n+1}}{\Delta t}\right) p_{i}^{n+1}+\left(C F_{i}^{n+1}\right)\left(\frac{d U}{d t}\right)^{n} \\
i=\overline{1, N N}
\end{gathered}
$$

where the superscript involving $(n)$ or $(n+1)$ indicates level of time evaluation. The term with level $(n+1) *$ indicates that the $(\hat{\rho g})$ term is evaluated at the $(n)$ time level on the first iteration, and at the most recent level on subsequent iterations. The other coefficients are evaluated at the $(n+1)$ time level by projection on the first iteration, and at the most recent level on subsequent iterations.

\section{Boundary conditions, fluid sources and sinks}

Specified pressures are obtained through the cellwise addition of a fluid flux, (see Figure 3.7), $Q_{B_{i}}\lfloor M / s\rfloor$ with reference to $(4.49)$ :

$$
Q_{B C_{i}}^{n+1}=v_{i}\left(p_{B C_{i}}^{n+1}-p_{i}^{n+1}\right)
$$

For a cell in which $v_{i}$ is specified as a large number, this flux term dominates the fluid mass balance and $p_{B C}^{n+1}=p_{i}^{n+1}$, achieving a specified pressure at the node representing cell $i$. Note that specified pressure may change each time step. For cells in which pressure is not specified, $v_{i}$ is set at zero, and no fluid is added to the cell by $(4.64)$.

Both fluid sources, $Q_{i}^{n+1}$, and fluid inflows across region boundaries, $\mathrm{q}_{\mathrm{IN}}^{\mathrm{n}+1}$, are specified cellwise. They directly add fluid mass to the node in 
cell 1. Thus, fluid sources and boundary inflows are indistinguishable in the model. Fluid sources and flows across boundarles are both accounted for by the vector $Q_{1}^{n+1}$ in SUTRA, and are referred to as fluid sources. Thus the term, $q_{I N}^{n+1}$, in (4.63) may be dropped and the definition of $Q_{1}^{n+1}$ may be generalized to include the boundary flows.

The form of the discretized fluid mass balance implemented in SUTRA is as follows:

$$
\begin{aligned}
& \text { NN } \\
& \sum_{j=1}\left[\left(\frac{A F_{i} \delta_{i j}}{\Delta t_{n+1}}\right)+B F_{i j}^{n+1}+v_{i} \delta_{i j}\right] p_{j}^{n+1}=Q_{i}^{n+1}+v_{i} p_{B C_{1}}^{n+1} \\
& +D F_{i}^{(n+1) *}+\left(\frac{A F_{1}^{n+1}}{\Delta t}\right) p_{1}^{n}+\left(C F_{1}^{n+1}\right)\left(\frac{d U}{d t} 1\right)^{n} \quad 1=\overline{1, N N}
\end{aligned}
$$

wherein $\delta_{1 j}$ is the Kronecker delta:

$$
\delta_{i j}=\left\{\begin{array}{lll}
0 & \text { if } & i \neq j \\
1 & \text { if } & i=j
\end{array}\right.
$$

4.5 Numerical Approximation of SUTRA Unified Solute Mass and Energy Balance

The governing equation representing the SUTRA unified energy and solute mass balance (2.52) is modified by the addition of a point source term which arises due to fluid inflows and outflows at points of specifled pressure:

$$
\begin{aligned}
& O_{u}(U)=\left[\varepsilon S_{w} \rho c_{w}+(1-\varepsilon) \rho_{s} c_{s}\right] \frac{\partial U}{\partial t}+\varepsilon S_{w} \rho c_{w} v \cdot \underline{\nabla} U \\
& -\underline{\nabla} \cdot\left\{\rho c_{w}\left[\varepsilon S_{w}\left(\sigma_{w} I+\underline{D}\right)+(1-\varepsilon) \sigma_{s} I\right] \cdot \underline{\nabla}\right\} \\
& -Q_{p} c_{w}\left(U^{*}-U\right)-\varepsilon S_{w} \rho \gamma_{1}^{w} U-(1-\varepsilon) \rho_{s} \gamma_{1}^{s} U_{s}-\varepsilon S_{w} \rho \gamma_{0}^{w}-(1-\varepsilon) \rho_{s}^{\gamma} \gamma_{0}^{8} \\
& -Q_{P B C} c_{w}\left(U_{B C}-U\right)=0
\end{aligned}
$$


The last term is the solute mass or energy source due to fluid inflow at a point of spectfied pressure, $Q_{P B C}\left[M / L^{3} \cdot s\right]$ is the mass fluid source rate given by (4.51), and $U_{B C}$ is the concentration or temperature of the flow. For outflow, $U_{B C}=U$, and the terms goes to zero. Relation (4.66) is numerically approximated in the following sections.

\section{Spatial integration}

When the equation for $o_{u}(U)$ in $(4.66)$ is approximated through nodewise, elementwise and cellwise discretizations, it no longer exactly equals zero. The approximate equation, $\hat{O}_{u}(U)$, equals a spatially varying residual, $R_{u}(x, y, t)$, as shown in (3.8). A weighted residual formulation may be written as:

$$
\int_{V} \widehat{o_{u}(u)} w_{i}(x, y) d V=0 \quad i=\overline{1, N N}
$$

where $W_{i}(x, y)$ is the weighting function, chosen to be either the basis function, $\phi_{i}(x, y)$ or the asymmetric weighting function, $\omega_{i}(x, y)$, depending on the term of the equation. Relation (4.66) is discretized and the approximation is substituted for $O_{u}(U)$ in (4.67). The resulting set of integral terms is evaluated, one term at a time, in the following paragraphs.

The first term is an integral of the temperature or concentration time derivative:

$$
\int_{V}\left\{\left[\varepsilon S_{w} \rho c_{w}+(1-\varepsilon) \rho_{s} c\right] \frac{\partial U}{\partial t}\right\} \phi_{i}(x, y) d V
$$

where the term in braces is discretized cellwise, and the weighting function is the basis function, (written in global coordinates). As the term with a carat in braces has constant value over a cell, $i$, the integral contains only the basis function and equals the cell volume, $V_{i}$, according to (3.15). Thus the term is: 


$$
\left[\varepsilon S_{w} p c_{w}+(1-\varepsilon) p_{s} c_{s}\right]_{i} \frac{\partial U}{\partial t} i v_{i}
$$

The second integral is:

$$
\int_{V}\left(\varepsilon S_{w} \hat{p c} \underline{v} \cdot \underline{\nabla v}\right) w_{i}(x, y) d V
$$

where the asymmetric welghting function is chosen to allow the use of 'upstream weighting' for this term representing advective transport. 'Upstream weighting' is intended for use only when the finite-element mesh has been designed too coarse for a particular level of dispersive and advective transport. The asymmetric function adds dispersion in an amount dependent on element length in the flow direction. As a result, it changes the parameters and thus changes the physics of the problem being solved. This term 18 written in general to allow upstream weighting, but simplifies to weighting with a basis function when upstream weight (UP in (4.23) and (4.24)) is set to zero. Thus, in order not to alter the physics for most simulation problems, this term will have symmetric weighting.

The coefficients in this term (except velocity) are evaluated at each Gauss point and are represented depending on nodewise discretization of $p$ and U. Porosity is discretized nodewise. Nodewise discretizations of $\varepsilon$ and $U$ are written:

$$
\begin{aligned}
\varepsilon(x, y) \simeq \hat{\varepsilon} & =\sum_{i=1}^{N N} \varepsilon_{1} \phi_{i}(x, y) \\
U(x, y, t) & =\sum_{i=1}^{N N} v_{i}(t) \phi_{i}(x, y)
\end{aligned}
$$


The velocity is evaluated at each Gauss point during numerical integration in a particular way that depends on consistent discretization of $\underline{\nabla}$ and $p g$ terms in Darcy's law. This consistent approximated velocity is denoted $\underline{v}^{*}$. Thus the term (4.70) is evaluated as:

$$
\sum_{j=1}^{N N} u_{j}(t) \int_{x} \int_{y}\left[\hat{\varepsilon}\left(s_{w} p\right) c_{w} \hat{v}^{*} \cdot \nabla_{\phi}\right] \omega_{i}(x, y) B(x, y) d y d x
$$

wherein $B(x, y)$ is the nodewise-discretized mesh thickness (4.47). Specific heat, $c_{w}$, is a constant.

The third term of $(4.67)$ is:

$$
-\int_{V} \underline{\nabla} \cdot\left\{\rho c_{w}\left[\varepsilon S_{w}\left(\sigma_{w=}+\underline{D}\right)+(1-\varepsilon) \sigma_{s} I\right] \cdot \nabla U\right\} \phi_{1}(x, y) d V
$$

where the basis function weights the integral. Green's Theorem (3.17) is applied to $(4.74)$ resulting in:

$$
\begin{aligned}
& -\int_{\Gamma}\left\{\rho c\left[\varepsilon S_{w}\left(\sigma_{w=}+\hat{D}\right)+(1-\varepsilon) \sigma_{s} I\right] \cdot \underline{\nabla U}\right\} \cdot \underline{n}_{\phi_{1}}(x, y) d \Gamma \\
& +\int_{V}\left\{\rho c\left[\varepsilon S_{w}\left(\sigma_{w=}+\underline{D}\right)+(1-\varepsilon) \sigma_{s} \underline{\underline{I}}\right] \cdot \underline{\nabla U}\right\} \cdot \underline{\nabla}_{\phi_{i}} d V
\end{aligned}
$$

where the carat refers to the entire terms in braces. The first term represents the diffusive/dispersive flux of solute mass or energy out across a system boundary in the region of node 1 . This term is denoted, $Y_{\text {OUT }} \cdot$. An influx would be ${ }^{-1}$ ouT $_{1}$ or ${ }_{I_{1}}$. The second term is based on nodewise discretization of $U$. The coefficients $\rho$ and $S_{w}$ are evaluated at Gauss points based on nodewise discretization of $U$ and $p$. Porosity, $E$ is discretized nodewise as in (4.71), and $c_{w}, \sigma_{w}$ and $\sigma_{s}$ are constants. The dispersion tensor, $D$, is evaluated at each 
Gauss point according to equations (2.38) through (2.40b). Velocities used in this evaluation are the consistent values, $\underline{\underline{v}}$, and dispersivities, $\alpha_{L}$ and $\alpha_{T}$, are discretized elementwise except that $\alpha_{L}$ is evaluated at each Gauss point for the anisotrtopic media model. The approximated $\underline{\underline{D}}$ is denoted, $\hat{\underline{D}}$ Thus, the term (4.74) is evaluated as:

$$
-\underline{Q}_{I N}+\sum_{j=1}^{N N} U_{j}(t) \iint_{x}\left\{\rho c{ }_{y}\left[\hat{\varepsilon S}\left(\sigma_{w} \underline{\underline{I}}+\underline{\underline{D}}\right)+(1-\hat{\varepsilon}) \sigma_{s} \underline{I}\right]\right\} \cdot \underline{\nabla}_{j} B(x, y) d y d x
$$

The remaining terms in (4.67) are discretized cellwise with the basis function as the weighting function:

$$
\begin{aligned}
& -\int\left[Q_{p} c_{w}\left(\hat{u^{*}}-U\right)\right] \phi_{i}(x, y) d V=-Q_{1} c_{w}\left(u_{i}^{*}-u_{i}\right) \\
& -\int_{V}\left[\varepsilon S_{w} \rho \gamma_{1}^{w} U\right] \phi_{i}(x, y) d V=-\left[\varepsilon S_{w} \rho \gamma_{1}^{w}\right]_{i} U_{i} v_{i} \\
& -\int_{V}\left[(1-\varepsilon) \rho_{s} \gamma_{1}^{s} u_{s}\right\rfloor \phi_{i}(x, y) d V=-\left\lfloor(1-\varepsilon) \rho_{s} \gamma_{1}^{s} U_{s}\right\rfloor_{i} v_{i} \\
& -\int_{V}\left[\varepsilon S_{w} \rho \gamma_{0}^{W}+\hat{(1-\varepsilon)} \rho_{s} \gamma_{0}^{s}\right\rfloor \phi_{1}(x, y) d V=-\left\lfloor\varepsilon S_{W} \rho \gamma_{0}^{W}+\left.(1-\varepsilon) \rho_{s} \gamma_{o}^{s}\right|_{i}\right. \\
& -\int_{V}\left[Q_{P B C}{ }^{c}\left(U_{B C}-U\right)\right] \phi_{i}(x, y) d V=-Q_{B C}{ }_{i}{ }_{w}\left(U_{B C}-U_{i}\right)
\end{aligned}
$$

where:

$$
\mathrm{Q}_{\mathrm{BC}}=v_{i}\left(\mathrm{p}_{\mathrm{BC}}-\mathrm{p}_{i}\right)
$$

and:

$$
\hat{Q}_{\mathrm{PBC}}=\sum_{i=1}^{N N}\left(\frac{\mathrm{Q}_{\mathrm{BC}}}{\mathrm{V}_{i}}\right)
$$


The relation, (4.79), is non-zero only for solute transport and the value of $U_{s}$ is given for solute trasport by the adsorption isotherms in the form:

$$
\mathrm{U}_{s}=\mathrm{C}_{s}=\mathrm{s}_{\mathrm{L}} \mathrm{C}+\mathrm{s}_{\mathrm{R}}
$$

where $s_{L}$ and $s_{R}$ are defined in section 4.7, "Temporal Evaluation of Adsorbate Mass Balance." In the above cellwise relations, $c_{w}, \rho_{8}, \gamma_{1}^{w}$, and $\gamma_{1}^{s}$ are constant, and $\gamma_{0}^{w}, \gamma_{0}^{s}, s_{L}$, and $s_{R}$ may vary cellwise and with time.

By combining and rearranging the evaluations of integrals in (4.67) and the definition (4.84), the following NN spatially discretized weighted residual relations are obtained:

$$
\begin{gathered}
A T_{i} \frac{d U}{d t} i+\sum_{j=1}^{N N} U_{j}(t) D_{i j}+\sum_{j=1}^{N N} U_{j}(t) B T_{i j}-\left(G T_{i}+G_{s} T L_{i}\right) U_{i}(t)+Q_{i} c_{w} U_{i}(t) \\
+Q_{B C} c_{i} U_{i}(t)=Q_{i} c_{w} U_{i}^{*}+Q_{B C_{i}} c_{w} U_{B C}+T_{i}+N_{i}+E T_{i}+G_{s} T_{i} \\
i=\overline{1, N N}
\end{gathered}
$$

where:

$$
\begin{aligned}
& \operatorname{AT}_{i}=\left[\varepsilon S_{w} \rho c_{w}+(1-\varepsilon) p_{s} c_{s}\right]_{i} v_{i} \\
& D T_{i j}=\int_{x} \int_{y}\left[\hat{\varepsilon}\left(S_{w} \rho\right) c_{w} \hat{v}^{*} \cdot \nabla \phi_{j}\right] \omega_{i} B d y d x \\
& B T_{i j}=\int_{x} \int_{y}\left\{\rho c\left[\hat{\varepsilon} S_{w}\left(\sigma_{w} \underline{\underline{I}}+\hat{D}\right)+(1-\hat{\varepsilon}) \sigma_{s=}\right] \cdot \underline{\nabla}_{j}\right\} \cdot \nabla \phi_{1} B d y d x \\
& \mathrm{GT}_{1}=\left(\varepsilon \mathrm{S}_{w} \circ \gamma_{1}^{w}\right)_{i} \mathrm{v}_{i}
\end{aligned}
$$




$$
\begin{aligned}
& G_{s} T L_{i}=\left\lfloor(1-\varepsilon) \rho_{s} \gamma_{1}^{s} s_{L}\right\rfloor_{i} v_{i} \\
& G_{s} T R_{1}=\left\lfloor(1-\varepsilon) \rho_{s} \gamma_{1}^{s} s_{R}\right\rfloor_{i} v_{i} \\
& \operatorname{ET}_{i}=\left\lfloor\varepsilon S_{w} p \gamma_{0}^{w}+(1-\varepsilon)_{s} \gamma_{0}^{s}\right\rfloor_{i} v_{1}
\end{aligned}
$$

The only integrals requiring Gaussian integration are $\mathrm{DT}_{i j}$ and $\mathrm{BT}_{i j}$. Note that these are evaluated in SUTRA subroutine ELEMEN, in an element by element manner. The remaining terms that do not involve $Q_{B C}$ are evaluated cellwise by SUTRA subroutine NODALB. Also note that the flux terms arising from specified pressure (those with $Q_{B C}$ ) are evaluated by subroutine $B C B$.

\section{Temporal discretization and iteration}

The time derivative in the spatially discretized and integrated equation is approximated by finite differences:

$$
\frac{\mathrm{dU}}{\mathrm{dt}} i=\frac{\mathrm{U}_{i}^{n+1}-\mathrm{U}_{i}^{\mathrm{n}}}{\Delta \mathrm{t}_{\mathrm{n}+1}}
$$

where:

$$
\begin{aligned}
& u_{i}^{n}=u_{i}\left(t^{n}\right) \\
& u_{t}^{n+1}=u_{i}\left(t^{n}+\Delta t_{n}\right)=u_{i}\left(t^{n+1}\right)
\end{aligned}
$$

All terms in (4.85) are evaluated at the new time level, $t^{n+1}$, except the velocity in (4.87) and the dispersion tensor in (4.88) which involves velocity are lagged on the first iteration. Because coefficients depend on the yet unknown values of $p$ and $U$ at the end of the time step, one or more iterations may be used to solve this non-linear problem. On the first iteration, and when only one iteration per time step is used, coefficients are based on a projected 
value of $p$ and $U$ as given by (4.61) and (4.62). On subsequent iterations coefficients are based on the most recent value of $p$ and $v$. Iterations end when the convergence criteria are satisfied.

On the first iteration, and when only one iteration per time step is used, the velocities are evaluated based on $p_{i}^{n}, U_{i}^{n-1}$ and $\rho_{i}^{n-1}$. This is because the pressure gradient in the velocity calculation, $\nabla p^{n}$, is based on pressures calculated when the fluid density was $\rho^{n-1}$. On subsequent iterations velocities are calculated using the pressure solution for the most recent iteration together with the densities resulting from the previous iteration upon which the most recent pressure solution was based. No spurious velocities, which arise from mismatched $P$ and $\rho$, are generated this way. The flux term, $Q_{B C}$, arising from the specified pressures is evaluated on the first iteration at the beginning of the time step in terms of $\mathrm{p}_{i}^{\mathrm{n}}$ and $\mathrm{p}_{\mathrm{BC}}^{\mathrm{n}}$. On subsequent iterations, it is based on the most recent pressure solution and $p_{B C}^{n+1}$.

The relations (4.85) may thus be written in a form which allows for solution of concentration or temperature at nodes, $v_{i}^{n+1}$, at the end of the present time step:

$$
\begin{aligned}
& \left(\frac{A T_{i}^{n+1}}{\Delta t}\right) U_{i}^{n+1}+\sum_{j=1}^{N N} U_{j}^{n+1} D T_{i j}^{(n+1) *}+\sum_{j=1}^{N N} U_{j}^{n+1} B T_{i j}^{n+1}+\left(G T_{i}^{n+1}+G_{s} T L_{i}^{n+1}\right) U_{i}^{n+1} \\
& +Q_{i}^{n+1} c_{w} U_{i}^{n+1}+Q_{B C_{i}}^{(n+1) *} c_{w} U_{i}^{n+1}=Q_{i}^{n+1} c_{w} U_{i}^{* n+1}+Q_{B C_{i}}^{(n+1) *} c_{w} U_{B C_{i}+1}^{n+1} \Psi_{i}^{n+1}+E T_{i}^{n+1} \\
& +G_{s}^{T R_{i}^{n+1}}+\left(\frac{A T_{i}^{n+1}}{\Delta t}\right)_{n+1}^{n} U_{i}^{n}
\end{aligned}
$$

The $(n+1) *$ level indicates that velocity and $Q_{B C}$ are evaluated on the first iteration at the time step $(n)$ and on subsequent iterations, at the most 
recent level. Other coefficients are evaluated at the $(n+1)$ time level by projection on the first iteration, and then at the most recent level on subsequent iterations .

\section{Boundary conditions, energy or solute mass sources and sinks}

Specified temperatures or concentrations at nodes are obtained numerically at the node $k$ by replacing the $k^{\text {th }}$ equation in (4.93) by:

$$
\mathrm{U}_{\mathrm{k}}^{\mathrm{n}+1}=\mathrm{U}_{\mathrm{BC}}^{\mathrm{n}+1}
$$

where $U_{B C}^{n+1}$ is the user-specified value of $U$ that node $k$ is to have during time step $(n+1)$. The specified value may change with each time step.

Source boundary conditions for $U$ arise whenever a fluid source $Q_{1}$ is specified. These may be either point sources of fluid or fluid flows across the boundaries. These fluid inflows must be assigned concentration or temperature values, $U_{i}^{*_{n}+1}$, which may change with each time step. Note that these sources are evaluated in SUTRA subroutine NODALB. Outflows of fluid result in the disappearance of the source term from the transport equation because $\left(U_{i}^{*_{n+1}}=U_{i}^{n+1}\right)$ the sink and aquifer have the same U-value.

Source boundary conditions for $U$ may arise at points of specified pressure when an inflow $Q_{B C}$ occurs at such a point. A value of $U$ must be specified for such fluid inflows as $\mathrm{U}_{\mathrm{BC}_{i}+1}^{\mathrm{n}}$. These values may change with each time step. This source term for $U$ disappears for outflow at a point of specified pressure. Note that specified pressure sources are evaluated in SUTRA subroutine BCB.

A source or sink at a boundary due to diffusion or dispersion appears in $(4.75):$

$$
\Psi_{I N_{i}}^{n+1}=\int_{\Gamma}\left\{\rho c_{w}\left[\varepsilon S_{w}\left(\sigma_{w} \underline{I}+\underline{\underline{D}}\right) \stackrel{\wedge}{+}(1-\varepsilon) \sigma_{s} \underline{I}\right] \cdot \underline{\nabla U}\right\}^{n+1} \cdot \underline{n} \phi_{i} d \Gamma
$$


where the carat refers to the entire term in braces. For solute transport, this term may represent molecular diffusion and dispersion of solute mass across a boundary. For energy transport, this term represents heat conduction and thermal dispersion across a boundary. This heat or solute flux is a user-specified value which may change each time step. If the term is set to zero, it implies no diffusion and no dispersion across a boundary for solute transport, or for energy transport it implies perfect thermal insulation and no dispersion across a boundary For an open boundary across which fluid flows, this term is not automatically evaluated by SUTRA. If no user-specified value exists at an open boundary, then this term is set to zero. This implicitly assumes that the largest part of solute or energy flux across an open boundary is advectively transported rather than diffusively or dispersively transported. In cases where this assumption is inappropriate, the code may be modified to evaluate this term at the new time level depending on the value of $\mathrm{U}^{\mathrm{n}+1}$.

The form of the discretized unified energy and solute mass balance equation which is implemented in SUTRA is as follows:

$$
\begin{aligned}
& \sum_{j=1}^{N N}\left\{\left(\frac{A T_{i}^{n+1} \delta_{i j}}{\Delta t_{n+1}}\right)+D T_{i j}^{(n+1) *}+B T_{i j}^{n+1}+\left[G T_{i}^{n+1}+G_{s} T L_{i}^{n+1}+\left(Q_{i}^{n+1}+Q_{B C_{i}}^{n}\right) c_{w} \mid \delta_{i j}\right\} U_{i}^{n+1}\right. \\
& =c_{w}\left(Q_{i}^{n+1} U_{i}^{(n+1) *}+Q_{B C}^{n} U_{B C}^{n+1}\right)+m_{i}^{n+1}+E T_{i}^{n+1}+G_{s} T R_{i}^{n+1}+\left(\frac{A T_{i}^{n+1}}{\Delta t_{n+1}}\right) U_{i}^{n} \\
& i=\overline{1, N N}
\end{aligned}
$$

wherein $\delta_{1 j}$ is the Kronecker delta. 


\subsection{Consistent Evaluation of Fluid Velocity}

Fluid velocity is defined by equation (2.19) as:

$$
\underline{v}=-\left(\frac{\underline{k} k_{r}}{\varepsilon S_{w} \mu}\right) \cdot(\underline{\nabla}-\rho g)
$$

This relation strictly holds true at a point in space. In order for the relation to hold true when discretized, the terms $\underline{\nabla} p$ and $\rho g$ must be given the same spatial variability. This avolds generation of spurious velocities which would be caused by local mismatching of the discretized pressure gradient term and density-gravity term. For example, in a hydrostatic system where densities vary spatially, $\underline{\nabla} p=\rho g$, to yield a zero vertical velocity. However, if $\underline{\nabla} p$ and $\rho g$ do not locally cancel because of the discretization chosen, then erroneous vertical velocities would be generated.

Such an error would occur over an element where $\underline{p}$ is allowed only a single constant value in a vertical section of the element, but where $p$ is allowed to vary linearly in the vertical direction. This would be the case in a standard finite-element approximation wherein both $p$ and $U$ vary linearly in the vertical direction across an element. Linear change in p implies a constant value $\underline{p}$, while linear change in $U$ implies a linear change in the value of $p$ according to (2.3) or (2.4). Thus a standard finite-element approximation over a bi-linear element results in inconsistent approximations in the vertical direction for $\underline{\nabla p}$ and $\rho g$ : constant $\underline{\nabla}$ and linearly varying $\rho$. This inconsistency generates 
spurious vertical velocities especially in regions of sharp vertical changes in

U. A consistent approximation of velocity is one in which $\underline{\nabla p}$ and $p g$ are allowed the same spatial variability, and further, are evaluated at the same time level.

A consistent evaluation of velocity is required by the transport solution In (4.87) and also requited in the evaluation of the dispersion tensor in (4.88), where velocity is required in each element, in particular, at the Gauss points for numerical integration. Also a consistent evaluation of the $p g$ term is required for the fluid mass balance solution in the integral shown in (4.56). The values are also required at the Gauss points in each element during numerical evaluation of this integral.

The coefficients for calculation of velocity in (4.97) are discretized as follows: Permeability, $k$, is discretized elementwise; porosity, $\varepsilon$, is discretized nodewise. Unsaturated flow parameters, $k_{r}$ and $S_{w}$, are given values depending on the nodewise-discretized pressure according to relations (2.8) and (2.21). Viscosity is either constant for solute transport or is given values depending on nodewise-discretized temperature according to (2.5).

To complete the discretization of velocity, values in global coordinates at the Gauss points are required for the term $(\underline{\nabla p}-\rho g)$. A consistent approximation is presented in the remainder of this section for this term based on the fact that this term will be discretized in a consistent manner in global coordinates in an arbitrarily oriented quadrilateral element whenever it is discretized consistently in local element coordinates $(\xi, \eta)$. Consistent discretization in local coordinates is obtained when the spatial dependence of $\frac{\partial p}{\partial \xi}$ and $\rho_{\xi}$ is the same, and when $\frac{\partial p}{\partial \eta}$ and $\rho g_{\eta}$ have the same spatial dependence. Because the discretization for $p(\xi, \eta)$ has already been chosen to be bi-linear, it is the discretization of the $\rho g$ term, in particular, which must be adjusted. First, 
in the following, a discretization of the $\rho g$ term is presented which is presented which is consistent with the discretization of $\underline{\nabla p}$ in local coordinates, and then both $\underline{\nabla p}$ and $\rho g$ are transformed to global coordinates while maintaining consistency.

The pressure gradient within an element in local coordinates is defined in terms of the derivatives with respect to the local coordinates:

$$
\begin{aligned}
& \frac{\partial p}{\partial \xi}(\xi, \eta)=\sum_{i=1}^{4} p_{i} \frac{\partial \Omega}{\partial \xi} i \\
& \frac{\partial p}{\partial \eta}(\xi, n)=\sum_{i=1}^{4} p_{i} \frac{\partial \Omega}{\partial \eta} i
\end{aligned}
$$

The summations may be expanded and written in detail by reference to relations (4.9) through (4.12) and (4.1) through (4.4).

A local discretization of $\rho g$, with a spatial functionality that is consistent with the local pressure derivatives, (4.98a) and (4.98b) is:

$$
\begin{aligned}
& (\rho g)_{\xi}(\xi, n)=\sum_{i=1}^{4} \rho_{i} g_{\xi_{i}}\left|\frac{\partial \Omega}{\partial \xi}\right| \\
& (\rho g)_{\eta}(\xi, n)=\sum_{i=1}^{4} \rho_{i} g_{n_{i}}\left|\frac{\partial \Omega}{\partial \eta} i\right|
\end{aligned}
$$

where the vertical bars indicate absolute value, $\rho_{i}$ is the value of $\rho$ at node $i$ in the element based on the value of $U$ at the node through relation (2.3) or (2.4), $g_{\xi_{i}}$ is the $\xi$-component of $g$ at node $i$, and $g_{\eta_{i}} i$ the $n$-component of $g$ at node $i$. The eight gravity vector componenets at the nodes in each element need be calculated only once for a given mesh and may be saved. This discretization is robust in that it allows both the density and (the direction and) the magnitude of gravity vector components to vary over an element. No particular 
significance should be attached to the absolute values of basis function derivatives, except that these happen to give the desired consistent approximations, as is shown shortly.

The gravity vector components in local coordinates at a point in the element are obtained from the global gravity components as:

$$
\left\{\begin{array}{l}
g_{\xi} \\
g_{n}
\end{array}\right\}=[J] \quad\left\{\begin{array}{l}
g_{x} \\
g_{y}
\end{array}\right\}
$$

where $[\mathrm{J}]$ is the Jacobian matrix defined by $(4.25)$.

The derivatives of pressure in local coordinates (4.98a) and (4.98b), and the consistent density-gravity term components in local coordinates, (4.99) and (4.100), are transformed to global coordinates for use in the evaluation of the integrals they appear in by:

$$
\begin{aligned}
& \left\{\begin{array}{c}
\frac{\partial p}{\partial x} \\
\frac{\partial p}{\partial y}
\end{array}\right\}=\left[J^{-1}\right]\left\{\begin{array}{c}
\frac{\partial p}{\partial \xi} \\
\frac{\partial p}{\partial \eta}
\end{array}\right\} \\
& \left\{\begin{array}{c}
(\hat{\rho g})_{x}^{*} \\
(\hat{\rho g})_{y}^{*}
\end{array}\right\}=\left[J^{-1}\right]\left\{\begin{array}{l}
(\rho g)_{\xi} \\
(\rho g)_{n}
\end{array}\right\}
\end{aligned}
$$

where $\left(\hat{\mathrm{pg}}_{\mathrm{g}}^{*}\right)_{\mathrm{x}}$ and $(\hat{\mathrm{\rho g}})_{\mathrm{y}}$ are the consistently discretized density-gravity term components in global coordinates, and $[\mathrm{J}]^{-1}$ is the inverse Jacobian matrix defined by (4.29).

The spatial consistency of these approximations may be seen by inspecting their expansions in local coordinates. For example, the $\xi$-components are: 


$$
\begin{aligned}
\frac{\partial p}{\partial \xi} & =\frac{1}{4}\left[\left(p_{2}-p_{1}\right)(1-n)+\left(p_{3}-p_{4}\right)(1+n)\right] \\
(\rho g)_{\xi} & =\frac{1}{4}\left[\left(\rho_{1} g_{\xi_{1}}+p_{2} g_{\xi}\right)(1-n)+\left(p_{3} g_{\xi}+p_{4} g_{\xi}\right)(1+n)\right]
\end{aligned}
$$

The terms in parentheses preceeding the terms containing $n$ all have a constant value for the element, and thus the approximations have consistent spatial dependences.

\subsection{Temporal Evaluation of Adsorbate Mass Balance}

The terms in the unified energy and solute mass balance equation which stem from the adsorbate mass balance require particular temporal evaluation because some are non-linear. The following terms of relation (4.93) are evaluated here: $A T_{i}^{n+1}, G T_{i}^{n+1}$, and $\mathrm{ET}_{i}^{\mathrm{n}+1}$. For solute transport, the coefficient, $\mathrm{c}_{s_{i}}$, in $\operatorname{AT}_{i}^{n+1}$ (4.86) becomes $\kappa_{1}^{n+1}$, according to (2.52b). The relation which defines $\kappa_{1}$ is given by either $(1.34 c),(1.35 c)$, or $(1.36 c)$ depending on the sorption isotherm. The variable, $U_{s_{i}}^{n+1}$, is expressed in terms of the concentration of adsorbate, $c_{s_{1}}^{n+1}$, in a form given by (4.84). The parameters in $(4.84)$, $s_{L}$ and $s_{R}$, are defined in this section and are based on either (1.34a), (1.35a) and (1.36a) depending again on the sorption isotherm. The temporal approximations of these parameters are described below for each isotherm.

For linear sorption, all terms and coefficients related to the adsorbate mass are linear and are evaluated at the new time level and strictly solved for at this level:

$$
v_{s_{1}}^{n+1}=c_{s_{1}}^{n+1}=x_{1} p_{0} c_{1}^{n+1}
$$




$$
\begin{aligned}
& c_{s_{i}+1}^{n+1}=\kappa_{1}^{n+1}=x_{1} p_{0} \\
& { }_{s_{L}}=x_{1} p_{0} \\
& s_{R}=0
\end{aligned}
$$

For Fruendlich sorption, the adsorbate concentration is split into a product of two parts for temporal evaluation. One part is treated as a first order term as is linear sorption. This part is evaluated strictly at the new time level and solved for on each tteration or time step. The remaining part is evaluated as a known quantity, either based on the projected value of $C_{1}$ at the end of the time step on the first iteration, or based on the most recent $C_{1}$ solution on any subsequent iteration.

$$
u_{s_{i}}^{n+1}=c_{s_{i}}^{n+1}=\left[\left(x_{1} \rho_{0}\right)^{\left(\frac{1}{x_{2}}\right)}\left(c_{i}^{p r o j}\right)^{\left(\frac{1-x_{2}}{x_{2}}\right)}\right] c_{i}^{n+1}
$$

Also:

$$
\begin{aligned}
& c_{s_{i}}^{n+1}=k_{1}^{n+1}=\left(\frac{x_{1}}{x_{2}}\right) \rho_{0}^{\left(\frac{1}{x_{2}}\right)}\left(c_{i}^{p r o j}\right)^{\left(\frac{1-x_{2}}{x_{2}}\right)} \\
& { }^{8}=\left(x_{1} \rho_{0}\right)^{\left(\frac{1}{x_{2}}\right)}\left(c_{1}^{p r o j}\right)\left(\frac{1-x_{2}}{x_{2}}\right) \\
& { }^{8} \mathrm{R}=0
\end{aligned}
$$

where the coefficient, ${ }{ }_{1}{ }_{i}^{n+1}$, is evaluated from the projected or most recent value of $C_{1}$, depending on the iteration. 
Finally, for Langmuir sorption the form used for the temporal evaluation preserves dependence on a linear relationship to $C_{i}$. However, the linear relationship is appropriate only at low solute concentrations. At high concentrations, the adsorbate concentration approaches $\left(x_{1} / x_{2}\right)$. Therefore, two temporal approximations are combined, (one for low $C$, and one for high $C$ ) in a manner depending on the magnitude of concentration. When $\left(x_{2} \rho_{0} c\right)<<1$, the following temporal approximation for low values of $C$, referred to as $C_{s}^{0}$, is employed:

$$
\left.c_{s}^{o}=\left(x_{1} \rho_{o} c^{n+1}\right)\left[1-\frac{x_{2} \rho_{o} c^{p r o j}}{\left(1+x_{2} \rho_{o} c^{p r o j}\right.}\right)\right]
$$

When $\left(x_{2} p C\right)>>1$, the following temporal approximation for high $C, C_{s}^{\infty}$ is employed:

$$
\left.c_{s}^{\infty}=\left(\frac{x_{1}}{x_{2}}\right)\left[1-\frac{1}{\left(1+x_{2} \rho_{o} c^{p r o j}\right.}\right)\right]
$$

Thus $\mathrm{C}_{s_{i}}^{\mathrm{n}+1}$ may be defined:

$$
\mathrm{v}_{s_{i}}^{\mathrm{n}+1}=\mathrm{C}_{s_{i}}^{\mathrm{n}+1}=\mathrm{w}_{0} \mathrm{C}_{s_{i}}^{0}+\mathrm{w}_{\infty} \mathrm{C}_{s_{i}}^{\infty}
$$

where the weights $W_{0}$ and $W_{\infty}$, are:

$$
\begin{aligned}
& w_{\infty}=\frac{x_{2} \rho_{0} c^{p r o j}}{\left(1+x_{2} \rho_{0} c^{p r o j}\right)} \\
& w_{0}=1-w_{\infty}
\end{aligned}
$$

By substituting $(4.108),(4.109),(4.111 a)$, and $(4.111 b)$ into $(4.110)$, the following temporal evaluation of $\mathrm{C}_{s_{i}}^{\mathrm{n}+1}$ is obtained after algebraic manipulation: 


$$
c_{s_{i}+1}^{n+1}=\frac{x_{1} \rho_{0} C_{i}^{n+1}}{\left(1+x_{2} \rho_{0} c_{i}^{p r o j}\right)^{2}}+\frac{\left(x_{1} \rho_{o} C_{i}^{p r o j}\right)\left(x_{2} \rho_{o} C_{i}^{p r o j}\right)}{\left(1+x_{2} \rho_{o} C_{i}^{p r o j}\right)^{2}}
$$

The coefficient, $k_{1}^{n+1}$, is defined as:

$$
\begin{aligned}
& c_{s_{i}^{n+1}}^{n+1}=k_{1_{i}^{n+1}}^{n+1}=\frac{x_{1} \rho_{0}}{\left(1+x_{2} \rho_{0} c_{i}^{p r o j}\right)^{2}} \\
s_{L} & =\frac{x_{1} \rho_{0}}{\left(1+x_{2} \rho_{0} c_{i}^{p r o j}\right)^{2}} \\
s_{R} & =\frac{\left.\left(x_{1} x_{2}\right)_{\rho_{0}} c_{i}^{p r o j}\right)^{2}}{\left(1+x_{2} \rho_{0} c_{i}^{p r o j}\right)^{2}}
\end{aligned}
$$

The first term in $(4.112 a)$ is solved for on each iteration and the second term is treated as a known. In the above four relations, $c_{i}^{p r o j}$ is based on a projection for the first iteration on a time step, and is the most recent value of $C_{i}$ on subsequent iterations for the time step. 
Chapter 5

Other Methods and Algorithms

\subsection{Rotation of Permeability Tensor}

The aquifer permeability may be anisotropic (as discussed in section 2.2 under the heading "Fluid flow and flow properties," and may vary in magnitude and direction from element to element (as shown in (4.45)). The permeability in each element is completely described by input data values for $k_{\max }$, $k_{\min }$ and $\theta$, the principal permeability values and the direction in degrees from the global $+x$ direction to the maximum direction of permeability. The evaluation of integrals (4.55) and (4.56) as well as the velocity evaluation (4.97) require the permeability tensor components in global coordinates as given by (4.45).

Thus a rotation of the tensor is required from principal directions $\left(x_{p}, x_{m}\right)$ to global directions $(x, y)$, as shown in Figure 2.2 .

The rotation is given by:

$$
\underline{k}^{L}=\underline{\underline{J}}^{T} \stackrel{k^{L}}{\underline{p}} \underline{\underline{J}}^{T^{-1}}
$$

where

$$
\begin{aligned}
& \underset{\mathrm{k}}{\mathrm{L}}=\left[\begin{array}{ll}
\mathrm{k}_{\max }^{\mathrm{L}} & 0 \\
0 & k_{\mathrm{min}}^{L}
\end{array}\right] \\
& \mathrm{J}^{\mathrm{T}}=\left[\begin{array}{ll}
\cos \theta & -\sin \theta \\
\sin \theta & \cos \theta
\end{array}\right] \\
& \mathrm{J}^{\mathrm{T}}=\left[\begin{array}{cc}
\cos \theta & \sin \theta \\
-\sin \theta & \cos \theta
\end{array}\right]
\end{aligned}
$$


and $\underline{k}^{L}$ is given by (4.45). The result is:

$$
\begin{aligned}
& k_{x x}^{L}=k_{\text {max }}^{L} \cos ^{2} \theta+k_{\text {min }}^{L} \sin ^{2} \theta \\
& k_{y y}^{L}=k_{\text {max }}^{L} \sin ^{2} \theta+k_{\text {min }}^{L} \cos ^{2} \theta \\
& k_{x y}^{L}=k_{y x}^{L}=\left(k_{\text {max }}-k_{\text {min }}\right) \sin \theta \cos \theta
\end{aligned}
$$

\subsection{Radial Coordinates}

SUTRA is written in terms of two-dimensional Cartesian coordinates $x$ and y. In general, the two-dimensional numerical methods are applied to Cartesian forms of the governing equations; however, because the mesh thickness, $B_{1}$, is allowed to vary from node to node, radial coordinates (cylindical coordinates), $r$ and $z$ are an exact alternate coordinate set.

A function, $f(r, z)$, of radius $r$, and vertical coordinate $z$, is integrated over a cylindrical volume as follows:

$$
R=\int_{z} \int_{r} \int_{\theta} f(r, z) r d \theta d r d z
$$

Assuming symmetry with respect to angular coordinate $\theta(f(r, z)$ does not depend on $\theta$ ), the integral becomes:

$$
R_{r}=\int_{z} \int_{r} f(r, z)(2 \pi r) d r d z
$$

This integration may be compared with a general integration of a function $g(x, y)$ in Cartesian coordinates as it is carried out in SUTRA methodology:

$$
R_{c}=\int_{y} \int_{x} g(x, y) B(x, y) d x d y
$$


Integrals $R_{r}$ and $R_{c}$ are exactly analogous if: $x=r, y=z$, and

$$
B(x, y)=2 \pi r
$$

Thus, by a simple redefinition of coordinate names, and by setting the mesh thickness, B, at each node, equal to the circumference of the circle it would sweep out when rotated about the $r=0$ axis of the cylinder $\left(B_{1}=2 \pi r_{i}\right)$, the SUTRA simulation is converted exactly to radial coordinates. Figure 5.1 shows a mesh and the volume it sweeps out when in radial coordinates. Each element becomes a three-dimensional ring when used in radial coordinates.

\section{3 Pinch Nodes}

Pinch nodes are employed to ease mesh design when large changes in the density of elements are desired over relatively short distances. See Figure 5.2, where pinch nodes are indicated by open nodal dots. This would aid in design of a mesh, for example, in which a large model region is required in order to properly simulate the ground-water flow system. However, only a small portion of this region need be simulated with transport. The fine mesh required in the transport region can be quickly coarsened to the region where only flow is of interest.

Unfortunately, use of pinch nodes tends to increase the band width of the simulation problem although it can significantly decrease the number of nodes in a simulation. Thus with a band-width matrix equation solver, as employed by SUTRA, the use of pinch nodes in a mesh does not always lead to an advantage of decreased computational time. The pinch node option is included, however, as the solver is modular and may be replaced by non-band-width-dependent methods. 


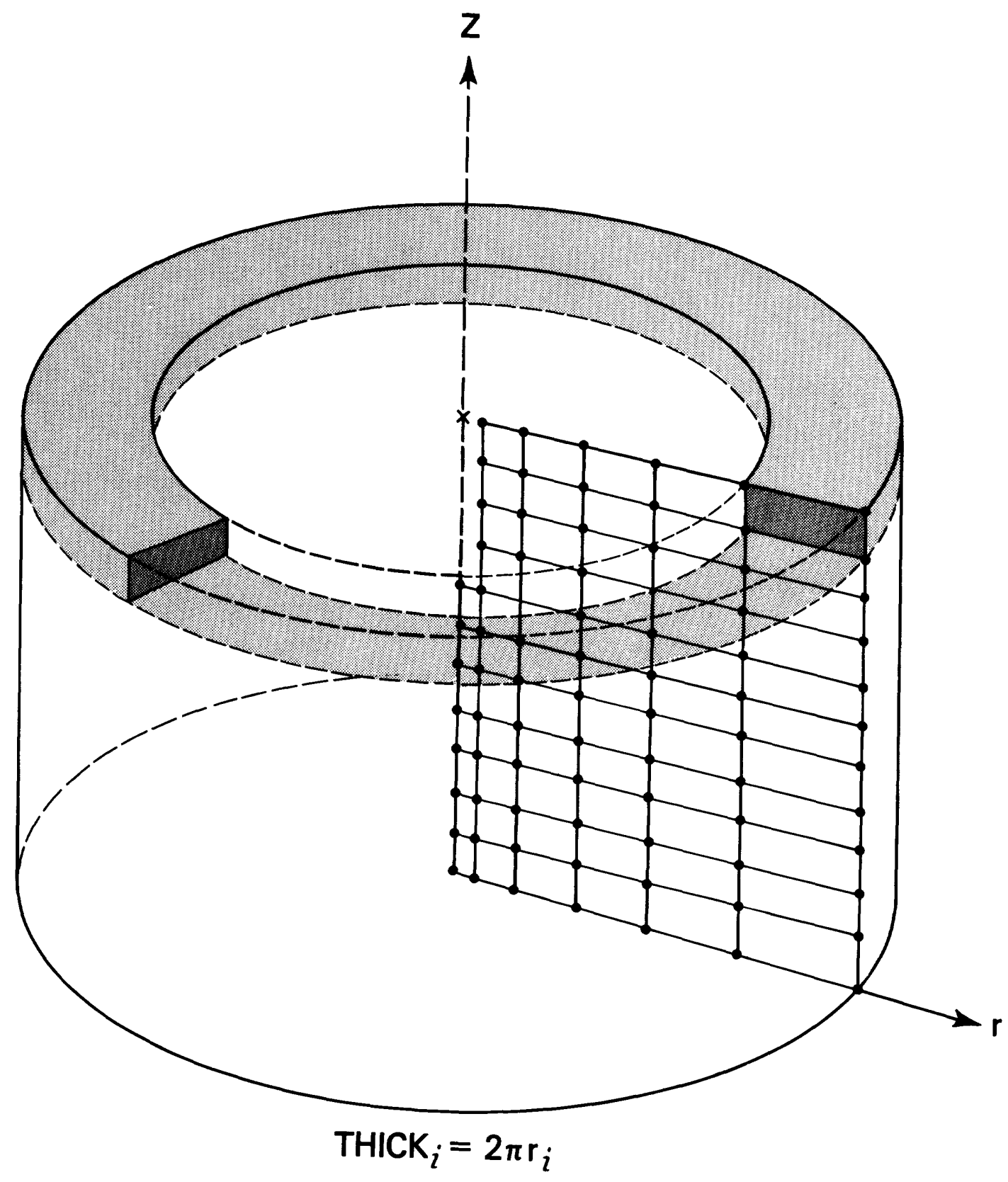

Figure 5.1

Finite-element mesh in radial coordinates. 


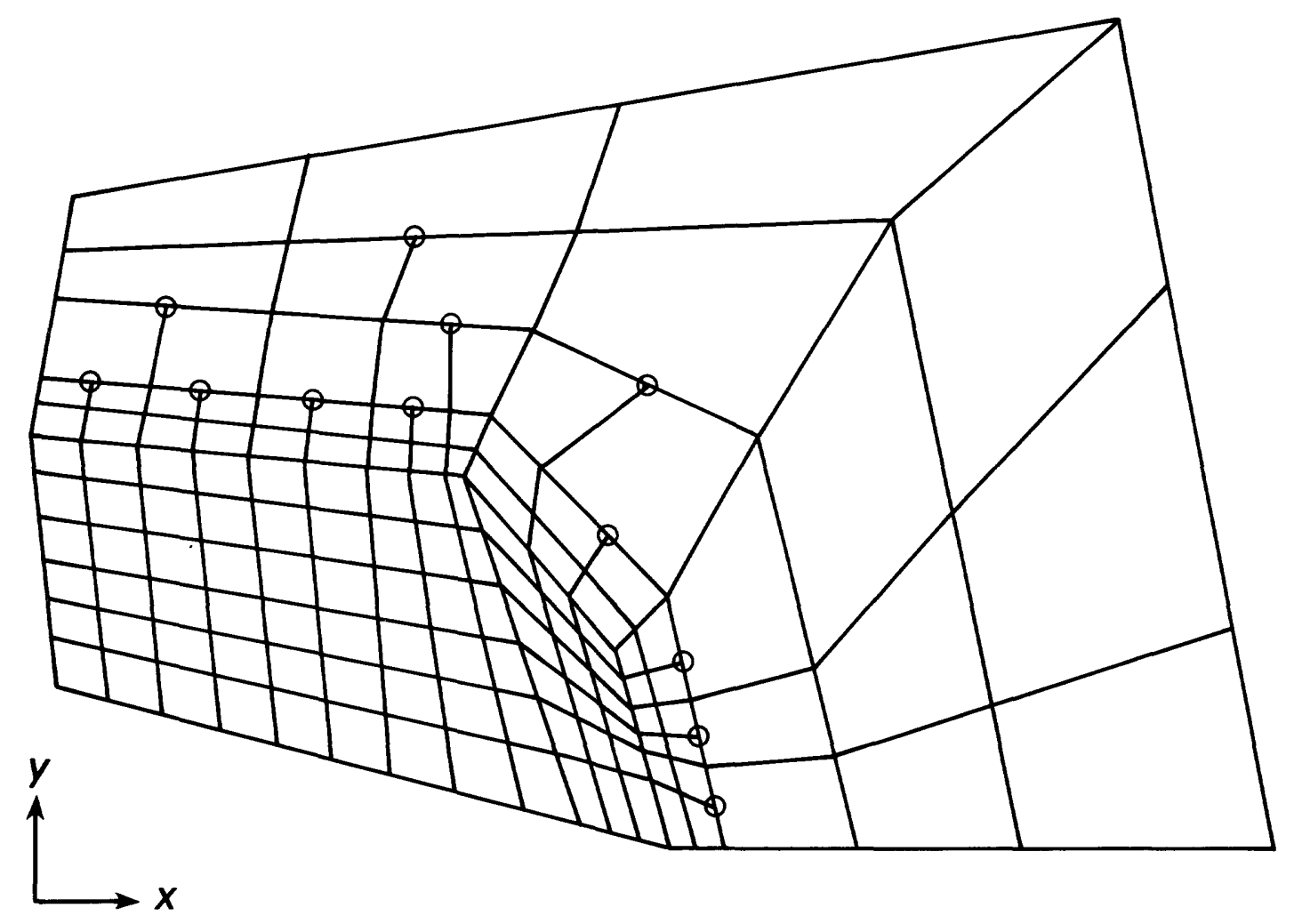

Figure 5.2

Finite-element mesh with pinch nodes. 
A pinch node is defined as a node in an element which is located at the mid-point of an element side, as shown in Figure 5.3. Each element has only four real nodes (at the corners) and four basis fuctions associated with these nodes. The pinch node has no basis function assigned to it in the element in which it appears on an element side. Values of variables and coefficients at the pinch node are determined as the average of the values of the real nodes at the end of the element side upon which the pinch node resides. Thus, no sources, sinks, or boundary conditions may be specified at a pinch node. The numerical solution at a pinch node depends entirely on the two nodes at the ends of its side.

Pinch nodes are handled by SUTRA as follows: All elementwise calculations are carried out as though a pinch node were a real node. In fact, each pinch node appears as a corner node in one or more elements. No special treatment is given pinch nodes through the entire matrix assembly process, and they enter the matrix through usual elementwise and nodewise calculations.

Just before solution of the matrix equation, pinch-node conditions are imposed on the matrix equation. For the pinch node, $k$, the right hand side of the equation for node $k$ is set to zero. The row of the final coefficient matrix for node $k$ is changed to all zeroes, except for two coefficients. These are in the two matrix columns related to the nodes at the ends of the element side upon which pinch node $k$ resides. They are set to a value, 0.50 , and the coefficent on the matrix diagonal (with subscript, kk) is set to a value, -1.0 . This sets an equation for pinch node $k$ as follows:

$$
\begin{aligned}
& p_{k}^{n+1}=\frac{1}{2}\left(p_{r}^{n+1}+p_{s}^{n+1}\right) \\
& v_{k}^{n+1}=\frac{1}{2}\left(v_{r}^{n+1}+v_{s}^{n+1}\right)
\end{aligned}
$$




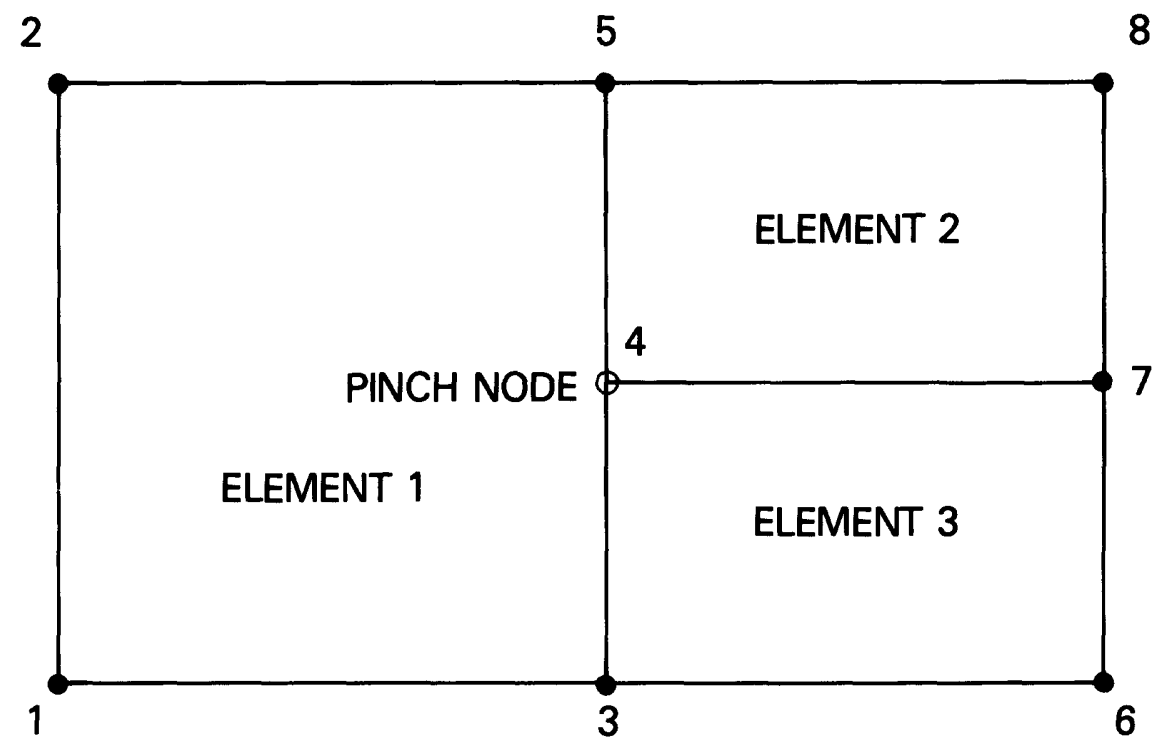

Figure 5.3

Detail of mesh with a pinch node. 
where subscripts $r$ and $s$ refer to the nodes at the ends of the pinch node element side.

Pinch nodes are specified in the data set containing the nodal incidence list for elements and the order of specification is related to the local element node numbering scheme as defined by Figure 5.4 .

\subsection{Solution Sequencing}

On any given time step, the matrix equations are created and solved in the following order: (1) the matrix equation for the fluid mass balance is set up, (2) the transport balance matrix equation is set up, (3) pressure is solved for, and (4) concentration or temperature are solved for. Both balances are set up on each pass such that the elementwise calculations only need be done once per pass. However, SUTRA allows the $p$ or $U$ equation to be set up and solved only every few time steps in a cyclic manner based on parameters NPCYC and NUCYC. These values represent the solution cycle in time steps. For example, setting up and solving for both $p$ and $U$ each time step $($ NPCYC $=$ NUCYC=1):

time step: 1234567 . . .

solve for: $\left\{\begin{array}{lllllll}p & p & p & p & p & p & p \\ U & U & U & \cup & U & U & U\end{array}\right.$

or solving for p every third time step and for $U$ each time step (NPCCYC=3 and NUCYC=1):

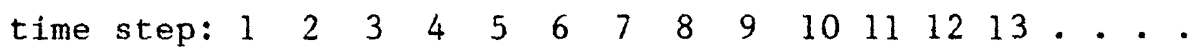

solve for: $\left\{\begin{array}{lllllllllllllllll}p & \cdot & p & \cdot & \cdot & p & \cdot & \cdot & p & \cdot & \cdot & p & \cdot \\ U & U & U & U & U & U & U & U & U & U & U & U & U\end{array}\right.$

However, either of $\mathrm{p}$ or $\mathrm{U}$ must be solved for on each time step and therefore 


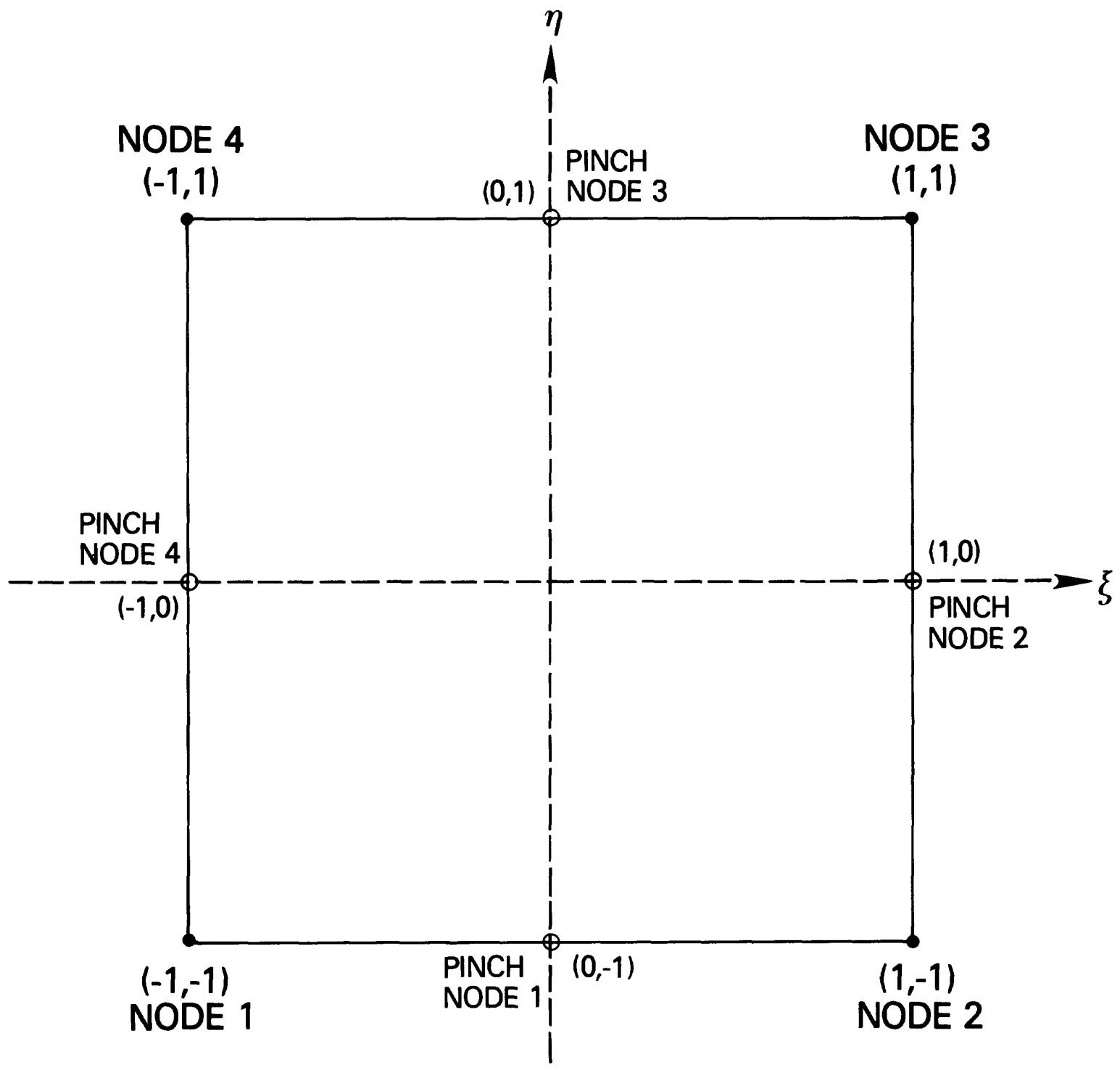

Figure 5.4

Finite element in local coordinates $(\xi, n)$ with pinch nodes. 
either NPCYC or NUCYC must be set to one.

For a simulation with steady state flow, the sequencing is:

time step: $0 \begin{array}{llllllll}0 & 1 & 2 & 3 & 4 & 5 & . & .\end{array}$

solve for: $\left\{\begin{array}{llllll}p & \cdot & \cdot & \cdot & \bullet \\ U & \cup & \cup & \cup & \cup & U\end{array}\right.$

For steady flow and transport:

time step: $0 \quad 1$

solve for: $\begin{cases}p & \text {. } \\ \cdot & \mathrm{U}\end{cases}$

The only exception to the cycling is that for non-steady cases, both unknowns are solved for on the first time step, as shown in the case for NPCYC=3, NUCYC=1, above.

It is computationally advantageous to allow a matrix equation solution for $U$ by back-substitution only, saving both equation construction and matrix decomposition steps. This is begun on the second time step solving for $U$ only after the step on which both $p$ and $U$ are solved for. In order to do this the matrix coefficients including the time step must remain constant. Thus, nonlinear variables and fluid velocity are held constant with values used on the first time step for $U$ after the step for $p$ and $U$. For example, when NPCYC=1, NUCYC=6:

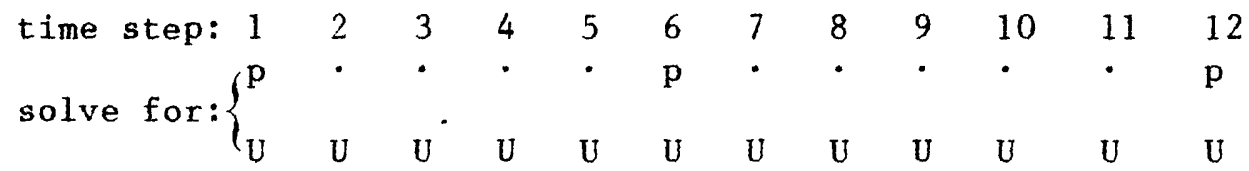
|constant values | constant values |

I back

substitute|

$\left\{\begin{array}{l}\text { back } \\ \text { substitute }\end{array}\right.$ 
A pressure-only solution may be obtained with $\mathrm{NPCYC} \approx 1$, and $\mathrm{NUCYC}=$ (number larger than the number of time steps). Note that $p$ and $U$ solutions must be set to occur on time steps when relevant boundary conditions, sources or sinks are set to change in value.

\subsection{Velocity Calculation for Output}

The velocities employed in the numerical solution of fluid mass, and solute mass or energy balances are those calculated at the Gauss points in each element (as described in section 4.6 "Consistent Evaluation of Fluid Velocity.") For purposes of output, however, only one velocity value per element is made available. This is the velocity at the element centroid as shown in Figure 5.5. The centroid is defined as the point in the element where the lines connecting the mid-point of opposite sides intersect.

The velocity at the centroid of an element is calculated by taking the average of the four global $x$-components of velocity at the Gauss points, as well as the average of the four global y-components of velocity at the Gauss points, and by constructing a velocity vector from these averaged components. This process gives the "true" velocity at the centroid that would be calculated employing the consistent velocity approximation evaluated at this point in the element. This may be seen by setting $\xi=n=0$ in (4.104) and (4.105).

\subsection{Budget Calculations}

A fluid mass and solute mass or energy budget provides information on the quantities of fluid mass and either solute mass or energy entering or exiting 


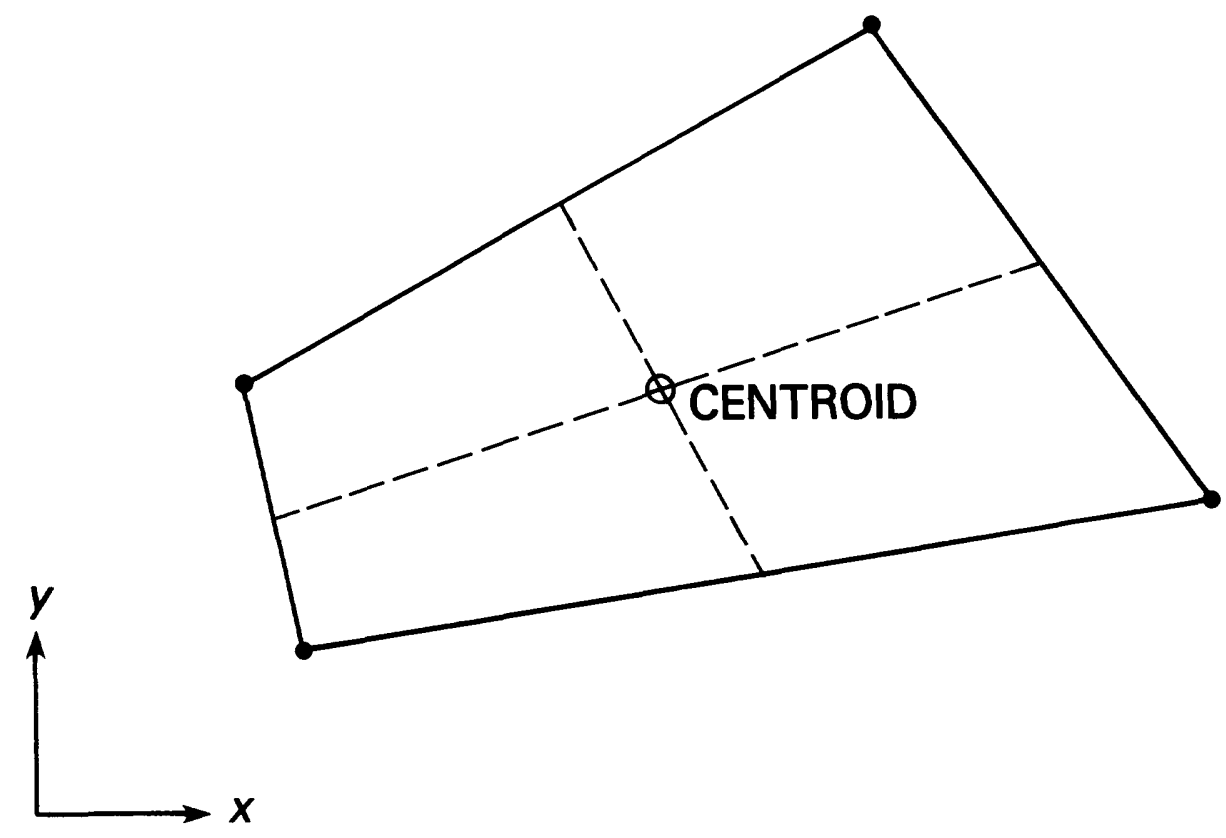

Figure 5.5

Finite element in global coordinates $(x, y)$ with element centrotd. 
the simulated region. It is not intended as a check on numerical accuracy, but rather as an aid in interpreting simulation results.

The fluid budget is calculated based on the terms of the integrateddiscretized fluid mass balance, (4.52), as approximated in time according to (4.65). After the solution to a time step makes available $p_{1}^{n+1}$ and $v_{1}^{n+1}$, the time derivatives of these, $\frac{d p}{d t} i$ and $\frac{d U}{d t} i$, are calculated according to $(4.57)$ and (4.91).

The total rate of change in stored fluid mass in the region due to pressure changes over the recent time step is:

$$
\sum_{i=1}^{N N} A F_{i}^{n+1} \frac{d p_{i}}{d t} \quad[M / s]
$$

where $A F_{1}$ is defined in (4.53), and the total rate of change in stored fluid due to changes in concentration or temperature is:

$$
\sum_{i=1}^{N N} C F_{i}^{n+1} \frac{d U_{i}}{d t} \quad[M / s]
$$

where $\mathrm{CF}_{1}$ as defined in (4.54).

The sum of (5.11) and (5.12) gives the total rate of change of fluid mass in the entire region.

Fluid sources, $Q_{i}^{n+1}$, may vary with time and those that do vary are reported by the budget at each source node. The sum of $Q_{i}^{n+1}$ :

$$
\sum_{i=1}^{N N} Q_{i}^{n+1} \quad[M / s]
$$

gives the total rate of fluid mass change due to all sources and sinks of fluid mass, as well as to specified fluxes across boundartes. Fluid sources due to specified pressure conditions, $Q_{B C}^{n+1}$, usually vary with time and are 
also reported by the budget at each node. This source is calculated at each node from (4.64). The sum of $Q_{B C}^{n+1}$ :

$$
\sum_{i=1}^{N N} Q_{B C_{1}^{n+1}}[M / s]
$$

gives the total rate of fluid mass change in the entire region due to inflows and outflows at all specified pressure nodes.

The sum of (5.13) and (5.14) should be close to the value given by the sum of (5.11) and (5.12). These may be expected to match better when iterations have been used and convergence achieved, as the budget is calculated for a time step with only one iteration with the $(n+1)$ time level values of non-linear coeffictents, and the solution was obtained with coefficients based on projected values of $p$ and $U$.

The solute mass or energy budget is calculated based on the terms of the integrated-discretized balance, (4.85), as approximated in the time according to (4.93). The total rate of change in stored solute mass or energy in the region over the recently computed time step is:

$$
\sum_{i=1}^{N N} \operatorname{AT}_{i}^{n+1} \frac{d U_{1}}{d t} \quad\left[M_{s} / s \text { or } E / s\right]
$$

where $\mathrm{AT}_{1}^{\mathrm{n}+1}$ is calculated from (4.86) using $\mathrm{U}_{1}^{\mathrm{n}+1}$ in all coefficients requiring a $U$ value (including adsorption isotherms for $c_{s} \kappa_{1}$ ). In reporting this portion of the budget, a separate value is given for the sum of the portion stemming from $\left(\varepsilon S_{w} \rho c_{w}\right)$ and for $(1-\varepsilon) \rho_{s} c_{s}$. The former sum relates to rate of solute mass or energy change in the fluid, and the latter relates to change in the solidimmobile portion. 
The total rate of first-order solute mass production in the fluid is calculated as:

$$
\sum_{i=1}^{N N} G T_{i}^{n+1} u_{i}^{n+1} \quad[M / s]
$$

and at the rate of first-order adsorbate production is calculated as:

$$
\sum_{i=1}^{N N} G_{s} T L_{i}^{n+1} U_{i}^{n+1}+G_{s} T R_{i}^{n+1} \quad[M / s]
$$

where $G T_{i}$ and $G_{s} T L_{1}$ and $G_{s} T_{i}$ are defined by $4.89 a, 4.89 b$ and $4.89 \mathrm{c}$ and all isotherms are based on $U_{1}^{n+l}$. Fluid and adsorbate rates are reported separately by the budget. These terms have no analogy for energy transport. The tęrms of zeroorder production of solute and adsorbate mass or energy production in the fluid and solid matrix are:

$$
\sum_{i=1}^{N N} \operatorname{ET}_{i}^{n+1} \quad\left[M_{s} / s \text { of } E / s\right]
$$

where $\mathrm{ET}_{i}$ is defined by $(4.90)$ and the fluid and immobile phase production rates are reported separately by the budget.

Solute mass and energy sources and sinks due to inflowing or outflowing fluid mass may vary with time and are reported by the budget at each fluid source node and at each specified pressure node. These are separately summed for the entire region:

$$
\begin{array}{ll}
\sum_{i=1}^{N N} Q_{i}^{n+1} c_{w} U_{i}^{* n+1} & {\left[M_{s} / s \text { or } E / s\right]} \\
\sum_{i=1}^{N N} Q_{B C}^{n+1} c_{w}{ }^{U_{B C}^{n+1}} & {\left[M_{i} / s \text { or } E / s\right]}
\end{array}
$$


Where $U_{i}^{*_{n}+1}$ and $U_{B C}^{n+1}$ take on the user-specified values of $U$ for fluid inflows, and the $U$ value of the ambient system fluid for outflows.

These sums give the total rate of change of solute mass or energy in the entire system due to these fluid sources and sinks.

Finally the diffusive-dispersive sources of solute mass or energy are summed for the entire system and are also reported by node as they may vary with time:

$$
\sum_{i=1}^{N N} \psi_{I N_{i}}^{n+1} \quad\left[M_{s} / s \text { or } E / s\right]
$$

The sum of $(5.16 a),(5.16 b),(5.17),(5.18),(5.19)$ and $(5.20)$ should be close to the value given by (5.15). These values may be expected to match best when iterations have been used and convergence achieved, as the budget. is calculated for a time step with only one iteration with all information at the $(n+1)$ time level, and the solution was obtained using non-linear coefficients based on projections of $p$ and $U$.

\subsection{Program Structure and Subroutine Descriptions}

SUTRA is structured in a modular, top-down programming style that allows for code readability, ease in tracing logic, and hopefully, ease in eventual modifications. Each subroutine carries out a primary function that is clearly distinguished from all other program functions. User-required program changes are limited to: 1) dimensioning three storage arrays in the main routine, and 
2) coding portions of a subroutine which is used to control time-dependent sources and boundary conditions (when they are used) and a subroutine which sets the unsaturated flow functions when unsaturated flow is simulated. The code consists of approximately 3000 statements and includes one main program and 24 subroutines. The program is commented to aid in tracing logic.

SUTRA is written in FORTRAN-77; however, few structures are used which are not compatible with FORTRAN-66. Modifications of the code required to compile in FORTRAN-66 would not be major.

The code runs accurately when it employs "double-precision" real variables ( 64 bit words with 47 bit mantissa) with a precision of about 15 significant figures, and 32 bit word integer variables. Should the code require modification to run on machines with other word lengths or other bit to byte ratios, the number of significant figures in a real variable should be preserved, if not increased.

Input and output is also somewhat modularized. Input is through Fortran unit numbers 5 and 55 . Unit-55 contains only data on initial conditions for a simulation at the nodes for $p$ and $U$. Unit -5 contains all other data required for a simulation. Output is to Fortran unit numbers 6 and 66 . Unit-66 receives the result of the final time step in a format equivalent to that of Unit-55, for later use as the intial conditions file if the simulation is to be restarted. Unit-6 receives all other simulation output usually to be printed on a line printer (as shown in Figure 5.6).

The main logic flow of the program is straightforward. A schematic diagram of the code is shown in Figure 5.7. The main routine sets up dimensions and calls the main control routine, SUTRA, which cycles the program tasks by calling most of the remaining subroutines in sequence. Subroutines are named to describe their main function. A description of each subroutine is given in the following sections. 


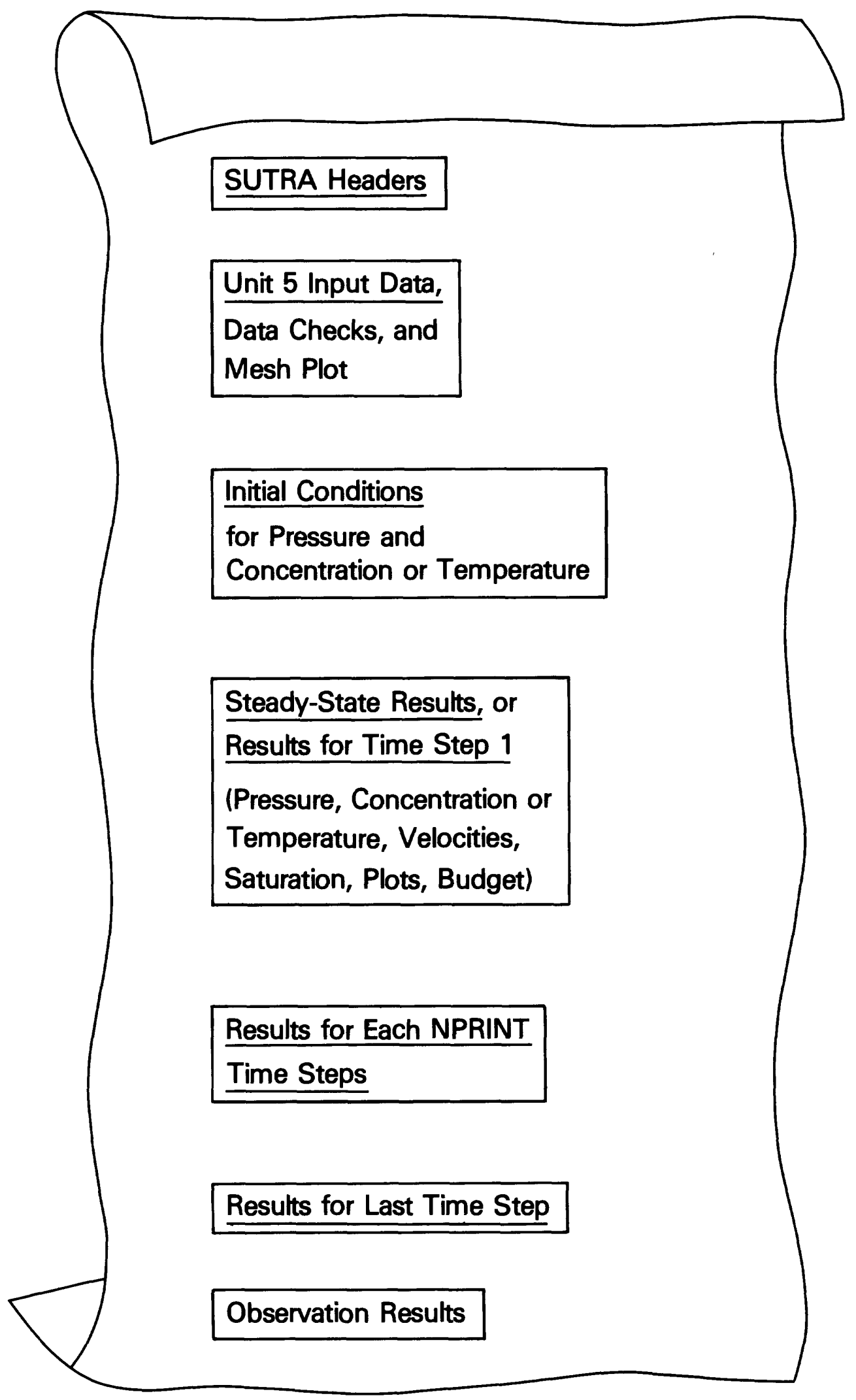

Figure 5.6

Schematic of SUTRA output. 


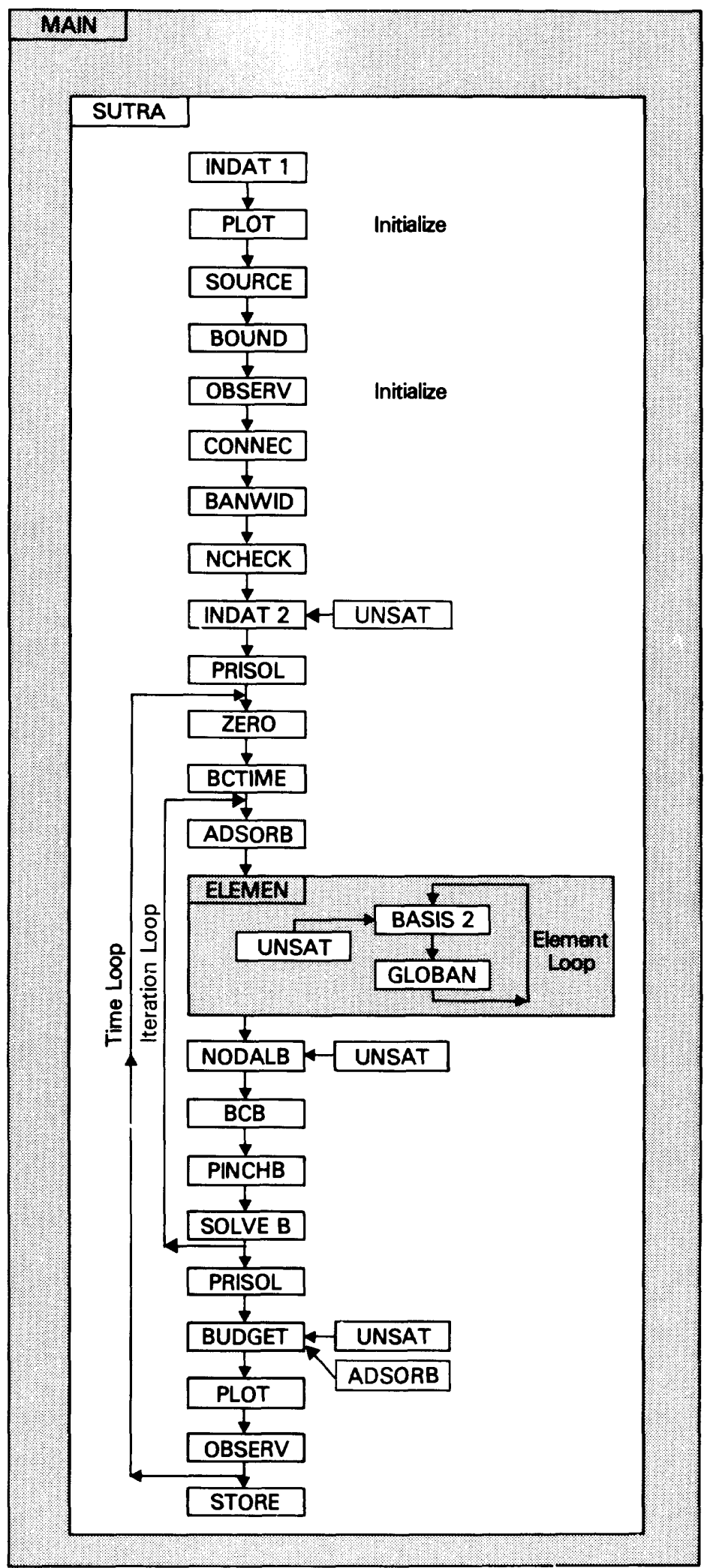

Figure 5.7

SUTRA logic flow. 


\section{Main Program}

\section{-Purpose:}

1) To dimension and allocate space for the main storage arrays.

2) To divide the storage arrays into their component arrays. (Set up pointers.)

3) To start and stop the simulation.

\section{-Ca11s to:}

SUTRA

\section{-Description:}

The main routine has three arrays that must be user-dimensioned: RM, RV and IMV. These are used for dynamic storage allocation and they contain almost all of the values required for SUTRA simulation. RM contains real matrices, RV contains real vectors, and IMV contains $\underline{\text { integer matrices }}$ and vectors. The dimensions required for RM, RV and IMV are RMDIM, RVDIM and IMVDIM, where the actual values are given in section 7.3 , "Program Dimensions."

After reading the actual Unit-5 input data for the variables listed above, the main routine sets up pointers which allocate the correct amount of space for each of the component arrays contained in the storage arrays. The pointers point to the position in the storage array of the starting element of each component array. The starting elements are passed to subroutine SUTRA as calling arguments Additional arrays which may be required by any modifications to SUTRA are added a1 
the bottom of the appropriate pointer lists in the call statement and in the pointer calculations. The values of NNV or NEV may need to be incressed, and the commented record of calculation of dimensions of storage arrays at the top of the routine should be increased accordingly.

\section{Subroutine SUTRA}

\section{-Purpose:}

1) To act as primary control on SUTRA simulation, cycling both iterations and time steps.

2) To sequence program operations by calling subroutines for input, output and most program calculations.

3) To carry out minor calculations.

\section{-Called by:}

Main routine

\section{-Calls to:}

INDAT 1, PLOT, SOURCE, BOUND, OBSERV, CONNEC, BANWID, NCHECK, INDAT2, PRISOL, ZERO, BCTIME, ADSORB, ELEMEN, NODALB, BCB, PINCHB, SOLVEB, BUDGET, STORE.

\section{-Description:}

Subroutine SUTRA receives pointers for all actual arrays and vectors which are dynamically allocated space by the main routine. These arrays are 
dummy dimensioned to actual sizes required for the simulation. Subroutine SUTRA initializes some constants and directs the reading of Unit-5 input data by calls to INDAT1, PLOT, SOURCE, BOUND, OBSERV, and CONNEC. It calls for band-width calculation (BANWID) and mesh data checks (NCHECK). The call to PLOT (after INDAT1) also plots the mesh. Then subroutine SUTRA directs a call to INDAT2 to read initial conditions from Unit-55, and calls PRISOL to print the initial conditions.

The subroutine decides on cycling parameters if steady state pressures will be calculated, and calls ZERO to inftialize arrays. For transient pressure solution steps, time-step cycling parameters are set and a decision is made as to which (or both) of $p$ and $U$ will be solved for on this time step. The decision depends on NPCYC and NUCYC, and subroutine SUTRA sets the switch, ML, as follows:

$$
M L= \begin{cases}0 & \text { solve for both } p \text { and } U \\ 1 & \text { solve for } p \text { only } \\ 2 & \text { solve for } U \text { only }\end{cases}
$$

The switch for steady state flow is ISSFLO, which is set as follows:

$$
\text { ISSFLO }= \begin{cases}0 & \text { steady flow not assumed } \\
1 & \text { steady flow assumed, before pressure time step } \\
2 & \begin{array}{l}
\text { steady flow assumed, after beginning of pres- } \\
\text { sure time step }
\end{array}\end{cases}
$$

Note that time step number, IT, is set to zero for the steady p solution, and increments to one for the first transport time step.

Subroutine SUTRA increments the simulation clock, TSEC, to the time at the end of the new time step, and shifts new vectors to previous level vectors which begins the time step. BCTIME is called to set time-dependent sources and boundary conditions if such exist. ADSORB is called if sorption is required. The element 
by element calculations required to construct the matrix equations are carried out by a call to ELEMEN. NODALB is called to carry out nodewise and cellwise calculations for the global matrices. $\mathrm{BCB}$ is called to modify the matrix equations for boundary conditions, and PINCHB is called to implement any pinch nodes in the matrices.

SOLVEB is called for $p$ and or $U$ solution (depending on the value of ML), and if iterations are underway, convergence is checked. If iterations are continued, control switches back to the step which shifts new to old vectors, and the sequence of calls is repeated. If no more iterations are required, SUTRA may call PRISOL and PLOT to print and plot results if these are requested on the present time step. OBSERV is called to remember values at observation nodes if any exist. BUDGET is called if requested output should occur this timê step.

If more time steps are to be undertaken, control switches back to the step which initializes arrays, and continues down from that point. If the simulation is complete, STORE is called if the store option has been selected to set up a restart file in Unit-66. OBSERV is called to print any observations that were taken. At this point, control returns to the main routine.

\section{Subroutine INDAT1}

\section{-Purpose:}

1) To read simulation and mesh data from the Unit-5 data file, and print out this information.

2) To intialize some variables and carry out minor calculations.

\section{-Called by:}

SUTRA 


\section{-Description:}

INDAT1 reads a portion of the Unit-5 input data file, ending with the elementwise data set. Most information is printed on the Unit-6 data file after reading, the amount of output depends on the user choice of long or short output format. Scale factors are multiplied with appropriate input data. Calculations are carried out for a thermal conductivity adjustment and for determination of $\underline{\underline{k}}$ matrix components of $\mathbf{k}$ in each element from $k_{\max }$, $k_{\min }$, and $\theta$.

\section{Subroutine PLOT}

\section{-Purpose:}

To provide maps on printer output paper of the finite-element mesh, pressure values at nodes, and $U$ values at nodes.

\section{-Ca1led by:}

SUTRA

\section{-Description:}

PLOT is called once for initialization to read plot set-up data from Unit-5, and to set up a plot of the mesh. PLOT is then called to plot the mesh. PLOT is called on each time step in which output is produced, once each for $p$ for $U$, if these plots have been requested.

The printer plot efther fits the longer plot direction across the output page, or along the output page, depending on the user choice. The plot is self-scaled to page size, and different scales may be chosen by the routine along and across the page. A blank border one tenth of the maximum $x$ and $y$ 
range surrounds the plotted region. Three figures of the solution value are plotted at each nodal location.

PLOT begins by ordering the nodes by plot line and saves the ordered results in $X X, Y Y$, and INDEX during the initialization call. Certain nodes fall in each line of the plot. During actual plotting, PLOT starts with nodes in the top plot line and places the values to be plotted in the proper position in the ine. The line is then printed. This is repeated for each Ine of the plot.

\section{Subroutine SOURCE}

-Purpose:

1) To read source node numbers and source values for fluid mass sources and boundary fluxes and for diffusive and productive $U$ sources, as well as fluxes of $\mathrm{U}$ at boundaries; to check the data, and to print information.

2) To set up pointer arrays which track the source nodes for the simulation.

-Called by:

SUTRA

-Description:

SOURCE reads and organizes, checks and prints information on source nodes for fluid mass, and for sources of solute mass or energy. Fluid mass source Information read is node number, mass source rate, and $U$ value of any Inflowing 
fluid at this node. If there are NSOP fluid source nodes, the node numbers become the first NSOP values in vector IQSOP. The rates are entered in the element corresponding to the nodes at which they are defined in vectors QIN and UIN which are NN long. The source information for $U$ read is node number and solute mass or energy source rate. If there are NSOU source nodes for $U$, the node numbers become the first NSOU values in IQSOU. Vector QUIN is NN Iong and contains the source rates in numerical order by node. Counts are made of each type of source and are checked against NSOP and NSOU for correctness. A blank (zero) node number ends the data set for QIN and then for QUIN. One blank element is left at the end of IQSOP and IQSOU so that a dimension of one is obtained even when no source nodes exist. These arrays are used primarily in NODALB and BUDGET.

Subroutine BOUND

-Purpose:

1) To read specified pressure node numbers and pressure values, check the data, and print information.

2) To read specified concentration or temperature node numbers and the values, to check the data, and print information.

3) To set up pointer arrays which track the specified $p$ and $U$ nodes for the simulation.

\section{-Called by:}

SUTRA 


\section{-Description:}

BOUND reads and organizes, checks and prints information on specified $p$ nodes and for specified $U$ nodes. Pressure information read is node number, pressure value and $U$ value of any inflow at this node. If there are NPBC specified pressure nodes, the above information becomes the first NPBC values in vectors $I P B C, P B C$ and $U B C$. Specified $U$ information read is node number and $U$ value. If there are NUBC specified concentration nodes, the above information begins in the (NPBC+1) position of IUBC and UBC, and ends in the (NUPBC+NUBC) position of UBC and IUBC. This is shown below:

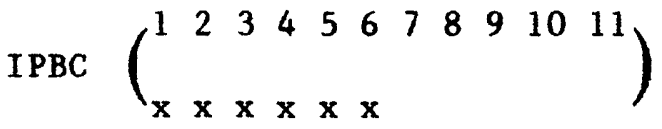

$$
\begin{aligned}
& \operatorname{PBC}\left(\begin{array}{lllllll}
1 & 2 & 3 & 4 & 5 & 6 \\
x & & & & & &
\end{array}\right. \\
& \operatorname{UBC}\left(\begin{array}{llllllllllll}
x & x & x & x & x & x & y & y & y & y & \\
1 & 2 & 3 & 4 & 5 & 6 & 7 & 8 & 9 & 10 & 11
\end{array}\right)
\end{aligned}
$$

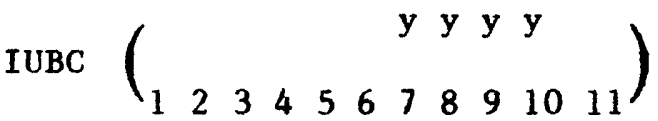

where $x$ refers to specified $p$ information, and $y$ refers to specified $U$ information.

Counts are made of each type of specification and are checked against NPBC and NUBC for correctness. A blank (zero) node number ends the data set for $p$ and then for $U$. One blank element is left at the end of each of these arrays in case there are no specified $p$ or $U$ nodes. The first NPBC elements of IUBC and UBC are blank. These arrays are used primarily by subroutines BCB and BUDGET. 


\section{-Purpose:}

1) To save $p$ and $U$ values at chosen observation nodes as a function of the time.

2) To report the observations after the simulation has been completed.

\section{-Called by:}

SUTRA

\section{-Description:}

On an initialization call from SUTRA, OBSERV reads observation node numbers and observation cycle, NOBCYC, in time steps from Unit-5 and outputs these values. Every NOBCYC time steps, when SUTRA calls OBSERV after a solution, OBSERV saves the current elapsed time, and $p$ and $U$ values at all observation nodes. When the simulation is completed, OBSERV is called to output the stored lists of: time step, elapsed time, $p$, and $U$.

\section{Subroutine CONNEC}

\section{-Purpose:}

1) To read, output, and organize node incidence data.

2) To read, output, and organize pinch-node incidence data.

\section{-Called by:}

SUTRA 
-Description:

CONNEC reads the nodal incidence list which describes how nodes are connected. The data is organized as array, IN, which contains the counterclockwise-ordered set of four node numbers in each element in order of element number. Thus the ninth through twelfth values in IN are the four nodes in element number three.

For an element with one or more pinch nodes, the pinch node numbers are entered in the first column of array IPINCH, and the node numbers at the ends of the side on which the pinch node resides are entered in columns two and three of IPINCH.

IPINCH is used in subroutine PINCH, and NCHECK. IN is used in BANWID, ELEMEN, and GLOBAN.

\section{Subroutine BANWID}

\section{-Purpose:}

To calculate the band width of the mesh and check the value spectfied by the user.

-Called by:

SUTRA

\section{-Description:}

BANWID checks the array, IN, in all elements for the maximum difference in node numbers contained in an element. This value, NDIFF, is used to calculate 
actual bandwidth, NBL, which is compared with the user-specified, NBI. IF NBL>NBI, the values are printed and the simulation is halted for corrections.

\section{Subroutine NCHECK}

\section{-Purpose:}

To check that pinch nodes are neither assigned sources, nor have specified p or $\mathrm{v}$.

-Called by:

SUTRA

\section{-Description:}

NCHECK compares the list of pinch node numbers with the list of source nodes, specifled pressure nodes and specified $U$ nodes. Any matches result in a printed report and the simulation halts.

\section{Subroutine INDAT2}

\section{-Purpose:}

1) To read Initial conditions from Unit-55.

2) To initialize some arrays.

-Called by:

SUTRA 
-Calls to:

UNSAT

-Description:

INDAT2 is the second major input data routine. It reads the data file, Unit-55, which contains initial conditions for $p$ and $U$. The warm-start section reads initial conditions and parameter values of a previous time step, all of which must have been stored by subroutine STORE on a previous simulation. For a cold-start, INDAT2 reads only initial $p$ and initial $U$. INDAT2 calls UNSAT for calculation of initial saturation values, on a cold start.

\section{Subroutine PRISOL}

\section{-Purpose:}

To output the following to Unit-6:

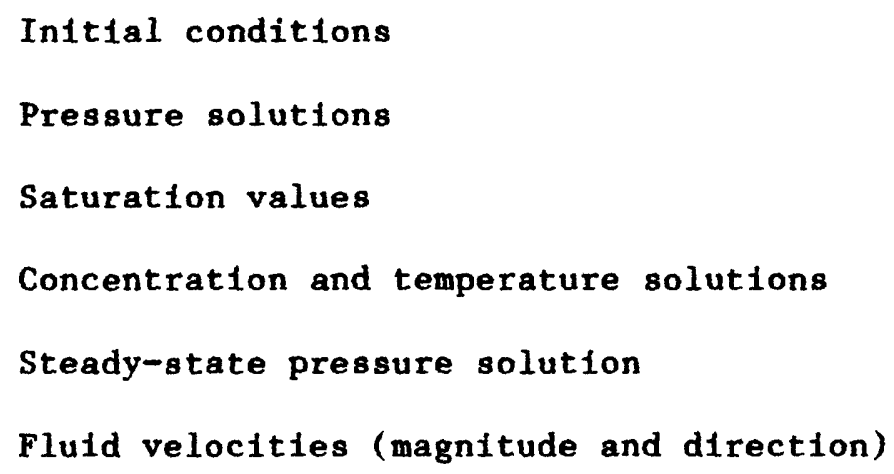

-Called by:

SUTRA 
-Description:

PRISOL is the main SUTRA output routine and is used for printing solutions.

Subroutine ZERO

-Purpose:

To fill a real array with a constant value.

-Called by:

Various routines

-Description:

ZERO fills an entire array with a specified value. This routine may be replaced with a machine-dependent assembly language routine in order to maximize efficiency.

Subroutine BCTIME

-Purpose:

A user-programmed routine in which time-dependent sources and boundary conditions are spectfled.

-Called by:

SUTRA 


\section{-Description:}

BCTIME is called on each time step when a time-dependent source or boundary condition is specified by the user. It allows the value of a source or boundary condition to be changed on any or all time steps.

BCTIME is divided into four sections. The first section allows the user to specify either time-dependent pressure and concentration or temperature of an inflow, or both, at spectfied pressure nodes (PBC or UBC). The second section allows user specification of time-dependent $\mathrm{U}$ at specified concentration/ temperature nodes. The third section allows user specification of time-dependent fluid source or source concentration/temperature. The fourth section allows userspecification of time-dependent solute mass or energy source.

The current time step number, IT, and current time (at the end of the present time step) in various units are available for use in the user-supplied programming. The user may program in any convenient way through data statements, calls to other programs, logical structures, 'read' or 'write' statements, or other preferred methods of specifying the time variability of sources or specified $p$ and $U$ conditions. More information may be found in section 7.5 , "UserSupplied Programming."

\section{Subroutine ADSORB}

\section{-Purpose:}

To calculate and supply values from adsorption isotherms to the simulation. 
-Called by:

SUTRA

\section{-Description:}

ADSORB calculates the sorption coefficient, $\kappa_{1}^{n+1},($ called $\operatorname{CS} 1$ ), and also $s_{L}$ (called SL) and $s_{R}$ (called $S R$ ) and $S R$ which are úsed in calculating) adsorbate concentrations, $U_{s}$, depending on the particular isotherm chosen: linear, Freundlich or Langmuir. The calculations are based on the description given in section 4.7, "Temporal Evaluation of Adsorbate Mass Balance." ADSORB is called once per time step for $U$, when sorption is employed in the simulation.

\section{Subroutine ELEMEN}

\section{-Purpose:}

1) To carry out all elementwise calculations required in the matrix equations .

2) To calculate element centroid velocities for output.

-Called by:

SUTRA

\section{- Calls to:}

BASIS2, GLOBAN

\section{-Description:}

ELEMEN undertakes a loop through all the elements in a mesh. For each element, subroutine BASIS2 is called four times, once for each Gauss point. 
BASIS2 provides basis-function information, and values of coefficients and velocities at each Gauss point, all of which is saved by ELEMEN for use in calculations for the present element.

Gaussian Integration (two by two points) as described in section 4.3 , is carried out for each integral in the fluid mass balance ((4.55) and (4.56)), and for each integral in the unified energy and solute mass balance ((4.87) and (4.88)). The portion of cell volume within the present element for node $I$, $\operatorname{VOLE}(I)$, is calculated with the fluid balance integrals. The values of the integrals are saved either as four-element vectors or as four-by-four arrays. Separate (nearly duplicate) sections of the integration code employ either basis functions for weighting or asymmetric weighting functions.

The vectors and arrays containing the values of integrals over the present element are passed to subroutine GLOBAN in order to add them to the global matrix equation (assembly process).

\section{Subroutine BASIS2}

\section{-Purpose:}

To calculate values of basis functions, weighting functions, their derivatives, Jacobians, and coefficients at a point in a quadrilateral element.

\section{-Called by:}

ELEMEN 
- Calls to:

UNSAT

\section{-Description:}

BASIS2 recetves the coordinates of a point in an element in local coordinates $(\xi, \eta)$, denoted $(X L O C, Y L O C)$ In the routine. At this point, BASIS2 determines the following: values of the four basis functions and their derivatives in each local coordinate direction, elements of the Jacobian matrix, the determinant of the Jacobian matrix, elements of the inverse Jacobian matrix, and if required, four values of the asymmetric weighting function (one for each node) and their derivatives. Also, the derivatives are transformed to global coordinates and passed out to ELEMEN. Values of nodewise-discretized parameters are formed at this location in the element, as are values of local and global velocity. Values of parameters dependent on $p$ or $U$ are calculated at this location. Unsaturated parameters are obtained by a call to UNSAT. The calculations are based on sections, 4.1 "Basis and Weighting Functions", 4.2 "Coordinate Transformations," and 4.6 "Consistent Evaluation of Fluid Velocity."

\section{Subroutine UNSAT}

\section{-Purpose:}

A user-programmed routine in which unsaturated flow functions are specified.

\section{-Called by:}

INDAT2, BASIS2, NODALB, BUDGET

\section{-Description:}


UNSAT is called by INDAT2 to calculate initial saturations at nodes, by BASIS2 at each Gauss point in each element during numerical integration, by NODALB for each cell, and by BUDGET for each cell. It allows the user to specify the functional dependence of relative permeability on saturation or pressure, and the dependence of saturation on pressure. UNSAT is divided into three sections. The first section requires the user to specify the saturation-pressure (or capillary pressure) function. The second section requires the user to specify the derivative of saturation with respect to pressure. The third section requires the user to specify the relative permeability dependence on saturation or capillary pressure. INDAT2 requires only values of saturation, BASIS2 requires only values of saturation and relative permeability, NODALB and BUDGET require values of saturation and its pressure derivative. These calculations are controlled in UNSAT by the parameter IUNSAT which INDAT2 sets to a value of three, which BASIS2 sets to a value of two, and NODALB and BUDGET set to one. For simulation of purely saturated flow, IUNSAT is set to zero by INDAT1, and UNSAT is never called. The user may program these functions in any convenient way, for example, through data statements, calls to other programs, logical structures, 'read' or 'write' statements, or other preferred methods. More information may be found in section 7.5 , "User-Supplied Programming."

\section{Subroutine GLOBAN}

\section{-Purpose:}

To assemble elementwise integrations into global matrix form.

\section{-Called by:}

\section{ELEMEN}




\section{-Description:}

GLOBAN carries out the sum over elements of integrals evaluated over each element by ELEMEN as suggested by relation (3.23). Both the matrix and right side vector terms involving integrals in the solution equations $(4.65 \mathrm{~b})$ and (4.96b) are constructed.

\section{Subroutine NODALB}

-Purpose:

To calculate and assemble all nodewise and cellwise terms in the matrix equation.

-Called by:

SUTRA

-Calls to:

UNSAT

\section{-Description:}

NODALB undertakes a loop through all nodes in the mesh and calculates values of all cellwise terms. For each node, time derivatives and a fluid source are added to the fluid mass balance matrix equation. The time derivative as well as terms due to fluid sources production and boundary fluxes of $U$ are prepared and added to the solute mass/energy balance matrix equation. Subroutine 
UNSAT is called for unsaturated flow parameters. The terms added by NODALB may be described as the non-integral terms of (4.52) and (4.85) (except for the specified pressure terms.)

Subroutine BCB

-Purpose:

1) To implement specified pressure node conditions in the matrix equations.

2) To implement specified temperature or concentration node conditions In the matrix equations.

-Called by:

SUTRA

\section{-Description:}

The source terms involving $v_{1}$ in (4.52) are added to fluid balance matrix equation in order to obtain specifled $p$ nodes. The unified energy-solute mass balance is modified by the addition of a source, QPL, (calculated with the most recent $p$ solution by subroutine SUTRA) with concentration or temperature value, UBC.

For a specified $U$ node, the discretized balance equation is modified by zeroing the row of the U-matrix which gives the equation for the specified node. A one is placed on the diagonal and the specified U-value, UBC, is placed in the same row of the right side vector. 


\section{Subroutine PINCHB}

-Purpose:

To implement pinch-node conditions in both matrix equations.

-Called by:

SUTRA

-Description:

PINCHB undertakes a loop through all pinch nodes. For each pinch node, the appropriate row of each matrix (for $p$ and $v$ ) is zeroed, a one is placed on the diagonal, -0.5 is placed in the two columns corresponding to the side neighbors of the pinch node, and the corresponding element of the right side vector is zeroed.

\section{Subroutine SOLVEB}

\section{-Purpose:}

To solve a matrix equation with a non-symmetric banded matrix.

-Called by:

SUTRA 
-Description:

SOLVEB expects the matrix band as a vertical rectangular block with the main diagonal in the center column, and minor diagonals in the other columns. The upper left-hand corner and lower right-hand corner of the matrix is blank.

The first section of the routine carries out an LU decomposition of the matrix which is saved within the original matrix space. The second section of the routine prepares the right side for solution and carries out back-substitution with a given right side vector.

\section{Subroutine BUDGET}

\section{-Purpose:}

1) To calculate and output a fluid mass budget on each time step with output.

2) To calculate and output a solute mass or energy budget on each time step with output.

\section{-Called by:}

SUTRA

-Calls to:

UNSAT, ADSORB

\section{-Description:}

BUDGET calculates and outputs a fluid mass, solute mass or energy budget on each output time step for whichever of $p$ and/or $U$ are solved for on the justcompleted time step. The calculations are done as described in section 5.6 "Budget Calculations." 


\section{-Purpose:}

To store $p$ and $U$ results as well as other parameters on Unit-66 in a format ready for use as initial conditions in Unit-55. This acts as a backup for re-start in case a simulation is unexpectedly terminated before completion by computer malfunction.

-Called by:

SUTRA

\section{-Description:}

STORE is called upon completion of each time step of a simulation, if the storage option has been chosen. STORE writes the most recent solution for $p$ and $U$ at the nodes on a file, Unit-66, in a format exactly equivalent to that of input data file Unit-55. Information is also written which is used in a warm start (restart) of the simulation. The results of only the most recent time step are stored on UNIT-66 as STORE rewinds the file each time before writing. 
SUTRA SIMULATION EXAMPLES

175

(p.177 follows) 


\section{Chapter 6}

\section{Simulation Examples}

This chapter outlines a number of example simulations which serve to demonstrate some of the capabilities of SUTRA modeling. Some of the examples show results which are compared with analytical solutions or numerical solutions available in the literature. These results serve to verify the accuracy of SUTRA algorithms for a broad range of flow and transport problems. The other examples demonstrate physical processes which SUTRA may simulate in systems where no other solutions are available. A complete SUTRA Input data set and model output is provided for the example of section 6.3, "Radial Flow with Energy Transport," in Appendix B and Appendix C.

\subsection{Pressure Solution for Radial Flow to a Well \\ (Theis Analytical Solution)}

\section{Physical Set-up:}

A confined, infinite aquifer contains a fully penetrating withdrawal well. Fluid is pumped out at a rate, $Q_{\text {TOT }}$.

\section{Objective:}

To simulate transient drawdown in this system which should match the Theis solution. The Theis solution (Lohman, 1979) is given in terms of variables used in SUTRA by:

$$
s^{*}=\frac{Q_{\operatorname{TOT}^{\mu}}}{4 \pi \rho^{2} \Delta z k|g|} W(u)
$$

where $s^{*}$ is the drawdown, $W(u)$ is the well function of $u$, and 


$$
u=\frac{r^{2} \mu s_{o p}}{4 k t}
$$

where $r$ is the radial distance from the well to an observation point and $t$ is the elapsed time since start of pumping.

\section{Simulation Set-up:}

The mesh contains one row of elements with element width expanding by a constant factor, 1.2915, with increasing distance from the well; other mesh dimensions are $\Delta r_{\min }=2.5[\mathrm{~m}], \Delta \mathrm{r}_{\max }=25 .[\mathrm{m}], \mathrm{r}_{\max }=500 .[\mathrm{m}], \Delta z=1 .[\mathrm{m}]$. Mesh thickness at node $i$, is given by $B_{i}=2 \pi r_{i}$, which provides a radial coordinate system. The number of nodes and elements in the mesh are: $N N=54$, $\mathrm{NE}=26$. See Figure 6.1.

The initial time step is, $\Delta t_{0}=1 .[s]$, with time steps increasing by a factor, 1.5 , on each subsequent step.

One pressure solution is obtained per time step, solutions for concentration are ignored; the cycling parameters are: NPCYC $=1$, NUCYC $=9999$.

\section{Parameters:}

$$
\begin{array}{ll}
\mathrm{S}_{\text {op }}=1.039 \times 10^{-6}\left[\mathrm{~m} \cdot \mathrm{s}^{2} / \mathrm{kg}\right] & \varepsilon=0.20 \\
\alpha=1.299 \times 10^{-6}\left[\mathrm{~m} \cdot \mathrm{s}^{2} / \mathrm{kg}\right] & \mathrm{k}=2.0387 \times 10^{-10}\left[\mathrm{~m}^{2}\right] \\
\beta=4.4 \times 10^{-10}\left[\mathrm{~m} \cdot \mathrm{s}^{2} / \mathrm{kg}\right] & \rho=1000 .\left[\mathrm{kg} / \mathrm{m}^{3}\right] \\
|\underline{g}|=9.81\left[\mathrm{~m} / \mathrm{s}^{2}\right] & \\
Q_{\text {TOT }}=0.6284[\mathrm{~kg} / \mathrm{s}] \quad \text { (one-half at each well node) }
\end{array}
$$




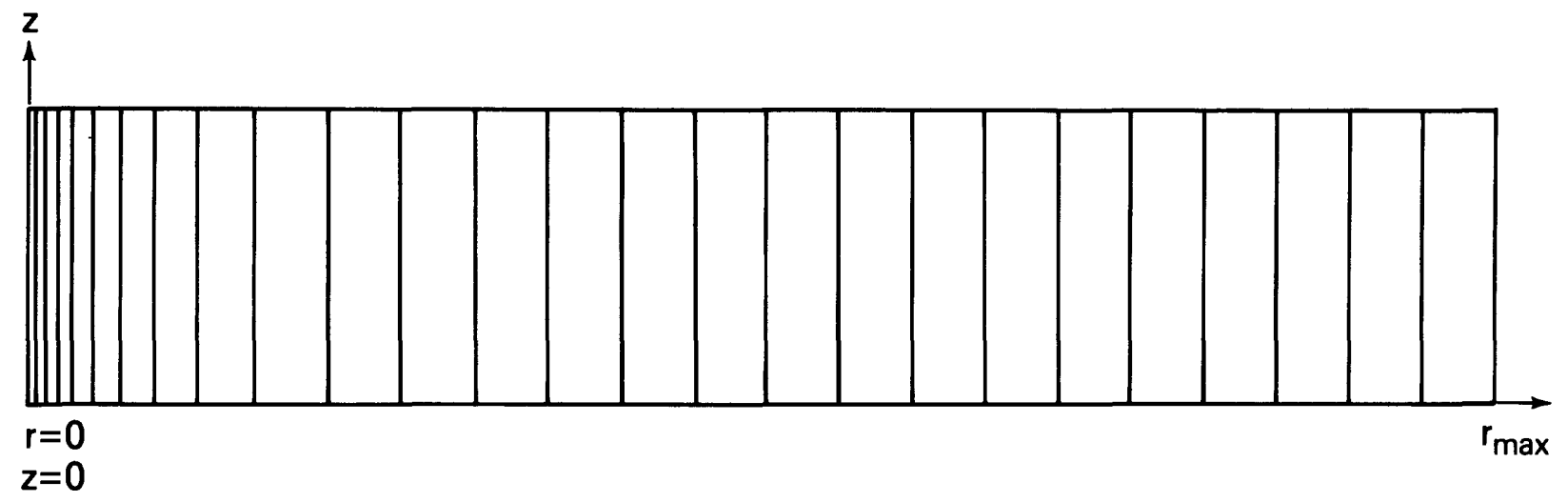

F1gure 6.1

Radial finite-element mesh for Theis solution. 


\section{Boundary Conditions:}

No flow occurs across any boundary except where hydrostatic pressure is specified at $r_{\max }$. At the top outside corner of the mesh, $r_{\max }$, pressure is held at zero. A sink is specified at $r=0$ to represent the well.

\section{Initial Conditions:}

Hydrostatic pressure with $p=0$ at the top of the aquifer is set initially.

\section{Results:}

SUTRA results are plotted for two locations in the mesh representing observation wells at $r=15.2852[\mathrm{~m}]$, and $r=301.0867[\mathrm{~m}]$. Both locations should plot on the same Theis curve. The match of SUTRA results between one and 6000 minutes with the Theis analytical solution shown in Figure 6.2 is good.

\subsection{Radial Flow with Solute Transport \\ (Analytical Solutions)}

\section{Physical Set-up:}

A confined infinite aquifer contains a fully penetrating infection well. Fluid is infected at a rate, QTOT, with a solute concentration, $c^{*}$, Into the aquifer initially containing fluid with solute concentration, $\mathrm{C}_{0}$. The fluid density does not vary with concentration.

\section{Objective:}

To simulate the transient propagation of the solute front as it moves radially away from the well. The concentrations should match the approximate 


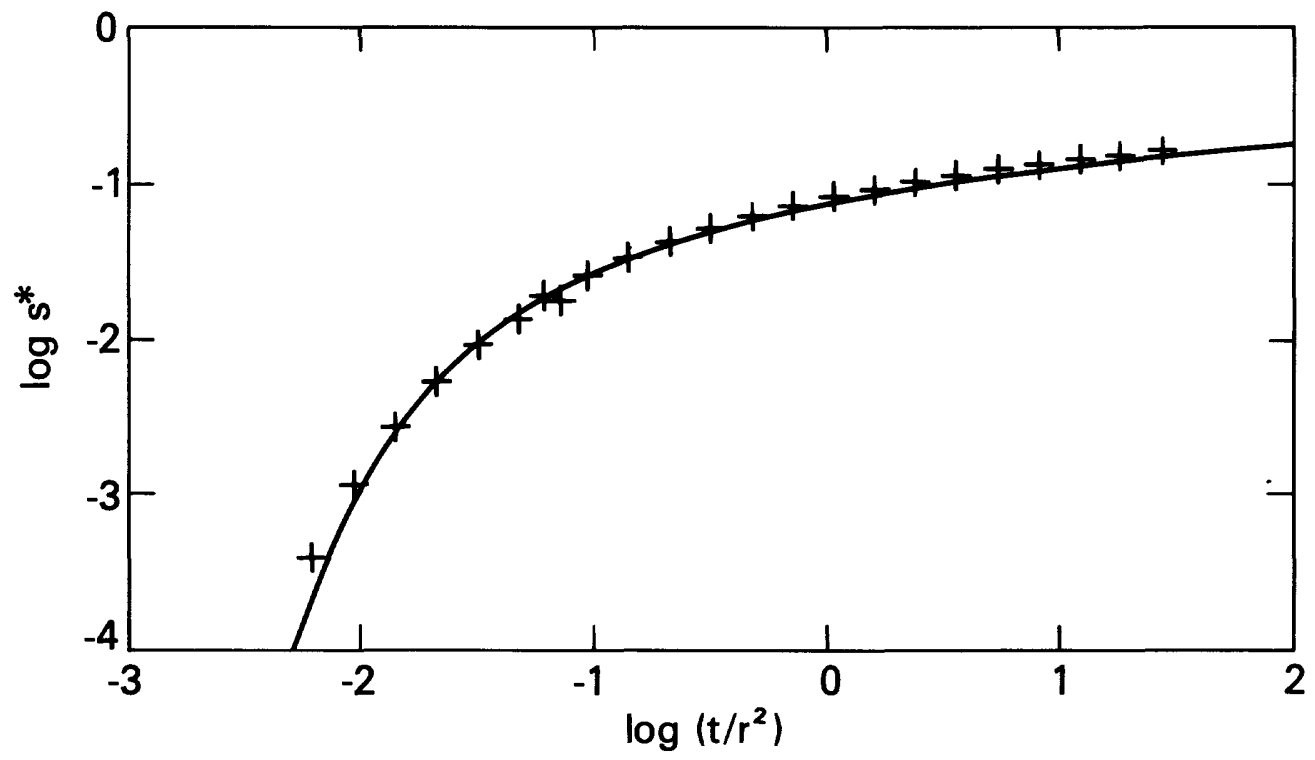

Figure 6.2

Match of Theis analytical solution (solid line) with SUTRA solution $(+)$. 
analytical solutions of Hoopes and Harleman (1967) and Gelhar and Collins (1971).

The solution of Gelhar and Collins (1971) is:

$$
\left(\frac{C-C_{0}}{C^{*}-C_{0}}\right)=\frac{1}{2} \operatorname{erfc}\left\{\frac{\left(r^{2}-r *^{2}\right)}{2\left[\left(\frac{4}{3} \alpha_{L}\right) r *^{3}+\left(\frac{D}{A}\right) r *^{4}\right]^{\frac{1}{2}}}\right\}
$$

where:

$$
\begin{aligned}
& r^{*}=(2 A t)^{\frac{1}{2}} \\
& A=\left(\frac{Q_{T O T}}{2 \pi \varepsilon b \rho}\right)
\end{aligned}
$$

The Hoopes and Harleman (1967) solution is obtained by replacing ${ }^{*}$ in the denominator of $(6.2)$ with $r$.

\section{Simulation Set-up:}

The mesh consists of one row of elements with element width expanding from $\Delta \mathrm{r}_{\min }=2.5[\mathrm{~m}]$ by a factor, 1.06 , to $\mathrm{r}=395 .[\mathrm{m}]$, and then maintaining constant element width of $\Delta r=24.2[\mathrm{~m}]$ to $r_{\max }=1000 .[\mathrm{m}]$. Element height, $\mathrm{b}$, is $10 .[\mathrm{m}$ ]. Mesh thickness is set for radial coordinates, $B_{i}=2 \pi r_{i}$, with the number of nodes and elements given by $\mathrm{NN}=132, \mathrm{NE}=65$. See Figure 6.3.

The time step is constant at $\Delta t=4021$. [s], and outputs are obtained for times steps numbered: $225,450,900,1800$. One pressure solution is carried out to obtain a steady-state, (ISSFLO $=1$ ), and one concentration solution is done per time step, $(\mathrm{NUCYC}=1)$.

\section{Parameters:}

$$
\begin{array}{ll}
S_{o p}=0.0 & \rho=1000 .\left[\mathrm{kg} / \mathrm{m}^{3}\right] \\
k=1.02 \times 10^{-11}\left[\mathrm{~m}^{2}\right] & D_{\mathrm{m}}=1 . \times 10^{-10}\left[\mathrm{~m}^{2} / \mathrm{s}\right]
\end{array}
$$




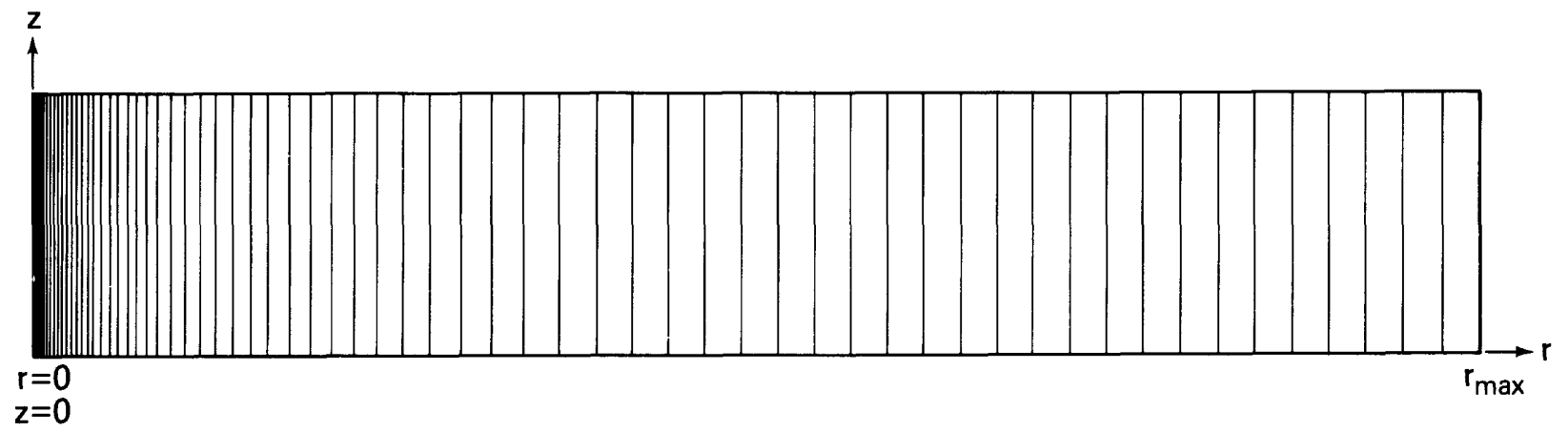

Figure 6.3

Radial finite-element mesh for constant-density

solute and energy transport examples. 


$$
\begin{array}{lrl}
\varepsilon=0.2 & \alpha_{\mathrm{L}}=10.0[\mathrm{~m}] \\
\mu=1.0 \times 10^{-3}[\mathrm{~kg} / \mathrm{m} \cdot \mathrm{s}] & \alpha_{\mathrm{T}}=0.0[\mathrm{~m}] \\
|\underline{g}|=9.8\left[\mathrm{~m} / \mathrm{s}^{2}\right] & \\
Q_{\text {TOT }}=62.5[\mathrm{~kg} / \mathrm{s}] & \text { (one half at each well node) } \\
C^{*}=1.0 &
\end{array}
$$

\section{Boundary Conditions:}

No flow occurs across any boundary except where hydrostatic pressure is specified at $r_{\max }$. At the top outside corner of the mesh, $r_{\max }$, pressure is held at zero. A source is specified at $r=0.0$ to represent the injection well.

\section{Initial Conditions:}

Initially hydrostatic pressure is set with $p=0.0$ at the aquifer top. Initial concentration, $C_{0}$, is set to zero.

\section{Rèsults:}

SUTRA results after $225,450,900$ and 1800 time steps are compared with the approximate analytical solutions of Gelhar and Collins (1971) and Hoopes and Harleman (1967) in Figure 6.4. The analytical solutions are approximate and they bound the SUTRA solution at the top and bottom of the solute front. All solutions compare well with each other and the SUTRA solution may be considered to be more accurate than either approximate analytic solution because it is based on a very fine spatial and temporal discretization of the governing equation. 


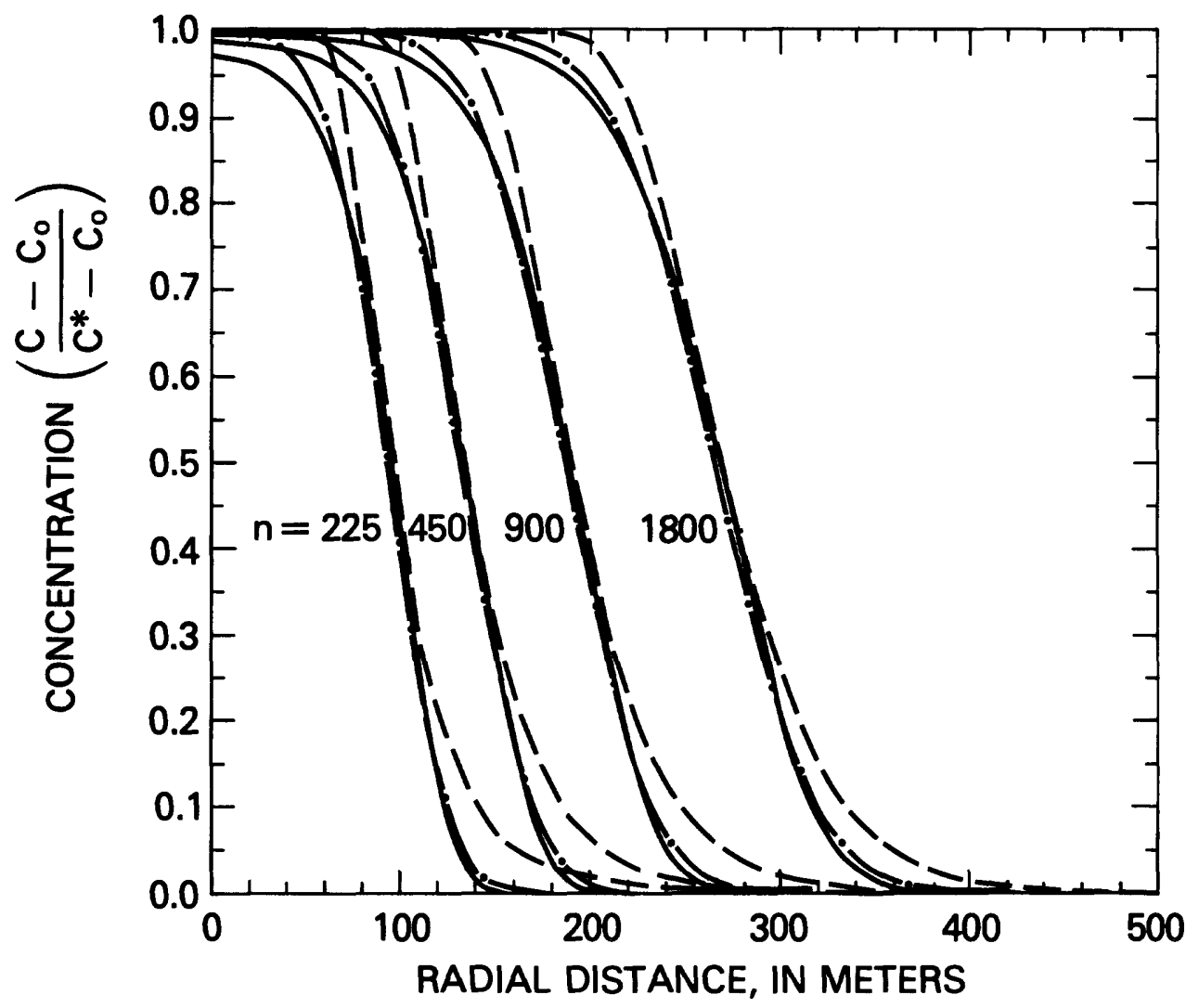

Figure 6.4

Match of analytical solutions for radial solute transport of Hoopes and Harleman (1967) (dashed), Gelhar and Collins (1971), (solid), and SUTRA solution (dash-dot). Number of elapsed time steps is $n$. 
6. 3 Radial Flow with Energy Transport

(Analytical Solution)

\section{Physical Set-up:}

A confined aquifer contains a fully penetrating infection well. Fluid is injected at a rate, QTOT, with a temperature, $T^{*}$, into the aquifer initially at a temperature, $T_{0}$. For this problem, density $\rho$, and viscosity $\mu$, are kept approximately constant by injecting fluid that only slightly differs in temperature from the ambient fluid; i.e. $\left(T^{*}-T_{0}\right)$ is small.

\section{Objective:}

To simulate the transient propagation of the temperature front as 1 t radially moves away from the well. The solution should match an approximate analytical solution of Gelhar and Collins (1971) modified for energy transport. The Gelhar and Collins (1971) solution, as modified for energy transport is:

$$
\begin{aligned}
& \left(\frac{T-T_{0}}{T^{*}-T_{0}}\right)=\frac{1}{2} \operatorname{erfc}\left\{\frac{\left(r^{2}-r *^{2}\right)}{2\left[\left(\frac{4}{3} \alpha_{L}\right) r *^{3}+\left(\frac{\lambda_{T O T}}{A_{T}}\right) r^{*}\right]^{\frac{1}{2}}}\right\} \\
& A=\frac{Q_{T O T}}{2 \pi \varepsilon B \rho} \\
& A_{T}=\left(\frac{\varepsilon \rho c_{w}}{c_{T O T}}\right) A \\
& c_{T O T}=\varepsilon \rho C_{w}+(1-\varepsilon) \rho c_{s} \\
& \lambda_{T O T}=\varepsilon \lambda_{w}+(1-\varepsilon) \lambda_{s} \\
& r *\left(2 A_{T} t\right)^{\frac{1}{2}}
\end{aligned}
$$


The energy solution above may be obtained from the solute solution by retarding the velocity of transport to represent movement of an 1sotherm rather than a parcel of solute. This is done by accounting for energy storage in the solid grains of the aquifer material in the storage term of the analytical solution.

\section{Simulation Set-up:}

The mesh used for this example is the same as for the radial solute transport example. Time steps and frequency of SUTRA outputs are the same as for the radial solute transport example. Further, cycling of the SUTRA solution is the same as for the radial solute transport example.

\section{Parameters:}

$$
\begin{array}{ll}
\mathrm{c}_{\mathrm{w}}=4182 \cdot\left[\mathrm{J} / \mathrm{kg} \cdot{ }^{\circ} \mathrm{C}\right] & \mathrm{S}_{\mathrm{op}}=0 . \\
\mathrm{c}_{\mathrm{s}}=840 \cdot\left[\mathrm{J} / \mathrm{kg} \cdot{ }^{\circ} \mathrm{C}\right] & \mathrm{k}=1.02 \times 10^{-11}\left[\mathrm{~m}^{2}\right] \\
\lambda_{\mathrm{W}}=0.6\left[\mathrm{~J} / \mathrm{s} \cdot \mathrm{m} \cdot{ }^{\circ} \mathrm{C}\right] & \varepsilon=0.2 \\
\rho=1000 \cdot\left[\mathrm{kg} / \mathrm{m}^{3}\right] & |\mathrm{g}|=9.8\left[\mathrm{~m} / \mathrm{s}^{2}\right] \\
\lambda_{\mathrm{S}}=3.5\left[\mathrm{~J} / \mathrm{s} \cdot \mathrm{m} \cdot{ }^{\circ} \mathrm{C}\right] & \alpha_{\mathrm{L}}=10 .[\mathrm{m}] \\
D_{\mathrm{S}}=2650 \cdot\left[\mathrm{kg} / \mathrm{m}^{3}\right] & \alpha_{\mathrm{T}}=0.0[\mathrm{~m}] \\
\frac{\partial \rho}{\partial \mathrm{T}}=0.0 & \\
\mu=\mu(\mathrm{T})(\mathrm{relation}(2.5)) & \text { (one half at each well node) } \\
Q_{\mathrm{TOT}}=312.5[\mathrm{~kg} / \mathrm{s}] & \\
T^{*}=1.0^{\circ} \mathrm{C} &
\end{array}
$$




\section{Boundary Conditions:}

No flow occurs across any boundary except where hydrostatic pressure is specified at $r_{\max }$. At the top outside corner of the mesh, pressure is held at zero. A source is specified at $r=0.0$ to represent the injection well. Further, the system is thermally insulated along the top and bottom of the mesh.

\section{Initial Conditions:}

Initially, hydrostatic pressure is set with $p=0.0$ at the top of the aquifer. The initial temperature is $\mathrm{T}_{0}=0.0^{\circ} \mathrm{C}$.

\section{Results:}

SUTRA results after $225,450,900$ and 1800 time steps are compared with the approximate (modified) analytical solution of Gelhar and Collins (1971) in Figure 6.5. The analytic solution has the same relation to the SUTRA solution as it does in Figure 6.4 for solute transport. Thus the match 18 good, and again the SUTRA result may be more accurate than the approximate analytic result because of the fine discretization employed.

6.4 Areal Constant-Density Solute Transport

(Example at Rocky Mountain Arsenal)

\section{Physical Set-up:}

This example involves a simple representation of ground-water flow and solute transport at the Rocky Mountain Arsenal, Denver, Colorado, which is based on the detailed model of the system by Konikow (1977). The simplified representa- 


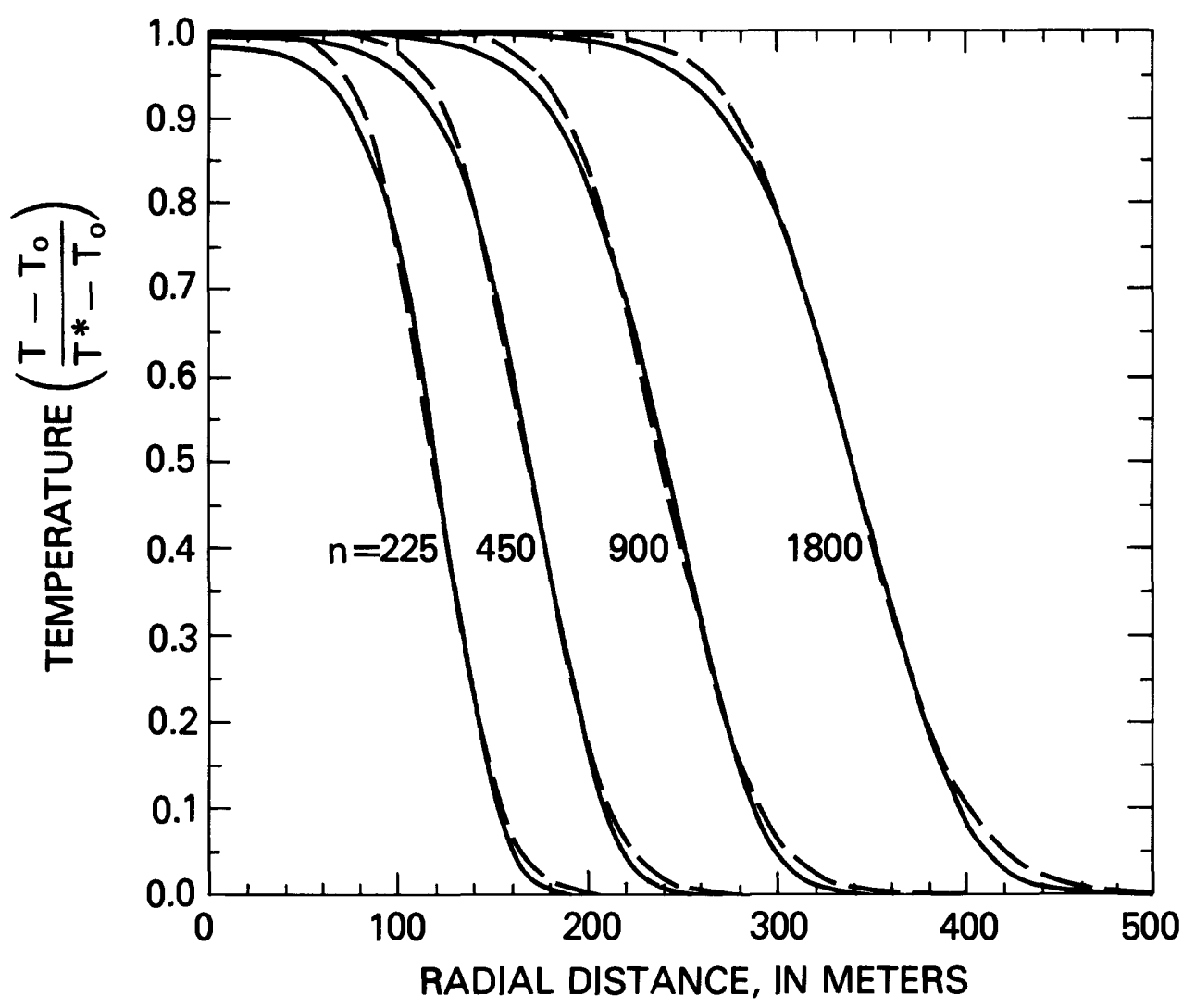

Figure 6.5

Match of analytical solution for radial energy

transport (modified from Gelhar and Collins (1971)

solid line) with SUTRA solution (dashed line).

Number of elapsed time steps is $n$. 
tion consists of an areal model of a rectangular alluvial aquifer with a constant transmissivity and two impermeable bedrock outcrops which influence groundwater flow. (See Figure 6.6.)

Regional flow is generally from the south-east to the north-west where some discharge occurs at the South Platte River. This is Idealized as flow originating in a constant head region at the top of the rectangle in Figure 6.6 , and discharging to the river at the bottom of the rectangle which also acts as a specified head region. Three wells pump from the aquifer (at a rate of QouT each), and contamination enters the system through a leaking waste isolation pond (at a rate of $Q_{I N}$, with concentration, $C^{*}$ ). The natural background concentration of the contaminant is $\mathrm{C}_{0}$.

\section{Objectives:}

1) To demonstrate the applicability of SUTRA to an areal constant density solute transport problem; 2) To convert SUTRA input data values so the pressure results represent heads, and the concentration results are in [ppm]; and 3 ) To simulate steady-state flow and hypothetical steady-state distributions of the contaminating solute, both as a conservative solute, and as a solute which undergoes first order decay, assuming that the contamination source in the idealized system is at a steady-state.

\section{Simulation Set-up:}

The rectangular mesh consists of 16 by 20 elements each of dimension 1000 . ft by 1000. ft, as shown in Figure 6.6. ( $N N=357, N E=320)$. Mesh thickness, $B$, is the actual aquifer thickness, assumed constant for the idealized model. One steady-state pressure solution is obtained (ISSFLO=1), and one concen- 


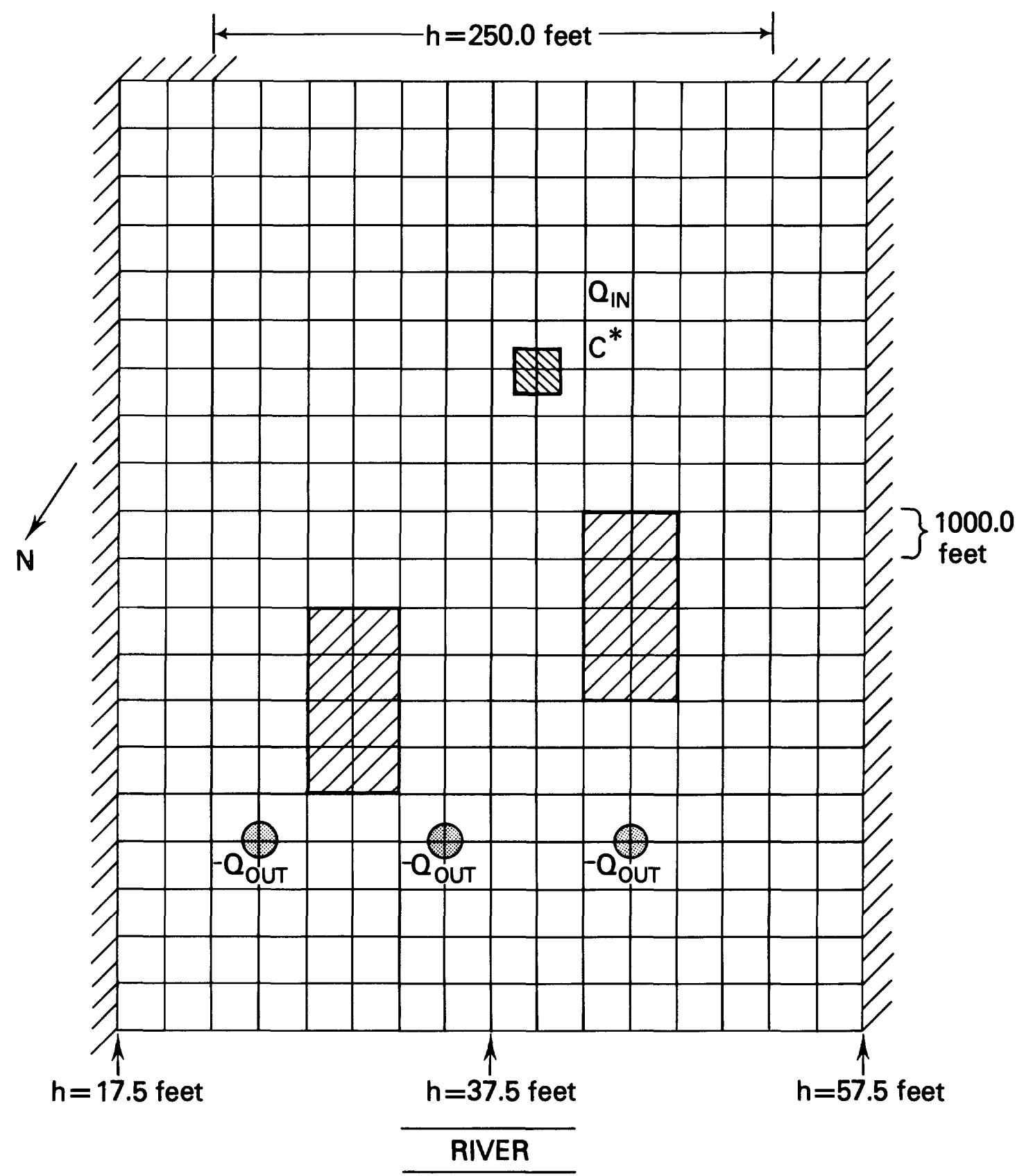

Figure 6.6

Idealized representation for example at Rocky

Mountain Arsenal, and finite-element mesh. 
tration solution is obtained. The concentration solution is obtained after a single time step of 1000 . years, which, for all practical purposes, brings the concentration distribution to a steady-state.

The leaky pond is simulated as an injection of fluid $\left(Q_{I N}, C^{*}\right)$ at a single node. Where the impermeable bedrock outcrop occurs, elements are assigned a conductivity value one-millionth of the aquifer values. A single value of constant head is specified along a portion of the top boundary, and a series of head values is specified along the bottom (river) boundary to represent changing elevation of the river.

In order to obtain results in terms of hydraulic head and $\lfloor\mathrm{ppm}\rfloor$, the following must be specified: $\rho=1.0, \frac{\partial \rho}{\partial C}=0.0,|g|=0.0, \mu=1.0$. Hydraulic conductivities are entered in the permeability input data set. Head values in [ft] are entered in data sets for pressure. Concentrations in [ppm \ are entered in data sets for mass fraction concentration. Sources and sinks are entered in units of volume per time.

\section{Parameters:}

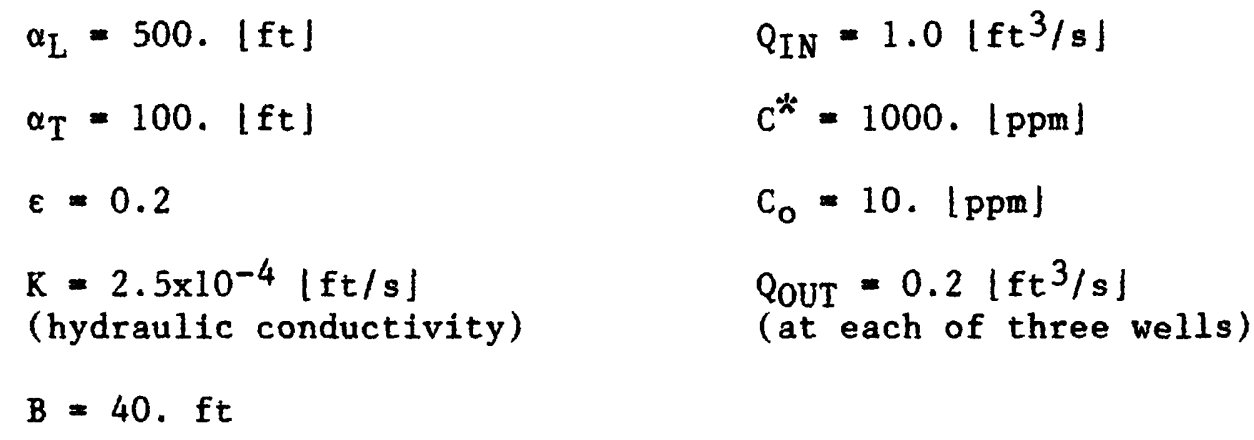

\section{Boundary Conditions:}

No flow occurs across any boundary except where constant head is spectfied 
at 250. [ft] at the top of the mesh and where constant head is specified as changing linearly between $17.5[\mathrm{ft}]$ at the bottom left corner, and $57.5[\mathrm{ft}$ ] at the bottom right corner of the mesh. Inflow at the top of the mesh is at background concentration, $c_{0}=10$. [ppm]. A source is specified at the leaky pond node, and a sink is specified at each well node.

\section{Initial Conditions:}

Initial pressures are arbitrary for steady-state simulation of pressure. Initial concentration is $\mathrm{C}_{0}=10$. [ppm].

\section{Results:}

A nearly steady-state solute plume for a conservative solute is obtained after a 1000 year time step shown in Figure 6.7. For a solute which undergoes first order decay with decay coefficient, $y=1.1 \times 10^{-9}\left[\mathrm{~s}^{-1}\right]$ (approximately a 20 year half-life), the nearly steady plume is shown in Figure 6.8. Just upstream of the plume envelope is a region in which concentration dips slightly below background levels. This is due to a numerical problem of insufficient spatial discretization in a region where the concentration must change sharply from fresh upstream values to contaminated plume values. Lower dispersivity values would exacerbate the problem in the upstream region, but minor upstream oscillations do not affect concentration values within the plume. 


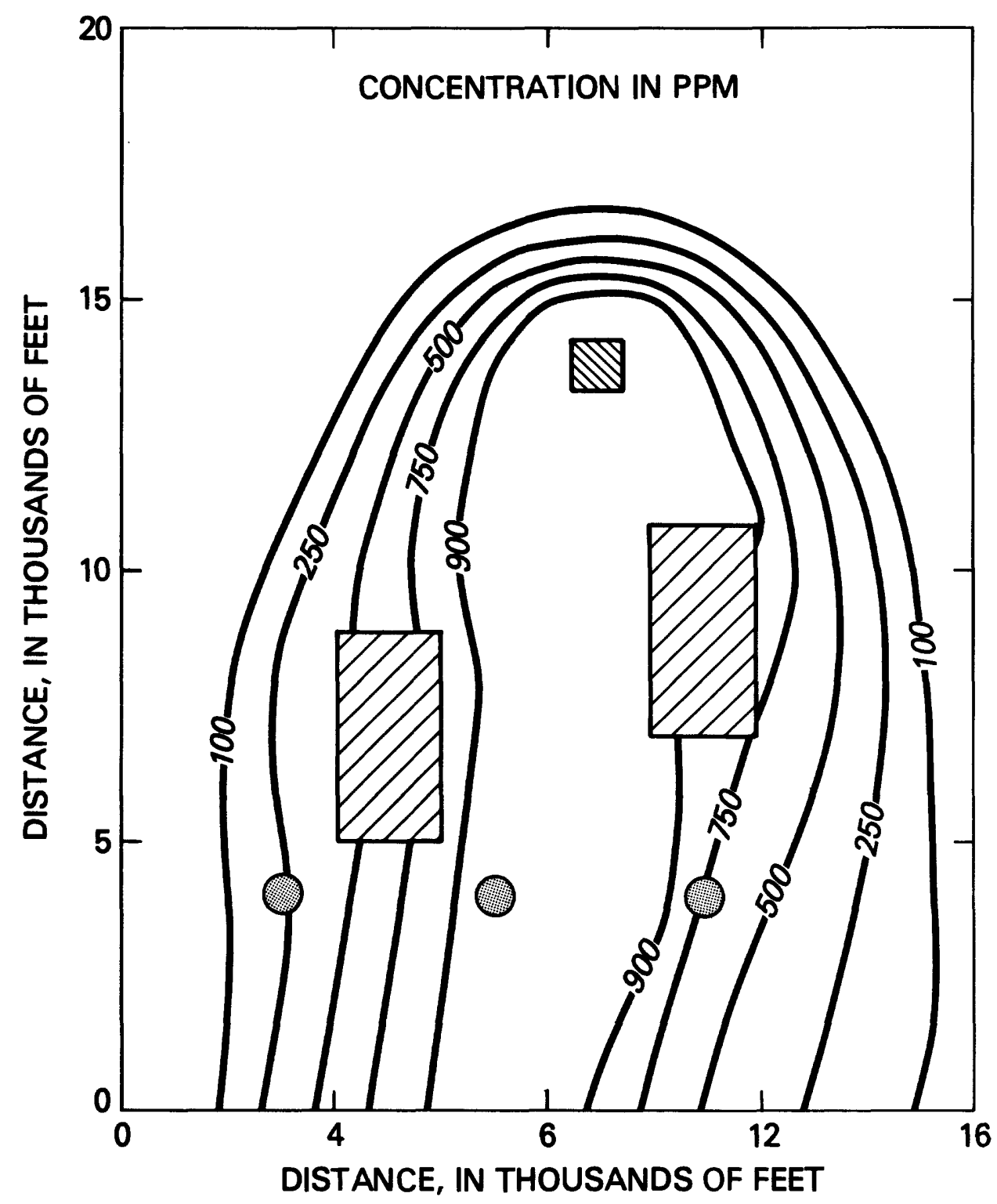

Figure 6.7 Nearly steady-state conservative solute plume as simulated for the Rocky Mountain Arsenal example by SUTRA. 


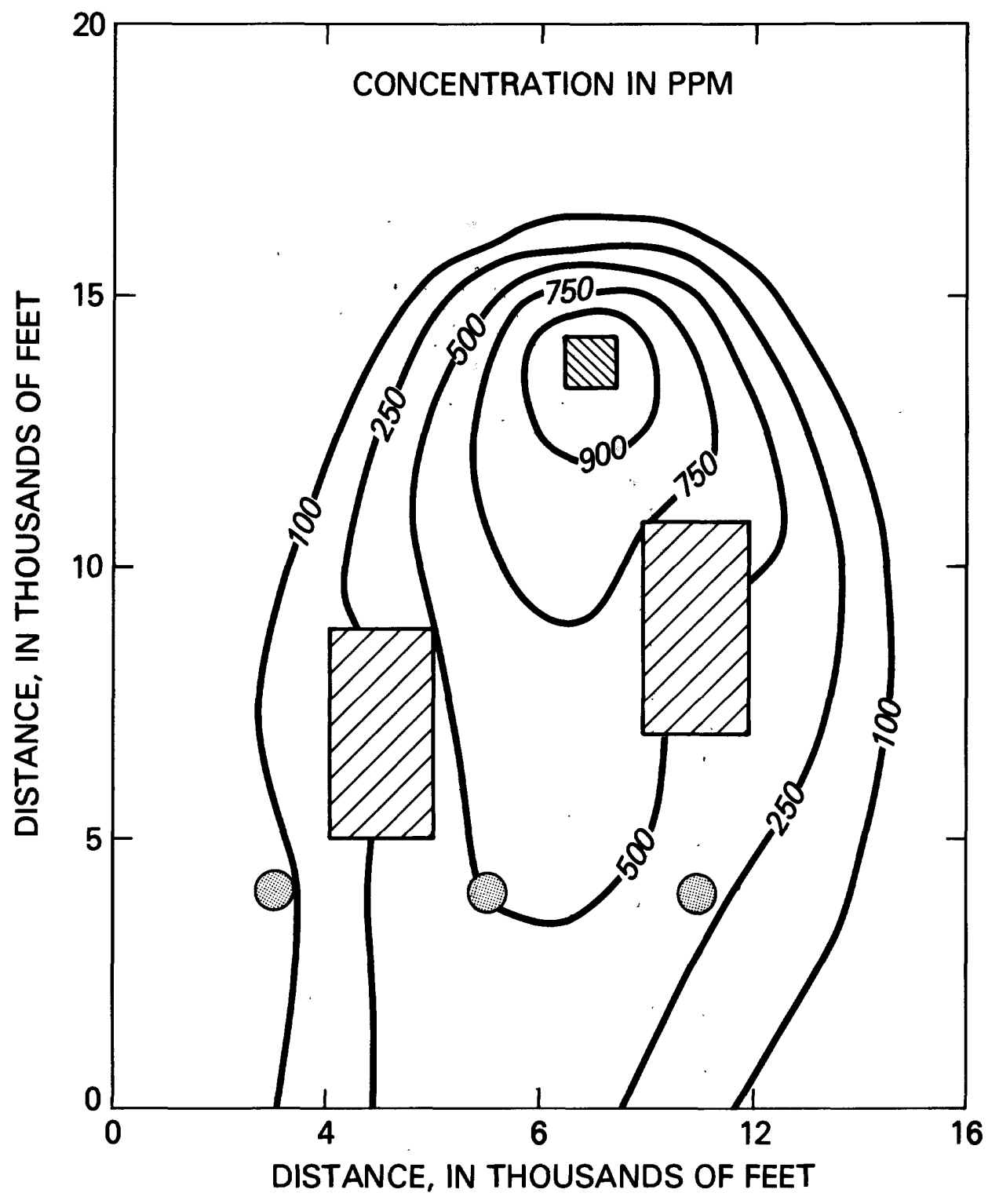

Figure 6.8

Nearly steady-state solute plume (with solute half-11fe $\sim 20$. years) as simulated for the

Rocky Mountain Arsenal example by SUTRA. 
6.5 Density-Dependent Flow and Solute Transport

(Henry (1964) Solution for Sea-Water Intrusion)

\section{Physical Set-up:}

This problem involves sea-water intruston into a confined aquifer studied In cross-section under steady conditions. Fresh-water recharge inland flows over salt water in the section and discharges at a vertical sea boundary.

The intrusion problem is non-linear and may be solved by approaching the steady state gradually with a series of time steps. Inftially there is no salt water in the aquifer, and at time zero, salt water begins to intrude the fresh water system by moving under the fresh water from the sea boundary. The intrusion is caused by the greater density of the salt water.

Dimensions of the problem are selected to make for simple comparison with the steady-state dimensionless solution of Henry (1964), and with a number of other published simulation models. A total simulation time of $t=100$. [min], is selected, which is sufficient time for the problem to essentially reach steady state at the scale simulated.

\section{Simulation Set-up:}

The mesh consists of twenty by ten elements, each of size $0.1[\mathrm{~m}]$ by $0.1[\mathrm{~m}]$, $(\mathrm{NN}=231, \mathrm{NE}=200)$. Mesh thickness, $B$, is $1 .[\mathrm{m}]$. See Figure 6.9. Time steps are of length $1 .[\mathrm{min}]$, and 100 time steps are taken in the simulation. Both pressure and concentration are solved for on each time step, (NUCYC=NPCYC=1).

A source of fresh water is implemented by employing source nodes at the left vertical boundary which inject fresh water at rate, $Q_{I N}$, and concentration, $C_{I N}$. The right vertical boundary is held at hydrostatic pressure of 


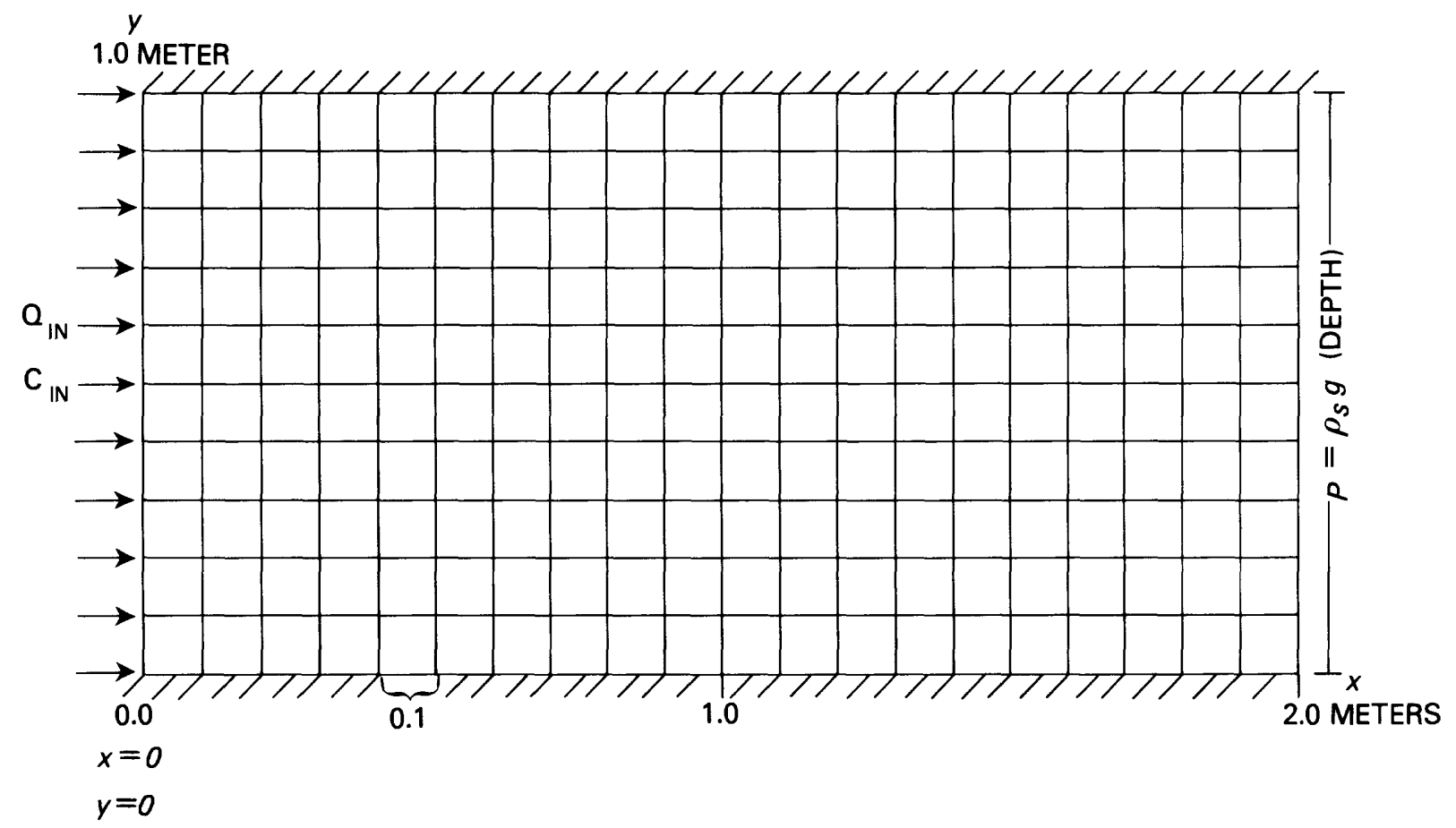

F1gure 6.9

Boundary conditions and finite-element mesh for Henry (1964) solution. 
sea water through use of spectfled pressure nodes. Any water which enters the section through these nodes has concentration $C_{B C}$ of sea water (equal to $C_{s}$ ).

\section{Parameters:}

$\varepsilon=0.35$

$\mathrm{C}_{\mathrm{s}}=0.0357\left[\frac{\mathrm{kg}(\mathrm{dissolved} \mathrm{solids})}{\mathrm{kg}(\text { seawater })}\right]$

$\rho_{s}=1025 .\left[\mathrm{kg} / \mathrm{m}^{3}\right]$

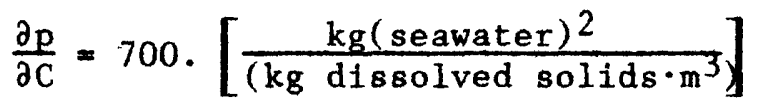

$\rho_{0}=700 .\left[\mathrm{kg} / \mathrm{m}^{3}\right]$

$Q_{\text {IN }}=6.6 \times 10^{-2}[\mathrm{~kg} / \mathrm{s}]$

(divided among 11 nodes

at left boundary)

$$
\begin{aligned}
& k=1.020408 \times 10^{-9}\left[\mathrm{~m}^{2}\right] \\
& \left(\text { based on } K=1.0 \times 10^{-2}[\mathrm{~m} / \mathrm{s}]\right)
\end{aligned}
$$$$
|\mathrm{g}|=9.8\left[\mathrm{~m} / \mathrm{s}^{2}\right]
$$$$
\alpha_{L}=\alpha_{T}=0.0
$$

$B=1.0[\mathrm{~m}]$

$$
\begin{aligned}
& \left.D=\begin{array}{l}
6.6 \times 10^{-6}\left[\mathrm{~m}^{2} / \mathrm{s}\right] \\
18.8571 \times 10^{-6}\left[\mathrm{~m}^{2} / \mathrm{s}\right]
\end{array}\right\} \begin{array}{l}
\text { two } \\
\text { cases }
\end{array} \\
& C_{\text {IN }}=0.0
\end{aligned}
$$

\section{Boundary Conditions:}

No flow occurs across the top and bottom boundaries. A fresh-water source is set along the left vertical boundary. Specified pressure is set at hydrostatic sea water pressure with $\left(\rho_{\mathrm{s}}=1025 .\left[\mathrm{kg} / \mathrm{m}^{3}\right]\right)$ along the right vertical boundary. Any inflowing fluid at this boundary has the concentration, $\mathrm{C}_{\mathrm{s}}=0.0357$ [kg(dissolved solids)/kg(seawater)], of sea water.

\section{Initial Conditions:}

Natural steady pressures are set everywhere in the aquifer based on the fresh-water inflow, zero concentration everywhere, and the specified pressures at the sea boundary. These initial conditions are abtained through an extra Initial simulation which calculates steady pressures under these conditions. 
Results:

Henry's solution assumes that dispersion is represented by a constant large coefficient of diffusion, rather than by velocity-dependent dispersivity. Two different values of this diffusivity have apparently been used in the literature by those testing simulators against Henry's solution. The total dispersion coefficient of Henry (1964), D, is equivalent to the product of porosity and molecular diffusivity in SUTRA, $D=\varepsilon D_{m}$.

Henry's results are given for his non-dimensional parameters: $\xi=2.0, b=0.1$, $a \simeq .264$ (page C80- Figure 34 in Henry (1964)). In order to match the Henry parameters using simulation parameters as 11 sted above, values of $D=6.6 \times 10^{-6}\left[\mathrm{~m}^{2} / \mathrm{s}\right]$ and $D_{m}=18.8571 \times 10^{-6}\left[\mathrm{~m}^{2} / \mathrm{s}\right]$ are required. Some authors, however, have apparently used a value equivalent to $D_{\mathrm{m}}=6.6 \times 10^{-6}\left[\mathrm{~m}^{2} / \mathrm{s}\right]$ and $D=2.31 \times 10^{-6}$ $\left[\mathrm{m}^{2} / \mathrm{s}\right]$, which differs from the Henry parameters by a factor equal to the porosity.

In the previous model solutions compared here, only Huyakorn and Taylor (1976) have employed the higher value which should match Henry's solution. A comparison of SUTRA results at $t=100$. [min], using the higher value with those of Huyakorn and Taylor (1976) along the bottom of the section is shown in Figure 6.10. Huyakorn and Taylor's results are for a number of simulation models based on significantly different numerical methods. SUTRA results are also shown for the lower diffusivity value. The results of simulations using the higher diffusivity value compare favorably. Results using the higher value have also been obtained with the INTERA (1979) finite-difference code at $t=100$. [min], (with centered-in-space and centered-in-time approximations). These are compared with SUTRA and the Henry solution for the 0.51 sochlor in Figure 6.11. The models match well but do not compare favorably with the analytic 


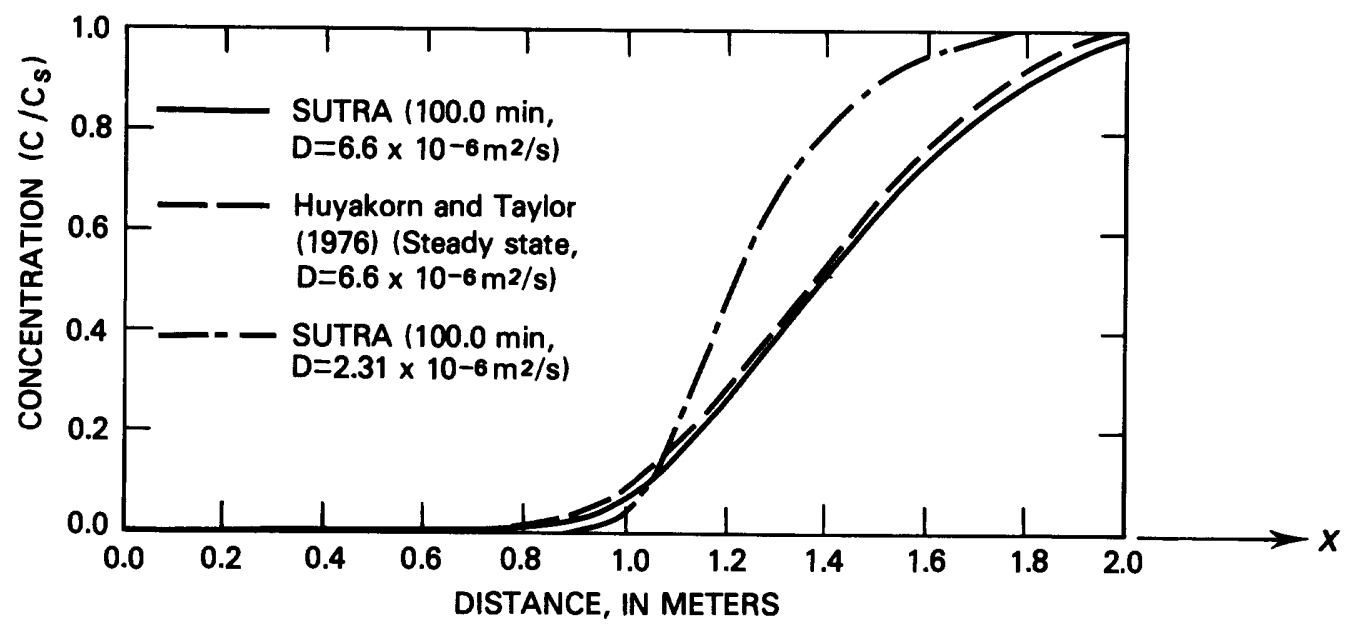

F1gure 6.10

Match of isochlors along bottom of aquifer for numerical results of Huyakorn and Taylor (1976) and SUTRA. 


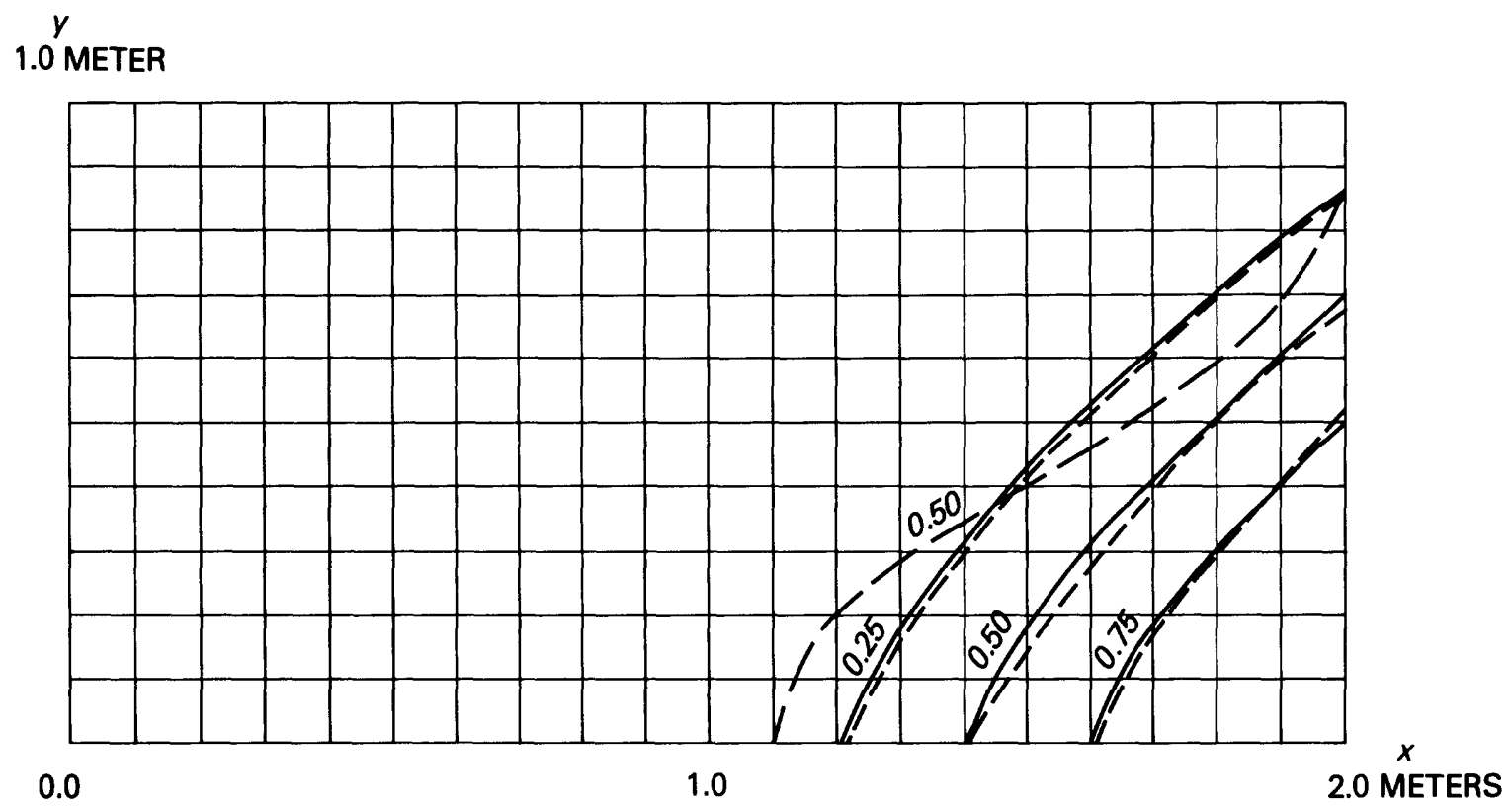

Figure 6.11

Match of isochlor contours for Henry analytical solution (for 0.50 isochlor) (long dashes), INTERA code solution (short dashes), SUTRA solution (solid line). 
solution, which is approximate and may not be as accurate as the numerical solutions.

For the lower value of diffusivity, $D_{m}=6.6 \times 10^{-6}\left[\mathrm{~m}^{2} / \mathrm{s}\right]$, (which should not compare with the Henry result), the SUTRA solution at $t=100$. [min] is compared in Figure 6.12 with that of Pinder and Cooper (1970) (method of characteristics), Segol et. al. (1975) (finite elements), Desai and Contractor (1977) (finite elements - coarse mesh), and Frind (1982) (finite elements). The match of the numerical 0.5 isochlor solutions is remarkably good; however, it should be noted that none of these match the analytical solution.

6.6 Density-Dependent Radial Flow and Energy Transport

(Aquifer Thermal Energy Storage Example)

Physical Set-up:

This is an example of aquifer thermal energy storage. Hot water is injected into an aquifer for storage and later withdrawn and used as an energy source. The fully penetrating injection wells are emplaced in a well-field in a hexagonal packing pattern. The wells are at the vertices of contiguous equilateral triangles with sides of $500 .[\mathrm{m}]$. This gives approximately radial symmetry to physical processes surrounding an interior well.

\section{Objective:}

To simulate the initial injection-withdrawal cycle at an interior well consisting of 90 days of injection (at $Q_{I N}$ ) of $60^{\circ} \mathrm{C}$ water into the aquifer initially at $20^{\circ} \mathrm{C}$, and 90 days of withdrawal (at $Q_{I N}$ ) producing the stored water. Degradation of recovered fluid temperature should occur due to thermal 
$y$

1.0 METER

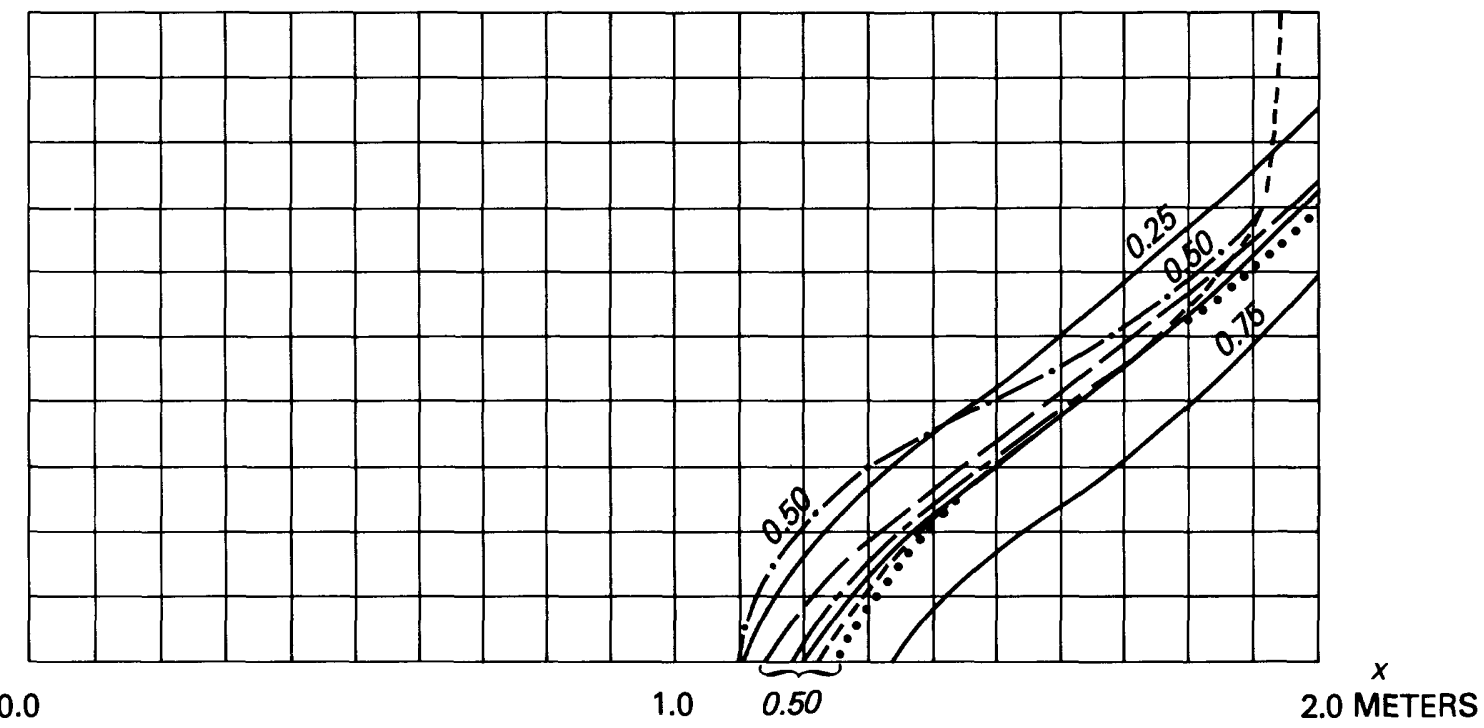

Figure 6.12

Match of 0.50 isochlor contours for Henry problem with simulated results for $D_{m}=6.6 \times 10^{-9}\left[\mathrm{~m}^{2} / \mathrm{s}\right]$ of Pinder and Cooper (1970), (short dashes), Segol, et al (1975) (dotted line), Frind (1982) (long and short dashes), Desai and Contractor (1977) (long dashes). SUTRA results at isochlors $(0.25,0.50,0.75)$ (solid line). Henry (1964) solution for $D_{m}=18.8571 \times 10^{-9}\left[\mathrm{~m}^{2} / \mathrm{s}\right]$, (0.50 isochlor, dash-dot). 
conduction, dispersion, and tipping of the thermal front. The front should tip as less dense, less viscous hot water rises over colder, denser, and more viscous formation water.

\section{Simulation Set-up:}

The mesh 18 30. [m] high with a vertical spacing between nodes of 3.0 [m]. The first column of elements has width $\Delta r_{m i n}=1.0[\mathrm{~m}]$, and element width increases with each column by a factor, 1.1593 , to a final column of width, $\Delta \mathrm{r}_{\max }=35 .[\mathrm{m}]$. The outside boundary of the mesh is at $r_{\max }=$ 246. [m]. See Figure 6.13. Mesh thickness, $B$, at any node 1 , is $B_{1}=$ $2 \pi r_{1}$, giving cylindrical symmetry. The number of nodes and elements in the mesh is given by $\mathrm{NN}=286, \mathrm{NE}=250$.

The time step is constant at $\Delta t=3.0$ [days]. One pressure solution and one temperature solution is obtained at each time step (NPCYC=NUCYC=1). The storage coefficient is assumed negligible resulting in a steady flow fleld at any time step. Subroutine BCTIME is programmed to control the well rate which changes after 90 days from fluid injection to fluid withdrawal.

A time-dependent fluid source is specified at the left vertical boundary (center axis) which injects $\left.60 .{ }^{\circ} \mathrm{C}\right]$ water for 90 days and then withdraws ambient water for 90 days. The right vertical boundary is held at hydrostatic pressure for water at $20 .\left[{ }^{\circ} \mathrm{C}\right]$. Any inflow at this boundary has a temperature of $20 .{ }^{\circ} \mathrm{C}$. Thermally insulated and impermeable conditions are held at the top and bottom of the mesh. 


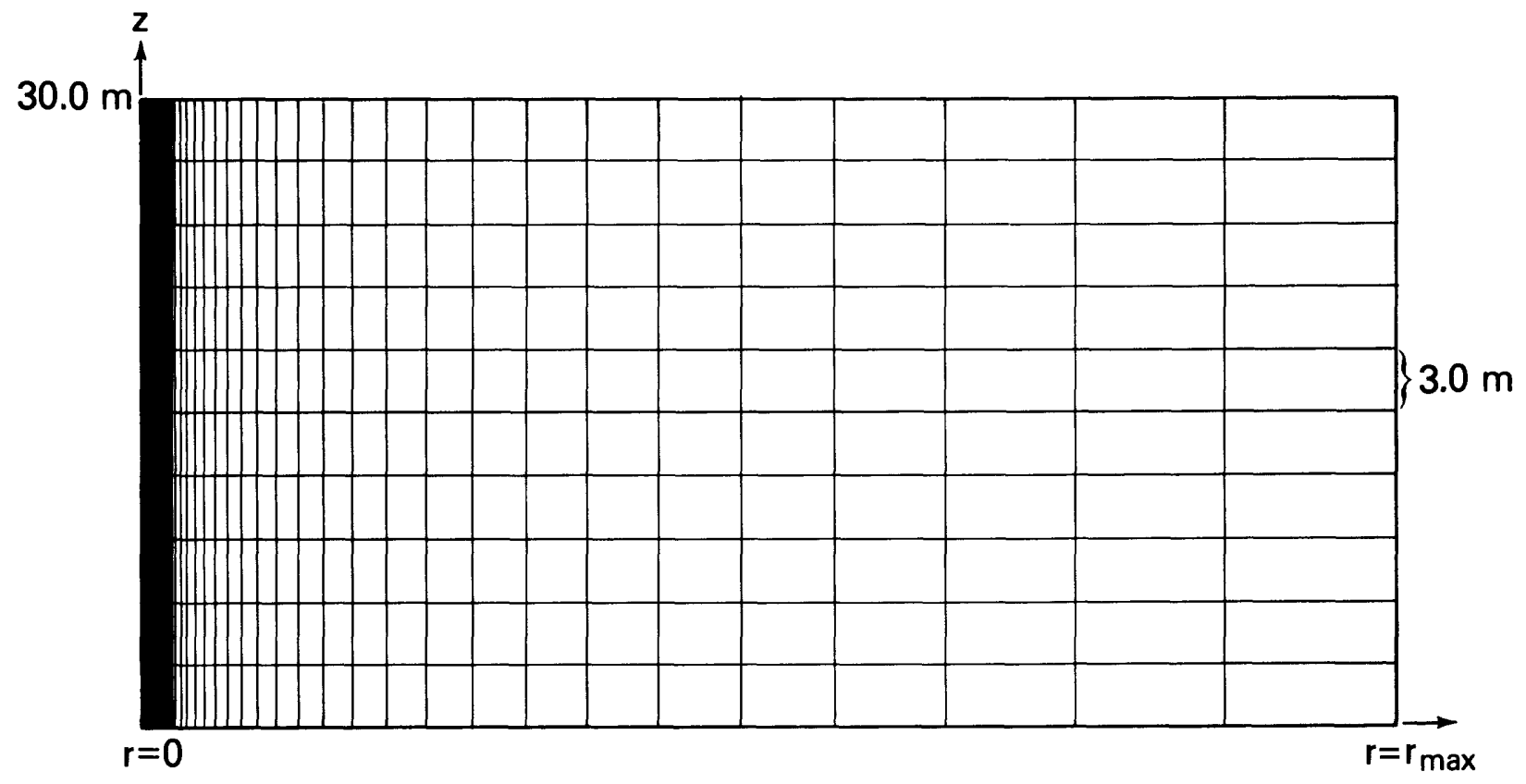

F1gure 6.13

Radial two-dimensional finite-element mesh for aquifer thermal energy storage example. 


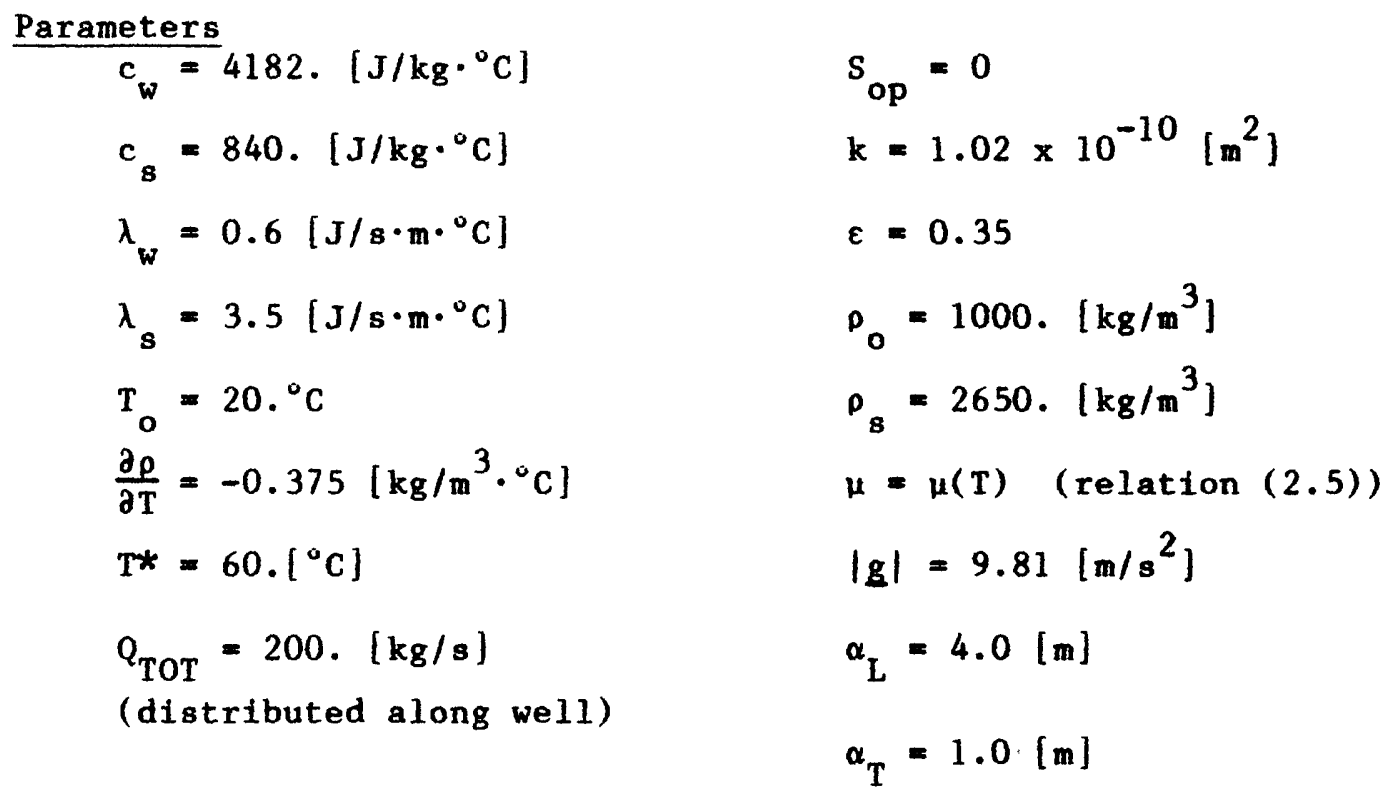

\section{Boundary Conditions:}

Conditions of no flow and thermal insulation are held at all boundaries except where hydrostatic pressure at $T=20 .\left[^{\circ} \mathrm{C}\right]$ is specified at $r_{\max }$. At the top outside corner of the mesh the pressure is held at zero. A time-dependent source is specified at $r=0.0$ to represent the injection-withdrawal well.

\section{Initial Conditions:}

Hydrostatic pressure is specified initially, with $p=0.0$ at the top of the aquifer. The initial temperature is set to $\mathrm{T}_{\mathrm{o}}=20 .\left[{ }^{\circ} \mathrm{C}\right]$.

\section{Results:}

SUTRA results during injection after 30 days and 90 days are shown in Figure 6.14 and Figure 6.15. Simulated results during withdrawal are shown in 


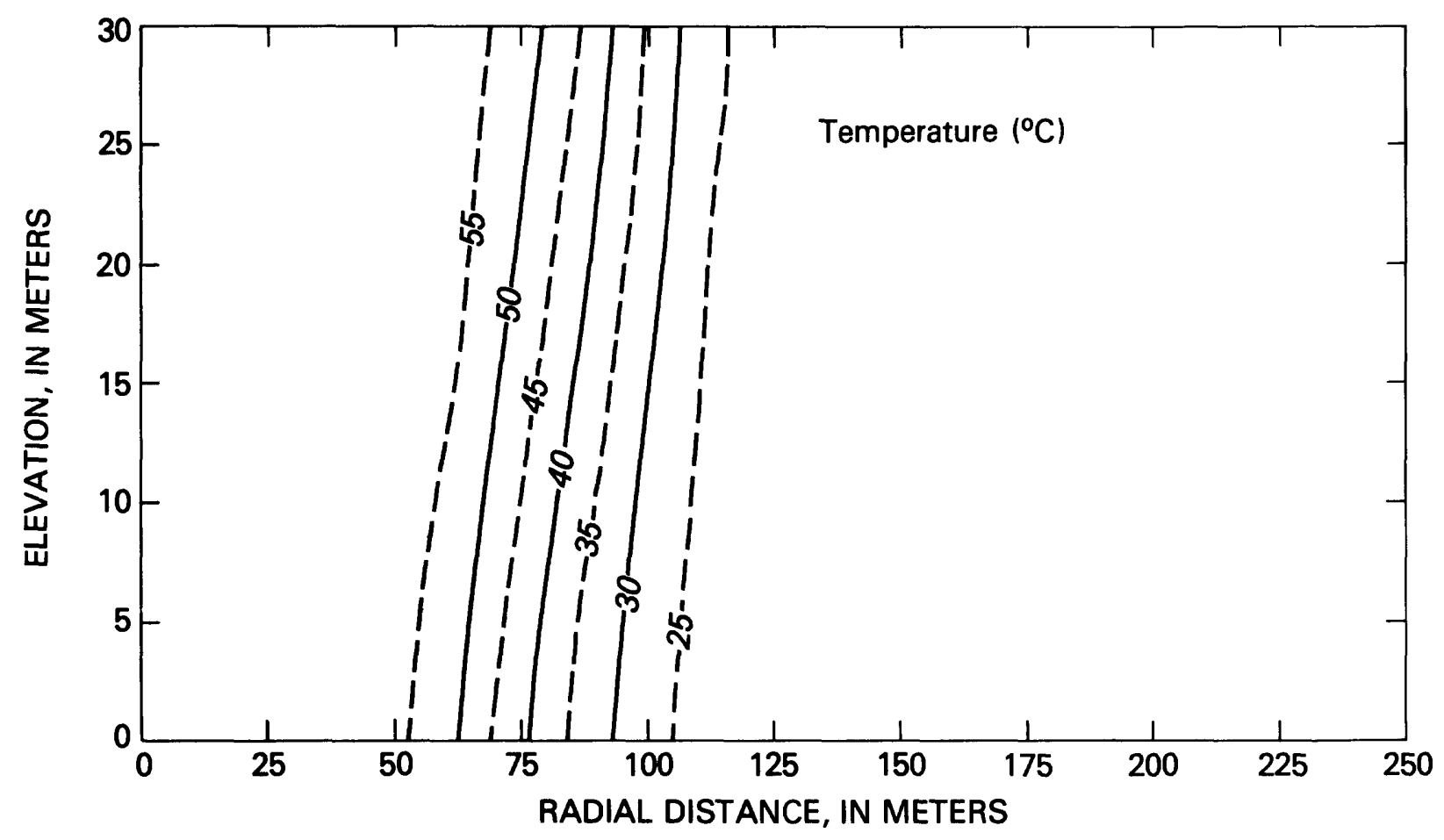

Figure 6.14

SUTRA results after 30 days of hot water injection. 


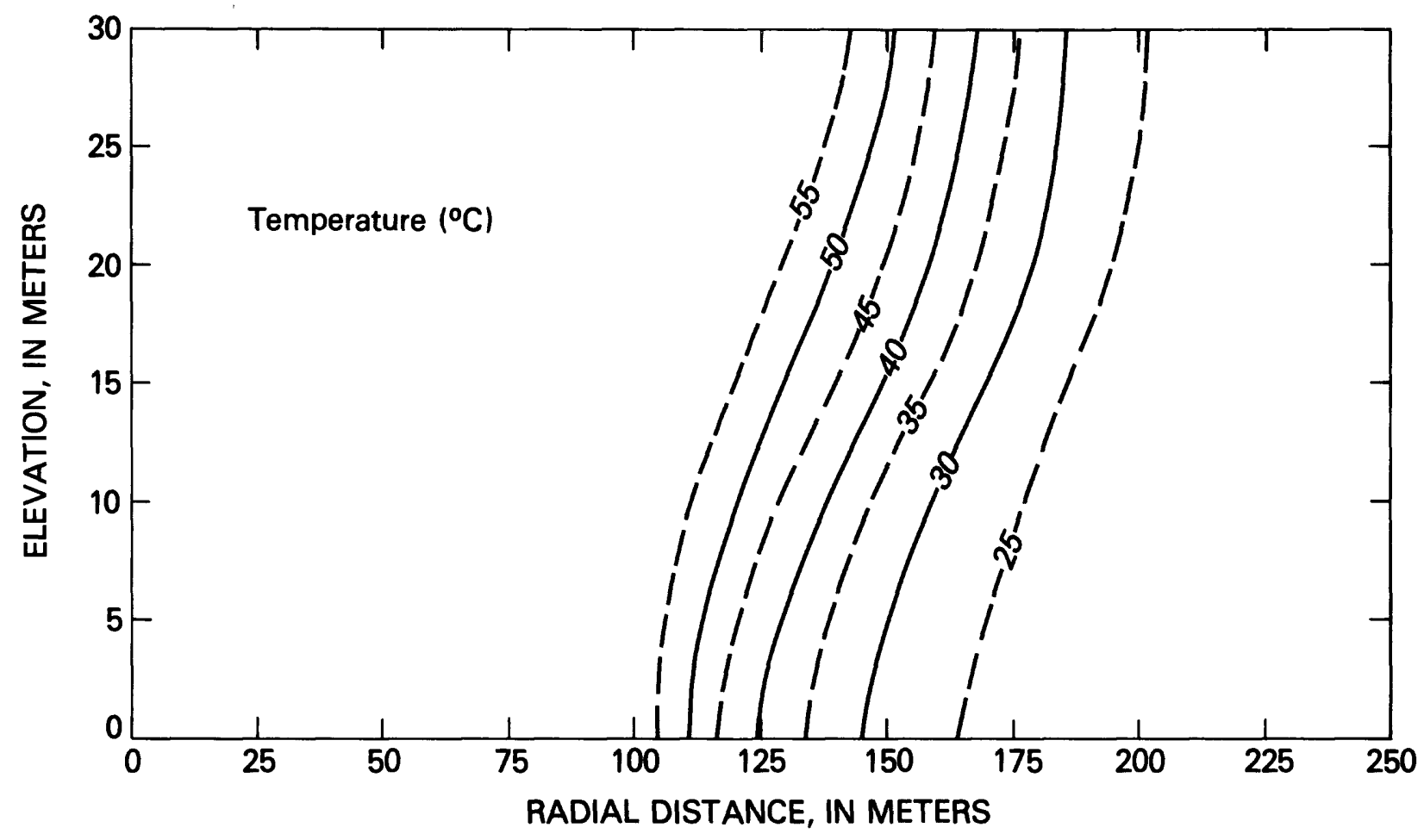

Figure 6.15

SUTRA results after 90 days of hot water injection. 
Figure 6.16, Figure 6.17 , and Figure 6.18 after 30 days, 60 days, and 90 days of withdrawal. The thermal transition zone (between hot and cold water) widens throughout the injection-production cycle, due to both dispersion and heat conduction. The top of the transition zone tips away from the well during the entire cycle, due to the bouyancy of the hotter water. These two effects combine to cause cooler water to reach the bottom of the withdrawal well much earlier than if no density differences or dispersion existed. Also, although the same quantity of water has been removed as injected, energy is lost to the aquifer during the cycle as seen at the end of simulation.

\subsection{Constant-Density Unsaturated Flow and Solute Transport (Example from Warrick, Biggar and Nielsen (1971))}

\section{Physical Set-up:}

Water containing solute infiltrates an initially unsaturated solute-free soll for about two hours. Solute-free water continues to infiltrate the soil after the initial two hours. The molsture front and a slug of solute move downwards through the soll column under conservative non-reactive constantdensity transport conditions, as described in a field experiment by Warrick, Biggar, and Nielsen (1971).

\section{Objective}

To simulate the transient propagation of the moisture front and solute slug as they move downwards through the soll column, under conditions of simulation equivalent to that used by Van Genuchten (1982) to represent the 


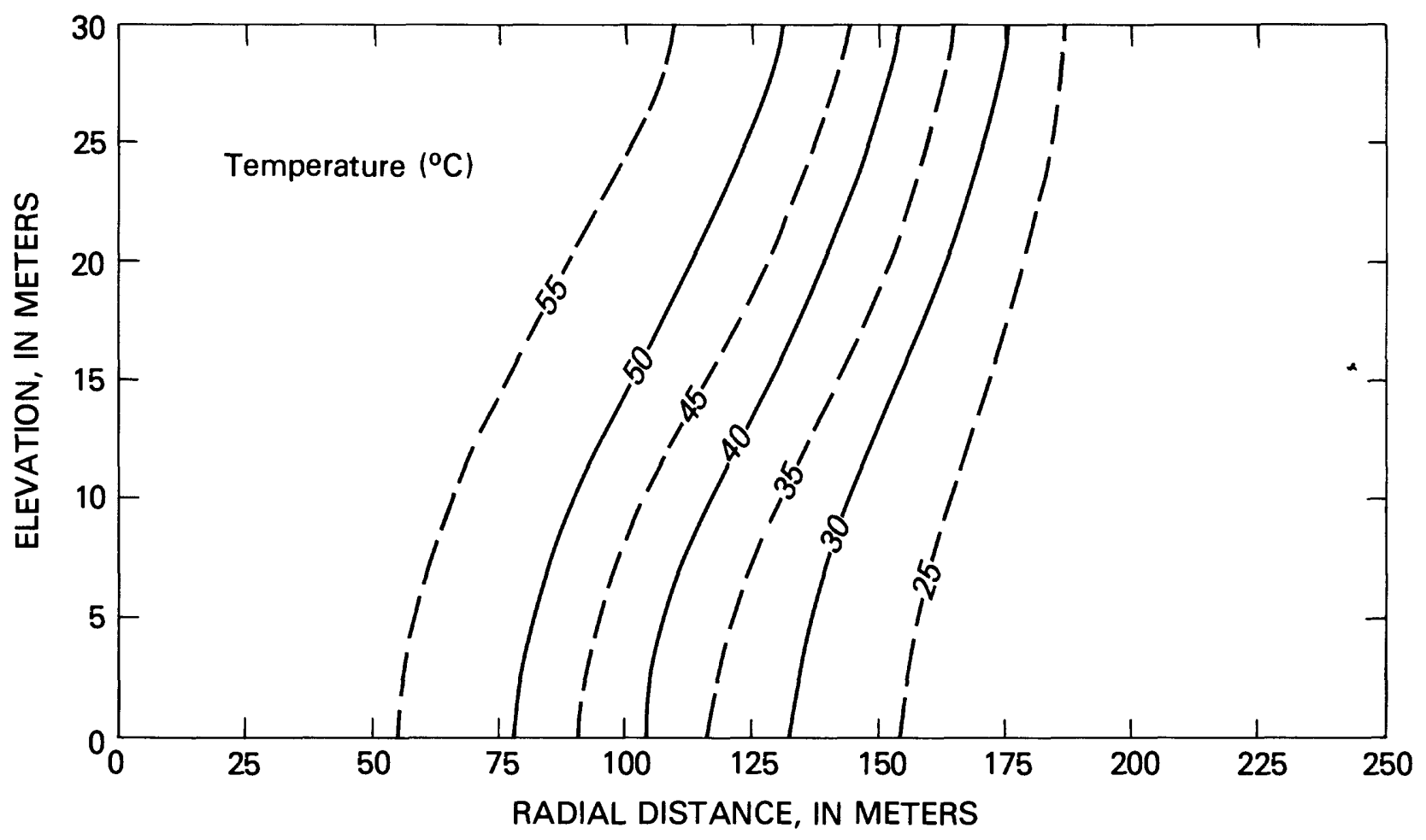

Figure 6.16 SUTRA results after 30 days of pumping, ( 120 days total elapsed time). 


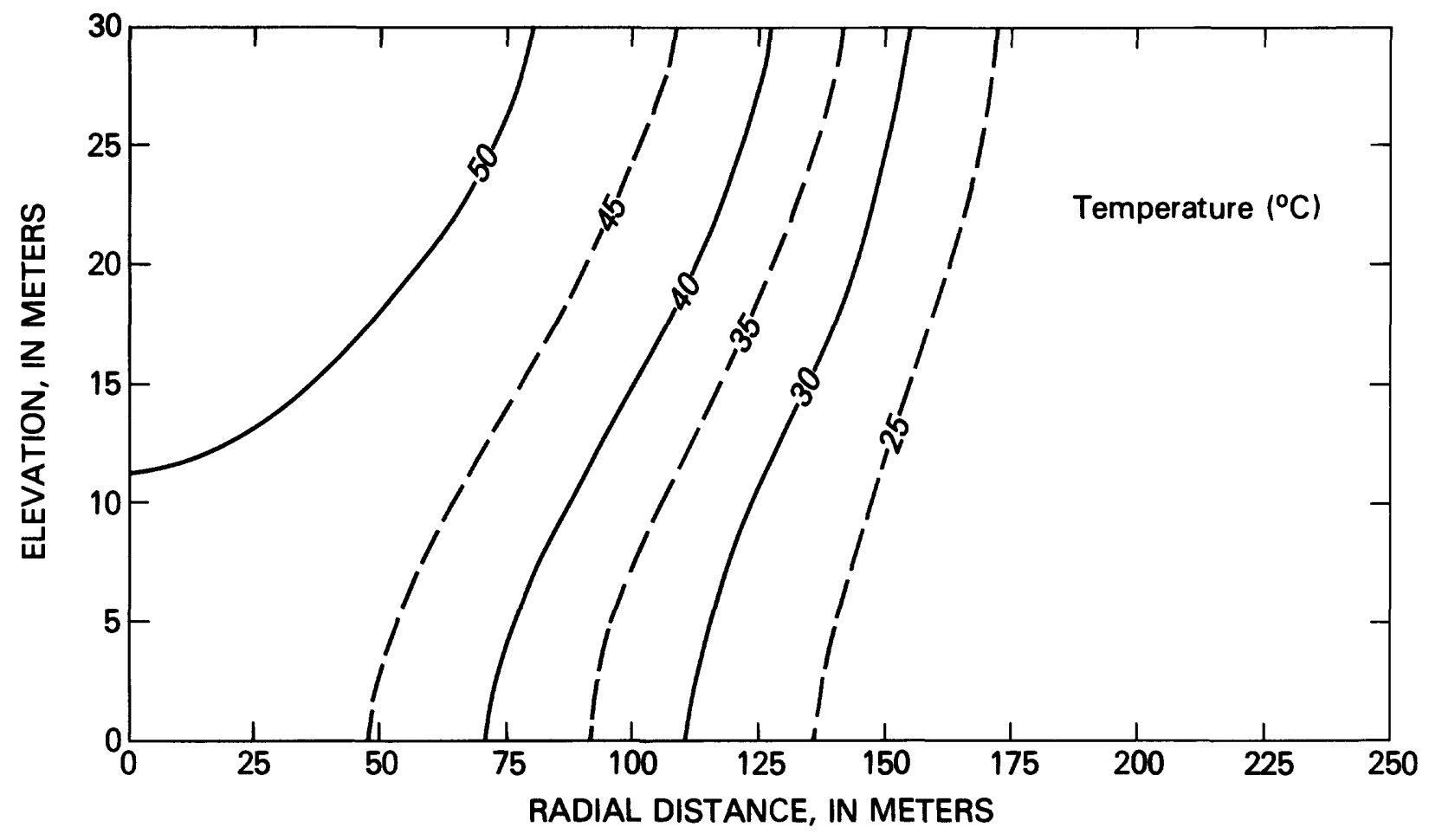

Figure 6.17 SUTRA results after 60 days of pumping, ( 150 days total elapsed time.) 


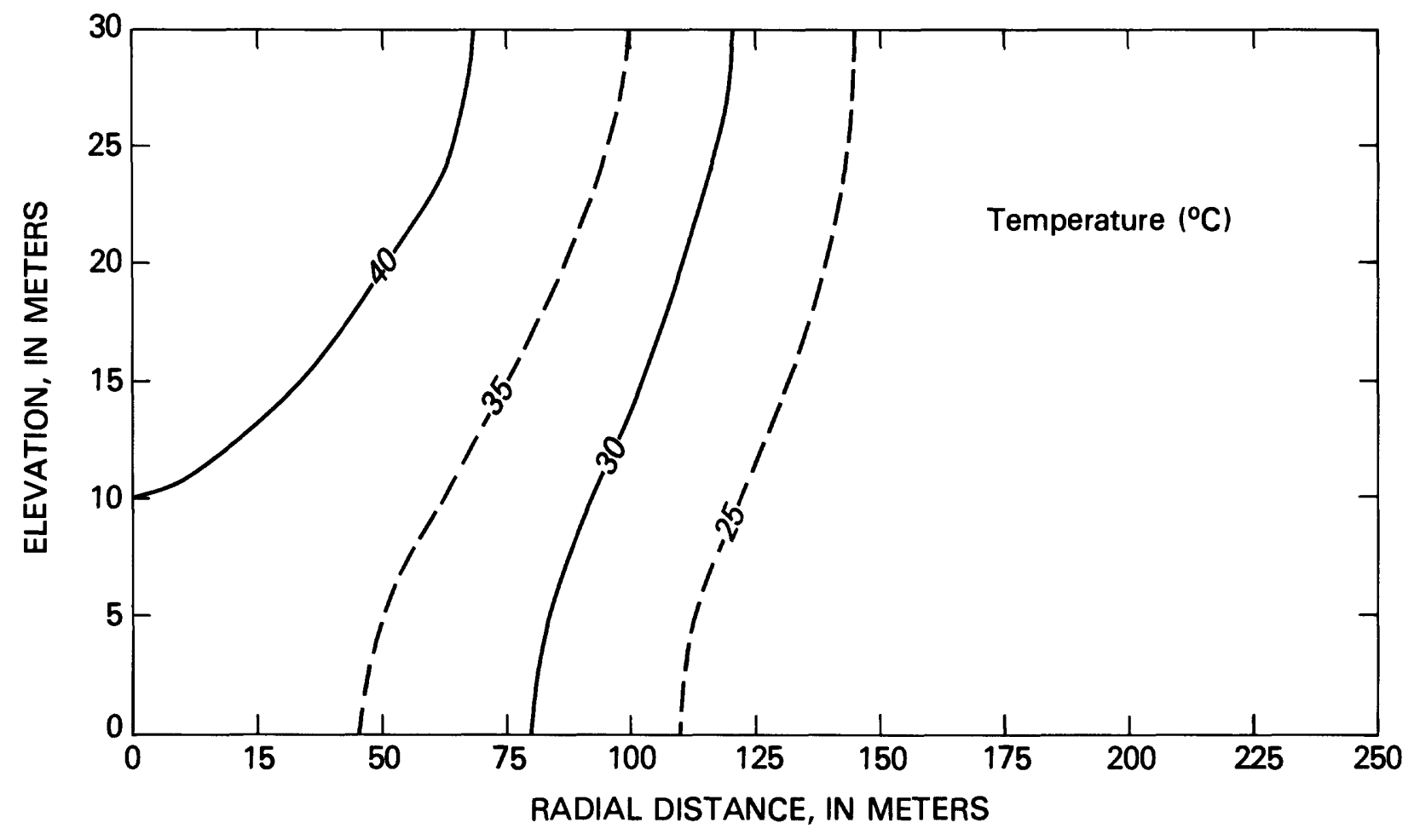

Figure 6.18

SUTRA results after 90 days of pumping, (180 days total elapsed time.) 
field experiment. The solutions should match the best fine grid - fine time step simulation results of Van Genuchten (1982) which were obtained with a number of different finite difference and finite element numerical methodologies.

\section{Simulation Set-up:}

The mesh consists of a single vertical column of 100 elements oriented in the direction of gravity, which is $2.0[\mathrm{~m}]$ long and $0.01[\mathrm{~m}]$ wide. The number of nodes and elements is: $N N=202, N E=100$. Each element is $0.01[\mathrm{~m}]$ wide and $0.02[\mathrm{~m}]$ high. Mesh thickness is unity. The vertical coordinate, $\mathrm{x}$, 1s measured downward from the top of the column.

The time step is constant at $\Delta t=30 .[\mathrm{s}]$, and because of the small time step, only one iteration is done per step. The simulation is carried out for nine hours of infiltration.

Outputs are obtained once each hour, but are only compared at two hours and nine hours. There is one pressure solution and one concentration solution each time step.

\section{$\underline{\text { Parameters }}$}

$$
\begin{aligned}
& k_{r}=1.235376 \times 10^{-6} \exp \left(13.604 \mathrm{~s}_{w}\right) \\
& S_{w}=1.52208-0.0718947 \ln (-\mathrm{p}) \\
& \quad \text { for }-2892.38<\mathrm{p} s-1421.96\left[\mathrm{~kg} /\left(\mathrm{m} \cdot \mathrm{s}^{2}\right)\right] \\
& S_{w}=2.94650-0.250632 \ln (-\mathrm{p}) \\
& \quad \text { for } \mathrm{p}<-2892.38\left[\mathrm{~kg} /\left(\mathrm{m} \cdot \mathrm{s}^{2}\right)\right] \\
& S_{\text {op }}=0.0 \quad \quad \rho=1000 .\left[\mathrm{kg} / \mathrm{m}^{3}\right] \\
& k=4.4558 \times 10^{-13 \quad\left[\mathrm{~m}^{2}\right]} \quad \sigma_{w}=0.0
\end{aligned}
$$




$$
\begin{array}{ll}
\varepsilon=0.38 & \alpha_{L}=0.01[\mathrm{~m}] \\
\mu=1.0 \times 10^{-3}[\mathrm{~kg} / \mathrm{m} \cdot \mathrm{s}] & \alpha_{\mathrm{T}}=0.0[\mathrm{~m}] \\
|\mathrm{g}|=9.81\left[\mathrm{~m} / \mathrm{s}^{2}\right] &
\end{array}
$$

\section{Boundary Conditions}

The top boundary representing an infiltration pond, is held fully saturated, $S_{w}=1.0$, (water content $\varepsilon S_{w}=0.38$ ) during the simulation by specification of pressure at $p=-1421.96\left[\mathrm{~kg} /\left(\mathrm{m} \cdot \mathrm{s}^{2}\right)\right]$. The bottom boundary is held at a specified saturation of $S_{w}=0.526316$, (water content $\varepsilon S_{w}=0.20$ ) by specification of pressure, $p=-15616.5\left[\mathrm{~kg} /\left(\mathrm{m} \cdot \mathrm{s}^{2}\right)\right]$. No flow occurs across either side boundary, but flow enters the top boundary due to the pressure specification. The concentration of inflowing fluid at the top is held at $c=209$. [meq/liter] until time $t=168.0[\mathrm{~min}]$, at which time the concentration of the inflow drops to $C=0.0$ [meq/liter]. Note that the concentration units are arbitrary (need not be mass fractions) because this is a constant density simulation.

\section{Initial Conditions:}

Initially, pressures are set to obtain the following initial distribution of saturation, shown in Figure 6.19:

$$
S_{w}(x, t=0)= \begin{cases}0.394737+0.219289 x & 0.0<x \leq 0.60[\mathrm{~m}] \\ 0.526316 & 0.6<x \leq 1.25[\mathrm{~m}]\end{cases}
$$

Initial concentrations are set to zero. 


\section{Results:}

SUTRA results after two hours and nine hours of infiltration are shown with the finely discretized solutions of Van Genuchten (1982) for saturation in Figure 6.19, and for concentration in Figure 6.20. The results coincide almost exactly for both early and late time so only one curve can be shown for each time. Although the SUTRA results are obtained with a non-iterative solution and small time steps, similar results may be obtained with longer time steps and a few iterations per step. The concentration front lags behind the moisture front as the volume between the concentration front and top boundary represents the water which has infiltrated. The volume of water between the moisture front and concentration front represents the initial water in the medium which has been displaced by the infiltrating water. 


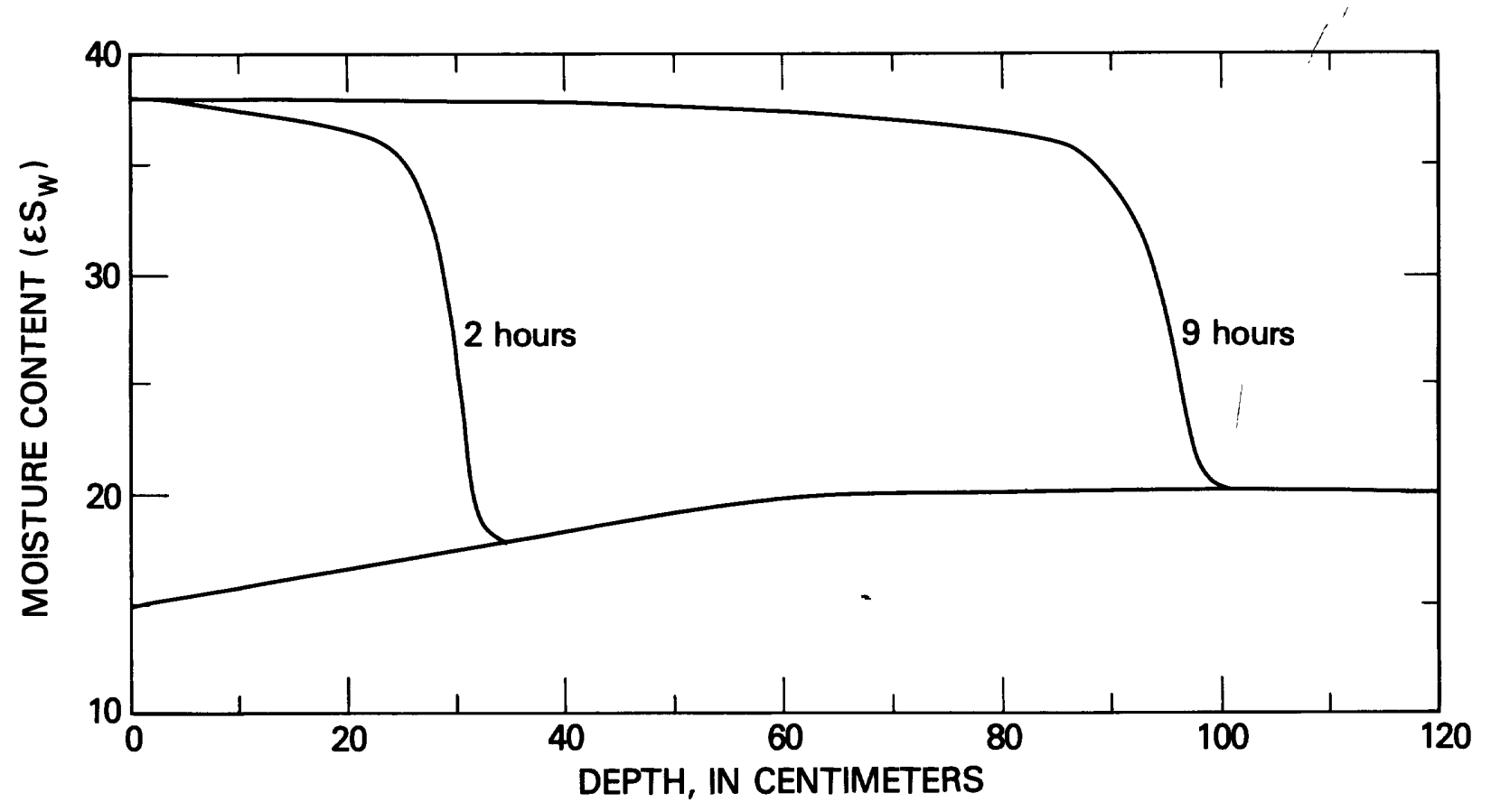

Figure 6.19

Propagation of moisture front for unsaturated flow and solute transport example. Results of Van Genuchten (1982) and SUTRA shown in same solid line. 


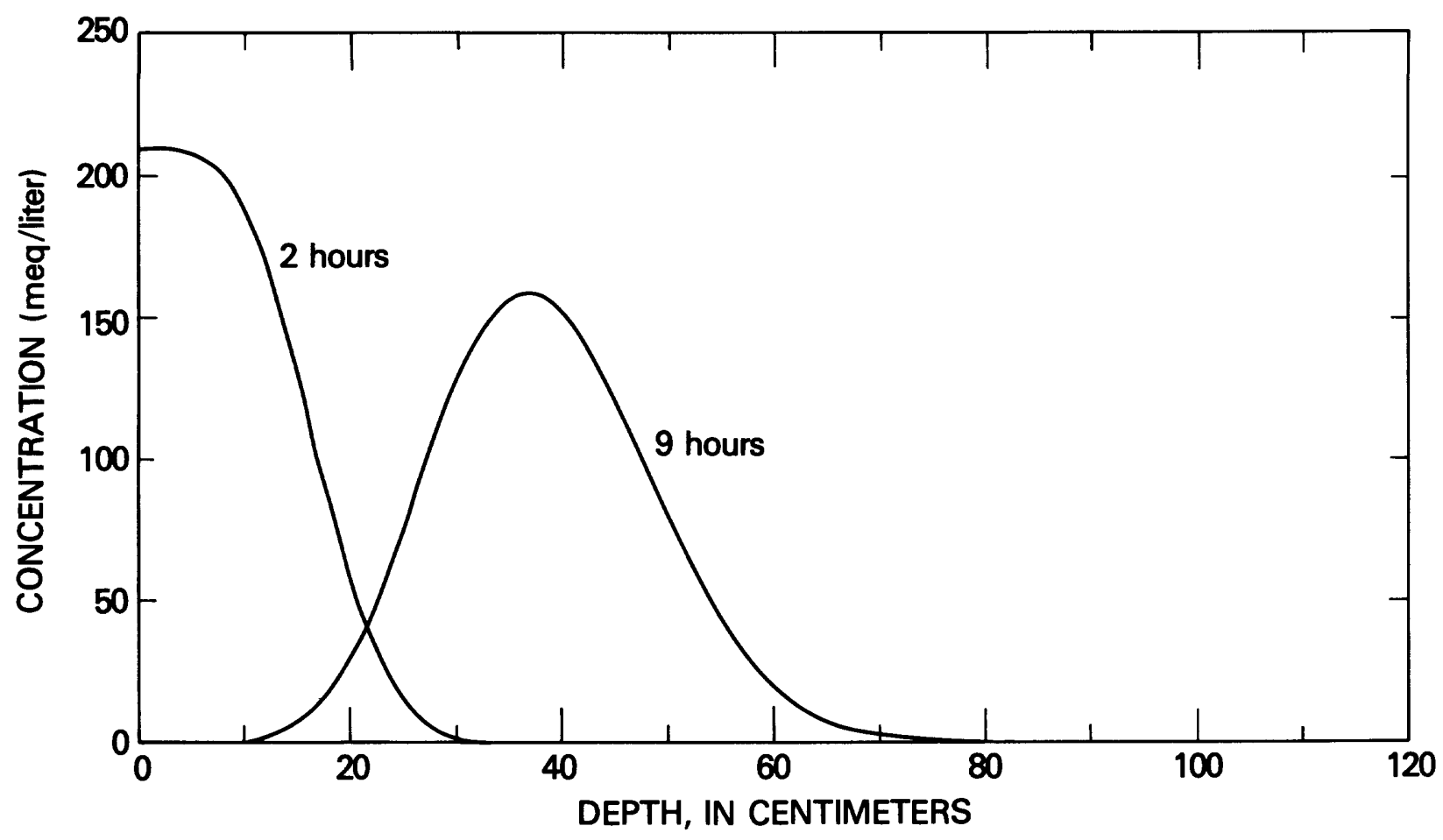

Figure 6.20

Propagation of solute slug for unsaturated flow and solute transport example. Results of Van Genuchten (1982) and SUTRA shown in same solid line. 
SUTRA SIMULATION SETUP

219

(p.221 follows) 


\section{Chapter 7}

\section{Simulation Setup}

\subsection{SUTRA Data Requirements}

The following is a complete list of data required to setup a simulation with SUTRA. (1) The information included in the list is the parameter name used in this report (if it has been mentioned), (2) the parameter units, (3) the parameter name in the input data list, and (4) a short explanation of the parameter.

\section{Mesh and coordinate data}

\begin{tabular}{|c|c|c|c|}
\hline \multirow[b]{2}{*}{$\mathbf{g}_{\mathbf{x}}$} & & \multirow[b]{2}{*}{ GRAVX } & \multirow[b]{2}{*}{$x$-component of gravity vector } \\
\hline & {$[\mathrm{L} / \mathrm{s}]$} & & \\
\hline $\mathrm{g}$ & {$\left[\mathrm{L} / \mathrm{s}^{2}\right]$} & GRAVY & $y$-component of gravity vector \\
\hline$x_{1}$ & {$[L]$} & $X(I)$ & $\begin{array}{l}x \text { coordinate of node } i, \text { for all } \\
\text { nodes in mesh }\end{array}$ \\
\hline$y_{1}$ & {$[\mathrm{~L}]$} & $\mathrm{Y}(\mathrm{I})$ & $\begin{array}{l}\text { y coordinate of node } 1 \text {, for all } \\
\text { nodes in mesh }\end{array}$ \\
\hline \multirow[t]{3}{*}{ NN } & & NN & total number of nodes in mesh \\
\hline & & $\operatorname{IIN}(1-4)$ & $\begin{array}{l}\text { counter-clockwise nodal incidence } \\
\text { list in each element }\end{array}$ \\
\hline & & $\operatorname{IEDGE}(1-4)$ & $\begin{array}{l}\text { ordered list of pinch nodes in each } \\
\text { element according to Figure } 5.5\end{array}$ \\
\hline \multirow[t]{3}{*}{$\mathrm{NE}$} & & $\mathrm{NE}$ & total number of elements in mesh \\
\hline & & NPINCH & total number of pinch nodes in mesh \\
\hline & & NBI & $\begin{array}{l}\text { full band-width of global banded } \\
\text { matrix }\end{array}$ \\
\hline
\end{tabular}




\begin{tabular}{|c|c|c|c|}
\hline B & {$\left[M /\left(L \cdot s^{2}\right)\right]^{-1}$} & COMPFL & fluid compressibility \\
\hline$\alpha$ & {$\left[M /\left(L \cdot s^{2}\right)\right]^{-1}$} & COMPMA & solid matrix compressibility \\
\hline$\varepsilon_{i}$ & {$[1]$} & $\operatorname{POR}(I)$ & $\begin{array}{l}\text { volumetric porosity of solid matrix } \\
\text { at each node }\end{array}$ \\
\hline $\mathrm{k}_{\max }$ & {$\left[L^{2}\right]$} & $\operatorname{PMAX}(I)$ & $\begin{array}{l}\text { maximum component of permeability in } \\
\text { each element }\end{array}$ \\
\hline $\mathrm{k}_{\mathrm{min}}$ & {$\left[L^{2}\right]$} & $\operatorname{PMIN}(L)$ & $\begin{array}{l}\text { minimum component of permeability in } \\
\text { each element }\end{array}$ \\
\hline${ }^{\theta} \mathrm{L}$ & {$\left[{ }^{\circ}\right]$} & $\operatorname{ANGLEX}(\mathrm{L})$ & $\begin{array}{l}\text { angle between } k_{\max } \text { and }+x-a x i s \text { in } \\
\text { each element }\end{array}$ \\
\hline Po & {$\left[M / L^{3}\right]$} & RHOWO & fluid base density \\
\hline \multirow[t]{2}{*}{$\frac{\partial \rho}{\partial U}$} & $\begin{array}{c}{\left[\mathrm{M} / \mathrm{L}^{3} \cdot{ }^{\circ} \mathrm{C}\right]} \\
\text { or }\end{array}$ & DRWDU & $\begin{array}{l}\text { for energy transport: coefficient } \\
\text { of fluid density change with temp- } \\
\text { erature }\end{array}$ \\
\hline & {$\left[M^{2} / L^{3} \cdot M_{s}\right]$} & DRWDU & $\begin{array}{l}\text { for solute transport: coefficient } \\
\text { of fluid density change with concen- } \\
\text { tration }\end{array}$ \\
\hline \multirow[b]{2}{*}{$\mathrm{U}_{\mathrm{o}}$} & {$\left[{ }^{\circ} \mathrm{C}\right]$} & URHOWO & $\begin{array}{l}\text { for energy transport: base tempera- } \\
\text { ture for density calculation }\end{array}$ \\
\hline & {$\left[M_{s} / M\right]$} & URHOWO & $\begin{array}{l}\text { for solute transport: base concen- } \\
\text { tration for density calculation }\end{array}$ \\
\hline
\end{tabular}

Transport parameters

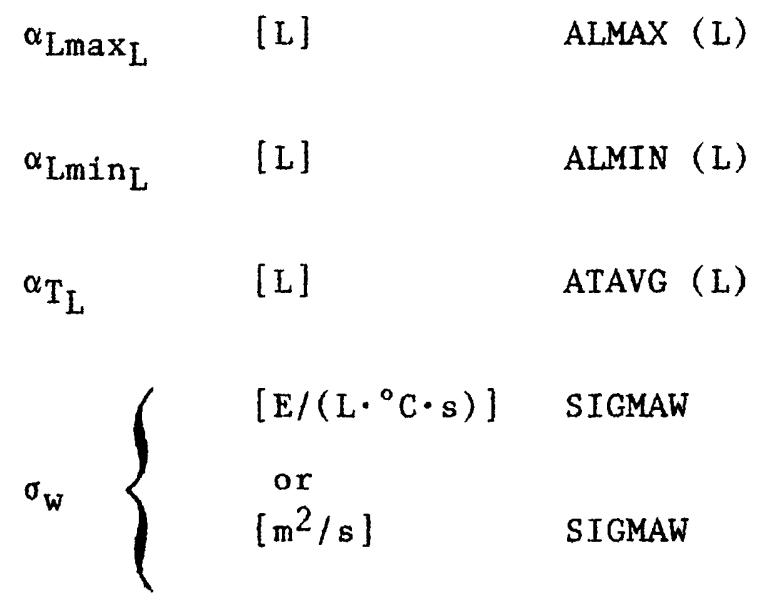

value of longitudinal dispersivity in direction of $k_{\max }$ in each element

value of longitudinal dispersivity in direction of $k_{\min }$ in each element

value of transverse dispersivity in each element

for energy transport: fluid thermal conductivity

for solute transport: molecular diffusivity of solute in fluid 


\begin{tabular}{|c|c|c|c|}
\hline$\sigma_{\mathbf{s}}$ & {$\left[\mathrm{E} /\left(\mathrm{L} \cdot{ }^{\circ} \mathrm{C} \cdot \mathrm{s}\right)\right]$} & SIGMAS & $\begin{array}{l}\text { for energy transport: solid grain } \\
\text { thermal conductivity (equals zero } \\
\text { for solute transport) }\end{array}$ \\
\hline$c_{w}$ & {$\left[E /\left(M \cdot{ }^{\circ} \mathrm{C}\right)\right]$} & CW & $\begin{array}{l}\text { for energy transport: fluid specific } \\
\text { heat capacity (equals one for solute } \\
\text { transport) }\end{array}$ \\
\hline$c_{8}$ & {$\left[\mathrm{E} /\left(\mathrm{M} \cdot{ }^{\circ} \mathrm{C}\right)\right]$} & Cs & $\begin{array}{l}\text { for energy transport: solid grain } \\
\text { specific heat capacity (not specified } \\
\text { in input data for solute transport) }\end{array}$ \\
\hline$P_{\mathbf{g}}$ & {$\left[\mathrm{M} / \mathrm{L}^{3}\right]$} & RHOS & $\begin{array}{l}\text { density of a solid grain in the solid } \\
\text { matrix }\end{array}$ \\
\hline
\end{tabular}

Reaction and production parameters

\section{Linear Sorption Isotherm}

$\begin{array}{lll}X_{1} & {\left[L_{f}^{3} / M_{G}\right] \quad \text { CHIl }} & \text { linear distribution coefficient }(2.34 a) \\ & \left(x_{2} \text { is zero for this isotherm) }\right.\end{array}$

\section{Freundlich Sorption Isotherm}

$\begin{array}{llll}x_{1} & {\left[L_{f}^{3} / M_{G}\right]} & \text { CHI } & \text { Freundlich distribution coefficient (2.35a) } \\ x_{2} & {[1]} & \text { CHI2 } & \text { Freundlich coefficient }(2.35 a)\end{array}$

\section{Langmuir Sorption Isotherm}

$\begin{array}{llll}x_{1} & {\left[L_{f}^{3} / M_{G}\right]} & \text { CHI } & \text { Langmuir distribution coefficient (2.36a) } \\ x_{2} & {\left[L_{f}^{3} / M_{s}\right]} & \text { CHI2 } & \text { Langmuir coefficient }(2.36 a)\end{array}$

\section{Production}

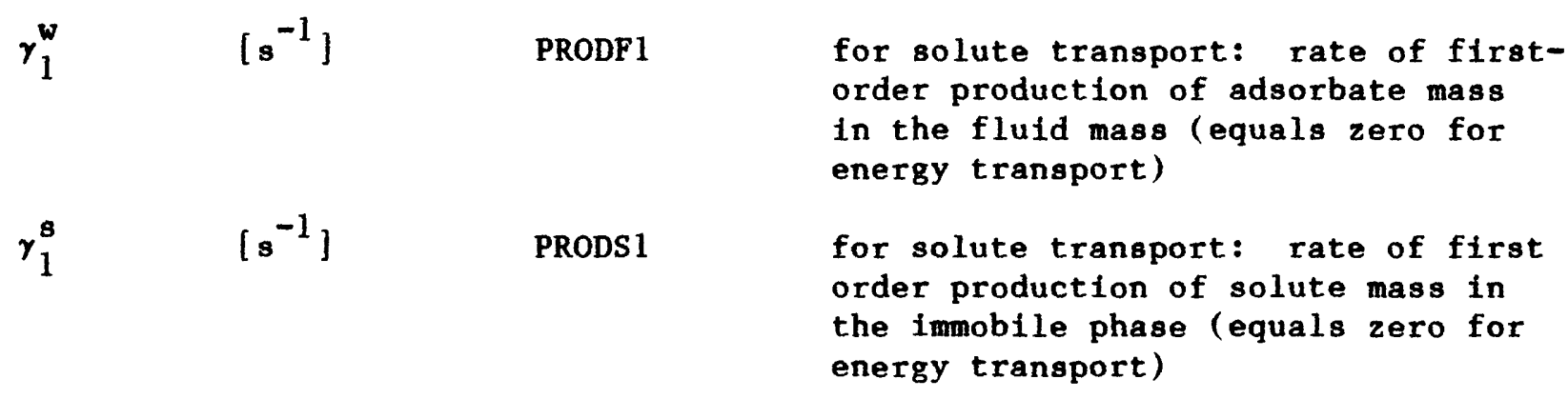




\begin{tabular}{|c|c|c|c|}
\hline$r_{0}^{w}$ & {$[(E / M) / s]$} & PRODFO & $\begin{array}{l}\text { for energy transport: zero-order } \\
\text { rate of energy production in the } \\
\text { fluid }\end{array}$ \\
\hline & {$\left[\left(M_{s} / M\right) / s\right]$} & PRODFO & $\begin{array}{l}\text { for solute transport: zero-order } \\
\text { rate of solute mass production in } \\
\text { the fluid }\end{array}$ \\
\hline & {$\left[\left(E / M_{G}\right) / s\right]$} & PRODSO & $\begin{array}{l}\text { for energy transport: zero-order } \\
\text { rate of energy production in the } \\
\text { immobile phase }\end{array}$ \\
\hline & {$\left[\left(M_{s} / M_{G}\right) / s\right]$} & PRODSO & $\begin{array}{l}\text { for solute transport: zero-order } \\
\text { rate of adsorbate mass production } \\
\text { in the immobile phase }\end{array}$ \\
\hline
\end{tabular}

Boundary conditions and source data

Flow Data - Specified Pressures

\begin{tabular}{|c|c|c|c|}
\hline NPBC & & NPBC & $\begin{array}{l}\text { number of nodes at which pressure is } \\
\text { a specified constant or function of } \\
\text { time }\end{array}$ \\
\hline IPBC $_{\text {ipu }}$ & & IPBC (IPU) & $\begin{array}{l}\text { node number at which pressure is } \\
\text { specified (for all NPBC nodes) }\end{array}$ \\
\hline $\mathrm{PBC}_{\text {ipu }}$ & {$\left[M /\left(L \cdot s^{2}\right)\right]$} & $\mathrm{PBC}(\mathrm{IPU})$ & $\begin{array}{l}\text { value of specified pressure at node } \\
\text { IPBC (for all NPBC nodes) }\end{array}$ \\
\hline UBC & {$\left[{ }^{\circ} \mathrm{C}\right]$} & UBC(IPU) & $\begin{array}{l}\text { for energy transport: value of temp- } \\
\text { erature of any fluid which enters } \\
\text { the system at node IPBC }\end{array}$ \\
\hline & {$\left[M_{s} / M\right]$} & UBC ( IPU) & $\begin{array}{l}\text { for solute transport: value of } \\
\text { concentration of any fluid which } \\
\text { enters the system at node IPBC }\end{array}$ \\
\hline & Flow Data & - Specifled I & Flows and Fluid Sources \\
\hline NSOP & & NSOP & $\begin{array}{l}\text { number of nodes at which a source } \\
\text { of fluid mass is specified }\end{array}$ \\
\hline $\mathrm{IQCP}_{\text {iqp }}$ & & $\begin{array}{l}\text { IQCP, } \\
\text { IQSOP (IQP) }\end{array}$ & $\begin{array}{l}\text { node number at which a fluid source } \\
\text { is specified (for all NSOP nodes) }\end{array}$ \\
\hline $\mathrm{Q}_{\mathrm{IN}_{1}}$ & {$[\mathrm{M} / \mathrm{s}]$} & QINC, QIN(I) & $\begin{array}{l}\text { fluid source rate at source node } \\
\text { IQCP (for all nodes) }\end{array}$ \\
\hline
\end{tabular}




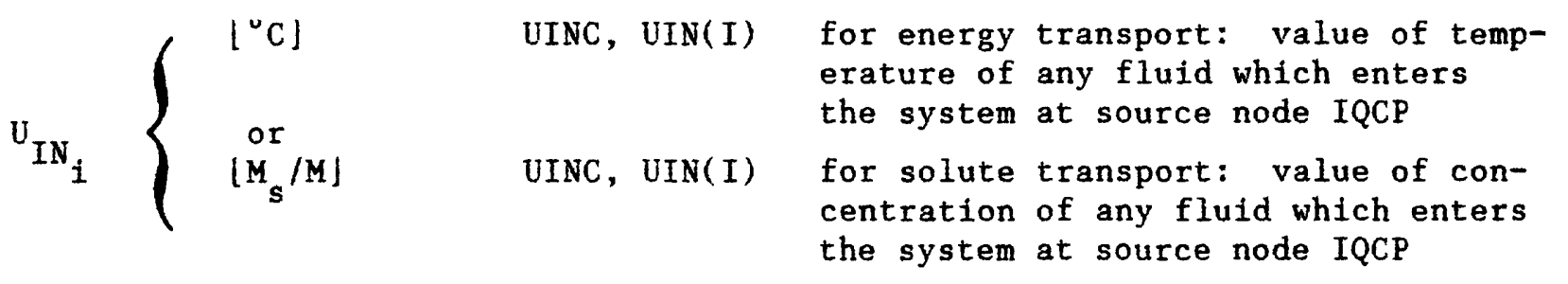

Energy or Solute Data -

Specified Temperatures or Concentrations

\begin{tabular}{|c|c|c|c|}
\hline \multicolumn{2}{|l|}{ NUBC } & NUBC & $\begin{array}{l}\text { number of nodes at which temperature } \\
\text { or concentration is a specified } \\
\text { constant or function of time }\end{array}$ \\
\hline \multicolumn{2}{|l|}{ IUBC $_{\text {ipu }}$} & IUBC (IPU) & $\begin{array}{l}\text { node number at which temperature } \\
\text { or concentration is specified (for } \\
\text { all NUBC nodes) }\end{array}$ \\
\hline \multirow[t]{2}{*}{ UBC } & {$\left[{ }^{\circ} \mathrm{C}\right\rfloor$} & $\mathrm{UBC}(\mathrm{IPU})$ & $\begin{array}{l}\text { for energy transport: value of spec- } \\
\text { ified temperature at node IUBC (for } \\
\text { all NUBC nodes) }\end{array}$ \\
\hline & {$\left[M_{s} / M\right]$} & $\mathrm{UBC}(\mathrm{IPU})$ & $\begin{array}{l}\text { for solute transport: value of spec- } \\
\text { ified concentration at node IUBC (for } \\
\text { all NUBC nodes) }\end{array}$ \\
\hline
\end{tabular}

Energy or Solute Data -

Diffusive Fluxes of Energy or Solute Mass at Boundaries

\begin{tabular}{|c|c|c|c|}
\hline \multicolumn{2}{|l|}{ NSOU } & NSOU & $\begin{array}{l}\text { number of nodes at which a diffusive } \\
\text { energy or solute mass flux (source) } \\
\text { is specified }\end{array}$ \\
\hline \multicolumn{2}{|l|}{ IQCU } & $\begin{array}{l}\text { IQCU, } \\
\text { IQSOU (IQU) }\end{array}$ & $\begin{array}{l}\text { node number at which a flux (source) } \\
\text { is specified (for all NSOU nodes) }\end{array}$ \\
\hline \multirow[t]{2}{*}{${ }^{I \mathrm{IN}_{i}}$} & $\lfloor E / s\rfloor$ & QUIN(I) & $\begin{array}{l}\text { for energy transport: energy flux } \\
\text { (source) rate at node IQCU (for all } \\
\text { NSOU nodes) }\end{array}$ \\
\hline & $\left\lfloor M_{s} / s\right\rfloor$ & QUIN(I) & $\begin{array}{l}\text { for solute transport: solute mass } \\
\text { flux (source) rate at node IQCU (for } \\
\text { all NSOU nodes) }\end{array}$ \\
\hline
\end{tabular}

Initial conditions

$\begin{array}{llll}t_{0} & {[s \mid} & \text { TSTART } & \text { starting time for simulation clock } \\ p_{i}\left(t=t_{0}\right) & \left\{M /\left(L \cdot s^{2}\right) \mid\right. & \operatorname{PVEC}(I I) & \text { initial pressure at all nodes in mesh }\end{array}$




$$
U_{i}\left(t=t_{0}\right)\left\{\begin{array}{l}
{\left[{ }^{\circ} \mathrm{C}\right]} \\
{\left[M_{s} / M\right]}
\end{array}\right.
$$

Numerical and temporal control data

\begin{tabular}{|c|c|c|}
\hline$v_{i}$ & {$[\mathrm{Ls}]$} & GNU \\
\hline UP & {$[1]$} & UP \\
\hline \multirow[t]{6}{*}{$\Delta t$} & [s] & DELT \\
\hline & [s] & TMAX \\
\hline & & ITMAX \\
\hline & & ITCYC \\
\hline & & DTMULT \\
\hline & & DTMAX \\
\hline NPCYC & & NPCYC \\
\hline \multirow[t]{5}{*}{ NUCYC } & & NUCYC \\
\hline & & ITRMAX \\
\hline & {$\left[M /\left(L \cdot s^{2}\right)\right]$} & RPMAX \\
\hline & {$\left[{ }^{\circ} \mathrm{C}\right]$} & RUMAX \\
\hline & {$\left[M_{s} / M\right]$} & RUMAX \\
\hline
\end{tabular}

\section{Data for options}

for energy transport: initial temperature at all NN nodes in the mesh for solute transport: initial concentration at all NN nodes in the mesh

specified pressure boundary condition 'conductance' factor (4.64)

fractional upstream weight for asymmetric weighting functions (4.23) (4.24)

initial time step

maximum allowed simulation time

maximum allowed number of time steps in a simulation

time step change cycle

multiplier for time step change cycle

maximum time step size allowed when using multiplier

time steps in pressure solution cycle

time steps in temperature or concen-

tration solution cycle

maximum number of iterations for nonlinearities per time step

pressure convergence criterion for iterations

for energy transport: temperature convergence criterion

for solute transport: concentration convergence criterion
IREAD

$\left\{\begin{array}{l}=+1 \text { new simulation - cold start } \\ =-1 \text { restart simulation - warm start }\end{array}\right.$

ISTORE

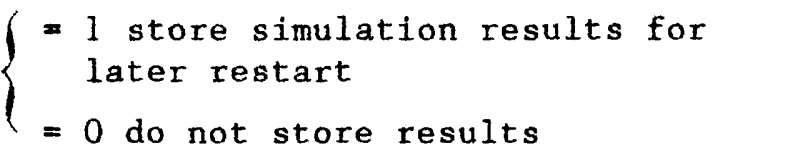


Simulation mode options

SIMULA

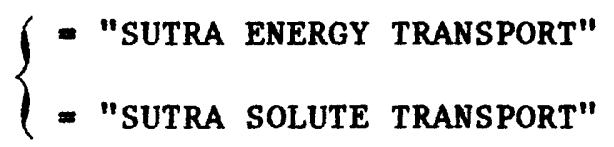

IUNSAT

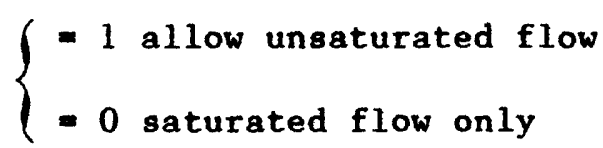

ISSFLO

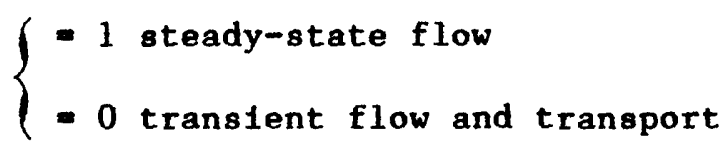

ISSTRA

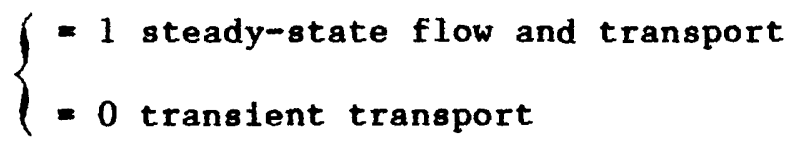

Veloc1ty Output Option

KVEL

$$
\left\{\begin{array}{l}
=1 \text { output fluid velocity at element } \\
\text { centroids } \\
=0 \text { no velocity output }
\end{array}\right.
$$

Printer Plot Output Option

KPLOTP

$$
\left\{\begin{array}{l}
=1 \text { output of pressure } \\
=0 \text { no pressure plots }
\end{array}\right.
$$

KPLOTU

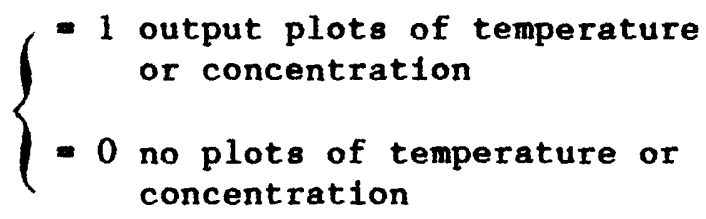

IDIREC

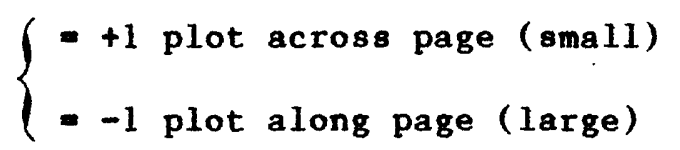

NLINPI

number of printer lines per inch 
NCHAPL number of printer characters per line

PBASE value for scaling plotted pressures

UBASE value for scaling plotted temperatures or concentrations

\section{Observation Option}

NOBS

number of nodes at which pressure and temperature or concentration will be observed (zero cancels the option)

NTOBS

maximum number of observation time steps

NOBCYC

observations are made every NOBCYC time steps

INOB(I) observation node numbers

\section{Budget Option}

KBUDG

$=1$ output fluid mass and energy or
solute mass budgets
$=0$ no budgets

\section{Output Controls}

KNODAL

KELMNT

KINCID

NPRINT $\left\{\begin{array}{l}1 \text { output nodewise input data } \\ 0 \text { cancel output }\end{array}\right.$
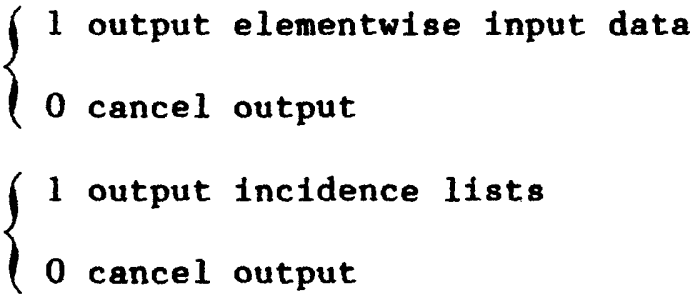

results are output every NPRINT time steps 


\subsection{Discretization Rules-of-Thumb}

Proper discretization in space and time is the vital factor in obtaining accurate simulation of the physics of flow and transport with a numerical model such as SUTRA. Adequate discretization is vital for two reasons: 1) The ability of a model to represent the variations in system parameters and to simulate complex processes depends on the fineness of discretization. 2) The accuracy and stability of the numerical methods used to represent system processes, in particular, transport, depends on the spatial and temporal discretization. This section describes some general guidelines for designing adequate discretization for simulation with SUTRA.

A 'sufficiently good' discretization allows for accurate simulation of the processes and parameter variations at the scale of interest, and thus the goodness of a discretization is a relative rather than absolute factor. A better discretization is always obtained by making existing discretization finer, but the finer the discretizations are, the more computationally expensive the simulations become.

Relative to a certain adequate level of fineness, even finer discretizations do not practically improve the accuracy of simulation. In contrast, discretization that is too coarse may completely obscure parameter variations and processes of interest in a simulation, and give highly inaccurate results. Unfortunately, simulation results based on inadequate discretization may appear to be a reasonably good representation of flow and transport physics in a particular system. The only way to explicitly check for inadequate discretization of a system is to simulate with a discretization that is assumed to be adequate and then with a significantly finer discretization and compare results. If there are no telling dif- 
ferences in the results, then the coarser simulation indeed has been adequately discretized.

Some general guidelines for obtaining adequate discretization, both for parameter representation and for accuracy and stability of numerical methods are given below.

1) Nodes are required where boundary conditions and sources are specified. Should accurate simulation of processes near these specified points be required, then a finer mesh is needed in these areas.

2) A finer mesh is required where parameters vary faster in space. This is often the case near sources or boundary conditions specifying inflows of fluid, solute or energy. The fineness required is that which makes the nodewise, cellwise, or elementwise discretization of the parameter values a good representation of the actual distributions. When a parameter distribution is known a priori, then this discretization is straightforward. However, when the parameter distribution depends on the simulation results then judgement must be exercised in discretization, and the result may be tested by experiment with various discretizations.

It is important to recognize that each node or element does not alone represent a physical entity in an aquifer system. This is demonstrated in the following example which shows that one layer of elements is not a good representation in cross section of a semi-confining layer or aquifer untt. Although permeability is specified elementwise and the permeability of two aquifer units separated by confining layer, viewed in cross-section, is clearly represented visually by three layers of elements, the numerical model does not 'see' three 
distinct layers of permeability. Each node at the boundary of these layers experiences some average of the two permeabilities rather than either one. Thus, no node in the system experiences the assigned low permeability of confining layer, and the three-layer discretization is inadequate. More layers of elements are required in each unit to obtain adequate discretization although the model always experiences an average permeability in the elements making up the boundaries of the units. Further refinement of discretization would be required to represent the pressure distribution should accurate simulation of sharply-varying pressures across the confining layer be required.

Discretization of the spatial distribution of transport variables, concentration or temperature, often is that which requires the finest mesh. The spatial distributions of these variables often include a 'front' at which the concentration or temperature changes sharply from high values on one side to low values on the other side. A rule-of-thumb is that at least five elements should divide the front in order to guarantee that the simulated front width arises from simulated physical processes rather than from spreading due to Inadequate discretization. When such fronts travel with the flow across a mesh during simulation, then the mesh must be designed fine enough to adequately represent the front at all points along its path. In regions external to the front path, coarser discretization is usually adequate, and an expanding mesh or pinch nodes may be used to design the discretization in this region.

3) The spatial stability of the numerical approximation of the unified transport equation (2.52) depends on the value of a mesh Peclet number, $\mathrm{Pe}_{\mathrm{m}}$, given by: 


$$
P e_{m}=\frac{\varepsilon S_{w}|\underline{v}| \Delta L_{L}}{\left[\varepsilon S_{w}\left(\sigma_{w}+\alpha_{L}|\underline{v}|\right)+(1-\varepsilon) \sigma_{s}\right]}
$$

where $\Delta \mathrm{L}_{\mathrm{L}}$ is the local distance between element sides along a streamilne of flow. Spatial instability appears as one or more oscillations in concentration or temperature. Stability is guaranteed in all cases when $P e_{m} \leq 2$, which gives a criterion for choosing a maximum allowable element dimension, $\Delta L_{L}$, along the local flow direction. This criterion significantly affects discretization. Spatial stability is usually obtained with SUTRA when

$$
\mathrm{Pe}_{\mathrm{m}} \leq 4
$$

which gives a less-stringent criterion. Mesh design according to the criterion is critical when concentrations or temperatures change significantly along streamlines, such as when a front is propagated in the direction of flow. When concentrations or temperatures exhibit small changes along streamlines, then the criterion, (7.2) may safely be violated, even by a few orders of magnitude, without inducing spatial instability. An example of this may be cross-sectional simulation of an aquifer containing fresh water and salt water. In such a case, flow often is directed parallel to the front between fresh water and salt water, allowing use of discretization with large mesh Peclet numbers.

In the typical case of solute or energy transport with longitudinal dispersion primarily due to longitudinal mixing, the mesh Peclet number becomes:

$$
\mathrm{Pe}_{\mathrm{m}} \approx\left(\frac{\Delta \mathrm{L}_{\mathrm{L}}}{\alpha_{\mathrm{L}}}\right)
$$

A discretization rule-of-thumb for simulation with SUTRA which guarantees spatial stability in most cases is:

$$
\Delta \mathrm{L}_{\mathrm{L}} \leq 4 \alpha_{\mathrm{L}}
$$


While (7.4) deals with adequate discretization for numerical stability it may be interpreted from another point of view. Taken in combination with the considerations of guideline (2) requiring at least five elements across a front, (7.4) implies that a minimum front width which may be simulated when the mesh is designed according to $\Delta \mathrm{L}_{\mathrm{L}} \sim 4 \alpha_{\mathrm{L}}$ is $20 \alpha_{\mathrm{L}}$. Thus for eariy times following onset of localized energy or solute source, the sharp front that should result may be simulated inaccurately as its width is less than $20 \alpha_{L}$.

4) Discretization for transverse dispersion also may be related to dispersivity. Although an exact guideline is not given, the object of transverse discretization is to make the local element dimension perpendicular to a streamline small relative to the total transverse dispersivity:

$$
\Delta \mathrm{L}_{\mathrm{T}}<\alpha_{\mathrm{T}}+\frac{1}{|\underline{\mathrm{v}}|}\left[\varepsilon \mathrm{S}_{\mathrm{w}} \sigma_{\mathrm{w}}+(1-\varepsilon) \sigma_{\mathrm{s}}\right]
$$

where $\Delta \mathrm{L}_{\mathrm{T}}$ is the local element dimension transverse to the flow direction. In the case where the transverse mixing rather than diffusion dominates the transverse dispersion an adequate but stringent rule-of-thumb may be, $\Delta \mathrm{L}_{\mathrm{T}}<10 \alpha_{\mathrm{T}}$, although simulation results should be compared for various transverse discretizations.

5) Radial/cylindrical meshes with a well require very fine discretization near the center axis to accommodate the sharply curving pressure distribution. The radial element dimensions may increase outward and become constant at, for example, a size of $4 \alpha_{L}$. 
6) Unsaturated flow simulation requires at least as fine discretization as does transport. Spatial instability appears as an oscillation in saturation values. Unsaturated flow parameters may vary sharply in space, especially during wetting events. A rule-of-thumb is to design the mesh to have at least five elements across a saturation front.

7) Discretization in time is done by choosing the size of time steps. Actual time step sizes may be as large as possible while providing adequate discretization of parameter changes in time. As with spatial discretization, the adequacy of a temporal discretization may be tested only by comparing results of simulations carried out with different time step sizes.

For saturated flow simulation, temporal aiscretization begins with fine time steps which may become significantly larger as the system response slows. The time-step multiplier feature is provided in SUTRA input data to allow this type of temporal discretization.

For unsaturated flow simulation with SUTRA, temporal discretization must be fine enough to keep saturation changes at each node to be small over any time step. A rule-of-thumb is that over a time step, the maximum saturation change is about 0.1 .

For transport simulation, temporal changes in concentration or temperature at a point in space are often due to the movement of fronts with the fluid flow. Therefore, adequate discretization of these parameters in time is often related to both fluid velocity and spatial gradients in the parameters. The higher the longitudinal spatial gradient and fluid velocity, the smaller the time step required for adequate temporal discretization. A general guideline is that relatively sharp fronts require time discretization which allows them to move only 
a fraction of an element per time step. Broad fronts with low gradient in concentration or temperature have adequate temporal discretization when time steps are chosen to move the front one or more elements per step.

Usually a constant time step size is chosen for transport simulation when flow velocities remain relatively constant during a simulation. For saturated flow and transport, if adequate temporal pressure discretization would allow larger time steps than the temporal transport discretization, then a pressure solution may be done only every $n$ time steps for transport. For example, if the adequate pressure time step is ten times that of transport, then SUTRA input data requires the specification: $\quad N P C Y C=10, \quad$ NUCYC $=1$.

\section{3 Program Dimensions}

All vector and array dimensions in the SUTRA computer code which may vary between simulations are combined for user convenience in three large arrays, RM, RV, and IMV. These arrays are dimensioned by the user in the main routine for SUTRA. No other arrays need be dimensioned. RM contains all of the real matrices in the code, RV contains all of the real vectors, and IMV contains all of the integer matrices and vectors. The dimensions required for these arrays, RMDIM, RVDIM, and IMVDIM, must be specified in the main program to values greater than or equal to those required. The required values are given by relations similar to:

$$
\operatorname{RMDIM}=2(\mathrm{NN})(\mathrm{NBI})
$$




$$
\begin{aligned}
\text { RVDIM }= & (\text { NNV })(\mathrm{NN})+(\mathrm{NEV}+8)(\mathrm{NE})+(\mathrm{NBCN}) 3 \\
& +(\mathrm{NOBS}+1)(\mathrm{NTOBS}+2) 2+\mathrm{NTOBS}+5 \\
\text { IMVDIM }= & (\mathrm{NE}) 8+\mathrm{NN}+(\mathrm{NPINCH}) 3+\mathrm{NSOP}+\mathrm{NSOU} \\
& +(\mathrm{NBCN}) 2+\mathrm{NOBS}+\mathrm{NTOBS}+12
\end{aligned}
$$

and

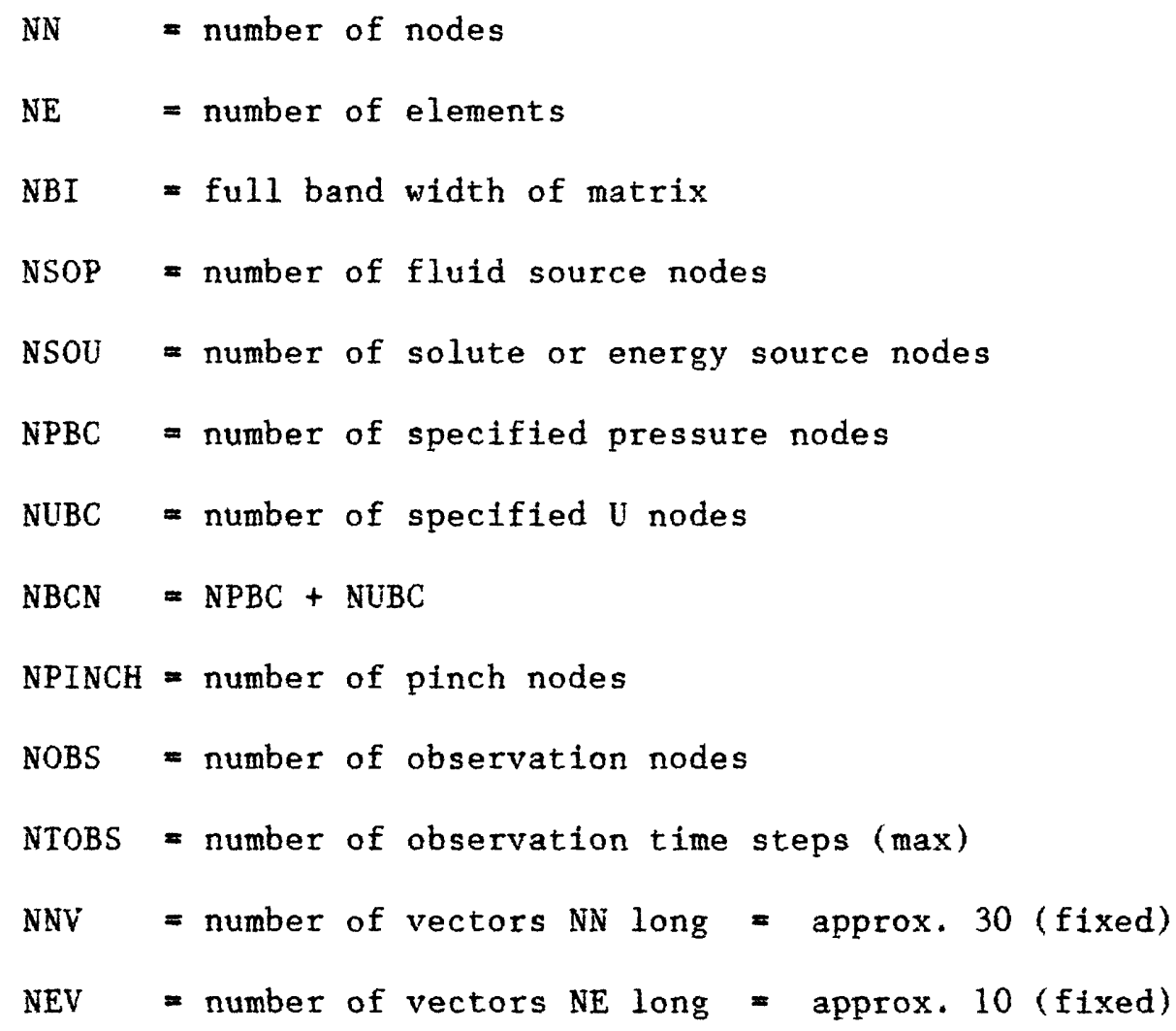

The actual relations and values are listed in the main routine and should be checked there for the most recent SUTRA model version. These dimensions may be greater than but not less than the values given by the relations equivalent to $(7.6),(7.7)$ and $(7.8)$ in the main routine. 
7.4 Input and output Files

The SUTRA computer code requires three or four files to be assigned on the computer in order to run simulations. Two of these are input files and one or two of these are output files.

\section{INPUT FILES:}

UNIT-5 A file must be assigned as fortran-unit-5 which contains SUTRA input data for UNIT-5. This file contains all of the data necessary for simulation except initial conditions.

UNIT-55 A file must be assigned as fortran-unit-55 which contains SUTRA input data for UNIT-55. This file contains initial conditions of pressure and concentration or temperature for the simulation to be done.

\section{OUTPUT FILES:}

UNIT-6 A file must be assigned as fortran-unit-6 on which printed output of the simulation will be placed.

UNIT-66 An optional output file must be assigned as fortran-unit-66 if the option to save the solution of the most recently completed time step for later restart is chosen in UNIT-5 when (ISTORE $=1$ ). Data will be written to this file in a format equivalent to UNIT-55 data so that this file may later be used as UNIT -55 .

The data lists and formats for the input files are given in section 7.7 , "SUTRA Input Data List." 


\subsection{User-Supplied Programming}

When SUTRA is used for simulation of systems with unsaturated flow, then the user must code the desired unsaturated flow functions in subroutine UNSAT. When SUTRA simulation includes time-dependent boundary conditions or sources, then the desired temporal varlations must be coded by the user in subroutine BCTIME.

\section{Subroutine UNSAT}

The general operation of this subroutine is described in section 5.7 , "Program Structure." Given a single value of pressure, UNSAT must provide values of $S_{w},\left(\partial S_{w} / \partial p\right)$, and $k_{r}$. UNSAT consists of three sections. The user must supply code in each of these sections. An example using the unsaturated flow functions $(2.8),(2.11)$, and $(2.21 a)$ and $(2.21 b)$ is given in the listing of Subroutine UNSAT in APPENDIX A, "SUTRA Progrem Listing."

The first section requires specification of saturation, $S_{w}$, as a function of pressure, $p$. The second section requires specification of the derivative of saturation with respect to pressure, $p$, or saturation, $S_{w}$. The third section requires specification of the relative permeability, $k_{r}$, as a function of efther saturation, $S_{w}$, or pressure, $p$. The pressure value which is passed to UNSAT is the projected value, the most recent iterate or the newly obtained solution. The values are either at Gauss points or at nodes.

Any convenient programing algorithm may be used to implement these functions In UNSAT. Some possibilities are: use of explicit expressions, as in the example; use of data statements; use of logical statements to give piecewise continuous 
functions; or use of READ statements to input new data to the functions from either UNIT-5 or a new data file. Sometimes functions with entry pressure or residual saturation require that functions used be switched when passing by these values. Logical statements which check $S_{w}$ or $p$ values may be used to switch to other functions or to constant values, as required.

\section{Subroutine BCTIME}

The general operation of this subroutine is described in section 5.7 , "Program Structure." At the beginning of each time step, BCTIME must provide: values of all specified time-varying pressure values and temperature or concentration values of fluid inflow at these nodes; values of specified time-varying temperature or concentration; values of specified time-varying fluid sources (or sinks) and temperatures or concentrations of these flows if they are inflows; and values of time of time-varying energy or solute mass sources (or sinks). BCTIME consists of four sections, each dealing with one of the above types of specification. The user must supply code in the section (or sections) of BCTIME which specifies the particular type of time-varying boundary condition or source desired.

The first section is used for spectfying either time variation of pressure, or time variation of the temperature or concentration of any flutd which enters the system at a point of specified pressure, or both. The coding must be entered within a loop which checks all NPBC specified pressure nodes for the time-variability flag. This flag is a negative node number in the list of specified pressure nodes IPBC(IP). The counter for the list is IP. When the loop finds that the IPth node number, IPBC(IP), is negative, then the actual node number is given by $I=$ -IPBC(IP). In this case, the user must supply code which specifies a value 
appropriate for the current time step, for both $\mathrm{PBC}(\mathrm{IP})$, which is the specified pressure for the IPth specified pressure node (node I), and for UBC(IP), which is the specified temperature or concentration of any inflow at the Ipth specified pressure node (node I). The loop skips over the positive node numbers in the list $\operatorname{IPBC}(I P)$.

The second section is used for specifying time variation of temperature or concentration. The coding must be entered within a loop which checks all NUBC specified temperature or concentration (U) nodes for the time-variability flag. This flag is a negative node number in the list of specified $U$ nodes, IUBC(IU). The list begins in the $(\text { NPBC }+1)^{\text {th }}$ element of IUBC as shown in the description of subroutine BOUND in section 5.7, "Program Structure." The first NPBC elements of IUBC are blank. The counter for the list is IU. If the loop finds that the IUth node number, IUBC $(N P B C+I U)$, is negative, then the actual node number is given by $I=-I U B C(N P B C+I U)$. In this case, the user must supply code which specifies a value, appropriate for the current time step, for $U B C(N P B C+I U)$, which is the specified temperature or concentration for the IUth specified $U$ node (node I). The loop skips over node numbers, IUBC(NPBC + IU), which are positive.

The third section is used for specifying time variation of either fluid sources (or sinks), temperature or concentration of inflowing fluid at sources, or both. The coding must be entered within a loop which checks all NSop fluid source nodes for the time-variability flag. This flag is a negative node number in the list of fluid source nodes, IQSOP(IQP). The counter for the list is IQP. If the loop finds that the IQPth node number IQSOP(IQP), is negative, then the actual node number is given by $I=-I Q S O P(I Q P)$. In this case, the user must supply code which specifies a value appropriate for the current time step, for 
both QIN(I), which is the specified fluid source for node I (the IQPth specified fluid source node), and for UIN(I), which is the temperature or concentration of inflowing fluid at node $I$. The loop skips over node numbers in the list, IQSOP(IQP), which are positive.

The fourth section is used for specifying time variation of energy or solute mass sources. The coding must be entered within a loop which checks all NSOU specified energy or solute mass source nodes for the time-variability flag. This flag is a negative node number in the list of specified energy or solute mass source nodes, IQSOU(IQU). The counter for the list is IQU. If the loop finds that the IQUth node number, IQSOU(IQU), is negative, then the actual node number is given by $I=-I Q S O U(I Q U)$. In this case, the user must supply code which specifies a value appropriate for the current time step, for QUIN(I), which is the specified energy or solute mass source for node I (the IQUth specified energy or solute mass source node). The loop skips over the positive node numbers in the list, IQSOU(IQU).

The current time at the end of the present time step in seconds, TSEC, and in other time units is available for use in specifying time variations. Any convenient programming algorithm may be used to implement the time-variations In BCTIME. Some possibilities are: use of expressions as explicit functions of time such as, for example, a sine function to represent tidal pressure variations; use of data statements and new arrays explicitly dimensioned in BCTIME; use of logical statements to give stepped or piecewise continuous functions; or use of READ statements to input the time-varying values directly from SUTRA UNIT-5 or a new data file. If different functions or values are to be specified at various nodes, then the user must also supply code to distinguish which functions apply to which specified node numbers. 
7.6 Modes and Options

\section{Simulation modes}

SUTRA may simulate flow and transport in three temporal modes for either energy or solute transport. The modes are: (1) transient flow and transport, (2) steady flow with transient transport, and (3) steady flow and steady transport, where mode (1) is the most computationally expensive and mode ( 3 ), the least expensive. Modes (2) and (3) are not applicable to all problems. The classes of problems amenable to solution by each node is given below.

\section{(1) Transient Flow and Transient Transport}

Allows for simulation of any physical problem which SUTRA deals with: either saturated or unsaturated flow or both; variable fluid density and viscosity; any sorption isotherm; energy or solute transport.

\section{(2) Steady-State Flow and Transient Transport}

Allows for simulation of a restricted class of SUTRA problems: saturated flow only; constant fluid density and viscosity; any sorption isotherm; energy transport with only small variations in temperature, or solute transport.

(3) Steady-State Flow and Steady-State Transport

Allows for simulation of the most restricted class of SUTRA problems: saturated flow only; constant fluid density and viscosity; linear sorption isotherm only; energy transport with only small variations in temperature, or solute transport. 
These modes are specified in UNIT-5 input data by the values of ISSFLO, ISSTRA, and SIMULA.

\section{Output options}

A number of output options are avallable which help to Interpret SUTRA simulation results. These are: (1) printer plots, (2) velocity output, (3) budget output, and (4) observation node output. The first three options require some extra computations and should be used only when necessary, as the extra calculations are done for each printed output.

(1) Printer Plots

Plots are available which are printed on each time step on which there is output. The plot is a map of three-digit pressures, temperatures or concentrations at the nodes which may be contoured by hand for an initial view of simulation results. Either a pressure plot or temperature (concentration) plot is output, or both on each time step with output. The plot consists of three significant figures of the pressure or temperature (or concentration) value at each node printed approximately at the nodal location in a map scaled to the printer paper. The map may be oriented either across the output page for a small plot, or along the page for a large plot. A plot of the locations of node numbers is provided with the input data print-out. Unfortunately, when some nodes in the mesh are grouped closely relative to the others, the printed three digits at clustered nodes may overlap and obscure the values. This typically occurs near the center axis for 
meshes in cylindrical coordinates. Use of the large plot may separate the values but the plot size can become unwieldy. Computer graphics contouring must then be employed, and is clearly more convenient than handcontoured printer plots when avallable.

\section{(2) Velocity Output}

An output of fluid velocity is avallable, the information in which may be used to plot velocity vectors everywhere in the simulated spatial region with computer graphics software supplied by the user. These velocities are calculated and output on each time step that a pressure solution is output. One velocity is calculated in each finite element, at the location of the element centroid, as described In section 5.5, "Velocity Calculation for Output." Velocity output occurs in two groups of values: first, the magnitude of the velocity vector at each element centroid, and second, the angle measured (with a counter-clockwise positive value) from the positive $x$-axis to the velocity vector direction. Note that velocity values are lagged one time step if a non-iterative solution is used. (In this case, they are calculated not with the new pressure solution, but with the solution of the previous time step and with fluid density values of the step before that. This keeps the velocity calculations consistent in time.) This option is controlled by UNIT -5 parameter, KVEL.

\section{(3) Budget Output}

A fluid mass and energy or solute mass budget output is available as an aid in tracking the simulated behavior of a system. The budget is not a check on numerical accuracy of the model as the calculations Involved in determining the budget are less accurate than the calcula- 
tions used to carry out the SUTRA simulation. The budget is output on each time step with printer output, and tallies total system changes in fluid mass, and energy or solute mass for the time step. Besides the totals of these quantities for the entire simulated region, the budget lists time step total gains or losses in these quantities at each specified pressure node, fluid source node, and energy or solute mass source node in the mesh. More information about the budget calculations is given in section 5.6, "Budget Calculations." The option is controlled by UNIT-5 parameter, KBUDG.

\section{(4) Observation Node Output}

An observation node output is available which observes pressure and temperature or concentration at particular nodes in the system during the simulation, and outputs the observations in table form after the last time step of the simulation has been completed. For each observed node, the table consists of three columns of numbers: the time of the observation, the observed pressure value, and the observed temperature or concentration value. Any number of observation nodes (NOBS) may be chosen, and observations may be requested every NOBCYC time steps. 
List of Input Data for UNIT 5

Model Series:

SUTRA

Model Version:

V1284-2D

Note that three arrays in the main routine of the code need to be dimensioned. The procedure for choosing dimensions is listed in the main routine itself, near the place where the dimensions need be specified.

DATASET 1: Input Data Heading (one card)

Variable

SIMULA

\section{Format}

$2 \mathrm{~A} 6$

\section{Description}

For energy transport simulation, write "SUTRA ENERGY TRANSPORT".

For solute transport simulation, write "SUTRA SOLUTE TRANSPORT".

The rest of the card is not used by SUTRA and may either be left blank or may be used to note an additional label for this UNIT 5 data list. 
DATASET 2: Output Heading (two cards)

$\begin{array}{lll}\text { Variable } & \text { Format } & \text { Description } \\ \text { TITLE1 } & 80 \mathrm{Al} & \begin{array}{l}\text { First line of a heading for the input data } \\ \text { set. }\end{array} \\ \text { TITLE2 } & 80 \mathrm{Al} & \begin{array}{l}\text { Second line of heading for the input data } \\ \text { set. }\end{array}\end{array}$

These two lines are printed as a heading on SUTRA output. 


\begin{tabular}{|c|c|c|}
\hline Variable & Format & Description \\
\hline NN & I5 & $\begin{array}{l}\text { Exact number of nodes in fintte element } \\
\text { mesh. }\end{array}$ \\
\hline NE & I5 & $\begin{array}{l}\text { Exact number of elements in finite element } \\
\text { mesh. }\end{array}$ \\
\hline NBI & I5 & $\begin{array}{l}\text { Full bandwidth of global banded matrix. NBI } \\
\text { is equal to one plus twice maximum difference } \\
\text { in node numbers in the element containing the } \\
\text { largest node number difference in the mesh. } \\
\text { This number is critical to computational } \\
\text { efficiency, and should be minimized by } \\
\text { careful numbering of the nodes ( see Figure } 7.1 \text { ). } \\
\text { Setting NBI too small causes SUTRA to automa- } \\
\text { tically print out the correct value and stop. }\end{array}$ \\
\hline NPINCH & I 5 & $\begin{array}{l}\text { Exact number of pinch nodes in the finite } \\
\text { element mesh. }\end{array}$ \\
\hline NPBC & I 5 & $\begin{array}{l}\text { Exact number of nodes at which pressure is a } \\
\text { spectfied constant value or function of time. }\end{array}$ \\
\hline NUBC & I 5 & $\begin{array}{l}\text { Exact number of nodes at which temperature or } \\
\text { concentration is a specified constant value or } \\
\text { function of time. }\end{array}$ \\
\hline NSOP & I 5 & $\begin{array}{l}\text { Exact number of nodes at which a fluid } \\
\text { source/sink is a specifted constant value } \\
\text { or function of time. }\end{array}$ \\
\hline NSOU & I 5 & $\begin{array}{l}\text { Exact number of nodes at which an energy or } \\
\text { solute mass source/sink is a specified constant } \\
\text { value or function of time. }\end{array}$ \\
\hline NOBS & I5 & $\begin{array}{l}\text { Exact number of nodes at which observations will } \\
\text { be made. Set to zero for no observations. }\end{array}$ \\
\hline NTOBS & I 5 & $\begin{array}{l}\text { Maximum number of time steps on which observations } \\
\text { will be made. This depends on both the number of } \\
\text { time steps in the simulation (DATASET 6), and on } \\
\text { the frequency of observations (DATASET 21). NTOBS } \\
\text { may be set to a value greater than that needed. } \\
\text { Set to zero for no observations. }\end{array}$ \\
\hline
\end{tabular}



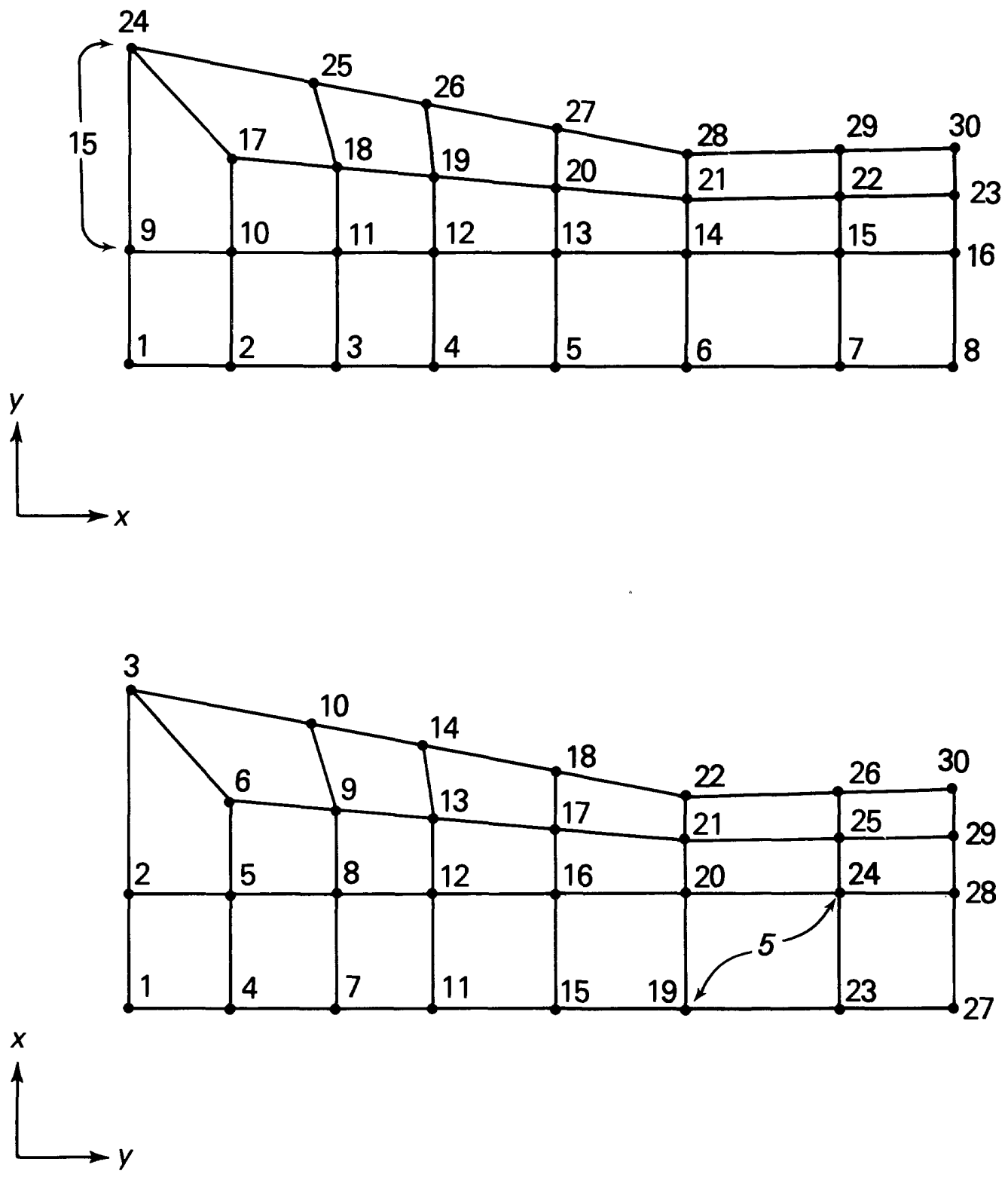

Figure 7.1

Minimization of band width by careful numbering of nodes. 


\begin{tabular}{|c|c|c|}
\hline Variable & Format & Description \\
\hline IUNSAT & I5 & $\begin{array}{l}\text { Set to }+1 \text { to allow simulation of unsaturated and } \\
\text { saturated flow. Set to } 0 \text { to allow simulation of } \\
\text { only saturated flow. When unsaturated flow is } \\
\text { allowed (IUNSAT }=1 \text { ) then the unsaturated flow } \\
\text { functions must be programmed by the user in } \\
\text { Subroutine UNSAT. }\end{array}$ \\
\hline ISSFLO & 15 & $\begin{array}{l}\text { Set to } 0 \text { for simulation with TRANSIENT } \\
\text { groundwater flow. Set to }+1 \text { for simulation } \\
\text { with STEADY-STATE groundwater flow. If } \\
\text { fluid density is to change with time, then } \\
\text { TRANSIENT flow must be selected. }\end{array}$ \\
\hline ISSTRA & I5 & $\begin{array}{l}\text { Set to } 0 \text { for simulation with TRANSIENT solute or } \\
\text { energy transport. Set to }+1 \text { for simulation of } \\
\text { STEADY-STATE transport. Note that steady-state } \\
\text { transport requires a steady-state flow field. } \\
\text { So, if ISSTRA }+1 \text {, then, also set ISSFLO }=+1\end{array}$ \\
\hline IREAD & I5 & $\begin{array}{l}\text { To read initial condition data (UNIT } 55 \text { ) } \\
\text { for cold start (first time step of a } \\
\text { simulation), set to }+1 \text {. To read initial } \\
\text { condition data (UNIT 55) for simulation } \\
\text { restart (to read data which has previously } \\
\text { been stored by SUTRA on UNIT } 66 \text { ), set to }-1 \text {. }\end{array}$ \\
\hline ISTORE & I5 & $\begin{array}{l}\text { To store results of most recently completed } \\
\text { time step on UNIT } 66 \text { for later use as initial } \\
\text { conditions on a restart, set to }+1 \text {. To cancel } \\
\text { storage, set to } 0 \text {. This option is recommended } \\
\text { as a backup for storage of results of intermediate } \\
\text { time steps during long simulations. Should the } \\
\text { execution halt unexpectedly, it may be restarted } \\
\text { with initial conditions consisting of results of } \\
\text { the last successfully completed time step stored } \\
\text { on UNIT } 66 \text {. }\end{array}$ \\
\hline
\end{tabular}


Variable

UP

Format

G10.0

GNU

G15.0

\section{Description}

Fractional upstream weight for stabilization of oscillations in results due to highly advective transport or unsaturated flow. UP may be given any value from 0.0 to +1.0 . UP $=0.0$ implies no upstream weighting (Galerkin method). UP $=0.5$ implies $50 \%$ upstream weighting. $U P=1.0$ implies full $(100 \%)$ upstream weighting. Recommended value is zero.

Warning: upstream weighting increases the local effective longitudinal dispersivity of the simulation by approximately $(U P \cdot(\Delta L) / 2$ ) where $\Delta \mathrm{L}$ is the local distance between element sides along the direction of flow. Note that the amount of this increase varles from place to place depending on flow direction and element size. Thus a non-zero value for UP actually changes the value of longitudinal dispersivity used by the simulation, and also broadens otherwise sharp saturation fronts.

Pressure boundary condition, 'conductance'. A high value causes SUTRA simulated pressure and specified pressure values at specified pressure nodes to be equal in all significant figures. A low value causes simulated pressures to deviate significantly from specifled values. The ideal value of GNU causes simulated and specified pressures to match in the largest six or seven significant figures only, and deviate in the rest. Trial-and-error is required to determine an ideal GNU value for a given simulation by comparing specified pressures with those calculated at the appropriate nodes for different values of GNU. An initial guess of 0.01 is suggested. 
DATASET 6: Temporal Control and Solution Cycling Data (one card)

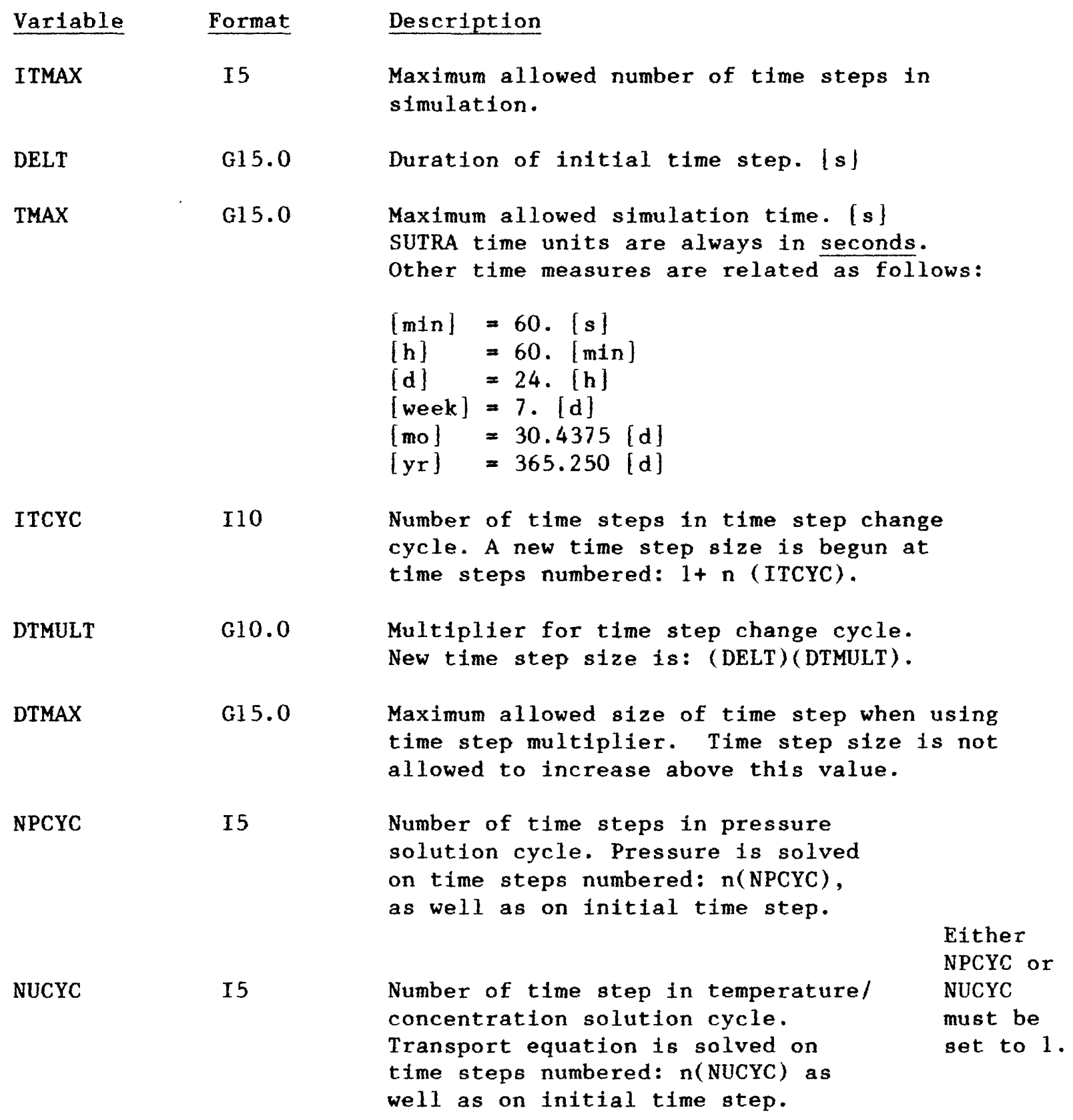


DATASET 7: Output Controls and Options (one card)

\begin{tabular}{|c|c|c|}
\hline Variable & Format & Description \\
\hline NPRINT & I5 & $\begin{array}{l}\text { Printed output is produced on time steps } \\
\text { numbered: n(NPRINT), as well as on first } \\
\text { and last time step. }\end{array}$ \\
\hline KNODAL & I5 & $\begin{array}{l}\text { A value of } 0 \text { cancels printout of node } \\
\text { coordinates, nodewise element thicknesses, } \\
\text { and nodewise porosities. Set to }+1 \text { for } \\
\text { full printout. }\end{array}$ \\
\hline KELMNT & I5 & $\begin{array}{l}\text { A value of } 0 \text { cancels printout of element- } \\
\text { wise permeabilities and elementwise dis- } \\
\text { persivities. } \\
\text { Set to }+1 \text { for full printout. }\end{array}$ \\
\hline KINCID & I5 & $\begin{array}{l}\text { A value of } 0 \text { cancels printout of node } \\
\text { incidences and pinch node incidences in } \\
\text { elements. Set to }+1 \text { for full printout. }\end{array}$ \\
\hline KPLOTP & I5 & $\begin{array}{l}\text { Set to a value of }+1 \text { for contourable } \\
\text { printer plot of pressures at all nodes } \\
\text { in mesh. Set to } 0 \text { to cancel pressure } \\
\text { plot. }\end{array}$ \\
\hline KPLOTU & I5 & $\begin{array}{l}\text { Set to a value of }+1 \text { for contourable } \\
\text { printer plot of concentrations or } \\
\text { temperatures at all nodes in mesh. } \\
\text { Set to } 0 \text { to cancel plot. }\end{array}$ \\
\hline KVEL & I5 & $\begin{array}{l}\text { Set to a value of }+1 \text { to calculate and } \\
\text { print fluid velocities at element centroids } \\
\text { each time printed output is produced. Note } \\
\text { that for non-steady state flow, velocities } \\
\text { are based on results and pressures of the } \\
\text { previous time step or iteration and not on } \\
\text { the newest values. } \\
\text { Set to } 0 \text { to cancel option. }\end{array}$ \\
\hline KBUDG & I5 & $\begin{array}{l}\text { Set to a value of }+1 \text { to calculate and print } \\
\text { a fluid mass budget and energy or solute } \\
\text { mass budget each time printed output is } \\
\text { produced. } \\
\text { A value of } 0 \text { cancels the option. }\end{array}$ \\
\hline
\end{tabular}


DATASET 8: Iteration Controls (one card)

\begin{tabular}{|c|c|c|}
\hline Variable & Format & Description \\
\hline ITRMAX & I10 & $\begin{array}{l}\text { Maximum number of iterations allowed per } \\
\text { time step to resolve non-linearities. } \\
\text { Set to a value of }+1 \text { for non-iterative } \\
\text { solution. Non-iterative solution may } \\
\text { be used for saturated aquifers when density } \\
\text { variability of the fluid is small, or for } \\
\text { unsaturated aquifers when time steps are chosen } \\
\text { to be small }\end{array}$ \\
\hline RPMAX & G10.0 & $\begin{array}{l}\text { Absolute iteration convergence criterion } \\
\text { for pressure solution. Pressure solution } \\
\text { has converged when largest pressure change } \\
\text { from the previous iteration's solution of } \\
\text { any node in mesh is less then RPMAX. May } \\
\text { be left blank for non-iterative solution. }\end{array}$ \\
\hline RUMAX & G10.0 & $\begin{array}{l}\text { Absolute iteration convergence criterion } \\
\text { for transport solution. Transport solution } \\
\text { has converged when largest concentration } \\
\text { on temperature change from the previous } \\
\text { Iteration's solution of any node in mesh } \\
\text { is less than RUMA. May be left blank } \\
\text { for non-iterative solution. }\end{array}$ \\
\hline
\end{tabular}




\begin{tabular}{|c|c|c|}
\hline Variable & Format & Description \\
\hline COMPFL & $\mathrm{G} 10.0$ & $\begin{array}{l}\text { Fluid compressibility, } \beta=(1 / \rho)(\partial \rho / \partial p) \text {. } \\
\left|\mathrm{M} /\left(L \cdot \mathrm{s}^{2}\right)\right|^{-1} \text {. Note, specific pressure } \\
\text { storativity is: } S_{o p}=(1-\varepsilon) \alpha+\varepsilon \beta\end{array}$ \\
\hline $\mathrm{CW}$ & G10.0 & $\begin{array}{l}\text { Fluid specific heat, } \mathrm{C}_{\mathrm{W}^{*}}\left|\mathrm{E} /\left(\mathrm{M} \cdot{ }^{\circ} \mathrm{C}\right)\right| \\
\text { (May be left blank for solute transport } \\
\text { simulation.) }\end{array}$ \\
\hline SIGMAW & G10.0 & $\begin{array}{l}\text { Fluid diffusivity, } \sigma_{\mathrm{W}} \text {. } \\
\text { For energy transport represents fluid } \\
\text { thermal conductivity, }\left|\mathrm{E} /\left(\mathrm{L} \cdot{ }^{\circ} \mathrm{C} \cdot \mathrm{s}\right)\right| \text {. } \\
\text { For solute transport represents molecular } \\
\text { diffusivity of solute in pure fluid. } \\
|\mathrm{L} 2 / \mathrm{s}|\end{array}$ \\
\hline RHOWФ & G10.0 & $\begin{array}{l}\text { Density of fluid at base concentration or } \\
\text { temperature. } \mid \mathrm{M} / \mathrm{L} / \text {. }\end{array}$ \\
\hline URHOW & G10.0 & $\begin{array}{l}\text { Base value of solute concentration (as mass } \\
\text { fraction) or temperature of fluid at which } \\
\text { base fluid density, RHOW } \emptyset \text { is specified. } \\
\left|M_{s} / M\right| \text { or } \mid{ }^{\circ} \mathrm{Cl} \text {. }\end{array}$ \\
\hline DRWDU & G10.0 & $\begin{array}{l}\text { Fluid coefficient of density change with } \\
\text { concentration (fraction) or temperature: } \\
0=\text { RHOW } \emptyset+\text { DRWDU (U-URHOW } \emptyset) \text {. } \\
\left|\mathrm{M} /\left(\mathrm{L}^{3} \cdot \mathrm{M}_{\mathrm{S}}\right)\right| \text { or }\left|\mathrm{M} /\left(\mathrm{L}^{3} \cdot{ }^{\mathrm{U}} \mathrm{C}\right)\right|\end{array}$ \\
\hline VISC $\emptyset$ & G10.0 & $\begin{array}{l}\text { For solute transport: fluid viscosity, } \mu \text {, } \\
|\mathrm{M} / \mathrm{L} \cdot \mathrm{s}| \text {. For energy transport. this value } \\
\text { is a scale factor. It multiplies the vis- } \\
\text { cosity which is calculated internally in } \\
\text { units of }|\mathrm{kg} / \mathrm{m} \cdot \mathrm{s}| \text {. VISC } \emptyset \text { may be used for } \\
\text { energy transport to convert units of } \\
|\mathrm{kg} / \mathrm{m} \cdot \mathrm{s}| \text { to desired units of viscosity. }\end{array}$ \\
\hline
\end{tabular}


DATASET 10: Solid Matrix Properties (one card)

\begin{tabular}{|c|c|c|}
\hline Variable & Format & Description \\
\hline COMPMA & G10.0 & $\begin{array}{l}\text { Solid matrix compressibility, } \alpha=(1-\varepsilon)^{-1} \partial \varepsilon / \partial p \\
{\left[M /\left(L \cdot s^{2}\right)\right]^{-1}}\end{array}$ \\
\hline cs & G10.0 & $\begin{array}{l}\text { Solid grain specific heat, } \mathrm{C}_{s} \cdot\left[\mathrm{E} /\left(\mathrm{M} \cdot{ }^{\circ} \mathrm{C}\right)\right] \\
\text { (May be left blank for solute transport } \\
\text { simulation.) }\end{array}$ \\
\hline SIGMAS & G10.0 & $\begin{array}{l}\text { Solid grain diffusivity, } \sigma_{s} \text {. } \\
\text { For energy transport represents thermal } \\
\text { conductivity of a solid grain. }\left[\mathrm{E} /\left(\mathrm{L} \cdot{ }^{\circ} \mathrm{C} \cdot \mathrm{s}\right)\right] \\
\text { (May be left blank for solute transport } \\
\text { simulation.) }\end{array}$ \\
\hline RHOS & G10.0 & Density of a solid grain, $\rho_{s} \cdot\left[M / L^{3}\right]$ \\
\hline
\end{tabular}


DATASET 11: Adsorption Parameters (one card)

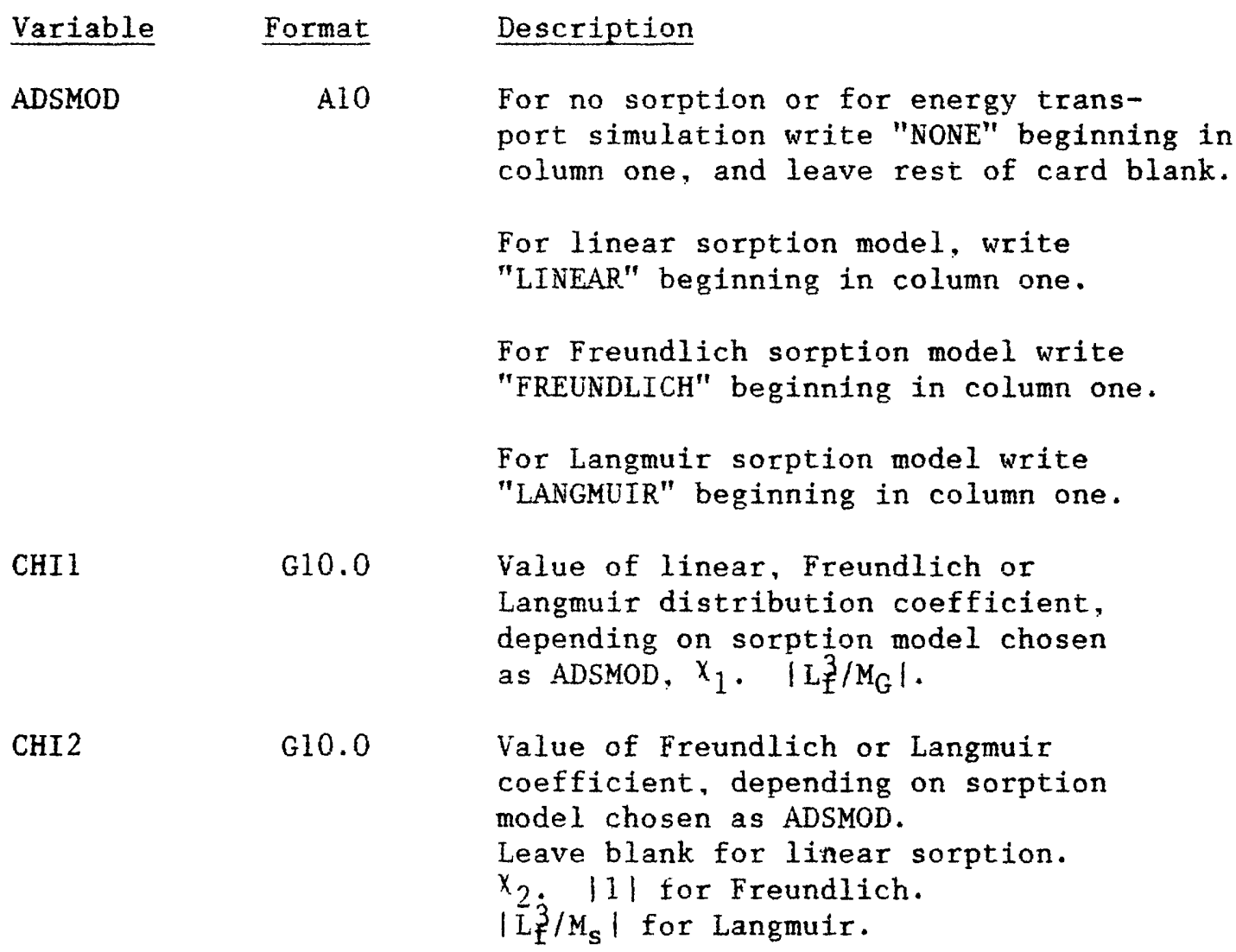


DATASET 12: Production of Energy or Solute Mass (one card)

\begin{tabular}{|c|c|c|}
\hline Variable & Format & Description \\
\hline PRODFO & G10.0 & $\begin{array}{l}\text { Zero-order rate of production in the fluid } \\
\gamma_{o}^{W}[(E / M) / s \mid \text { for energy production, } \\
{\left[\left(M_{g} / M\right) / s\right] \text { for solute mass production. }}\end{array}$ \\
\hline PRODSO & G10.0 & $\begin{array}{l}\text { Zero-order rate of production in the immobile } \\
\text { phase, } \gamma_{0}^{g} \cdot\left[\left(E / M_{G}\right) / s\right] \text { for energy } \\
\text { production, }\left[\left(M_{s} / M_{G}\right) / s\right] \text { for adsorbate mass } \\
\text { production. }\end{array}$ \\
\hline PRODF1 & G10.0 & $\begin{array}{l}\text { First-order rate of solute mass production in } \\
\text { the fluid, } \gamma_{1} \text {. }\left[s^{-1}\right] \\
\text { Leave blank for energy transport. }\end{array}$ \\
\hline PRODS1 & G10.0 & $\begin{array}{l}\text { First-order rate of adsorbate mass production in } \\
\text { the immobile phase, } \gamma_{1}^{\mathrm{s}} \cdot[\mathrm{s}] \\
\text { Leave blank for energy transport. }\end{array}$ \\
\hline
\end{tabular}


DATASET 13: Orientation of Coordinates to Gravity (one card)

\begin{tabular}{|c|c|c|}
\hline Variable & Format & Description \\
\hline GRAVX & G10.0 & 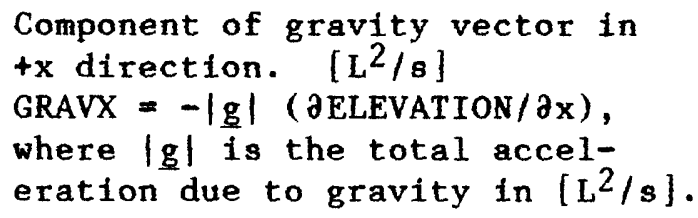 \\
\hline GRAVY & G10.0 & 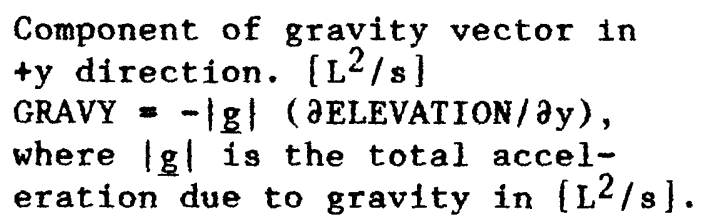 \\
\hline
\end{tabular}


DATASET 14A: Scale Factor for Nodewise Data (one card)

\begin{tabular}{|c|c|c|}
\hline Variable & Format & Description \\
\hline & $5 X$ & $\begin{array}{l}\text { In the first five columns of this card } \\
\text { write "NODE ", leaving one column blank. }\end{array}$ \\
\hline SCALX & G10.0 & $\begin{array}{l}\text { The scaled } x \text {-coordinates of nodes in } \\
\text { DATASET } 14 \mathrm{~B} \text { are multiplied by SCALX in SUTRA. } \\
\text { May be used to change from map to field } \\
\text { scales, or from English to SI units. A value } \\
\text { of } 1.0 \text { gives no scaling. }\end{array}$ \\
\hline SCALY & G10.0 & $\begin{array}{l}\text { The scaled y-coordinates of nodes in } \\
\text { DATASET } 14 \mathrm{~B} \text { are multiplied by SCALY in SUTRA. } \\
\text { May be used to change from map to field } \\
\text { scales, or from English to SI units. A } \\
\text { value of } 1.0 \text { gives no scaling. }\end{array}$ \\
\hline SCALTH & $\mathrm{G} 10.0$ & $\begin{array}{l}\text { The scaled element (mesh) thicknesses at } \\
\text { nodes in DATASET } 14 \mathrm{~B} \text { are multiplied by SCALTH } \\
\text { in SUTRA. May be used to easily change } \\
\text { entire mesh thickness or to convert English } \\
\text { to SI units. A value of } 1.0 \text { gives no scaling. }\end{array}$ \\
\hline PORFAC & G10.0 & $\begin{array}{l}\text { The scaled nodewise porosities of DATASET } 14 \mathrm{~B} \\
\text { are multiplied by PORFAC in SUTRA. May } \\
\text { be used to easily assign a constant porosity } \\
\text { value to all nodes by setting PORFAC=porosity, } \\
\text { and all POR(II)=1.0 in DATASET } 14 \mathrm{~B} \text {. }\end{array}$ \\
\hline
\end{tabular}


DATASET 14B: Nodewise Data (one card for each of NN nodes)

\begin{tabular}{|c|c|c|}
\hline Variable & Format & Description \\
\hline II & I5 & $\begin{array}{l}\text { Number of node to which data on this card } \\
\text { refers, } 1 \text {. }\end{array}$ \\
\hline $\mathrm{X}(\mathrm{II})$ & $\mathrm{G} 10.0$ & Scaled $x$-coordinate of node II, $x_{1},[L]$ \\
\hline $\mathrm{Y}(\mathrm{II})$ & G10.0 & Scaled $y$-coordinate of node II, $y_{i} \cdot[L]$ \\
\hline THICK(II) & G10.0 & $\begin{array}{l}\text { Scaled thickness of mesh at node } I I .[L] \\
\text { In order to simulate radial cross-sections } \\
\text { set THICK(II) }=(2 \pi) \text { (radius }) \text {, where } \\
\text { radius } 1 \text { s the radial distance from the } \\
\text { vertical center axis to node } 1 \text {. }\end{array}$ \\
\hline $\operatorname{POR}(\mathrm{II})$ & G10.0 & Scaled porosity value at node II, $\varepsilon_{i} \cdot$ [1] \\
\hline
\end{tabular}


DATASET 15A: Scale Factors for Elementwise Data (one card)

\begin{tabular}{|c|c|c|}
\hline Variable & Format & Description \\
\hline & $10 x$ & $\begin{array}{l}\text { In the first ten columns of this card } \\
\text { write "ELEMENT ", leaving three } \\
\text { columns blank. }\end{array}$ \\
\hline PMAXFA & G10.0 & $\begin{array}{l}\text { The scaled maximum permeability values } \\
\text { of elements in DATASET } 15 \mathrm{~B} \text { are multiplied } \\
\text { by PMAXFA in SUTRA. May be used to } \\
\text { convert units or to aid in assignment of } \\
\text { maximum permeability values in elements. }\end{array}$ \\
\hline PMINFA & G10.0 & $\begin{array}{l}\text { The scaled minimum permeability values } \\
\text { of elements in DATASET } 15 B \text { are multiplied } \\
\text { by PMINFA in SUTRA. May be used to convert } \\
\text { units or to aid assignment of minimum } \\
\text { permeability values in elements. }\end{array}$ \\
\hline ANGFAC & G10.0 & $\begin{array}{l}\text { The scaled angles between the maximum } \\
\text { permeability direction and the x-axis } \\
\text { of elements in DATASET } 15 \mathrm{~B} \text { are multi- } \\
\text { plied by ANGFAC in SUTRA. May be used } \\
\text { to easily assign a uniform direction } \\
\text { of anisotropy by setting ANGFAC= angle, } \\
\text { and all ANGLEX(L)=1.0 in DATASET 15B. }\end{array}$ \\
\hline ALMAXF & $\mathrm{G} 10.0$ & $\begin{array}{l}\text { The scaled maximum longitudinal dis- } \\
\text { persivities of elements in DATASET 15B } \\
\text { are multiplied by ALMAXF in SUTRA. May } \\
\text { be used to convert units or to aid in } \\
\text { assignment of dispersivities. }\end{array}$ \\
\hline ALMINF & G10.0 & $\begin{array}{l}\text { The scaled minimum longitudinal dis- } \\
\text { persivities of elements in DATASET 15B } \\
\text { are multiplied by ALMINF in SUTRA. May } \\
\text { be used to convert units or to aid in } \\
\text { assignment of dispersivities. }\end{array}$ \\
\hline ATAVGF & G10.0 & $\begin{array}{l}\text { The scaled average transverse disper- } \\
\text { sivities of elements in DATASET } 15 B \text { are } \\
\text { multiplied by ATAVGF in SUTRA. May be } \\
\text { used to convert units or to aid in } \\
\text { assignment of dispersivity. }\end{array}$ \\
\hline
\end{tabular}


DATASET 15B: Elementwise Data (one card for each of NE elements)

\begin{tabular}{|c|c|c|}
\hline Variable & Format & Description \\
\hline L & 110 & $\begin{array}{l}\text { Number of element to which data on this } \\
\text { card refers. }\end{array}$ \\
\hline $\operatorname{PMAX}(\mathrm{L})$ & G10.0 & $\begin{array}{l}\text { Scaled maximum permeability value of } \\
\text { element } L, k_{\max }(L) \cdot\left[L^{2}\right]\end{array}$ \\
\hline $\operatorname{PMIN}(L)$ & G10.0 & $\begin{array}{l}\text { Scaled minimum permeability value of } \\
\text { element } L, k_{\min }(L) .\left[L^{2}\right] \\
\text { Isotropic permeability requires: } \\
\text { PMIN }(L)=\operatorname{PMAX}(L) \text {. }\end{array}$ \\
\hline $\operatorname{ANGLEX(L)}$ & G10.0 & $\begin{array}{l}\text { Angle measured in counterclockwise direction } \\
\text { from +x-direction to maximum permeability } \\
\text { direction in element } L, \theta_{L} \cdot\left[{ }^{\circ}\right] \\
\text { Arbitrary when both } \operatorname{PMIN}(\mathrm{L})=\operatorname{PMAX}(\mathrm{L}) \text {, and } \\
\text { ALMAX(L) - ALMIN(L). }\end{array}$ \\
\hline $\operatorname{ALMAX}(L)$ & G10.0 & $\begin{array}{l}\text { Scaled longitudinal dispersivity value of } \\
\text { element } L \text { in the direction of maximum } \\
\text { permeability } \operatorname{PMAX}(\mathrm{L}), \alpha_{\mathrm{Lmax}}(\mathrm{L}) .[\mathrm{L}]\end{array}$ \\
\hline ALMIN (L) & G10.0 & $\begin{array}{l}\text { Scaled longitudinal dispersivity value of } \\
\text { element } L \text { in the direction of minimum } \\
\text { permeability } \operatorname{PMIN}(L), \alpha_{\operatorname{Lmin}}(L) .[L]\end{array}$ \\
\hline $\operatorname{ATAVG}(L)$ & G10.0 & $\begin{array}{l}\text { Scaled average transverse dispersivity value } \\
\text { of element } L, \alpha_{T}(L) .[L]\end{array}$ \\
\hline
\end{tabular}


DATASET 16: Data for Printer Plot (Two or three cards when plot has been requested by DATASET 7 )

OMI T when no plot is requested

Variable Format Description

Card 1: (always required when plot is requested)

IDIREC I5 Chooses plot direction:

Set to -1 for small plot which fits across

the output page.

Set to +1 for larger plot which is oriented along the output page.

NLINPI I5 Number of printer lines per inch.

NCHAPI I5 Number of printer characters per inch.

NCHAPL I5 Number of printer characters per output line.

The plotting routine prints three digits

of the nodal value to be plotted at the $(x, y)$

location of the node on a map of the mesh which

the routine constructs. The three digits are not

necessarily the first three digits of the value

to be plotted, but are always one digit to the

left and two digits to the right of the decimal

point. Thus, if the value to be plotted is

1234.567 , then the digits 456 , are printed

at the nodal location on the output.

Card 2: (include this card only when pressure plots are requested in DATASET 7)

PBASE G13.0 Value for scaling plotted pressures.

The pressure value to be plotted, PPLOT,

is calculated by SUTRA as

PPLOT $=$ (true pressure $p_{i} /$ PBASE)

PBASE should be used to scale out powers of

ten and to shift the scaled digits of interest

to the position of the three plotted digits. 
Card 3: (include this card only when temperature or concentration plots are requested in DATASET 7)

UBASE

G13.0

Value for scaling plotted temperature or concentration values.

The value to be plotted UPLOT, is calculated by SUTRA as:

$U_{\text {PLOT }}=$ ( $t$ rue value $U_{i} /$ UBASE). For example, UBASE may be set to one-tenth of the highest source concentration in the system; then fractional concentrations relative to the highest concentration are plotted with digits ranging from 000 to 999 which represents a relative concentration of $1.000(\sim 0.999)$. 
DATASET 17: Data for Fluid Source and Sinks (one card for each of NSOP fluid source nodes as specified in DATASET 3, plus one blank card)

0 M I $T$ when there are no fluid source nodes

Variable Format Description

IQCP I10 Number of node to which source/sink data on this card refers.

Specifying the node number with a negative sign indicates to SUTRA that the source flow rate or concentration or temperature of the source fluid vary in a specified manner with time. Information regarding a time-dependent source node must be programmed by the user in Subroutine BCTIME, and should not be included on this card.

QINC G15.0 Fluid source (or sink) which is a specified constant value at node IQCP, QIN. $|\mathrm{M} / \mathrm{s}|$ A positive value is a source of fluid to the aquifer. Leave blank if this value is specified as time-dependent in subroutine BCTIME.

Sources are allocated by cell as shown in Figure 7.2 for equal-sized elements. For unequal-sized elements, sources are allocated in proportion to the cell length, area or volume over which the source fluid enters the system.

UINC G15.0 Temperature or solute concentration (mass fraction) of fluid entering the aquifer which is a specified constant value for a fluid source at node IQCP, UIN. $1{ }^{\circ} \mathrm{Cl}$ or $\left|M_{S} / M\right|$

Leave blank if this value is specified as time-dependent in Subroutine BCTIME.

Last card:

$B L A N K \quad C A R \quad D$

Placed immediately following all NSOP fluid source node cards. 


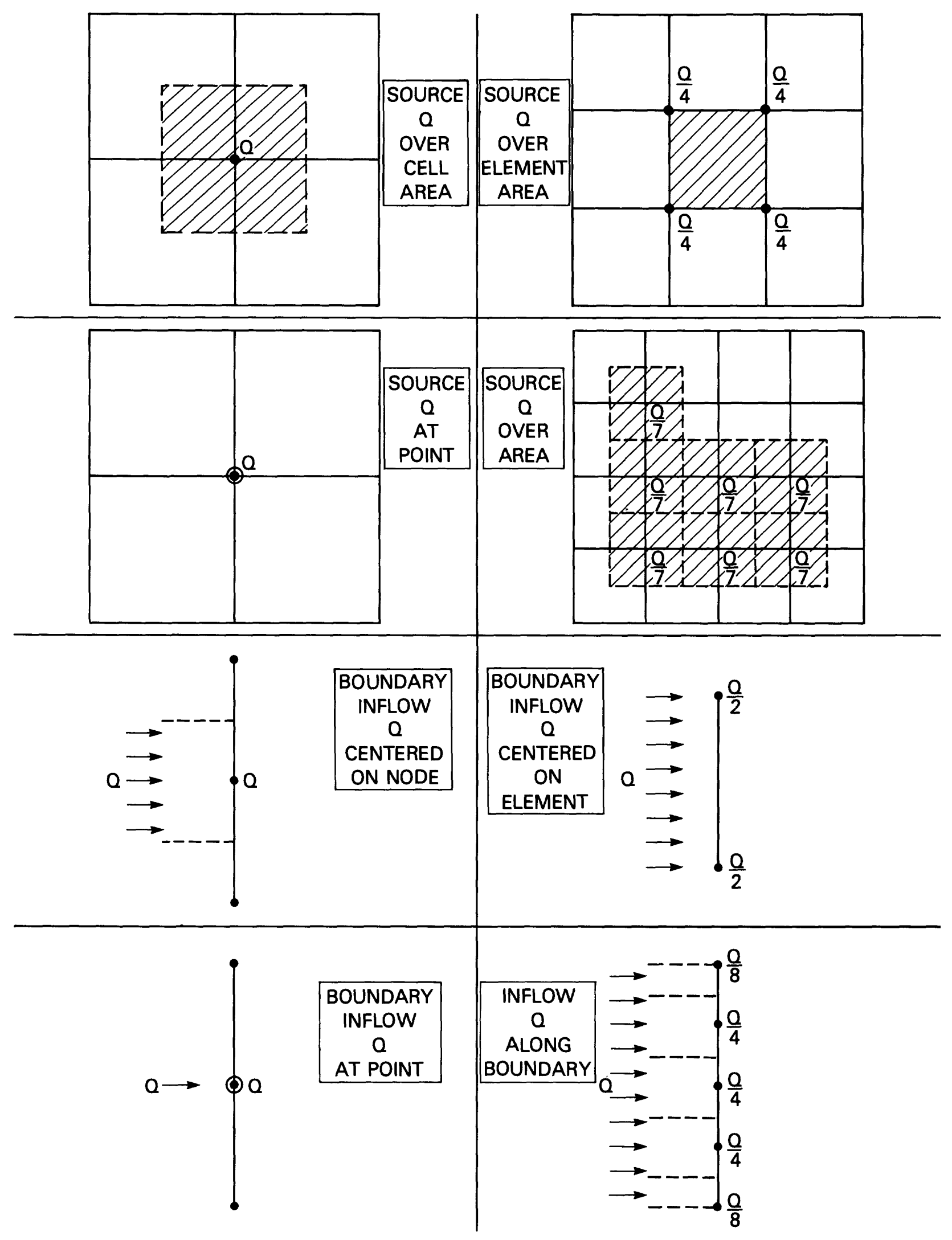

Figure 7.2

Allocation of sources and boundary fluxes in equal-sized elements. 
DATASET 18: Data for Energy or Solute Mass Sources and Sinks

(one card for each NSOU energy or solute source nodes as specified in DATASET 3, plus one blank card)

$\underline{0 M I T}$ when there are no energy or solute source nodes

Variable Format Description

IQCU I10

Number of node to which source/sink data on this card refers.

Specifying the node number with a negative sign indicates to SUTRA that the source rate varies in a specified manner with time. All information regarding a time-dependent source node must be programmed by the user in

Subroutine BCTIME, and a value should not be included in this card.

Sources are allocated by cell as shown in Figure 7.2 for equal-sized elements. For unequal-sized elements, sources are allocated in proportion to the cell length, area or volume over which the source energy or solute mass enters the system.

QUINC G15.0

Source (or sink) which is a specified constant value at node IQCU. $I N$. $|E / S|$ for energy transport, $\left|M_{S} / s\right|$ for solute transport. A positive value is a source to the aquifer. Leave blank if IQCU is negative, and this value is specified as time-dependent in Subroutine BCTIME.

\section{Last card:}

B L A N K C A R D

Placed immediately following all NSOU energy or solute mass source node cards. 
DATASET 19: Data for Specified Pressure Nodes (one card for each of NPBC specified pressure nodes as indicated in DATASET 3 , plus one blank card)

OMI I when there are no specified pressure nodes

Variable Format Description

Cards 1 to NPBC:

IPBC

Number of node to which specified pressure data on this card refers.

Specifying the node number with a negative sign indicates to SUTRA that the specified pressure value or inflow concentration or temperature at this node vary in a specified manner with time. Information regarding a time-dependent specified pressure node must be programmed by the user in Subroutine BCTIME, and should not be included on this card.

PBC G20.0

UBC $\quad$ G20.0

Pressure value which is a specified constant at node IPBC. $\left|M /\left(L \cdot s^{2}\right)\right|$

Leave blank if this value is specified as time-dependent in Subroutine BCTIME.

Temperature or solute concentration of any external fluid which enters the aquifer at node IPBC. UBC is a specified constant value. $\left|{ }^{\mathrm{C}} \mathrm{C}\right|$ or $\left|\mathrm{M}_{\mathrm{S}} / \mathrm{M}\right|$ Leave blank if this value is specified as time-dependent in subroutine BCTIME.

Last card:

B L A N K C A R D

Placed immediately following all NPBC specified pressure cards. 
DATASET 20: Data for Specified Concentration or Temperature Nodes

(one card for each of NUBC specified concentration or temperature nodes indicated in DATASET 3 , plus one blank card)

OMI T when there are no specified concentration or temperature nodes

Variable Format $\quad \underline{\text { Description }}$

Cards 1 to NUBC:

IUBC

Number of node to which specified concentration or temperature data on this card refers. Specifying the node number with a negative sign indicates to SUTRA that the specified value at this node varies in a specified manner with time. This time-dependence must be programmed by the user in Subroutine BCTIME, and a value should not be included on this card.

UBC $\quad$ G20.0

Temperature or solute concentration value which is a specified constant at node IUBC. $\left[{ }^{\circ} \mathrm{C}\right]$ or $\left[M_{s} / M\right]$

Leave blank if IUBC is negative and this value is specified as time-dependent in Subroutine BCTIME.

Last card:

B L A N K C A R D

Placed immediately following all NUBC specified temperature or concentration cards. 
DATASET 21: Observation Node Data (one card plus one card for each (NOBS+16)/16 (integer arithmetic) observation nodes as specified in DATASET 3)

$\underline{O M I T}$ when there are no observation nodes

Variable Format $\underline{\text { Description }}$

Card 1:

NOBCYC I10 Observations of pressure and temperature or concentration will be made at all observation nodes specified below every NOBCYC time steps.

Cards 2 to (NOBS+16)/16

INOB

1615

Node numbers of observation nodes. (Sixteen nodes per card.)

Enter a value of zero as an extra observation node number following the last real observation node in order to indicate to SUTRA that there are no more observation nodes. This will require one extra card if there is an exact multiple of 16 observation nodes. 
DATASET 22: Element Inctdence and Pinch Node Data (one or two cards for each of $\mathrm{NE}$ elements)

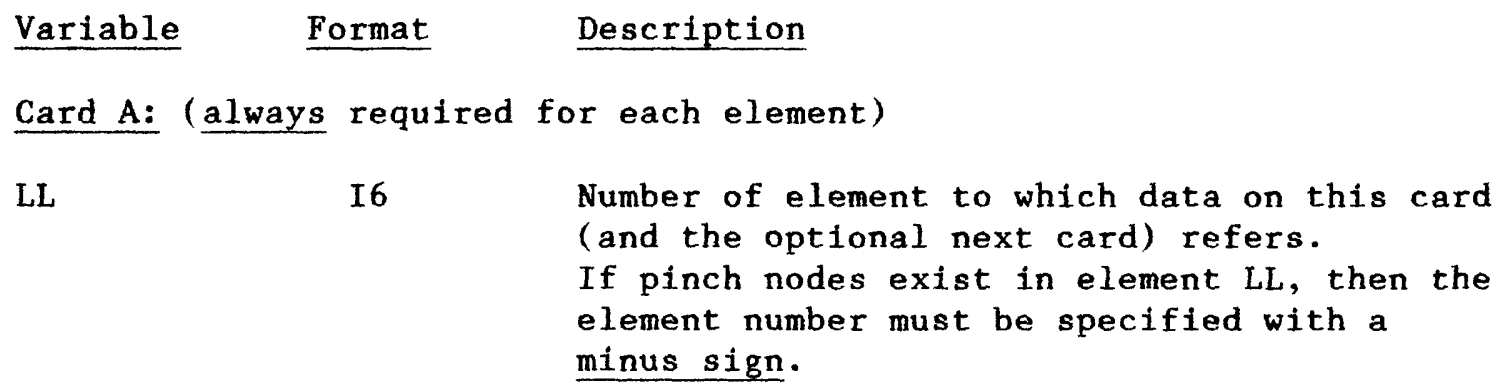
only when LL is negative, $O M I T$ when LL is positive)

$\left.\begin{array}{lc}\text { IEDGE( 1) } & \text { I6 } \\ \operatorname{IEDGE(~2)~} & \text { I6 } \\ \operatorname{IEDGE(3)} & \text { I6 } \\ \operatorname{IEDGE(4)} & \text { I6 }\end{array}\right\} \begin{aligned} & \text { PINCH-NODE INCIDENCE LIST } \\ & \text { pinch node at } \\ & \text { mid-point of } \\ & \text { edge between } \\ & \text { nodes: }\end{aligned}$

A blank in the list of pinch node numbers indicates that no pinch node exists on that particular edge element LL. 
Model Series:

Model Version:
SUTRA

V1284-2D

The data in UNIT 55 need be created by the user only for Cold-Starts of SUTRA simulation (1.e.: for the first time step of a given simulation).

The Restart options are controlled by IREAD and ISTORE in DATASET 4 of UNIT 5 data. SUTRA will optionally store final results of a simulation in a form directly useable as UNIT 55 for later restarts.

DATASET 1: Simulation Starting Time (one card)

Variable Format $\quad \underline{\text { Description }}$

TSTART

G20.0

Elapsed time at which the initial conditions for simulation specified in UNIT 55 are given. [s]

This sets the simulation clock starting time. Usually set to a value of zero for Cold-Start. 
DATASET 2: Initial Pressure Values at Nodes

Requires ( $N N+3) / 4$ cards. (Done by integer arithmetic.)

$\underline{\text { Variable }}$

PVEC(II)
Format

$4 \mathrm{G} 20.0$

\section{Description}

Initial (starting) pressure values at time,

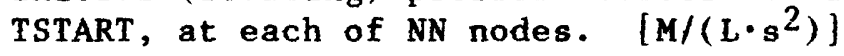
Four values per card, in exact order of node numbers. These values are arbitrary and may be left blank if the steady-state flow option in DATASET 4 of UNIT 5 has been chosen. Inttial hydrostatic or natural pressures in a crosssection may be obtained by running a single steady-flow time step with the store option. Then the natural pressures are calculated and stored on UNIT 66, and may be copied to the ColdStart UNIT 55 file without change in format, as initial conditions for a transient run. 
DATASET 3: Initial Temperature or Concentration Values at Nodes

Requires $(\mathrm{NN}+3) / 4$ cards. (Done by integer arithmetic.)

Variable Format Description

UVEC(II) 4G20.0 Initial (starting) temperature or solute concentration (mass fraction) values at time, TSTART, at each of NN nodes.

$\left[{ }^{\circ} \mathrm{C}\right]$ or $\left[M_{s} / M\right]$

Four values per card, in exact order of node numbers. 
REFERENCES

279

(p. 281 follows) 


\section{REFERENCES}

Bear, Jacob, 1979, Hydraulics of Groundwater, McGraw-Hill, New York, 567 p. Desai, C. S., and Contractor, D. N., 1977, Finite element analysis of flow, diffusion, and salt water intrusion in porous media: in Formulation and Computational Algorithms in Finite Element Analysis, by Bathe, K. J., (editor) and others, MIT Press, p. 958-983.

Frind, E. O., 1982, Simulation of long-term transient density-dependent transport in groundwater, Advances in Water Resources, v. 5, p. 73-97.

Gelhar, L. W., and Axness, Carl L., 1983, Three-dimensional stochastic analysis of macrodispersion in aquifers, Water Resources Research, v. 19, no. 1 , p. $161-180$.

Gelhar, L. W., and Collins, M. A., 1971, General analysis of longitudinal dispersion in nonuniform flow, Water Resources Research, v. 7, no. 6, p. 1511-1521.

Henry, H. R., 1964, Effects of dispersion on salt encroachment in coastal aquifers: in Sea Water in Coastal Aquifers, U.S. Geological Survey WaterSupply Paper 1613-C, p. C71-C84.

Hoopes, J. A., and Harleman, D. R. F., 1967, Dispersion in radial flow from a recharge well, Journal of Geophysical Research, v. 72, no. 14, p. 3595-3607.

Huyakorn, P. S., and Pinder, George F., 1983, Computational Methods in Subsurface Flow, Academic Press, New York, 473 p. 
Huyakorn, P., and Taylor, C., 1976, Finite element models for coupled groundwater flow and convective dispersion: in Finite Elements in Water Resources by Gray, W.G., Pinder, G. F., and Brebbia, C. A., (editors), Pentech Press, London, 1.131-1.151.

INTERA, 1979, Revision of the documentation for a model for calculating effects of liquid waste disposal in deep saline aquifers, U.S. Geological Survey Water Resources Investigations $79-96,73 \mathrm{p}$.

Konikow, L. F., 1977, Modeling chloride movement in the alluvial aquifer at the Rocky Mountain Arsenal, Colorado, U.S. Geological Survey Water Supply Paper 2044, 43 p.

Lohman, S. W., 1972, Ground Water Hydraulics, U.S. Geological Survey Professional Paper 708, 70 p.

Pinder, G. F., and Cooper, H. H., Jr., 1970, A numerical technique for calculating the transient position of the saltwater front, Water Resources Research, v. 6, no. 3, p. 875-882.

Pinder, G. F., and Gray, W. G., 1977, Finite Element Simulation in Surface and Subsurface Hydrology, Academic Press, New York, 295 p.

Segol, G., Pinder, G. F., Gray, W. G., 1975, A galerkin-finite element technique for calculating the transient position of the saltwater front, Water Resources Research, v. 11, no. 2, p. 343-346.

Van Genuchten, M. Th., 1980, A closed-form equation for predicting the hydraulic conductivity of unsaturated soils, Soil Science Society of America Journal, v. 44 , no. 5, p. $892-898$. 
Van Genuchten, M. Th., 1982, A comparison of numerical solutions of the onedimensional unsaturated-saturated flow and mass transport equations, Advances in Water Resources, v. 5, no. 1, p. 47-55.

Wang, H. F., and Anderson, M. P., 1982, Introduction to Groundwater Modeling, Freeman and Co., San Francisco, 237 p.

Warrick, A. W., Biggar, J. W., and Nielsen, D. R., 1971, Simultaneous solute and water transfer for an unsaturated soil, Water Resources Research, v. 7, no. 5, p. $1216-1225$. 


\section{APPENDICES}

\section{5}

(p. 287 follows) 


\section{Nomenclature}

\section{Generic Units}

$\begin{array}{ll}{[1]} & \text { unity - implies dimensionless or }\left[\mathrm{L}^{0}\right] \\ {[E]} & \text { energy units or }\left[\mathrm{M} \cdot \mathrm{L}^{2} / \mathrm{s}^{2}\right] \\ {[\mathrm{L}]} & \text { length units } \\ {\left[\mathrm{L}_{\mathrm{B}}^{3}\right]} & \text { fluid volume } \\ {\left[\mathrm{L}_{\mathrm{G}}^{3}\right]} & \text { solid grain volume } \\ {[\mathrm{M}]} & \text { fluid mass units } \\ {\left[\mathrm{M}_{\mathrm{G}}\right]} & \text { solid grain mass units } \\ {\left[\mathrm{M}_{\mathrm{S}}\right]} & \text { solute mass units }\end{array}$

Units

$\begin{array}{ll}{\left[{ }^{\circ} \mathrm{C}\right]} & \text { degrees Celcius } \\ {[\mathrm{cm}]} & \text { centimeters } \\ {[\mathrm{d}]} & \text { days } \\ {[\mathrm{gr}]} & \text { grams } \\ {[\mathrm{h}]} & \text { hours } \\ {[\mathrm{J}]} & \text { Joules or }\left[\mathrm{kg}^{\circ} \mathrm{m}^{2} / \mathrm{s}^{2}\right. \\ {[\mathrm{kg}]} & \text { kilograms } \mathrm{mass} \\ {[\mathrm{lbm}]} & \text { pounds mass } \\ {[\mathrm{m}]} & \text { meters } \\ {[\mathrm{min}]} & \text { minutes } \\ {[\mathrm{mo}]} & \text { months } \\ {[\mathrm{s}]} & \text { seconds }\end{array}$




$$
\begin{aligned}
& \frac{\partial q}{\partial t} \text { or } \frac{d \Psi}{d t} \\
& \underline{v}=\underline{i} v_{x}+j v_{y}+\underline{k} v_{z} \\
& \underline{\nabla}=\underline{i} \frac{\partial \Psi}{\partial x}+j \frac{\partial \Psi}{\partial y}+\underline{k} \frac{\partial \Psi}{\partial z} \\
& \underline{\nabla} \cdot \underline{v}=\frac{\partial v}{\partial x}+\frac{\partial v}{\partial y} y+\frac{\partial v}{\partial z} z \\
& i=\frac{1, N N}{1}=1,2,3,4, \ldots, N N \\
& |\Psi| \\
& |\underline{v}| \\
& \underline{\Psi} \text { or } \mid
\end{aligned}
$$$$
\Delta \Psi
$$$$
\text { 甲 }
$$$$
\Psi_{\text {BC }}
$$$$
\Psi_{1} \text { or } \Psi_{j}
$$$$
\Psi_{\text {IN }}
$$$$
\Psi_{\text {KG }}
$$$$
\text { I }
$$$$
\text { v }
$$$$
\mathbf{v}_{\mathbf{x}}
$$$$
\mathbf{v}
$$

time derivative of $\$$

vector $\mathbf{v}$ with components in $\underline{i}, \underline{j}$, and $\underline{k}$ directions

gradient of scale

divergence of vector $\underline{v}$

index 1 takes on all integer values between one and $\mathrm{NN}$

absolute value of scalar

magnitude of vector $\underline{v}$

approximate or discretized value of $\Psi$

discrete change in value of (e.g: $\left.\Delta \Psi=\Psi_{1}-\Psi_{2}\right)$

initial condition or zeroth value of

value of as spectified at a boundary condition node

value of at node or cell $i$ or $j$

value of in inflow

value of at the $\mathrm{KG}^{\text {th }}$ Gauss point

value of in element $L$

value of a vector $\underline{v}$ along

a stream line

value of a vector $\underline{v}$ in $x$ direction

value of a vector $\underline{v}$ in $y$ direction 


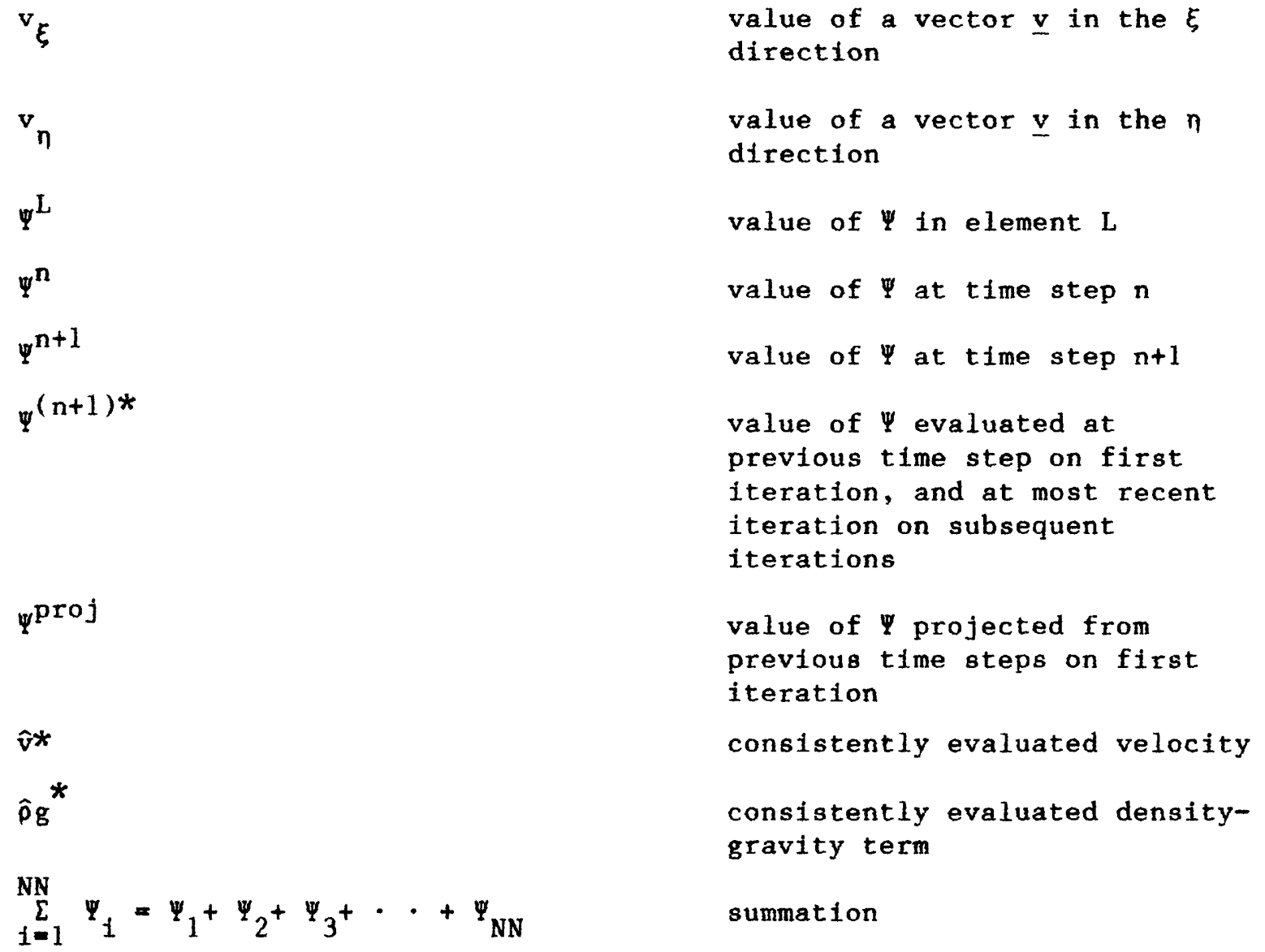

\section{Greek Lowercase}

\begin{tabular}{|c|c|c|c|}
\hline$\alpha$ & $(2.17)$ & {$\left[M /\left(L \cdot s^{2}\right)\right]^{-1}$} & Porous matrix compressibility \\
\hline$\alpha_{L}(x, y, t)$ & $\begin{array}{l}(2.40 \mathrm{~b}) \\
(2.41)\end{array}$ & {$[L]$} & $\begin{array}{l}\text { Longitudinal dispersivity of } \\
\text { solid matrix }\end{array}$ \\
\hline$\alpha_{\operatorname{Lmax}}(x, y)$ & $(2.42 b)$ & {$[\mathrm{L}]$} & $\begin{array}{l}\text { Longitudinal dispersivity in } \\
\text { the maximum permeability } \\
\text { direction, } x_{p}\end{array}$ \\
\hline${ }^{\alpha} \operatorname{Lmin}(x, y)$ & $(2.42 b)$ & {$[L]$} & $\begin{array}{l}\text { Longitudinal dispersivity in } \\
\text { the minimum permeability } \\
\text { direction, } x_{m}\end{array}$ \\
\hline$\alpha_{T}(x, y)$ & $(2.40 \mathrm{~b})$ & {$[\mathrm{L}]$} & $\begin{array}{l}\text { Transverse dispersivity of } \\
\text { solid matrix }\end{array}$ \\
\hline B & $(2.15)$ & {$\left[M /\left(L \cdot s^{2}\right)\right]^{-1}$} & Fluid compressibility \\
\hline$\gamma_{0}^{s}(x, y, t)$ & $(2.25)$ & {$\left[\mathrm{E} / \mathrm{M}_{\mathrm{G}} \cdot \mathrm{s}\right]$} & $\begin{array}{l}\text { Energy source in solid } \\
\text { grains }\end{array}$ \\
\hline
\end{tabular}




\begin{tabular}{|c|c|c|c|}
\hline$\gamma_{0}^{s}$ & $(2.37 b)$ & {$\left[\left(M_{s} / M\right) / s\right]$} & $\begin{array}{l}\text { Zero-order adsorbate mass } \\
\text { production rate }\end{array}$ \\
\hline$\gamma_{0}^{w}(x, y, t)$ & $(2.25)$ & {$[E / M \cdot s]$} & Energy source in fluid \\
\hline$\gamma_{0}^{w}$ & $(2.37 b)$ & {$\left[\left(M_{s} / M\right) / s\right]$} & $\begin{array}{l}\text { Zero-order solute mass } \\
\text { production rate }\end{array}$ \\
\hline$\gamma_{1}^{s}$ & $(2.37 b)$ & {$\left[s^{-1}\right]$} & $\begin{array}{l}\text { First-order mass production } \\
\text { rate of adsorbate }\end{array}$ \\
\hline$\gamma_{1}^{w}$ & $(2.37 b)$ & {$\left[s^{-1}\right]$} & $\begin{array}{l}\text { First order mass production } \\
\text { rate of solute }\end{array}$ \\
\hline$\delta_{i j}$ & $(4.65 a)$ & & Kronecker delta \\
\hline$\varepsilon(x, y, t)$ & $(2.6)$ & {$[1]$} & Porosity \\
\hline$\eta$ & $(4.3)$ & & n local coordinate \\
\hline $\mathrm{K}_{1}\left(\mathrm{C}, \mathrm{C} \mathrm{s}_{\mathrm{s}}\right)$ & $(2.32 b)$ & {$\left[\mathrm{M} / \mathrm{M}_{\mathrm{G}}\right]$} & $\begin{array}{l}\text { First general sorption } \\
\text { coefficient }\end{array}$ \\
\hline $\mathrm{x}_{2}\left(\mathrm{C}, \mathrm{C}_{\mathrm{s}}\right)$ & $(2.32 b)$ & {$\left[M / M_{G} \cdot s\right]$} & $\begin{array}{l}\text { Second general sorption } \\
\text { coefficlent }\end{array}$ \\
\hline$\kappa_{3}\left(c, c_{s}\right)$ & $(2.32 b)$ & {$\left[\mathrm{M}_{\mathrm{s}} / \mathrm{M}_{\mathrm{G}} \cdot \mathrm{s}\right]$} & $\begin{array}{l}\text { Third general sorption } \\
\text { coefficient }\end{array}$ \\
\hline$\lambda(x, y, t)$ & $(2.25)$ & {$\left[E /\left(s \cdot L \cdot{ }^{\circ} \mathrm{C}\right)\right]$} & $\begin{array}{l}\text { Bulk thermal conductivity } \\
\text { of solid matrix plus fluid }\end{array}$ \\
\hline$\lambda_{\mathrm{s}}$ & $(2.26)$ & {$\left[E /\left(s \cdot L \cdot{ }^{\circ} C\right)\right]$} & $\begin{array}{l}\text { Solid thermal conductivity } \\
\text { (about } \lambda \sim 0.6\left[\mathrm{~J} /\left(\mathrm{s} \cdot \mathrm{m} \cdot{ }^{\circ} \mathrm{C}\right)\right] \\
\left.\text { at } 20^{\circ} \mathrm{C}\right)\end{array}$ \\
\hline$\lambda_{w}$ & $(2.26)$ & {$\left[\mathrm{E} /\left(\mathrm{s} \cdot \mathrm{L} \cdot{ }^{\circ} \mathrm{C}\right)\right]$} & $\begin{array}{l}\text { Fluid thermal conductivity } \\
\text { (about } \lambda \sim 0.6\left[\mathrm{~J} /\left(\mathrm{s} \cdot \mathrm{m} \cdot{ }^{\circ} \mathrm{C}\right)\right] \\
\left.\text { at } 20^{\circ} \mathrm{C}\right)\end{array}$ \\
\hline$\mu$ & $(2.5),(2.6)$ & & Fluid viscosity \\
\hline$v_{1}$ & $(4.51)$ & & $\begin{array}{l}\text { Pressure-based conductance } \\
\text { for specified pressure in } \\
\text { cell } i\end{array}$ \\
\hline$v_{p}$ & $(4.38)$ & & $\begin{array}{l}\text { Conductance for spectfied } \\
\text { pressure nodes }\end{array}$ \\
\hline
\end{tabular}




\begin{tabular}{|c|c|c|c|}
\hline$\xi$ & $(4.1)$ & & $\xi$ local coordinate \\
\hline$p_{0}$ & $(2.4)$ & $\left|M / L_{f}^{3}\right|$ & $\begin{array}{l}\text { Base fluid density at } \\
\mathrm{C}=\mathrm{C}_{0} \text { or } \mathrm{T}=\mathrm{T}_{0}\end{array}$ \\
\hline$\rho(x, y, t)$ & $(2.1)$ & $\left|M / L_{f}^{3}\right|$ & Fluid density \\
\hline$\rho_{s}$ & $\begin{array}{l}(2.24) \\
(2.30)\end{array}$ & $\left|M_{G}^{3} / L_{G}^{3}\right|$ & $\begin{array}{l}\text { Density of solid grains } \\
\text { in solid matrix }\end{array}$ \\
\hline$\sigma^{\prime}$ & $(2.17)$ & $\left|M /\left(L \cdot s^{2}\right)\right|$ & Integranular stress \\
\hline$\sigma_{s}$ & $(2.47)$ & & $\begin{array}{l}\text { Diffusion in solid phase in } \\
\text { unified transport equation }\end{array}$ \\
\hline$\sigma_{w}$ & $(2.47)$ & & $\begin{array}{l}\text { Diffusion in fluid phase in } \\
\text { unified transport equation }\end{array}$ \\
\hline$\theta(x, y)$ & $(2.21 a)$ & 101 & $\begin{array}{l}\text { Angle from }+x \text {-coordinate } \\
\text { axis to direction of } \\
\text { maximum permeability, } x_{p}\end{array}$ \\
\hline$\phi_{k v}(x, y, t)$ & $(2.42 b)$ & 101 & $\begin{array}{l}\text { Angle from maximum permea- } \\
\text { bility direction, } x \text { to } \\
\text { local flow directioh, ( } /|| \underline{v} \mid)\end{array}$ \\
\hline$\phi_{j}$ & $(3.4)$ & & $\begin{array}{l}\text { Symmetric bi-linear basis } \\
\text { function in global coordinates } \\
\text { at node } i\end{array}$ \\
\hline$x_{1}$ & $(2.34 \mathrm{~b})$ & $\left\lfloor L_{f}^{3} / M_{G}\right\rfloor$ & $\begin{array}{l}\text { Linear distribution } \\
\text { coefficient }\end{array}$ \\
\hline$x_{1}$ & $(2.35 b)$ & $\left|L_{f}^{3} / M_{G}\right|$ & $\begin{array}{l}\text { A Freundlich distribution } \\
\text { coefficient }\end{array}$ \\
\hline$x_{1}$ & $(2.36 b)$ & $\mid L_{f}^{3} / M_{G} !$ & $\begin{array}{l}\text { A Langmuir distribution } \\
\text { coefficient }\end{array}$ \\
\hline$x_{2}$ & $(2.36 \mathrm{~b})$ & $\left|L_{f}^{3} / M_{s}\right|$ & Langmuir coefficient \\
\hline$x_{2}$ & $(2.35 b)$ & 111 & Freundlich coefficient \\
\hline${ }^{\psi} \mathrm{IN}_{i}$ & $(4.75)$ & & $\begin{array}{l}\text { Energy source }|E / s| \text { or } \\
\text { solute mass source }\left|M_{s} / M \cdot s\right| \\
\text { at node } i\end{array}$ \\
\hline$\psi_{\text {OUT }_{i}}$ & $(4.75)$ & & $\begin{array}{l}\text { Sink of energy or solute } \\
\text { mass at node } i\end{array}$ \\
\hline$\omega_{i}$ & $(4.43)$ & & $\begin{array}{l}\text { Asymmetric weighting } \\
\text { function in global } \\
\text { coordinates at node } i\end{array}$ \\
\hline
\end{tabular}


$$
\Gamma
$$

$\Gamma_{s}(x, y, t)$

$\Gamma_{w}(x, y, t)$

$\Delta t$

$\Delta \mathrm{L}_{\mathrm{L}}$

$\Delta \mathrm{L}_{\mathrm{T}}$

$\Delta t_{n}$

$\Delta t_{n+1}$

$\mathrm{H}_{+}$

$\mathrm{H}_{-}$

$\mathrm{H}^{* *}$

$\theta_{i}$

$\varepsilon_{+}$

$\Xi$

$\Xi^{*}$
$\left|M_{s} / M \cdot s\right|$

$\left|M_{s} / M_{G} \cdot s\right|$

$|s|$

(7.4)
External boundary of simulated region

Adsorbate mass source (per unit solid matrix mass) due to production reactions within adsorbed material itself

Solute mass source in fluid (per unit fluid mass) due to production reactions

Length of time step

Distance between sides of element $L$ along stream line

Distance between sides of element L perpendicular to stream line

Time step $n$

Time step $n+1$

One-dimensional basis function in $n$ direction

One-dimensional basis function in $n$ direction

Asymmetric portion of $n$ weighting function

Asymmetric weighting function at node $i$

One-dimensional basis function in $\xi$ direction

One-dimensional basis function in $\xi$ direction

Asymmetric portion of $\xi$ weighting function 


$\begin{array}{lll}T(x, y, t)(2.22) \quad\left[M /\left(L^{3} \cdot s\right)\right] \quad & \begin{array}{l}\text { Solute mass source }(e \cdot g ., \\ \text { dissolution of solid } \\ \text { matrix or desoprtion })\end{array} \\ & \begin{array}{l}\text { Bi-linear symmetric basis } \\ \Omega_{1}\end{array} & \text { function at node } 1\end{array}$

\section{Roman Lowercase}

\begin{tabular}{|c|c|c|c|}
\hline$a_{\xi}$ & $(4.23)$ & & $\begin{array}{l}\text { Asymmetric weighting } \\
\text { function coefficient }\end{array}$ \\
\hline$c(x, y, t)$ & $(2.1)$ & {$\left[M_{s} / L_{f}^{3}\right]$} & $\begin{array}{l}\text { Solute volumetric concen- } \\
\text { tration (mass solute per } \\
\text { volume total fluid) }\end{array}$ \\
\hline$c_{s}$ & $(2.27 b)$ & {$\left[E /\left(M_{G} \cdot{ }^{\circ} \mathrm{C}\right)\right]$} & $\begin{array}{l}\text { Solid grain specific heat } \\
\text { (about } \mathrm{c} \sim 8.4 \mathrm{X} 10^{2}\left[\mathrm{~J} /\left(\mathrm{kg} \cdot{ }^{\circ} \mathrm{C}\right]\right. \\
\left.\text { for sandstone at } 20^{\circ} \mathrm{C}\right)\end{array}$ \\
\hline$c_{w}$ & $(2.25)$ & {$\left[E /\left(M \cdot{ }^{\circ} \mathrm{C}\right)\right]$} & $\begin{array}{l}\text { Specific heat of water (about } \\
\text { c } \sim 4.182 \times 10^{3}\left[\mathrm{~J} /\left(\mathrm{kg} \cdot{ }^{\circ} \mathrm{C}\right)\right] \\
\text { at } 20^{\circ} \mathrm{C}\end{array}$ \\
\hline$d_{L}(x, y, t)$ & $(2.39 c)$ & {$\left[L^{2} / s\right]$} & $\begin{array}{l}\text { Longitudinal dispersion } \\
\text { coefficient }\end{array}$ \\
\hline$d_{T}(x, y, t)$ & $(2.39 c)$ & {$\left[L^{2} / s\right]$} & $\begin{array}{l}\text { Transverse dispersion } \\
\text { coefficient }\end{array}$ \\
\hline $\operatorname{det} J$ & $(4.30)$ & & $\begin{array}{l}\text { Determinant of Jacobian } \\
\text { matrix }\end{array}$ \\
\hline$e_{s}$ & $(2.24)$ & {$\left[E / M_{G}\right]$} & $\begin{array}{l}\text { Energy per unit mass solid } \\
\text { matrix }\end{array}$ \\
\hline$e_{w}$ & $(2.24)$ & {$[E / M]$} & Energy per unit mass water \\
\hline$f(x, y, t)$ & $(2.30)$ & {$\left[M_{s} /\left(L^{3} \cdot s\right)\right]$} & $\begin{array}{l}\text { Volumetric adsorbate source } \\
\text { (gain of adsorbed species by } \\
\text { transfer from fluid per untt } \\
\text { from fluid per unit total } \\
\text { volume) }\end{array}$ \\
\hline$f_{s}(x, y, t)$ & $(2.32 a)$ & {$\left[M_{s} / M_{G} \cdot s\right]$} & $\begin{array}{l}\text { Specific solute mass } \\
\text { adsorption rate (per unit } \\
\text { mass solid matrix) }\end{array}$ \\
\hline g & $(2.19 b)$ & {$\left[L / s^{2}\right]$} & $\begin{array}{l}\text { Gravitational acceleration } \\
\text { (gravity vector) }\end{array}$ \\
\hline
\end{tabular}




\begin{tabular}{|c|c|c|c|}
\hline$h(x, y, t)$ & $\begin{array}{l}(2.20) \\
(3.1)\end{array}$ & $|L|$ & $\begin{array}{l}\text { Hydraulic head (sum of } \\
\text { pressure head and } \\
\text { elevation head) }\end{array}$ \\
\hline$\underline{\underline{k}}(x, y)$ & $(2.19 a)$ & $\left|L^{2}\right|$ & Solid matrix permeability \\
\hline $\mathrm{k}_{\max }(\mathrm{x}, \mathrm{y})($ & $(2.21 \mathrm{a})$ & $\left|L^{2}\right|$ & $\begin{array}{l}\text { Absolute maximum value of } \\
\text { permeability }\end{array}$ \\
\hline$k_{\min }(x, y)$ & $(2.21 \mathrm{a})$ & $\left|L^{2}\right|$ & $\begin{array}{l}\text { Absolute minimum value of } \\
\text { permeability }\end{array}$ \\
\hline$k_{r}(x, y, t)$ & $(2.19)$ & 111 & $\begin{array}{l}\text { Relative permeability to } \\
\text { fluid flow (assumed to be } \\
\text { independent of direction). }\end{array}$ \\
\hline$p(x, y, t)$ & $(2.1)$ & $\left\lfloor M /\left(L \cdot s^{2}\right) !\right.$ & Fluid pressure \\
\hline$P_{c}(x, y, t)$ & $(2.7)$ & $\left|M /\left(L \cdot s^{2}\right)\right|$ & Capillary pressure \\
\hline Pcent & $(2.7)$ & $\left\lfloor M /\left(L \cdot s^{2}\right) \mid\right.$ & Entry capillary pressure \\
\hline $\mathrm{p}_{\mathrm{BC}}$ & $(4.38)$ & & $\begin{array}{l}\text { Specified pressure value } \\
\text { at node } i\end{array}$ \\
\hline${ }^{\mathrm{q}} \mathrm{IN}_{\mathrm{i}}$ & $(4.44)$ & & $\begin{array}{l}\text { Fluid mass flux in across } \\
\text { boundary at node } i\end{array}$ \\
\hline${ }^{\mathrm{q}} \mathrm{OUT}_{i}$ & $(4.44)$ & & $\begin{array}{l}\text { Fluid mass flux out across } \\
\text { boundary node } i\end{array}$ \\
\hline$r^{*}$ & $(6.3 a)$ & & $\begin{array}{l}\text { Parameter in analytical } \\
\text { solution for radial } \\
\text { transport }\end{array}$ \\
\hline $\mathbf{s}_{L}$ & $(4.84)$ & & $\begin{array}{l}\text { Left side coefficient } \\
\text { contribution of sorption } \\
\text { isotherm to } U \text { equation }\end{array}$ \\
\hline $\mathbf{s}_{\mathrm{R}}$ & $(4.84)$ & & $\begin{array}{l}\text { Right side contribution of } \\
\text { isotherm to } U \text { equation }\end{array}$ \\
\hline t & $(3.4)$ & & Time \\
\hline$v(x, y, t)$ & $(2.39 c)$ & $|L / s|$ & Magnitude of velocity $\underline{\mathrm{v}}$ \\
\hline$\underline{v}(x, y, t)$ & $(2.19 a)$ & $\lfloor L / s \mid$ & Average fluid velocity \\
\hline$\underline{v}_{s}$ & $(2.49)$ & $|L / s|$ & Net solid matrix velocity \\
\hline$v_{x}(x, y, t)$ & $(2.39 c)$ & $|L / s|$ & Magnitude of $\mathrm{x}$-component of $\underline{\mathrm{v}}$ \\
\hline$v_{y}(x, y, t)$ & $(2.39 c)$ & $\lfloor L / s \mid$ & Magnitude of $y$-component of $\underline{v}$ \\
\hline
\end{tabular}


$\mathrm{x}$

$\mathrm{x}_{\mathrm{m}}$

${ }^{\mathrm{x}} \mathrm{p}$

y

Roman Uppercase

A

(6.3b)

$\mathrm{AF}_{i}$

$(4.53)$

$\mathrm{AT}_{i}$

$B(x, y, t)$

$\operatorname{BASE}(x, y)$

$\mathrm{BF}_{i j}$

$\mathrm{BT}_{i j}$

c

$C(x, y, t)$

$|\mathrm{L}|$

$\left|M_{s}\right| M \mid$

$\left|M_{s} / M\right|$

$c_{s}(x, y, t)$

$\left|M_{s} / M_{G}\right|$

$c^{*}(x, y, t)$

$\left|M_{s} / M\right|$

$(4.54)$ $\mathrm{x}$ coordinate

Minor principal direction

Major principal direction

y coordinate

Factor in analytical solution for radial transport

Matrix coefficient of pressure time derivative

Matrix coefficient of $U$ time derivative

Aquifer thickness

Elevation of aquifer base for example problem

Matrix coefficient in pressure equation

Matrix coefficient in $U$ equation

Base fluid solute concentration

Fluid solute mass fraction (or solute concentration) (mass solute per mass total fluid)

Specific concentration of adsorbate on solid grains (mass adsorbate/(mass solid grains plus adsorbate))

Solute concentration of fluid sources (mass fraction))

Matrix coefficient of $U$ time derivative in pressure equation 


\begin{tabular}{|c|c|c|c|}
\hline$\underline{D}(x, y, t)$ & $(2.25),(2.29)$ & $\left|L^{2} / s\right|$ & Dispersion tensor \\
\hline $\mathrm{D}_{\mathrm{m}}$ & $(2.29)$ & $\left|L^{2} / s\right|$ & $\begin{array}{l}\text { Apparent molecular } \\
\text { diffusivity of solute in } \\
\text { solution in a porous medium } \\
\text { including tortuosity ffects, } \\
\text { (about, } \mathrm{D}_{\mathrm{m}} \sim \mathrm{l} \text {. } 10(\mathrm{~m} / \mathrm{s}) \\
\text { for } \mathrm{NaCl} \text { at } 20 .{ }^{\circ} \mathrm{C} \text { ) }\end{array}$ \\
\hline$D_{i j}$ & $(2.39 c)$ & $\left|L^{2} / s\right|$ & Element of dispersion tensor \\
\hline$D_{x x}$ & $(2.39 a)$ & $\left|L^{2} / s\right|$ & Element of dispersion tensor \\
\hline$D_{\text {yy }}$ & $(2.39 b)$ & $\left|L^{2} / s\right|$ & Element of dispersion tensor \\
\hline $\mathrm{DF}_{i}$ & $(4.56)$ & & $\begin{array}{l}\text { Element of vector on right } \\
\text { side of pressure equation }\end{array}$ \\
\hline $\mathrm{DT}_{i j}$ & $(4.87)$ & & $\begin{array}{l}\text { Matrix coefficient of } u \\
\text { equation }\end{array}$ \\
\hline $\mathrm{ET}_{i}$ & $(4.90)$ & & $\begin{array}{l}\text { Element of vector on right } \\
\text { side of } U \text { equation }\end{array}$ \\
\hline$F_{m}$ & $(2.42 b)$ & & $\begin{array}{l}\text { Dispersive flux in } \\
\text { principal direction } m\end{array}$ \\
\hline$F_{p}$ & $(2.42 a)$ & & $\begin{array}{l}\text { Dispersive flux in } \\
\text { principal direction } p\end{array}$ \\
\hline$F_{s}$ & $(2.41)$ & & $\begin{array}{l}\text { Dispersive flux along } \\
\text { stream line }\end{array}$ \\
\hline $\mathrm{G}_{\mathrm{KG}}$ & $(4.32)$ & & $\begin{array}{l}\text { Coefficient of Gauss } \\
\text { integration }\end{array}$ \\
\hline$G_{s} T L$ & $(4.89 b)$ & & $\begin{array}{l}\text { Element of vector on left } \\
\text { side of } U \text { equation }\end{array}$ \\
\hline$G_{s} T R$ & $(4.89 c)$ & & $\begin{array}{l}\text { Element of vector on right } \\
\text { side of } U \text { equation }\end{array}$ \\
\hline $\mathrm{GT}_{\mathbf{i}}$ & $(4.89 a)$ & & $\begin{array}{l}\text { Element of vector on left } \\
\text { side of } U \text { equation }\end{array}$ \\
\hline$\underline{I}$ & $(2.25)$ & $\lfloor 1\rfloor$ & $\begin{array}{l}\text { Identity tensor (ones on } \\
\text { diagonal, zeroes elsewhere) }\end{array}$ \\
\hline$I_{i j}$ & $(3.23)$ & & $\begin{array}{l}\text { Matrix arising from } \\
\text { integral in example } \\
\text { problem }\end{array}$ \\
\hline
\end{tabular}




\begin{tabular}{|c|c|c|c|}
\hline IMVDIM & $(7.8)$ & & Program dimension \\
\hline $\mathrm{K}(\mathrm{x}, \mathrm{y})$ & $\begin{array}{l}(2.20) \\
(3.1)\end{array}$ & $|L / s|$ & Hydraulic conductivity \\
\hline KG & $(4.32)$ & & Number of Gauss point \\
\hline NE & $(3.3)$ & & Number of elements in mesh \\
\hline NN & $(3.4)$ & & Number of nodes in mesh \\
\hline NP & $(4.32)$ & & Number of Gauss points \\
\hline NPBC & $(7.1)$ & & $\begin{array}{l}\text { Number of specified pres- } \\
\text { sure nodes in mesh }\end{array}$ \\
\hline NSOP & $(7.1)$ & & $\begin{array}{l}\text { Number of specified fluid } \\
\text { source nodes in mesh }\end{array}$ \\
\hline NSOU & $(7.1)$ & & $\begin{array}{l}\text { Number of specified U } \\
\text { source nodes in mesh }\end{array}$ \\
\hline NUBC & $(7.1)$ & & $\begin{array}{l}\text { Number of specified } U \\
\text { nodes in mesh }\end{array}$ \\
\hline NPCYC & $(7.1)$ & & Pressure solution cycle \\
\hline NUCYC & $(7.1)$ & & U solution cycle \\
\hline 0 & $(3.7)$ & & $\begin{array}{l}\text { The governing equation of } \\
\text { the example problem }\end{array}$ \\
\hline$o_{p}$ & $(4.38)$ & & $\begin{array}{l}\text { The fluid mass balance } \\
\text { equation }\end{array}$ \\
\hline$o_{u}$ & $(4.66)$ & & $\begin{array}{l}\text { The energy or solute mass } \\
\text { balance equation }\end{array}$ \\
\hline $\mathrm{Pe}_{\mathrm{m}}$ & $(7.1)$ & & The mesh Peclet number \\
\hline $\mathrm{PBC}_{\text {ipu }}$ & $(7.1)$ & & $\begin{array}{l}\text { The ipu }{ }^{\text {th }} \text { pressure boundary } \\
\text { condition value }\end{array}$ \\
\hline$Q_{i}$ & $(4.50)$ & $|\mathrm{M} / \mathrm{s}|$ & $\begin{array}{l}\text { Total fluid mass source to } \\
\text { cell } i\end{array}$ \\
\hline$Q_{p}(x, y, t)$ & $(2.22)$ & $\left|M /\left(L^{3} \cdot s\right)\right|$ & $\begin{array}{l}\text { Fluid mass source (including } \\
\text { pure water mass plus solute } \\
\text { mass dissolved in source } \\
\text { water) }\end{array}$ \\
\hline$Q^{* *}(x, y)$ & $(3.1)$ & $\left|s^{-1}\right|$ & $\begin{array}{l}\text { Volumetric fluid source for } \\
\text { example problem (volume } \\
\text { fluid injected per time } /\end{array}$ \\
\hline
\end{tabular}




\begin{tabular}{|c|c|c|c|}
\hline & & & $\begin{array}{l}\text { example problem (volume } \\
\text { fluid injected per time } \\
\text { volume aquifer) }\end{array}$ \\
\hline $\mathrm{Q}_{\mathrm{PBC}}$ & $(4.51)$ & {$\left[M / L^{3} \cdot s\right]$} & $\begin{array}{l}\text { Fluid mass source rate due } \\
\text { to a specified pressure }\end{array}$ \\
\hline $\mathrm{Q}_{\mathrm{BC}}$ & $(3.38)$ & & $\begin{array}{l}\text { Fluid volumetric source } \\
\text { due to a specified head } \\
\text { in the example problem }\end{array}$ \\
\hline $\mathrm{Q}_{\mathrm{BC}}$ & $(4.64)$ & {$[\mathrm{M} / \mathrm{s}]$} & $\begin{array}{l}\text { Fluid mass source due to } \\
\text { a specified pressure node }\end{array}$ \\
\hline $\mathrm{Q}_{\mathrm{IN}_{\mathrm{i}}}$ & $(3.20)$ & & $\begin{array}{l}\text { Fluid volume efflux at } \\
\text { boundary for example } \\
\text { problem }\end{array}$ \\
\hline $\mathrm{Q}_{\text {TOT }}$ & $(6.12)$ & & $\begin{array}{l}\text { Total pumping rate for } \\
\text { pump-test example }\end{array}$ \\
\hline$Q_{i}^{*}$ & $(3.28)$ & & $\begin{array}{l}\text { Fluid volumetric source } \\
\text { for example problem }\end{array}$ \\
\hline $\mathbf{R}$ & $(3.8)$ & & $\begin{array}{l}\text { Residual of discretized } \\
\text { equation }\end{array}$ \\
\hline RMDIM & $(7.6)$ & & Program matrix dimension \\
\hline RVDIM & $(7.7)$ & & Program matrix dimension \\
\hline$s_{o p}(x, y)$ & $(2.13)$ & {$\left[M_{f} /\left(L \cdot s^{2}\right)\right]^{-1}$} & Specific pressure storativity \\
\hline$S(x, y)$ & $(3.1)$ & {$\left[L^{-1}\right]$} & $\begin{array}{l}\text { Specific storativity for } \\
\text { example problem }\end{array}$ \\
\hline$s^{*}$ & $(6.1 a)$ & & $\begin{array}{l}\text { Dimensionless drawdown for } \\
\text { pump test example }\end{array}$ \\
\hline$s_{w}(x, y, t)$ & $(2.6)$ & [1] & $\begin{array}{l}\text { Water saturation (saturation) } \\
\text { (volume of water per volume } \\
\text { of voids) }\end{array}$ \\
\hline $\mathrm{T}_{\mathrm{o}}$ & $(2.3)$ & {$\left[{ }^{\circ} \mathrm{C}\right]$} & Base fluid temperature \\
\hline$T(x, y, t)$ & $(2.1)$ & {$\left[{ }^{\circ} \mathrm{C}\right]$} & $\begin{array}{l}\text { Fluid temperature (degrees } \\
\text { Celcius) }\end{array}$ \\
\hline$\pi(x, y, t)$ & $(3.2)$ & {$\left[L^{2} / s\right]$} & $\begin{array}{l}\text { Aquifer transmissivity for } \\
\text { example problem }\end{array}$ \\
\hline$T^{*}(x, y, t)$ & $(2.25)$ & {$\left[{ }^{\circ} \mathrm{C}\right]$} & Temperature of source fluid \\
\hline
\end{tabular}




\begin{tabular}{|c|c|c|}
\hline $\mathrm{U}$ & $(2.47)$ & $\begin{array}{l}\text { either } T \text { or } C \text { depending on } \\
\text { type of simulation }\end{array}$ \\
\hline $\mathrm{U}_{\mathrm{BC}}$ & $(4.66)$ & $\begin{array}{l}\mathrm{U} \text { value of inflow at point } \\
\text { of specified pressure }\end{array}$ \\
\hline$u^{*}$ & $(2.47 a)$ & $U$ value of fluid source \\
\hline UP & $(4.23)$ & Upstream weighting factor \\
\hline$v_{i}$ & $(3.15)$ & Cell volume at node 1 \\
\hline VOL & $(2.9)$ & Volume (total) \\
\hline VOL $_{w}$ & $(2.13)$ & Fluid volume \\
\hline $\mathbf{w}_{0}$ & $(4.111 b)$ & Weight for Langmuir lsotherm \\
\hline$W(u)$ & $(6.1 a)$ & $\begin{array}{l}\text { Well function for pump test } \\
\text { example }\end{array}$ \\
\hline$w_{i}$ & $(4.39)$ & Weighting function \\
\hline$W_{\infty}$ & $(4.111 a)$ & Weight for Langmuir isotherm \\
\hline
\end{tabular}


Appendix B:

SUTRA Program Listing

(Model version V1284-2D)

301

(p.303 follows) 


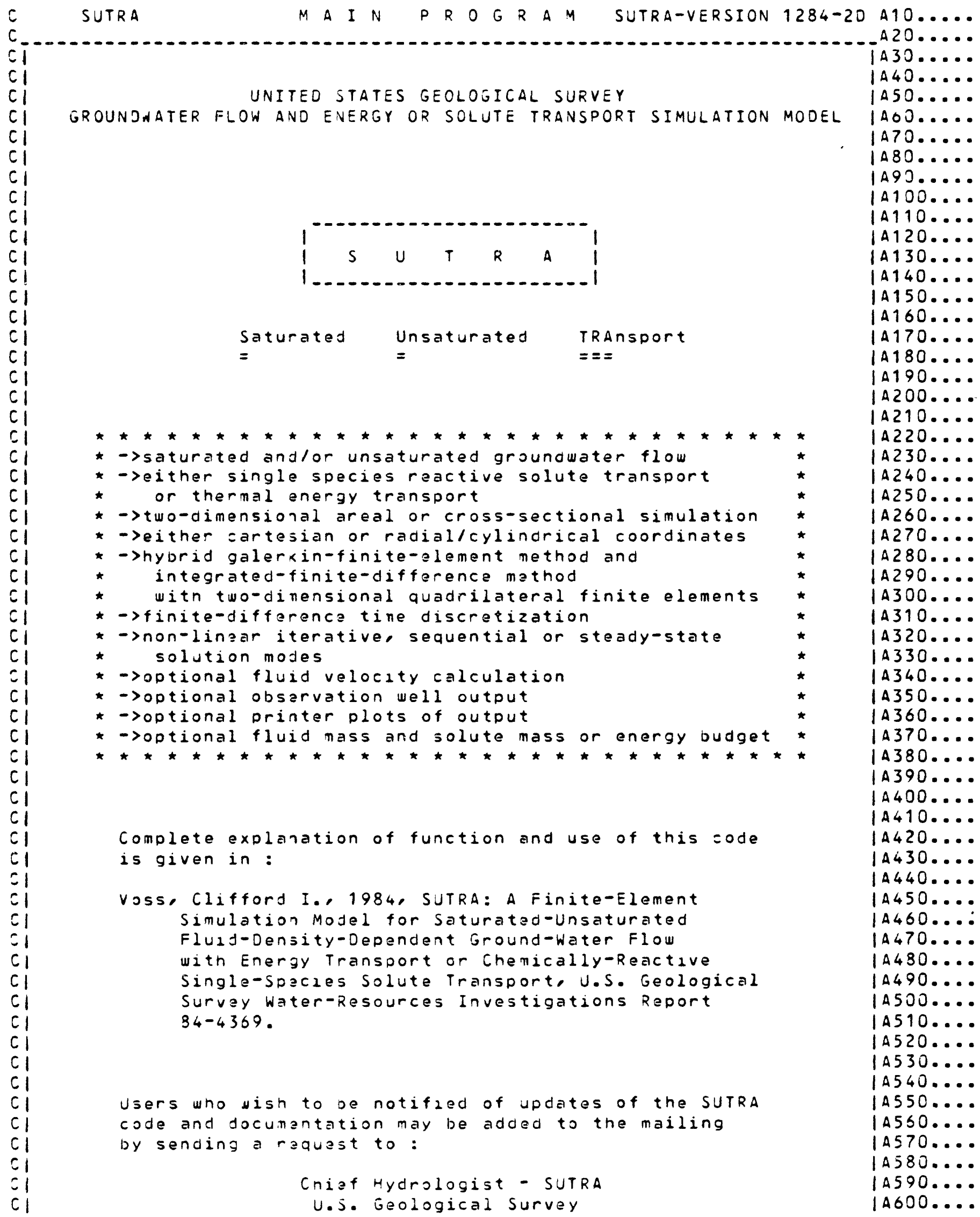


431 National ienter

Reston. Virginia 22072 USA

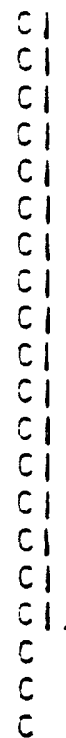

$c$

$c$

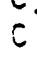

$C C^{*} * *$

$C \mid * *$

$01 * *$

$C 1 * *$

$C 1 * *$

$C l * *$

$C 1 * *$

$C 1 * *$

$C 1 * *$

$C \mid * *$

$C 1 * *$

$C 1 * *$

$C 1 * *$

$01 * *$

$C \mid * *$

$C 1 * *$

$C 1 * *$

(1**

CI**

CI**

$C 1 * *$

$C l * \star$

$61 *$ *
* * th * *

$\star j$

$\star$

* Japartinent of

* Engineering and Servics

* Florida.

* availabie for

* * * * * * * *
* Engineering and Services

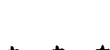

IMPLICIT DOUBLE PRECISION (A-H,O-Z)

COMMON/LGEM/ RM

COMMON/LGEV/ RV

COMMON/LGEMVI IMV

COMMON/DIMS/ NN,NE,NIN,NBI,NB,NBHALF,NPINCH,NPBC,NUBC,

1 NSOP,NSOU,NBCN

COMMON/CONTRL/ GNJ,UP,OTMULT, OTMAX, ME, ISSFLO,ISSTRA,ITCYC,

1 NPCYC,NUCYC,NPRINT, IREAO,ISTORE,N JUMAT, IUNSAT

COMMON/OBS/ NOBSN,NTOSSN, VOBCYC,ITCNT

CHARACTER 1 TITLE1 (80),TITLE2(30)

CHARACTER $* 6$ SIMULA(2)

DIMEVSION KRV(100)

\section{The three arrays that need be
are dimensioned as follows:}

\section{DIYENSION RM( RMOIY), RV( RVDIM), IMV(IMVDIM)}

RMDIM $>=2 * N N * N 3 I$

RVIIM $>=((N N V * N N+(N E V+8) * N E+N B C N * 3$

$+(N J B S+1) *(N T O B S+2) * 2+N T O B S+5))$

IMVOIM $>=(6 N E \star 8+N N+N P I N C H * 3+N S O P+N S O U$ $+N B E N \star 2+N O B S+N T O B S+12)$ )

where:

$$
N N V=30
$$

$N E V=10$

$N B C N=N P B C+N U B C$

and:

$N V=$ number of nodes in finite element mesh
$1 A 010 \ldots$

$14020 \ldots$.

$14030 \ldots$

1 $4640 \ldots$

1 $A 650 \ldots \ldots$

$14050 \ldots$.

$14070 \ldots$.

14680....

1 $A 690 \ldots$.

| $A 700 \ldots$.

| $4710 \ldots$.

|A>20....

| $A 730 \ldots$.

IA740....

IA750....

| $A 760 \ldots$

I $A 770 \ldots$

A780....

A790....

$\triangle 800 \ldots$.

A810....

A820....

A $830 \ldots$.

A840...

A850....

$\triangle 860 \ldots$

$A 870 \ldots$

$4880 \ldots$

A890....

A900....

A910....

A920....

A930....

A940....

A950....

A960....

A970....

A980....

A990....

A1000...

A1010...

A1020...

A1030...

A1040...

A1050...

A1060...

A1070...

A1080...

$41090 \ldots$

A1100...

A1110...

A1120...

A1130...

A1140...

A1150...

A1160...

A1170...

A1180...

A1190...

A1200... 


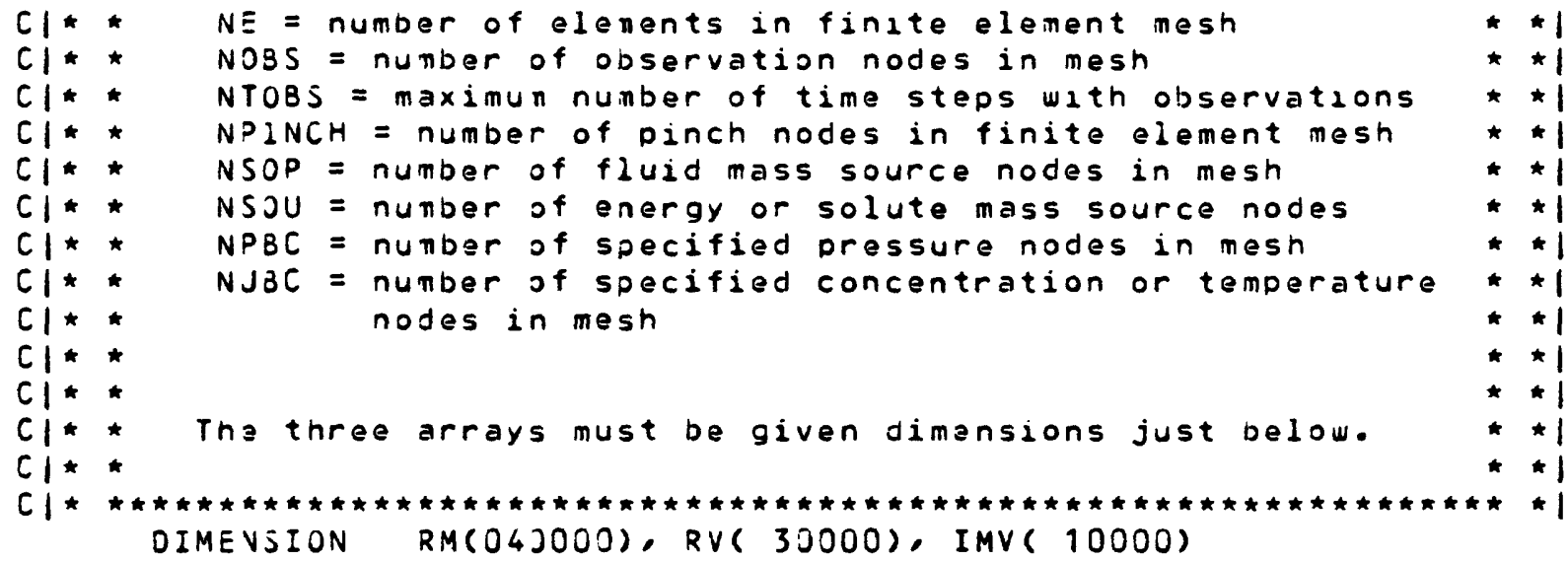

CI

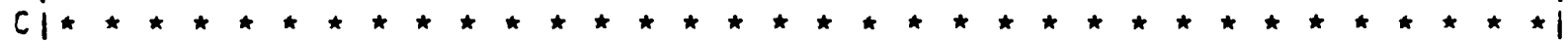

C

C..... INPUT DATASET 1: INPUT DATA HEADING

C..... ( SET ME $=-1$ FOR SOLUTE TRANSPJRT, ME $=+1$ FOR ENERGY TRANSPORT) REAJ $(5,100)$ SIMULA

100 FORMAT $(2 A 6)$ WRITE $(6,110)$

110 FORMAT $(1 H 1,132(1 H *) / / / / 3(1 X, 132(1 H *) / / / /) / / / /$

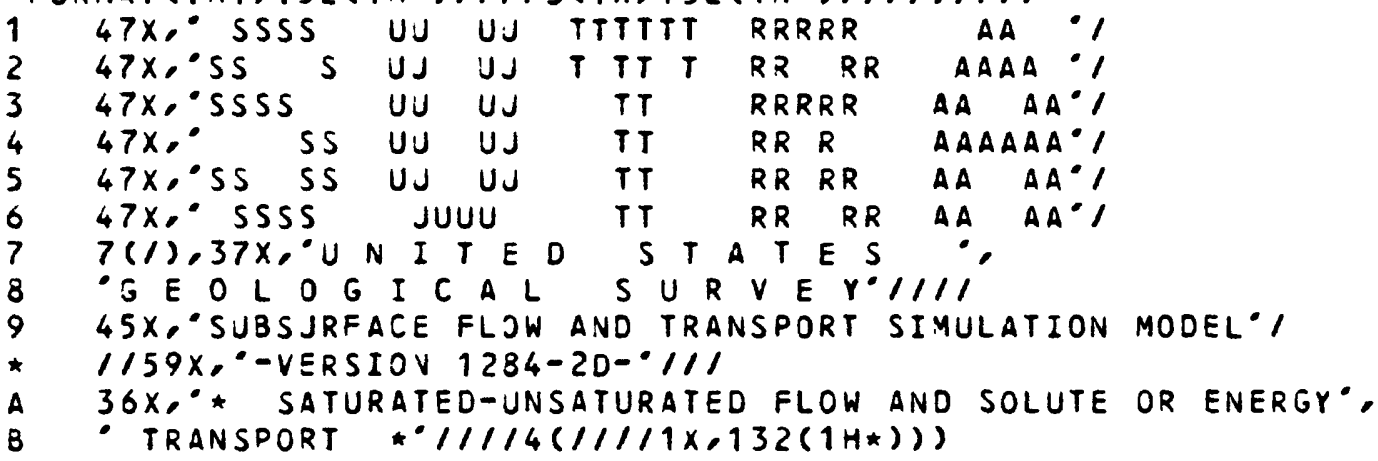

$c$

IF(SIMULA(1).NE. SUTRA ') GOTO 115

IF(SIMULA(2).EQ. "SOLUTE') GOTO 120

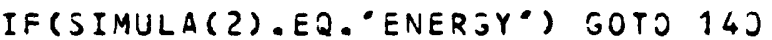

115 WRITE $(6,116)$

116 FORMAT $(1 H 1 / / / / / 20 x, \circ \star \star \star \star$ ERROR IN FIRST DATA CARD--*,

1 - - - DATA INPUT HALTED FJR CORRECTIONS * * * * *) STOP

$120 M E=-1$

WRITE $(6,130)$

130 FORMAT(1H1//132(1H*)///20X,***** SUTRA SOLU

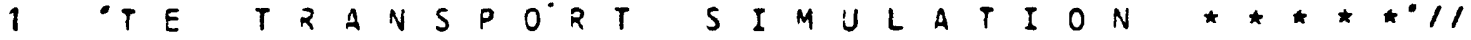

$5 / 132(1 H *) /)$

GOTO 160

$140 M E=+1$

WRITE $(6,150)$

150 FORMAT $\left(1 H 1 / / 132(14 *) / / / 20 x^{\circ} * \star \star \star \star *\right.$ SUTRA E NER

1 I Y T R A N S P ORT S I MULATION * * *

$21132(1 H *) / 2$
A $1210 \ldots$ A1220... A1230... A1240... A1250... A1260... A1270... A1200... A1290... A1300... A1310... A1320... A1330... A1340... A1350... A1360... A1370... A1380... A1390... A1400... A1410... A1420... A1430... A1440... A1450... A1460... A1470... A1480... A1490... A1500... A1510... A1520... A1530... A1540... A1550... A1560... A1570... A1580... A1590... A1600... A1610... A1620... A1630... $41640 \ldots$ \$1650... A1660... A1670... A1680... A1690... A1700... A1710... A1720... A1730... $41740 \ldots$ A1750... A1760... $\Delta 1770 \ldots$ A1780... A1790... A1800... 
C..... IVPUT DATASET 2: OUTPUT HEAOING $R \equiv A J(5,170)$ TITLE1, TITLE2

170 FORMAT (8OA1/80A1) WRITE $(6,180)$ TITLE1,TITLE2

183 FORMAT (////1X,131(1H-)//25X,8JA1//26X,30A1//1X,131(14-)) REAO(S, 200) NV,NE,N3I,NPIVCH,VPBC, VUSC,NSOP, NSOU,NOBS, NTOSS READ(5,200) IUNSAT,ISSFLO,ISSTRA, IREAD, ISTORE

200 FORMAT (16I5) WRITS(5,205)

205 EJRMATC(////11X, S I Y U L A T I ON MO DE 1 O P T I J N $\left.S^{\circ} /\right)$

$I=(I S S T R A . E Q .1 . A N J$. ISSFLO.NE.1) THEN WRITE $(0,210)$

210 FORYAT(///111X, SSTEADY-STATE TRANSPORT ALSO REQUIRES THAT'

1 "Flow IS aT STEAOY STATE. $/ 111 X$, PLEASE CORRECT ISSFLO $"$

2 -AND ISSTRA IV THE INPUT JATA, ANO RERUN. 111111111

$345 X_{0}^{\circ} S I M U$ I $T$ I O N HAL TE D DUE TO INPUT ERROR') ENDFILE( $(0)$

STOD

ENDIF

IF (IJNSAT.EQ.+1) NRITE $(6,215)$

IF (I JNSAT.EQ.0) WRITE $(6,216)$

215 FORMAT(11X,-- ALLOW UVSATURATED AND SATURATED FLOW: UNSATURATEO 1 - PROPERTIES ARE USER-PROGRAMMED IN SUBROUTINE U N S A $T^{\circ}$ )

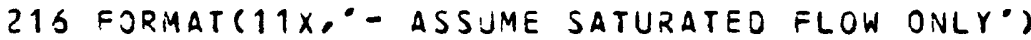

IF(ISSFLO.EQ.+1.AVO.ME.EQ.-1) WRITE $(5,219)$

IF (ISSFLO.EQ.+1.AVD.ME.EQ.+1) WRITE $(6,220)$

IF(ISSFLO.EQ.0) WRITE $(6,221)$

219 FORMAT $111 X_{0} \cdot-$ ASSJME STEAOY - STATE FLOW FIELO CONSISTENT WITH $\bullet$

1 - INITIAL EONCEVTRATION (ONJITIONS ${ }^{\circ}$ )

220 FORMAT( $11 X_{0} 0^{-}$ASSUME STEAOY STATE FLOW FIELD CONSISTENT WITH,

1 - INITIAL TEMPEZATURE CONOITIONS')

221 =ORMAT( $11 X_{2},-\triangle L L O W$ TIME-JEPEVDENT FLOW FIELO')

IF (ISSTRA.EQ.+1) NRITE $(6,225)$

IF (ISSTRA.EQ.0) WRITE $(6,226)$

225 FORMAT $\left(11 X_{0},-\right.$ ASSJME STEAJY-STATE TRANSPJRT')

226 FORMAT $\left(11 x, 0^{\circ-}\right.$ - ALLOW TIME-DEPENDENT TRANSPORT')

IF (IREAD.EQ.-1) WRITE $(6,230)$

IF(IREAD.EQ.+1) WRITE $(6,231)$

230 FORMATC11X,-- WARY START - SIYULATION IS TO BE ",

1 CONTINUEO FROY PREVIOUSLY-STORED DATA')

231 FORMAT $\left(11 X^{\circ},-\right.$ COLD START - BESIN NEW SIMULATION $\left.{ }^{\circ}\right)$

IF(ISTORE.EQ.+1) WRITE $(0,240)$

IF (ISTORE.EQ.0) WRITE $(6,241)$

240 FORMAT(11X,-- STOZE RESULTS AFTER EACH TIME STEP ON UNIT-06,"

1 - AS BACK-UP AVO FJR USE IV A SIMULATION RE-START')

241 =ORMAT $\left(11 X_{\circ}^{\circ}-\right.$ DO NOT STORE RESULTS FOR USE IN A ',

$c$

1 -RE-START OF SIMULATION')

IF (ME.EQ.-1)

1 WRITE (6,245) NV,NE,NBI,NPINCH,NPBC,NUBC,NSOP, NSOU,NOBS, NTOBS

245 FORMAT $/ / 1 / 11 X,{ }^{\circ} S$ I Y U L A T I O N C O N T R O L

1 - $V$ U M 3 E $2 S^{\circ} / 111 \mathrm{x}, 10,5 x,{ }^{\circ}$ NUMBER OF NODES IN FINITE- ${ }^{\circ}$,

2 ELEMENT MESH*111X,I0,5X, NUMBER OF ELEMENTS IN MESH*

3 11X,I6,5X, ESTIMATED MAXIMJM FJLL BAND WIOTH FOR YESH'/1

$11 X, I 6,5 X, \circ E X A=T$ NJMBER OF PINCH NODES IN MESH* /

$11 X, I 6,5 X,{ }^{\circ}$ EXACT NJMBER OF NODES IN MESH AT WHICH $\bullet$

- PRESSURE IS A SPECIFIED CONSTANT OR FuNCTION OF TIME*/

$11 X, 16,5 X$, EXAET NJMBER OF NODES IN MESH AT WHICH ',

- SQLUUTE CONCENTRATION IS A SPECIFIED CONSTANT OR "
A1810...

A1820...

A $1830 \ldots$

A1840...

$\triangle 1350 \ldots$

A1360...

A $1870 \ldots$

A1380...

A1890...

A1900...

A1910...

$41920 \ldots$.

A1930...

A1940...

A $1950 \ldots$

A1960...

$\$ 1970 \ldots$

A1980...

A1990...

A $2000 \ldots$

$\triangle 2010 \ldots$

A2020...

, $A 2030 \ldots$

A2040...

A2050...

A2 $2060 \ldots$

A $2370 \ldots$

A2080...

A2090...

A2100...

A2110...

A $2120 \ldots$

A2130...

A2140...

A2150...

A2160...

A2170...

A2180...

A2190...

A2200...

A2?10...

A $2220 \ldots$

A2230...

A2240...

A2250...

A2260...

A2270...

A $2280 \ldots$

A2290...

A2300...

A2310...

A2320...

A2330...

$\Delta 2340 \ldots$

A2350...

A2360...

A2370...

A2380...

A2390...

A2400... 
IF (ME.EQ. + 1)

$$
1
$$

- = UNCTION OF TIME*//11X,I6,5X, EXACT NUMBER OF NODES at',

- WHICH FLUIO INFLOW OR OUTFLOW IS A SPECIFIED CONSTANT',

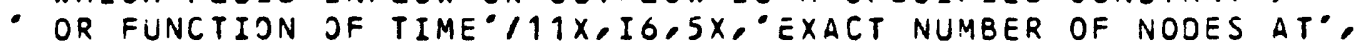

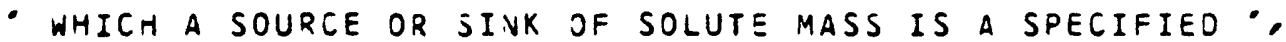

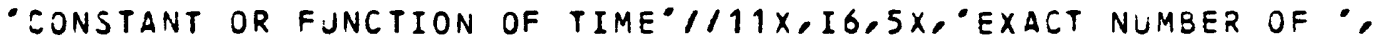

- NODES AT WHICH PRESSURE AND CONCENTRATION WILL BE OBSERVEO०,

$111 X$, IO,5X, MAXIMUY NUMBER OF TIME STEPS ON WHICH,

- obSERVATIONS WILL be MAOE*)

WRITE (6,255) VN,NE,NBI,NPINCH,NPBC,NUBC,NSOP,NSOU,NUBS,NTOBS

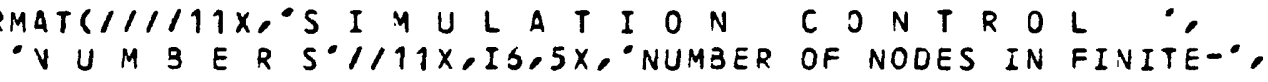

- ELEMENT MESH॰ $/ 11 X$, I6,5X, "NUMBER OF ELEMENTS IN MESH*

$11 X, 16,5 X, \circ E S T I M A T E D$ MAXIMUM FULL BAND WIDTH FOR MESH* $/ 1$

$11 X, I 6,5 X, \circ E X A E T$ NJMBER OF PINCH NODES IN MESH* 1

$11 X, I 6,5 X, \circ E X A=T$ NJMBER OF NODES IN MESH AT WHICH -

- PRESSURE IS A SPECIFIED CONSTANT OR FUNCTION OF TIME',

$11 X, I 6,5 X, \bullet E X A C T$ NJMBER OF NODES IN MESH AT WHICH '

- TEMPERATURE IS A SPECIFIED CONSTANT OR "

- FUNCTION OF TIME*//11X,I6,5X, EXACT NUMBER OF NODES AT',

- NHICH FLUIO INFLJW OR OUTFLOW IS A SPECIFIED CONSTANT',

- or function jF TIME /11X,I6,5X,

- WHICH A SOUREE OR SINK OF ENERGY IS A SPECIFIED CONSTANT',

- or FUNCTION JF TIME //11X,I6,5X, EXACT NJMBER OF NODES ",

- AT WHICH PRESSURE AND TEMPERATURE WILL BE OBSERVED

$111 X$, I6,5X, MAXIMUM NUMBER OF TIME STEPS ON WHICH •,

- OBSERVATIONS WILL BE MADE')

\section{calculate dimensions for pointers}

$N B C N=N P B C+N U B C+1$

$N S O P=N S O P+1$

NSOU $=N S O U+1$

$N P I N=H=N P I N C H+1$

MATDIM $=N N \star N B I$

$N I N=N E \star 8$

NOBSN $=$ NOBS +1

NTOB SN $=$ NTOBS +2

MATOBS $=$ NOBSN $* N T O B S N$

$N E 4=V E \star 4$

- SET UP POINTERS FJR REAL MATRICES

$K R M 1=1$

$K R M 2=K R M 1+\quad M A T D I M$

KRM $=K R M 2+\quad M A T O I M$

NOTE: THE LAST POINTER IN THE AGOVE LIST, CURRENTLY, KRM3, MAY N E V E R BE PASSED TO SUTRA. IT POINTS TO THE STARTING ELEMENT OF THE NEXT NEW MATRIX TO BE ADDED. PRESENTLY, SPACE IS ALLOCATEO FOR (C) MATRICES.

\section{SET UP POINTERS FJR REAL VECTORS}

NNV IS NUMBEZ OF REAL VECTORS THAT ARE NN LONG $N N V=30$

NEV IS NUMBER OF REAL VECTORS THAT ARE NE LONG
A2410...

$\Delta 2420 \ldots$

A2430...

A2440...

A2450...

A2460...

A2470...

A2480...

A2490...

A2500...

A $2510 \ldots$

A2520...

A2530...

A2540...

A2550...

A2560...

A2570...

A2580...

A2590...

A2600...

A2610...

A2620...

A2630...

$42640 \ldots$

A2650...

A2660...

A2670...

A2680...

A2690...

A2700...

A2710...

A2720...

A2730...

A2740...

A2750...

A2760...

A $2770 \ldots$

A2780...

A2790...

A2800...

A2810...

A2820...

A2830...

A2840...

A2850...

A2860...

A2870...

A2880...

A2890...

A2900...

A2910...

A2920...

A2930...

A2940...

A2950...

A2960...

A2970...

A2980...

A2790...

$23000 \ldots$ 


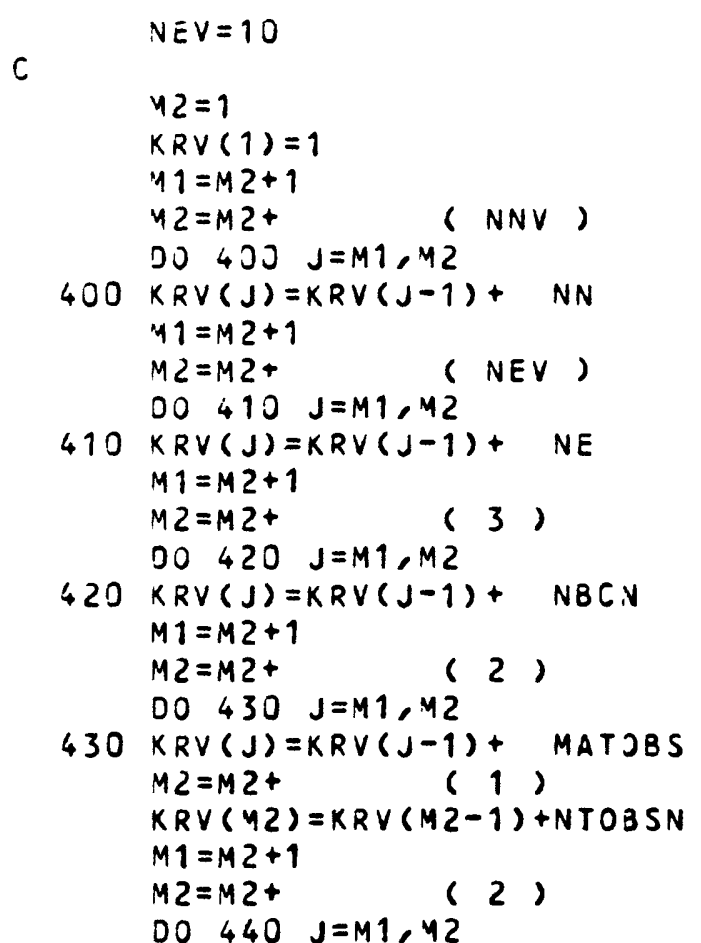
$440 \operatorname{KRV}(J)=\operatorname{KRV}(J-1)+N E 4$

c

\section{$K I M \vee 1=1$}

$K I M V 2=K I M V 1+$ $K I M V 3=K I M V 2+$ $K I M \vee 4=K I M \vee 3+$ $K I M \vee 5=K I M \vee 4+$ $K I M \vee 6=K I M \vee 5+$ $K I M \vee 7=K I M V 6+$ $K I M V S=K I M V 7+$ $K I M \vee 9=K I M \vee 8+$ C KIMV10=KIMV9+

NIN NPINCH $\$ 3$ NSOP

NSOU

NBCN

NBCN

NV NOBSN NTOBSN

NOTE: THE LAST POINTER IN THE ABOVE LIST, CURRENTLY, KIMV10, maY N E V E R bE PASSED TO SUTRA. IT POINTS TO THE STARTING ELEMENT OF THE NEXT NEW INTEGER VECTOR TO BE
PRESENTLY, SPAE IS ALLOCATEO FOR (8) INTEGER VECTORS.

\section{PASS POINTERS TO MAIN CONTROL ROUTINE, SUTRA}

CALL SUTRA RM(XRM1),RM(KRMZ), $R \vee(K R \vee(1)), R \vee(K R \vee(2)), R \vee(K R \vee(3)), R \vee(K R \vee(4)), R \vee(K R \vee(5))$, $R \vee(K R V(6)), R \vee(K R \vee(7)), R \vee(K R V(8)), R \vee(K R \vee(9)), R V(K R V(10))$, $R \vee(K R V(11)), R \vee(K R V(12)), R \vee(K R V(13)), R V(K R V(14)), R V(K R V(15))$, $R \vee(K R V(16)), R V(K R V(17)), R V(K R V(18)), R V(K R V(19)), R V(K R V(20))$, $R \vee(K R V(21)), R \vee(K R V(22)), R V(K R V(23)), R V(K R V(24)), R V(K R V(25))$, $R \vee(K R \vee(26)), R \vee(K R \vee(27)), R \vee(K R \vee(28)), R \vee(K R \vee(29)), R \vee(K R V(30))$, $R \vee(K R V(31)), R V(K R V(32)), R V(K R V(33)), R \vee(K R V(34)), R V(K R V(35))$, $R \vee(K R \vee(36)), R \vee(K R V(37)), R \vee(K R V(38)), R \vee(K R \vee(39)), R \vee(K R V(40))$,
A3010... A3020... A $3030 \ldots$ $A 3040 \ldots$ A3050... A3060... $\triangle 3070 \ldots$ A3080... A3090... A3100... A $3110 \ldots$ A3120... A3130... $\triangle 3140 \ldots$ $\triangle 3150 \ldots$ A3160... A3170... A3180... A $3190 \ldots$ A3200... A3210... A3220... A $3230 \ldots$ $\triangle 3240 \ldots$ $\triangle 3250 \ldots$ A3260... A3270... A3280... A3290... A $3300 \ldots$ A3310... A3320... A3330... A3340... A3350... A3360... A3370... A3380... A3390... A3400... A3410... A3420... A3430... A3440... A3450... A3460... ADDED.A3470 ... A $3480 \ldots$ A3490... A3500... A $3510 \ldots$ A3520... A3530... A3540... A3550... A3560... A3570... A3580... A3590... $43600 \ldots$ 
$9 \quad \operatorname{RV}(K R V(41)), \operatorname{RV}(K R V(42)), \operatorname{RV}(K R V(43)), \operatorname{RV}(\operatorname{KRV}(44)), \operatorname{RV}(K R V(45))$,

* RV(KRV(40)), RV(KRV(47)),RV(KRV(48)),

1 I $Y V(K I M V 1), I M V(K I M V 2), I M V(K I M V 3), I M V(K I M V 4), I M V(K I M V 5)$,

$2 \operatorname{IMV}(K I M \vee 6), \operatorname{IMV}(K I M V 7), \operatorname{IMV}(K \operatorname{IMV} 8), \operatorname{IMV}(K I M \vee 9))$

A3610... A3520... A3630... A 3640 ... A $3650 \ldots$ A3660... A3670... A3680... A3690... 
C

C

c

C..... INPUT SIMULATION JATA FROM UNIT-5 (DATASETS 3 THROUGH 15B) CALL INDAT1 (X,Y,THICK, POR, ALMAX, ALMIN,ATAVG,PERMXX,PERMXY, 1 PERMYX,PERIYY,PANGLE,SOP)

C

C.....PLOT MESH (IVPUT JATASET 16)

IF(KPLOTP+KPLOTU.JT.O) CALL PLOT $(0,1, X, Y, C C, I N D E X, X X, Y Y, P V E C)$

C

C..... INPUT FLUID MASS, AND ENERGY JR SOLUTE MASS SOURCES

C (JATASETS 17 AVD 18)

CALL ZERO(QIN,NN,0.00O)

CALL ZERO(UIV,NN,0.0DO)

CALL ZERO(QUIN,NN,0.00O) 
C SUBRJUTINE S S T R A SUTRA - VERSION 1284-20 B10....

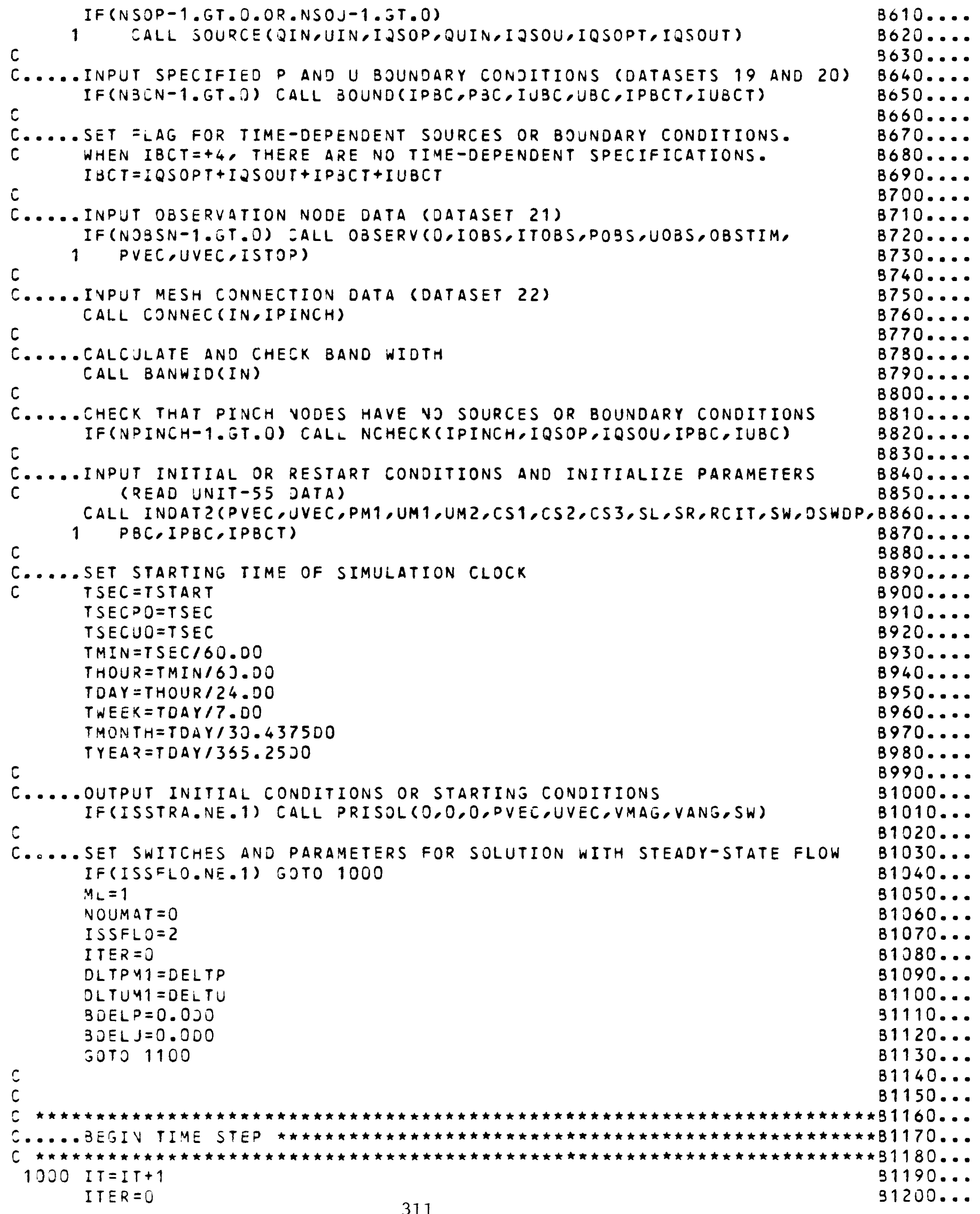


$M L=0$

NOUMAT $=0$

C......SET VOUMAT TO OBTAIN U SOLUTIJN BY SIMPLE BACK SUBSTITUTION

c

C BEGINNING ON SECONO TIME STEP AFTER A PRESSURE SOLUTION IF THE SOLUTIOV IS NON-ITERATIVE (ITRMAX $=1$ ) IF (MJO(IT-1,VPCYE).NE.O.ANO.MOO(IT,NPCYC).NE.O.AND.IT.GT.Z 1 - ANO. ITRMAX.EQ.1) NOUHAT $=1$

C..... CHOOSE SOLUTION VARIABLE ON THIS TIME STEP:

C $M L=0$ FOR P AND U, YL=1 FOR O ONLY, $\triangle N D M L=2$ FOR U ONLY. IF (IT.EQ.1.AVO.ISSFLO.NE.2) GOTO 1005

$I F(M J O(I T, N P C Y C)$. NE.OS $M L=2$ $I F(M J D(I T, N U C Y C)$.NE.O) $M L=1$

C..... YULTIPLY TIME STEP SIZE BY DTMULT EACH ITCYC TIME STEPS IF (MOD (IT,ITCYC).EQ.O.AND.IT.GT .1) DELT=DELT*DTMULT

C......SET TIME STEP SIZE TO MAXIMUM ALLOWED SIZE, OTMAX IF (DELT.GT.OTYAX) DELT = DTMAX

C..... INCREMENT SIYULATION CLOCK, TSEC, TO END OF NEW TIME STEP

IJJS TSEC $=$ TSEC+DELT

TMIN $=$ TSEC/60.00

THOUR $=$ TMIN $/ 60.00$

TOAY $=$ THOUR/24.00

TWEER = TOAY 7 . DO

TMONTH $=$ TOAY/ 30.437500

TYEAR $=$ TOAY/365.2500

$c$

C.....SET TIME STEP. FOR P AND/OR U. WHICHEVER ARE SOLVED FOR

C

ON THIS TIME STEP

IF (ML-1) $1010,1020,1030$

1010 DLTUM1=DELTU

DLTPM1 = DELTP

GOTO 1040

1020 DLTP Y1= DELTP

GOTO 1040

1030 DLTUY1=DELTU

1040 CONTINUE

DELTP $=$ TSEC-TSECPO

DELTJ $=T S E C-T S E C U O$

C..... SET PROJECTION FACTORS USEO ON FIRST ITERATION TO EXTRAPOLATE

C AHEAD ONE-HALF TIME STEP

$B D E L P=(D E L T P / O L T P Y 1) \star J .5000$

$B D E L U=(D E L T U / D L T U Y 1) * 3.5000$

$B D E L P 1=B O E L P+1.00 J$

$B D E L J 1=B D E L U+1.000$

C..... INCREMENT CLOCK FOR WHICHEVER OF P AND U WILL BE SOLVED FOR

C ON THIS TIME STEP

IF $(M L-1) \quad 1060,1073,1080$

1060 TSECPO $=$ TSEC

TSEC JO $=$ TSEC

GOTO 1090

1070 TSECPO=TSEC GOTO 1090

1380 TSECJO=TSEC

1090 CONTINUE

B1210...

$31220 \ldots$

$\$ 1230 \ldots$

B1240...

B1250...

B1260...

$\$ 1270 \ldots$

B1280...

$81290 \ldots$

B1300...

B1310...

$81320 \ldots$

B1330...

B1340...

B1350...

B1360...

$B 1370 \ldots$

B1380...

B1390...

B1400...

B1410...

B1420...

B1430...

$81440 \ldots$

$81450 \ldots$

B1460...

B1470...

$81480 \ldots$

B1490...

B1500...

B1510...

B1520...

B1530...

B1540...

B1550...

B1560...

81570...

B1580...

B1590...

B1600...

B1610...

B1620...

$81530 \ldots$

$31640 \ldots$

B1650...

B1560...

B1670...

$81680 \ldots$

$91690 \ldots$

\$1700...

$81710 \ldots$

$81720 \ldots$

$31730 \ldots$

B1740...

B1750...

C.... BEGIV ITERATION - - - - - - - - -

C - - - - - - - - - - - - - - - . - - - - - - B1770...

1100 ITER $=$ ITER+1

こ

IF (ML-1) 2000,220J,2400
$81780 \ldots$

B1790...

B1800... 
C.....SHIFT AND SET VECTORS FOR TIME STEP WITH BOTH P AND J SOLUTIONS

$2000002025 \quad I=1, N N$

PITER(I) $=P V E C(I)$

PVEL $(I)=P \vee E C(I)$

UITER (I) = UVEE(I)

$R C I T Y I(I)=R C I T(I)$

$2325 R C I T(I)=R H O W J+J R W J U *(J I T E R(I)-U R H O W O)$

DO 2050 IP $=1, N P B C$

$I=I A 3 S(I P B C(I P))$

$2 P L I T R(I P)=G N U *(P B C(I P)-P I T E R(I))$

2050 CONTINUE

IF(ITER.GT.1) GOTJ $26 J 0$

DO $2075 \quad I=1, N N$

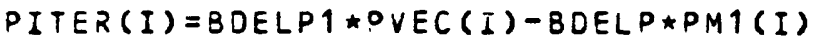

$\operatorname{UITER}(I)=B$ JELU $1 \star U V E C(I)-B$ JELUネUM $1(I)$

PMI (I) =PVEC (I)

$\operatorname{UM} 2(I)=U M T(I)$

2075 UMT (I) $=U V E C(I)$

GOTO 2600

C.....SHIFT AND SET VECTORS FOR TIME STEP WITH P SOLUTION ONLY

2200 DO $2225 \quad I=1, N N$

PVEL $(I)=P \vee E C(I)$

2225 PITER (I) =PVEZ (I)

IF(ITER.GT.1) GOTO 2600

DO $2250 \quad I=1, N N$

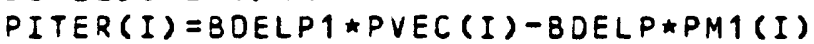

$\operatorname{UITER}(I)=U V E S(I)$

RCITYI (I) $=$ RCIT (I)

$R C I T(I)=R H O W J+D R W J U \star(J I T E R(I)-U R H J W O)$

2250 PMI (I) $=$ PVEC (I)

GOTO 2600

C.....SHIFT AND SET VECTORS FOR TIME STEP NITH U SOLUTION ONLY

2400 IF(NJUMAT.EQ.1) GJTO 2480

DO $2425 I=1, N$,

2425 UITER (I) =UVEC (I)

IF(ITER.GT.1) GOTJ 2600

DO $2450 \quad I=1, N N$

PITER (I) = PVES (I)

$P \vee E L(I)=P \vee E C(I)$

$\operatorname{UITER}(I)=8 D E L U 1 \star U V E C(I)-B D E L U \star U M 1(I)$

2450 RCITHI (I) $=$ RCIT(I)

JO 2475 IP $=1$,NPBC

$I=I A B S(I P B C(I P))$

2475 CONTINUE

QPLITR (IP) $=G N U *(P B C(I P)-P I T E R(I))$

2480 DO $2500 \quad I=1, V N$

$U M 2(I)=U M T(I)$

$2500 \cup M 1(I)=U V E C(I)$

2500 CONTINUE

$c$

C..... INITIALIZE arRAYS WITH VALUE JF zERO

MATOIM $=N N \star N B I$

IF $(M L-1) 3000,3000,3300$

3000 CALL ZERO(PMAT,MATDIM,0:000)

CALL ZERO(PVEC,NN,O.ODO)

CALL ZERO(VOL,NN,J.ODJ)

IF (ML-1) 330J.340J.33J0

3300 IF(NJJMAT) $3350,3350,3375$

3350 CALL ZERO(UMAT,MATOIM,O.OJO)

3375 CALL ZERO(UVEL,NN,O.DJO)

$31810 \ldots$ $B 1820 \ldots$

B1830...

B1840...

B1850...

B1860...

31870...

B1880...

$B 1890 \ldots$

B1900...

B1910...

B1920...

B1930...

B1940...

B1950...

B1960...

B1970...

$31980 \ldots$

$31990 \ldots$

$32000 \ldots$

B2010...

B2320...

B2030...

B2040...

B2050...

B2060...

$82070 \ldots$

B2080...

B2090...

B2100...

B2110...

B2120...

B2130...

B2140...

$82150 \ldots$

$82160 \ldots$

B2170...

B2180...

$82190 \ldots$

B2200...

B2210...

B2220...

$82230 \ldots$

B2240...

$82250 \ldots$

32260...

B2270...

B2280...

B2290...

B2300...

B2310...

$82320 \ldots$

B2330...

B2340...

$82350 \ldots$

$32360 \ldots$

B2370...

$32380 \ldots$

$82390 \ldots$

$82400 \ldots$ 
$3+30$ CONTINUE

C

C..... SET TIME-DEPENJENT BOUNOARY CJNDITIONS, SCURCES AND SINKS

C

C..... SET SORPTION PARAMETERS FOR THIS TIME STEP

IF (ML.NE.1.AND.ME.EQ.-1.AND.NOUMAT.EQ.0.AND.

1 AJSMOD.NE. NOVE

C..... OO ELEMENTWISE CALCULATIONS IN MATRIX EQUATION FOR P ANDIOR U

IF (N JUMAT.EQ.O)

1 CALL ELEMEN (ML, I $Y, X, Y, T H I C K, P I T E R, U I T E R, R C I T, R C I T M 1, P O R$,

2 ALMAX,ALMIN,ATAVG,PERMXX,PERMXY,PERMYX,PERMYY,PANGLE,

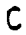

3 VYAG,VANG,VOL,PMAT,PVEC,UMAT, UVEC, GXSI, GETA,PVEL)

C.....DO NODEWISE CALCULATIONS IN MATRIX EQUATION FOR P AND/OR J CALL NOOALB (ML, VOL,PMAT, PVEC, UMAT, UVEC,PITER, UITER, PM1,UM1,UMZ, 1 POR, QIN,UIN, QUIN,CS1,CS2,CS3,SL,SR,SW,OSWDP,RHO,SOP)

C

C..... SET SPECIFIED P AND U CONOITIONS IN MATRIX EQUATION FOR P ANO/OR CALL BCB (ML, PMAT, PVEC,UMAT, UVEC, IPBC,PBC, IUBC, UBC, QPLITR)

$c$

C..... SET PINCH NODE CONDITIONS IN MATRIX EQUATION FOR P ANDIOR U IF (NPINCH-1) 4200,4200,4000

4000 CALL PINCHB (ML, IPINCH,PMAT,PVEC,UMAT,UVEC)

$42 J 0$ CONTINUE

C

C.....MATRIX EQUATION FOR P ANDIOR J ARE COMPLETE, SOLVE EQUATIONS:

c

C

WHEN KKK $=0$, DECOMPOSE AND BACK-SUBSTITUTE,

WHEN KKK $=2$, BACK-SJBSTITUTE ONLY.

IHALFB $=$ NBHALF -1

IF (ML-1) 500J,5000.55J0

C..... SOLVE FOR P

$5000 K K K=300000$

CALL SOLVEB (KKK, PMAT, PVEC, NN, IHALFB, NN,NBI)

C...... SOLUTION NOW IN PVEL

IF (ML-1) 5500,600J.5500

C.....SOLVE FOR U

5500 KKK $=300000$

IF (NOUMAT) $5700,5700,5600$

$5500 \quad K K K=2$

5700 CALL SOLVEB (KKK, UMAT, UVEC,NN, IHALFB,NN,NBI)

C.....U SOLUTION NON IN UVEC

6000 CONTINUE

$c$
$c$
$c$
$c$
$c$
$c$
$c$
$c$
$c$
$c$
$c$

C...... CHECK PROGRESS ANJ CONVERGENCE OF ITERATIONS

C AND SET STOP AVD GJ FLAGS:

C ISTOD $=-1$

ISTOP $=0$

C ISTOP $=1$

C ISTOP $=2$

C IGOI $=0$

C IGOI $=1$

C IGOI $=2$
NOT CONVERSEO - STOP SIMULATION

ITERATIONS LEFT OR CONVERGED - KEEP SIMULATING LAST TIME STEP REACHED - STOP SIMULATION MAXIMUM TIYE REACTED - STOP SIMULATION $P$ ANO U ZONVERGEJ, OR NO ITERATIONS DONE JNLY P HAS NOT YET CONVERGED TO CRITERION JNLY U HAS NOT YET CONVERGED TO CRITERION BOTH P AND IJ HAVE NOT YET CONVERGEO TO CRITERIA $I G O I=3$

ISTOP $=0$

I SOI $=\mathcal{J}$

$32410 \ldots$ $32420 \ldots$ $82430 \ldots$ $82440 \ldots$ $32450 \ldots$ $82460 \ldots$ $32470 \ldots$ $82480 \ldots$ $82490 \ldots$ $32500 \ldots$ B2510... $82520 \ldots$ B2530... B2540... $82550 \ldots$ $82560 \ldots$ B2570... $82580 \ldots$ B2590... B2600... B2610... $82620 \ldots$ UB2630... B2640... B2650... B2660... $82670 \ldots$ B2680... B2690... $82700 \ldots$ $82710 \ldots$ B2720... B2730... B2740... B2750... B2760... B2770... B2780... B2790... B2800... $82810 \ldots$ B $2820 \ldots$ B2830... B2840... B2850... B2860... $32870 \ldots$ B2880... B2890... B? $2900 \ldots$ B2910... $92920 \ldots$ B2930... B2940... B2750... B2960... $B 2970 \ldots$ B2980... B2990... B3 3000 ... 


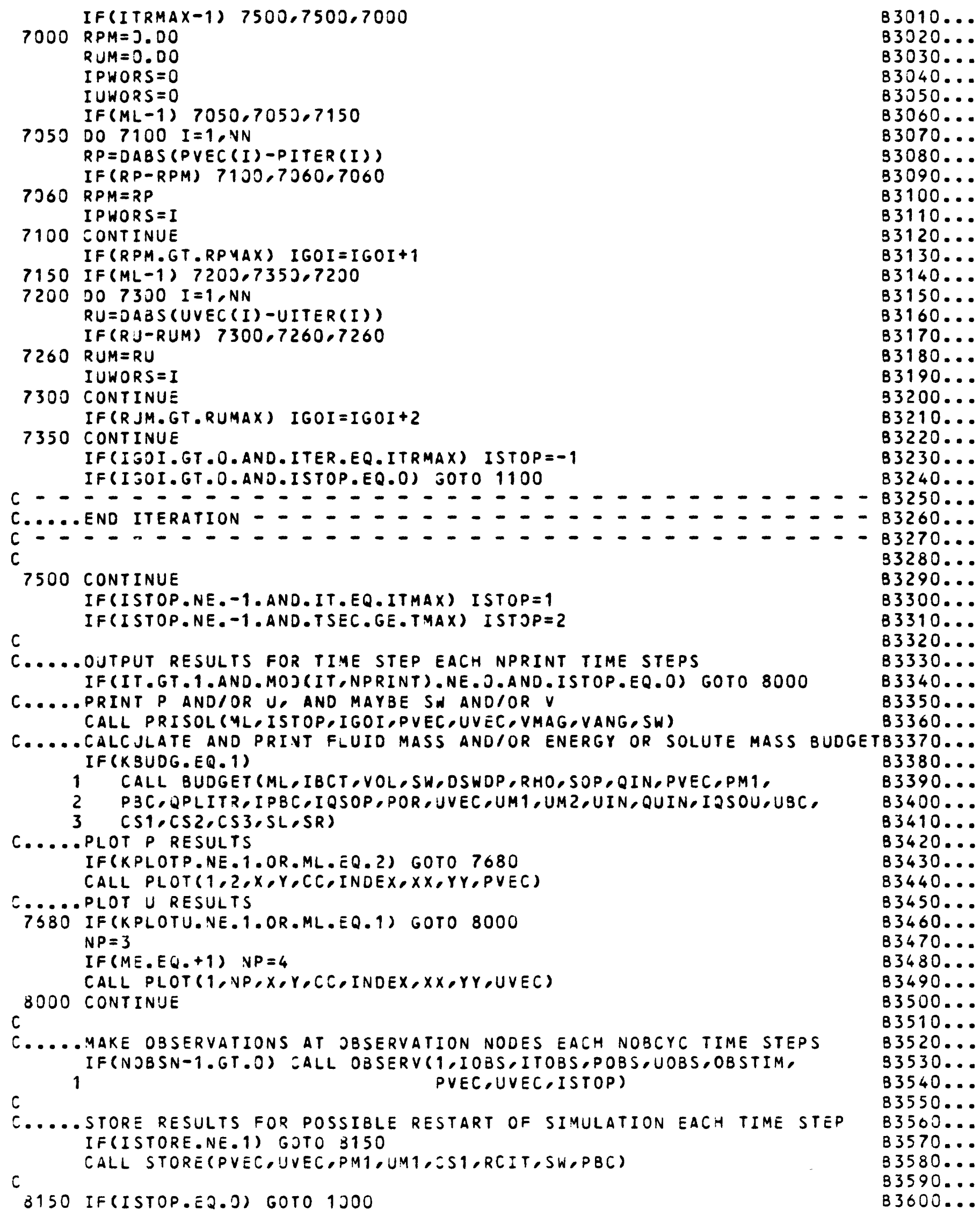

B3010... B3020... B3030... $83040 \ldots$ B3050... B3060... $83070 \ldots$ B3080... B3090... B3100... B $3110 \ldots$ B3120... B3130... B3140... B3150... B3160... B3170... B3180... B3190... B3200... B3210... B3220... B3230... $83240 \ldots$ B3250... $83260 \ldots$ $83270 \ldots$ B3280... B3290... B3300... B3310... B3320... B3330... B3340... B3350... B3360... B3380... B3390... B3400... B3410... B3420... $83430 \ldots$ $83440 \ldots$ B3450... B3460... B3470... B3480... B3490... B3500... B3510... B3520... B3530... B3540... $83550 \ldots$ B3500... B3570... B3580... B3590... B3600... 
c

c

C..... COMPLETE OUTPUT AND TERMINATE SIMULATION IF(ISTORE.EQ.1) WRITE 60,8100$)$

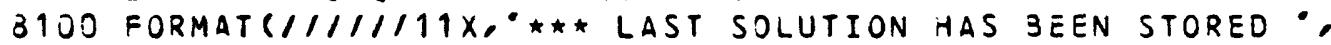
C 1 ON UNIT $66 \star 6^{\circ}$ )

C.....OJTPUT RESULTS OF OBSERVATIONS

8200 IF (NOBSN-1.GT.O) LALL OBSERV(2, IOBS, ITOBS,POBS,UOBS,OBSTIM, 1 PVEC,UVEC, ISTOP)

$C$

C.....OUTPUT END OF SIMJLATION MESSAGE AND RETURN TO MAIN FOR STOP IF(ISTOP.GT.O) GOTO 8400 IF (ISOI-2) $8230,8260,8290$

8230 WRITE $(6,8235)$

3235 FORMAT $/ / / / / / 111 \mathrm{X}$, - SIMULATION TERMIVATED DUE TO * 1 NON-CONVERGENT PRESSURE',

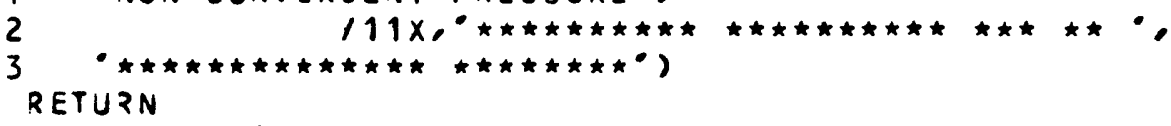
- of TIME PERIOJ*, 
c

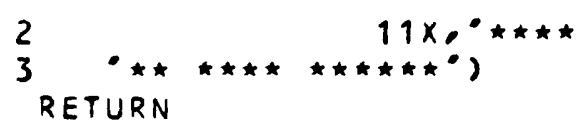

B4210... B4220... $84230 \ldots$ END B4240... $84250 \ldots$ 


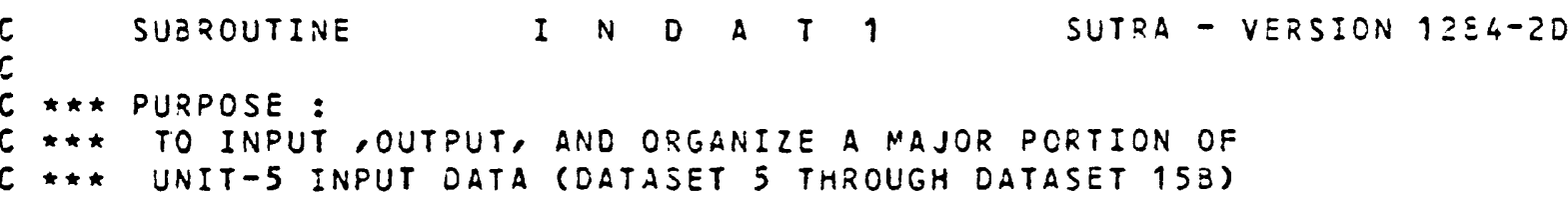


140 IF (NPCYC.EQ.1.OR.NUCYC.EQ.1) GCTO 160 WRITE $(6,150)$

150 FORMATC//11X, * * ERROR DETECTED: EITHER NPCYC OR ',

1 -NUCYC MUST EE SET TO $\left.1.0^{\circ}\right)$

INSTOP $=$ INSTOP-1

$1 S O$ CONTINUE

C..... SET MAXIMUM ALLOWEO TIME STEPS IN SIMULATION FOR

C

STSAOY-STATE FLOW AND STEACY-STATE TRANSPORT SCLUTION MCOES

IF(ISSFLO.EQ.1) THEN

NPCYC $=$ ITMAX+1

NUCYC $=1$

ENDIF

IF(ISSTRA.EQ.1) ITMAX $=1$

c

C..... INPUT DATASET 7: OUTPUT CONTROLS AND OPTIONS

READ (5,170) NPRINT, KNOOAL, KELMNT, KINCIO,KPLOTP, KPLOTU,KVEL, KBULG

170 FORMAT $(16 I 5)$

WRITE $(6,172)$ NPRINT

132 FORMAT(///11X, O U T P UT C ON T ROLS A N

1 OP T I O N S*/111X,I6,SX, PPRINTEO OUTPUT CYCLE ',

2 (IN TIME STEPS)

IF (KNOOAL.EQ.+1) WRITE $(6,174)$

IF (KNOOAL.EQ.0) WRITE $(6,175)$

174 FORMAT(/11X,- - PRINT NODE COORDINATES, 'THICKNESSES ANO",

1 POROSITIES )

175 FORMAT(/11X,- CANCEL PRINT OF NOOE COOROINATES, THICKNESSES ANO

1 - PCROSITIES )

IF (KELMNT.EQ.+1) WRITE $(6,176)$

IF(KELMNT.EQ.0) WRITE $(6,177)$

176 FORMAT $(11 X, \circ-$ PRINT ELEMENT PERMEAEILITIES AND OISPERSIVITIES $)$

177 FORMAT(11X," - CANCEL PRINT CF ELEMENT PERMEABILITIES AND",

1 OISPERSIVITIES $\left.S^{\circ}\right)$

IF (KINCIO.EQ.+1) WRITE $(6,178)$

IF(KINCID.EQ.0) WRITE $(6,179)$

178 FORMAT $111 \times,-$ - PRINT NOCE AND PINCH NOCE INCIOENCES IN EACH ', 1 'ELEMENT')

179 FORMAT(11X,- - CANCEL PRINT OF NODE AND PINCH NODE INCIDENCES,

1 'IN EACH ELEMENT')

IF (KPLOTP.EQ.+1) WRITE $(6,180)$

IF(KPLOTP.EQ.0) WIRITE $(6,181)$

180 FORMAT( $111 X, 0^{\circ}-$ PLCT PRESSURES ON EACH TIME STEP WITH OUTPUT')

181 FORMAT $\left(111 X, 0^{\circ}\right.$ - CANCEL PLOT OF PRESSURES')

$I M E=2$

$I F(M E \cdot E Q \cdot+1) \quad I M E=1$

IF(KPLOTU. EQ.+1) WRITE $(6,1 \Xi 2)$ UTYPE(IME)

IF(KPLOTU.EQ.0) WRITE $(6,183)$ UTYPE(IME)

132 FORMAT $\left(11 X, 0^{\circ}-\right.$ PLCT $, A 14,0^{\circ}$ ON EACH TIME STEP WITH OUTPUT')

$1 E 3$ FORMAT $(11 X, \circ$ - CANCEL PLOT OF ',A14)

IF (KVEL.EQ.+1) WRITE $(6,184)$

IF(KVEL.EQ.0) WRITE $(6,185)$

134 FORMAT $(111 \mathrm{X}, \circ$ - CALCULATE ANO PRINT VELOCITIES AT ELEMENT ',

1 -CENTRCIOS ON EACH TIME STEP HITH OUTPUT',

1 SS FORMAT $\left(/ 11 X^{\circ},-\right.$ CANCEL PRINT OF VELOCITIES')

IF(KBUDG.EQ.+1) WRITE(6,186) STYPE(IME)

IF (KBUCG.EG.0) WRITE $(6,187)$

186 FORMAT $/ 111 \times,-$ CALCULATE AND PRINT FLUID AND ',A6, BUDGETS,

1 ON EACH TIME STEP WITH OUTPUT')

C

187 FORMAT(/11X,"- CANCEL FRINT OF EUDGETS')

C..... INPUT DATASET 8: ITERATION CONTROLS

cs10....

$\cos 20 \ldots$

$\operatorname{cs} 30 \ldots$

$c \leqslant 40 \ldots$.

$\cos 5 \ldots$.

$c t 00 \ldots$.

C $570 \ldots$.

$\cos 0 \ldots$.

CSP0....

C>00....

$C>12 \ldots$

c? $? 20 \ldots$

c $730 \ldots$.

C $740 \ldots$.

C750....

c $760 \ldots$.

C>70....

C780....

C790....

$c \geq 00 \ldots$.

C $\varepsilon 10 \ldots$.

C $320 . .$.

C $330 \ldots$.

C $\equiv 40 \ldots$.

C350....

C860....

C $370 . .$.

C830....

C8.90....

C900....

c910....

c $920 \ldots$.

c $930 \ldots$.

C940....

c950....

c $950 \ldots$.

c $>>0 . .$.

С $¥ 80 . .$.

Сจ9०....

C100c...

c1010...

C1020...

c10 $10 . .$.

C1040...

C1C5C...

c1060...

C1070...

C1030...

c1090...

C1100...

c111c...

C1120...

c1130...

C1140...

C1150...

c1150...

c1170...

C11BC...

C119C...

C120C... 
c

196 CONTINUE

190 FORMAT $(10,2510.0)$

IF(ITRMAX-1) $192,192,194$

192 WRITE $(6,193)$

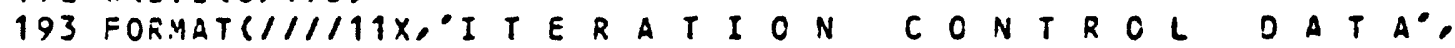
$1 / / 11 \times 0^{\circ}$ NON-ITERATIVE SOLUTION ${ }^{\circ}$ )

GOTO 196

194 WRITE $(6,195)$ ITRMAX,RPMAX,RUMAX

195 FORMAT(//I/11X, ITE R A T I C N C O NTR C L DATA*, $1 / / 11 X, I 15,5 x^{\circ}$ MAXIMUM NUMBER OF ITERATIONS FER TIME STEP०,

2 /11X,1P015.4,5X, ABSOLUTE CCNVERGENCE CRITERION FOR FLOH',

3 - SCLUTION*111X.1PO15.4.5X, ABSOLUTE CONVERGENCE CRITERICN',

4 - FOR TRANSPORT SOLUTION*)

C..... INPUT DATASET 9: FLUID PROPERTIES

REAO (5,200) COMPFL,CH,SIGMAW,RHOWO,URHOWC, ORWOU, VISCO

C.... INPUT DATASET 10: SOLID MATRIX PROPERTIES

READ (5,200) COMPMA,CS,SIGMAS,RHOS

200 FORMAT $(8610.0)$

IF (ME.EQ.+1)

1 WRITE $(6,210)$ COMPFL,COMPMA,CH,CS, VISCO,RHOS,RHOWO,ORWDU,URHCHO, 2 SIGMAH,SIGMAS

210 FORMAT(1H1////11X, ${ }^{\circ}$ C O N S T A N T P R O P E R T I E S OF',
1
- F L U I D
A $N D$
$\begin{array}{lllll}2 & 0 & L & I & 0\end{array}$
M A T R I $X$

2

$1 / 11 X, 1 P 015.4,5 x,{ }^{\circ}$ COMPRESSIEILITY OF FLUID*111X,1P015.4,5X,

- COMPRESSISILITY OF POROUS MATRIX*/111X,1P015.4,5X,

- SPECIFIC HEAT CAPACITY OF FLUID, $111 \mathrm{X}, 1$ PO15.4.5X,

- SPECIFIC HEAT CAPACITY OF SOLIO GRAIN*1/13X, "FLUID VISCOSITY"

- is CalCulated bY SUTRA as a function CF TEMPERATURe in "

-UNITS OF [Kg/(m*S)]*//11X.1P015.4,5X,*VISCC, CONVERSION *

- FACTOR FOR VISCOSITY UNITS, [OESIREd unitS] = VISCO* "

- $[\mathrm{kg} /(\mathrm{m} * \mathrm{~s})]^{*} / / 11 \mathrm{X}, 18015.4,5 x$. OENSITY OF A SOLID GRAIN*

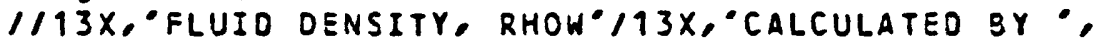

- SUTRA in terms OF TEMPERATURE, U, AS: $113 X, \circ$ RHOH $=$ RHOHO +

-ORHOU* (U-URHOWO $)^{\circ} / 111 X, 1$ PO15.4,5X."FLUID BASE DENSITY, RHOWO"

$111 X, 1 P 015.4,5 X,{ }^{\circ}$ COEFFICIENT OF DENSITY CHANUE WITH "

-TEMPERATURE, ORWDUं/11X,1PD15.4.5X."TEMPERATURE, URHOWO.",

- AT WHICh FLUID DENSITY is at BASE VALUE, RHOWO

/ $111 X, 1 P 015.4,5 X 0^{\circ}$ THERMAL CCNDUCTIVITY OF FLUID॰

$111 X, 1 P 015.4,5 \times$. THERMAL CONDUETIVITY OF SOLID GRAIN')

IF $(M E . E Q,-1)$

1 WRITE 6,220$)$ COMPFL,COMPMA,VISCC,RHOS,RHOWO, ORWDU,URHOWO, SIEMAWC 220 FORMAT 1 HI////11X, ${ }^{\circ}$ C O N S T A T P R O P E R T I E S O F ,

$1 . F L U I D$ A N D SOLLI D M A T R I $X^{\circ}$

$2 / 111 \times, 1 P 015.4,5 x, \circ$ COMPRESSIEILITY OF FLUIO० $/ 11 X, 1 P 015.4,5 x$,

3 'COMPRESSIBILITY OF POROUS MATRIX"

4 /111X,1P015.4,5X, 'FLUID VISCOSITY*

$4 / 111 x, 1 P 015,4,5 x, \circ$ DENSITY OF A SOLID GRAIN*

$5 / 113 X, \circ$ FLUID DENSITY, RHOW'/13X, CALCULATED

6 SUTRA IN TERMS OF SOLUTE CONCENTRATION, U. AS:",

$7113 \times,{ }^{\circ}$ RHOW $=$ RHOWO + CRWCU* (U-URHOWO)

$8 \% / 11 X, 1 P 015.4,5 X, \circ F L U I D$ BASE DENSITY, RHOWO

$9 \quad 111 x, 1$ PD $15.4,5 x, \circ$ COEFFICIENT OF DENSITY CHANGE WITH ${ }^{\circ}$,

* "SOlUTE CONCENTRATION. CRWOU"

1 /11X.1PD15.4.5X."SOLUTE CONCENTRATION, URHOWO. "

4 -AT WHICH FLUID DENSITY IS AT BASE VALUE, RHOWO"

C

5 / /11X,1PD15.4,5X, MCLECULAR CIFFUSIVITY OF SOLUTE IN FLUID')

C..... INPUT DATASET 11: ADSORPTION PARAMETERS
C1210...

C1 $1220 \ldots$

C1230...

C1240...

c1350...

C12 $12 \ldots$

C127C...

C123C...

C1270...

C1300...

C1310...

C1320...

C1330...

C1340...

C135C...

c1350...

C1370...

C1380...

C1390...

C1400...

c1410...

C1420...

C1430...

C144C...

C1450...

C1450...

C1470...

C1430...

C1490...

C150C...

C1510...

C1520...

C1530...

C1540...

C1550...

C1560...

C157c...

C158C...

C159C...

C1500...

C1610...

C1620...

WC1630...

C1640...

c1550...

c1660...

C1570...

C1630...

c1690...

C1700...

C1710...

c1720...

C1730...

c1740...

C1750...

C1750...

C1770...

C1730...

C1790...

C150C... 
$R E A D(5,230) \quad \triangle D S M O D, C H I 1, C H I 2$

23C FORMAT $(A 10,2610.0)$

IF (ME.EQ.+1) GOTO 248

IF (AOSMOD.EQ.'NONE

WRITE $(6,232)$ ADSMOD

232 FORMATC//111X, A D S OR P T I O N PARAMETER S

$1 /\left(16 X, A 10,5 X,{ }^{\circ}\right.$ EQUILIERIUM SCRPTION ISOTHERM')

GOTO 236

234 WRITE $(6,235)$

235 FORMAT(///11X, A D S OR P TION PARAMETER $S^{\circ}$

1 (/16X, "NON-SORBING SOLUTE')

236 IF( (ADSMOD.EQ. NNONE ').OR. (ADSMOD.EQ. 'LINEAR ').OR.

1 (2DSMOD.EQ. 'FRELNOLICH').OR.(ADSMOD.EQ. LANGMUIR ')) GOTO 238 WRITE $(6,237)$

237 FORMATC/111X, * * * ERROR DETECTED: TYPE OF SORPTION MOOEL,

1 - IS NOT SPECIFIEO CORRECTLY. $111 X,{ }^{\circ}$ CHECK FOR TYPE ANO,

2 -SPELLING, ANO THAT TYPE IS LEFT-JUSTIFIED IN INPUT FIELO०)

INSTOP $=$ INSTOP-1

238 IF(ADSMOD.EQ. LINEAR P) WRITE(6.242) CHI1

242 FORMAT $11 \mathrm{X}, 1$ PO15.4,5X, LINEAR DISTRIEUTION COEFFICIENT')

IF(ADSMOD.EQ. 'FREUNDLICH') WRITE $(6,244)$ CHI1,CHI2

244 FORMAT $(11 X, 1$ PD $15.4,5 X$, FREUNDLICH DISTRIBUTION COEFFICIENT'

$1 / 11 X, 1 P D 15,4,5 x, \circ$ SECOND FREUNDLICH COEFFICIENT')

IF(ADSMOD.EQ.'FREUNDLICH'.AND.CHI2.LE.0'.DO) THEN WRITE $(6,245)$

245 FORMAT $111 \times, *$ * * * ERROR DETECTED : SECCND COEFFICIENT $\bullet$

1 MUUST BE GREATER THAN ZER0\%)

INSTOP $=$ INSTOP-1

ENDIF

IF(ADSMOD.EQ. LANGMUIR ') WRITE(5,246) CHI1,CHI2

246 FORMAT $11 \mathrm{X}, 1$ PO15.4.5X, LANGMUIR CISTRISUTICN CCEFFICIENT"

C

$1111 x, 1$ PO15.4,5X, SECOND LANGMUIR (OEFFICIENT')

C..... INPUT DATASET 12: PRODUCTICN OF ENERGY OR SCLUTE MASS

243 READ (5,200) PRODFO,PRODSO,PRODF1,PROJS1

IF(ME.EQ.-1) WRITE(6,250) PRODFO,PROOSO,PROOF1,PROOS1

250 FORMATC///11X, P R O D U C T I O N A N D DECA Y OF

1 'S P E C I ES MAS S / /13X, PROOUCTION RATE (+) $113 X$,

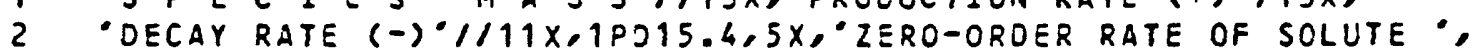

3 -MASS PROOUCTION/OECAY IN FLUIC $/ 11 \mathrm{X}, 1$ FC15.4.5X,

4 ZERO-ORDER RATE OF ADSCRBATE MASS PROCUCTION/DECAY IN",

5 -IMMOZILE PHASE*111X,1PD15.4,5X, FIRST-ORDER RATE CF SOLUTE ',

3 -MASS PRODUCTION/OECAY IN FLUIO /11X,1PD15.4,5X,

4 -FIRST-ORDER RATE OF AOSCRBATE MASS PRODUCTION/DECAY IN *

- IMMOSILE PHASE')

IF (ME.EQ.+1) WRITE(6, 260) PROCFC,PROOSO

250 FORMATI/IIIIX, P R O OUCTION ANO LOSS OF

1 -E N E R G Y /113X, PRODUCTICN RATE $(+) \cdot 113 X$,

2 -LOSS RATE (-) \%111X,1PC15.4,5X, ZERO-ORDER RATE OF ENERGY -,

3 PPRCOUCTION/LOSS IN FLUIO /11X,1PO15.4,5X,

4 IERO-OROER RATE OF ENERGY FROZUCTION/LOSS IN",

5 'SOLIO GRAINS',

C..... SET' PARAMETER SWITCHES FOR EITHER ENERGY OR SOLUTE TRANSPORT IF (ME) $272,272,274$

C FOR SOLUTE TRANSPORT:

$272 C S=0.000$

$C H=1.0 C O$

SIGMAS $=0.000$

GOTO 278

FOR ENERGY TRANSPORT:

C1 $1510 \ldots$

C1820...

C1830...

C1840...

C1250...

c1350...

C187C...

C158C...

C1E90...

c1900...

C191C...

C1920...

C1930...

C194C...

C1950...

C196C...

C1970...

C1980...

C1990...

C2000...

C201C...

C2020...

C203C...

C204C...

C205C...

C2060...

C207C...

C2020...

C209C...

C210C...

C211C...

C 212 ...

C2130...

C2140...

C215C...

C215C...

, c2170...

$c \geq 180 \ldots$

C2190...

C2200...

C2210...

C222C...

C2230...

C2240...

C2250...

C225C...

C227c...

c2230...

C2200...

C230C...

C $2310 . .$.

C232C...

C2330...

C2340...

C2350...

C2 $360 \ldots$

C $237 \mathrm{C} \ldots$

C2380...

C2390...

C2400... 


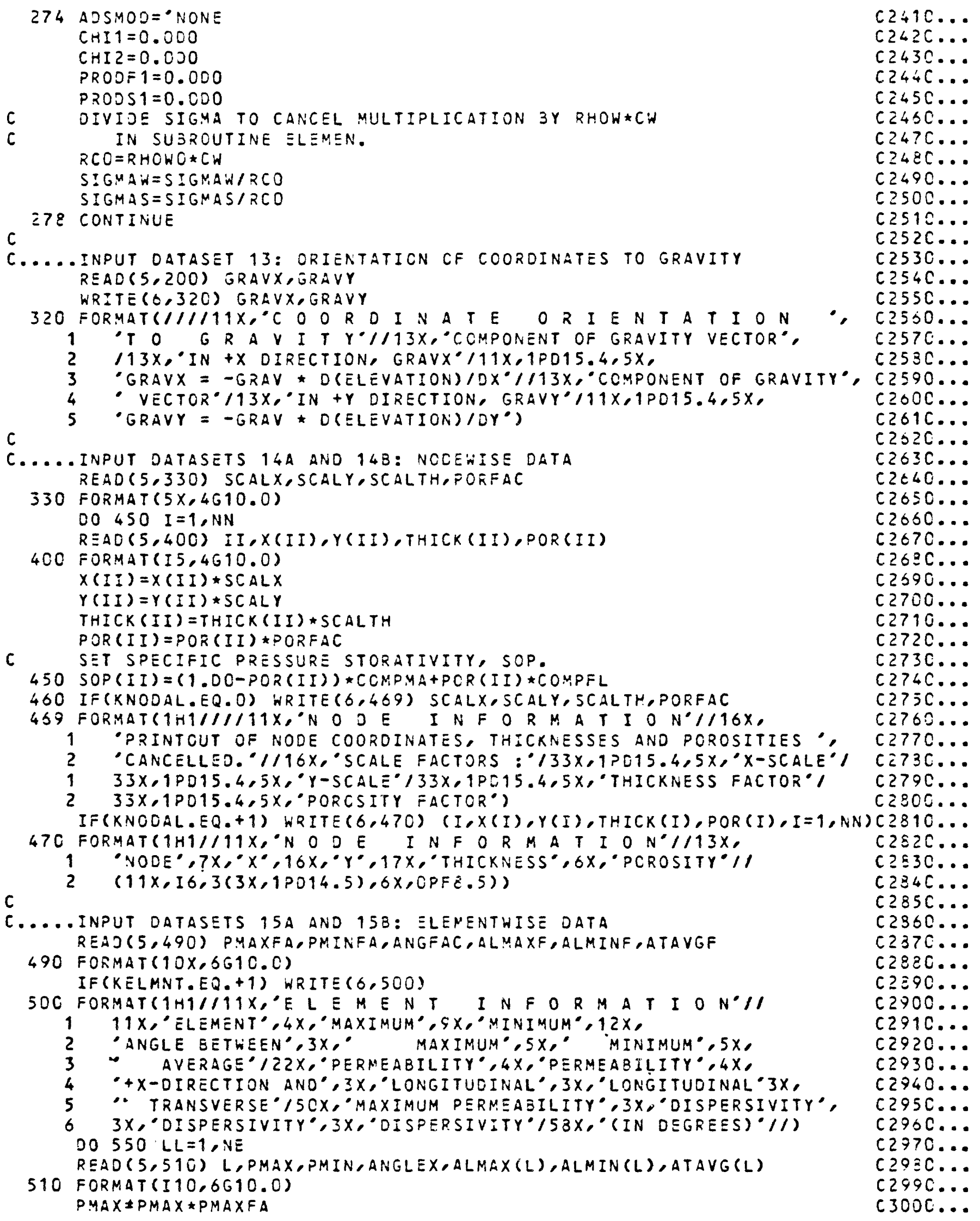


c 520 FORMAT $(11 X, I 7,2 X, 2(1$ PO14.5,2X),8X,4(OPF10,3,5X))

C.....ROTATE PERMEABILITY FROM MAXIMUMIMINIMUM TO X/Y DIRECTIONS RADIAX $=1.7453290-2 \times A N G L E X$

SINA = DSIN (RADIAX)

$\operatorname{COS} A=D \operatorname{COS}(R A D I A X)$

SINA $=$ SINA* SINA

$\operatorname{CCSA2}=\operatorname{COS} A * \operatorname{COS} A$

$P E R M X X(L)=P M A X * \operatorname{COSAZ}+P M I N * S I N A Z$

PERMYY $(L)=P M A X \star S I N A Z+P M I N * C O S A Z$

PERMXY $(L)=(P M A X-P M I N) \star S I N A \star C O S A$

PERMYX $(L)=P E R M X Y(L)$

PANGLE(L) $=$ RADIAX

550 CONTINUE

IF (KELMNT.EQ.O)

1 WRITE 6,569$)$ PMAXFA,PMINFA, ANGFAC, ALMAXF, ALMINF, ATAVGF

569 FORMAT $/ / / 111 X, 0^{\circ} E L E M E N T$ I N F O R M A T I O N $/ 1$

$116 X$, PRINTOUT OF ELEMENT PERMEABILITIES AND DISPERSIVITIES ',

2 -CANCELLED. $/ 116 \mathrm{X}$, SCALE FACTORS:"/33X,1P015.4,5X, MAXIMUM",

1 PPERMEABILITY FACTOR'/33X,1PO15.4\%,5X, MINIMLM PERMEASILITY "

2 'FACTOR'/33X,1PD15.4,5X, ANGLE FROM +X TO MAXIMUM OIRECTION",

3 FACTOR०133X,1PD15.4,5X, MAXIMUM LONGITUDINAL OISPERSIVITY*,

4 - FACTOR'133X,1PD15.4,5X, -MINIMUM LONGITUOINAL OISPERSIVITY",

C

5 - FACTOR' $133 X, 1$ PD15.4,5X, ${ }^{\circ}$ TRANSVERSE OISPERSIVITY FACTCR $\left.{ }^{\circ}\right)$

C.....ENO SIMULATION FOR CORRECTIONS TO UNIT-S DATA IF NECESSARY IF(INSTOP.EQ.O) GOTO 1000

WRITE $(6,999)$

999 FORMAT $/ / / / / / 111$, PLEASE CORRECT INPUT DATA ANO RERUN.",

1

2

ENDFILE(6)

$1 / 122 x$, S I M U L A T I O N

STOP

$c$

c

1000 RETURN END
C3010... C302C... C $3030 \ldots$ c $3040 \ldots$ C305C... C 3 CSC... C $3070 \ldots$ c $3030 . .$. c309C... c $3100 \ldots$ C $3110 \ldots$ C $312 C \ldots$ c $3130 \ldots$ C $3140 \ldots$ C $3150 \ldots$ C315C... C 317 C... C $3180 \ldots$ C $3190 \ldots$ c $3200 \ldots$ C $3210 \ldots$ C $5220 \ldots$ C $3236 \ldots$ C $3240 \ldots$ C $3250 \ldots$ C $3260 \ldots$ C $3270 \ldots$ C $3280 . .$. C $3290 \ldots$ C $3300 \ldots$ c $3310 .$. c $3320 \ldots$ c $3330 \ldots$ c $3340 \ldots$ C $3350 \ldots$ c $3360 .$. C $337 C \ldots$ C $3380 . .$. C $3390 \ldots$ c $3400 \ldots$ C $3410 \ldots$ C $3420 \ldots$ c $3430 \ldots$ c $3440 \ldots$ 
SUBRJUTINE PLOT (ICALL,NP, X,Y,CC,INOEX, XX,YY,CVEC)

IMPLICIT DOUSLE PRECISION $(A-H, O-Z)$

COMMON/KPRINT/ KNODAL, KELMNT, KINCID,KPLOTP, KPLOTU,KVEL,KBUOG

COMMON/CONTRL/ GVJ,UP,DTMULT, JTMAX,ME, ISSFLO,ISSTRA, ITCYC,

1 NPCYC,NUCYC,NPRINT, IREAD, ISTORE, NOUMAT, IUNSAT

COMMON/OIMS/ NN,NE,NIN,NBI,NG,NBHALF,NPIVCH,NPBC,NUBC,

1 NSOP,NSOU,NBCN

CHARACTER*1 PRNT $(122)$, SYM(17), BLANK (60)

DOUBLE PRECISION NX(500), NY(14)

CHARACTER 4 OIGIT $(82), V F 1(6), V F 2(6), V F 3(7)$

DIMEVSION $K(10), N(10)$

CHARACTER 30 TITLE $(1,4)$

OIMENSION $X(N N), Y(N N), C C(N N), X X(N N), Y Y(N N), I N D E X(N N), C V E C(N N)$

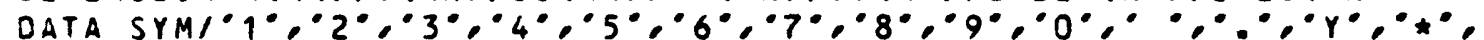

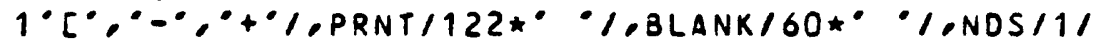

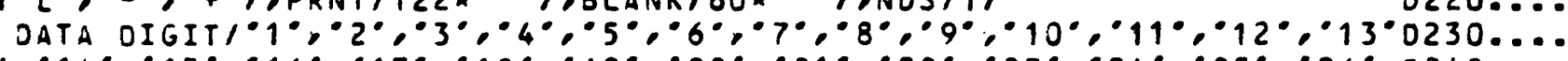

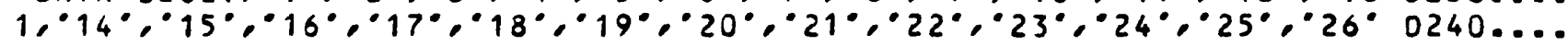
$20^{\circ} 27^{\circ}, 28^{\circ}, 29^{\circ}, 30^{\circ}, 31^{\circ} \cdot 32^{\circ}, 33^{\circ} \cdot 34^{\circ}, 35^{\circ}, 36^{\circ} \cdot 37^{\circ}, 38^{\circ},-39^{\circ}, 0250 \ldots \ldots$ $2^{\circ} 40^{\circ}, \quad \cdot 41^{\circ}, 82^{\circ}, 83^{\circ}, 84^{\circ}, 8^{\circ}, \cdot 85^{\circ}, \cdot 87^{\circ}, 8^{\circ} 88^{\circ}, 89^{\circ}, 90^{\circ}, \cdot 91^{\circ}, 0260 \ldots \ldots$

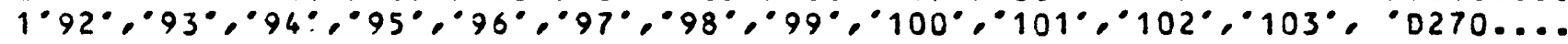

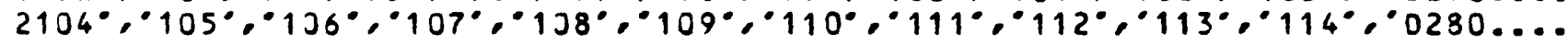
$3115^{\circ}, \cdot 116^{\circ}, 117^{\circ}, 118^{\circ}, \circ 119^{\circ}, \cdot 120^{\circ}, \cdot 121^{\circ}, 122^{\circ}$,

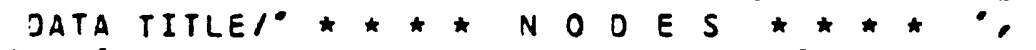

$1 * *$ * PRESSJRE/PBASE * * *

2 * * CONCENTRATION/CBASE * * *

3 - * teMPERATURE/TBASE * * " 1

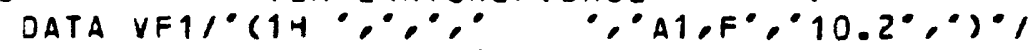

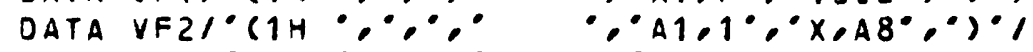

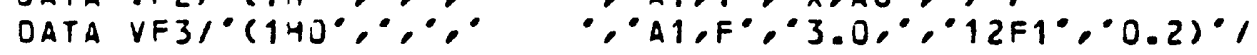

$c$

IF(ICALL) $1100,1130,1$

C.....READ PLOT SETUP DATA (DATASET 16)

1100 READ $(5,1200)$ IOIREC,NLINPI,NCHAPI,NCHAPL

1200 FORMAT (4I5)

PLTWID = (DOLE (NCHAPL) -13.000$) / D B L E(N C H A P I)$

$N 1=N L I N P I$

$N 2=N C H A P I$

$N 3=N C H A P L$

$X N 1=1.00 /(2.00 * N 1)$

$N \times S=1$

$N Y S=1$

$N I N Y=P L T W I D$

c

$K(1)=N N$

IF(KPLOTP.NE.1) GJTO 1400

REAJ 5,1300$)$ PBASE

1300 FORMAT (013.0)

1400 IF(KPLOTU.NE.1) GOTO 1500

READ 5,1300$)$ U3ASE

1500 CONTINUE

WRITE (6,1520) IOIREC, VLINPI , NCHAPI, NEHAPL

1520 FORMATC///11X, P L OT I N F ORMATION II

$0290 \ldots$.

$0300 \ldots$.

$0310 \ldots$.

$0320 \ldots .$.

$0330 \ldots . .$.

$0340 \ldots$.

$0350 \ldots$.

$0360 \ldots$.

$0370 \ldots$

$0380 \ldots$.

$0390 \ldots$.

$0400 \ldots .$.

$0410 \ldots$

$0420 \ldots$

$0430 \ldots$.

$0440 \ldots$.

$0450 \ldots$.

$0460 \ldots .$.

$0470 \ldots .$.

$0430 \ldots$.

$0490 \ldots$.

$0500 \ldots$.

$0510 \ldots$.

$0520 \ldots$.

$0530 \ldots$.

$0540 \ldots$.

$0550 \ldots$.

$0560 \ldots$.

$0570 \ldots$.

$0580 \ldots$.

$0590 \ldots$.

$0600 \ldots$. 
$113 \times, \circ P L O T$ ORIEVTATION'1

2 I $15,5 X, \bullet I O I R E C \ldots \ldots=-1$ SMALL PLOT ACROSS PAPER, $=+1 \bullet$

3 -LARGE PLOT ALJNG PAPER*//13x. "LINE PRINTER CHARACTERISTICS*/

4 11X,I15,5X, NUMBER OF OUTPUT,

5 -LINES PER INCH $/ 11 X$, I 15,5X, "NUMBER OF OUTPUT CHARACTERS

6 - PER INCH'111X,I15,5X,"MAXIMUM NUMBER OF OUTPUT'"

7 : EHARACTERS PER LINE')

IF(KPLOTP.NE.1) GOTO 1540

WRITE $(6,1530)$ PBASE

1530 FORMAT //13X,"PRESSURE PLOT DATA*/11X,1P015.4,5X,

1 -PBASE.....PLOTTED PRESSURE VALUE IS PRESSURE/PBASE ${ }^{\circ}$ )

1540 IF (KPLOTU.NE.1) GJTO 1580

IF (ME) $1550,1550,1560$

1550 WRITE $(6,1555)$ UBASE

1555 FORMATC//13X, CONCENTRATION PLOT OATA*/11X,1PO15.4,5X,

1 -JBASE.... PLOTTED CONCENTRATION VALUE IS CONCENTRATION/UBASE*) GOTO 1580

1560 WRITE $(6,1565)$ UBASE

1565 FORMAT $/ / 13 \mathrm{X}, \circ$ TEMPERATURE PLOT DATA /11 X, 1PD15.4,5X,

1 -UBASE.... PLOTTED TEMPERATURE VALUE IS TEMPERATURE/UBASE")

1580 WRITE $(6,1590)$

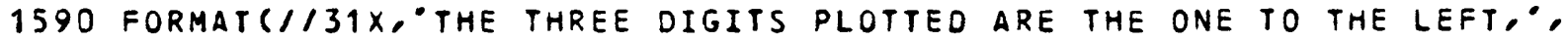
$c$

1 /31X, AND THE TWO TO THE RIGHT OF THE DE(IMAL POINT')

C..... SET LONGER PLOT AXIS DOWN (IDIREC $=+1$ )

c

SMALLX $=0.00$

OR AIROSS PAPER (IDIREC $=-1$ )

SMALLY $=0.00$

$B I G X=0 . D O$

$B I G Y=0.00$

DO $1600 \quad I=1, N N$

$I F(X(I) . G T . B I G X) \quad 3 I G X=X(I)$

IF $(X(I), L T$. SMALL $X)$ SMALL $X=X(I)$

IF $(Y(I) . G T$. BIGY) BIGY=Y(I)

1600 IF $(Y(I) . L T$. SMALLY) SMALLY $=Y(I)$

XRANGE = BIGX-SMALLX

YRANJE = BIJY-SMALLY

TENT $+X=X R A N G E / 10$. JDO

TENT +Y $=$ YRANGE/10. JDO

IF (XRANGE.GE.YRANSE.AVD. IDIREC.NE. - 1) KKKKK $=+1$

IF ( XRANGE.GE.YRANSE.AVD. IDIREC.EQ.-1) KKKKK $=-1$

IF (XRANGE.LT.YRANJE.AVO.IJIREG.NE. - 1) KKKKK $=-1$

IF (XRANGE.LT.YRANJE.AND. IDIREC.EQ.-1) KKKKK =+1

IF (KKKKK.EQ.-1) GJTO 344

XMIN $=$ SMALLX-TENTHX

$X M A X=B I G X+T E N T H X$

YMIN $=$ SMALLY $-T E N T H Y$

C YMAX $=B I G Y+T E N T H Y$

GOTO 345

344 XMIN $=$ SMALLY TENTHY

XMAX $=B I G Y+T E V T H Y$

YMIN = SMALLX-TENTHX

C

YMAX $=8$ I GX +TENTHX

345 CONTINUE

XRANGE = XRANGE*1.2J0O

YRANSE $=$ YRANGE*1.2JOO

IF $(K K K K K . E Q .+1)$ NINX $=(N I N Y / Y R A N G E) \star X R A N G E+0.5000$

IF $(K K K K K . E Q .-1) \quad N I N X=(N I N Y / X R A N G E) \star Y R A N G E+0.5 J D O$

$0610 \ldots$.

$0620 \ldots$.

$0630 \ldots$.

$0640 \ldots$

$0650 \ldots$

D060....

$0670 \ldots$.

$0080 \ldots$

D690....

$0700 \ldots$.

$0710 \ldots$.

$0720 \ldots$.

$0730 \ldots$.

$0740 \ldots$

$0750 \ldots$

$0760 \ldots$

$0770 \ldots$

0780....

$0790 \ldots$.

$0800 \ldots .$.

D810....

$0820 \ldots$

$0830 \ldots$

$0840 \ldots$.

$0850 \ldots$.

$0860 \ldots$.

D870....

D880....

$0890 \ldots$.

$0900 \ldots$.

D910....

$0920 \ldots$.

D930....

D940....

$0950 \ldots$.

$0960 \ldots$.

$0970 \ldots$.

$0980 \ldots$.

0990....

$01000 \ldots$

$01010 \ldots$

$01020 \ldots$

D1030...

$01040 \ldots$

$01050 \ldots$

$01060 \ldots$

$01370 \ldots$

$01080 \ldots$

$01090 \ldots$

01100...

D1110...

$01120 \ldots$

$01130 \ldots$

$01140 \ldots$

$01150 \ldots$

$01160 \ldots$

01170...

$01180 \ldots$

$01190 \ldots$

$01200 \ldots$ 
IF $(K K K K K . E 2 .-1)$ SOTO 361

$00352 \quad I=1, N V$

$X X(I)=X(I)$

$Y Y(I)=Y(I)$

362 INDEX $(I)=I$ SOTO 368

$36130353 \quad I=1, N \mathrm{NV}$

$X X(I)=+Y(I)$

$Y Y(I)=+X(I)$

C NOTE THAT THE SIGV OF YY IS REVERSEO LATER

C

363 IN OROER TO

c

368 CONTINUE

$c$

C.... INITIALIZE VARIABLES

$N \times D=V X S=N I N X$

NYO $=$ NYS $\#$ NINY

IF $(N X D . G E .((N Y D+1) / 2))$ GOTO 11

$N I N X=1+((N Y D-1) /(2 \star N X S))$

$N \times D=V \times S * N I N X$

$11 \times S F=X R A N G E / N \times D$

$Y S F=Y R A N G E / N Y D$

IF (KKKKK.EQ.+1) GOTO 12

$X S F=Y R A N G E / N X D$

12 CONTINUE

$Y S F=X R A N G E / N Y D$

$N 4=N \times D \star N 1+1$

$N 5=N \times D+1$

$N G=N Y D+1$

$N 7=N 1 * N I N X$

$N 8=N 2 * N Y O+1$

$N 9=N 2 \star N I N Y$

$N R=N 8-1$

$N A=N 4 / 2-2$

$N B B=N 4 / 2+4$

$N C=(N 3-N 8-10) / 2$

$N D=N C+N 8$

$N E E=Y A X O(N 5, N 6)$

$V F 1(3)=0 I G I T(N D-40)$

$\operatorname{VF} 2(3)=0 \operatorname{IGIT}(N O-4)$

$\operatorname{VFZ}(3)=$ IIGIT (NC)

C.....ARRAVGe EACH DATA SET IN DESCENDING VAlues of $X$ DO $9 J L=1$, NDS

$N N N=R(L)$

$003 J \quad I=1, N N N$

$B I G=X X(I)$

\section{$K K=I$}

DO $2 J \mathrm{~J}=I, N N N$

$I F(X X(J) . G T \cdot 3 I G)$ jo TO 15

GO TO 20

$15 B I G=X X(J)$

$K K=J$

20 CONTINUE

TEMPI $=Y Y(I)$

TEMPII $=X X(I)$

TEMP $3=$ INDEX $(I)$

$Y Y(I)=Y Y(K K)$

$X X(I)=X X(K K)$

INDEX $(I)=$ INDEX $(K K)$

$01210 \ldots$

$01220 \ldots$

$01230 \ldots$

$01240 \ldots$

$01250 \ldots$

$01260 \ldots$

$01270 \ldots$

$01280 \ldots$

$01290 \ldots$

$01300 \ldots$

$01310 \ldots$

$01320 \ldots$

$01330 \ldots$

$01340 \ldots$

$01350 \ldots$

$01360 \ldots$

$01370 \ldots$

$01380 \ldots$

$01390 \ldots$

$01400 \ldots$

$01410 \ldots$.

$01420 \ldots$

$01430 \ldots$

$01440 \ldots$

$01450 \ldots$

$01460 \ldots$

$01470 \ldots$

$01480 \ldots$

$01490 \ldots$

$01500 \ldots$

$01510 \ldots$

$01520 \ldots$

$01530 \ldots$

$01540 \ldots$

$01550 \ldots$

$01560 \ldots$

$01570 \ldots$

$01580 \ldots$

$01590 \ldots$

$01600 \ldots$

$01610 \ldots$

$01620 \ldots$

$01630 \ldots$

$01640 \ldots$

$01650 \ldots$

$01660 \ldots$

$01670 \ldots$

$01680 \ldots$

$01690 \ldots$

$01700 \ldots$

$01710 \ldots$

$01720 \ldots$

$01730 \ldots$

$01740 \ldots$

$01750 \ldots$

$01760 \ldots$

$01770 \ldots$

$01780 \ldots$

$01790 \ldots$

$01800 \ldots$ 
C

INDEX $(K K)=$ TEMP3

$Y Y(K K)=T E M P I$

$30 \times X(K K)=$ TEMPII

90 CONTINUE

C..... COMPJTE LABEL NUMBERS FOR X AND Y AXES

DO 1 J $I=1, N E E$

$N N X=V 5-I$

$N N Y=N O-I$

IF (NNY.LT.O) 60 TO 95

$N Y(I)=Y S F \star N N Y+Y M I N$

IF $(K K K K K \cdot E Q \cdot-1) \quad N Y(I)=Y M I N+(I-1) \star Y S F$

95 IF $(N V X . L T .0)$ :0 TJ 100

$N X(I)=X S F \star N N X+X M I N$

C

100 CONTINUE

C....SET JP PLOT OF MESH

OO $105 \quad I=1, N N$

c

$105 \operatorname{CVEC}(I)=I \star 00.010000$

C

C

C..... ENTRY FOR PRESSURE ANJ CONCENTRATION OR TEMPERATURE PLOTS

C.......-. - - -

1 CONTINUE

C.......-....-

C.... nOR yalile variable to be plotteo

CCNORM $=1.000$

IF (NP.EQ.2) CCNORY =PBASE

IF (NP.GT.2) CCNORM=UBASE

DO $2 I=1, N N$

$2 C C(I)=C V E C(I N D E X(I)) / C C N O R M$

c

C.....INITIALIZE VARIABLES

$Z=X M A X$

WRITE $(6,40)$

DO $15 \mathrm{I}=1$, NOS

$10 N(I)=1$

DO $210 \quad I=1, N 4$

C

C....LLCATE X AXES

IF (I.EQ.1.OR.I.EQ.N4) GO TO 110

DO $114 \mathrm{~J}=1, \mathrm{~N} 8, \mathrm{NQ}$

c

$114 \operatorname{PRNT}(J)=S Y M(15)$

C.... LOCATE Y AXES

IF $((I-1) / N 1 * N 1 . N E . I-1)$ GO TO 117

115 PRNT $(1)=S Y M(14)$

PRNT $(N 8)=S Y M(14)$

117 IF $((I-1) / N 7 \star N 7 . N E . I-1)$ GO TO 130

כ० $118 \quad J=2, N R$

$\operatorname{IF}((J-1) / N 9 * N 9 . E Q . J-1) P R N T(J)=S Y M(17)$

118 IF $((J-1) / N 9 \star N 9 . N E . J-1) P R N T(J)=S Y M(10)$

GO TO 130

110 DO $120 \mathrm{~J}=1, \mathrm{NB}$

IF $((J-1) / N 2 \star N 2 . E Q . J-1) \quad P R N T(J)=S Y M(14)$

$c$

120 IF $((J-1) / N 2 * N 2 . N E . J-1) \quad \operatorname{PRNT}(J)=\operatorname{SYM}(10)$

C..... COMPJTE LOCATION JF PJINTS
$01810 \ldots$

$01820 \ldots$

$01830 \ldots$

$01840 \ldots$

$01850 \ldots$

D1860...

$01870 \ldots$

$01880 \ldots$

$01890 \ldots$

$01900 \ldots$

$01910 \ldots$

$01920 \ldots$.

$01930 \ldots$.

$01940 \ldots$

$01950 \ldots$

$01960 \ldots$

$01970 \ldots$

$01980 \ldots$

$01990 \ldots$

$02000 \ldots$

$02010 \ldots$

D2020...

$02030 \ldots$

$02040 \ldots$

$02050 \ldots$

$02060 \ldots$

$02070 \ldots$

$02080 \ldots$

$02090 \ldots$

D2100...

D2110...

$02120 \ldots$

$02130 \ldots$

$02140 \ldots$

$02150 \ldots$

$02160 \ldots$

$02170 \ldots$

$02180 \ldots$

$02190 \ldots$

D2200...

D2210...

D2220...

D2230...

D2240...

D2250...

D2260...

$02270 \ldots$

$02280 \ldots$

$02290 \ldots$

D2300...

D2310...

$02320 \ldots$

$02330 \ldots$

D2340...

D2350...

D2360...

$02370 \ldots$

$02380 \ldots$

$02390 \ldots$

D2400... 
$13000150 \mathrm{~J}=1, \mathrm{NOS}$

135 IF ( $V(J) . \equiv Q . K(J)+1)$ GO TO 150

IF(I.ST.1) GO TO 137

$I F(X X(N(J)) \cdot .-E \cdot 2+X N 1 * X S F) \quad$ GO TO 137

$N(J)=N(J)+1$

GO TO 135

137 IF $(X X(N(J)) . L E . Z+X N 1 * X S F . A N J . X X(N(J)) . G E . Z-X N 1 * X S F)$ GO TO 140

GO TO 150

C $140 M=N R+0.500-((Y Y(N(J))-Y M I N) \star N 2) / Y S F$

140 DELYL $=$ $((Y Y(Y(J))-Y M I N) * N 2) / Y S F$

$M=N R+0.50 J-D E L Y=$

REVERSE SIGN OF YY (I.E. REVERSE PLOTTING DIRECTION) IF

GRAPH IS TO BE TRANSPJSEO....

IF $(K K K K K . E Q .-1) \quad M=0.500+D E L Y C$

C

IF(M.LT.O.OR.M.GT.NR) GO TO 145

IF(CC(N(J))) 142,146,147

142 IF (M.NE.O) PRNT $(M)=5 Y M(16)$

NUM $=(-C C(N(J))+.00500) * 10.00$

GO TO 141

$147 N U M=(C C(N(J))+0.00500) \star 100.00$

IF (NUM.GT.979) NUM=MJO(NUM, 1000)

141 IF(NUM.LT.100) GO TO 143

INDX $3=$ NUM $/ 10$ J

IF (M.NE.O.AND.CC (N(J)).GT.O.) PRNT (M) $=5 Y M(I N D \times 3)$

$N \cup M=N U M-I N D \times 3 * 100$

$143 I N D X 1=M O D(N \cup M, 10)$

IF (INOXI.EQ.O) INJXI $=10$

INOX $2=N U M / 10$

IF (INOX2.EQ.J) INOX2 $=10$

GO TO 144

146 IND $\times 1=14$

INDX $2=14$

144 PRNT $(M+1)=5 Y M(I N D \times 2)$

PRNT $(y+2)=S Y M(I N D \times 1)$

$145 N(J)=N(J)+1$

IF $(N(J) \cdot E Q \cdot K(J)+1)$ GJ TO 150

IF $(X X(N(J)), L E . Z+X N 1 * X S F . A N D . X X(N(J)), G E . Z-X N 1 * X S F)$ GO TO 140

c

150 CONTINUE

C......PRINT AXES,LABELS, AND POINTS

C IF (I-NA.EQ.O) GO TO 170

C IF (I-NBB.EQ.O) GO TO 180

IF $((I-1) / N 1 * N 1-(I-1)) \quad 190,160,190$

160 WRITE $(6, V F 1)(B L A N K(J), J=1, N C),(P R N T(J), J=1, N 8), N X(1+(I-1) / N 1)$ GO TO 200

C 170 WRITE $(6, V F 2)(B L A N K(J), J=1, N C),(P R N T(J), J=1, N 8)$

C GOTO 200

C 130 NRITE $(6, V F 2)(B L A N K(J), J=1, N C),(P R N T(J), J=1, N 8)$

C GO TO 200

190 WRITE $(6, V F 2)(B L A N K(J), J=1, N C),(P R N T(J), J=1, N 8)$

c

C..... COMPUTE NEW VALUE FOR $Z$ AND INITIALIZE PRNT

$200 Z=Z-2 . D O * X N 1 * X S F$

DO $210 \mathrm{~J}=1, \mathrm{~N} 8$

c

210 PRNT $(J)=S Y M(11)$

C..... NUMBER ANO LABEL Y AXIS ANO PRINT TITLE

WRITE $(6, V F 3)(B L A Y K(J), J=1, N C),(N Y(I), I=1, N 6)$

$02410 \ldots$

$02420 \ldots$

$02430 \ldots$

$02440 \ldots$

$02450 \ldots$

$02460 \ldots$

$02470 \ldots$

$02480 \ldots$

$02490 \ldots$

$02500 \ldots$

$02510 \ldots$

$02520 \ldots$

$02530 \ldots$

$02540 \ldots$

$02550 \ldots$

$02560 \ldots$

$02570 \ldots$

$02580 \ldots$

$02590 \ldots$.

$02600 \ldots$.

$02610 \ldots$

$02620 \ldots$

$02630 \ldots$.

$02640 \ldots$

$02650 \ldots$

$02660 \ldots$

$02670 \ldots$

$02680 \ldots$

$02690 \ldots$

$02700 \ldots$

$02710 \ldots$

$02720 \ldots$

$02730 \ldots$

$02740 \ldots$

$02750 \ldots$

$02760 \ldots$

$02770 \ldots$

$02780 \ldots$.

$02790 \ldots$

$02800 \ldots$

$02810 \ldots$

$02820 \ldots$

$02830 \ldots$

$02840 \ldots$

$02850 \ldots$

$02860 \ldots$

$02870 \ldots$

$02880 \ldots$

$02890 \ldots$

$02900 \ldots$

$02910 \ldots$

$02920 \ldots$

$02930 \ldots$

$02940 \ldots$.

$02950 \ldots$

$02960 \ldots$

$02970 \ldots$

$02980 \ldots$

$02990 \ldots$

$03000 \ldots$ 
C SUBRTUTINE

$P \quad L \quad O \quad T$

SUTRA - VERSION 1284-20 010...

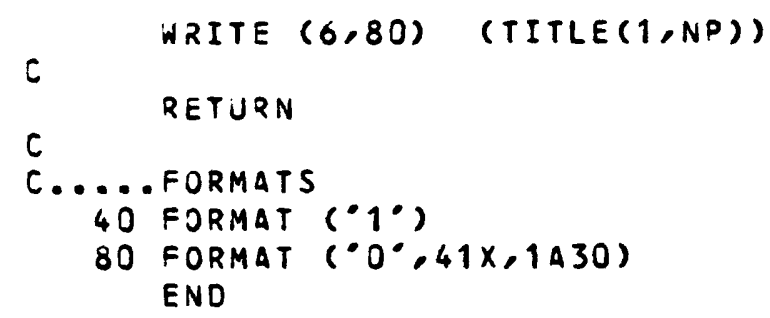

$03010 \ldots$

$03020 \ldots$ $03030 \ldots$ $03040 \ldots$ $03050 \ldots$ $03060 \ldots$ $03070 \ldots$ $03080 \ldots$ 


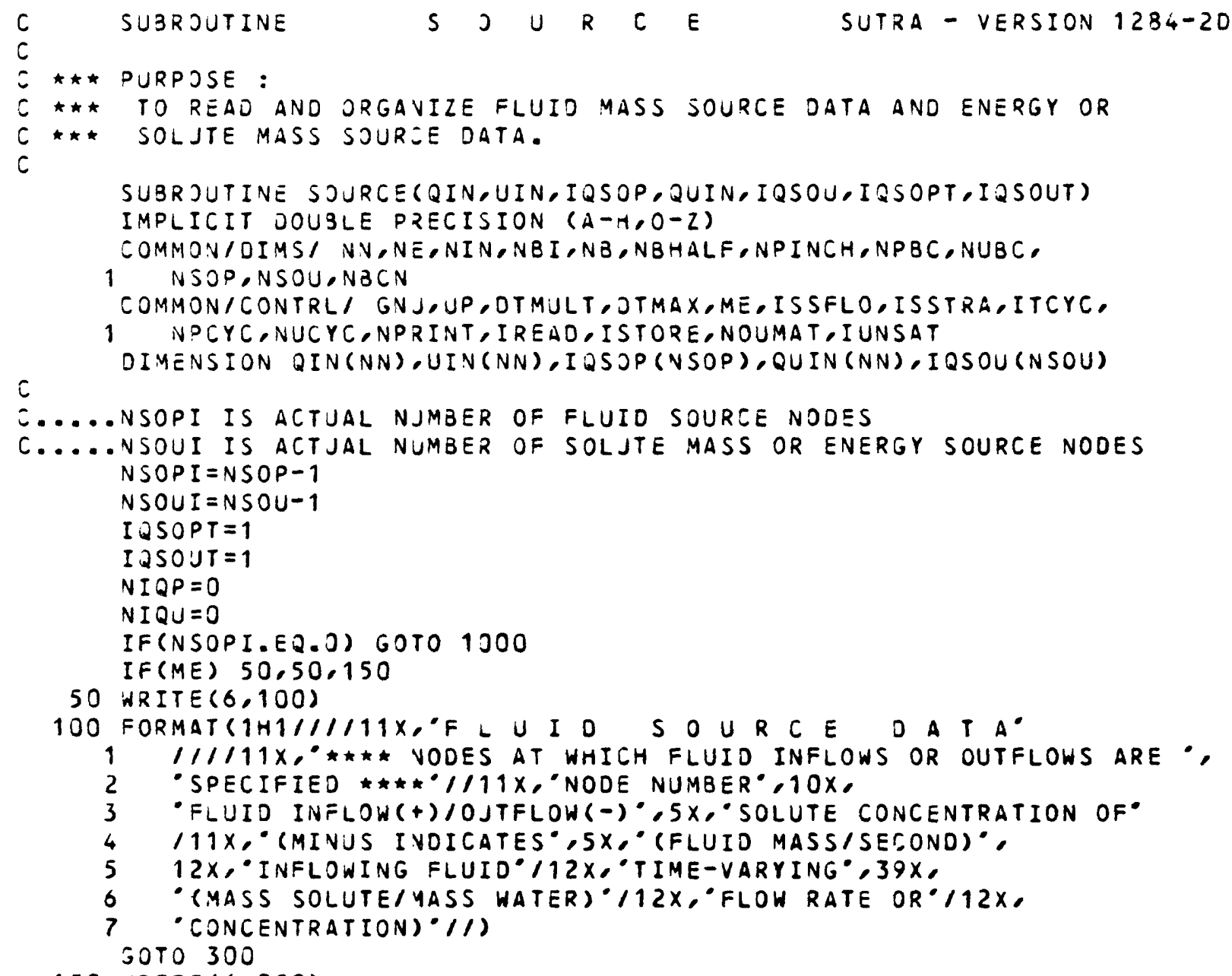

150 WRITE $(6,200)$

230 FORMATGIH1////11X, ${ }^{\circ} F L U$ I $D$ S O U R C E D A T A

$1 / / / 111 x, * \star \star \star$ NOOES AT WHICH FLUIO INFLOWS OR OUTFLOWS ARE $\bullet$,

2 SPECIFIEJ $\star * * * / / 111 x$, NOOE NUMBER*,10X, - FLUID INFLOW(+)/OJTFLOW(-): $5 x, \circ$ TEMPERATURE [DEGREES CELCIUS] $111 \times 0^{\circ}$ (MINUS IVDICATES $, 5 X, \circ$ (FLUID MASS/SECOND), $12 X$, - OF INFLOWING FLUID* $112 x, \circ$ TIME-VARYING $112 x$, FlOW OR* $112 x$,

c - temperature) ( / /)

C.... INPUT DATASET 17

300 CONTINUE

READ $(5,400)$ IQCP, ZINC,UINE

400 FORMAT (I $10,2315.0)$

IF (IJCP.EQ.OS) GOTJ 700

$V I Q P=N I Q P+1$

$I Q S O P(N I Q P)=I Q C P$

IF (IQCP.LT.O) IQSOPT $=-1$

$I Q P=I A B S(I \cap C P)$

$Q I N(I Q P)=2 I N C$

$U I N(I Q P)=U I N E$

IF(IQCP.GT.O) GOTJ $45 J$

NRITE $(6,500)$ IQCP

GOTO 600

450 IF(QINC.GT.O) GOTO 460

WRIT $\equiv(6,500)$ I $2 C P, Q I N=$

जOTO OOO

$\cdots$

E20....

E30.....

E40....

E50.....

E60.....

E70......

E80.....

E9J......

E100....

E110....

E120....

E130...

E140...

E150...

E160....

E170....

E180....

E190....

E200....

E210....

E220....

E230....

E240....

E250....

E260....

E270....

E280....

E290...

E300....

E310....

E320....

E330....

E340...

E350....

E360...

E370....

E380....

E E390...

E400....

E410...

$5420 \ldots$

E430...

E440....

E450....

E460...

E470...

E480...

E490...

E500....

E510....

E520...

E530...

ES40....

ES50...

E560...

$5570 \ldots$.

E580....

E590....

E600.... 
450 WRITE $(6,500)$ IQCD,QINC,UINC

500 FORMAT $(11 X, 110,13 X, 1 P E 14.7,16 X, 1 P E 14.7)$

SOO GOTO 300

700 IF (NIQP.EQ.NSOPI) GOTJ 890

C..... ENO SIMULATION IF THERE NEEO BE CORRECTIONS TO DATASET 17 WRIT $(6,750)$ NIQP,NSOPI

750 FJRMAT $/ / / 111 \mathrm{X}, \cdot T$ TE NJMBER OF FLUID SOURCE NODES READ, ,IS,

1 IS NOT EQUAL TO THE NUMBER SPECIFIED, , IS//1/

2 11X, PLEASE CORRECT DATA AND RERUN $1 / \| 1 / 11$

$322 x, \circ$ I I M U L A T I D N H A L T E D I

$422 \times, 0$

ENDFILE $(\overline{6})$

STOP

390 IF (I 2SOPT.EQ.-1) NRITE $(6,900)$

900 FORMAT(///11X, THE SPECIFIED TIME VARIATIONS ARE • $c$

1 - JSER-PROGRAMMED IN SUBROUTINE B C T I ME $\left.\cdot{ }^{\circ}\right)$

c

1000 IF(NSOUI.EQ.O) GOTO 9300

IF (ME) $1050,1050,1150$

1050 WRITE $(6,1100)$

1100 FORMAT $/ /\|/\| / 111 X^{\circ} S O L U T E$ S O U R C E D A T A.

$1 / \| / 11 x_{1} \cdot * \star \star \star$ NODES AT WHICH SOUREES OR SINKS OF SOLUTE $\cdots$

2 - MASS ARE SPECIFIED $\star \star \star \star * * 1 / 11 x, \cdot$ NODE NUMBER, $10 x$,

3 SOLUTE SOURCE(+)/SINK(-)"111 $x^{\circ}$ (MINUS INDICATES, $5 X$,

4 (SOLUTE MASS/SECOVD) $112 X$, TIME-VARYING"112X,

5 - SOURCE OR SINK) $/ 1 /$ )

GOTO 1300

1150 WRITE $(6,1200)$

1200 FORMAT $/ / 1 / 1 / 111 X,{ }^{\circ} E N$ E R S Y S O U R C E DA T A

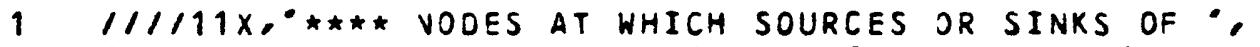

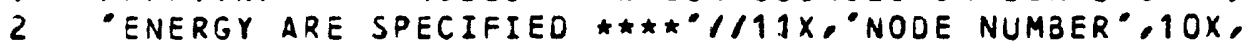

3 -ENERGY SOURCE (+)/SINK $(-) \cdot 111 x_{*}^{\circ}$ (YINUS INOICATES, $5 X^{\circ}$,

4 - (ENERGY/SECONJ) $112 X,-T I M E-V A R Y I N G * 112 X$,

c

- SOURCE OR SINK)・/1)

C..... INPUT DATASET 18

1300 CONTINUE

READ(5,400) IQCU.2UINE

IF(I2CU.EQ.O) GOTJ $17 J 0$

$N I Q U=N I Q U+1$

$I Q S O U(N I Q U)=I Q C U$

IF $I$ ICU.LT.O) IQSOUT $=-1$

$I Q U=I A B S$ (IQCU)

ZUIN (IQU) $=$ QUINC

IF(IQCU.GT.O) GOTO 1450

WRITE $(6,1500)$ IQCJ

GOTO 1600

1450 WRITE $(6,1500)$ IQCJ,QUINC

1500 FORMAT $(11 X, I 10,13 X, 1$ PE 14.7$)$

1500 GOTO 1300

1700 IF(NIQU.EQ.NSOUI) GOTO 1890

C..... END SIMULATION IF THERE NEED BE CORRECTIONS TO DATASET 18

IF (ME) $1740,1740,1760$

1740 WRITE $(6,1750)$ NI2J,NSOUI

1750 FORMATC////11X, THE NJMBER OF SOLUTE SOURCE NOOES READ, 'I5,

1 IS NOT EQUAL TO THE NUMBER SPECIFIED, -IS/1/1

2 11X, PLEASE CORRECT DATA AND RERUN*1/11/11

$322 \times 0^{\circ} S$ I $M U L A T I O N$ H A L T E D*I

$422 \times$, ,

$\leq 610 \ldots$

E620....

E630....

E640....

E050....

E660....

E670....

E680....

E090....

E700....

ET10...

E720...

E730....

ET40....

E750....

E760....

E770....

E780....

E790....

E800....

E810....

E820....

E830....

E840....

E850....

E860....

E870....

E880....

E890....

E9O0....

E910....

E920....

E930....

E940....

E950....

E960....

E970....

E980....

E990....

E1000...

E1010...

E1020...

E1030...

E1040...

E1050...

E1060...

E1070...

E1080...

E1090...

E1100...

E1110...

E1120...

E1130...

E1140...

E1150...

E1160...

E1170...

E1180...

E1190...

E1200... 
ENDFILE(6)

STOP

1760 WRITE $(6,1770)$ NI2J,NSOUI

1770 FORMAT $/ / / / 11 X, \cdot T$ TE NJMBER OF ENERGY SOURCE NODES READ, 'IS,

1 IS NOT EQUAL TO THE NUMBER SPECIFIEO, II5/1/1

2 11X,PPLEASE CORRECT DATA AVD RERUV $1 / 111111$

$322 x, \circ$ I I U L A T I ON HALTE

$422 x_{0}^{\circ}$

ENDFILE $(\overline{6})$

STOP

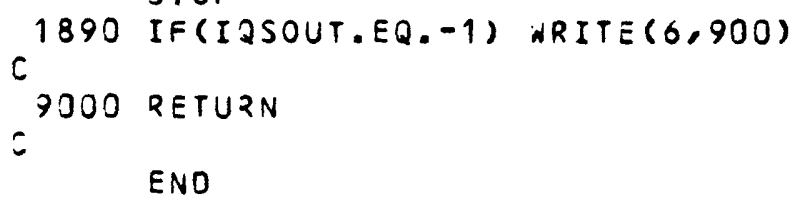

ENO

E1210...

E1220...

E1230...

E1240...

E1250...

E1260...

E1270...

E1280...

E1290...

E1300...

E1310...

E1320...

E1330...

E1340...

E1350... 


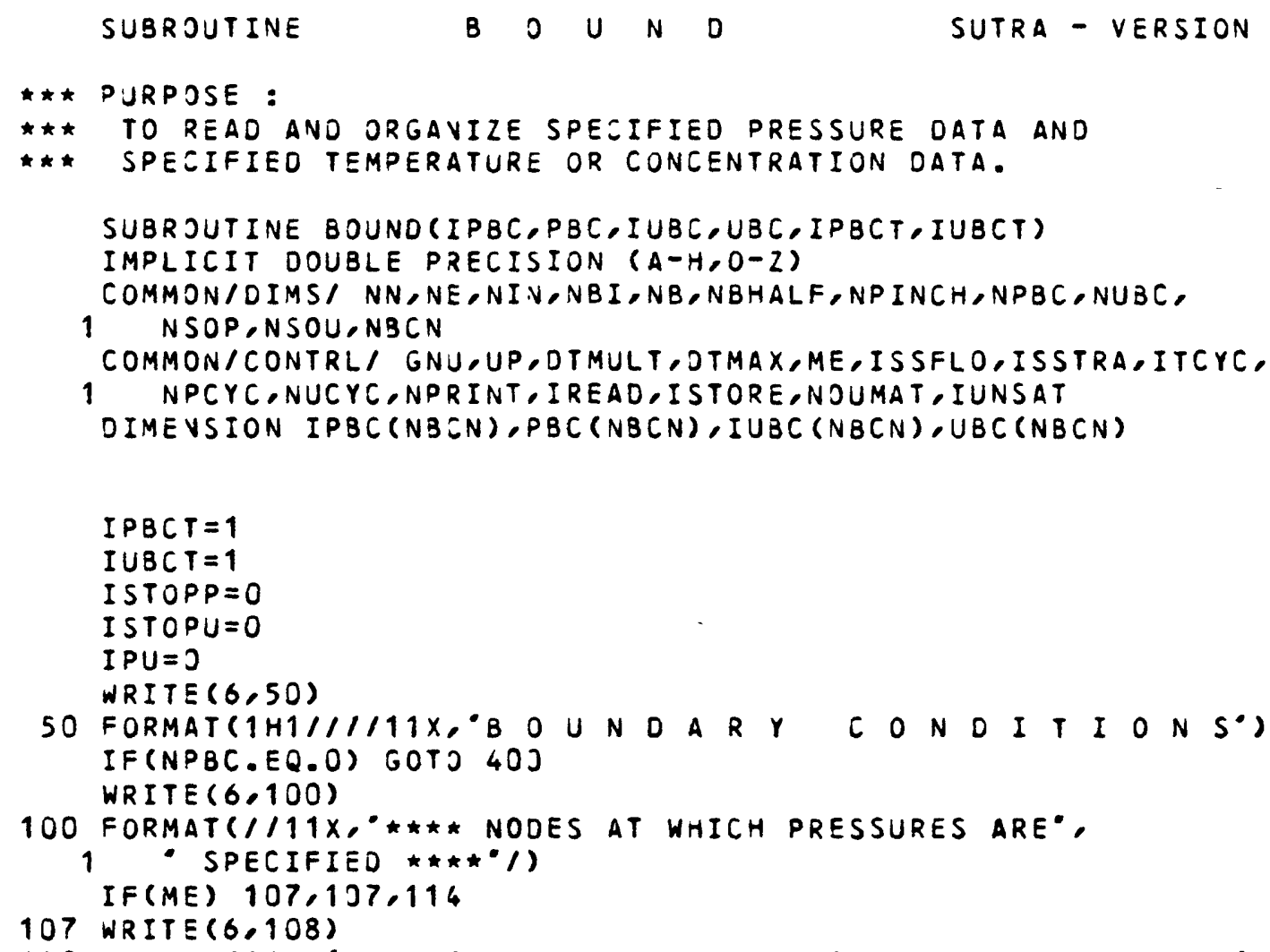

108 FORMAT $111 \mathrm{X}^{\circ}$ (AS WELL AS SOLUTE CONCENTRATION OF ANY

$1 / 16 x,-$ FLUID IVFLON WHICH MAY OCCUR AT THE POINT'

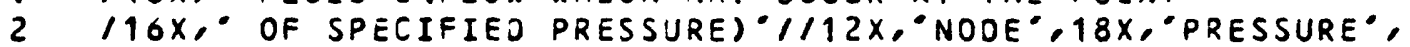


216 FORMAT(//11X,०TIME-DEPENDENT SPECIFIEO PRESSURE*/12X,०OR INFLOW

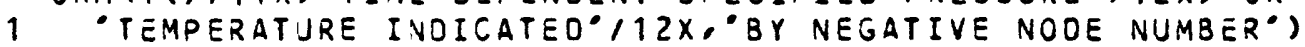

C

400 IF (NJBC.EJ.0) GOTJ 2000

\section{IF (ME) $500,500.553$}

500 WRITE 6,1000$)$

1000 FORMAT $/ / / / 11 x,,^{\circ} \star \star \star *$ NOOES AT WHIEH SOLUTE CONCENTRATIONS ARE $\bullet$,

1 SPECIFIED TO BE IVDEPENDENT OF LOCAL FLOWS AND FLUID SOURCES

$2 \cdot * \star \star \star^{\circ} / 112 x, \cdot$ VOOE $, 13 x, \cdot$ CONCENTRATION* $\left./ /\right)$

GOTO 1120

550 WRITE $(6,1001)$

1001 FORMAT $/ / / / 11 x_{0}^{\circ} \star \star \star \star *$ NODES AT WHICH TEMPERATURES ARE $\bullet$

1 SPECIFIEO TO $3 E$ INDEPENDENT OF LOCAL FLOWS AND FLUID SOURCES*,

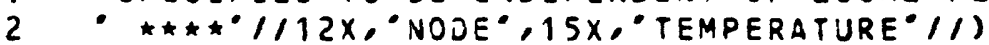

$c$

C..... INPUT DATASET 20

$1120 \quad I P U=I P U+1$

READ (5,150) IUBC (IPU) ,UBC (IPU)

IF(IJBC (IPU). LT.O) IUBCT $=-1$

IF (IJBC (IPU).EQ.O) GOTO 1180

IF(IUBC (IPU).GT.0) WRITE(6.1150) IUBC(IPU),UBC (IPU)

IF (IJBC (IPU).LT.0) WRITE $(6,1150)$ IUBC (IPU)

1150 FORMAT $(11 X, 15,6 X, 1$ POZJ.13)

GOTO 1120

1180 IPU $=I P U-1$

$I U=I P U-I P$

IF(IU.EQ.NUBC) GOTO 1200

ISTOPU $=1$

1200 IF(IUBCT.NE.-1) GOTO 2000

IF (ME) $1205,1205,1215$

1205 WRITE $(6,1206)$

1206 FORMAT $(/ / 12 x, \cdot T I M E-D E P E N D E N T$ SPECIFIED CONCENTRATION*/12X, IS *

1 INDICATED BY VEGATIVE NODE NUMBER ') SOTO 2000

1215 WRITE $(6,1216)$

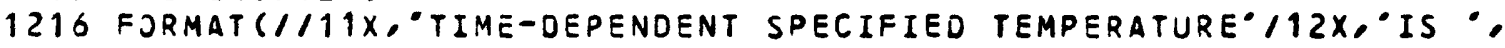
$c$

1 'INDICATED BY VEGATIVE NODE NUMBER ${ }^{\circ}$ )

C..... END SIMULATION IF THERE NEED BE CORRECTIONS TO DATASET 19 OR 20

2000 IF (ISTOPP.EQ.O.AND.ISTOPU.EQ.O) GOTO 6000

IF (ISTOPP.EQ.1) WRITE $(6.3000)$ IP,NPBC

3000 FORMAT $\left(/ / / 11 \times,{ }^{\circ} 4\right.$ TTUAL NUMBER OF SPECIFIED PRESSURE NOOES ${ }^{\circ}$,

1 "READ, "IS." IS NOT EQUAL TO NUMBER SPECIFIED IN",

2 - INPUT, $\because$ (5)

IF (ME) $3500,3500,4600$

3500 IF (ISTOPU.EQ.1) WRITE $(6,4000)$ IU.NUBC

4000 FORMAT $/ / / 111 \mathrm{X}, 0^{\circ}$ AETUAL NUMBER OF SPECIFIED CONCENTRATION NODES,

1 READ,, IS,, IS NOT EQUAL TO NUMSER SPECIFIED IN",

2 INPUT,, (5)

GOTO 4800

4600 IF(ISTOPU.EQ.1) WRITE $(6,4700)$ IU,NUBC

4700 FORMAT $(1 / 1 / 111, \cdot$ ACTUAL NUMBER OF SPECIFIED TEMPERATURE NODES,

1 READ, -I I5, ${ }^{\circ}$ IS NOT EQUAL TO NUMBER SPECIFIEO IN"

2 INPUT, $($ IS)

4300 WRITE $(6,5000)$

5000 FORMAT $/ / / / 11 X_{0}^{\circ}$ PLEASE CORREET DATA AND RERUN. $/ / / / 1 / / 1$

$122 x$, , I I U L A T I ON H A L T E $0^{\circ}$ I

$222 x,-$ ENDF ILE $(\overline{6})$

STOP
,F610....

F620....

F630....

Fo40....

F650....

F660....

Fo70....

,F630....

F690....

F700....

F710....

$F 720 \ldots$.

F730....

F740...

F750....

F760....

F770....

F780....

F790....

F800....

F810....

F820....

F830....

F840....

F850....

F860....

F870....

F880....

F890....

F900....

F910....

F920....

F930....

F940....

F950....

F960....

F970....

F980....

F990....

F1000...

F1010...

F1020...

F1030...

F1040...

F1050...

F1060...

F1070...

F1080...

F1090...

F1100...

F1110...

F1120...

F1130...

F1140...

F1150...

F1160...

F1170...

F1180...

F1190...

F1200... 
c

6J00 IF(IPBCT.EQ. -1.0R IUBLT.E2.-1) WRITE $(6,7000)$

7000 FJRMATC///11X, THE SPECIFIEJ TIME VARIATIONS ARE "

1 - USER-PROGRAMMED IN SUBROUTINE B C T I M E .*)

c

RETURN

END

F1210...

F1220...

F1230...

F1240...

F1250...

F1260...

F1270...

F1280... 


SUSRTUTINE 03 S E R V SUTRA - VERSION 1284-20

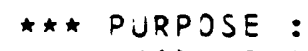


c

400 RETURN

300 FORMAT $((11 x, 16(3 x, I 6)))$

IF(JSTOP.EQ.O) GOTO 400

C.... END SIMULATION IF CORRECTIONS ARE NECESSARY IN DATASET 21 WRITE $(6,350)$ IOB, NOBS

350 FORMAT $/ / / / 111 X 0^{\circ}$ AETUAL NUMBER OF OBSERVATION NODES

1 READ, -I5,, IS NOT EQUAL TO NJMBER SPECIFIED IN",

2 INPUT, $\because 5 / / 1 / 11 X, \circ$ PLEASE CORRECT DATA AND RERUN.,

$3 / 1 / 1 / 1 / 122 x$, S I M U L A T I O N H A L T E O I

$422 x$

STOP

C.... MAKE OBSERVATIONS EACA NOBCYC TIME STEPS

500 CONTINUE

IF (MOD (IT,NOBCYC).NE.J.AND.IT.GT.1.AND.ISTOP.EQ.O) RETURN

IF(IT.EQ.O) RETUR.V

$I T C N T=I T C N T+1$

ITOBS (ITCNT) =IT

OBSTIM (ITCNT) =T SEE

DO $1300, J J=1$. NOBS

$I=\operatorname{IOS} 2(\mathrm{~J})$

POBS (JJ,ITCNT) =PVEC (I)

1000 CONTINUE

UOBS (JJ,ITCNT) =UVEC (I)

c

RETURN

C.... OUTPUT OBSERVATIONS

SOOO CONTINUE

$M N=2$

IF $\left(M E, E Q_{0}-1\right) \quad M N=1$

$J J Z=0$

$M L O O P=(N O B S+3) / 4$

DO $7000 \quad L O O P=1, M L J O P$

$J J 1=J J 2+1$

$J J 2=J J 2+4$

IF ( $L O O P . E Q \cdot M L O O P) \quad J J 2=N O B S$

WRITE $(6,5999) \quad($ IOBS (JJ),JJ JJJ1,JJ2)

5999 FORMAT $\left(1 H_{1} / / 1 / 5 X_{0} \cdot 0\right.$ S S E R V A T I $O N$ N

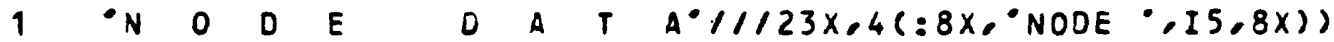

WRITE $(6,6000)$ (UNJERS, JJ $=J J 1, J J 2)$

6000 FORMATS $23 x, 4(: 8 x, A 10,8 x)$ )

WRITE $(6,6001)$ (UNAME $(Y N), J J=J J 1, J J 2)$

6001 FORMAT $\left(11 X, 0^{\circ}\right.$ TIME STEP, $4 X 0^{\circ}$ TIME $(S E C)^{\circ}, 4\left(: 2 x, 0^{\circ}\right.$ PRESSURE $\left.\left., 3 X, A 13\right)\right)$

DO 6500 ITT $=1$, ITC.VT

WRITE (6.6100) ITO3S(ITT),OBSTIM(ITT),

1 (POBS(JJ,ITT), JOBS(JJ,ITT),JJ=JJ1,JJ2)

6100 FORMAT $(5 x, I 5,1 x, 1$ PD12.5.8(1X,1PD12.5))

6500 CONTINUE

7000 SONTINUE

RETURN

$c$

END
G610....

$6620 \ldots$.

$6630 \ldots$.

G640....

$6650 \ldots$.

G660....

$6670 \ldots$.

G680....

G690....

G700....

G710....

G720....

$6730 \ldots$.

G740....

G750....

$6760 \ldots$.

$6770 \ldots$

G780....

G790....

G800....

G810....

G820....

$6830 \ldots$.

G840....

G850....

G860....

$6870 \ldots$.

G880....

G890....

$6900 \ldots$.

G910....

G920....

G930....

$6940 \ldots$.

$6950 \ldots$.

G960....

$6970 \ldots$.

$6980 \ldots$.

$6990 \ldots$.

G1000...

G1010...

G1020...

G1030...

G1040...

61050...

$61060 \ldots$

G1070...

G1080...

G1090...

G1100...

G1110...

G1120...

G1130...

G1140... 
C SUbRoUtine $C$ J $N$ N E $C$ SUTRA - VERSION 1284-20 H10....

IF (KINCID.EQ.0) GJTO 1000

WRITE $(6,650)$ L, (IN $(M), M=M 1, M 4)$

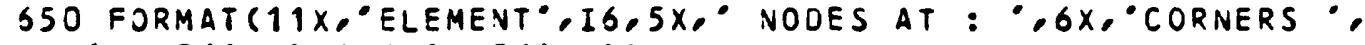

$15(1 H *), 4 I 6,1 X, 5(1 H *))$

IF(LL.LT.0) WRITE $(6,750)$ (IEOGE (M),M=1,4)

c

POO FORMAT $(11 \times, \cdot$ EOGES, 416$)$

1000 CONTINUE

IF(IPIN.EQ.O) GOTO 5030

IF (IPIN.EQ.NPINCH-1) JOTO 1500

WRITE $(6,1450)$ IPIN,NPINCH

1450 FORMAT $/ / / / 111 x_{0}^{\circ}$ ACTJAL NUMBER OF PINCH NODES,, 14,

$1 \%$ DIFFERS FROM NUMBER ALLOWED AS SPECIFIED IN INPUT,, 1411

$211 X$, PLEASE CORREET INPUT OATA ANOIOR OIMENSIONS AND RERUN."

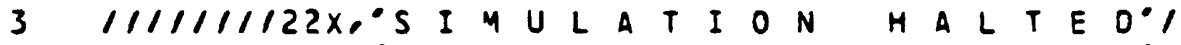

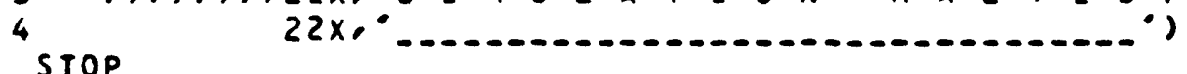

C

1500 CONTINUE

IF (KINCID.EQ.0) GOTO 5000

WRITE $(6,3000)$

3000 FORMAT $/ / / 1 / 111 x^{\circ} * \star \star \star$ PINCH NODE CONNECTIONS $\star \star \star \star^{*} / 17 x^{\circ}$

1 PINCH NODE', $17 \times, \circ$ CONNECTED NODES $/ 1 / 1)$

$004000 I=1, I P I N$

4000 WRITE $(6,4500)$ (IPINCH(I,NP),NP=1,3)

4500 FORMAT $(11 X, 16,10 X, 216)$

C

C

5000 RETURN

END 


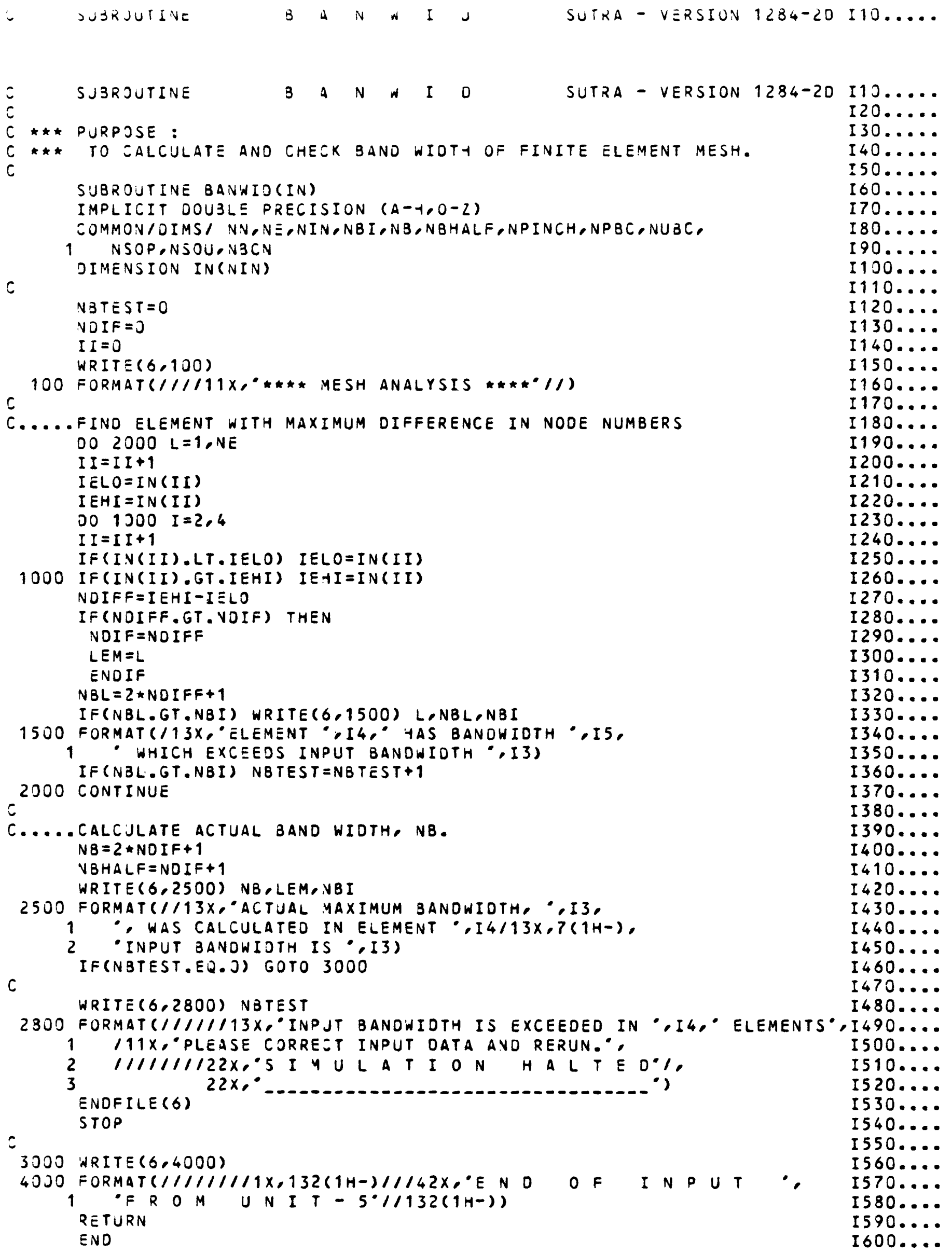


SUBROUTINE

J10....

PURPJSE :

TO EHECK THAT PIVCH NODES ARE NOT ASSIGNED SPECIFIED

* * PRESSURES, CONCENTRATIONS, TEMPERATURES OR SOURCES.

SUBRJUTINE NEHECK(IPIVCH,IQSOP, IQSOU,IPBC,IUBC)

IMPLICIT DOUBLE PRECISION $(A-H, O-Z)$

COMMON/OIMS/ NN,NE,NIN,NBI,NB,NBHALF,NPINCH,NPBC,NUBC,

1 NSDP,NSOU, NBCN

COMMON/CONTRL/ GNU,UP, DTMULT, OTMAX, ME, ISSFLO, ISSTRA, ITCYC,

1 NPCYC,NUCYC,NPRINT, IREAD, ISTORE, NOUMAT, IUNSAT

OIMEVSION JQOX 30$), J Q J X(3)), J P X(30), J U X(30)$

OIMENSION IPINCH(NPINEH, 3), IQSOP(NSOP), IQSOU (NSOU),

1 IPBC (NBCN),IUBC (NBLN)

c

$I Q P X=0$

$I Q U X=0$

$I P X=J$

IUX $=0$

NPIN $=$ NPINCH-1

NSOPI $=$ NSOP -1

NSOUI $=$ NSOU -1

DO $1000 I=1, N P I N$

I PIN = IPINCH $(I, 1)$

C..... MATCH PINCH NODES HITH FLUIO SOURCE NODES

IF (NSOPI.EQ.O) GOTO 200

DO 1 JO IQP $=1$,NSOP

IF(IPIN-IABS(IQSOP(IQP)) $100,50,100$

50 I $Q P X=I Q P X+1$

$J Q P X(I Q P X)=I P I N$

100 CONTINUE

200 IF(NSOUI.EQ.J) GOTO 400

C..... MATCH PINCH NODES WITH ENERGY OR SOLUTE MASS SOURCE NODES

DO 330 IQU $=1$,NSOU

IF(IPIN-IABS(IQSOU(IQU))) $300,250,300$

250 I QUX $=I Q U X+1$

$J Q U X(I Q U X)=I P I N$

300 CONTINUE

400 IF (NPBC.EQ.0) GOTO 6JJ

C..... MATCH PINCH NOOES WITH SPECIFIED PRESSURE NODES

$D O 5 J 0$ I $P=1, N P B C$

IF(IPIN-IABS(IPBC(IP)) ) $500,450,500$

450 IPX $=I P X+1$

$J P X(I P X)=I P I N$

500 CONTINUE

600 IF (NUBC.EQ.O) GOTO 1000

C..... MATCH PINCH NODES WITH SPECIFIED TEMPERATURE OR

C CONCENTRATION VODES

$D O 7 J O I U=1, N U B C$

$I U P=I U+N P B C$

IF (IPIN-IABS(IUBC(IUD))) $700,050,700$

350 I $\cup X=I \cup X+1$

$J \cup X(I \cup X)=I P I V$

700 CONTINUE

1000 CONTINUE

$c$

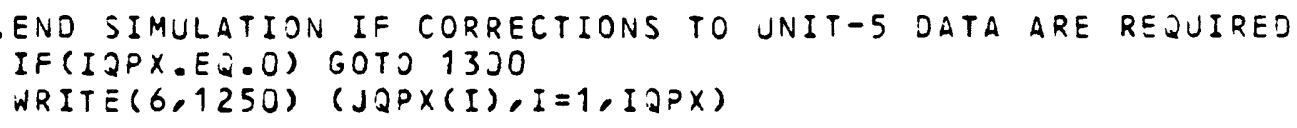


1250 FJRMATC////11X, THE FOLLOWINJ NODES MAY NOT 3 E SPECIFIED AS

1 - FLUIO SJURCE NODES : "115X,2(20I6/))

WRIT $\equiv(6,1251)$

1251 FORMAT $/ 11 X, \circ$ PLEASE REOISTRIBUTE SOURCES OR CHANGE THESE PINCH ${ }^{\circ}$,

1 - NOJES TO NORYAL CORNER MESH NODES ANO THEN RERUN.')

1300 IF (I JUX.EQ.O) GOTJ 1430

IF (ME.EQ.-1) WRITE $(0,1350)$ (JQUX(I), I=1,IQUX)

1350 FORMAT $/ / / / / 11 X$, "THE FOLLOWING NODES MAY NOT BE SPECIFIED AS,

1 - SOLUTE SJURCE NOJES : $/ 15 X, 2(20 I 5 /))$

IF (ME.EQ.+1) WRITE $(0,1355)$ (JJUX(I),I I I,IQUX)

1355 FORMAT ////11X, "THE FOLLOWING NODES MAY NOT BE SPECIFIED AS

1 - ENERGY SOURCE NOJES: $\left./ 15 x_{0} 2(20 I 6 /)\right)$

WRIT $\leqq(6,1251)$

1400 IF (IPX.EQ.0) GOTO 1500

WRITE $(6,1450) \quad(J P X(I), I=1, I P X)$

1450 FORMAT $/ / / / 119 x_{0}$ "THE FOLLOWINS NODES MAY NOT BE INPUT AS

1 SPECIFIED PRESSURE NODES:, $115 \times, 2(2016 / 1)$ WRITE $(6,1451)$

1451 FORMAT $/ 11 X_{1}$, PLEASE REMOVE SPECIFIED PRESSURE RESTRICTION OR०

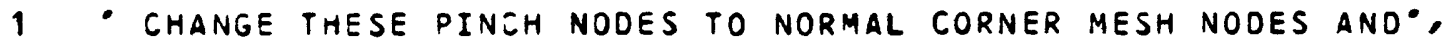

2 - THEN RERUN.')

1500 IF (ME) $1600,1500,1660$

1600 IF (IJX.EQ.0) GOTO 1683

WRITE $(6,1650) \quad(J U X(I), I=1, I U X)$

1550 FORMATC///111X, 'THE FOLLOWIN' NODES MAY NOT BE INPUT AS

1 SPECIFIED COVCENTRATION NODES:, $115 \times, 2(20 I 6 / 1)$ WRITE $(6,1651)$

1551 FORMATC/11X, PLEASE REMOVE SPECIFIED CONCENTRATION RESTRICTION ',

1 OR CHANG THESE PINCH NODES TO NORMAL CORNER NOOES AND".

2 - THEN RERUN.')

GOTO 1680

1660 IF(IJX.EQ.O) GOTO 1680

WRITE $(6,1670)$ (JUX(I),I $=1, I U X)$

1570 FORMAT $/ / / / 11 X$, "THE FOLLOWING NODES MAY NOT BE INPUT AS

1 - SPECIFIED TEMPERATURE NODES:, $115 \times, 2(20 I 6 /))$ WRITE $(6,1671)$

1571 FORMAT $/ 111 \times$, PLEASE REMOVE SPECIFIED TEMPERATURE RESTRICTION OR*,

C

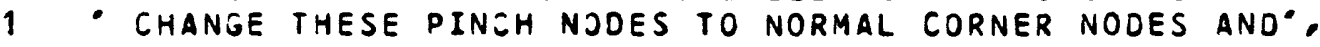

2 - THEN RERUN.',

1580 IF $(I 2 X+I P X+I U X) \quad 1800,1800,1700$

17JO WRIT $\leqq(6,1750)$

1750 FORMAT $/ / / / / / / 11 x_{0}^{\circ}$ S I MULATI ON HALTE $0^{\circ} /$,

1

ENOFILE(6)

c

STOP

1300 RETURN

END
J610...

$J 620 \ldots$.

Jo30....

$1040 \ldots$.

J650....

J660....

J670....

J630....

J690....

J700....

J710....

$J 720 \ldots$.

$J 730 \ldots$

$J 740 \ldots$.

$J 750 \ldots$

J760....

J770....

J780....

J790....

J800....

J810....

$J 820 \ldots$.

J830....

$J 840 \ldots .$.

J850....

J860....

$\mathrm{J} 870 \ldots$

J880....

J890....

J900....

J910....

J920....

J930....

J940....

J950....

J960....

J970....

J980....

J990....

J1000...

J1010...

J1020...

J1030...

J1040...

J1050...

J1060...

J1070...

J1080...

J1090...

J1100... 
SUBRJUTINE INDAT2 (PVEE,UVEC,PM1,UM1,UM2,CS1,CS2,CS3,SL, SR,RCIT, 1 SW,DSWDP,PBC,IPBC,IPBCT)

IMPLICIT DOUBLE PRECISION (A- $-1,0-Z)$

COMMJN/DIMS/ NN,NE,NIV,NBI,NB,NBHALF,NPINCH,NPBC,NUBC,

1 NSOP,NSOU,NBCN

COMMON/CONTRL/ GNU,UP, DTMJLT, DTMAX,ME, ISSFLO, ISSTRA, ITCYC,

1 NPCYC,NUCYC,NPRINT, IREAD, ISTORE,NOUMAT, IUNSAT

COMMON/TIME/ DELT, TSEC,TMIN, THOUR, TDAY, TWEEK, TMONTH, TYEAR,

1 TMAX,DELTP,DELTU,DLTPM1,DLTUM1,IT, ITMAX

COMMON / PARAMS/ COMPFL, COMPMA,DRWOU,CW,CS,RHOS, DECAY, SIGMAW, SIGMAS

1 RHOWO,URHOWO, VISCO,PRODF1,PRODS1, PRODFO,PRODSO,CHI $1, C H I 2$

DIMENSION PVEC(NN), UVEC (NN),PYI (NN),UM1 (NN),UMZ (NN),SL(NN),SR (NN),

$1 \quad C S 1(N N), C S 2(N N), C S 3(N N), R C I T(N N), S W(N V), D S W D P(N N)$,

c

$2 \quad P B C(N B C N), I P B C(N B C V)$

IF(IREAD) $500,500.620$

C..... INPUT INITIAL CONJITIONS FOR NARM START (UNIT-55 DATA)

500 READ $(55.510)$ TSTART,DELTP,DELTU

510 FORMAT $(4620.10)$

READ $(55,510)$ (PVEE (I), I $=1, N N)$

$R E A D(55,510)$ (UVEE(I), I = $1, N N)$

READ(55,510) (PM1 (I), I=1,NN)

$R E A D(55,510)$ (UM1 (I),I I I,NN)

READ $(55,510)(C 51(I), I=1, N N)$

$\operatorname{READ}(55,510)(R C I T(I), I=1, N N)$

$\operatorname{READ}(55,510) \quad(S W(I), I=1, N N)$

READ $(55,510) \quad(P B C(I P U), I P U=1, N B C N)$

C CALL ZERO(CSZ,NN.O.ODJ)

C CALL LERO(CS3,NN,O.ODJ)

CALL ZERO(SL,NN.0.0DO)

CALL ZERO(SR, VN,0.00O)

CALL ZERO(DSWDP,NN.0.JDO)

DO $550 \quad I=1, N N$

$550 \cup M 2(I)=U M 1(I)$

GOTO 1000

C

C..... INPUT INITIAL CONJITIONS FOR LOLD START (UNIT-55 DATA)

620 READ $(55,510)$ TSTART

READ $(55,510)$ (PVE: $(I), I=1, N N)$

READ $(55,510) \quad(J V E=(I), I=1, N N)$

C..... START-UP WITH NO PROJECTIONS BY SETTING BOELP $=B O E L U=1 . D-10$

C IN PRJJECTIOV FORMULAE FOJND IN SUBROUTINE SUTRA.

$D E L T P=D E L T \star 1.016$

DELT J $=$ OELT*1.010

C.... INITIALIZE SPECIFIED TIME-VARYING PRESSURES TO INITIAL PRESSURE

C VALUES FOR START-JP CALCULATION OF INFLOWS OR OUTFLOWS

C (SET $2 P L I T R=0)$

IF (IPBCT $) \quad 580,740,740$

68000730 IP $=1$, VPBC

, $I=I P 3 C(I P)$

$I F(I) \quad 700.703 .730$

$700 D_{B C}(I P)=D V \equiv C(-I)$ 
730 CONTINUE

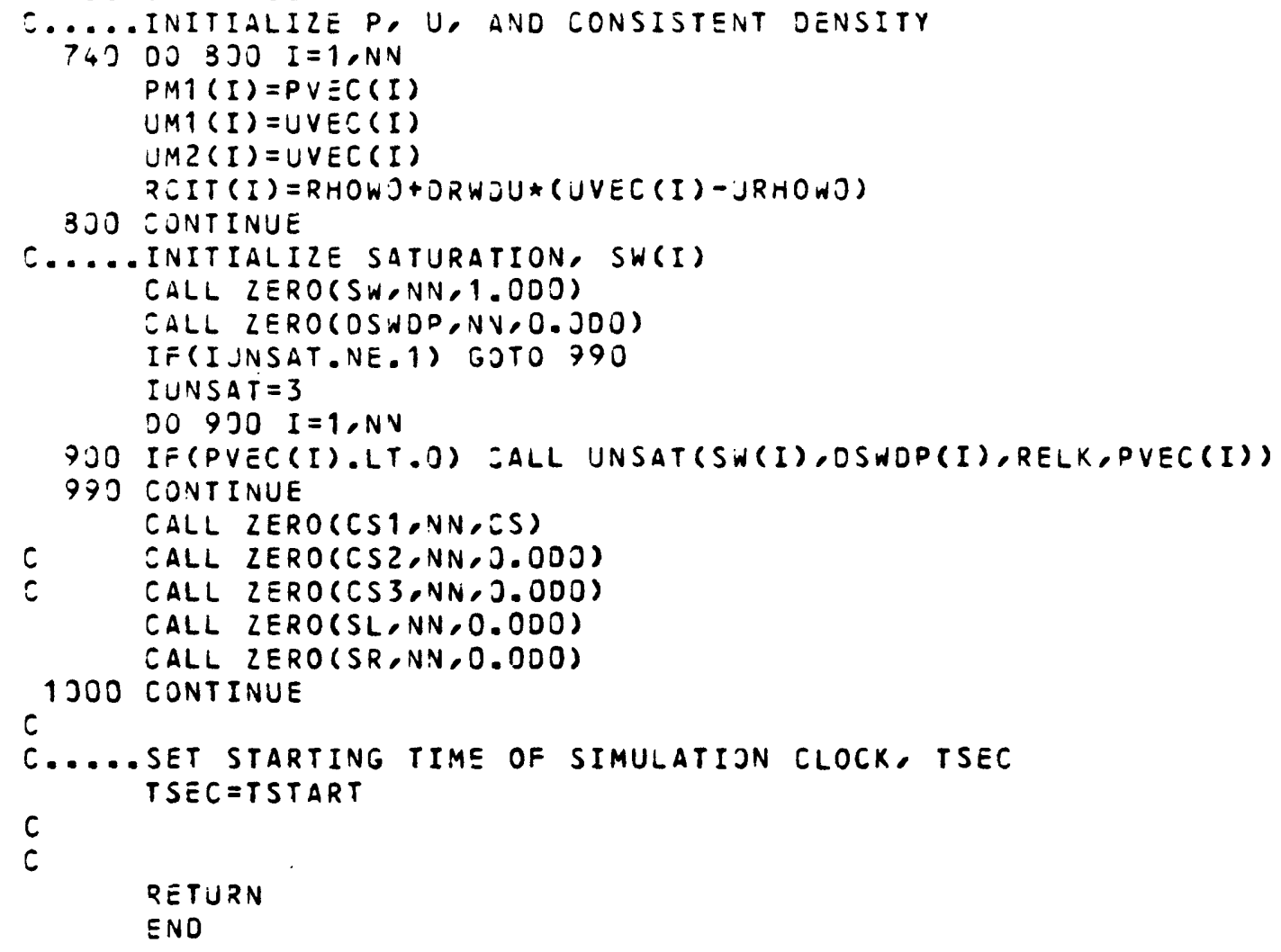

K610.... K620....

ko30.... K640.... k650.... k660.... k670.... K680.... k690.... K700.... $k 710 \ldots$. $k>20 \ldots$ $k 730 \ldots$ $k 740 \ldots$ K750... K760.... K770.... K780.... K790.... K800.... K810.... K820.... K830... K840... K850.... K860.... K870.... K830.... K890... 
SUSROUTINE $\quad P \quad I \quad S O L$ SUTRA - VERSION $1234-20$

PURPJSE :

TO PRINT PRESSURE ANJ TEMPERATURE OR CONCENTRATION

SOLJTIONS AND TO OUTPUT INFORMATION ON TIME STEP, ITERATIONS,

SATURATIONS, AND FLUID VELOCITIES.

SJBROUTINE PRISOL (ML, ISTOP, IGOI, PVEC,UVEC, VMAG, VANG, SW)

IMPLICIT DOUSLE PRECISION $(A-H, O-Z)$

COMMON/DIMS/ NN,NE, NI, , NBI, NB,NBHALF, NPINCH,NPBC, NUBC,

1 NSOP,NSOU,NBCN

COMMON/CONTRL/ GNJ,UP, OTMULT, JTMAX, ME, ISSFLO, ISSTRA, ITCYC,

1 NPCYC,NUCYC,NPRINT, IREAD, ISTORE, NOUMAT, IUNSAT

COMMON/TIME/ DELT, TSEZ, TMIN, T HOUR, TDAY, TWEEK, TMONTH, TYEAR,

1 TMAX,DELTP, DELTU, OLTPMI, DLTUM1, IT, ITMAX

COMMON/ITERAT/ RPY,RPYAX, RUM, RUMAX, ITER, ITRMAX, IPWORS, IUWORS

COMMON/KPRINT/ KCJORD,KELINF,KINCID,KPLOTP,KPLOTU,KVEL,KBUDG

$c$

OIMEVSION PVEC (NN),UVEC (NN), VYAG (NE), VANG (NE), SW (NN)

C.... OUTPUT MAJOR HEADINGS FOR CURRENT TIME STEP

IF(IT.GT.O.OR.ISSFLO.EQ.2.OR. ISSTRA.EQ.1) GOTO 100

WRITE $(6,60)$

60 FORMAT $\left(1 H_{1} / 1 / 111 X^{\circ}\right.$ I V I T I A L C O N O I T I O N S :

1 /11X,

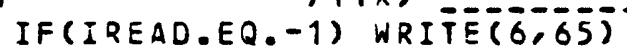

65 FORMAT(//11X, INITIAL CONDITIJNS RETRIEVED FROM STORAGE ",

1 ON UNIT $\left.55.0^{\circ}\right)$

C SOTO 500

100 IF (IJOI.NE.O.AND.ISTJP.EQ.O) NRITE(6,150) ITER,IT

150 FORMAT(///////11X,"ITERATIOV',I3," SOLJTION FOR TIME STEP ",I4)

IF(ISTOP.EQ.-1) WRITE(6,250) IT, ITER

250 FORMAT(IHI//11X, SOLUTION FOR TIME STEP ', I4,

1 "NOT CONVERGEJ AFTER ",I3," ITERATIONS.")

IF(ISTOP.GE.0) WRITE 6,35 J) IT

350 FORMAT (1H1//11X, RESULTS =OR TIME STEP, $14 /$

$111 x_{1}$

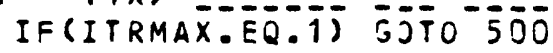

IF(ISTOP.GE.J.ANO.IT.ST.O) NRITE $(0,355)$ ITER

IF(IT.EQ.O.AND. ISTOP.SE.O.AND.ISSFLO.EQ.2) WRITE(6.355) ITER

355 FORMAT $\left(11 \mathrm{X}, 0^{\circ}\right.$ (AFTER 'II3," ITERATIONS) :")

WRITE $(6,450)$ RPM, IPWORS, RUM, IUWORS

450 FORMAT $/ / 11 \mathrm{X}$, MMAXIMUM P CHANJE FROM PREVIJUS ITERATION ',

1 1DO14.5, AT NJOE '-I5/11X, MMAXIMJM U CHANGE FROM PREVIOUS ',

2 ITERATION , 1PO14.5," AT NODE $\bullet$ I5)

500 IF(IT.EQ.O.AVD.ISSFLO.EQ.2) GOTO 580

IF (ISSTRA.EQ.1) GOTO $B O 0$

WRITE 6,550$)$ JELT, TSEC,TMINं, THOUR,TDAY,TNEEK,

1 TMONTH, TY $\equiv A R$

550 FORMAT ( $/ 1111 \mathrm{X}, \circ$ TIME INCREMENT :",T27,1P015.4, SECONOS $1 / 11 \mathrm{X}$,

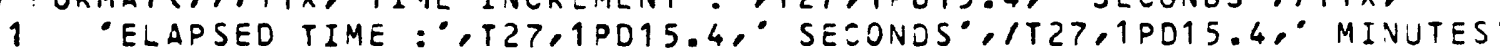

2 /T27,1PD15.4, HOURS /T27,1P015.4, DAYS /T27,1PD15.4, WEEKS $/$

3 T27,1PD15.4, YONTHS $/ T 27,1 P D 15.41^{\circ}$ YEARS $)$

- OUTPUT PRESSURES FOR TRANSIENT FLOW SOLUTION (AND POSSIBLY, SATURATIOV AND VELOCITY)

IF(ML.EQ.2.AVD.ISTOP.SE.O) GOTO 700 
IF(ISSFLO.GT.O) GOTO 700

WRITE $(6,65 C)$ (I,PVEC $(I), I=1, N N)$

6SC FORMAT(//111X, P $R$ E S S U R E*//8X,6('NODE*,17X)/

$1(7 X, 6(1 X, I 4,1 X, 1 P C 15.8)))$

IF(IUNSAT.NE.O) WRITE $(6, t 51) \quad(I, S W(I), I=1, N N)$

651 FORMAT(//111X,'S A T U R A T I O N $T / 18 \times, 6\left(^{\circ} N^{\circ} O E^{\circ}, 17 X\right) /$

$1(7 X, 6(1 X, I 4,1 X, 1 P D 15,8)))$

IF(XVEL.EQ.1.AVD.IT.GT.O) WRITES 6.655) (L,VMAG(L),LL1,NE)

IF (KVEL. $\subseteq 0.1$. AND.IT.GT.O) WRITE( 6,556) (L,VANG $(L), L=1, N \equiv)$

555 FORMATC//111X, ${ }^{\circ} F$ L U I $D$ V $E L$

$111 \times, M A G N$ I T U D E AT CENTROID OF ELEMENT'H

$\left.25 X, 6\left(^{\circ} E L E M E N T^{\circ}, 14 X\right) /(7 X, 6(1 X, I 4,1 X, 1 P C 15.3))\right)$

656 FORMAT //111X, $F$ L U I D $V$ E $L$ O $C$ I $T$ Y./1

$111 X,-A N G L E$ IN DEGREES FROM +X-AXIS TO FLOW DIRECTION,

2 - AT CENTROID OF ELEMENT'I

$35 X, 6\left(^{\circ}\right.$ ELEMENT',14X)/(7X,6(1X,I4,1X,1PO15.8))

$c$ Goro 700

C..... OUTPUT PRESSURES FOR STEAOY-STATE FLOW SOLUTION

$6 B O$ WRITE $(6,690)$ (I,PVEC (I), I=1,NN)

690 FORMATC/1/11X, S T E A D Y - S T A T E P R E

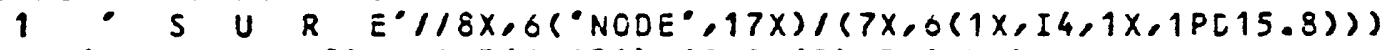

IF(IUNSAT.NE.0) WRITE $(6,651)(I, S W(I), I=1, N N)$

C GOTO 1000

C.... OUTPUT CONCENTRATIONS OR TEMPERATURES FOR

C TRANSIENT TRANSPORT SCLUTION

7OC IF(ML.EQ.1.AND. ISTOP.GE.O) GOTO 1000

IF (ME) $720,720,730$

720 WRITE $(6,725)$ (I,UVEC (I), I $=1, N N$ )

725 FORMAT(//11X, ${ }^{\circ} \mathrm{C} N \mathrm{~N} C$ E N T R A T I O N $N^{*} / 18 \mathrm{X}$,

$16($ NODE, $17 X) /(7 X, 6(1 X, I 4,1 X, 1 P 015.8)))$ GOTO 900

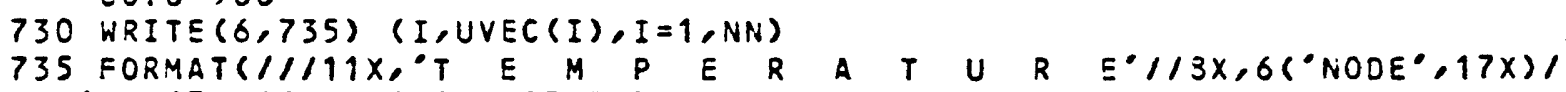

$1(7 x, 6(1 X, I 4,1 X, F 15.9)))$

GOTO 900

$c$

C..... OUTPUT CONCENTRATIONS OR TEMPERATURES FOR

C STEAOY-STATE TRANSPORT SOLUTION

800 IF (ME) $820,320,830$

320 WRITE $(C, 825)$ (I,UVEC $(I), I=1, N N)$

E25 FORMAT (I/II1X, S T E A D Y - S TA T E C O N C.,

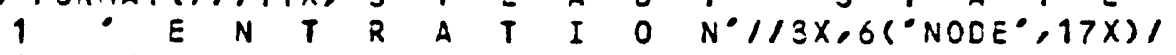

$2(7 x, 6(1 x, 14,1 x, 1 P 015,8)))$

GOTO 900

830 WRITE 6.835$)$ (I,UVEC (I), I $=1, N N)$

835 FORMAT(/1/11X, S T E A O Y - S T A T E T E M P.,

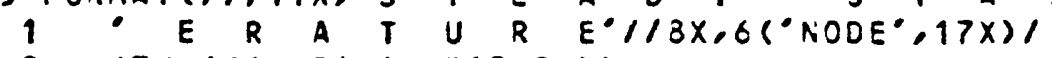

C

$2(7 X, 6(1 X, I 4,1 X, F 15.9)))$

C.... OUTPUT VELOCITIES FOR STEAOY-STATE FLOW SOLUTION

900 IF(ISSFLO.NE.2.OR.IT.NE.1.OR.KVEL.NE.1) GOTO 10.00 WRITE $(6,925)$ (L,VMAG $(L), L=1, N E)$

WRITE 6,950$)$ (L,VANG $(L), L=1, N E)$

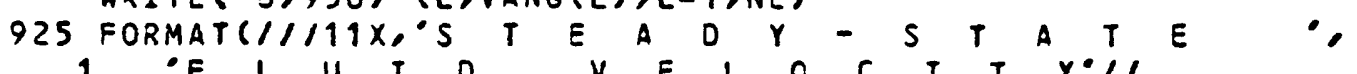

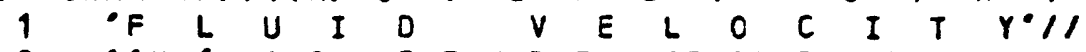

$211 X,-M A G N I T U D E$ AT CENTROID OF ELENENT 11

$\left.35 X, 6\left(^{\circ} E L E M E N T^{\circ}, 14 X\right) /(7 X, 6(1 X, I 4,1 X, 1 P D 15.8))\right)$

950 FORMATC//111X, S T E A D Y - S T A T E

LS10....

L620....

LS3C....

$1540 \ldots$.

LS50....

Ls60....

LS70....

L630....

L590....

L700....

L710....

L720....

$L 730 \ldots$.

L740...

$L 750 \ldots$

L760....

$L 770 \ldots$.

L780....

L790....

L800....

$S^{\circ}, L E 10 \ldots$.

Lo20....

L830....

L340....

L850....

Lร $60 . .$. .

L $370 . \ldots$.

L380....

L390....

L900....

L910....

L720....

L930....

L940....

L750....

L780....

L970....

L930....

L990....

L1000...

L1010...

L102C...

L103C...

L1C4C...

L105C...

L106C...

L1070...

L108C...

L109C...

L1100...

L1110...

L1120...

L1130...

L1140...

L1150...

L1160...

L1170...

L1130...

L1190...

L1200... 
C

1000 RETURN

c $\because F$ I $U$ I $\quad 0$ V E L

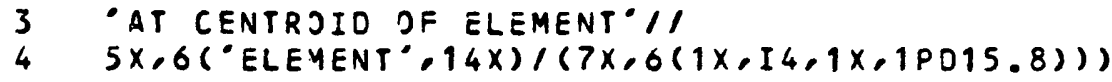
3 AAT CENTRJID DF ELEMENT'/1
4 5X,6("ELEMENT', $14 X) /(7 X, 6(1 X, 14,1 X, 1 P D 15,8)))$ $211 X, \triangle A N$ J L IV OESREES FROM +X-AXIS TO FLOW OIRECTION,$~ L 1220 \ldots$

L1210...

L1230... L1240... L1250... L1260... L1270... L1280... 

$M 20 \ldots \ldots$

$c$

C.....fill arrar a with valje in variable 'fillOO 10 I $=1$, IAJIM

$10 \quad A(I)=F I L L$

$c$

RETURN

ENO

$M 30 \ldots \ldots$

M50....

M60.....

M70.....

M80.....

M90.....

M100....

M110....

M120....

$M 130 \ldots$.

M140....

M150....

M160.... 
purpose :

JSER-PROGRAMMED SUBROUTINE WHICA ALLOWS THE JSER TO SPECIFY:

(1) TIME-DEPENDENT SPECIFIED PRESSURES AND TIME-DEPENDENT CONCENTRATIONS OR TEMPERATURES OF INFLOWS AT THESE POINTS

(2) TIME-DEPENDENT SPECIFIED CONCENTRATIONS OR TEMPERATURES

(3) TIME-DEPENDENT FLUIO SOURCES AND CONCENTRATIONS

OR TEMPERATURES OF INFLOWS AT THESE POINTS

(4) TIME-DEPENDENT ENERGY OR SOLUTE MASS SOURCES

SUBR OUTINE BCTIME(IPBE,PBE,IUBC,UBC, QIN,UIN, QUIN,IQSOP,IQSOU,

1 IPBCT,IUBCT, IQSOPT, IQSOUT)

IMPLICIT DOUBLE PRECISION $(A-4,0-Z)$

COMMON/DIMS/ NN,NE,NIN,NBI,NB,NBHALF,NPINCH,NPBC,NUBC,

1 NSOP,NSOU,NBCN

COMMON/TIMEI DELT, TSEZ,TMIN, THOUR, TDAY, TWEEK, TMONTH, TYEAR,

1 TYAX,DELTP,DELTU,DLTPM1, DLTUM1,IT, ITMAX

DIMENSION IPBC (NBCN),PBC (NBCN),IUBC (NBCN),UBC(NBCN),

C

$1 Q I N(N N), U I N(N N), Q U I N(N V), I 2 S O P(N S O P), I Q S O U(N S O U)$

C..... DEFINITION OF REQUIREO VARIABLES

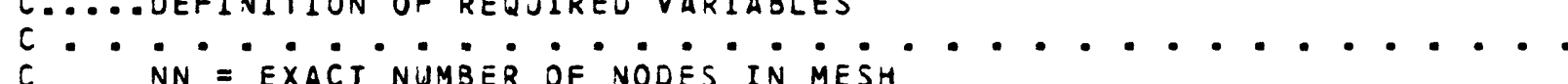

c

NN = EXACT NUMBER OF NODES IN MESH

NPBC = EXACT NUMBER OF SPECIFIED PRESSURE NODES

NUBC = EXACT NUMBER OF SPECIFIED CONCENTRATION OR TEMPERATURE NODES

IT $=$ NUMBER OF CURRENT TIME STEP

TSEC = TIME AT END OF CURRENT TIME STEP IN SECONDS

TMIN = TIME AT ENJ OF CURRENT TIME STEP IN MINUTES

THOUR = TIME AT END OF CURRENT TIME STEP IN HOURS

TOAY = TIME AT END OF CURRENT TIME STEP IN DAYS

TWEEK = TIME AT EVD OF CURRENT TIME STEP IN WEEKS

TMONTH = TIME AT END OF CURRENT TIME STEP IN MONTHS

TYEAZ = TIME AT END OF CURRENT TIME STEP IN YEARS

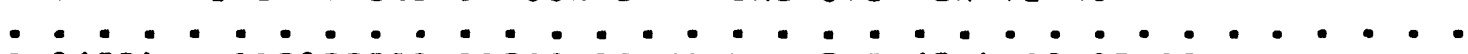

PBC(IP) = SPECIFIED PRESSURE VALUE AT IP(TH) SPECIFIED PRESSURE NOJE

UBC(IP) = SPECIFIED CONCENTRATION OR TEMPERATURE VALUE OF ANY INFLOW OCCURRING AT IP(TH) SPECIFIED PRESSURE NODE

IPBC (IP) = ACTUAL NODE NUMBER OF IP(TH) SPECIFIED PRESSURE NODE [NHEN NODE NUMBER I = IPBC(IP) IS NEGATIVE $(I<0)$, VALUES MUST BE SPECIFIED FOR PBC AND UBC.]

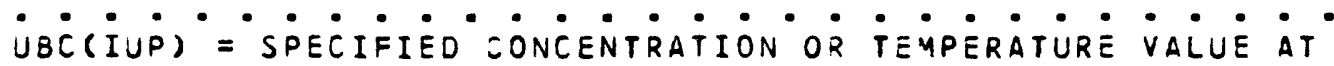
IU(TH) SPESIFIED ZONCENTRATION OR TEMPERATURE NODE (WHERE IUP $=$ IU +NPBC)

IUBC (IUP) = ACTUAL NODE NUMBER OF IU(TH) SPECIFIED CONCENTRATION OR TEYPERATURE NODE (WHERE IUP =IU+NPBC)

[WHEN NODE NUMBER I =IUBC (IU) IS NEGATIVE $(I<0)$,

A VALJE MJST BE SPECIFIED FOR UBC.]

IQSOP $\dot{I Q P})=$ NODE NUMJER OPF IQPP(TH) FLUID SOURCE NODE.

[WHEV NODE NJMBER I=IQSOP(IQP) IS NEGATIVE $(I<O)$,

VALUES MJST $3 E$ SPECIFIEO FOR QIN AND UIN.J

QIN $(-I)=$ SPECIFIED FLUID SOURCE VALUE AT NODE $(-I)$

UIN $(-I)=$ SPECIFI DD LONCENTRATION OR TEMPERATJRE VALJE OF ANY INELOW JCCURRING AT FLUID SOURCE NODE (-I)

N20.....

N30.....

$\mathrm{N} 40 . . .$.

N50.....

N60.....

N70.....

N80.....

N9O.....

N100....

N110....

N120...

N130...

N140....

N150....

N160....

N170....

N1 $80 . .$.

N190....

N200....

N210....

N220....

N230...

N240....

N250....

N260....

N270....

N280...

N290....

N300....

N310....

N320....

N330....

N340....

N350....

N360....

N370....

N380....

N390....

N400....

N410....

N420....

N430....

N440....

N450....

N460....

N470....

N480....

N490....

N500....

N510....

N520...

N530....

N540....

N550....

N560....

N570....

N5 $30 . .$.

N590....

NOOO.... 

ARE SENSITIVE TO $U$ " THEN A FLOW SOLUTION MUST OCCUR AS WELN12OO... 
$c$

UBC (IUP) $=(6$, )

N1210... 400 CONTINUE

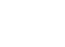

) N1220... c c $c$ c

c

c c

440 IF(I2SOPT) $450,640,640$

- $N 1230 \ldots$

- $N 1240 \ldots$ N1250... N1260... N1270... N1280... N1290... N1300... N1310... C - - - - - - - - - - - - - - - - - - - - - - N N1320... c- - - - - - - - - - - - - - - - - - - - - - - N - - $330 .$. C......SECTION (3): SET TIME-DEPENDENT FLUIO SOURCES/SINKS, N1340... C OR IONCENTRATIONS (TEMPERATURES) OF SOURCE FLUID C

450 CONTINUE DO 630 IQP $=1$, NSOPI $I=I Q S O P(I Q P)$ IF (I) $500,600,600$

\section{CONTINUE}

$c$ NOTE : A TIME STEP IN WHICH QINS, CHANGES.

\section{QIN $(-I)=(6)$}

NOTE : A TRANSPORT SOLUTION MUST OCCUR FOR ANY

C

c

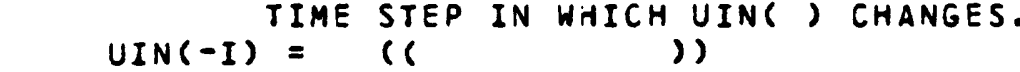

))$$
\text { ST }
$$
N1450... N1460... N1470... N1480... N1490... N1500... N1510... N1520... N1530... N1540... N1550... N1560... N1570... N1580... N1590... N1600... N1610... N1620... N1630... N1640... N1650... N1660... N1670... N1680... N1690... N1700... N1710... N1720... N1730... N1740... N1750... N1760... N1770... N1780... N1790... N1800... 


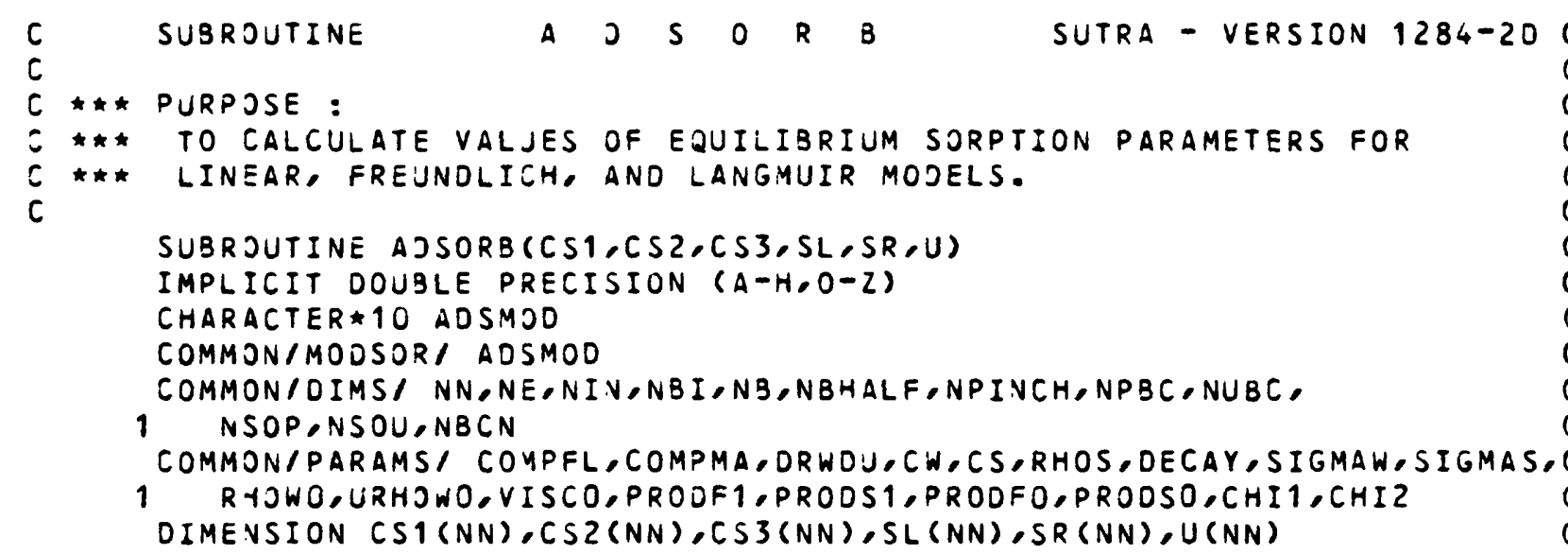




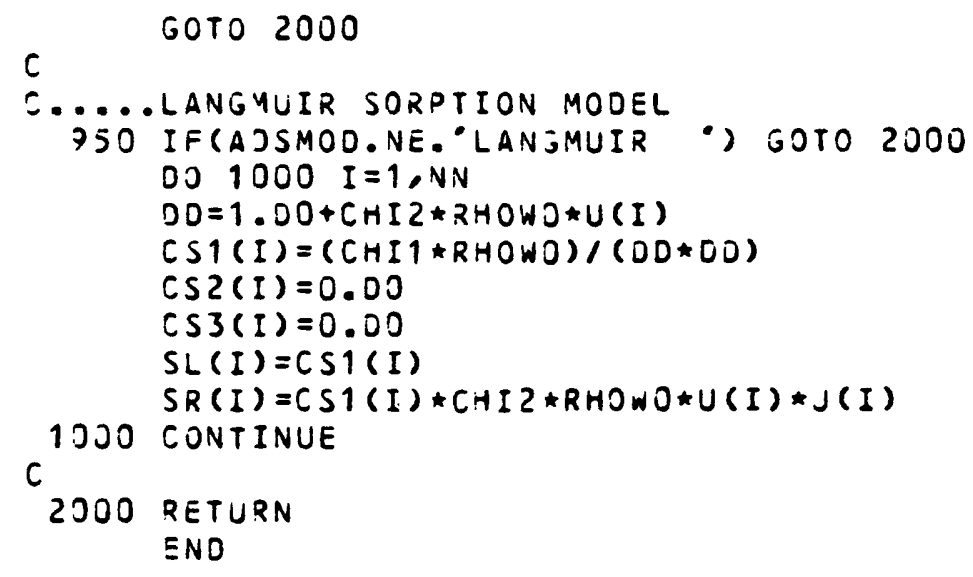

$0610 \ldots$

$0020 \ldots$.

$0630 \ldots$.

$0640 \ldots$.

$0650 \ldots$.

$0660 \ldots$.

$0670 \ldots$.

$0680 \ldots .$.

$0690 \ldots$.

$0700 \ldots$.

$0710 \ldots$.

$0720 \ldots$

$0730 \ldots$

$0740 \ldots$

$0750 \ldots$. 
SUBROUTINE ELEMENCML, IN, X, Y, THICK,PITER, UITER, RCIT,RCITM1,POR,

1 ALMAX,ALMIN,ATAVG,PERMXX,PERMXY,PERMYX,PERMYY,PANGLE,

2 VYAG, VANG, VOL, PMAT, PVEZ, UYAT, UVEC, GXSI, GETA,PVEL)

IMPLICIT DOUBLE PRECISION ( $A-H, O-Z)$

COMMON/OIMS/ NN, NE,NIN,NBI,NB,NBHALF,NPINCH,NPBC,NUBC,

1 NSOP, NSOU,NBCN

COMMON/TENSOR/ GRAVX, SRAVY

$P 20 \ldots . .$.

P30.....

P40.....

$P 50 \ldots . .$.

P60.....

$P 70 \ldots . .$.

P80......

P90.....

P100....

P110....

P $120 \ldots$

P130....

P140....

P150....

P160....

$P 170 \ldots$

COMMON/PARAMS/ COMPFL,COMPMA,DRWDU,CW,CS,RHOS,DECAY,SIGMAW,SIGMAS,P18O...

1 R TOWO,URHJWO, VISCO,PRODF1,PRODS1,PRODFO,PRODSO,CHI1,CHI 2

COMMON/TIME/ DELT,TSEZ,TMIN,THOUR,TDAY,TWEEK, TMONTH,TYEAR,

1 TMAX,DELTP, JELTU,OLTPM1,DLTUM1,IT,ITMAX

COMMON/CONTRL/ GNJ,UP,OTMULT,DTMAX, ME, ISSFLO,ISSTRA,ITCYC,

1 NPCYC, NUCYC, NPRINT, IREAD, ISTORE, NOUMAT, IUNSAT

COMMON/KPRINT/ KNODAL,KELMNT,KINCID,KPLOTP,KPLOTU,KVEL,KBUDG

DIMENSION IN(NIN),X(NV),Y(NN),THICK(NN),PITER(NN),

1 UITER(NN),RCIT(NN),RCITMI (NN),POR (NN),PVEL (NN)

DIMEVSION PERMXX (VE),PERMXY (NE),PERMYX (NE),PERMYY (NE),PANGLE (NE),

1 ALMAX (NE), ALMIN(NE), ATAVG (NE), VMAG (NE), VANG (NE),

2 GXSI (NE, 4), GETA (NE, 4)

DIMEVSION VOL (NN), PMAT (NN,NBI), PVEC (NN),UMAT (NN,NBI),UVEC (NN)

DIMENSION BFLOWE $(4,4)$, DFLOWE (4), BTRANE $(4,4)$, DTRANE $(4,4), V O L E(4)$

DIMEVSION $F(4,4), N(4,4)$, DET $(4), \operatorname{UFOXG}(4,4)$, OFOYG $(4,4)$,

1 DNDXG $(4,4)$, DWDYG $(4,4)$

DIMENSION SWG(4),RHOG (4), VISCG (4),PORG (4),VXG(4),VYG (4),

1 RELKG (4),RGXG(4),RJYG (4),VSMAG (4),THICKG (4)

DIMENSION RXXG(4),RXYj(4),RYXG(4),RYYG(4)

DIMENSION BXXG(4),BXYj(4),BYXj(4),BYYG(4),

1 EXG(4),EYS(4)

DIMENSION GXLOC (4), GYLOC (4)

DATA GLOC/O.57735026918962600/

DATA INTIM/O/,ISTOP/O/.GXLOC/-1.00,1.00,1.00,-1.00/,

C

1 GYLOCI-1.00.-1.00.1.00.1.00/

C.... DECIDE WHETHER TO CALIULATE CENTROID VElocities ON THIS CALL

IVPRVT $=0$

IF (MJO(IT,NPRINT) .EQ.J.AND.ML.NE.2.AND.IT.NE.J) IVPRNT=1

IF(IT.EQ.1) IVPRNT =1

C

KVPR VT =IVPRNT+KVEL

C.... ON FIRST TIME STEP, PREPARE GRAVITY VECTOR COMPONENTS,

C GXSI AND JETA, FOR CONSISTENT VELOCITIES,

ANU CHECK ELEMENT SHAPES

IF (INTIM) $100,100,2000$

100 INTI $M=1$

C..... LOOP THROUGH ALL ELEMENTS TO OBTAIN THE INVERSE JACOBIAN

C AT EACH OF THE FOUR NOJES IN EACH ELEMENT

DO $1300 \mathrm{~L}=1$, VE

DO $500 \quad I L=1,4$

$X L O C=G X L O C(I L)$

$Y L O C=G Y L O C(I L)$

P $200 . .0$

P210....

$P 220 \ldots$

$P 230 \ldots$

$P 240 \ldots$

P250....

P260....

P270....

P280....

P290....

P300....

P310....

P320....

P330...

P340...

P350....

P360....

P370....

P380....

P390....

P400....

P410....

P420....

P $430 \ldots$

P440....

P450....

$P 460 \ldots$

$P 470 \ldots$

P $480 \ldots$

P490....

P500....

P510....

P $520 \ldots$

P530....

P540....

P550...

P560....

P570....

P580....

P570....

P600.... 
CALL BASISZ (COCO,L,XLCC,YLOC,IN,X,Y,F $(1, I L), W(1, I L), D E T(I L)$, OFDXG $(1, I L), D F O Y G(1, I L), O W D X G(1, I L), O W O Y G(1, I L)$, PITER,UITER,PVEL,POR,THICK, THICKG(IL),VXG(IL),VYE(IL), SWG(IL), RHOG(IL), VISCG(IL),PORG(IL),VGMAÜ(IL),RELKG(IL), PERMXX,PERMXY,PERMYX,PERMYY,CJ11,CJ12,CJ21,CJZ2,

GXSI, GETA,RCIT,RCITMI, RGXG(IL), RGYG(IL))

$G X S I(L, I L)=C J 11 \star G R A V X+C J 12 \star E R A V Y$

GETA $(L, I L)=C J 21 \star G R A V X+C J 22 \star G R A V Y$

C..... CHECK FOR NEGATIVE- OR ZERO-AREA ERRORS IN ELEMENT SHAPES

IF (DET(IL)) $200,200,500$

200 ISTOP $=$ ISTOP +1

WRITE $(6,400)$ IN $((L-1) \star 4+I L), L, D E T(I L)$

400 FORMAT $111 \times$. THE DETERMINANT OF THE JACOBIAN AT GAUSS POINT 500 CONTINUE

IN ELEMENT ', I4, IS NEGATIVE OR ZERO, , 1PE15.7)

c

1000 CONTINUE

IF(ISTCP.EQ.O) GOTO 2000

WRITE $(6,1500)$

1500 FORMAT $/ / / / 111 X,-5 O M E$ ELEMENTS HAVE INCORRECT GEOMETRY.-

1 / $111 X, " P L E A S E$ CHECK THE NODE COORDINATES AND ',

- INC:DENCE LIST, MAKE CORRECTIONS, AND THEN RERUN. $11 / 11111$

3 11X, S I M U L A T I ON HA L TE D '

ENDFILE $(\overline{6})$

STOP

$c$

C.....LOOP THROUGH ALL ELEMENTS TO CARRY OUT SPATIAL INTEGRATION

C OF FLUX TERMS IN P AND/CR U EQUATIONS

2000 IF (IUNSAT.NE.0) IUNSAT $=2$

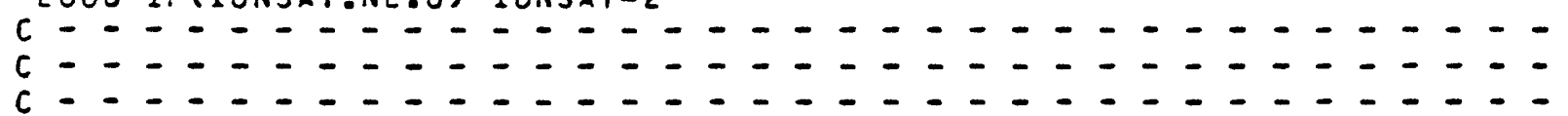

$009999 \mathrm{~L}=1, \mathrm{NE}$

$X I X=-1.00$

$Y I Y=-1.00$

$K G=0$

C.... OSTAIN BASIS FUNCTION AND RELATED INFORMATION AT EACH OF

C FOUR GAUSS POINTS IN THE ELEMENT

DO 2200 IYL $=1,2$

DO 2100 IXL $=1,2$

$X G=K G+1$

$X L O C=X I X \star G L O C$

$Y L O C=Y I Y * G L O C$

CALL BASIS2 $(0001, L, X L O C, Y L O C, I N, X, Y, F(1, K G), W(1, K G), D E T(K G)$,

1

2

3 $X I X=-X I X$ OFEXG $(1, K G), D F C Y G(1, K G), O W D X G(1, K G), O W D Y G(1, K G)$, PITER, UITER, PVEL,POR, THICK, THICKG (KG), VXG (KG), VYG (KG), SWG $(K G), R H O G(K G), V I S C G(K G), P O R G(K G), V G M A G(K G), R E L K G(K G)$, PERMXX,PERMXY, PERMYX,PERMYY,CJ11,CJ12,CJ21,CJ22, $G X S I, G \equiv T A, R C I T, R C I T M 1, R G X G(K G), R G Y G(K G))$

c

C..... CALCULATE VELOCITY AT ELEMENT CENTROID WHEN RECUIREO IF (K VPRNT-2) $3000,2300,3000$

2300 AXSUM $=0.000$

AYSUM $=0.000$

DO $2400 \quad K G=1,4$ $A \times S U M=A \times S U M+V \times G(K G)$

$2400 \quad A Y S U M=A Y S U M+V Y G(K G)$
$P \leqslant 10 \ldots$ PS $20 . \ldots$. $P 630 \ldots$. P640.... P550.... PS60.... FSTD.... PSBO.... $P \leqslant 90 \ldots$. F>00.... P710.... P>20.... , I $4,8730 \ldots$ $p>40 \ldots$. P750.... P760.... P770.... P780.... P790.... P200.... PE $10 \ldots .$. $P \equiv 20 \ldots$. P3 $30 \ldots$. $P ح 40 \ldots$. $P \geqq 50 \ldots$ P\&50.... P870.... P\&80.... $P \& 90 \ldots$. $P=00 \ldots$. P710.... $P 720 \ldots$ P930.... PQ40... $P 950 \ldots$. P960.... P $70 \ldots$. P780.... P790.... P1000... P101C... P1C20... P1C3C... P104C... P105C... P106C... F1O7C... P1080... P1C9C... F1100... P111C... P1120... P1130... P1140... P1150... P1160... P1170... P1180... P119C... P1200... 


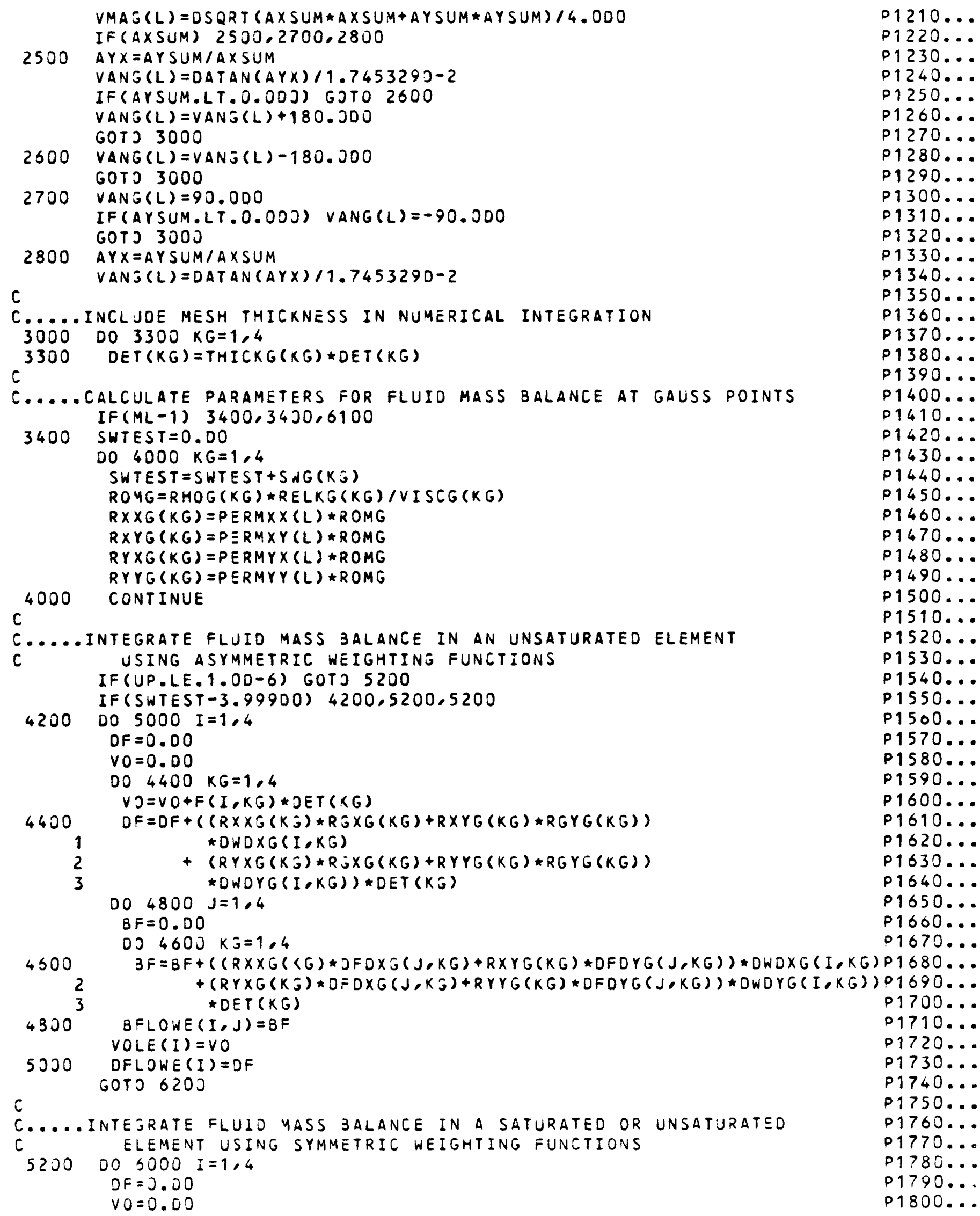




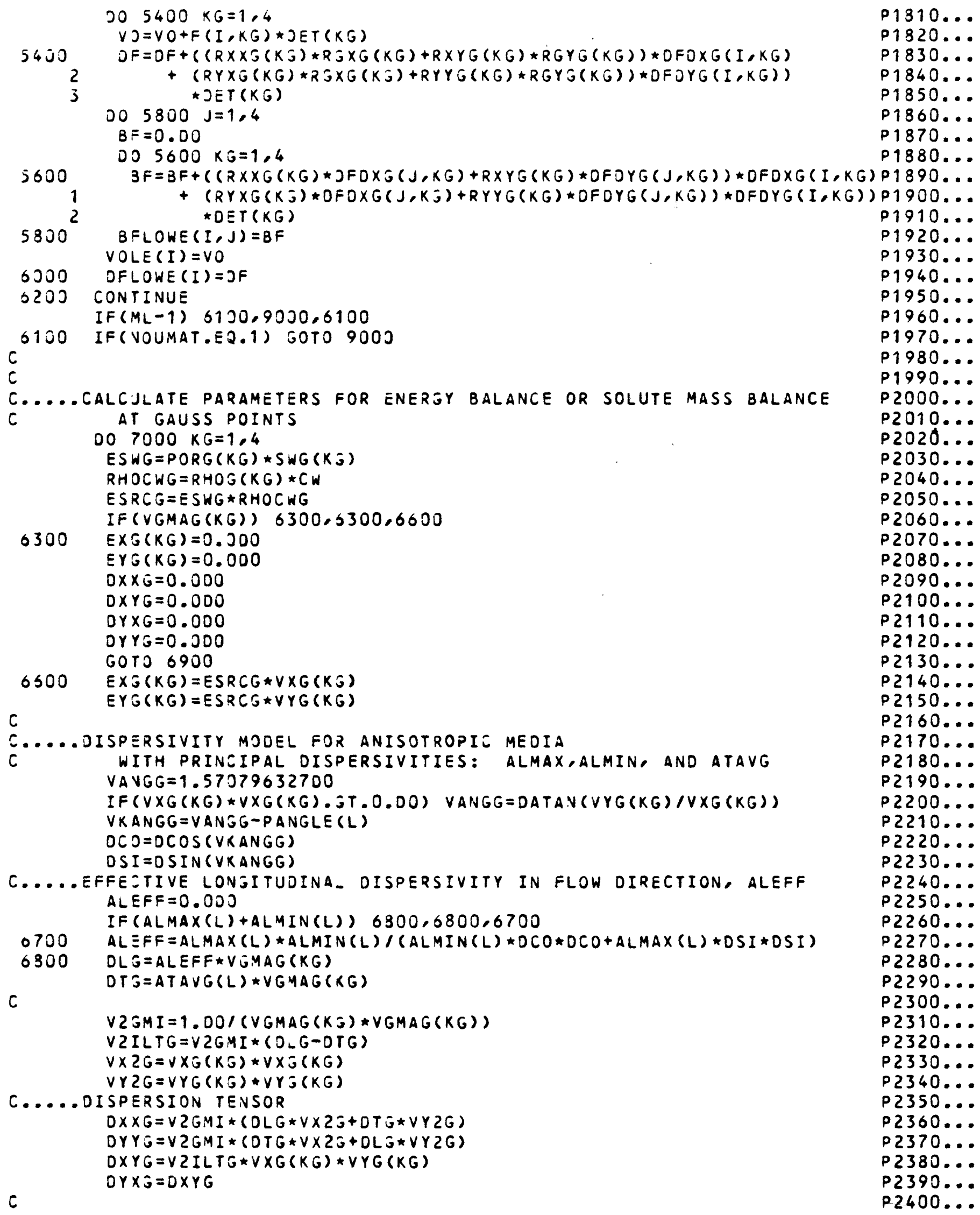


C..... IN-PARALLEL CONOUETIVITIES (OIFFUSIVITIES) FORMULA

6900 ESE =ESRCG*SIGMAW+(1.DO-PORG (KG))*RHOCWG*SIGMAS

C.....ADD JIFFUSION AND DISPERSION TERMS TO TOTAL OISPERSION TENSOR $B \times X G(K G)=E S R C G \star J \times X G+E S E$

$B \times Y G(K G)=E S R C G \star J X Y G$

$B Y X G(K G)=E S R C G * D Y \times G$

7000 BYYG(KG) $=E S R C G \star J Y Y G+E S E$

c

C..... INTEjRATE SOLUTE MASS BALANCE OR ENERGY BALANCE

C USING SYMMETRIC WEIGHTING FUNCTIONS FOR DISPERSION TERM AND

C USING EITHER SYMMETRIC OR ASYMMETRIC WEIGHTING FUNCTIONS

C FOR AOVECTION TERM

$008000 \quad I=1.4$

$008000 \mathrm{~J}=1,4$

$B T=0.00$

$D T=0.00$

DO 7500 KG $=1,4$

P2410...

P2420...

P2430...

P2440...

$P 2450 \ldots$

P2460...

P2470...

P2480...

P2490...

P2500...

P2510...

P2520...

P2530...

P2540...

P2550...

P2560...

$B T=B T+((3 \times X G(K G) \star D F D X G(J, K G)+B \times Y G(K G) \star D F D Y G(J, K G)) \star D F D \times G(I, K G) P 2580 \ldots$

$+(B Y X G(K S) \star D F D X G(J, K G)+B Y Y G(K G) \star D F D Y G(J, K G)) \star D F D Y G(I, K G)) P 2590 \ldots$ $\star D E T(K G)$ $7500^{2} \quad D T=D T+(E X G(K G) \star D F J X G(J, K G)+E Y G(K G) \star D F O Y G(J, K G))$

$P 2600 \ldots$

BTRANE $(I, J)=B T$

P2610...

P2620...

P2630...

8000 DTRANE $(I, J)=0 T$

9000 CONTINUE

$c$

c

C..... SEND RESULTS OF INTEGRATIONS FOR THIS ELEMENT TO

c

GLOBAL ASSEMBLY ROJTINE

9999 CALL GLOBANCL,ML, VOLE, BFLOWE, OFLOWE, BTRANE, DTRANE,

P2640...

P2650...

P2660...

P2670...

P2680...

P2690...

P2700...

1 IN,VOL,PMAT,PVEC OJMAT, UVEC)

P2710...

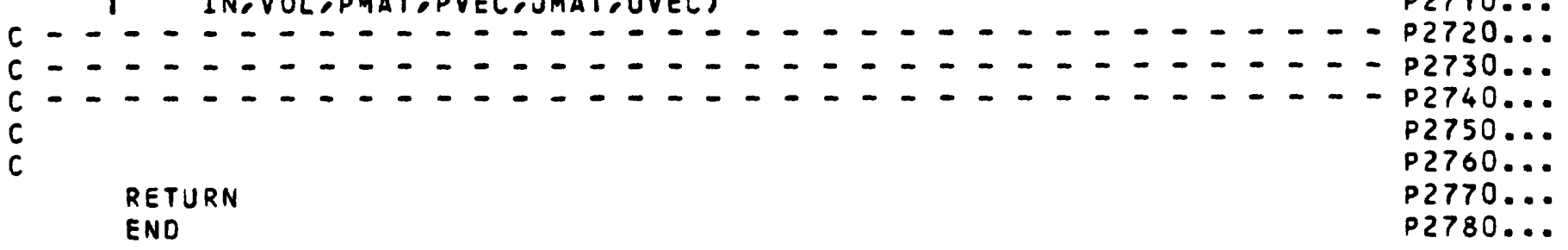




$C$ SUBROUTINE
$C$

SUEROUTINE BASIS2(ICALL,L,XLOC,YLOC,IN, X,Y,F,W,DET,

1 DFOXG,DFOYG,OWOXG,OWOYG,PITER,UITER,PVEL,POR, THICK, THICKG,

$2 V X G, V Y G, S W G, R H O G, V I S C G, P O R G, V G M A G, R E L K G$,

3 PERMXX,PERMXY,PERMYX,PERMYY,CJ11, CJ12,CJ21,CJ22,

4 GXSI, GETA,RCIT,RCITMI,RGXG,RGYG)

IMPLICIT COUBLE PRECISION (A-H,C-Z)

COMMON/OIMS/ NN, NE, NIN,NBI, NB, NEHALF, NPINCH,NPBC,NUBC,

1 NSOP,NSOU,NBCN

COMMON/CONTRL/ GNU,UP,CTMULT, DTMAX, ME, ISSFLO,ISSTRA, ITCYC,

1 NPCYC, NUCYC, NPRINT, IREAD, ISTORE, NOUMAT, IUNSAT

COMMON/SATPARI PCENT, SWRES, PCRES, SSLOPE, SINCPT

COMMON/PARAMS/ COMPFL, COMIPMA, ORWDU,CH,CS, RHOS, DECAY, SIGMAW, SIGMAS,

1 RHOWO,URHOWO, VISCO,PRODF1, PRODS1, PRODFO, PRODSO, CHI1, CHI2

COMMON/TENSOR/ GRAVX,GRAVY

DOUBLE PRECISION XLOC, YLOC

OI YENSION IN (NIN), X (NN), Y (NN), UITER (NNS,PITER (NN), PVEL (NN),

1 POR (NN), PERMXX (NE), PERMXY (NE), PERMYX (NE),PERMYY (NE), THICK (NN)

DIMENSION GXSI (NE, 4), GETA (NE, 4), RCIT (NN), RCITMI (NN)

DIMENSION $F(4), W(4), D F D X G(4)$, OFCYG (4), OWDXG (4), DNDYG (4)

DIMENSION FX (4),FY(4),AFX(4),AFY (4),

1 DFDXL (4), OFOYL (4), DWDXL(4), OWDYL (4),

$2 X O H(4), Y D W(4), X I I X(4), Y I I Y(4)$

OATA XIIXI-1.00,+1.00,+1.00,-1.001,

$c$

$1 Y I I Y /-1.00,-1.00,+1.00,+1.00 /$

c

C.....AT THIS LOCATION IN LOCAL COOROINATES, (XLOC, YLOC),

C CALCULATE SYMMETRIC WEIGHTING FUNCTIONS, $F(I)$,

C SPACE DERIVATIVES, DFOXG(I) AND DFOYG(I), AND

C DETERMINANT OF JACOBIAN, DET.

C

$X F 1=1.00-X L O C$

$X F 2=1.00+X L O C$

$Y F 1=1.00-Y L O C$

C

$Y F Z=1.00+Y L O C$

C......CALCULATE BASIS FUNCTION, F.

$F X(1)=X F 1$

$F X(2)=X F 2$

$F X(3)=X F 2$

$F X(4)=X F 1$

$F Y(1)=Y F 1$

$F Y(2)=Y F 1$

$F Y(3)=Y F 2$

$F Y(4)=Y F 2$

DO $10 \quad I=1,4$

C

$10 F(I)=0.25000 \star F X(I) \star F Y(I)$

C..... CALCULATE DERIVATIVES HITH RESPECT TO LOCAL COORDINATES. DO $20 \quad I=1,4$ OFDXL $(I)=X I I X(I) * 0.25000 * F Y(I)$

20 OFDYL $(I)=Y I I Y(I) * 0.25000 * F X(I)$ 
C SUBROUTINE $\quad$ B A S I S 2 SUTRA - VERSION 1284-20 Q10....

C

C...... CALCULATE ELEMENTS of jaCosian matrix, CJ.

CJ11 $=0.00$

$\operatorname{cs} 12=0.00$

$\operatorname{cs} 21=0.00$

$C J 22=0.00$

DO $100 \quad I L=1,4$

$I I=(L-1) * 4+I L$

$I=I N(I I)$

CJ11 $C J 11+C F D X L(I L) \star X(I)$

$C J 12=C J 12+C F D X L(I L) * Y(I)$

$C J 21=C J 21+D F D Y L(I L) * X(I)$

c

$100 C J 22=C J 22+D F D Y L(I L) * Y(I)$

C..... CALCULATE OETERMINANT OF JACOBIAN MATRIX.

$D E T=C J 11 \star C J 22-C J 21 * C J 12$

c

C.....RETURN TO ELEMEN WITH JACOBIAN MATRIX ON FIRST TIME STEP. IF(ICALL.EQ.O) RETURN

c

C...... CALCulate elements of inVerse jacobian matrix, CiJ. ODET $=1.00 / D E T$

CIJ11 $=+00 E T \star C J 22$

CIJ12 $=-O D E T \star C J 12$

$C I J 21=-0 D E T \star C J 21$

C CIJ $22=+00 E T \star C J 11$

C...... CALCULATE DERIVATIVES WITH RESPECT TO gLOBal COOROINATES DO $200 \quad I=1,4$ OFDXG(I) $=$ CIJ11*DFOXL (I) +CIJ1 2*CFDYL(I)

c

200 DFDYG $(I)=C I J 21 * C F D X L(I)+C I J 22 * C F D Y L(I)$

C.... CALCULATE CONSISTENT COMPONENTS OF (RHO\#GRAV) TERM IN LOCAL

C COOROINATES AT THIS LCCATION, (XLOC,YLOC)

$R G \times L=0.00$

$R G Y L=0 . D O$

$R G \times L M 1=0.00$

RGYLM1 $=0.00$

DO $300 \quad I L=1,4$

$I I=(L-I) * 4+I L$

$I=I N(I I)$

$A D F O X L=D A B S(D F O X L(I L))$

$A D F D Y L=D A B S$ (DFOYL(IL))

$R G X L=R G X L+R C I T(I) \approx G \times S I(L, I L) \star A D F D \times L$

$R G Y L=R G Y L+R C I T(I) \star G E T A(L, I L) \star A C F D Y L$

$R G X L M 1=R G X L M 1+R C I T M I(I) * G X S I(L, I L) * A D F O X L$

$R G Y L M 1=R G Y L M 1+R C I T M 1(I) * G E T A(L, I L) * A D F D Y L$

BOO CONTINUE

C

C..... TRANSFORM CONSISTENT COMPONENTS OF (RHO*GRAV) TERM TO

C GLOEAL COOROINATES

$R G \times G=C I J 11 * R G X L+C I J 12 \star R G Y L$

$R G Y G=C I J 21 * R G X L+C I J 22 * R G Y L$

$R G X G M 1=C I J 11 * R G X L M 1+C I J 12 \star R G Y L M 1$

$c$

$R G Y G M 1=C I J 21 * R G X L M 1+C I J 22 \star R G Y L M 1$

C..... Calculate pafameter values at this location, (Xloc,yloc)

PITERG $=0.00$

UITERG $=0.00$

$6510 \ldots$

cs20....

$6630 \ldots$.

$0640 \ldots$.

$0650 \ldots$.

$0 \leq 50 \ldots$.

G670....

$0520 \ldots .$.

$0590 \ldots$.

Q700....

$6710 \ldots$.

c7?2....

Q $730 . .$.

$6740 . \ldots$

c750....

$6760 \ldots$

Q770....

$6780 \ldots$.

$0790 \ldots$.

C800....

C310....

$6 \equiv 20 \ldots$

$5830 \ldots$.

$6 \equiv 40 \ldots$.

QE50....

$6560 \ldots$.

cद>⿰丨....

6.8ิ80....

Q990....

$6700 \ldots$

$0 \geqslant 10 . .$.

$6920 \ldots$.

$6930 . .$.

$6940 . .$.

$6950 . .$.

$0960 \ldots$

Q970....

c\$30....

c390....

Q100C...

0101C...

61020...

Q103C...

Q104C...

61050...

Q1060...

Q107C...

Q1080...

$61090 .$.

$\$ 1100 \ldots$

6111C...

Q1120...

c1130...

6114C...

61150...

Q1160...

61170...

61150...

Q1170...

$6120 \mathrm{C} \ldots$ 


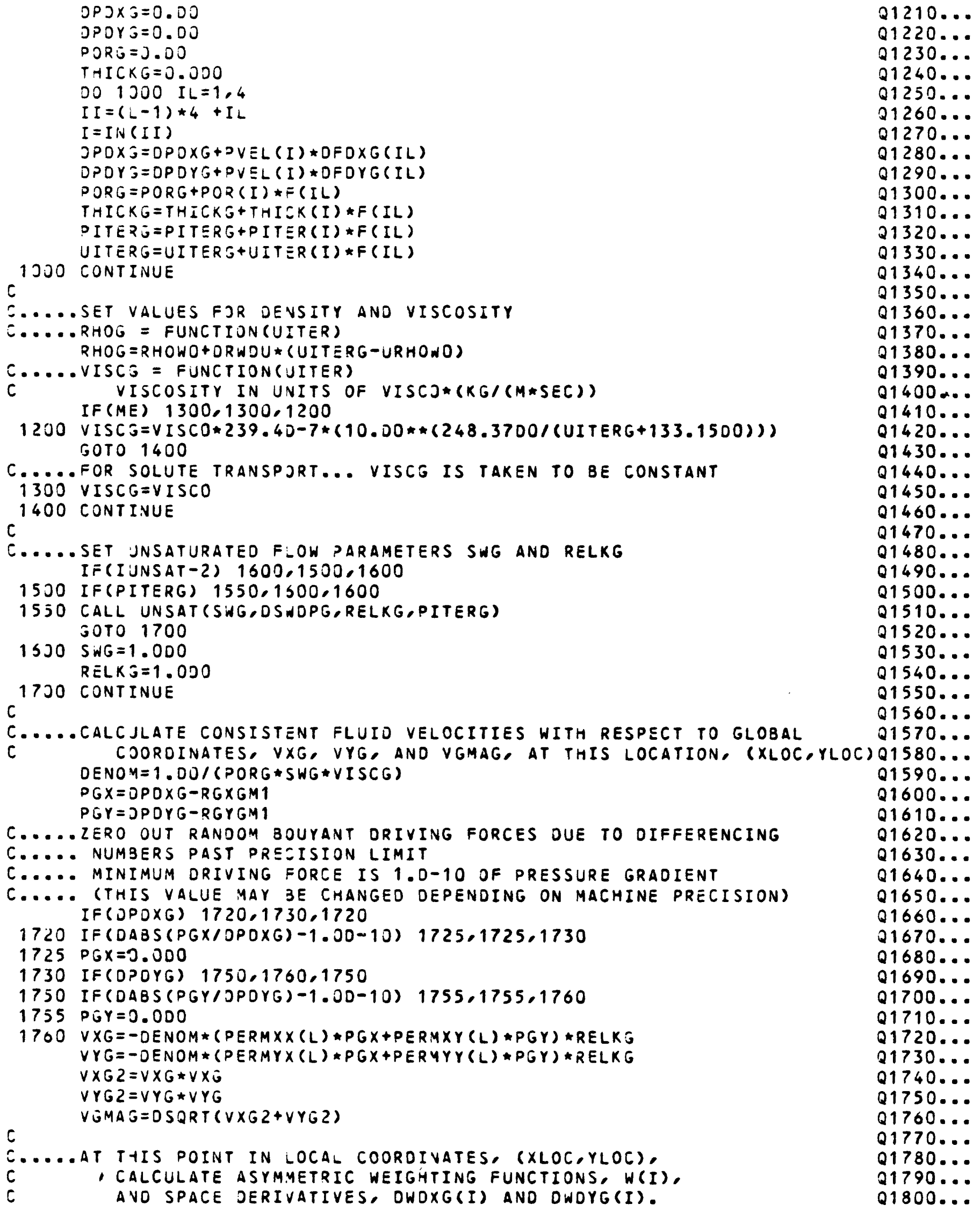


C SUBRTUTINE B A S I S 2 SUTRA-VERSION 1284-20 Q10....

C

C.....ASYMUETRIC FUNCTIONS SIMPLIFY WHEN UP=0.0

IF(UP.GT.1.OJ-6.AVD.NJUMAT.EQ.O) GOTO 1790

DO $1780 \quad I=1,4$

$W(I)=F(I)$

$D W D X J(I)=D F D \times G(I)$

DWDY $S(I)=D F O Y G(I)$

1780 CONTINUE

C..... RETURN WHEN JNLY SYMMETRIC WEIGHTING FUNCTIONS ARE USED RETURN

$\mathrm{C}$

C..... Calc Jlate fluid velocities with respect to local coOrdinates,

C..... VXL, VYL, ANO VLMAG, AT THIS LOCATION, (XLOC,YLOC).

$1790 \vee X L=C I J 11 * V \times G+C I J 21 * V Y G$

$V Y L=C I J 12 \star V X G+C I J 22 \star V Y G$

C

VLMAG =DSQRT $(V X L \star V X L+V Y L \star V Y L)$

$A A=0.000$

$B 3=0.000$

IF (VLMAG) $1900,1900,1300$

$1800 \quad A A=U P \star V X L / V L M A G$

C

$B B=U P * V Y L / V L M A G$

$1900 \times I X I=.75000 * A A \star X F 1 \star X F 2$

$Y I Y I=.75000 * 8 B * Y F 1 * Y F 2$

$002000 \quad I=1,4$

$A F X(I)=.5000 * F X(I)+X I I X(I) * X I X I$

$2000 A F Y(I)=.5000 \star F Y(I)+Y I I Y(I) \star Y I Y I$

C

C..... CALCJLATE ASYMMETRIC WEIGTTINJ FUNCTION, W.

DO $3000 \quad I=1,4$

$3000 W(I)=A F X(I) \star A F Y(I)$

c

THAAX $=0.50 D 0-1.50 J 0 \star A A \star X L O C$

$T H B B Y=0.5000-1.50 J 0 * B B * Y L O C$

$004000 \quad I=1.4$

$X O W(I)=X I I X(I) \star T H A A X$

4000 YOW (I) $=$ YIIY (I)*TH3BY

C

C..... CALCJLATE DERIVATIVES WITH RESPECT TO LOCAL COOROINATES. DO $5 J 00 \quad I=1.4$

DWDXL (I) $=X D W(I) \star A F Y(I)$

5000 DWDYL $(I)=Y O W(I) * A F X(I)$

c

C..... Calculate oerivatives with respect to global coOrdinates. DO $6 J 00 \quad I=1,4$

$D N D X G(I)=C I J 11 \star D W J X L(I)+C I J 12 \star D W D Y L(I)$

6000 DWDYG(I) $=C I J 21 * D W D X L(I)+C I J 22 * D W D Y L(I)$

$c$

$c$

RETURN

END 
(3) RELATIVE PERMEABILITY AS A FUNCTION OF EITHER

PRESSURE OR SATURATION (REL(PRES) OR RELK(SW) )

code between dasted lines must be replaced to give the PARTICULAR UNSATJRATED RELATIONSHIPS DESIREO.

SUBR JUTINE UNSAT (SW,DSWDP, RELK, PRES)

IMPLICIT DOUBLE PRECISION (A-H,O-Z)

COMMON/CONTRL/ GNJ,UP,DTMULT, DTMAX, ME, ISSFLO, ISSTRA, ITCYC, NPCYC, NUCYC, NPRINT, IREAD, ISTORE, NOUMAT, IUNSAT

R20.....

R30.....

R40.....

RSO.....

R60.....

R70.....

R80.....

R90.....

$R 100 \ldots$.

R110....

R120....

$R 130 \ldots$.

R140....

R150....

R160....

R170....

R180....

R190....

THREE PARAMETERS FOR JNSATURATED FLOW RELATIONSHIPS OF

VAN SENUCHTEV (198J)

RESIDUAL SATURATION, SWRES, GIVEN IN UNITS [L*\#O]

PARAMETER, AA, GIVEN IN INVERSE PRESSURE UNITS [m*(5**2)/kg] PARAMETER, VN, GIVEN IN UNITS [L**O]

c

DATA SWRESIO.3000/,

$A A / 5.00-51 \%$ VN/2.0001

$-R 200 \ldots$.

R210....

$R 220 \ldots \ldots$

$R 230 \ldots$.

$R 240 \ldots$.

R250....

$R 260 \ldots$

C

$c$

c

\section{$c$}

c

C......SECTION (1):

C SW VS. PRES (VALUe ialCulated ON EACH CALl TO UNSAT)

C CODING MUST Give a Va-Ue to SaTURATION, SW.

$c--$

THREE PARAMETER MODEL OF VAN JENUCHTEN(1980)

SWRM $1=1 . D 0-S W R E S$

$\triangle A P V N=1.00+(A A \star(-P R E S)) \star \star V N$

$V N F=(V N-1 . D 0) / V N$

$\triangle A P V N N=A A P V N * \star V N F$

$S W=$ SWRES+SNRM1/AAPVNN

$R 270 \ldots . .$.

$R 280 \ldots$.

$R 290 \ldots .$.

$R 300 \ldots$.

R310....

$R 320 \ldots$.

$R 330 \ldots$.

$\star \star \star R 340 \ldots \ldots$

-

$R 360 \ldots$

R370....

$R 380 \ldots$.

$R 390 \ldots$.

R400....

R410....

$R 420 \ldots .$.

$R 430 \ldots$.

$R 440 \ldots .$.

$R 450 \ldots$.

$R 460 \ldots$.

$c$

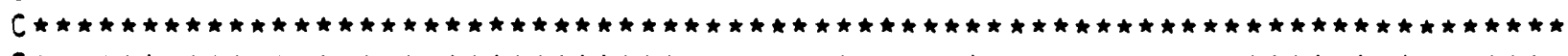

C

$c$

C

i

IF(IJNSAT-2) $600,1200,1800$

$R 470 \ldots$.

$R 480 \ldots$ RSO0.... RS10.... RS20.... RS 30 .... R540.... RS50.... $R 560 . \ldots$

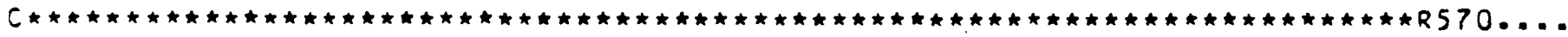

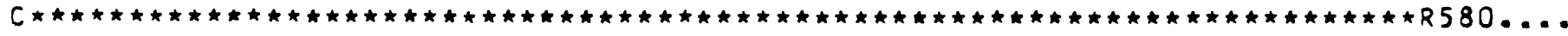
C.......SECTION (2): 
C CODIVG MUST jIVE a VALUe to jerivative of saturation with

C RESPECT TO PRESSURE, DSWDP.

$c$

6OO CONTINUE

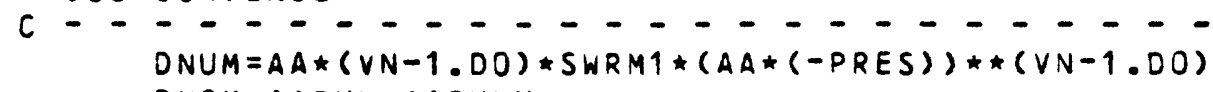

$D N O M=A A P V N \star A A P V N N$

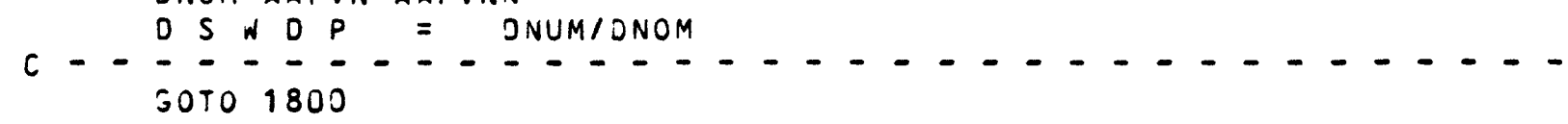

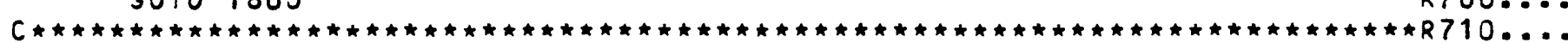

C

C

$c$

c

c

c

$c$

$R 730 \ldots$

$R 740 \ldots$

$R 750 \ldots$

$R 760 \ldots$.

$R 770 \ldots$.

$R 780 \ldots$.

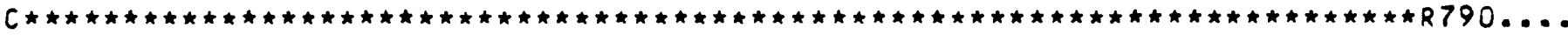

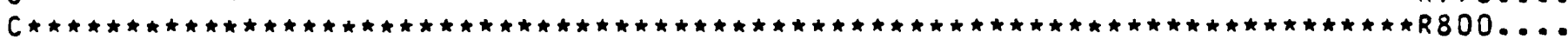

C......SECTION (3):

C RELK VS. P, OR RELK VS. SW (CALCULATED ONLY WHEN IUNSAT=2)

C CODING MUSt GIVE a VALUE tO RELATIVE PERMEABILITY, RELK.

c

1200 CONTINUE

C-- - - - - - - - - - - - - - - - - - - - - - - - - - - - - - - - - - - - - - SWSTAR $=(S W-S W R E S) /$ SWR 1

RELK = OSQRT (SWSTAR)*

1

$(1 . D 0-(1 . D O-S W S T A R \star \star(1 . D O / V N F)) \star \star(V N F)) \star \star 2.00$$$
c
$$$$
c
$$

C

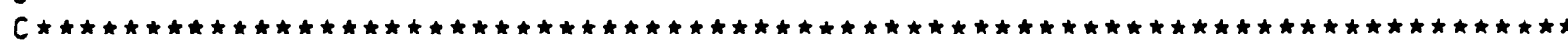
$c$

$c$

$C$

c

c

C

1300 RETURN

c

END $R 810 \ldots$. R820.... $R 830 \ldots$. $R 840 \ldots$. R850.... R860.... $R 870 \ldots$. R8BO.... $R 890 \ldots .$. $R 900 \ldots .$. R910.... R920.... $R 930 \ldots$. R940.... $R 950 \ldots$. R960.... $R 970 \ldots$. R930.... R990.... R1000... R1010... R1020... R1030... 


$$
\text { SUBRJUTINE G L } O B \text { B A }
$$

C

$N 1=(L-1) * 4+1$

$N 4=N 1+3$

$c$

C.....ADD RESULTS OF INTEGRATIONS OVER ELEMENT L TO GLOBAL

C P-MATRIX AND P-VECTOR

IF (ML-1) 50.50 .150

50 IE $=0$

DO 1 J J II $=\mathrm{N} 1, \mathrm{~N} 4$

$I E=I E+1$

$I B=I V(I I)$

VOL $(I B)=V O L(I B)+V O L E(I E)$

PVEC (IB) =PVEC (IB) + DFLOWE (IE)

$J E=0$

DO $100 \mathrm{JJ}=\mathrm{N} 1, \mathrm{~N} 4$

$J E=J E+1$

$J B=I N(J J)-I B+N B H A L F$

$100 \operatorname{PMAT}(I B, J B)=P M A T(I B, J B)+B F L O W E(I E, J E)$

IF $(M L-1) 150,300,150$

$c$

C.....ADD RESULTS OF INTEGRATIONS OVER ELEMENT L TO GLOBAL

C U-MATRIX

150 IF(NOUMAT.EQ.1) GOTO 300

$I E=0$

DO $200 \quad I I=N 1, N 4$

$I E=I E+1$

$I B=I N(I I)$

C.....POSITION FOR ADDITION TO U-VECTOR

$J E=0$

DO $230 J J=N_{1}, N_{4}$

$J E=J E+1$

$J B=I V(J J)-I B+N B H A L F$

c

200 JMAT (IB,JB) =UMAT (IB,J3) +OTRANE (IE, JE) +BTRANE (IE, JE)

c

300 CONTINUE

RETURN

END 


$C$ SUBROUTINE
$C$ N

SUBROUTINE NODAL3 (ML, VOL,PMAT,PVEC,UMAT,UVEC,PITER,UITER,PMI,UM1,

1 UM2,POR,QIN,UIN,QUIN,CS1,CS2,CS3,SL,SR,SW,DSWDP,RHO,SOP)

IMPLICIT DOUBLE PRECISION $(A-1,0-Z)$

COMMON/DIMS/ NN,NE,NIN,NBI,NB,NBHALF, NPINCH,NPBC,NUBC,

1 NSOP,NSOU,NSCN

COMMON/TIME/ DELT, TSEE,TMIN, THOUR, TDAY,TWEEK, TMONTH, TYEAR,

1 TMAX,OELTP,OELTU,OLTPMI,DLTUM1,IT, ITMAX

COMMON/PARAMS/ COMPFL,COMPMA,ORWDU.CH,CS,RHOS, DECAY,SIGMAW,SIGMAS,

1 R HOWO, URHOWO, VISCO,PROJF 1 , PRODS1 , PRODFO,PRJOSO,CHI 1 , CHI 2

COMMON/SATPARI PCENT, SWRES, PCRES, SSLOPE, SINCPT

COMMON/CONTRL/ GNU.UP,DTMULT,DTMAX,ME,ISSFLO,ISSTRA,ITCYC,

1 NPCYC,NUCYC, NPRINT, IREAD, ISTORE, NOUMAT, IUNSAT

OIMEVSION VOL (NN), PMAT (NN,NBI), PVEC (NN),UMAT (NN,NBI),UVEC (NN)

DIMEVSION PITER (NV),UITER (NN),PM1 (NN),UM1 (NN),UMZ (NN),

1 PJR (NN), QIN (NN),UIN (NN),QUIN(NN),CS1(NN),CS2(NN), CS3(NN),

$c$

$2 \quad S L(N N), S R(N N), S W(N N), R H O(N V), O S H O P(N N), S O P(N N)$

C$$
\text { C }
$$

C.... SET

IF (IUNSAT . NE . O) IJNSAT $=1$

....DO NOT UPDATE NODAL PARAMETERS ON A TIME STEP WHEN ONLY U IS SOLVEJ FOR BY BACS SUBSTITUTION (IE: WHEN NOUMAT=1)

IF (NJUMAT) 5J,50,200

50 OO $120 \quad I=1, N N$

IF(IUNSAT-1) $120,100,120$

100 IF(PITER(I)) 110.120 .120

110 CALL UNSATCSW(I),DSWOP(I),RELK,PITER(I))

120 CONTINUE

C..... SET FLUID DENSITY AT NODES, RHO(I)

C RHO = F (UITER(I))

DO $150 \quad I=1, N N$

150 RHO(I) $=$ RHOWO+DRWOU* (UITER(I)-URHOWO)

c

200 CONTINUE

DO $1300 \quad I=1, N N$

$S W R H O N=S W(I) \star R H O(I)$

C

IF $(M L-1) \quad 220,220,230$

$c$

C...... CALCJLATE CELLWISE TERMS FOR P EQUATION

C.....FOR STEADY-STATE FLOW, ISSFLO=2; FOR TRAVSIENT FLOW, ISSFLO=O

220 AFLN $=(1-I S S F L O / 2)$ *

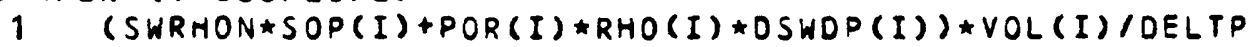

$C F L N=P O R(I) * S W(I) * O R W J U * V O L(I)$

DUDT $=(1-I S S F L O / 2) *(U M 1(I)-U M 2(I)) / D L T U M I$

$C F L N=C F L N \star D U O T$

C.....ADD EELLWISE TERMS ANO FLUID SOURCES OR FLUXES TO P EQUATION

PMAT (I,NBHALE) = PMAT (I,N3HALF) + AFLN

$P V E C(I)=P V E C(I)-C F L N+A F L N \star P M I(I)+2 I N(I)$
T10.....

T20....

T30.....

T40....

T50....

T60.....

T70.....

T80.....

T90.....

T100....

T110...

T120...

T130....

T140....

r150....

T160....

T170....

T180....

T190....

T200....

T210....

T220....

T230....

T240....

$1250 \ldots .$.

T260...

T270....

T280....

T290....

T300....

T310....

T320....

T330....

T340....

T350....

T360....

T370....

T380....

T390....

T400....

T410....

T420...

$T 430 \ldots$.

T440....

$T 450 \ldots$

T460....

$1470 \ldots$.

T480....

T490....

T500....

TS10....

T520....

T530...

T540....

T550....

T560...

T570....

T580....

T590....

1600.... 


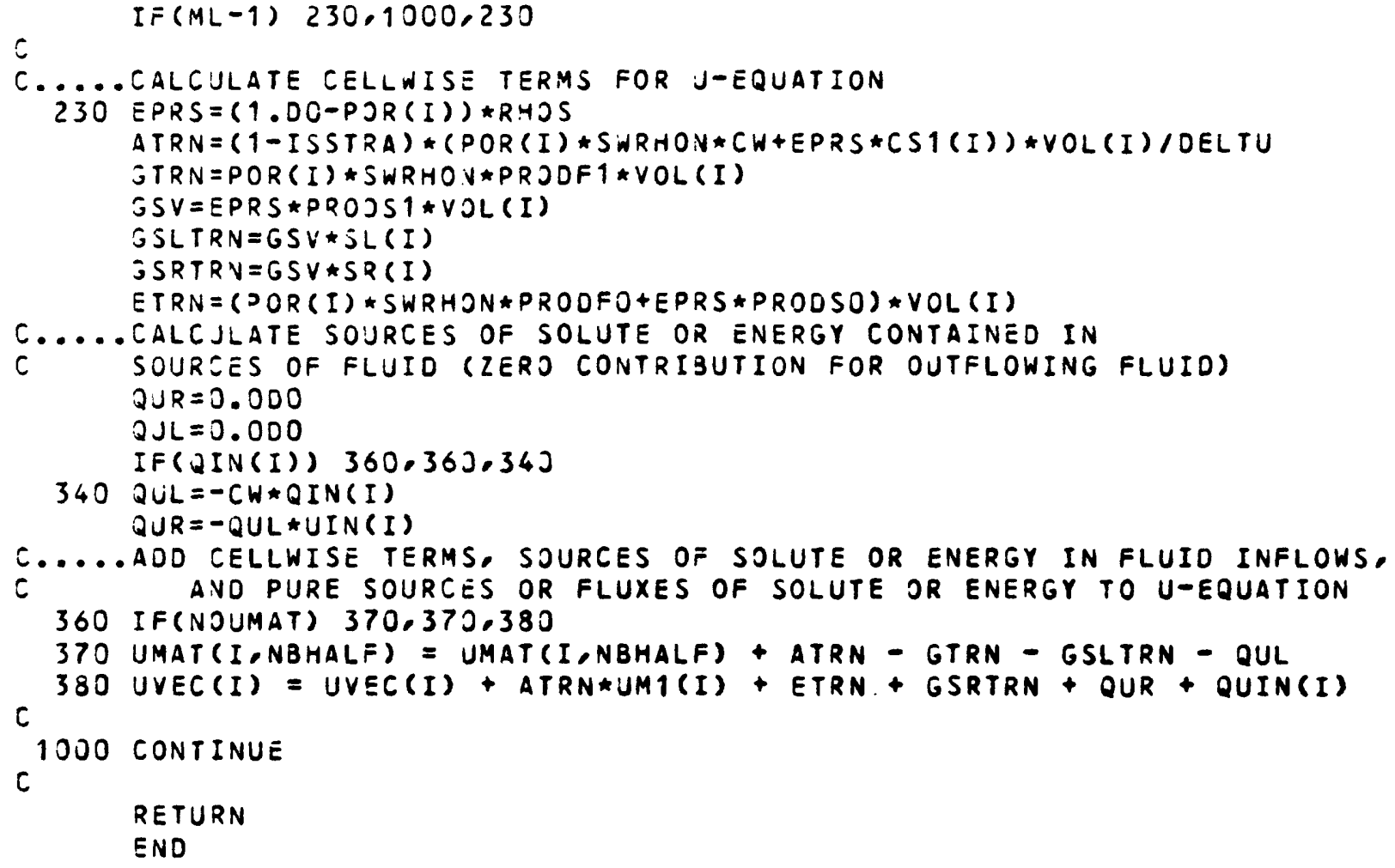

T610.... T620....

T630....

T640....

T650....

T660....

T670....

T680....

T690....

T700....

T710....

T720....

T730...

T740....

T750....

T760....

T770....

T780....

T790....

T800....

T810....

T820....

T830...

T840....

T850....

T860....

T870.... 


$$
\text { SUBRJUTINE } B \quad C \text { B }
$$

SUTRA - VERSION $1284-20$ U10....

SUBR JUTINE BCB (ML,PMAT, PVEC, UMAT, UVEC, IPBC,PBC,IUBC,UBC, QPLITR) IMPLICIT DOUBLE PRECISION $(A-H, O-Z)$

COMMON/DIMS/ NN,NE,NIV,NBI,N3,NBHALF,NPINCH,NPBC,NUBC,

1 NSOP,NSOU.NBCN

COMMON/TIME/ DELT, TSEC,TMIN, THOUR, TDAY, TWEEK, TMONTH, TYEAR,

1 THAX, OELTP, DELTU,OLTPM1, OLTUM1,IT , ITMAX

U20....

U30.....

$440 \ldots \ldots$

U50.....

U60.....

$470 \ldots \ldots$

$480 \ldots . .$.

490.....

U100....

U110....

U120...

U130....

COMMON/PARAMSI COMPFL,COMPMA,DRWDU,CW,CS,RHOS,DECAY,SIGMAW,SIGMAS,U14O....

1 RHOWO,JRHOWO,VISCO,PROJF1,PRODS1,PRODFO,PRODSO,CHI $1, C H I 2$

COMMON/CONTRL/ GNJ UUP,DTMULT, DTMAX,ME, ISSFLO,ISSTRA,ITCYC,

1 NPCYC,NUCYC,NPRINT, IREAD, ISTORE,NOUMAT, IUNSAT

DIMENSION PMAT (NN, NBI), PVEC (NN), UMAT (NN,NBI) ,UVEC (NN),

1 IPBC(NBCN),PBC (NBCV), IUBC (NBCN),USC(NBCN),2PLITR(NBCN)

$c$

IF (NPBC.EQ.O) GOTO 1050

C......SPECIFIED P BOUNDARY GONDITIONS

$D O 1 J 00 \quad I P=1, N P B C$

$I=I A B S(I P B C(I P))$

C

IF (ML-1) $100,100,200$

C..... MODIFY EQUATION FOR P BY ADOING FLUID SOURCE AT SPECIFIED

C

100 GINL $=-G N U$ PRESSURE NODE

GINR $=G N U \star P B C(I P)$

PMAT (I,NBHALF) =PMAT (I,NBHALF) - GINL

C

$\operatorname{PVEC}(I)=\operatorname{PVEC}(I)+G I N R$

IF (ML-1) $200,1000,200$

C..... MODIFY EQUATION FOR U BY ADOING U SOUREE WHEN FLUID FLOWS IN

C

\section{AT SPECIFIED PRESSJRE NODE}

200 GUR $=0.000$

GUL $=0.000$

IF(QPLITR(IP)) 360.360 .340

$340 \mathrm{GUL}=-C W \star Q P L I T R$ (IP)

GUR $=-\tilde{S U L} \star J B C(I P)$

360 IF (NOUMAT) 370.370 .380

370 UMAT (I NBHALF) =UMAT (I NBHALF) -GUL

380 UVEC (I) $=$ UVEC (I) $+G J R$

1000 CONTINUE

c

c

1050 IF (ML-1) 1100.3000 .1100

C.....SPECIFIED U BOUNDARY CONDITIONS

C MODIFY U EQUATION AT SPECIFIEO U NODE TO READ: $U=U B C$

1100 IF (NJBC.EQ.O) GOTO $30 J 0$

$D O 2000$ IU $=1, N U B C$

$I U P=I U+N P B C$

$I=I A B S(I \cup B C(I \cup P))$

IF (NOUMAT) $1200,1200,2000$

1200 DO $1500 \quad \mathrm{JB}=1, \mathrm{NB}$

1500 JMAT $(I, J B)=0.000$

UMAT $(I, N B H A L F)=1.000$

2000 UVEC (I $)=U B C($ IUP $)$ 
$c$ 3000 CONTINUE

c

RETURN

ENO
U610.... U620. U630.... U640.... U650.... U660.... 
$c$
$C$
$c$
$C$
$c$
$c$

$* *$ * PURPJSE :

* * TO IMPLEMENT PINEH NJDE CONDITIONS BY MODIFYING THE

** GLOBAL FlOW AND TRANSPORT MATRIX EQUATIONS.

SUBRJUTINE PINCHB (ML, IPINCH,PMAT, PVEC, UMAT, UVEC)

IMPLICIT DOUBLE PRECISION $(A-H, O-Z)$

COMMON/DIMS/ NN,NE,NIN,NBI,NB,NBHALF,NPINCH,NPBC,NUBC,

1 NSOP,NSOU,NBCN

COMMON/CONTRL/ GNU,UP, DTMULT, JTMAX,ME, ISSFLO, ISSTRA,ITCYC,

1 NPCYC,NUCYC,NPRINT, IREAD, ISTORE, NOUMAT, IUNSAT

DIMENSION IPINCH(NPINZH,3), PMAT (NN,NBI), PVEC (NN),

$c$

1 UYAT(NN,NBI), UVEC (NN)

C.... NPIN IS ACTUAL NUMBER OF PINCH NODES IN MESH

NPIN $=$ NP I NCH-1

DO 1300 IPIN=1,NPIN

SET NUMBERS OF PINCH NODE AND NEIGHBOR NODES

$I=I P I N C H(I P I N, 1)$

$I C O R 1=I P I N C H(I P I N, 2)$

ICOR $2=I P I N C H(I P I N, 3)$

$J C 1=I C O R I-I+N B H A L F$

$C$

$J C 2=I C O R 2-I+N B H A L F$

IF (ML-1) $50,50,253$

C.....ADJUST P EQUATION FOR PINCH NODE CONDITIONS

$5000100 \mathrm{JB}=1$-NB

$100 \operatorname{PMAT}(I, J B)=0.000$

PVEC $(I)=0.000$

PMAT (I,NBHALF) $=1.0000$

PMAT $(I, J C 1)=-0.5000$

PMAT $(I, J C 2)=-0.5000$

IF (ML-1) $250,1000,250$

C.....ADJUST U EQUATION FOR PINEH NJDE CONDITIONS

250 IF (NJUMAT) 300.303 .500

300 DO $400 \mathrm{JB}=1, \mathrm{NB}$

$400 \operatorname{UMAT}(I, J B)=0.000$

JMAT (I,NBHALE) $=1.3000$

UMAT $(I, J C 1)=-0.5000$

UMAT $(I, J C 2)=-0.5000$

500 UVEC $(I)=0.000$

c

1000 CONTINUE

C

C

RETURN

END
SUTRA - VERSION 1284-20 V10.....

$\checkmark 20 \ldots \ldots$

$\checkmark 30 \ldots \ldots$

$\checkmark 40 \ldots \ldots$

$\checkmark 50 \ldots \ldots$

$\checkmark 60 \ldots .$.

$\vee 70 \ldots .$.

$\vee 80 \ldots .$.

v90.....

$v 100 \ldots$.

$\checkmark 110 \ldots$

v120....

$v 130 \ldots$

$\vee 140 \ldots$

$v 150 \ldots$

$v 160 \ldots$

$v 170 \ldots$

$\vee 180 \ldots$

v190....

$\checkmark 200 \ldots$

$v 210 \ldots$.

$\checkmark 220 \ldots$

$v 230 \ldots$.

v240....

$v 250 \ldots$.

v260....

$v 270 \ldots$.

v $280 \ldots$

$v 290 \ldots$.

v300....

$\checkmark 310 \ldots$.

$v 320 \ldots$.

$v 330 \ldots$.

v340....

$v 350 \ldots$.

$v 360 \ldots$.

$v 370 \ldots$

v380....

V390....

$\checkmark 400 \ldots$.

$v 410 \ldots$

$v 420 \ldots$

$v 430 \ldots$

$v 440 \ldots$.

$v 450 \ldots$

$v 460 \ldots$

$v 470 \ldots$

$\checkmark 480 \ldots$ 


$$
\begin{aligned}
& \vdots \\
& \vdots \\
& \vdots \\
& \vdots \\
& \vdots \\
& \vdots
\end{aligned}
$$

$c$
$c$
$C$
$c *$
$c *$
$C *$
$C *$
$c$

SUBROUTINE

$\begin{array}{llllll}5 & \supset & L & V & E & 8\end{array}$

PURPJSE :

TO SOLVE THE MATRIX EQUATION BY:

(1) DECOMPOSING THE MATRIX

(2) MODI=YING THE RIGHT-HANJ SIJE

(3) BACK-SUBSTITUTING FOR THE SOLUTION

SUBROUTINE SOLVEB (KKK,C,R,NNP, IHALFB, MAXNP,MAXBW)

IMPLICIT DOUBLE PRECISION (A-H,O-Z)

OIMEVSION C(YAXNP, MAXZW),R (MAXNP)

$C$ I $M B P=I H A L F B+1$

C.... OECOYPOSE MATRIX : BY BANDED GAUSSIAN ELIMINATION FOR

c NON-SYMMETRIC YATRIX

IF $(K K K-1) \quad 5,5,50$

$5 N U=N V P-I H A L F B$

DO $20 \mathrm{NI}=1$, NU

PIVOTI $=1.00 / C(N I, I H B P)$

$N J=N I+1$

$I B=I H B P$

$N K=N I+I H A L F B$

DO $10 \mathrm{NL}=\mathrm{NJ}, \mathrm{NK}$

I $B=I 3-1$

$A=-C(N L, I B) \star D I V O T I$

$C(N L, I B)=A$

$J B=I B+1$

$K B=I B+I H A L F B$

$L B=I+B P-I B$

$D 010 M B=J B, K B$

$N B=L B+M B$

$10 C(N L, M B)=C(N L, M B)+A * C(N I \cap N B)$

20 CONTINUE

$N R=N J+1$

$N U=N N P-1$

$N K=N$ N

$D O 4 J N I=N R, N U$

PIVOTI $=1 . D O /(C(N I, I H B P))$

$N J=N I+1$

$I 3=I+B P$

$003 J \mathrm{NL}=\mathrm{NJ}, \mathrm{NK}$

I $B=I 3-1$

$A=-C(N L, I B) * P I \vee O T I$

$C(N L, I B)=A$

$J B=I B+1$

$K B=I 3+I H A L F B$

$L B=I H B P-I B$

$D O 3 J M B=J 3, K B$

$N B=L B+M B$

$30 C(N L, M B)=C(N L, M B)+A \star C(N I, N B)$

40 CONTINUE

IF $(K K K-1) \quad 50,44,50$

$c$

44 RETURN

C.....JPDATE RIGHT-HAND SIDE VECTOR, R

$50 \quad N U=N V P+1$

I $B A N D=2 * I H A L F B+1$

$D O 7 J N I=2$, IHBP

$I B=I H B P-N I+1$

$\mathrm{NJ}=1$
SUTRA - VERSION 1284-20 W10....

$w 20 \ldots$.

w30.....

W40....

W50.....

w60.....

W70.....

w80.....

W90.....

w100....

W110....

w120...

w130....

w140...

w150....

w160....

W170....

$w 180 \ldots$.

w190....

W200....

W210....

$w 220 \ldots$.

W230....

w240....

$w 250 \ldots$.

$w 260 \ldots$.

$w 270 \ldots$.

W280....

W290....

w300....

W310....

W320...

w330...

W340....

w350....

w360....

W370....

W380....

W390....

W400....

W410....

W420....

W430....

$W 440 \ldots$.

W450....

W460....

W470....

W480....

W490....

W500....

W510....

W520....

W530....

W540...

W550....

W560...

W570....

W580....

W590....

W600.... 
SUM $=3.000$

$D O 60 \mathrm{JB}=I B, I H A L F B$

$S J M=S U M+C(N I, J B) \star R(N J)$

$60 \quad N J=N J+1$

$70 R(N I)=R(N I)+5 U M$

$I B=1$

$N L=I+B P+1$

DO $90 \mathrm{NI}=\mathrm{NL}, \mathrm{NNP}$

$N J=N I-I H B P+1$

SUM $=0.00$

$D O 8 J \mathrm{~J} B=I B, I H A L F B$

$S U M=S J M+C(N I, J B) \star R(N J)$

$80 N J=N J+1$

$c$

$90 R(N I)=R(N I)+5 U M$

\section{C.... BACK SOLVE}

$R(N N P)=R(N N P) / C(N N P, I+B P)$

OU 110 I $B=2$, IHBP

$N I=N U-I B$

$N J=N I$

$M B=I+A L F B+I B$

SUM $=0.00$

$D O 100 \quad J B=N L, M B$

$N J=N J+1$

$100 \quad S U M=S U M+C(N I, J B) \star R(N J)$

$110 R(N I)=(R(N I)-S U M) / C(N I, I H B P)$

$M B=I B A N D$

$D O 130 I B=N L, N N P$

$N I=N J-I B$

$N J=N I$

SUM $=3 . D O$

$D O 120 \mathrm{JB}=\mathrm{NL}, M 3$

$N J=N J+1$

$120 S U M=S U M+C(N I, J B) \star R(N J)$

$130 R(N I)=(R(N I)-S U M) / C(N I, I H B P)$

$c$

c

RETURN

END 
SUBRJUTINE BJOJET CML, I BCT,VOL,SW,OSWOP,RHO,SOP,QIN,PVEC,PMI,

1 PBC, QPLITZ,IPSE,IQSOP,POR,UVEC,UM1,UMZ,UIN, QUIN,I JSOU,UBC,

$2 \quad(S 1, C S 2, C S 3, S L, S R)$

IMPLICIT DOUBLE PRECISION $(A-H, O-Z)$

CHARALTER 10 ADSMOD

COMMON/MOOSOR/ ADSMOO

COMMON/OIMS/ NN,NE,NIN,NBI, NB, NBHALF,NPINCH,NPBC,NUBC,

1 NSOP,NSOU,NBCN

COMMON/TIMEI DELT,TSEE,TMIN,T HOUR, TDAY,TWEEK, TMONTH,TYEAR,

1 TYAX, DELTP,DELTU, DLTPMT, OLTUM1, IT, ITMAX

1 R TOWO,URHOWJ, VISCO,PROJF1,PRODS1,PRODFO,PROOSO,CHI1,CHI 2

COMMON/CONTRL/ GNU,UP, OTMULT,DTMAX,ME, ISSFLO, ISSTRA, ITCYC,

1 NPCYC,NUCYC,NPRINT, IREAD, ISTORE, NOUMAT, IUNSAT

CHARACTER 13 UNAME (2)

DIMEVSION QIN(NN),UIN(NN),IQSJP(NSOP), QUIN(NN), IQSOU(NSOU)

DIMEVSION IPBC (NBEN), JBC (NBCN),QPLITR (NBCN),PBC(NBCN)

DIMENSION POR(NN), VOL (NN), PVEZ(NN),UVEC(NN), SW(NN),OSWDP(NN),

$1 \quad R+O(N N), S O P(N N), P M 1(N N), U M 1(N N), U M Z(N N)$,

$2 \quad \operatorname{CS} 1(N N), C S 2(N N), C S 3(N N), S L(N N), S R(N N)$

C

DATA UNAME(1) 1 CONCENTRATION: 1 ,UNAME $(2) /$ : TEMPERATURE $/$

$M N=2$

IF (IUNSAT.NE.J) IUNSAT $=1$

IF (ME.EQ.-1) $M N=1$

WRITE $(6,10)$

10 FORMAT (1H1)

C..... SET JNSATURATED. FLOW PARAMETERS, SW(I) ANO OSWOP(I) IF (I JNSAT-1) $40,2 J, 40$

$200030 I=1, N N$ IF (PVEC (I)) $\cdot 25,27,27$

25 CALL UNSAT (SW (I), DSWOP(I),RELK,PVEC(I)) GOTO 30

$27 \operatorname{SW}(I)=1.000$

DSWDP (I ) $=0.000$

C

30 CONTINUE

C..... CALCULATE COMPONEVTS JF FluIJ MASS BUDGET

40 IF $(M L-1) \quad 50,50,1030$

50 CONTINUE

STPT JT $=0.00$

STUT JT $=0.00$

QINT $T T=0.00$

DO $100 \mathrm{I}=1, \mathrm{NN}$

STPT JT =STPTOT+(1-ISSFLO/2)*RHJ (I)*VOL (I)*

1 (SW(I) $1 S O P(I)+P O R(I) \star D S W D P(I)) \star(P V E C(I)-P M I(I)) / D E L T P$

STUTJT =STUTOT + $(1-I S S F L O / 2) \star P O R(I) \star S W(I) \star D R W O U * V O L(I) \star$

1 (JMI(I)-UYZ(I)) /OLTUM1

QINTOT $=Q I N T O T+Q I N(I)$

C

130 CONTINUE

QPLT JT $=0.00$

DO $2 J 0$ IP $=1$, NPBC 
c

200

$I=I A B S(I P B C(I P))$

QPLITR(IP) $=G V U *(P B C(I P)-P V E C(I))$

QPLTOT $=Q P L T O T+Q P L I T R(I P)$

C..... OUTPUT FLUID MASS BUDJET

WRITE (6,300) IT,STPTOT,STUTOT ,UNAME (YN), QINTOT,QPLTOT

300 FORMATC//11X, F L U I D

$M A S S B$ U D GE T AFTER TIME',

1 STEP IIS, ${ }^{\circ}$ IN (MASS/SECONO) ///11X,1P015.7.5X,

2 - RATE OF CHANGE IN TOTAL STORED FLUID DUE TO PRESSURE CHANGe',

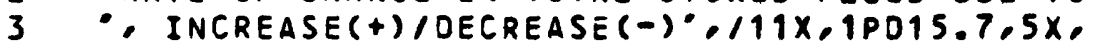

2

3

3

4

5

c

- RATE of CHANGE IN TOTAL STORED FLUID DUE TO

- INCREASE(+) / DECREASE(-)"

$111 X, 1 P 015.7,5 X, 0$ TOTAL OF FLUID SOURCES ANO SINKS,

- NET INFLOW(+)/NET OUTFLOW(-) $111 X, 1 P 015.7,5 X$,

- TOTAL Of Fluido flows at pJints of SPECIFIED PRESSURE, •

- NET INFLOH(+)/NET OUTFLOH(-) •)

IF(IBCT.EQ.4) GOTJ 603

NSOPI $=$ NSOP -1

INEG $T=0$

DO 500 IQP $=1$,NSOPI

$I=I Q S O P(I Q P)$

IF(I) $325,500,500$

325 INEG:T $=$ INEGCT+1

IF (IVEGCT.EQ.1) WRITE $(6,350)$

350 FORMAT( $/ / / 22 x_{0}$ "TIME-DEPENDENT FLUID SOURCES OR SINKS $/ 122 x$,

1 NODE, $5 X_{0}$ - INFLOW $(+)$ /OUTFLOW $(-) \cdot 137 x_{\circ} \cdot$ (MASS/SECOND) 111$)$

WRITE $(6,450)-I, Q I N(-I)$

450 FORMAT $(22 x, I 5,10 X, 1 P 015.7)$

c

500 CONTINUE

600 IF (NPBC.EQ.0) GOTJ 800

WRITE $(6,650)$

650 FORMAT $/ / / 22 x, 0^{\circ} F L J I D$ SOURCES OR SINKS DUE TO SPECIFIED PRESSURES

$1 / 122 x_{0}^{\circ}$ NODE, $5 x_{0}^{\circ}$ INFLOW $(+) / O U T F L O W(-) \cdot 137 x_{0}^{\circ}$

$D O 700$ IP $=1$, NPBC

$I=I A B S(I P B C(I P))$

c

700 CONTINUE

WRITE $(6,450)$ I, QPLITR (IP)

C..... CALCULATE COMPONENTS JF ENERGY OR SOLUTE MASS BUDGet

BOO IF(ML-1) $1000,4503,1030$

1000 CONTINUE

FLDT JT $=0.00$

SLOTJT $=0.00$

PIFTOT $=0.00$

P1STOT $=0.00$

POFT OT $=0.00$

POSTOT $=0.00$

QQUTOT $=0.00$

QIUTOT $=0.00$

C.....SET ADSORPTION PARAMETERS

IF (ME.EQ. -1. AND.AJSMOJ.NE. "NONE

1 CALL $\triangle D S O R B(C S 1, C S 2, C S 3, S L, S R, U V E C)$

DO $1300 \quad I=1, V N$

$E S R V=P O R(I) \star S W(I) \star R H O(I) \star V O L(I)$

$E P R S V=(1.00-D O R(I)) * R+0 S * V O L(I)$

DUDT $=(1-I S S T R A) *(J V E C(I)-J M 1(I)) / D E L T U$

FLDTJT $=F L O T O T+E S R V \star C W \star D U D T$ $\times 610 \ldots$

$\times 620 \ldots$

$\times 630 \ldots$

$\times 640 \ldots$

$\times 650 \ldots$

$\times 660 \ldots$

$\times 670 \ldots$

$\times 680 \ldots$

$\times 690 \ldots$

$x>00 \ldots$

$x>10 \ldots$.

$x>20 \ldots$

$x>30 \ldots$

$x>40 \ldots$.

$x>50 \ldots$

$x>60 \ldots$.

$x>70 \ldots$

$x>80 \ldots$.

$x>90 \ldots \ldots$

$\times 800 \ldots$.

$\times 810 \ldots$.

$\times 820 \ldots$

$\times 830 \ldots$.

$\times 840 \ldots$.

$\times 850 \ldots$

$\times 860 \ldots$

$\times 870 \ldots$.

$\times 880 \ldots$.

$\times 890 \ldots$.

$\times 900 \ldots$.

$\times 910 \ldots$.

$\times 920 \ldots$.

$\times 930 \ldots$.

$\times 940 \ldots$.

(MASS/SECOND) 11$) \times 960 \ldots$.

$\times 970 \ldots$.

$\times 980 \ldots$

$\times 990 \ldots$

$\times 1000 \ldots$

$\times 1010 \ldots$

$\times 1020 \ldots$

$\times 1030 \ldots$

$\times 1040 \ldots$

$\times 1050 \ldots$

$\times 1060 \ldots$

$\times 1070 \ldots$

$\times 1080 \ldots$

$\times 1090 \ldots$

$\times 1100 \ldots$

$\times 1110 \ldots$

$\times 1120 \ldots$

$\times 1130 \ldots$

$\times 1140 \ldots$

$\times 1150 \ldots$

$\times 1160 \ldots$

$\times 1170 \ldots$

$\times 1180 \ldots$

$\times 1190 \ldots$

$\times 1200 \ldots$. 
$S L O T J T=S L D T O T+E P R S V \star C S 1(I) \star D U D T$

$P$ PFT $T T=P 1 F T O T+E S R V \star P R J D F 1$

$P 1 S T J T=P 1 S T O T+E P R S V \star P R O D S 1 *(S L(I) \star U V E C(I)+S R(I))$

$P O F T J T=P O F T O T+E S R V \star P R J D F O$

POSTOT $=P O S T O T+E P R S V \star P R O D S J$

QQUT JT $=$ QQUTOT + QUIN $(I)$

IF(OIN(I)) $1200,1200,1250$

1200 QIUTOT=QIUTOT+QIN (I) * SW*UVEC (I)

GOTO 1300

1250 2IUT JT $=Q I U T O T+2 I N(I) \star E W * U I N(I)$

1300 CONTINUE

c

QPUTOT $=0.00$

DO 1500 I $P=1, N P B C$

IF(QPLITR(IP)) $1400,1400,1450$

$1400 I=I A B S(I P B C(I P))$

QPUTOT $=$ QPUTOT+QPLITR $(I P) \star E W \star U V E C(I)$

GOTO 1500

1450 QPUTOT $=Q P U T O T+Q P L I T R(I P) \star C W * U 3 C(I P)$

1500 CONTINUE

乞

IF (ME) $1550,1550.1615$

$C$

C.....OUTPUT SOLUTE MASS BUDGET

1550 WRITE $(6,1600)$ IT,FLOTOT,SLOTOT,P1FTOT,P1STOT,POFTOT,POSTOT,

1 QIUTOT,QPUTOT, ZQUTJT

1600 FORMAT $/ / 11 x_{0}$ 'S OLUTE BU D G E T AFTER TIME STEP OISAX

$1 \%$ IN (SOLUTE MASS/SECONO) $/ / / 11 \mathrm{x}, 1$ P015.7,5X, NET RATE OF $\because$

2 INCREASE( +) /DECREASE(-) OF SOLUTE / 11 X, 1P015.7.5X,

3 - NET RATE OF INCREASE(+)/DECREASE(-) OF ADSORBATE /11X,1PD15.7, 1

4 5X, NET FIRST-ORDER PRODUCTION $(+)$ /OECAY $(-)$ OF SOLUTE $/ 11 X^{\circ}$.

5 1PD15.7.5, NET FIRST-ORDER PRODUCTION(+)/DECAY(-) OF $\cdots$

6 -ADSORBATE $/ 11 X, 1 P J 15.7 .5 X$, NET ZERO-ORDER PRODUCTION( $+11^{\circ}$,

7 -DECAY(-) OF SOLUTE /11X,1PD15.7,5X, NET ZERO-ORDER ?

8 - PROOUCTION(+)/DECAY ( - ) OF AOSORBATE /11X,1PD15.7,5X,

9 -NET GAIN(+)/LOSS(-) OF SOLUTE THROUGH FLUIO SOURCES AND SINKS ${ }^{\circ}$

* /11X,1PD15.7.5 $x_{0}$.NET GAIN(+)/LOSS(-) JF SOLUTE THROUGH •

1 -INFLOWS OR OUTFLOWS AT POINTS OF SPECIFIEO PRESSURE

2 /11X,1PO15.7,5X, NET GAIN(+)/LOSS(-) JF SOLUTE THROUGH,

3 - SOLUTE SOURCES AND SINKS')

C GOTO 1645

C.....OUTPUT ENERGY BUDGET

1615 WRITE(5.1635) IT,FLOTOT,SLOTOT,POFTOT,POSTOT,DIUTOT,QPUTOT, QQUTOT X1640...

1635

FORMAT (//11X, - E N E R G Y B U O GET

IN (ENERGY/SECOND) ///11X,1PD15.7,5X, -NET RATE OF ?

2 - INCREASE( +) /DECREASE(-) OF ENERGY IN FLUID $111 \mathrm{X}, 1$ PO15.7.5X

- VET RATE OF INCREASE(+) / JECREASE(-) DF ENERGY IN SOLID GRAINS $\times 16800^{\circ}$

$111 X, 1$ PO15.7,5X,-NET ZERO-OROER PRODUCTION(+)/LOSS(-) OF $\bullet$,

- ENERGI IV FLUID*111x,1PD15.7.5X, "NET ZERO-ORDER "

- PRODUCTION(+)/LOSS(-) OF ENERGY IN SOLIO GRAINS

$111 x, 1 P 015.7,5 x, 0 N E T$ GAIN(+)/LOSS(-) JF ENERGY THROUGH FLUID

- SOURCES ANJ SINKS $/ 11 X, 1$ PD15.7,5X, NNET GAIN(+)/LOSS(-) OF

- ENERGY THROUGH INFLOWS OR OUTFLOWS AT POINTS OF SPECIFIED -

- PRESSURE /11X,1PD15.7,5X,-NET GaIN(+)/LOSS(-) OF ENERGY?,

c

- THROUGH ENERGY SOURCES ANJ SINKS')

$15 \div 5$ NSOPI $=$ NSOP-1

IF (NSOPI.EQ.O) GOTO 2300

IF (ME) $1649,1649,1659$

$\times 1210 \ldots$

$\times 1220 \ldots$

$\times 1230 \ldots$

$\times 1240 \ldots$

$\times 1250 \ldots$

$\times 1260 \ldots$

$\times 1270 \ldots$

$\times 1280 \ldots$

$\times 1290 \ldots$

$\times 1300 \ldots$

$\times 1310 \ldots$

$\times 1320 \ldots$

$\times 1330 \ldots$

$\times 1340 \ldots$

$\times 1350 \ldots$

$\times 1360 \ldots$

$\times 1370 \ldots$

$\times 1380 \ldots$

$\times 1390 \ldots$

$\times 1400 \ldots$

$\times 1410 \ldots$

$\times 1420 \ldots$

$\times 1430 \ldots$

$\times 1440 \ldots$

$\times 1450 \ldots$

$\times 1460 \ldots$

$\times 1470 \ldots$

$\times 1480 \ldots$

$\times 1490 \ldots$

$\times 1510 \ldots$

$\times 1520 \ldots$

$\times 1530 \ldots$

$\times 1540 \ldots$

$\times 1550 \ldots$.

$\times 1560 \ldots$

$\times 1570 \ldots$

$\times 1580 \ldots$

$\times 1590 \ldots$

$\times 1600 \ldots$

$\times 1610 \ldots$

$\times 1620 \ldots$

$\times 1630 \ldots$

$\times 1650 \ldots$

$\times 1660 \ldots$

$\times 1670 \ldots$

$\times 1690 \ldots$

$\times 1700 \ldots$

$\times 1710 \ldots$

,$\times 1720 \ldots$

$\times 1730 \ldots$

$\times 1740 \ldots$

$\times 1750 \ldots$

$\times 1760 \ldots$

$\times 1770 \ldots$

$\times 1780 \ldots$

$\times 1790 \ldots$

$\times 1800 \ldots$ 
1549 WRITE $(6,1650)$

1550 FORMATC///22X, SOLUTE SOURCES OR SINKS AT FLUID SOURCES AND " 1 'SINKS*/122X," NODE*,8X, SOURCE(+)/SINK(-) $1 / 32 x_{\text {, }}$

2 - (SOLUTE YASS/SECOND) 1$)$ GOTO 1680

1659 WRITE $(6,1660)$

1660 FORMAT $/ / / 22 X, \circ E N E R G Y$ SOURCES OR SINKS AT FLUID SOURCES AND -

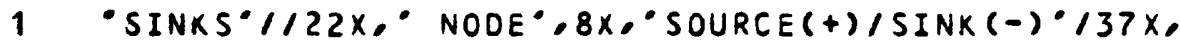

2 (ENERGY/SECONJ) (1)

1680 OO 1900 IQP $=1$, NSOPI $I=I A B S(I Q S O P(I Q P))$ $I F(Q I N(I)) \quad 1700,1700,1750$

1700 QU $=Q I N(I) \star C W \star U V E C(I)$ GOTO 1800

1750 QU=QIN(I)*CW*UIN(I)

1800 WRITE $(6,450)$ I,QU

1900 CONTINUE c

2000 IF (NPBC.EQ.0) GOTO 4500

IF (ME) $2090,2090,2150$

2090 WRITE $(6,2100)$

2100 FORMAT $/ / / 22 x, \circ$ SOLUTE SOURCES OR SINKS DUE TO FLUIO INFLOWS OR 1 JUTFLOWS AT POINTS OF SPECIFIED PRESSURE $1 / 22 x_{1}{ }^{\circ}$ NOOE, $8 X^{\circ}$, 2 -SOURCE $(+) /$ SINK $(-) \cdot 132 X^{\circ}$ " (SOLUTE MASS/SECONO) $\%$ ) GOTO 2190

2150 WRITE $(6,2160)$

2160 FORMAT///22X, ENERGY SOURCES OR SINKS DUE TO FLUID INFLOWS OR

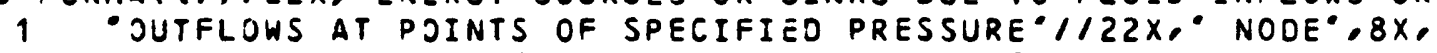

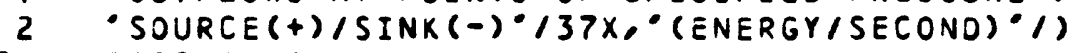

2190002400 IP $=1, N P B C$ $I=I A B S(I P B C(I P))$

$I F(Q P L I T R(I P)) \quad 2230,2200,2250$

$2200 Q P U=2 P L I T R(I P) \star C W \star U V E E(I)$ GOTO 2300

$2250 Q P U=Q P L I T R(I P) \star C W * U B C(I P)$

2300 WRITE $(6,450)$ I,QPU

2400 CONTINUE c

IF(IBCT.EQ.4) GOTO 4500

NSOUI $=$ NSOU -1

INEG $=T=0$

DO 3500 I $2 U=1$, VSOUI

$I=I Q S O U(I Q U)$

$I F(I) \quad 3400,3500,3500$

3400 INEGET $=$ INEGCT +1

I $F(M \equiv) \quad 3450,3450,3460$

3450 IF (INEGCT.EQ.1) WRITE $(6,3455)$

3455 FORMAT $/ 1 / 22 x_{0}$ "TI IE-JEPENJENT SOLUTE SOURCES AND SINKS $1 / 22 X^{\circ}$ 1 NOJE*, 1JX, GAIN(t)/LOSS(-) $130 X_{\circ}^{\circ}$ (SOLUTE MASS/SECOND) 11 ) GOTO 3475

3400 IF (INEGCT.EQ.1) WRITE(6,3465)

3455 FORMAT( $/ / 122 x, \circ$ TIME-DEPENJENT ENERGY SOURCES AND SINKS"1/22X,

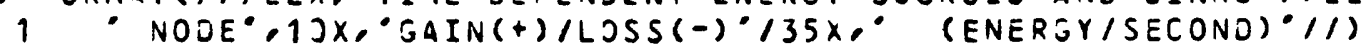

3475 CONTINUE WRITE $(6,3490)-I, 2 U I N(-I)$

3490 FORMAT $(22 x, 15,10 X, 1 P 015.7)$

3500 CONTINUE

c

4500 CONTINUE

$x 1810 \ldots$ $\times 1820 \ldots$ $\times 1830 \ldots$ $\times 1840 \ldots$ $\times 1350 \ldots$ $\times 1860 \ldots$ $\times 1870 \ldots$ $\times 1880 \ldots$ $\times 1890 \ldots$ $\times 1900 \ldots$ $\times 1910 \ldots$ $\times 1920 \ldots$ $\times 1930 \ldots$ $\times 1940 \ldots$ $\times 1950 \ldots$ $\times 1960 \ldots$ $\times 1970 \ldots$ $\times 1980 \ldots$ $\times 1990 \ldots$ $\times 2000 \ldots$ $\times 2010 \ldots$ $\times 2020 \ldots$ $\times 2030 \ldots$ $\times 2040 \ldots$ $\times 2050 \ldots$ $\times 2060 \ldots$ $\times 2070 \ldots$ $\times 2080 \ldots$ $\times 2090 \ldots$ $\times 2100 \ldots$ $\times 2110 \ldots$ $\times 2120 \ldots$ $\times 2130 \ldots$ $\times 2140 \ldots$ $\times 2150 \ldots$ $\times 2160 \ldots$ $\times 2170 \ldots$ $\times 2180 \ldots$ $\times 2190 \ldots$ $\times 2200 \ldots$ $\times 2210 \ldots$ $\times 2220 \ldots$ $\times 2230 \ldots$ $\times 2240 \ldots$ $\times 2250 \ldots$ $\times 2260 \ldots$ $\times 2270 \ldots$ $\times 2280 \ldots$ $\times 2290 \ldots$ $\times 2300 \ldots$ $\times 2310 \ldots$ $\times 2320 \ldots$ $\times 2330 \ldots$ $\times 2340 \ldots$ $\times 2350 \ldots$ $\times 2360 \ldots$ $\times 2370 \ldots$ $\times 2380 \ldots$ $\times 2390 \ldots$ $\times 2400 \ldots$ 
C SUBRJJTINE

$=$

RETURN

END
B J J J $\Xi T$

SUTRA - VERSION 1234-20 $\times 10 \ldots$

$\times 2410 \ldots$

$\times 2420 \ldots$

$\times 2430 \ldots$ 


$$
\text { SUBR JUTINE }
$$

$Y 10 \ldots . .$. $Y 20 \ldots$. $Y 30 \ldots . .$.

purpose:

* * to store results that may later be jSEO to re-start

*** THE SIMULATION.

SUBROUTINE STORE (PVEC,UVEC,PM1,UM1,CS1,RCIT,SW,PSC) IMPLICIT DOU $3 L E$ PRECISION $(A-H, O-Z)$

COMMON/DIMS/ NN,NE,NIN,NBI,NB,NBHALF,NPINCH,NPBC,NUBC,

1 NSOP,NSOU,NBCN

COMMON/TIMEI DELT OTSEC,TMIN, T HOUR , TDAY , TWEEK , TMONTH ITYEAR,

1 TYAX,OELTO,DELTU,DLTPM1,OLTUM1,IT, ITMAX

OIMEVSION PVEC (NN),UVEC (NN), PMI (NN),UM1 (NN), CS1(NN),RCIT (NN),

1 SN (NN), PBC (NBCV)

C

C.....REWIND UNIT-SO FOR WRITING RESULTS OF CURRENT TIME STEP REWIND(66)

$c$

C....STORE TIME INFORMATIOV

WRITE $(60.100)$ TSEZ,DELTP,JELTJ

C

100 FORMAT $(4020.10)$

C..... STORE SOLUTIJN

WRITE $(60.110)$ (PVEC(I),I $=1, N N)$

WRITE $(66,110)$ (UVEC(I),I $=1, N N$ )

WRITE $(60, i 10) \quad(P M I(I), I=1, N N)$

WRITE $(66,110)$ (UMI (I), I $=1, N N)$

WRITE $(66,110) \quad(C S 1(I), I=1, N N)$

WRITE $(66,110) \quad(R C I T(I), I=1, N N)$

WRITE $(66,110) \quad(S W(I), I=1, N N)$

WRITE $(60,110) \quad(P B C(I P), I P=1, N 3 C N)$

c

110 FORMAT $(4(1$ PO20.13))

ENDFILE(66)

C

RETURN

END

$Y 40 \ldots .$.

$Y 50 \ldots .$.

YoO.....

$Y>0 \ldots . .$.

Y $80 \ldots . .$.

Y $90 \ldots . .$.

Y100...

$Y 110 \ldots$

Y120....

Y130....

$Y 140 \ldots$

Y150....

Y160....

$Y 170 \ldots$.

$Y 180 \ldots$.

Y190....

$r 200 \ldots$

Y $210 \ldots$.

$r 220 \ldots$.

Y $230 \ldots$.

$Y 240 \ldots$

r250....

$Y 260 \ldots$

$Y 270 \ldots$.

Y $230 \ldots$.

$Y 290 \ldots$.

Y300....

$Y 310 \ldots$.

Y320...

$Y 330 \ldots$.

Y340....

r350....

Y $360 \ldots$.

Y $370 \ldots$. 
Appendix C:

Data File Listing for

Radial Energy Transport

Example

(p.383 follows) 
SUTRA ENERGY TRANSDORT

\# INPUT DATA HEADING

........ STEADY RADIAL FLDW WITH EVERGY TRANSPORT - SOLUTION CHECK ........

++++++++ EXAMPLE RUN FOR SUTRA DOCUMENTATION - SECTION 6.3 , PAGE $186++++++++$

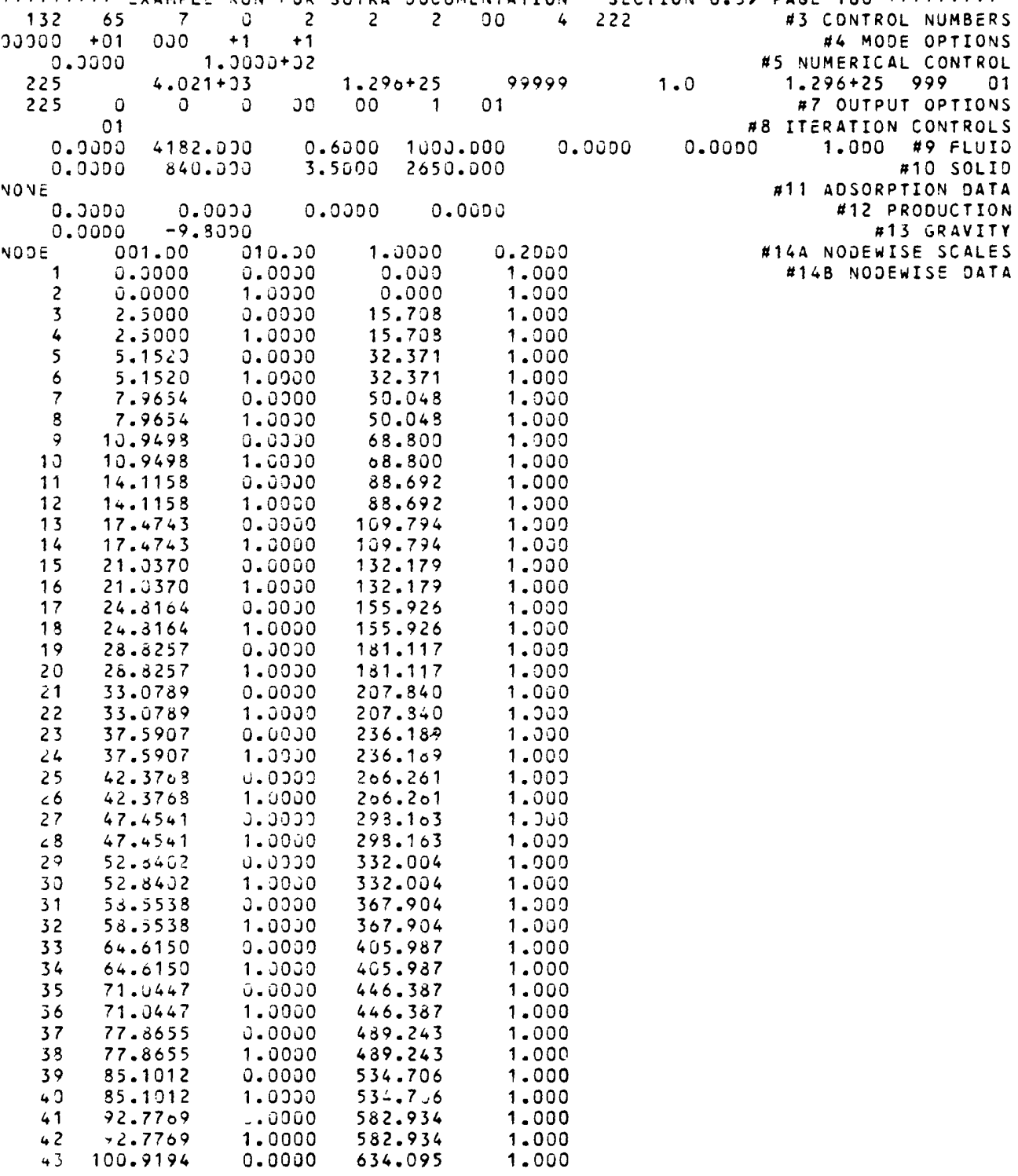




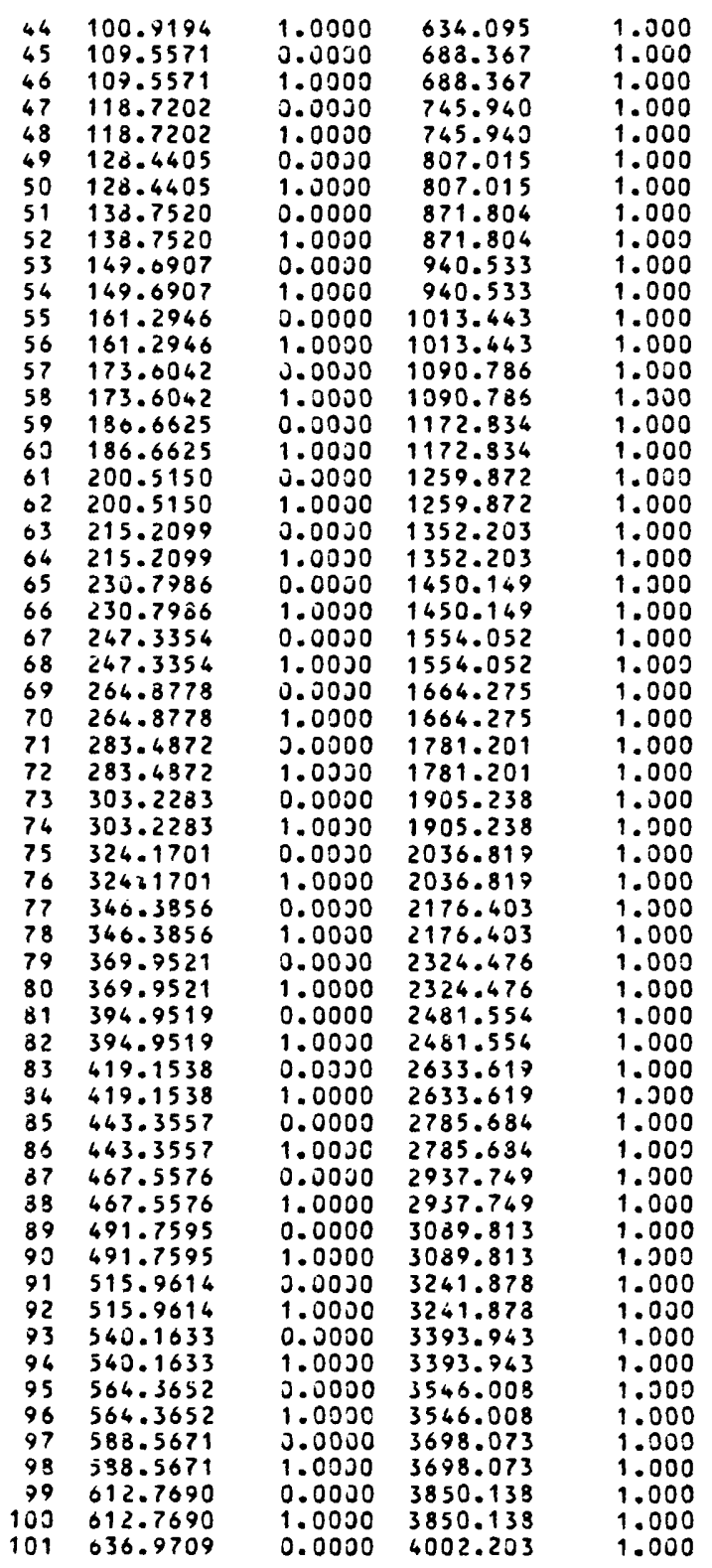




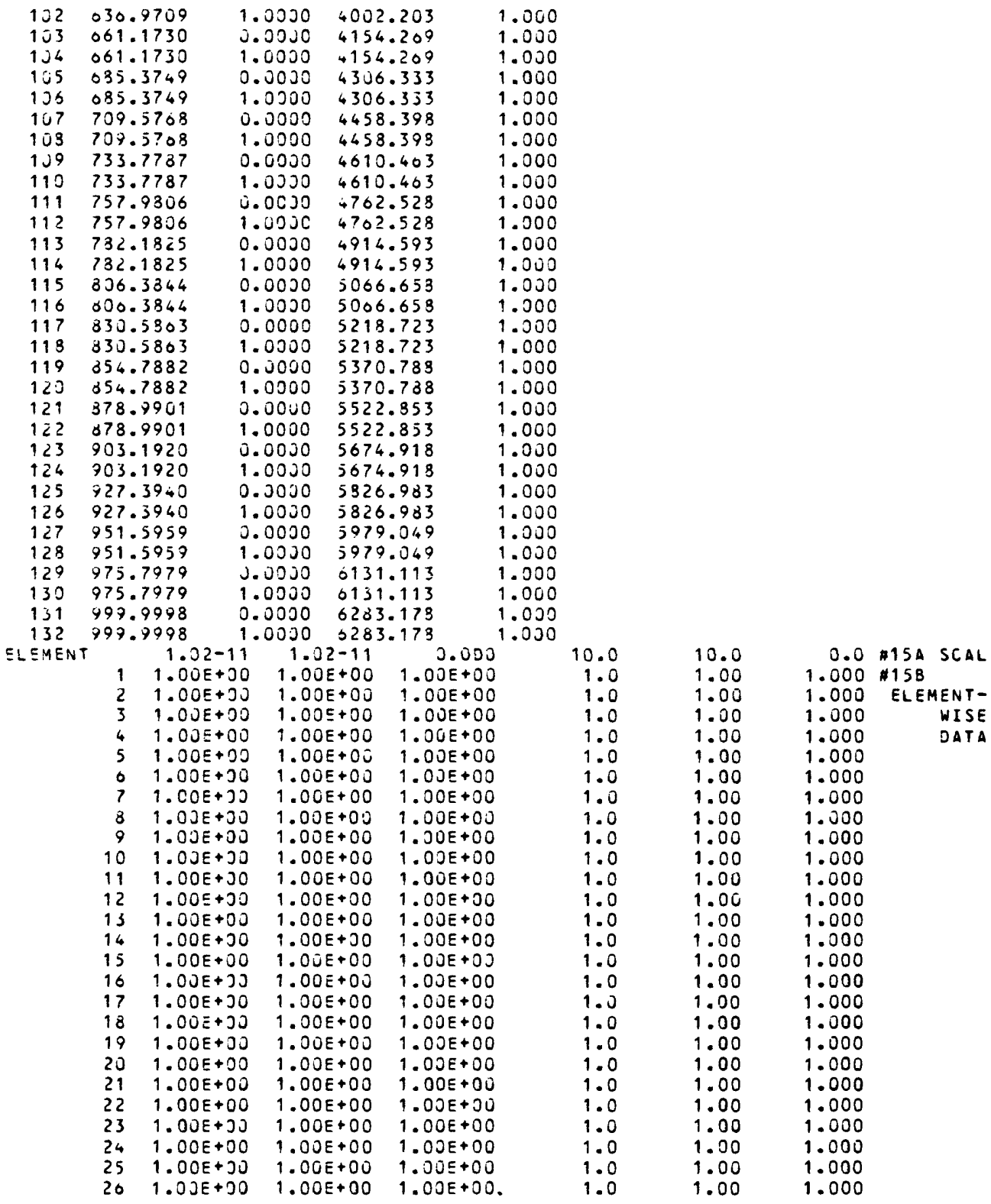




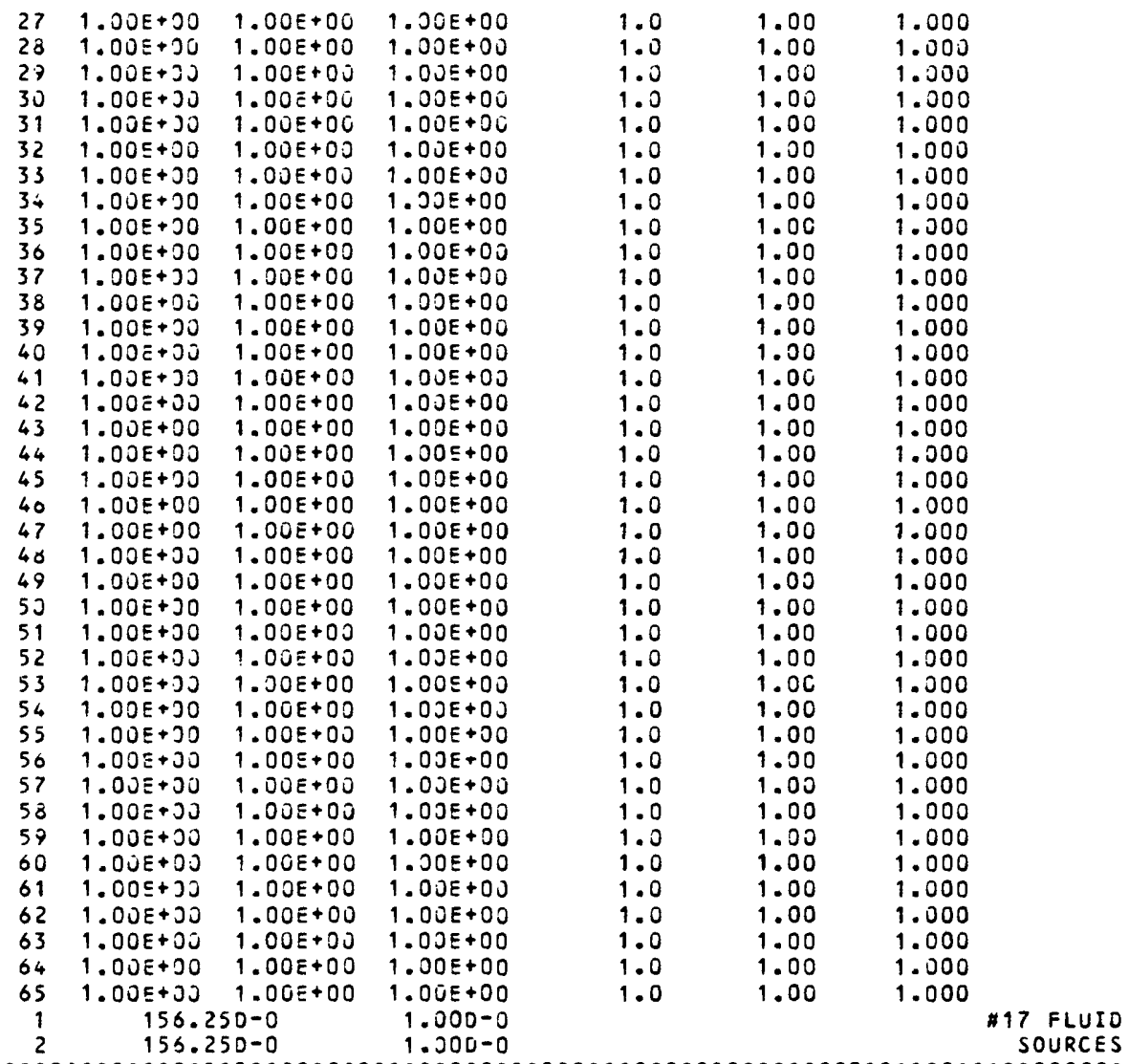
0000000000J0000000JjJ00J00000000000J00000000000000000000000000000000000000000000 $1320.00000050 \quad$ H19 SPECIFIEO

$13198000.00000000 \quad$ PRESSURES
OU0000ग0000000000000J00J000J0000000000000000000000000000000000000000000000000000

$\begin{array}{llll}1 & 1.000500 J 0 & \text { H 2O SPECIFIED } \\ 2 & 1.000000 J 0 & \text { SEMPERATURES }\end{array}$

00000000000000000000300000000000000000000000000000000000000000000000000000000000

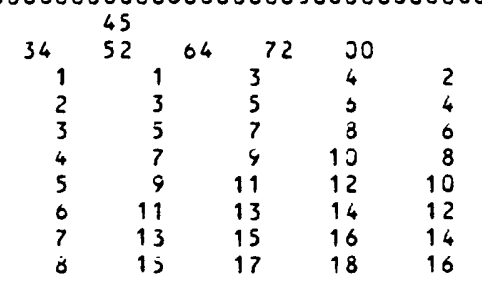

\# 21 OBSERVATION NODES

*22 nodal incidences 


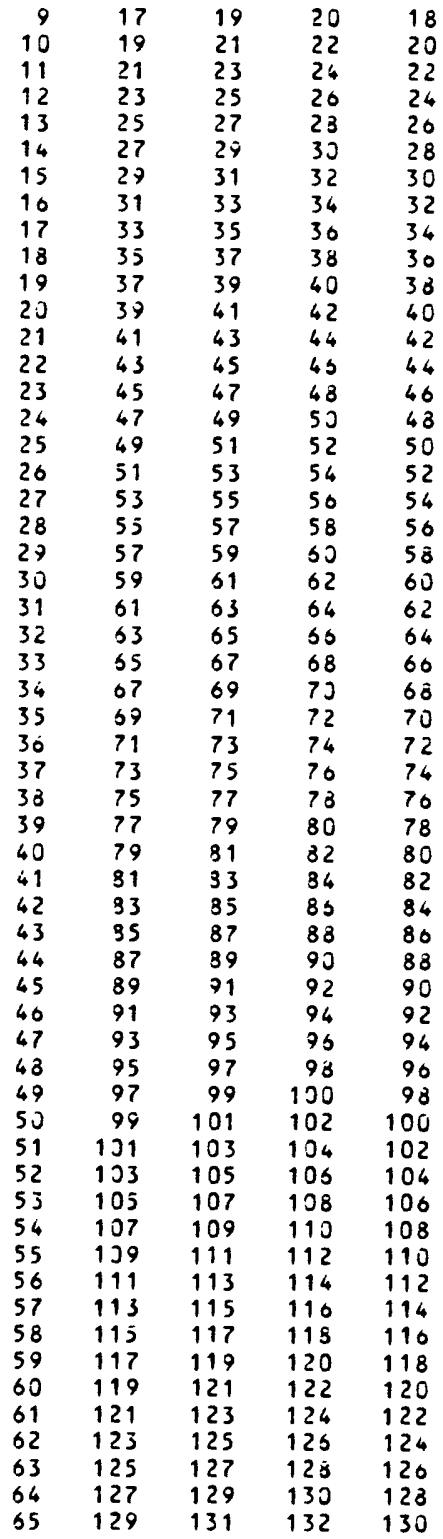


UNIT-55

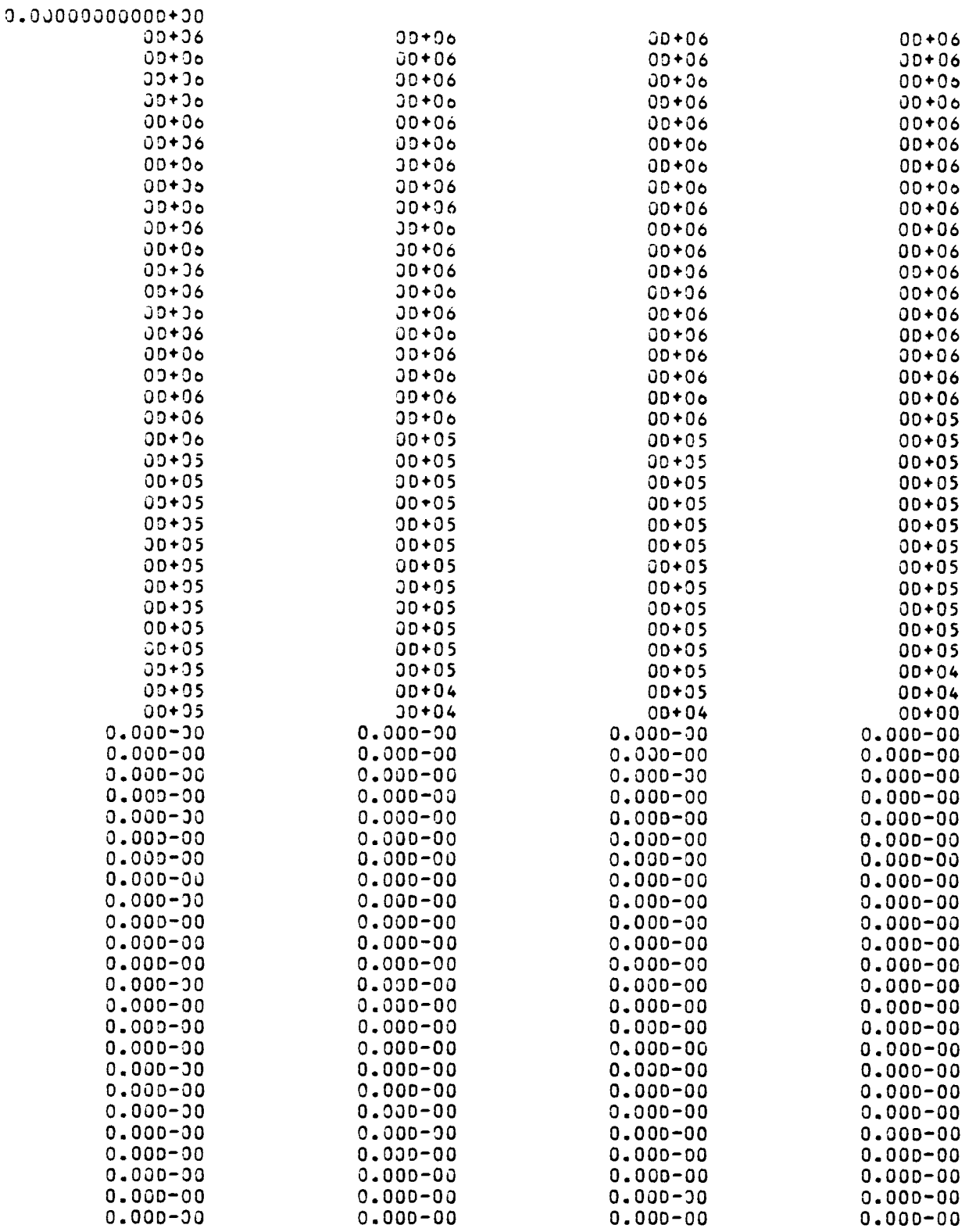



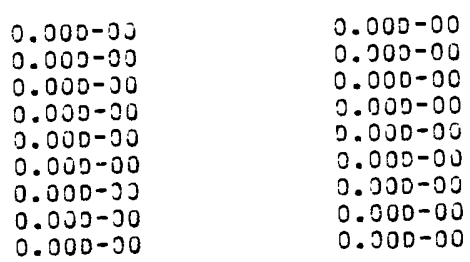

$0.000-00$

$0.000-00$

$0.000-00$

$0.000-00$

$0.000-00$

0.000-00

$0.000-00$

$0.000-00$

$0.000-00$

$0.000-00$

$0.000-00$

$0.000-00$

$0.000-00$

$0.000-00$

$0.000-00$

$0.000-00$

$0.000-00$

$0.000-00$ 
Appendix D:

Output Listing for

Radial Energy Transport

Example 


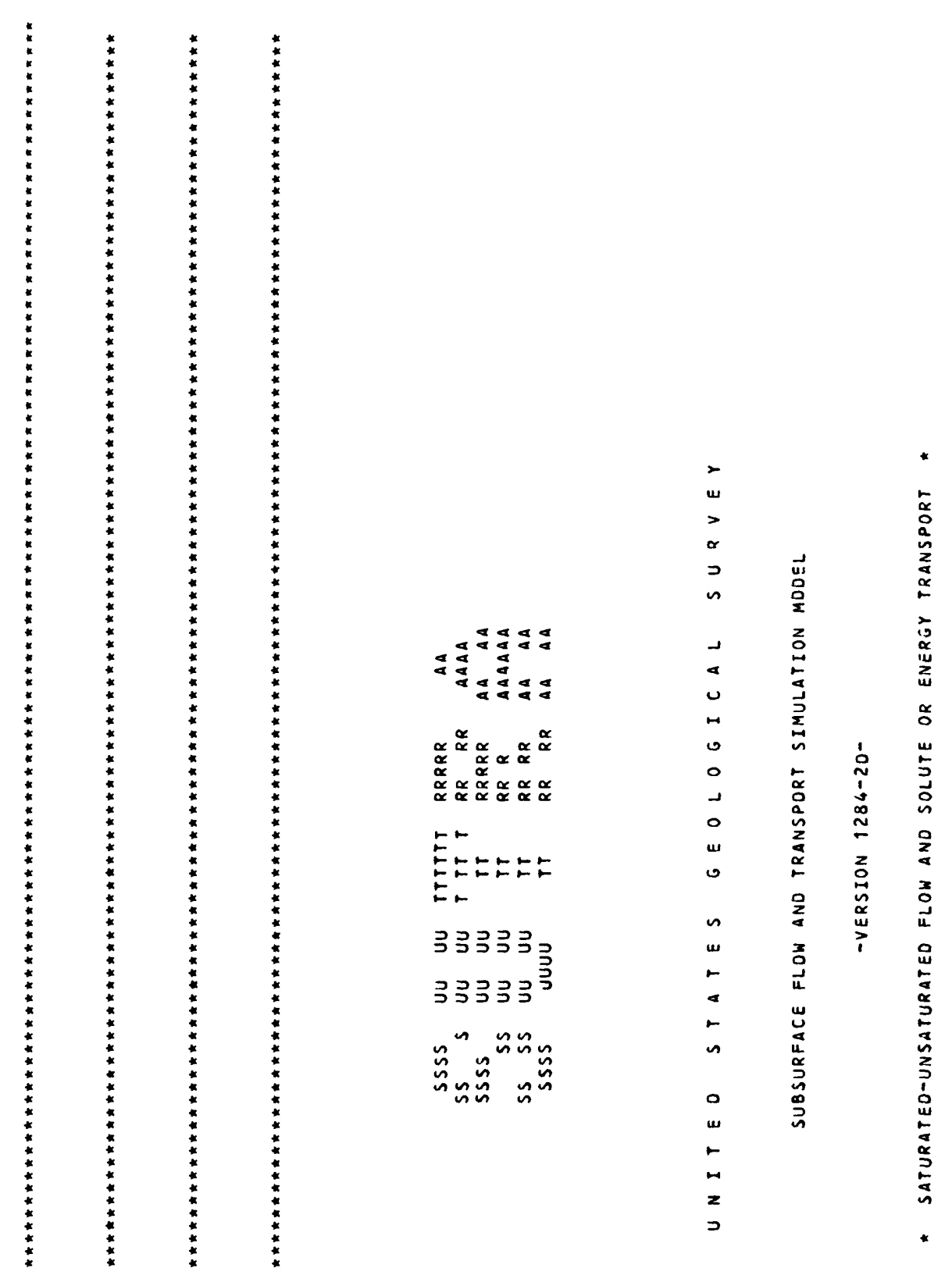




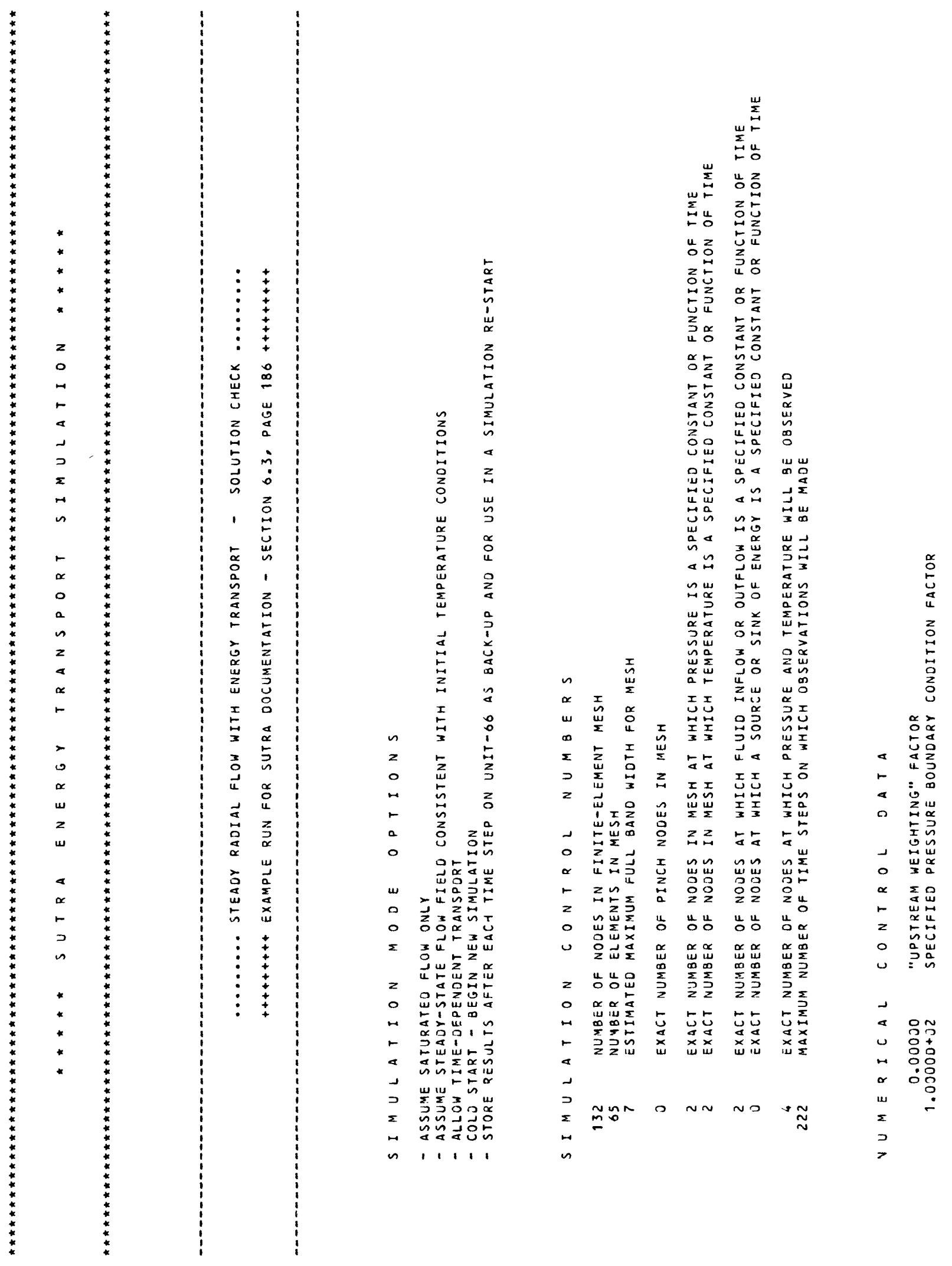




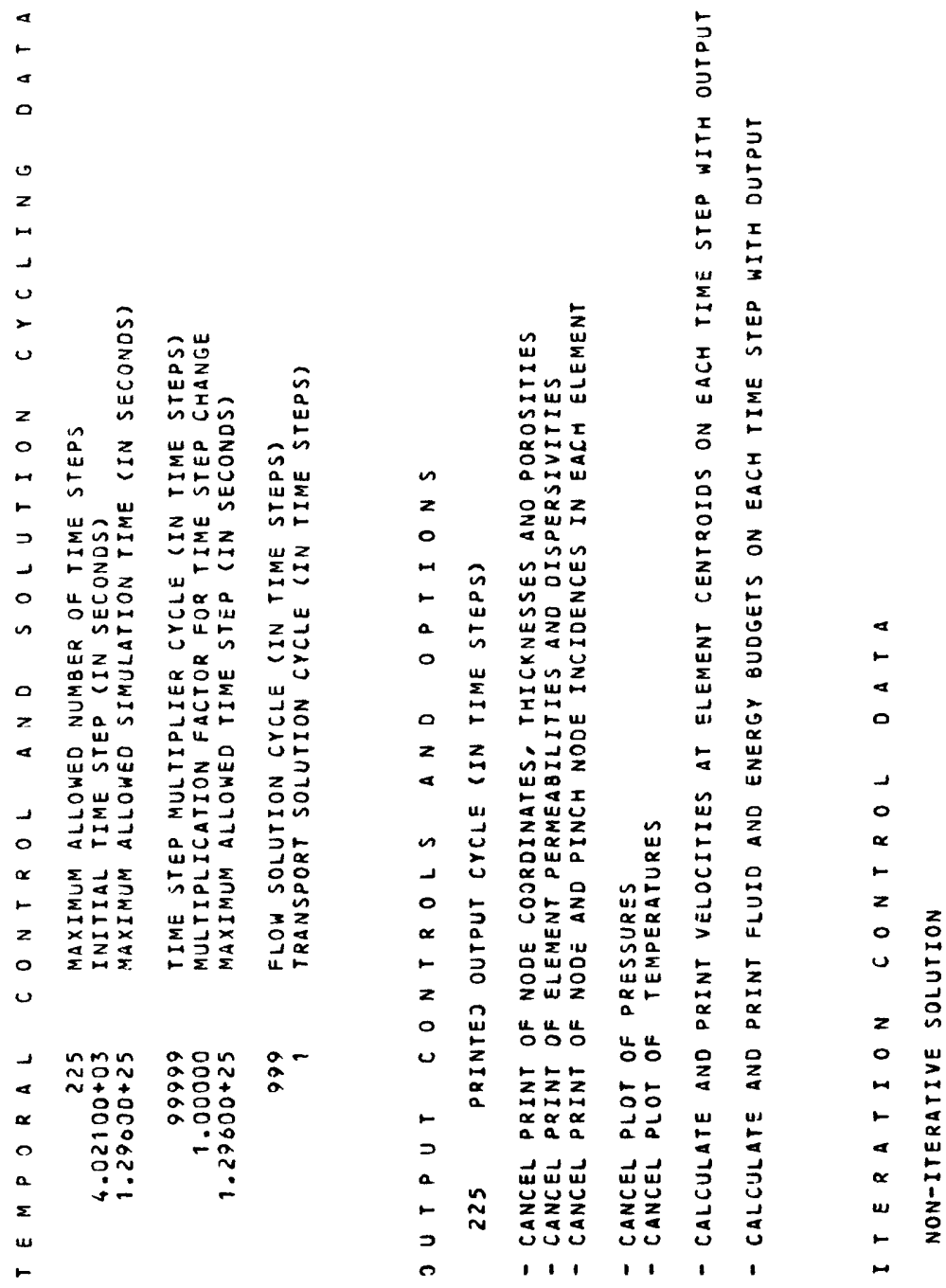




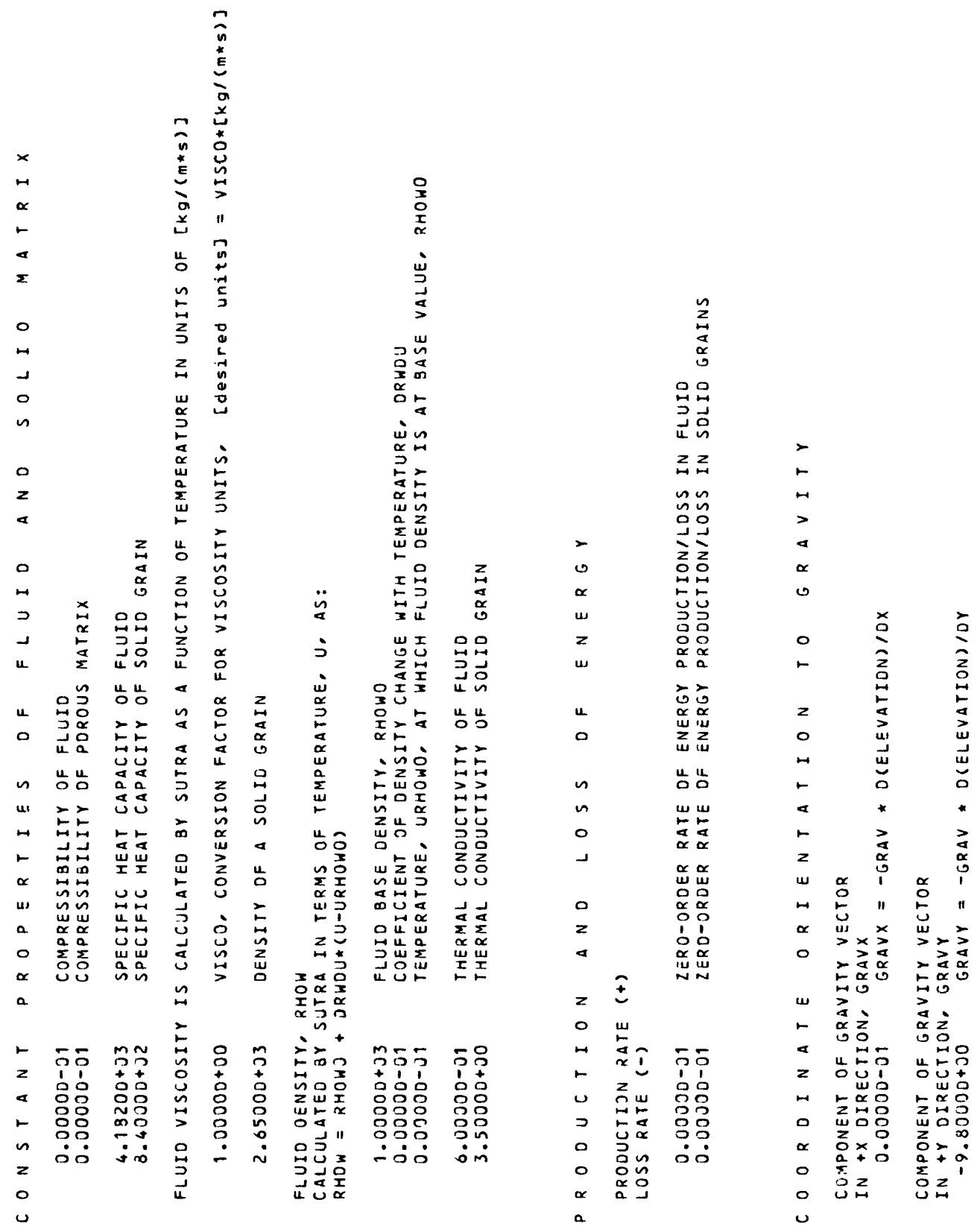




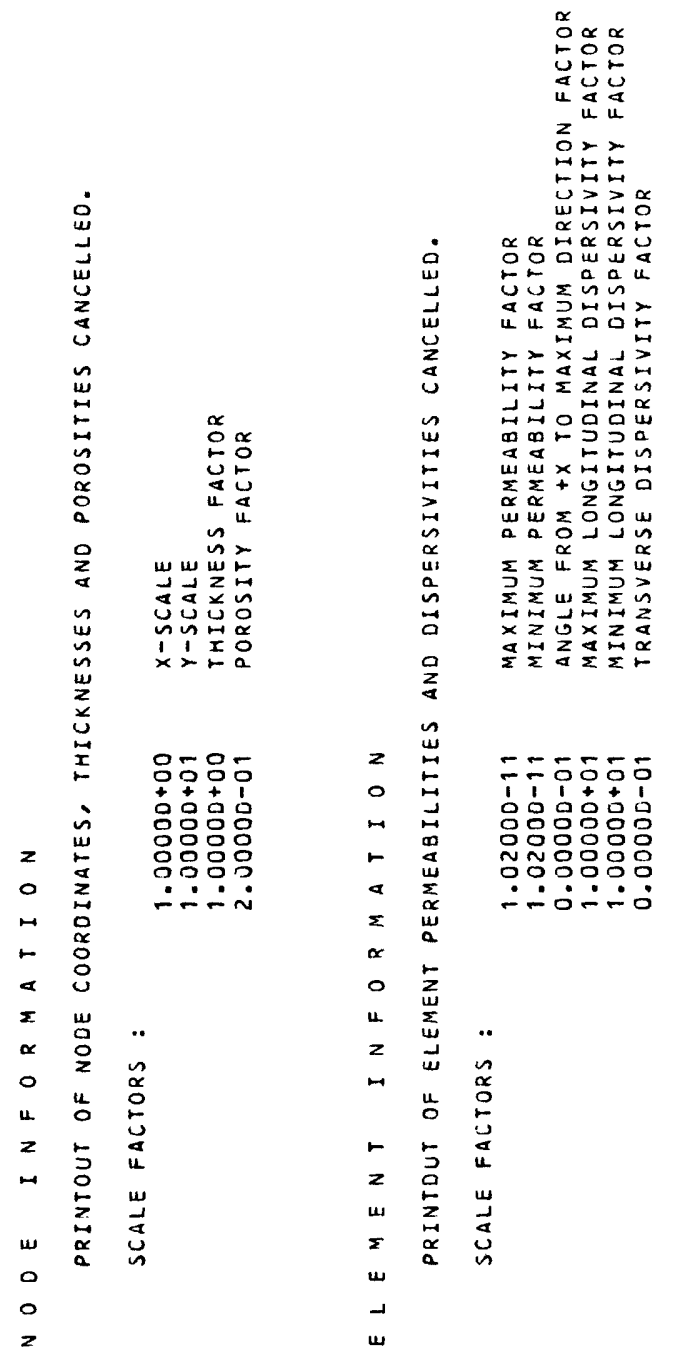




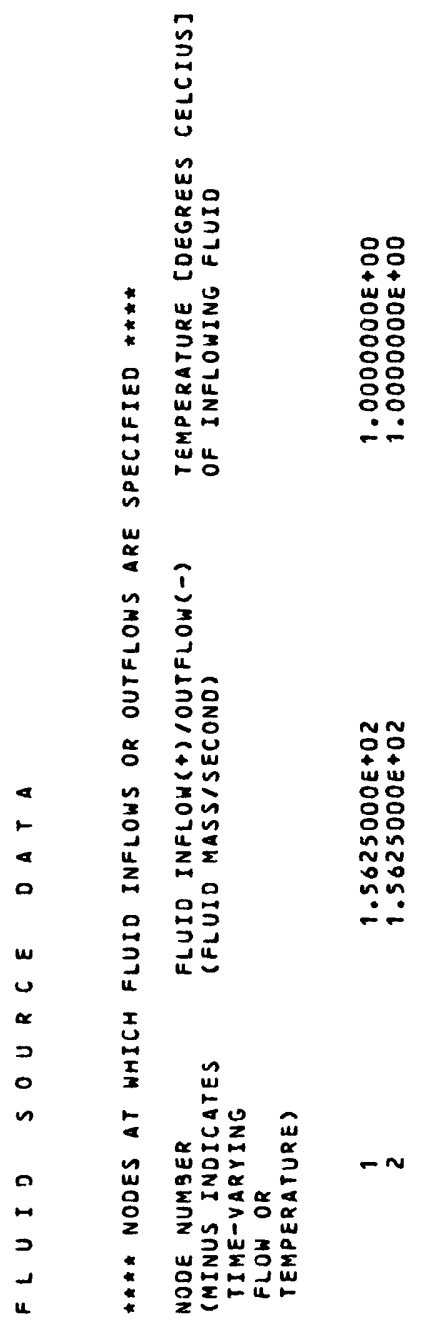




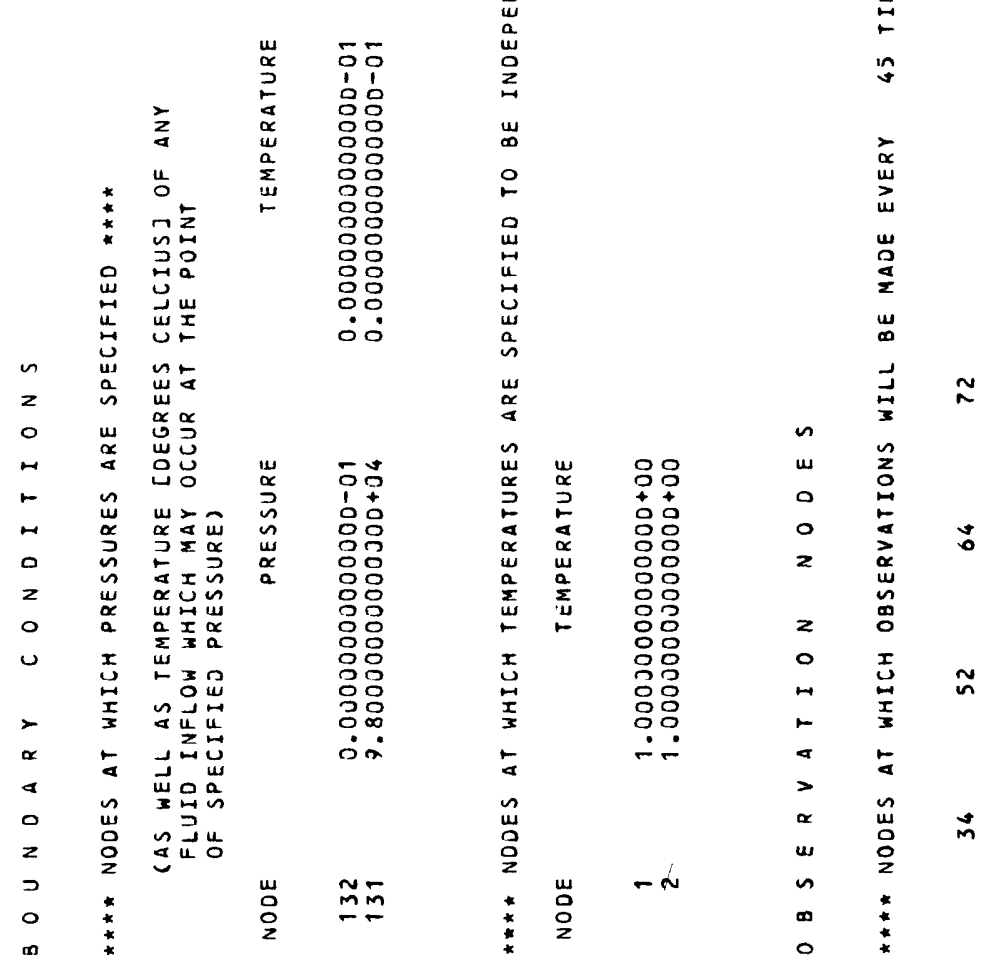




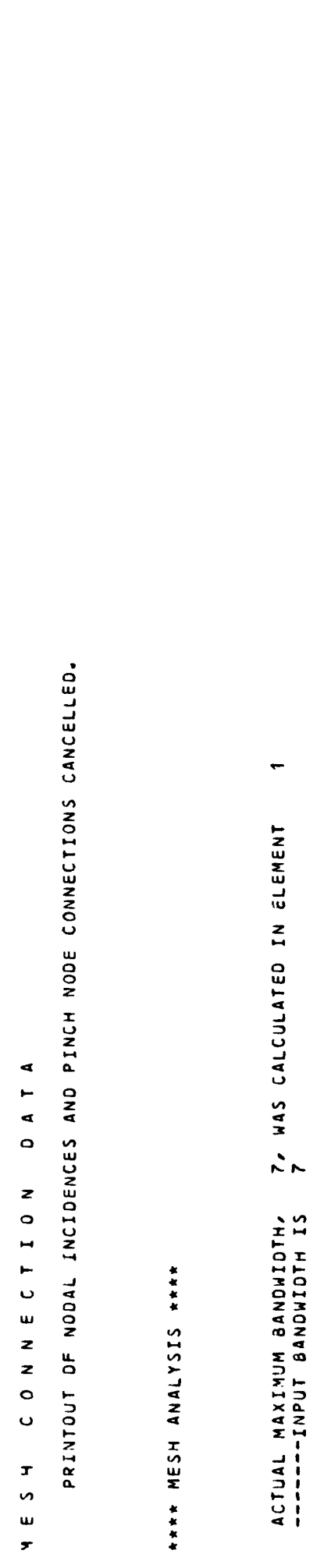




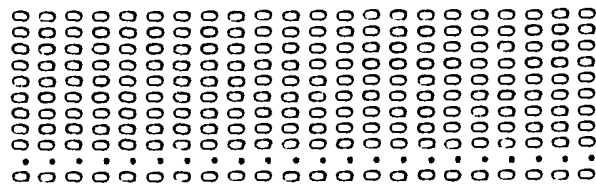

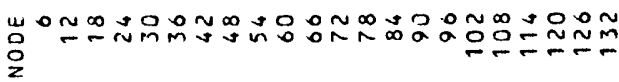

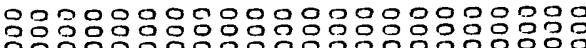

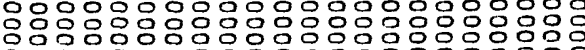

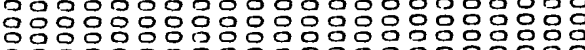

í0.000000000000000

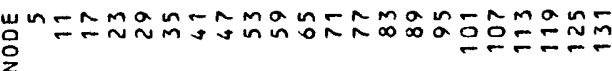

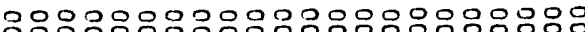

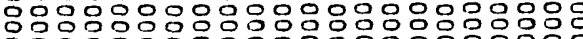

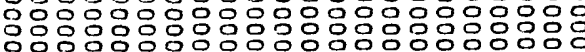

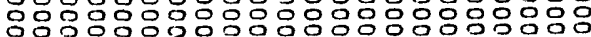

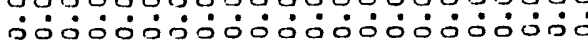

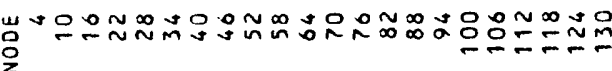

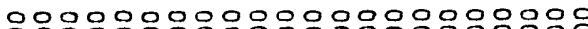

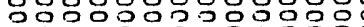

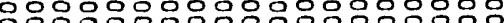

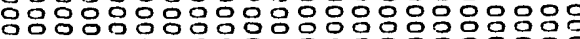

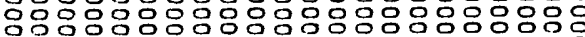

-000000000000000000

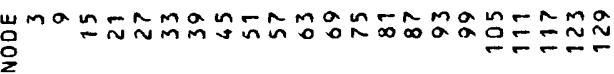

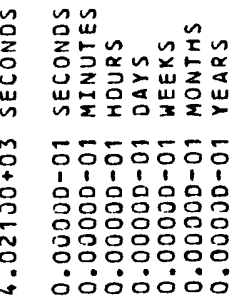

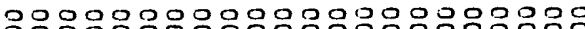

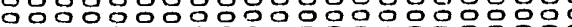

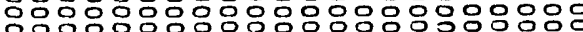

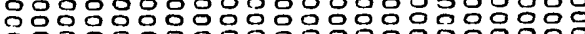

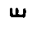

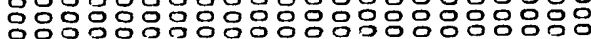

- 容

$\propto$

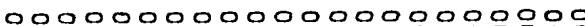

ш

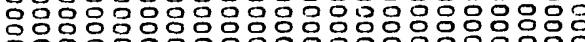

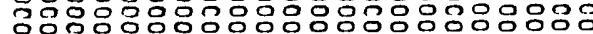

응응응응응응뭉응응응응응응

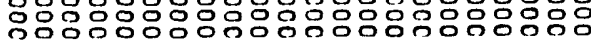

= 000000000000000000

is

- 亗 M - 


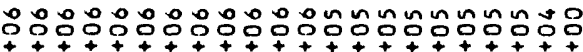

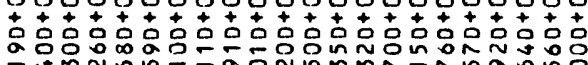

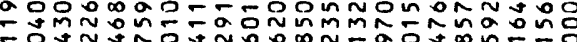

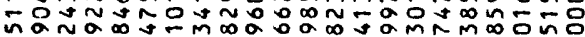

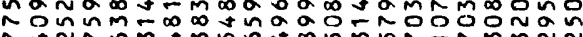
aป

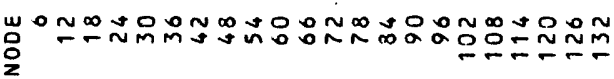

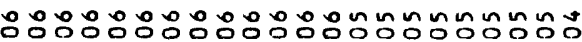

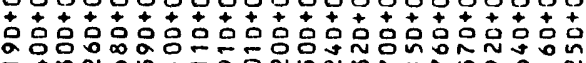
- $m \sim ⿻ 上 丨$

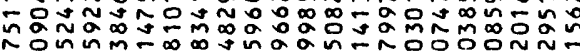
nN 0 U

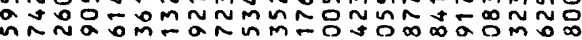

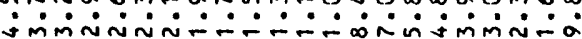

แั

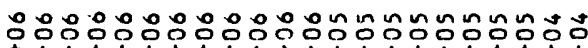

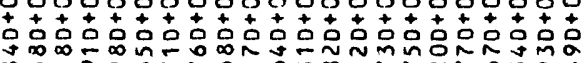

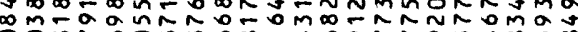

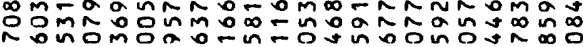
= aำ

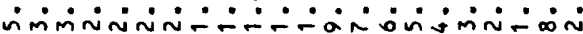

w

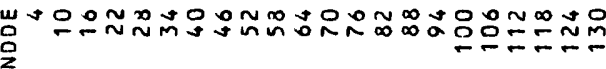

$\propto$

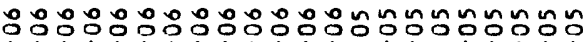

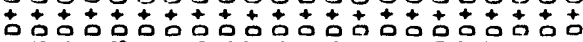
央

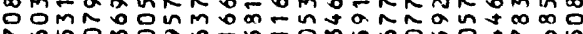
Nonomoao-n-omnoonosa on -

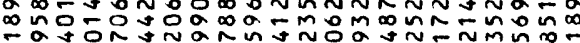

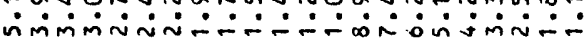

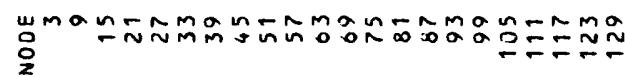

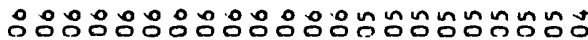

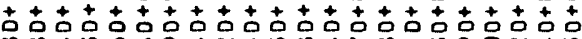

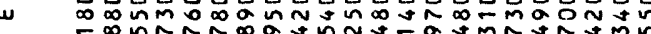

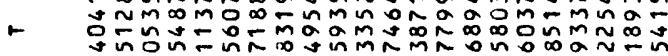

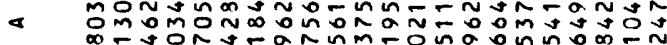

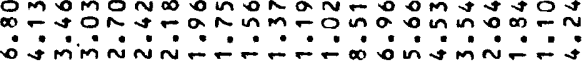

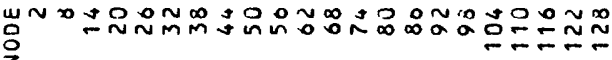
12

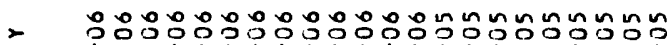

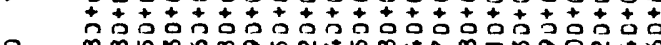

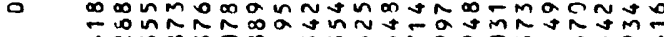

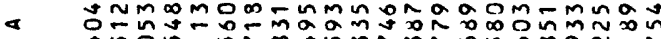
ง

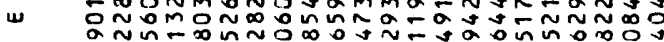

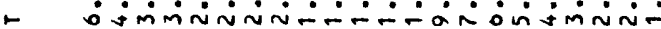

$\sim$

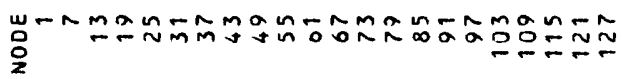




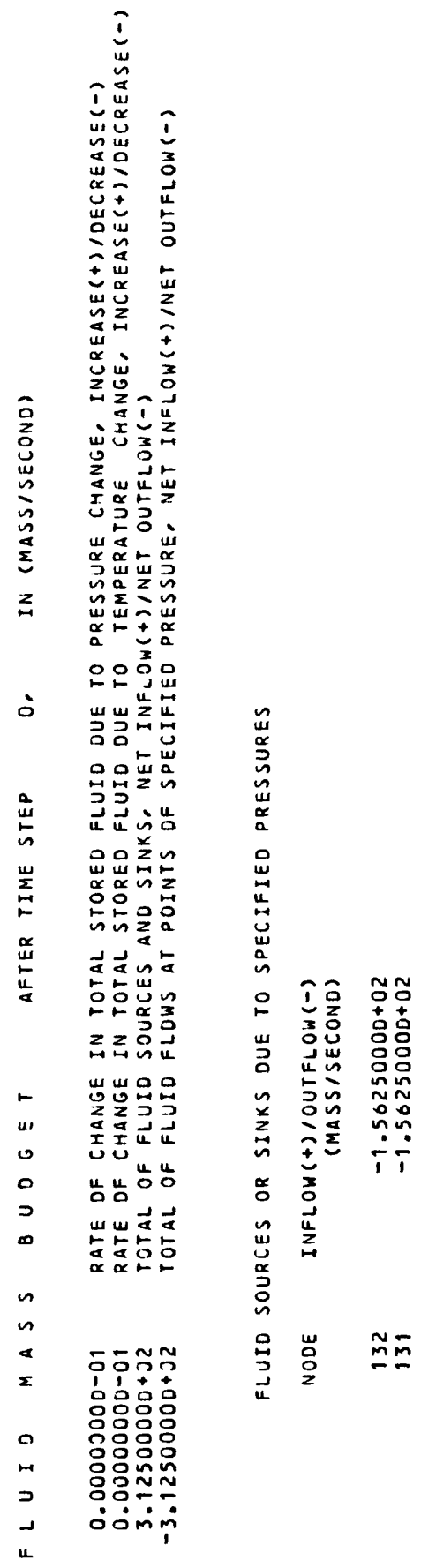




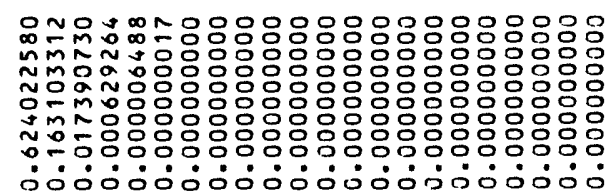

แ山ำ

운둔누

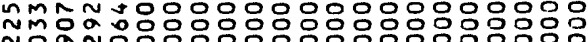

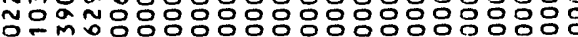
는 는응ㅇㅇㅇㅇㅇㅇㅇㅇㅇㅇㅇㅇㅇㅇㅇㅇㅇㅇㅇㅇㅇㅇㅇㅇㅇㅇㅇㅇㅇㅇㅇㅁㅇㅇㅇㅇㅇㅇㅇㅇㅇㅇㅇ

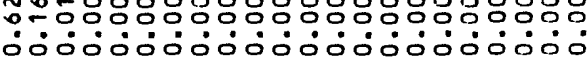

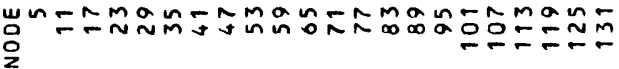

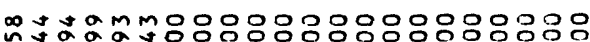

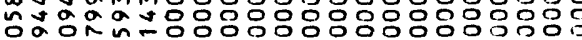
N N N

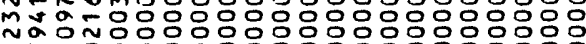

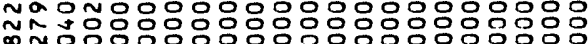

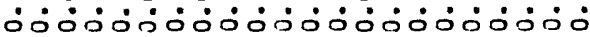

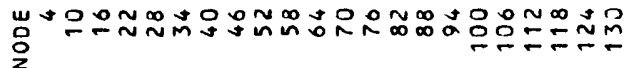

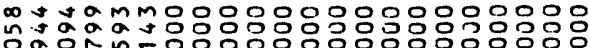

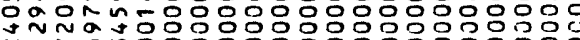

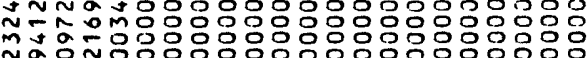

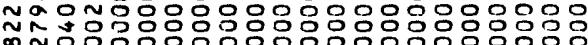

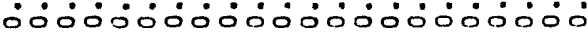

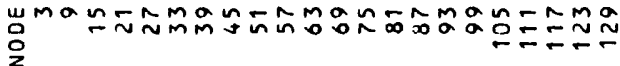
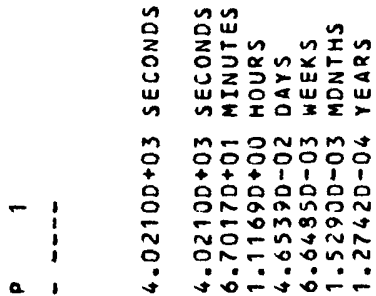

$\stackrel{a}{\leftarrow}$

$\sum_{i=1}^{\infty}$

然

$\stackrel{5}{ \pm}$

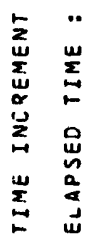

$\omega$

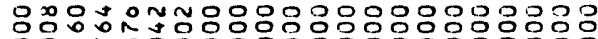

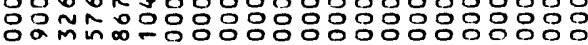
응수 눈

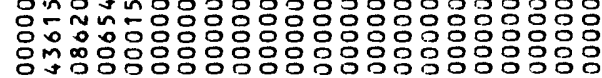

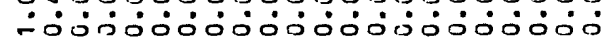

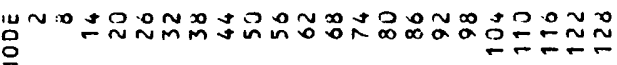

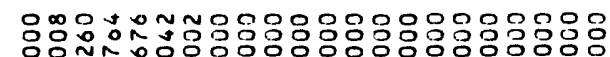

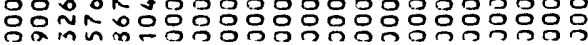

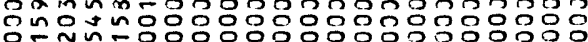

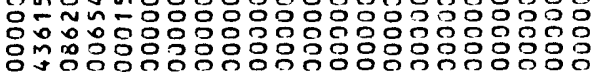

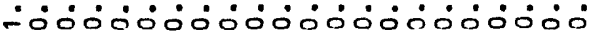

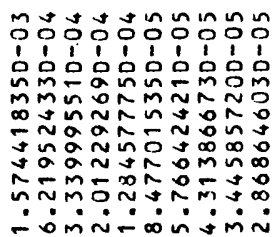

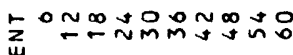

$\sum_{w}^{m}$

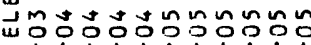

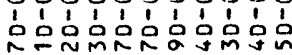

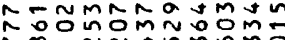

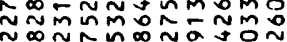

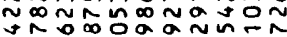

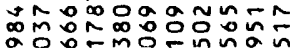
- $\dot{m} \dot{m} \dot{0} \dot{0} \dot{m} \dot{\sim} \dot{\sim}$

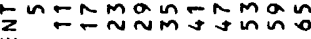
$\sum_{w}^{w}$

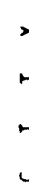

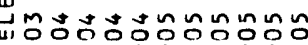

ilipipipi

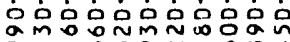

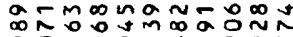

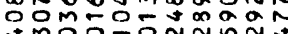

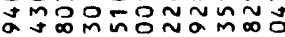

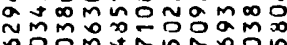
$\dot{\sim} \dot{0} \dot{i} \dot{0} \dot{0} \dot{0} \dot{m} \dot{m}$

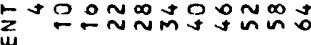
$\sum_{w}^{m}$

$>$

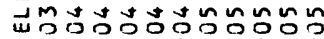
b́tón

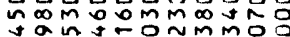

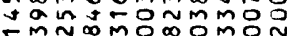

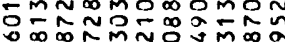
- - Táom n-o

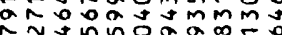

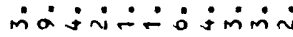

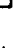

แั

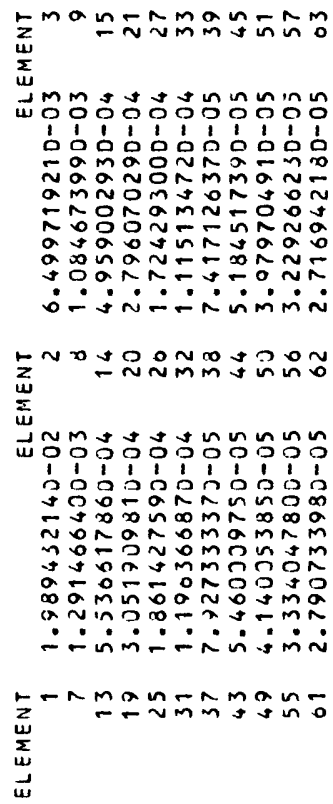




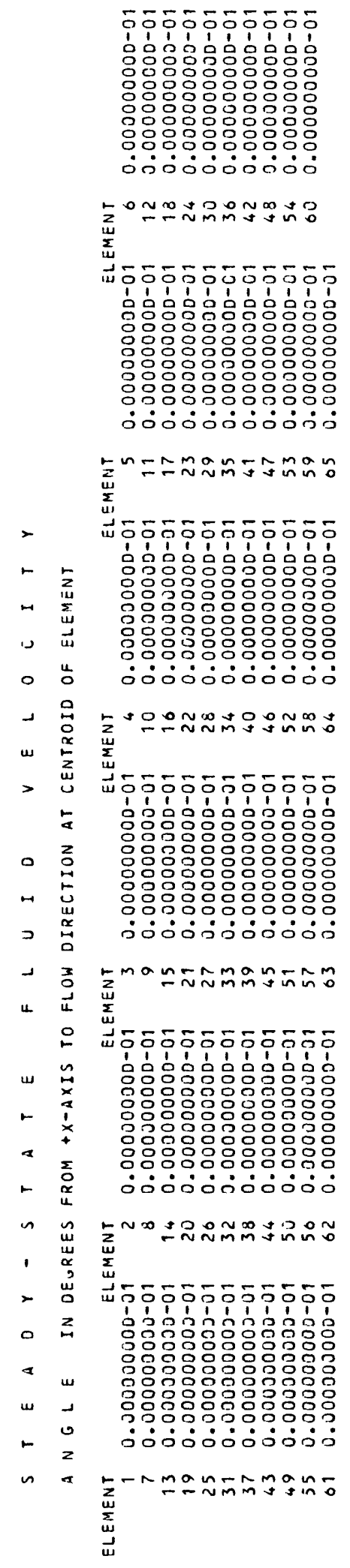




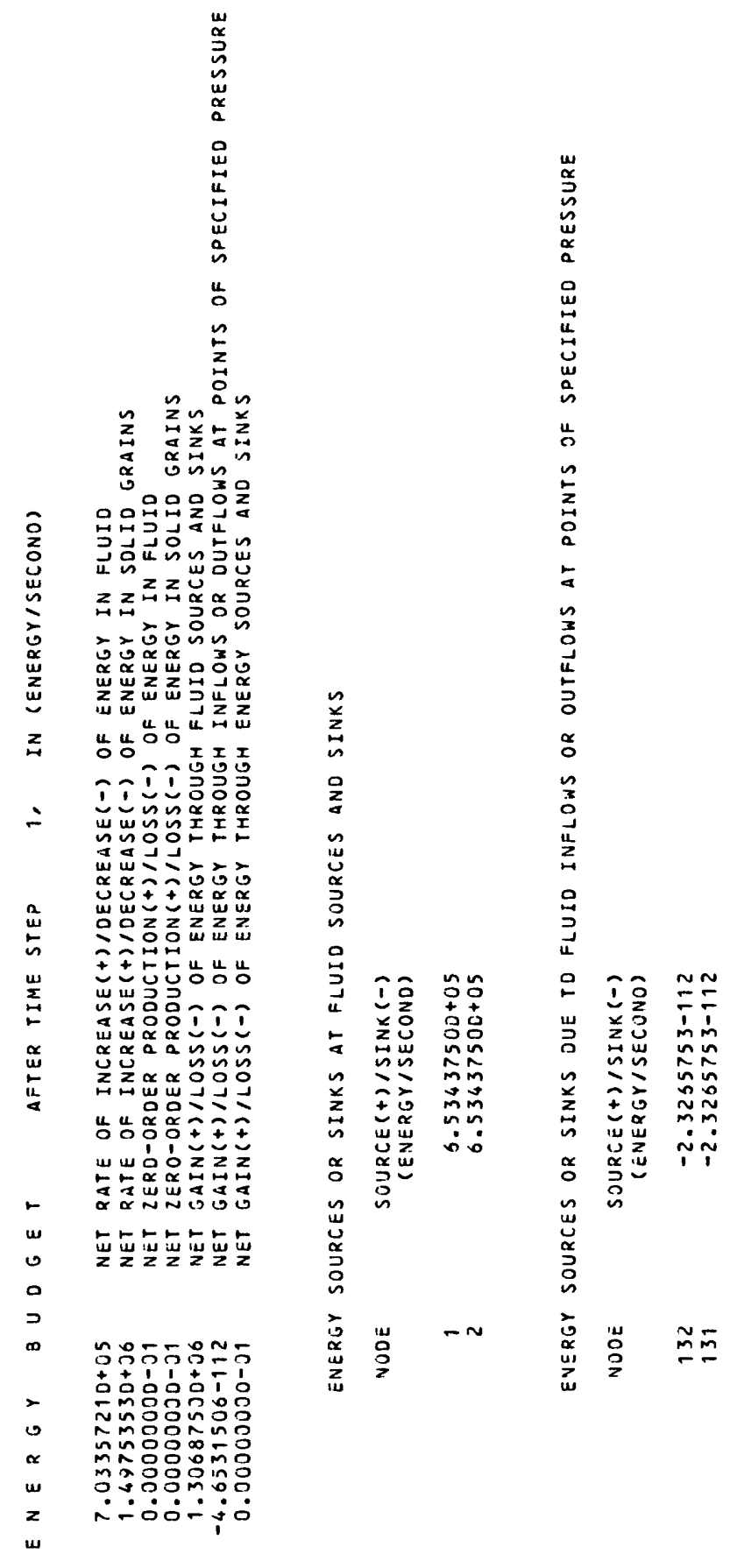



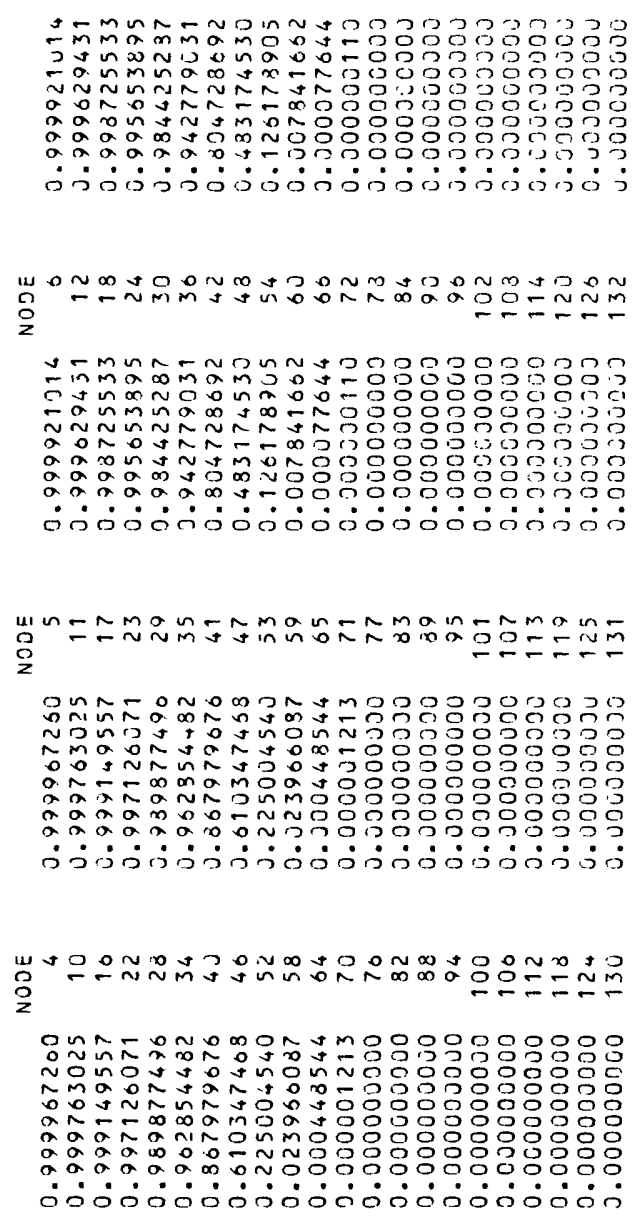

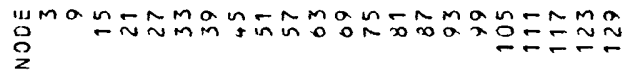
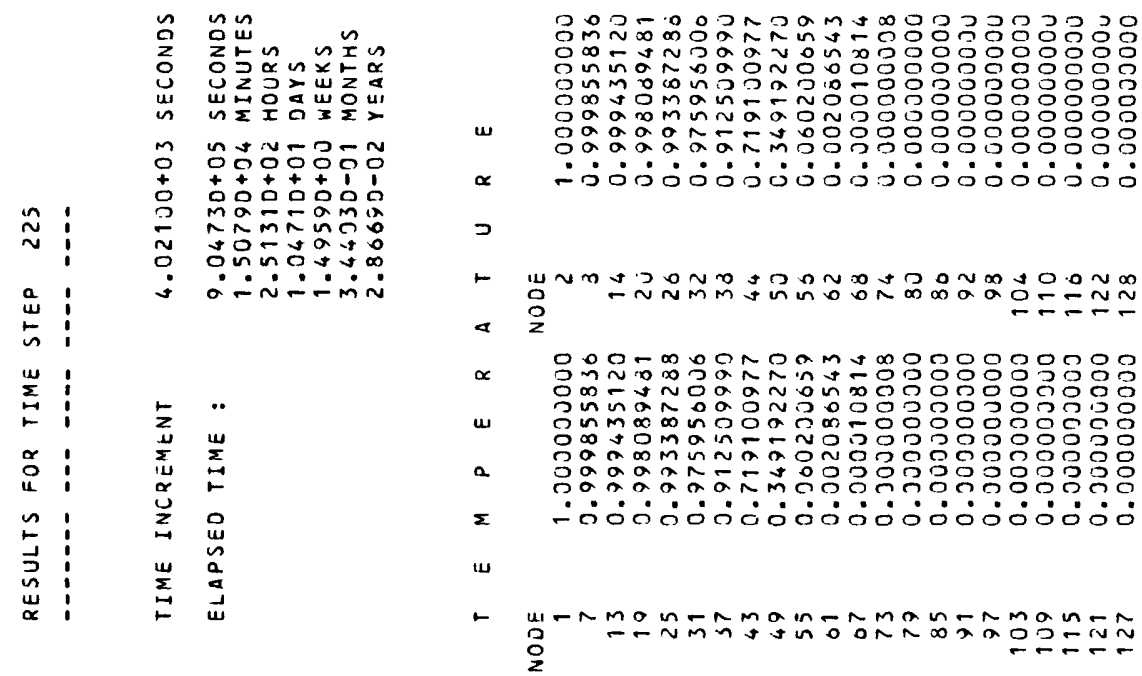


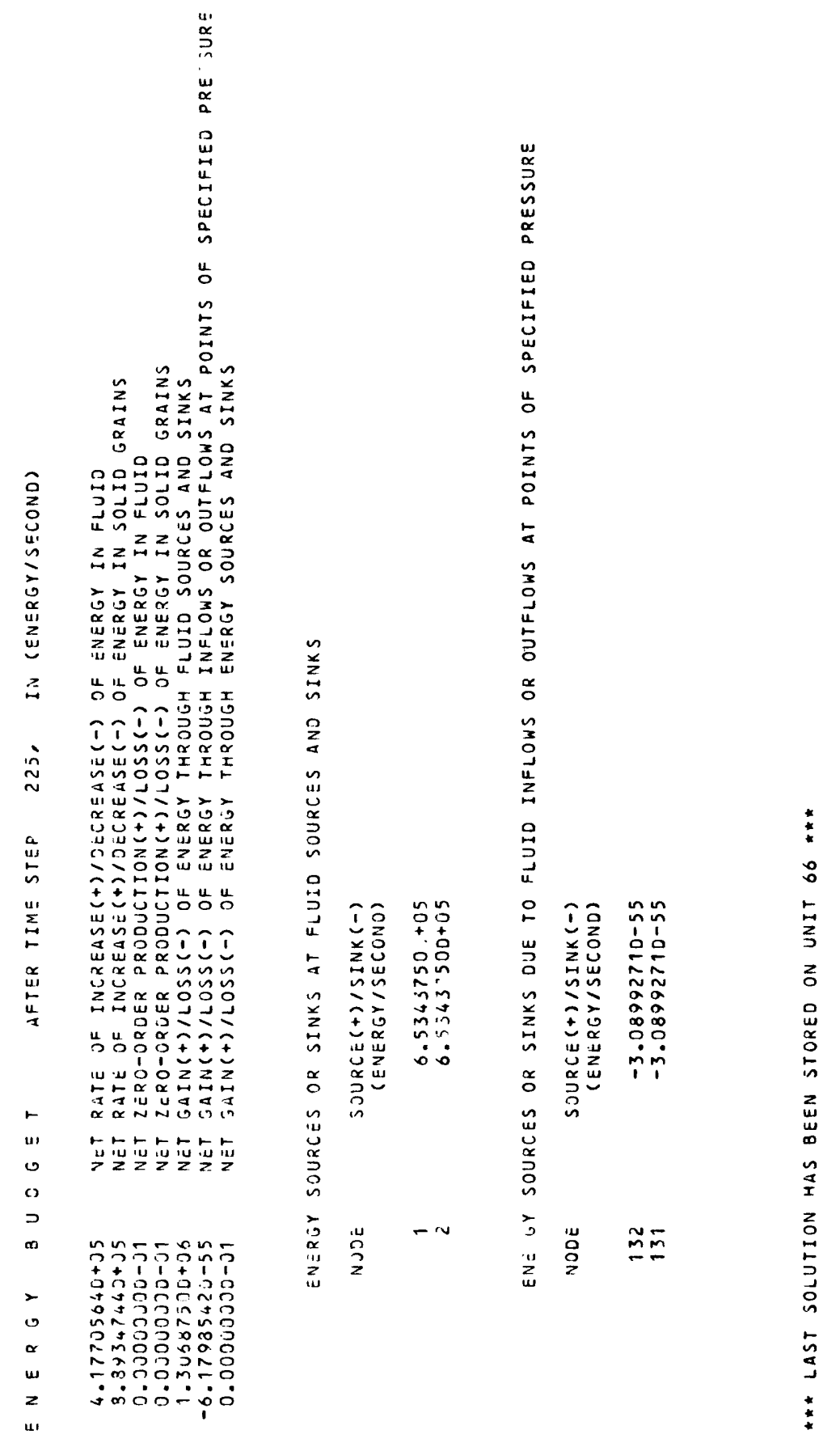



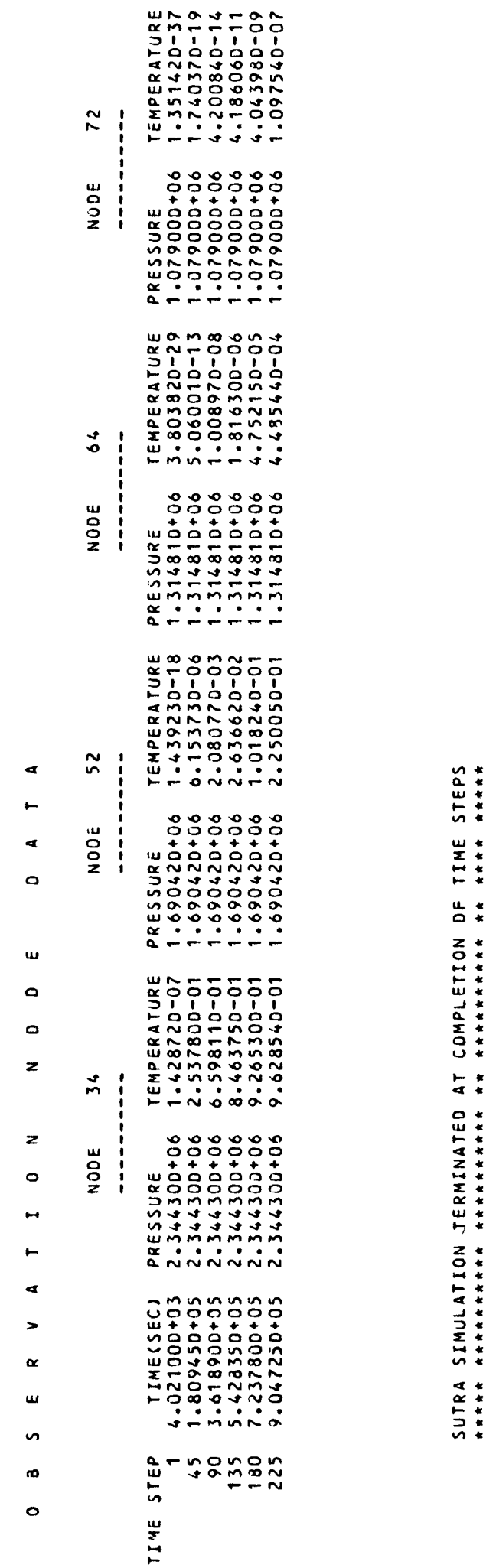